\title{
norden
}

\section{Arctic Human Development Report}

Regional Processes and Global Linkages

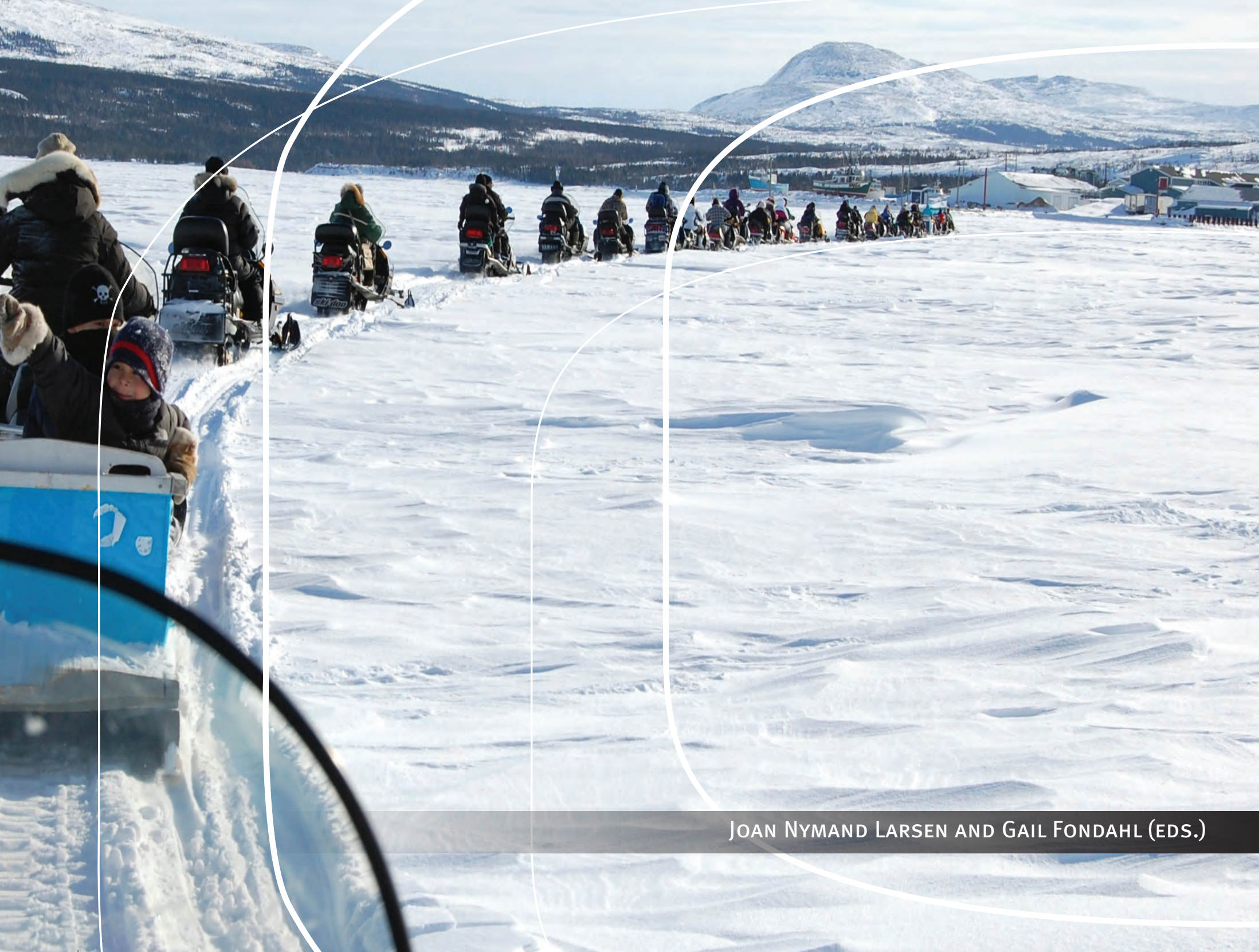



2 norden 



\section{Arctic Human Development Report}

Regional Processes and Global Linkages 
Arctic Human Development Report

Regional Processes and Global Linkages

ISBN 978-92-893-3881-3 (PRINT)

ISBN 978-92-893-3883-7 (PDF)

ISBN 978-92-893-3882-0 (EPUB)

http://dx.doi.org/10.6027/TN2014-567

TemaNord 2014:567

ISSN 0908-6692

(C) Nordic Council of Ministers 2014

Layout: Hanne Lebech

Cover photo: Rudolf Riedlsperger

Print: Rosendahls-Schultz Grafisk

Copies: 200

Printed in Denmark

This publication has been published with financial support by the Nordic Council of Ministers. However, the contents of this publication do not necessarily reflect the views, policies or recommendations of the Nordic Council of Ministers.

www.norden.org/en/publications

\section{Nordic co-operation}

Nordic co-operation is one of the world's most extensive forms of regional collaboration, involving Denmark, Finland, Iceland, Norway, Sweden, and the Faroe Islands, Greenland, and Åland.

Nordic co-operation has firm traditions in politics, the economy, and culture. It plays an important role in European and international collaboration, and aims at creating a strong Nordic community in a strong Europe.

Nordic co-operation seeks to safeguard Nordic and regional interests and principles in the global community. Common Nordic values help the region solidify its position as one of the world's most innovative and competitive.

\section{Nordic Council of Ministers}

Ved Stranden 18

DK-1061 Copenhagen K

Phone (+45) 33960200

www.norden.org 


\section{Content}

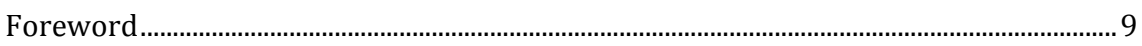

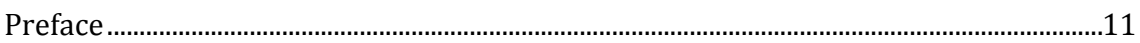

AHDR-II Acknowledgements ......................................................................................

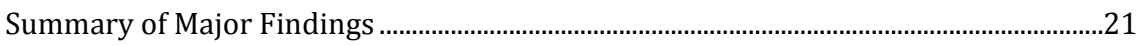

1. Introduction.

Gail Fondahl and Joan Nymand Larsen.............................................................................2

1.1 Why a second Arctic Human Development Report? Why now? .....................29

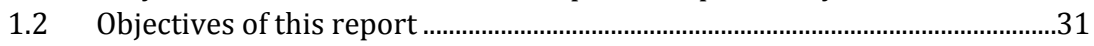

1.3 The rationale for a second Arctic Human Development Report.....................32

1.4 Progress on monitoring human development in the Arctic ............................38

1.5 AHDR-II's sub-title: regional processes and global linkages...........................42

1.6 Producing AHDR-II: scope, choices and challenges .........................................4

1.7 An overview of the chapters ..............................................................................46

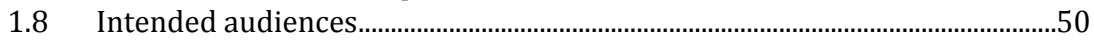

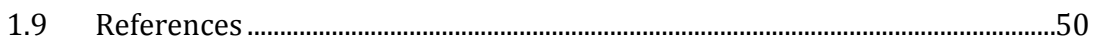

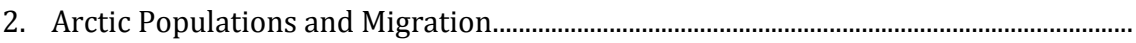

Timothy Helenia

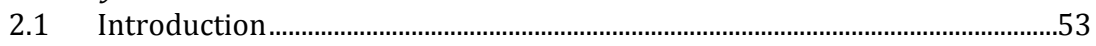

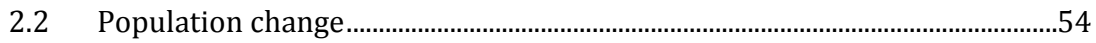

2.3 Changing composition of the Arctic population ...............................................69

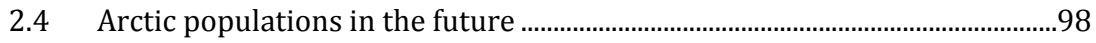

2.5 Summary of major trends ........................................................................101

2.6 Key conclusions and gaps in knowledge......................................................102

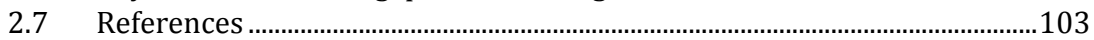

3. Cultures and Identities...................................................................................................

Peter Schweitzer, Peter Sköld and Olga Ulturgasheva..............................................105

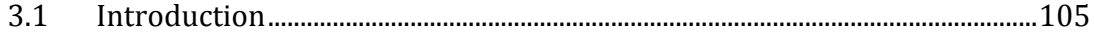

3.2 Brief historical background to circumpolar cultures and identities..........107

3.3 Aspects of circumpolar cultures....................................................................113

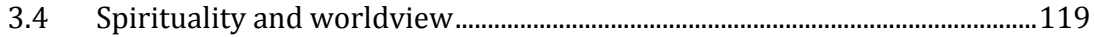

3.5 The arts and sports .........................................................................................122

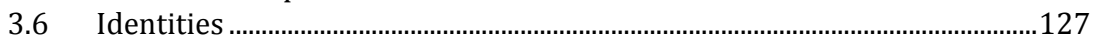

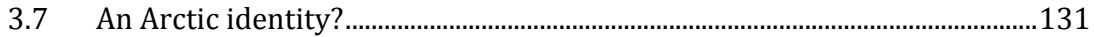

3.8 Quantifying ethnicity........................................................................................134

3.9 Cultural identities and "traditional" activities..................................................135

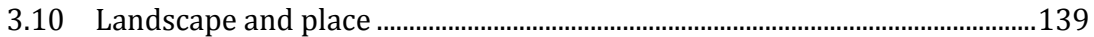

3.11 Identities in a changing Arctic........................................................................140

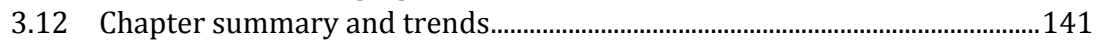

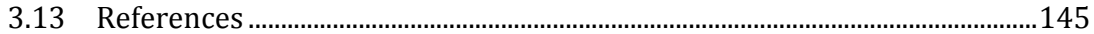


4. Economic Systems.

Lee Huskey, Ilmo Mäenpää and Alexander Pelyasov ……...........................................151

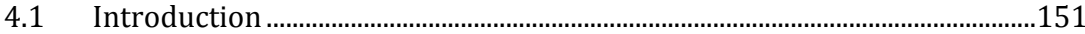

4.2 Arctic regions.............................................................................................................153

4.3 General overview of the Arctic economy.......................................................154

4.4 The three pillars of the Arctic economy .........................................................164

4.5 Variation in production around the North .......................................................169

4.6 The economy and the well-being of northern residents ...............................171

4.7 The future of the Arctic......................................................................................176

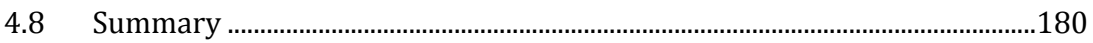

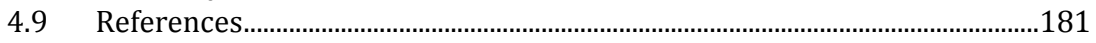

5. Governance in the Arctic: Political Systems and Geopolitics ..........................................

Greg Poelzer and Gary N. Wilson .................................................................................185

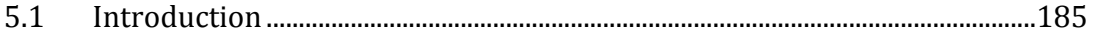

5.2 National political systems in comparative perspective ..................................188

5.3 Levels of Arctic governance: local, regional and national..............................194

5.4 Geopolitics and governance among Arctic political communities ..............209

5.5 Regional collaboration....................................................................................215

5.6 Key conclusions ............................................................................................219

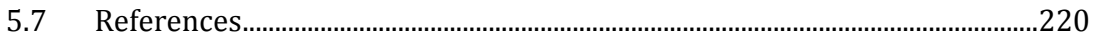

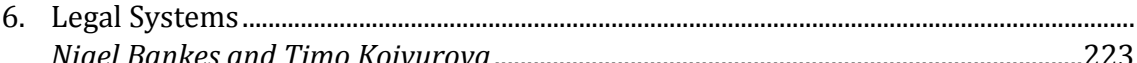

6.1 The multiple legal orders of the Arctic .......................................................225

6.2 Developments in international law pertaining to Indigenous peoples .........................234

6.3 The delimitation of continental shelf boundaries between Arctic states and the extended continental shelf entitlements of Arctic

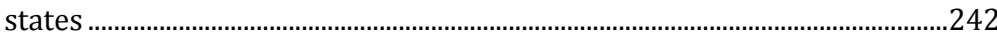

6.4 Chapter conclusions and gaps in knowledge ................................................248

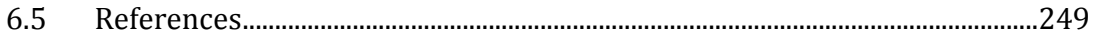

7. Resource Governance

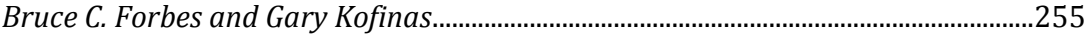

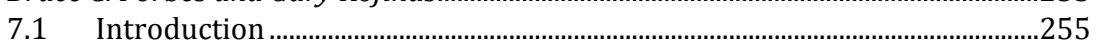

7.2 Putting Arctic resource governance in a global context..............................258

7.3 Local resource management systems ……..................................................258

7.4 From co-management to adaptive co-management? ..................................259

7.5 Responding to climate change........................................................................260

7.6 Integrating knowledge systems in Arctic resource governance ..................260

7.7 Case studies............................................................................................................26

7.8 Conclusion....................................................................................................28

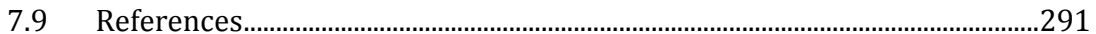


8. Human Health and Well-Being

Arja Rautio, Birger Poppel and Kue Young.............................................................299

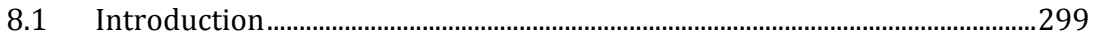

8.2 Health and well-being in the Circumpolar North............................................. 300

8.3 Continuing health disparities .............................................................................302

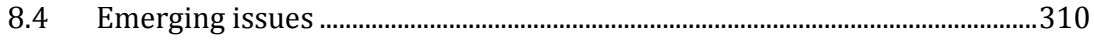

8.5 Changing quality of life......................................................................................315

8.6 Application of the United Nations' Human Development Index

(HDI) to the Circumpolar Arctic .........................................................................318

8.7 Including additional dimensions in the assessment of human development.

8.8 What can we learn about human development from different community, regional, national, circumpolar studies? .

8.9 Findings from studies assessing Arctic Human Development by a number of key social indicators

8.10 Studies assessing Arctic human development by measuring subjective or community well-being/quality of life..

8.11 Findings from studies assessing Arctic human development by measuring subjective or community well-being/quality of life....................329

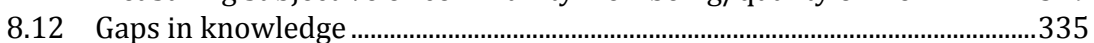

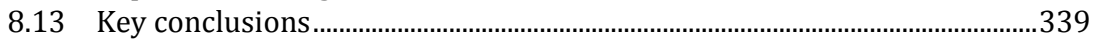

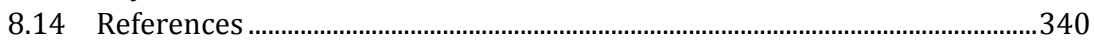

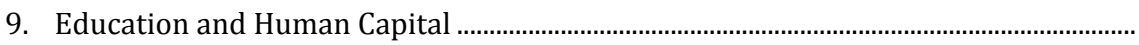

Diane Hirshberg and Andrey N. Petrov ........................................................................349

9.1 Introduction...........................................................................................................349

9.2 Education and educational systems in the Arctic ...........................................351

9.3 Human capital and knowledge in the Arctic ....................................................365

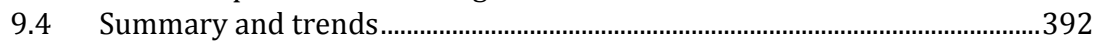

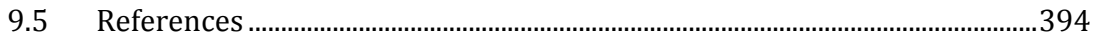

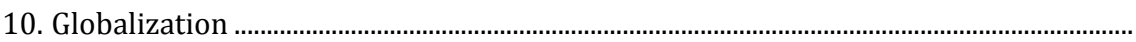

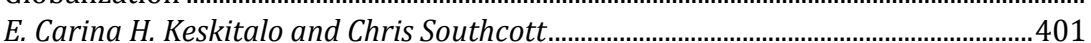

10.1 Introduction..................................................................................................... 401

10.2 Globalization as a concept and its potential impacts on the Arctic ............ 402

10.3 Economic globalization: local interests, external actors and unstable markets ......................................................................................................... 403

10.4 Political globalization: are increasingly large scales of governance both empowering and limiting? ..........................................................................413

10.5 Cultural and social globalization: potentially increased

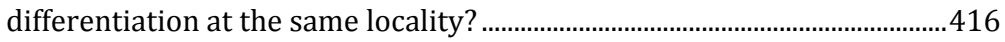

10.6 Summary of major trends and gaps in knowledge .........................................419

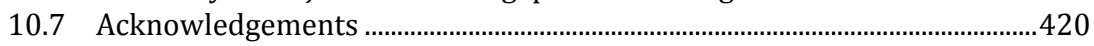

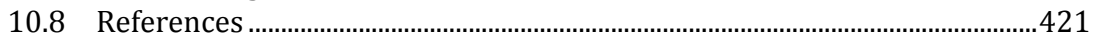


11. Community Viability and Adaptation.

Rasmus Ole Rasmussen, Grete K. Hovelsrud and Shari Gearheard .............................427

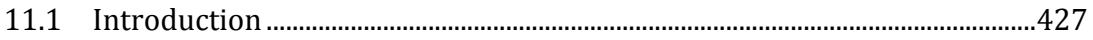

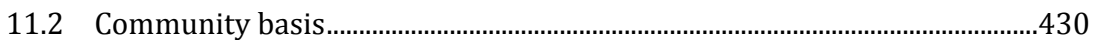

11.3 Community dynamics ......................................................................................44

11.4 Community perspectives .................................................................................461

11.5 Challenges influencing community viability in the Arctic ............................466

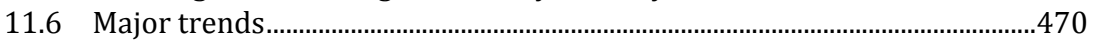

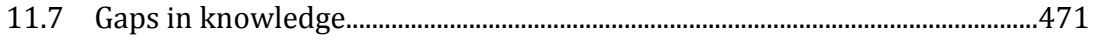

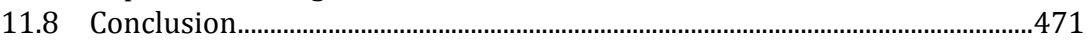

11.9 References.....................................................................................................4

12. Major Findings and Emerging Trends in Arctic Human Development.......................... Joan Nymand Larsen and Gail Fondahl .......................................................................479

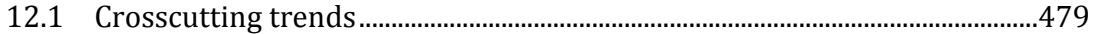

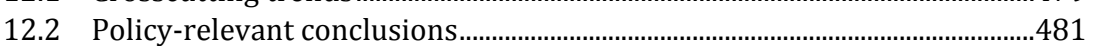

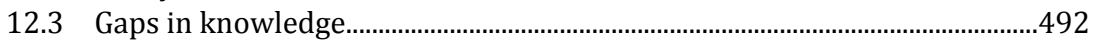

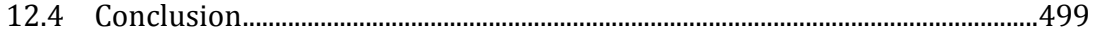

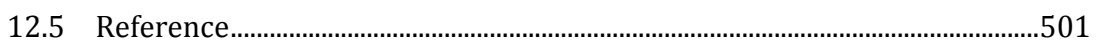

AHDR Sammenfatning .........................................................................................................503 


\section{Foreword}

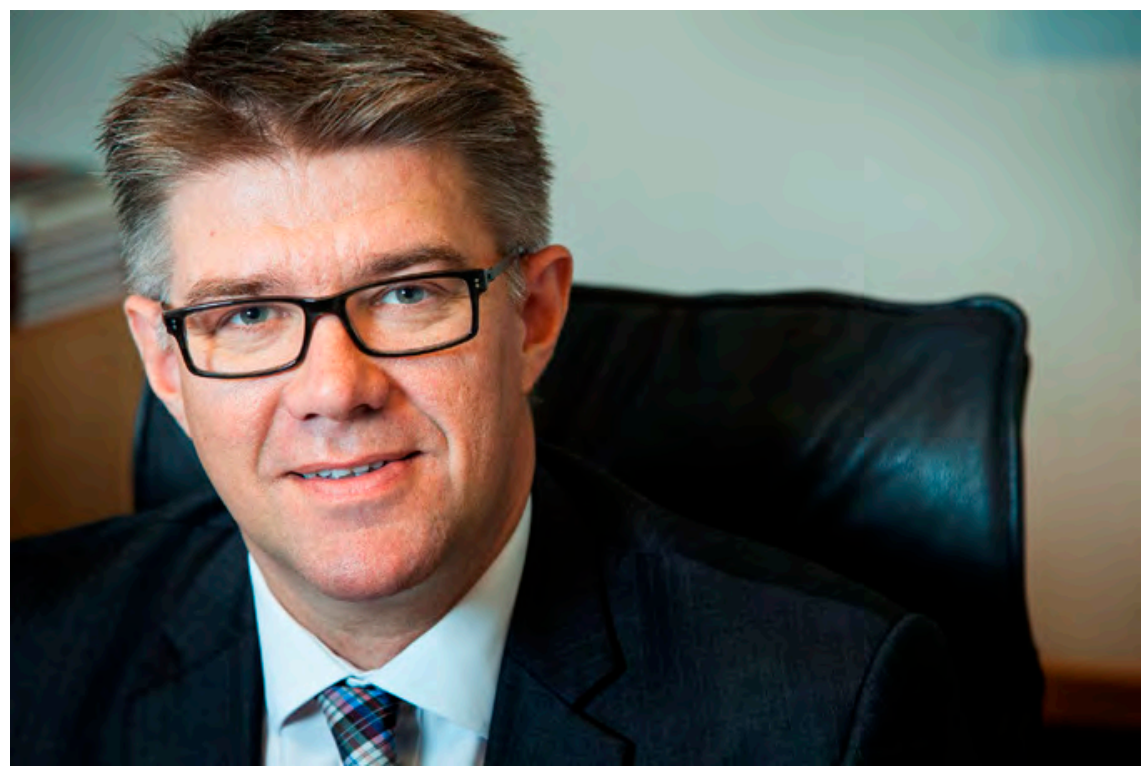

It is with great pleasure that I present this second volume of the Arctic Human Development Report (AHDR): Regional Processes and Global Linkages, initiated by the Stefansson Arctic Institute, Iceland, and presented to the Sustainable Development Working Group of the Arctic Council for project endorsement in the fall of 2011.

On the tenth anniversary of the first Arctic Human Development Report this second volume offers an overview of the contrasts in an era marked by changes, at both far more rapid pace and to a greater extent than anticipated. People living in the Arctic are aware of the region's dynamics that have shaped our livelihood and history through time. Therefore the authors of this report make a welcome effort to cover many of the critical and emerging challenges we are facing in the region and thus deepening our knowledge about the impacts of global changes that are interlinked and have profound implications for sustainable development in the Arctic.

Preparation and writing of the Arctic Human Development Report II was carried out by an international group of leading scientific experts on Arctic issues. The management and scientific coordination of the project 
was led by the Stefansson Arctic Institute, which hosted the Secretariat supported by an Executive and Advisory Committee. In particular I would like to thank project leader Dr. Joan Nymand Larsen from Iceland and project co-leaders Dr. Gail Fondahl from Canada and Ms. Henriette Rasmussen from Greenland for their great dedication, efforts and skills in leading and coordinating this ambitious and challenging work. I would also like to thank the numerous lead authors and contributing authors who gave so generously of their time and expertise and without whom this volume would not have been possible.

In presenting the Arctic Human Development Report II, I acknowledge that the present report, like the first one, is an academic report and the fruit of a close and long-term collaboration of an extensive network of experts in Arctic sciences. Although the report reflects neither the policies of the Arctic States nor the position of the Arctic Council, this report, like other scientific research reports can serve as an important source of valuable information to help deepen our understanding of human development in the Arctic. Furthermore, our role is to ensure that the work of the scientific community feeds into public debate, whereas public policy is supported by a strong knowledge base. Improved understanding of our circumstances helps governments identify problems and strengthens our ability to implement solutions aimed at supporting strong and sustainable communities in the Arctic.

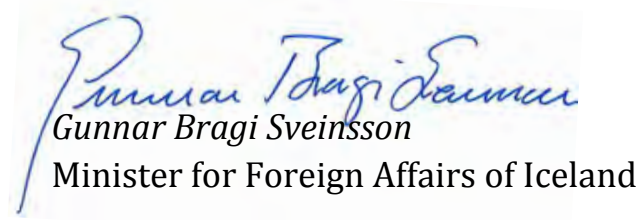




\section{Preface}

We are pleased to present this second volume of the Arctic Human Development Report: Regional Processes and Global Linkages (AHDR-II). The report was initiated by the Stefansson Arctic Institute in 2010, and together with the Icelandic representation on the Sustainable Development Working Group (SDWG) of the Arctic Council it was presented to the Council in 2011 for project endorsement. The startup work on this second volume was met with great enthusiasm, including by members of the Arctic Council, Parliamentarians of the Arctic Region, the scientific community and educators throughout the North.

The first $A H D R$ - the priority project of the Icelandic Chairmanship of the Arctic Council 2002-2004 - was launched in November, 2004. The production of $A H D R-I I$ on the tenth anniversary of the first $A H D R$ makes it possible to move beyond the baseline assessment to make valuable comparisons and contrasts across a decade of persistent and rapid change in the North. This new volume addresses critical issues and emerging challenges in Arctic living conditions, quality of life in the North, global change impacts and adaptation, and Indigenous livelihoods. The report is designed to integrate new knowledge from a diverse set of disciplines, to highlight key messages and major current and emerging trends, and to identify gaps in our knowledge.

AHDR-II was prepared by a working group consisting of 25 lead authors and 57 contributing experts. The volume is organized into ten substantive chapters. Eight are devoted to a thematic area of key relevance to Arctic human development and two chapters present issues that cut across these themes - Globalization and Community Viability \& Adaptability. The volume focuses on changes in each of these areas since the first $A H D R$ was published in 2004.

The $A H D R$ process began in 2002, with a mandate to present the most up-to-date information about the state of Arctic human development and the major trends unfolding in the region. The AHDR and its follow-up projects on Arctic social indicators (ASI) have since then played an important role in facilitating discussions about impacts of change in Arctic societies. AHDR (2004) provided important baseline information for the Arctic Council, policymakers, and others who deal 
with issues of human development and societal transformation in a time of rapid environmental, cultural, political, economic and social change.

Discussion about producing a sequel to the first AHDR commenced in mid-2010. Once funding had been assured from the Nordic Council of Ministers, and dialogue initiated with the Arctic Council's SDWG, the Northern Forum, and other key organizations, a call for nomination of Arctic experts to serve as lead authors was disseminated widely via several Arctic electronic mailing lists. The call resulted in over 120 nominations. From these names, the project lead and co-leads together with the Report's Executive Committee selected lead authors, using criteria of representation of all of the Arctic states, various academic disciplines, both genders, and balancing the desire to have a number of authors who had participated in the first $A H D R$ with a number of new contributors. Lead authors, in turn, were responsible for recruiting contributing authors to offer further expertise.

We aspired to have strong representation of Indigenous scholars among the lead authors. However, among the several invitations forwarded to Indigenous scholars, only one responded positively: others declined due to the many demands they face on other fronts. We then entreated lead authors to consider including Indigenous scholars among their contributing authors. Among the report's contributing authors are nine Indigenous scholars. To further address the desire for representation of Indigenous perspectives, on advice of the SDWG's Permanent Participants, we sought to incorporate more Indigenous persons in the review of the report's chapters.

Lead authors reviewed each other's chapter outlines; these outlines were also provided to the SDWG for review. Second stage drafts of the chapters were externally peer-reviewed, in a process handled by the International Arctic Science Committee (IASC), whose help we gratefully acknowledge. Two or more scholars reviewed each chapter. The second draft chapters were also provided to SDWG for review. During the writing of the report, several authors presented their work at conferences, in order to receive further input and review from colleagues.

The growing challenges to human well-being in the Arctic provide the context that underlies this second volume, as seen in the explicit coverage of a larger number of topics, including one new chapter and the inclusion of new and emerging issues in several others. Increasing knowledge, expressed in a growing volume of published literature in particular since the International Polar Year (IPY) 2007-2008, enables our deeper assessment of a range of topics. We have also benefited from developments in Arctic social sciences since the first $A H D R$, including the 
greater participation of indigenous peoples, the increased volume of social science research on Arctic issues, the better integration of traditional and local knowledge in research, and the broader discussion of the complexity of Arctic change. Thus this second volume aims to contribute to a richer, more nuanced understanding of Arctic human development, and to help expand our knowledge of the thematic areas presented in the first $A H D R$.

In terms of its scope, AHDR-II spans a wide range of topics: Arctic populations and migration, culture and identities, economy, politics, legal issues, resource governance, health and well-being, and education and human capital. Interwoven through all of these topics are issues of gender and the influences of a changing climate, and changing global and regional political, economic, cultural and social processes. With the deepening of knowledge about Arctic human development, we see more connectivity among these areas. Early research focused more on the immediate changes within Arctic regions and local communities, whereas new evidence points increasingly to the regional processes, the global linkages, and the complex interactions between different sources and scales of changes. Thus, the individual chapters address observed and projected climate change impacts and their interaction with other sources of change, global processes other than climate/environmental change, and gendered dimensions of each chapter's thematic area.

$A H D R$ II is an academic report written for several audiences - governments, communities, and other stakeholders at all levels, but also very much with the Arctic Council in mind: the report aims to help inform its work and that of the SDWG in particular, in furthering sustainable development in the Arctic. As a second circumpolar assessment of human development and quality of life in the Arctic that identifies important and emerging issues relating to sustainable human development in the Arctic, the report provides a basis for the development of policies and actions to address these issues. In addition, the report will hopefully be an important contribution to the instructional material for northern colleges and universities, and an accessible handbook for non-Arctic stakeholders seeking to know more about change and emerging trends in the North. 
The work of organizing and preparing the AHDR-II report was supported by an Executive Committee, and the Secretariat for this project was located at the Stefansson Arctic Institute. This volume was made possible thanks to the commitment and voluntarism of a large number of leading Arctic social scientists. We express our gratitude to all the authors and contributing experts, and others who contributed to this process.

Joan Nymand Larsen Project Leader (Iceland)

Stefansson Arctic Institute
Gail Fondahl

Co-lead (Canada)

University of Northern British Columbia 


\section{AHDR-II Acknowledgements}

\section{Project Leadership}

Joan Nymand Larsen, Iceland (Chair)

Gail Fondahl, Canada (Co-Chair)

Henriette Rasmussen, Denmark/Greenland (Co-Chair)

\section{Editors}

Joan Nymand Larsen

Gail Fondahl

Editorial Assistant

Mara Kimmel

Executive Committee

Einarsson, Níels

Fondahl, Gail

Hovelsrud, Grete

Larsen, Joan Nymand

Petrov, Andrey

Rasmussen, Henriette

Rautio, Arja

Retter, Gunn-Britt

Lead Authors

Bankes, Nigel

Fondahl, Gail

Forbes, Bruce C.

Gearheard, Shari

Heleniak, Timothy

Hirshberg, Diane

Hovelsrud, Grete K.

Huskey, Lee

Keskitalo, E. Carina H.

Kofinas, Gary

Koivurova, Timo

Larsen, Joan N.

Mäenpää, Ilmo 
Pelyasov, Alexander

Petrov, Andrey

Poelzer, Greg

Poppel, Birger

Rasmussen, Rasmus Ole

Rautio, Arja

Schweitzer, Peter

Sköld, Peter

Southcott, Chris

Ulturgasheva, Olga

Wilson, Gary N.

Young, Kue

\section{Contributing Authors and Experts}

Allard, C.

Amundsen, $\mathrm{H}$.

Baker, B.

Barnhardt, R.

Beach, $\mathrm{H}$.

Berkman, P.

Berman, M.

Bogoyavlenskiy, D.

Brattland, C.

Broderstad, E. G.

Cavin, P.

Crate, S.

Dale, B.

Duyck, S.

Emelyanova, A.

Ford, V.

Hansen, K.G.

Hansen, T. T.

Haraldsdóttir, K.

Henriksen, T.

Hodgkins, A.

Hoogensen Gjörv, G.

Howe, L.

Huntington, $\mathrm{H}$.

Ignatyeva, I.

Ingimundarson, J.H.

Ivanova, A.

Johnson, N. 
Joona, J.

Juutilainen, S.

Kankaanpää, P.

Keskitalo, J.H.

Kimmel, M.

Krupnik, I.

Kryazhkov, V.

Leary, D.

Lennert, $\mathrm{M}$.

Loukacheva, N.

Maheux, G.

McGregor, $\mathrm{H}$.

Meek, C.L.

Metcalf, V.K.

Mortensen, B.O.G.

Olsen, J.

Olsen, P.C.C.

Paul, V.

Ravna, Ø.

Rayfuse. R.

Robards, M.D.

Rönkä, A.

Roto, J.

Shadian, J.

Sleptsov, A.

Sloan, L.

Stammler, F.

Stepien, A.

Sunnari, V.

Tollefsen, A.

Uitangak, E.

VanderZwaag, D.

Ventsel, A.

Young, O.R.

Maps

Johanna Roto (Nordregio maps)

Julien Gruenfelder

José Sterlings

Yulia Zykova

Winfried Dallmann (AHDR Arctic map) 
Special thanks to Nordregio for the production of maps for AHDR-II.

\section{Peer Reviewers}

Anderson, D.

Berman, M.

Bjerregaard, P.

Cameron, E.

Chapin, $\mathrm{T}$.

Christensen, J.

Collignon, $\mathrm{B}$.

Conde Perez, E.

Crate, S.

Eningowuk, K.

Evengård, $\mathrm{B}$.

Haftendorn, $\mathrm{H}$.

Hamilton, L.

Hansen, G.H.

Helander, E.

Hickel, J.

Huntington, $\mathrm{H}$.

Kotyrlo, E.

Luszczuk, M.

Natcher, D.

Sambo Dorough, D.

Takakura, $\mathrm{H}$.

Vlasova, T.

We also extend our special thanks to Volker Rachold of the International Arctic Science Committee (IASC) for organizing the international peer review process.

The cooperation of the International Arctic Social Science Association (IASSA) and the University of the Arctic (UArctic) is gratefully acknowledged.

We thank the members of the Arctic Council's Sustainable Development Working Group, including the Permanent Participants, for their review and input at several stages of the project. Many individuals offered their expert comments through the States and organizations listed below.

- Aleut International Association (AIA)

- Arctic Athabaskan Council (AAC)

- Canada

- Denmark/Greenland/Faroe Islands 
- Finland

- Gwich'in Council International (GCI)

- Iceland

- Indigenous People's Secretariat (IPS)

- Inuit Circumpolar Conference (ICC)

- Norway

- Russian Association of Indigenous Peoples of the North (RAIPON)

- Russian Federation

- Saami Council

- Sweden

- United States of America

Special thanks are due to Harald Finkler, Jónas Gunnar Allansson and Atli Már Sigurðsson and Jutta Wark.

\section{Student Research Assistants}

Kormos, J.

Lorentzen, R.L.

Ohman, T.

Laiho, M.

Meetings - Organizational Support

Bitsch, S.

Ingimundarson, J. H.

Ólafsdottir, L.

\section{Financial Support}

The Arctic Human Development Report II could not have been completed without the generous financial support from the following:

- Government of Iceland. Ministry for Foreign Affairs

- Icelandic Ministry for the Environment and Natural Resources

- Stefansson Arctic Institute

- Nordic Council of Ministers, Arctic Cooperation Programme

- U.S. National Science Foundation

- University of Northern British Columbia

- Academy of Finland

- Alaska, State of

- Finnish Ministry of the Environment

- Government of Canada. Department of Foreign Affairs and International Trade and Department of Aboriginal Affairs and Northern Development 
- International Arctic Science Committee (IASC), including its Social and Human Sciences Working Group

- MISTRA, Swedish Foundation for Strategic Environmental Research

- Nordland Research Institute

- Norwegian Institute of Cultural Heritage Research (NIKU)

- Norwegian Ministry of Foreign Affairs

- Oak Foundation

- Trust for Mutual Understanding

- U.S. Fish and Wildlife Service

- U.S. National Aeronautics and Space Administration

- US National Park Service Shared Heritage Beringia Program

The above list of contributors to the AHDR-II is not comprehensive; numerous other individuals have contributed to the project in many different ways but are not mentioned by name. 


\title{
Summary of Major Findings
}

\author{
Joan Nymand Larsen and Gail Fondahl
}

The goals of the second volume of the (AHDR-II) Arctic Human Development Report: Regional Processes and Global Linkages - are to provide an update to the first AHDR (2004) in terms of an assessment of the state of Arctic human development; to highlight the major trends and changes unfolding related to the various issues and thematic areas of human development in the Arctic over the past decade; and, based on this assessment, to identify policy relevant conclusions and key gaps in knowledge, new and emerging Arctic success stories, and important AHDR-II follow-up activities.

\section{Policy-relevant conclusions}

The report contains a broad range of policy-relevant conclusions, many of which are specific to individual thematic areas covered in the chapters. Based on these findings we offer a set of cross-cutting trends with relevance for policy makers at various levels, including priority setting by the Arctic Council and its Sustainable Development Working Group:

- The combination of rapid and stressful changes highlighted in the first $A H D R$ (2004) continues today, amplified in rate and magnitude. These societal and environmental changes confront Arctic residents, local communities, and socioeconomic sectors and challenge their wellbeing. Gaps in development and human well-being persist between different groups, genders, levels, and geographical locations. At the same time, we note trends of increased indigenous empowerment and improved local political and economic autonomy.

- Urbanization in the Arctic is accelerating, propelled both by local and global forces. Outmigration from rural communities toward larger settlements and urban centers, increased concentration of the population and the emergence of "climigration" are notable trends. Rural depopulation, "brain drain" and "brain waves" related to changes in the economy and resource development and to growing 
aspirations for formal education, population aging and associated changing dependency ratios, new flows of immigrants to the Arctic and related cultural negotiations and contestations - in short the magnitude and complexity of migration and urbanization - pose multifaceted challenges to human development in the Arctic.

- Interest in the Arctic has mushroomed, due in large part to climate change and expected resource development opportunities, and the media hype regarding these developments. The Arctic is becoming more "marketable" and Arctic identities are seen increasingly as an asset. Concomitantly Arctic residents are themselves redefining what it means to be "a Northerner".

- An increasing trend of legitimate participation in Arctic decisionmaking and continued innovation in governance can be observed at all scales. At the same time, as we witness greater self-determination and autonomy, and improved indigenous representation in regional, national and international bodies, the demands on local and indigenous representatives are stretching resources, human and fiscal, to the limit. These are challenges that need to be resolved if the positive trajectories are to be maintained.

- Expectations are high for the expansion of resource extractive industries; we note also the growth in importance of non-resource extractive industries. Interest in economic diversification, within and beyond extractive industries, is rising. While optimism surrounding resource extractive activities remains, so does the high cost of doing business in the North. Climate change is not likely to change this reality, as benefits related to increased accessibility will be balanced or even outweighed by infrastructure damage.

\section{Arctic success stories}

The current assessment of human development in the Arctic has shown that the region continues to face significant challenges, yet at the same time, there are many examples of Arctic success stories that must be told, and they include:

- The increasing use of Indigenous knowledge: There is an increasing use of Indigenous languages in formal schooling in the Arctic, and a growing recognition of the importance of local and Indigenous knowledge in many aspects of Arctic life. Indigenous knowledge is 
increasingly employed in education, governance, and other spheres of Arctic life.

- The increase in local participation, control and ownership: Recent institutional changes in the North have increased the local control and ownership of northern resources in some parts of the Arctic, and have had an important effect on the relationship between international and local economies.

- The continued growth of innovative governance arrangements: The efforts of indigenous peoples to achieve self-determination and forms of self-government have been fundamental to the success of the emergence of new and innovative forms of governance, and the growth of regional autonomy.

- The emergence of Arctic identities and a sense of Indigenous and, more broadly, Northern identity becoming an asset: Culture, especially indigenous culture in the North, has increasingly become a resource, both in the sense of a commodity and in the sense of a tool that makes external recognition easier. Also, there is a growing sense of the marketability of the North, which may present certain advantages to being in the North.

\section{Gaps in knowledge}

This second assessment of the state of human development in the Arctic, and its focus on the major trends unfolding in these times of rapid change, has brought attention to a number of critical gaps in knowledge about human development and living conditions in the North. These include:

- Understanding the needs of Arctic youth and elderly - The population is aging in many parts of the Arctic, triggering the need to better understand the social, cultural, economic and political role this segment of the population does and could play. Qualitative research is needed to investigate both the needs of older people, including their gendered dimensions, and available capacities of regional policy-makers to respond to those needs. At the same time, the future of the Arctic is dependent on its youth. The aspirations and ambitions of Arctic youth (and their gendered dimensions) need to be understood.

- Arctic social indicators and monitoring - As a direct follow-up to recommendations made in the first AHDR (2004), the Arctic Social 
Indicators project developed a set of social indicators to track and monitor human development in the Arctic. Further work is needed on these indicators and their technical definitions to increase their strength and applicability; to reevaluate and to readjust them as needed to better reflect the impact of global changes, as well as the complex interactions between bio-physical changes and changes in human system. Such improvement will ensure ASI's ability to effectively contribute to informing successive volumes of the $A H D R$. In addition, efforts are needed at the national and international as well as regional scales to address the persistent gaps in data availability, the lack of common data protocols and the lack of primary data collection, in order to enable the application of Arctic social indicators for tracking human development in the Arctic.

- Gendered dimensions of Arctic change - While the past decade has seen a substantial expansion of research on gender in the Arctic, we still note significant gaps in knowledge. Men and women are not affected equally by climate change and globalization, and their impacts on traditional and non-traditional economic activities in the Arctic, on political systems, on education and health care provision, etc.. We lack knowledge about the gendered dimensions of contemporary cultural practices and expressions, domestic violence's gendered dimensions, the gendered characteristics of decisionmaking regarding resources at different scales, the gendered nature of aging in the Arctic (see above), the gendered experience of food and other (in)securities (see below) and the gendered nature of Arctic geopolitics.

- "Soft securities": Food, water, and energy - Food security is declining in the Arctic. Both environmental change and globalization play a role, as local foods become less available or attractive, and imported foodstuffs of lesser nutritional value and high cost gain popularity. We need to better understand the evolving role of traditional activities that produce "country foods" in northern societies, and their evolving interdependencies with the industrial sector. Climate and other environmental changes affect water security as well, while global shifts in energy prices and related transportation costs compromise energy security in many Arctic settlements dependent imported fuel. Research is needed here on innovative, economically effective ways of providing these key physical components to human well-being.

- Arctic settlements, cities, and communities. - Questions remain on the economic role of large settlements in the economic growth of 
Northern regions. Further knowledge about the relation of Arctic "diasporas" - and their impact on the culture, economics and politics of the communities from which they originate, is needed, as is greater knowledge about how "new newcomers" are also affecting these same spheres. We also need more knowledge on the impacts of global change on Arctic cities and urban areas.

- Institutional arrangements - While the introduction of a variety of new institutions represents one of the most significant changes in the Northern economy, we lack knowledge on what types of institutions work best to improve the economic well-being of northern residents; and what roles institutions, formal and informal, will play in meeting future needs of human development in the North.

- Global linkages and new Arctic actors - We need to continue to work toward a more comprehensive understanding of the social, cultural, political, economic and environmental linkages between the Arctic and the rest of the world. How can we better evaluate the changing importance of the Arctic to the globe - economically, environmentally, and politically? We need to better understand the ambitions of non-Arctic states in the Arctic, and the impacts these may have on Arctic futures.

- Promulgating and adopting best practices - The Arctic has generated many innovations: in adaptive governance practices, in the inclusion of local and indigenous voices in decision-making and also in research, in the development of power- and economic benefit sharing structures, and in initiatives related to distance education and telemedicine. We need greater insight into the conditions under which these innovations can be transferred to other northern places and what adaptations are required. We need to develop best practices for evaluating the impact of economic development initiatives in the Arctic, which fully calculate environmental, social and cultural costs and benefits.

\section{Regional perspectives on human development - Arctic specific social indicators}

AHDR (2004) identified the need for follow-up activities on devising specific social indicators for the Arctic region. In two follow-up projects to the first $A H D R$ indicators were devised in the work of the Arctic Social Indicators projects (ASI I and II). The chosen ASI domains and the corresponding selected indicators have provided the framework for measur- 
ing and tracking change in Arctic human development while emphasizing those aspects of well-being that are particularly important to Arctic residents. The construction of an ASI suite of indicators that reflect wellbeing in the Arctic, and which goes beyond the standard and universal Human Development Index of the United Nations, has enabled the analysis and discussion of Arctic human development from an Arctic regional perspective. The domains and suite of ASI indicators are:

- Fate control - guiding one's own destiny (\% of surface lands legally controlled by the inhabitants through public governments and Native corporations).

- Cultural integrity - belonging to a viable local culture (language retention).

- Contact with nature - interacting closely with the natural world (consumption or harvest of local foods).

- Material wellbeing (per capita household income).

- Health and Population (infant mortality and net-migration).

- Education (ratio of students successfully completing post-secondary education).

These indicators have been cited and applied recurrently in AHDR-II and have been employed in other recent studies of the Arctic. They require further refinement as important region-specific measures of human development in the Arctic.

\section{Follow-up activities}

We offer a number of suggestions for follow-up activities to AHDR-II, based on discussions with lead and contributing authors.

- Dissemination: A series of well-targeted town hall meetings, addressing a range of issues and challenges discussed and brought to the forefront in this volume, would be a highly effective AHDR-II dissemination and outreach activity.

- Monitoring: We propose the further implementation of the ASI monitoring system. There remains a critical need to refine the regionspecific indicators and then to implement a system for collecting, storing, and tracking data on such Arctic social indicators, to provide a tool for measuring changes in human development in the Arctic. 
- Science Plan: Efforts should be made to support and undertake the drafting of an AHDR science plan. The AHDR team is in a unique position to present a science plan for addressing critical gaps in knowledge on Arctic human development and living conditions in the circumpolar region.

- AHDR and the youth: The future of the Arctic will be determined by the choices of the youth and their aspirations and priorities as concerns culture and identities, where to study and where to live and work. Thus, we suggest the production of a follow-up version of the AHDR-II that targets specifically the youth and puts the focus on issues and topics of interest and importance to Arctic youth. 



\title{
1. Introduction
}

\author{
Lead authors \\ Gail Fondahl, University of Northern British Columbia, Canada and \\ Joan Nymand Larsen, Stefansson Arctic Institute, Iceland
}

\section{Contributing authors}

Igor Krupnik (Smithsonian Institution, USA), Jingchao Peng (Graduate School of Asia-Pacific Studies, Japan), Vijay Sakhuja (National Maritime Foundation, India)

\subsection{Why a second Arctic Human Development Report? Why now?}

A decade has passed since the publication of the Arctic Human Development Report (AHDR, 2004). That report, the first assessment of human development in the Arctic, described the unprecedented combination of rapid changes confronting Arctic societies, including environmental and economic changes, cultural transformations, industrial expansion and political developments. It provided a snapshot of the Arctic region at the beginning of the 21st century, and a baseline to "serve as a point of departure for assessing progress in the future" (AHDR, 2004: 15).

During the intervening decade, we have witnessed a dramatic increase in interest in the Arctic, in part due to the continued rapid and even accelerating socio-economic and climatic changes in this region. A record low sea ice extent in the Arctic ocean in 2007, followed by a new record minimum in 2012, have both alarmed and excited the global community, as concern over climate change and its negative impacts is counterpoised by the seeming promise of greater access to the vast natural resources of the region and the anticipation of seasonal commercial shipping through Arctic waters. Evolving technologies and shifting geopolitics also suggest that the Arctic may provide more economical resources and safer shipping routes to the world's population. Yet Northerners continue to face the "double exposure" (O'Brien and Leichenko, 2000) of environmental change and globalization, which in cases is testing their capacity to cope and adapt. Indeed, given these challenges, sev- 
en years after the publication of first Arctic Human Development Report the Arctic Council's Nuuk Declaration identified "the need for a comprehensive overview of human development in the Arctic and call[ed] for an assessment of the current state of human development in the Arctic and its relationship with climate change and other factors affecting Arctic communities" (Arctic Council, 2011: 2). The Parliamentarians of the Arctic Region also advocated for a second AHDR in the concluding conference statement of their 9th Conference: "Support the Icelandic initiative toward a second Arctic Human Development Report in 2014, bringing together state-of-the-art knowledge from the IPY that covers Arctic societies and their welfare in a global context" (CPAR, 2010).

To develop policies and practices that will reduce the vulnerabilities of Northerners and increase the benefits they experience from the various changes, we need to understand trends in Arctic human development. A decadal checkup allows us to begin to map such trends. We hope that by documenting developments over the past decade and identifying such trends this second Arctic Human Development Report (AHDR-II) will further encourage scientists to invest efforts in addressing their sources and potential trajectories, and policy makers to identify ways of encouraging positive paths and reversing negative ones. We imagine that AHDR-II will be followed, on a regular basis, by further assessments of human development in this rapidly transforming region.

\section{Torshavn, capital of Faroe Islands, 2010}

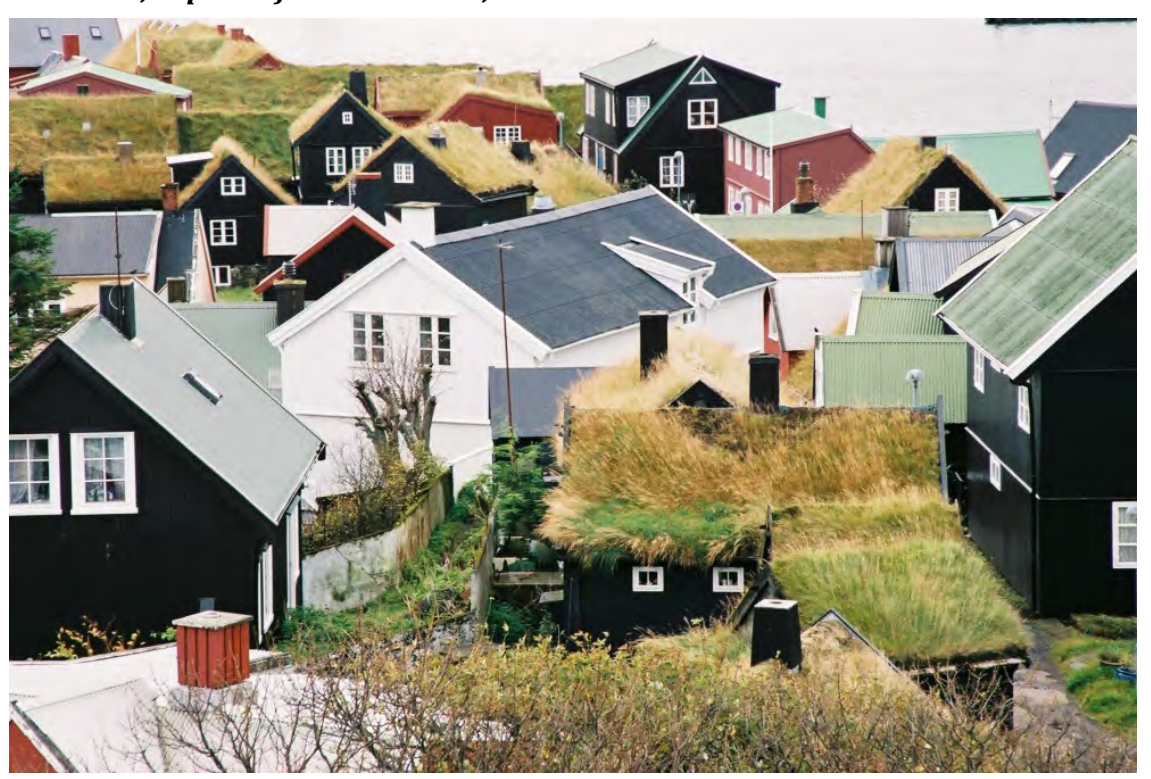

Credit: Harald Finkler. 


\subsection{Objectives of this report}

AHDR-II has six key objectives:

- To deliver a broad and inclusive update to the baseline of human development in the Arctic provided by the first $A H D R$, in a time of rapid regional and global change.

- To offer an instrument that can be used in assessing progress toward sustainable human development in the Arctic.

- To allow for the comparison and contrasting of cultural, social, economic and political conditions in the Arctic with similar conditions in the non-Arctic parts of the Arctic states, as well as in other parts of the world.

- To inform the public and provide valuable material for educational instruction, especially for, but not limited to, the University of the Arctic and northern universities and colleges.

- To serve as a handbook for policy makers concerned with the Arctic.

- To provide a circumpolar assessment that the Arctic Council's Sustainable Development Working Group (SDWG) can use to better understand major issues related to sustainable human development in the Arctic, in order to identify priorities and to develop policies and plans to address these issues.

This new assessment contributes to our understanding of the interplay and consequences of physical and social change processes affecting Arctic residents' quality of life, at both the regional and global scales. As a second assessment it begins to reveal trends in human development. It identifies important gaps that remain in our knowledge and the data needed to better understand the preconditions for sustainable human development in the Arctic region. Also, it offers examples of a number of Arctic success stories and in doing so emphasizes that while there are many challenges unfolding in the Arctic today, there is also a growing number of achievements related to the increasing political and economic autonomy, changing governance structures, and improved livelihoods for Indigenous peoples in the Arctic. 
University of Svalbard in Longyearbyen, Svalbard, Norway, 2014

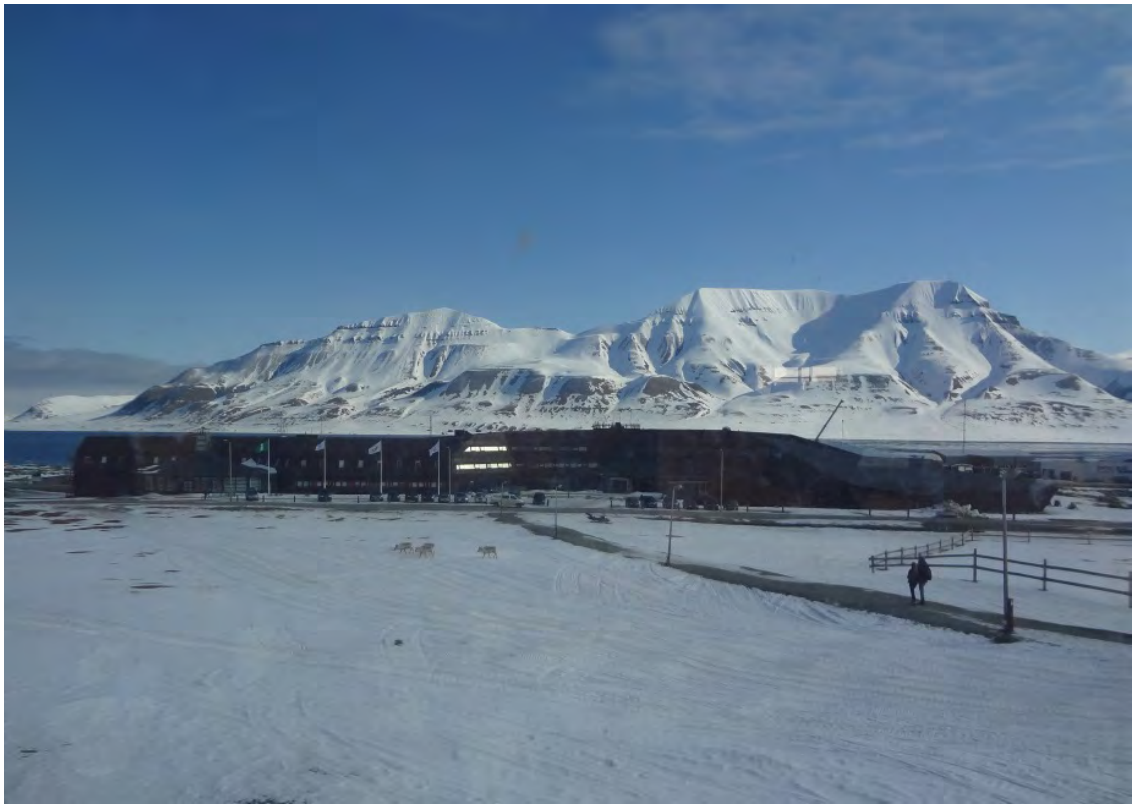

Credit: Joan Nymand Larsen.

\subsection{The rationale for a second Arctic Human Development Report}

Within the past decade, we have observed increasing environmental and social change in the Arctic. We have also witnessed increasing interest in, and attention to the Arctic. Interest on behalf of politicians, governmental officials, resource corporations, and others has been complemented by increased study of both the human and the physical dimensions of the Arctic: we are of course especially interested in the former in this report. Together these trends provide incentive for a new assessment of the state of human development in the Arctic.

\subsubsection{A decade of accelerating change in the Arctic}

"The Arctic is changing rapidly in ways that interact and fundamentally affect the region's ecosystems and societies. Climate change is important, but it is not the only driver of rapid changes in the Arctic. In many contexts, social, political and economic drivers may be of greater importance than global warming."

(Arctic Council 2013a, p. x) 
The first $A H D R$ reported rapid change: the past decade has witnessed yet further intensification of such change. Melting ice, thawing permafrost, increased forest fires, more variability in weather, and other changes pose challenges to Arctic residents. The Arctic is warming at twice the rate of the rest of the planet. But, as noted above in the quote from the Arctic Resilience Interim Report, a range of social, economic and political forces also challenge human development. The chapters of this second Arctic Human Development Report document:

- intensified migrations, both within the Arctic, and between the Arctic and other global regions

- the increasing penetration of new ideas, norms and values into and across Arctic space due to new communications technologies and educational opportunities

- growing interest in Arctic resource development from a variety of players, Arctic and non-Arctic

- (d)evolving governance structures that both enable and challenge northern communities

- accelerating geopolitical maneuverings and policy developments incited by both an increasingly accessible Arctic and by changes in political rapport between the (non-Arctic) centers of the Arctic states, as well as by relations between Arctic and non-Arctic states

- trends and needs in the development of human capital.

In short, we observe an Arctic affected by accelerating movements of people, ideas and capital as well as by a "destabilized ecosystem" (Sejersen 2009; see also Megatrends, 2011). The cumulative and imbricated effects of these myriad contributors to change pose heightened challenges: a new assessment of human development in the Arctic will contribute to understanding their nature and impacts.

\subsubsection{A decade of increased attention to the Arctic}

"The Arctic is hot," declared Gustav Lind, then Chair of the Arctic Council's Senior Arctic Officials, in 2012. Interest in the region has surged, giving rise to many new and potential "stakeholders" and players in the region's future. New resource extraction projects are underway or being drafted, although not without difficulties. While much is made of the changes in accessibility due to climatic warming, global commodity markets and price fluctuations play a definitive role in these developments. Illustrative of such resource projects is the initiation of the Mary 
River Iron Ore Project on Baffin Island - a rich deposit long known is now being mined, though at a scaled-back version of original plans due to multiple challenges (Conley, 2013).

Interest in the Arctic Ocean as both a shipping route and a tourism destination is also growing. Observations suggest increased maritime accessibility: in the periods between 1979-1988 and 1988-2007 the number of ice free days along the Northern Sea Route in the Russian Arctic increased by 22 days (Mokhow and Khon, 2008). Increased cruise tourism is already observed in Greenland, Norway, Alaska, and Canada (Stewart et al., 2010). Tourism, research and government support vessels contribute to the growth of Arctic shipping more than do ships for resource development and for the shipping of goods through the Arctic Ocean (Dawson and Pizzolato, 2014). Still, northern newsfeeds report the growing interest in using the Northern Sea Route and Northwest Passage for commercial purposes by both Arctic States and non-Arctic, and preliminary voyages have been undertaken by several such states. Expectations for increased use in turn have given impetus to increased international cooperation on Arctic response to shipping (and other) disasters in the region, as observed in the Arctic Search and Rescue Treaty, concluded in 2012 by the eight Arctic states. Projections indicate that the five Arctic littoral states will gain increased maritime access to their exclusive economic zones: Greenland's access will grow by an estimated $28 \%$, Canada's by $19 \%$, and Russia's by $16 \%$ by mid-century (Stephenson et al., 2011; Larsen et al., 2014).

The increasing geopolitical importance of the Arctic also has stimulated interest on the part of the governments of the Arctic states to attend more to their northern regions. In the past decade all eight Arctic states have crafted new policy strategies regarding these regions (see Chapter 5, Political Systems and Geopolitics). Non-Arctic states also have pursued much more active roles in the Arctic: witness, for instance, the successful petition of five new non-Arctic states for observer status to the Arctic Council (Arctic Council, 2013b; see also Chaturvedi, 2013; Lackenbauer, 2013; Xing and Bertelsen, 2013; and Textboxes 1.1 and 1.2). 


\section{Textbox 1.1}

\section{Rising interest in the Arctic: a Chinese perspective \\ Jingchao Peng}

In recent years, the Arctic region has attracted a great deal of attention from China. The ice level of the Arctic is decreasing due to rising temperature caused by climate change, opening up energy resources and shipping routes. China's policymakers consider these changes in the Arctic will impact all countries in its periphery, thus legitimizing China's demand for a greater voice in Arctic governance. Beijing has publicly announced itself as a 'near-Arctic state'. It is now a permanent observer in the Arctic Council.

Beijing's primary interests in the Arctic focus at the environment, energy resources and the new shipping route. To understand the consequences the warming Arctic has on China's domestic environment and climate, the government has sponsored six Arctic expeditions. It has also set up a scientific station in the Svalbard Islands and is building a second expedition icebreaker. China is also gearing up to tap the Arctic's economic potential. The Northern Sea Route decreases the navigation time between China and north Europe by one-third. In the summer of 2013, China's state shipping company COSCO sent cargo to Rotterdam via the Northern Sea Route for the first time. In October 2014, China's Ministry of Transport released its first-ever guidebook on Arctic shipping to the public. Noteworthy moves were also made in the energy sector. China's state oil firm, China National Petroleum Corp, is reported to be negotiating with Rosneft a joint exploration of the oil resources in Barents and Pechora Seas' continental shelves.

China's rising presence in the Arctic has been met with misgivings and even outright objection from the local population. Perhaps with the hope to diffuse local negative perceptions, Chinese policymakers insist that monitoring climate change in the Arctic remain China's Arctic priority and it fully recognizes the sovereignty and sovereign rights of Arctic states in the Arctic. On the ground, however, it is clear that economic interests are driving China's Arctic investment as well. Looking ahead, China's investment in the Arctic is slated to grow. Against this backdrop, how to promote and manage China's Arctic stakes while avoiding conflicts with local interests remains one of Beijing's biggest Arctic policy challenges.

The past decade also witnessed a considerable expansion of conferences, academic and otherwise, focusing wholly or partially on issues of human development in the Arctic (e.g., the Second International 
Conference on Arctic Research Planning [ICARP-II] in 2005; ArcticNet Annual Scientific Meetings, since 2004; Arctic Frontiers, since 2007; the Polar Law Symposium, since 2008: the Arctic Futures Symposium, since 2010, Arctic Circle, since 2013). We have seen several new journals and yearbooks launched (e.g., Yearbook of Polar Law [launched in 2009], Polar Journal [2011], Arctic Yearbook [2012], Arkticheskie Vedomosti/Arctic Herald [2012]). We also observe the expansion of academic programming, especially notable at the Masters level, through University of the Arctic, as well as the development of new academic programs (e.g., the Polar Law program at University of Akureyri). New centers focused on the Arctic proliferated (e.g., the International Centre for Northern Governance and Development, Canada, opened 2008; the Centre for Innovation and Research in Culture and Learning in the Arctic (CIRCLA) at Aalborg University in Denmark, 2012; the Arctic Research Center at Umeå University (ARCUM) in Sweden, 2012). The increased focus on socio-economic, cultural and political issues can also be discerned in the International Arctic Science Committee's (IASC) establishment, in 2011, of a Social and Human Sciences Working Group as one of its five working groups.

\section{Textbox 1.2}

Indian perspective on Asia-Arctic relations and transnational connections

Vijay Sakhuja

The growing importance of the Arctic in Indian discourse is recognizable; the narrative focuses on the scientific-politico-economic-strategic developments in the region. India has established a research station, 'Himadri,' on Svalbard and 14 science related institutions in India are engaged in research on the Arctic. The studies of these relate to the polar ice, climate, and weather but India can be expected to expand its interest in the field of resource development. This can be attributed to the evolving geo-economic shift to the North pivoting on living and non-living resources, which would be of immense economic value. India also has interest in the navigability of the Northern Sea Route, which would help transport resources from the Arctic. India advocates making the Arctic a region of peace and stability, demilitarized sea space, and would like to play a vital role in promoting the idea of a nuclear free Arctic. India's entry into the Arctic Council as an Observer is noteworthy, and it hopes to engage other members and play a constructive role. In essence, the Arctic is not at the margins of India's mental map. India is a responsible stakeholder willing to contribute to the evolving Arctic order. 


\subsubsection{A decade of substantial research on the Arctic}

Concomitant with this general upsurge of interest in the Arctic, we have also witnessed a substantial increase in the study of all facets of the Arctic over the past decade, including those focusing on various aspects of human development. A wide range of circumpolar assessments have been completed since the first $A H D R$; while many focus on natu$\mathrm{ral} /$ physical sciences, increasingly these include consideration of the socalled "human dimensions" (e.g., ACIA, 2005; AMAP, 2011; AMAP, 2014; CAFF, 2013; PAME, 2011; Forbes 2011). Also contributing greatly to our knowledge of the Arctic regions in the past decade were the efforts of thousands of researchers, from 60 countries, involved in the International Polar Year 2007-2008 (Krupnik et al. 2011; see Textbox 1.3). IPY 2007-2008 was the first International Polar Year in which social scientists took an active role in research projects, and in which the social life of humans in the Arctic received significant scholarly attention. Arctic research in the past decade has increasingly involved collaboration between visiting scientists and local community members, enhancing its relevance and accessibility to Northerners.

\section{Textbox 1.3}

\section{IPY 2007-2008}

Igor Krupnik

The (fourth) International Polar Year (IPY) 2007-2008, co-sponsored by ICSU and WMO, became the largest coordinated research program in the Earth's polar regions and followed in the footsteps of its predecessors, the International Geophysical Year 1957-1958, the Second International Polar Year 1932-1933, and the First International Polar Year 1882-1883. An estimated 50,000 researchers, local observers, educators, students, and support personnel from more than 60 nations were involved in 228 international IPY 2007-2008 projects (170 in science, one in data management, and 57 in education and outreach) and related national efforts. IPY generated an intensive spike of research and observation activities in the Arctic and Antarctica from 1 March 2007 till 1 March 2009, with many efforts continuing beyond that date. 
IPY 2007-2008 involved a large range of disciplines, from geophysics to ecology, human health, social sciences, and the humanities. Introduction of the social sciences and humanities, as well as the inclusion of polar residents, particularly Indigenous people, marked a radical shift from the earlier IPY/IGY efforts, which had excluded research in the socio-economic and humanities field, except for medical and psychological studies focused on the personnel of polar stations. All IPY 2007-2008 projects included partners from several nations and/or from Indigenous communities and polar residents' organizations. IPY demonstrated a new trans-disciplinary approach that included biology, human health, social sciences, and the humanities, in addition to meteorology, glaciology, oceanography, geophysics, geology, and other traditional polar research fields. It sent a powerful message about the societal value of advanced research on rapid environmental change across the polar regions, and it reached out to many new constituencies, including polar residents, Arctic Indigenous nations, and millions of people on the planet.

IPY activities in social science disciplines (anthropology, archaeology, economics, human geography, linguistics, political science) and the humanities (history, literature, arts) included 35 endorsed international research projects, plus several initiatives in education and outreach directly related to the social science and humanities themes. For the first time, projects were proposed and implemented by polar Indigenous organizations. All Arctic Council's 'permanent participants'-the Inuit Circumpolar Council, Aleut International Association, Arctic Athabaskan Council, Gwich'in Council International, Russian Association of Indigenous Peoples of the North, and Sámi Council-and their national and local chapters were involved in IPY as partners, logistical and public supporters, but also as initiators and lead institutions. Altogether, IPY social science and humanities projects engaged more than 1500 researchers, students, Indigenous experts and monitors, and representatives of polar Indigenous organizations.

\subsection{Progress on monitoring human development in the Arctic}

The first Arctic Human Development Report called for the development of small, tractable and robust set of indicators to track human development in the Arctic. In response, the follow-on Arctic Social Indicators project proposed such a set of indicators (ASI, 2010), and performed preliminary testing on them (ASI, 2014) (See Textbox 1.4). These indicators provide a basis for tracking change between subsequent volumes of the 
AHDR. Other initiatives to measure human development, such as the Arctic Observation Network Social Indicator Project (2007-2011) contribute to this goal as well.

\section{Textbox 1.4}

Arctic Social Indicators (ASI): Monitoring Human Development in the Arctic

The Arctic Social Indicators (ASI) project was formulated to fill a critical gap identified by the AHDR to devise a set of Arctic specific social indicators to help facilitate monitoring of change in human development. A key objective was to devise a small suite of affordable indicators that would enable policy makers and others to take the quick pulse on the state of human development, while other objectives included the development and implementation of the first ASI monitoring system.

The work on devising Arctic specific indicators dates back to the time of the first $A H D R$ (2004). One of the original objectives of the first $A H D R$ had been to devise a set of Arctic social indicators. However, during the process of completing the first $A H D R$ this task soon proved too ambitious in terms of time and other resources. Still, the $A H D R$ identified a number of key domains of particular relevance to Arctic residents and well-being for devising such indicators, and in its conclusion the $A H D R$ recommended that "a small number of tractable indicators to be used in tracking changes in key elements of human development in the Arctic over time" be developed (AHDR, 2004:242). In 2006, and as follow-up to the first $A H D R$, the work on devising Arctic social indicators commenced. An ASI working group was put in place with representation from a broad range of disciplines, and from different geographical locations. The ASI working group met in a series of workshops with the objectives to discuss, identify, select and measure ASI indicators, and to formulate the framework for an ASI monitoring system. By 2014 the working group had produced two reports on the development, measurement and application of Arctic specific social indicators.

The work on the ASI constitutes a significant step forward in moving us toward an ability to track trends in key elements of human development in the Arctic and, as a result, guiding discussions regarding questions of policy. The bulk of ASI activities have coincided with the research initiatives of the International Polar Year (IPY) 2007-2008, which officially lasted to March of 2009. ASI was endorsed as an IPY project in the Arctic social sciences. While in some respects still relatively rudimentary, the ASI monitoring system has been hailed as pioneering and as a forerunner in discussions and applications of Arctic indicators and the monitoring of human societies and the impacts of Arctic change. In this regard ASI has made an important contribution to the study of Arctic social indicators and human based monitoring in the Arctic. 
In its work the ASI working group arrived at six domains for Arctic social indicator selection, of which three domains had been identified during the process of the first $A H D R$ as being particularly important for capturing those aspects of wellbeing in the Arctic that Arctic residents view as particularly prominent (fate control, cultural integrity, contact with nature) and three domains that may be considered more universal in terms of measuring well-being, and that are prominent in the measurement of the Human Development Index of the United Nations (material well-being, education, and health/demography) (AHDR, 2004).

A common list of key criteria for the selection of indicators was developed by the ASI working group, and they included: data availability, data affordability, ease of measurement, robustness, scalability and inclusiveness. A wide range of indicators was identified for each domain, and the best indicators in terms of the set of selection criteria were identified. In particular, the ASI team agreed that the chosen indicators should be affordable in terms of time and other resources (ASI, 2010; ASI, 2014).

While a large number of indicators were identified during the process, and subsequently included in the two ASI volumes, special attention was placed on identifying a small suite of ASI indicators which would be particularly strong in terms of meeting the selection criteria, and which as a collective would provide a holistic picture of the state of Arctic human well-being. This suite of ASI indicators is composed of the following indicators: infant mortality, net-migration, consumption/harvest of local foods, per capita household income, ratio of students successfully completing post-secondary education, language retention, fate control index. Challenges do persist however in terms of those indicators that reply on primary data collection (e.g. country food) and those that are formulated in terms of an index value (e.g. fate control) (ASI, 2014).

Following the success of ASI-I to develop a set of Arctic specific social indicators, ASI-II was implemented in 2011. The objectives of the ASI-II follow-up project were to systematically identify and describe data challenges of the final set of recommended ASI indicators; to conduct a series of regional case studies to illustrate and further test their strength and applicability; and to formulate recommendations for a long-term monitoring system. ASI-II constitutes the implementation phase of the set of Arctic social indicators developed during the ASI-I project; and together with ASI-I, the results serve as input into the process of the AHDR-II. 
The suite of ASI indicators has been applied to five case studies representing different regions in the Arctic: Sakha Republic (Yakutia), Russian Federation; West-Nordic region; North West Territories, Canada; Inuit regions of Alaska, USA; and the Inuit world - an application of SLiCA data (Survey of Living Conditions in the Arctic). A general conclusion of the ASI case studies is that significant data challenges persist in the Arctic, although some regions are more data rich than others; and significant trade-offs are encountered when we rely on a single indicator (ASI, 2014).

The original ambition of the ASI project had been to produce extensive sets of comparable data featuring ASI indicators for each of the six ASI domains. However, this task has proved to be impossible given the current state of data quality and lack of data availability both at the pan-arctic level and at different geographical scales. Indicators cannot be compared between regions in any meaningful way given existing differences in data protocols in addition to other data issues. It is necessary to make adjustments to tailor the analysis to meet the regional availability of data, and hence, to settle for best possible proxies, or in some cases substitute with second-best alternative indicators - though without compromising the validity of the analysis.

The implementation of a more complete system of ASI monitoring might be costly indeed, but as far as addressing existing data limitations, the direct and indirect cost savings in terms of the potential value created by such a system should not be ignored. The core pillars of an ASI monitoring system are: the use of high quality data that meets standards for robustness, validity, reliability, comparability, scalability and ease of measurement and interpretation. The data needs to be clearly relevant to one or more of the six domains of Arctic human development (health and demography, material well-being, education, cultural integrity, contact with nature, and fate control), be sensitive to change over time, be available at least down to a regional level, and be applicable to, and reported separately for, Indigenous and non-Indigenous populations; and preference is for available and affordable data. Any monitoring system will need to rely on existing and continuously collected data available from national, regional and local agencies, and other sources that provide such data at no or small cost (ASI, 2014).

Notwithstanding existing data challenges, the ASI team has been able to not only draw important conclusions about human well-being for each of the five case study regions, but also demonstrate the strength, applicability, and value of the suite of ASI indicators and the proposed ASI monitoring system. The ASI analysis has demonstrated that if more complete data sets were available, including 
primary data collection on harvest, the ASI system could provide an even stronger tool to inform the policy level, and for engaging in regional and local priority setting.

There is no doubt that the long-term monitoring of human development in the Arctic would be greatly facilitated by the regular and frequent collection and reporting of relevant data, including those required for the proposed small set of ASI indicators.

\section{Katuaq, the cultural centre in Nuuk, Greenland}

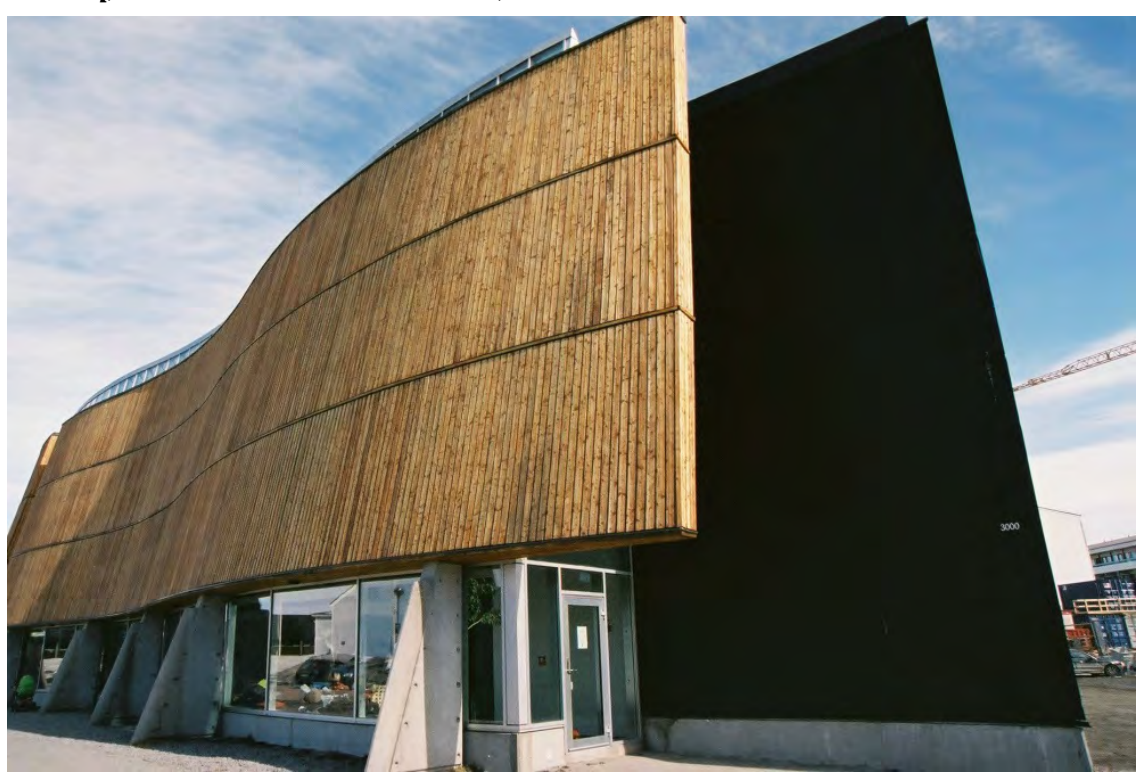

Credit: Harald Finkler.

\subsection{AHDR-II's sub-title: regional processes and global linkages}

The subtitle of this second assessment of human development in the Arctic, Regional processes and global linkages, underscores how Arctic transformations must be understood as intimately linked to global change - both the environmental changes brought on by global warming and the cultural, social, economic, political and environmental transformations driven by other global change processes, often lumped together under the term "globalization". Connections between communities of the north and the national and global souths are expanding, both due to 
communications infrastructure and to increases in physical mobility. These can lead to cultural enrichment - and to social fragmentation (Megatrends, 2011). The connectivity, and its impacts, are uneven across the regions of the North.

The Arctic contains a number of globally important and increasingly desired resources, which nevertheless remain expensive to develop. Hydrocarbons on- and off-shore across the Arctic, rare earths in Greenland, diamonds in Sakha Republic and Northwest Territories, and many other reserves beckon outside investors and developers. "The scope and size of [resource development] projects makes them inherently global in nature as only the largest of corporations can pull them off" (Heinenen et al., 2013: 29). Such corporations have the financial capital, technology, and human resources required in highly capital intensive projects, and tend to be able to operate under the kind of conditions of greater risk and uncertainty that typify resource development in the Arctic. Protest to such projects has also assumed a global nature, organized most notably by transnational environmental NGOs (Heinenen et al., 2013). At the regional level, job opportunities, in-migration, infrastructural developments, and environmental damage reshape Arctic places.

Global and regional developments in Indigenous rights are greatly informed by Arctic persons and peoples. Self-determination movements have transformed the Arctic's geopolitical landscape, most notably in Greenland, but with examples from all corners of the Arctic. Arctic Indigenous groups increasingly influence regions beyond the Arctic and their rights agendas, by actions such as assuming leadership roles in international Indigenous forums, and mounting political campaigns when actions beyond the Arctic affect their well-being (e.g., opposing the European Union's application for observer status at the Arctic Council).

Changes in the Arctic - political, economic, and cultural have global effects, potentially impacting human development in other parts of the world (Arctic Council, 2013a), while we also see increasing evidence of changes outside the Arctic spurring transformations within the region. This report's chapters attend to these regional processes and global linkages in acknowledgement of the increasingly intertwined social and ecological systems of the Arctic with the rest of the world. 


\subsection{Producing AHDR-II: scope, choices and challenges}

AHDR-II builds on the first Arctic Human Development Report, and mostly avoids repetition of the former. For those unfamiliar with the first report, the two might best be read together (http://www.thearctic.is/ AHDR\%20chapters.htm). This report focuses predominantly on the first decade of the 21st century.

As an overview of human development, AHDR-II cannot be allencompassing. Rather, its goal is to identify the major trends for each topic under consideration. Concrete examples from various regions of the Arctic illuminate such trends. It is not the report's intention to deal with all regions of the Arctic within each sub-topic, but rather to offer a limited number of illustrations to ground the observations.

Figure 1.1: AHDR map

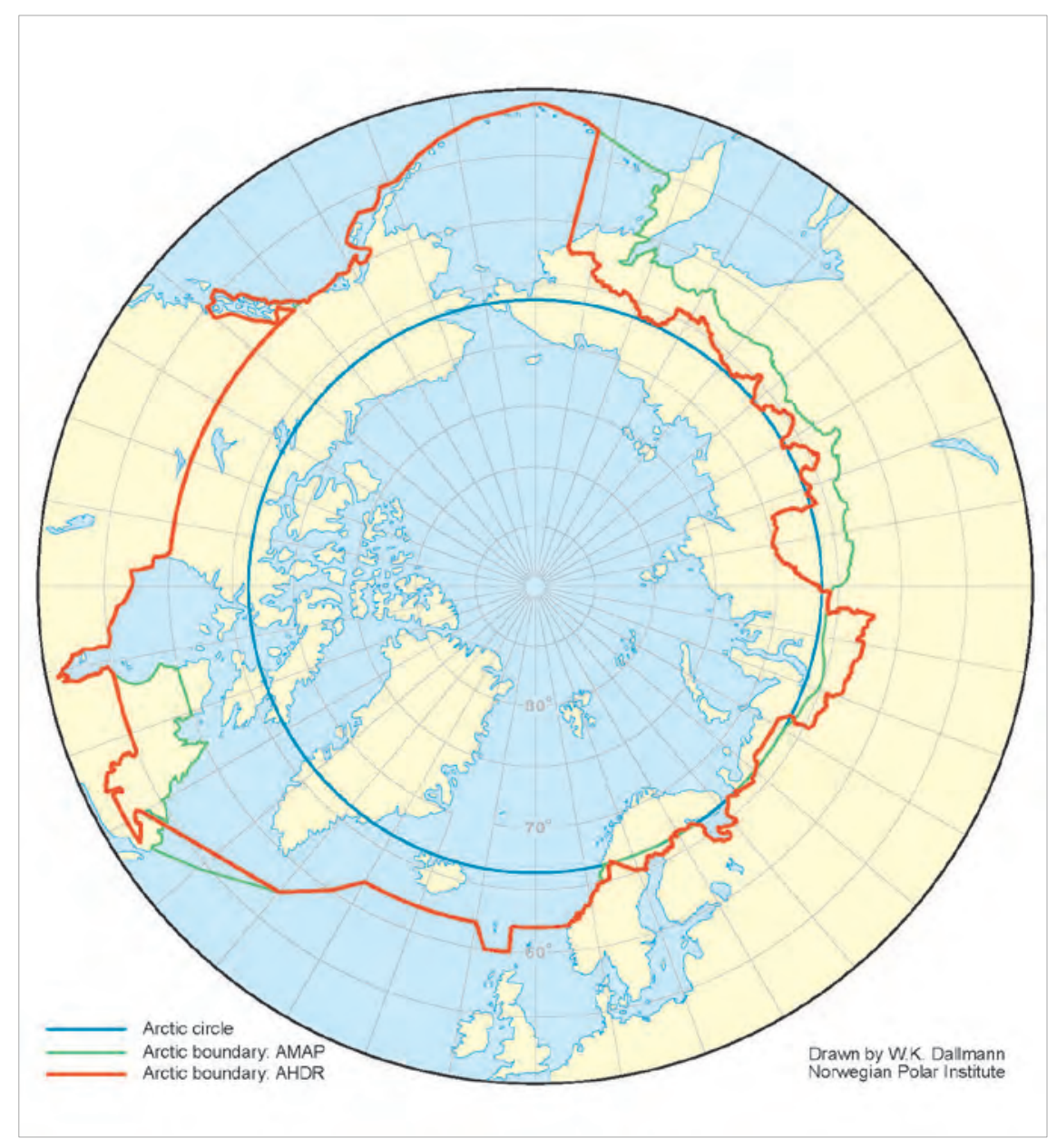




\subsubsection{The Arctic(s)}

AHDR-II employs the same delimitation of the Arctic as did the first AHDR (Figure 1.1). As the first report discussed the various ways of defining the Arctic (AHDR, 2004: 17-18), we will not repeat those here. As in the first report, in some cases authors have needed to diverge from these common geographical boundaries, due to data availability and other reasons.

Yet while we consider the Arctic a distinctive region (a notion that the first $A H D R$ noted is contested from some quarters) we also underscore the great sub-regional differentiation across this territory. Students of the Arctic often note that there is not one Arctic, but several. We acknowledge this heterogeneity, and note that the trends identified in each chapter in certain cases apply to only some areas of the Arctic. Still, Arctic residents do face many of the same challenges across the entire region.

\subsubsection{The Content}

This assessment of human development in the Arctic is organized similarly to the first $A H D R$, to allow for easy comparison. Developments in the Arctic over the past decade, however, suggested some need for modifications, which we outline below. We asked authors to attend to a specific set of questions in addressing their topic and crafting their chapter for this report. These included the effect of indigeneity, ethnicity, geography, age and gender on perceptions and experiences of change in the Arctic; the impacts of climate and global change on the system (i.e., chapter topic) under consideration; variance across the Arctic; and main regional processes and global connections relevant to their topic. Authors were also tasked with identifying the major changes in the past decade and the major trends unfolding related to their topics.

\section{The "common threads" of gender, globalization and climate change} Central to our analysis of changes and trends were three overarching themes of great importance to today's Arctic: climate change, globalization, and gender. All chapters were expected to deal with these "common threads". In addition, AHDR-II includes a specific chapter on globalization, as a major force in Arctic human development, and as force less scrutinized than climate change. Given the focus on climate change in numerous recent studies of the Arctic (e.g., AMAP, 2011; Larsen et al., 2014), we judged it desirable to include consideration of climate change within the chapters but not necessary to allocate a separate chapter to this topic. 
The decision to not include a specific chapter on gender in this second $A H D R$ was made purposefully and after considerable deliberation. Gender affects all domains of human development in the Arctic. Women and men often experience and accommodate change in distinct ways. They are differently affected by climate change (e.g., Preet et al., 2010); perceive health risks from contaminated food differently; enumerate different reasons for migration from rural to urban areas (e.g., Christensen, 2013); and participate differentially in governance and decisionmaking bodies in the North, to name just a few divergences along gender lines. Lead authors were tasked with considering the gender dimensions of developments and trends for their specific topic, be it politics, economics, education, etc., over the past decade. The chapters in this volume document the difference that gender makes .We continue to assert that gender equality is a pre-requisite for human development, wellbeing and dignity in the Arctic.

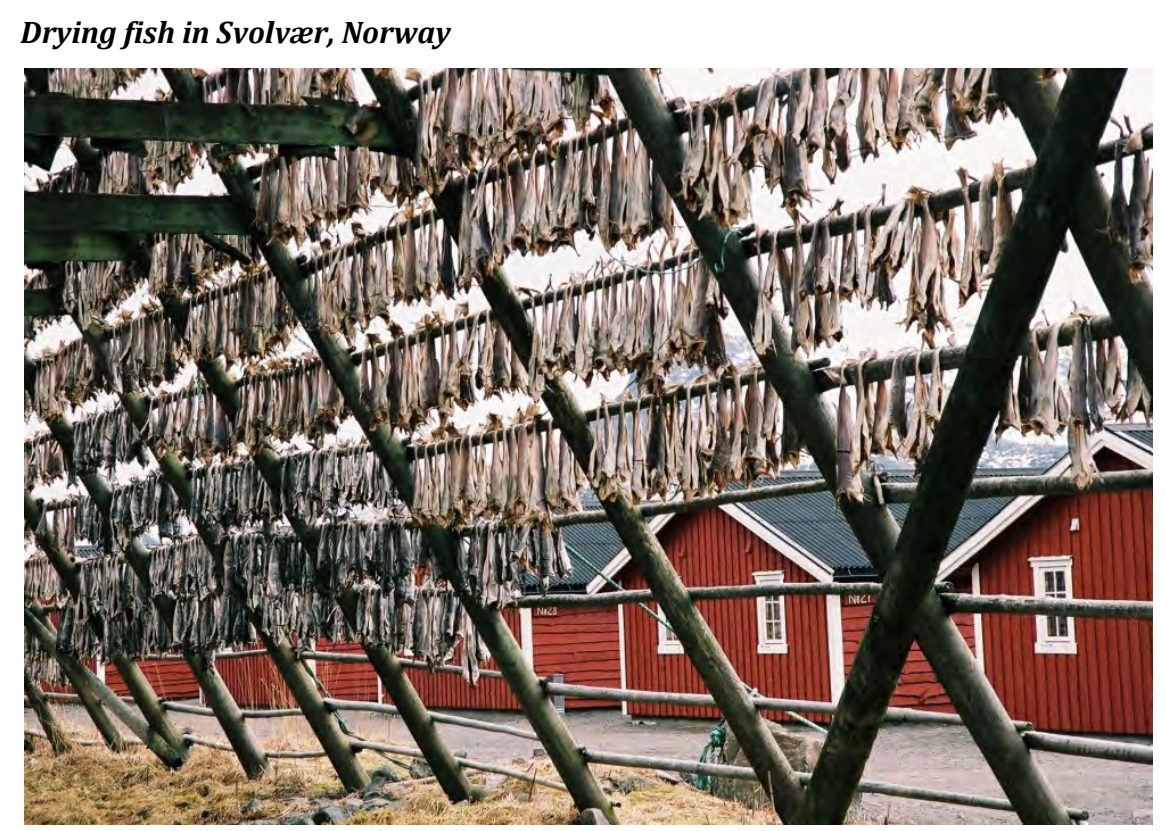

Credit: Harald Finkler.

\subsection{An overview of the chapters}

As with the first $A H D R$, we start with a chapter on demographics that lays out who lives in the Arctic at the end of the first decade of the 21st century, and charts the changes in demographic indicators (birth rates, 
death rates, fertility, etc.) over the past decade for the Arctic region and its sub-regions. In addition, the chapter focuses more attention on migration. The Arctic population is increasingly mobile. The chapter documents the continued rural-urban migration (a trend common for the whole Arctic region), describes the loss of population from urban centers in the Russian North, and notes the relocation of whole communities necessitated by climate change.

In place of a chapter on Societies and Cultures, AHDR-II offers a chapter on Cultures and Identities. The cultural politics of identities are outlined, with attention to the complex and multiple identities negotiated and claimed by many Arctic residents. These identities are in part legacies of the colonial history of the Arctic: thus, the chapter adds a brief historical overview of the Arctic's various sub-regions to help contextualize current developments. Increased communication, travel and trade connect Arctic residents to each other and other populations, influencing their cultures. The chapter adds a section on arts and sports. It highlights the heightened interest in, and marketability of the North, identifying this as an advantage and resource for northern residents.

Chapter 4, on Economic Systems, recaps the Arctic's wealth of natural resources, which to date have remained largely unexploited due to the remoteness and harsh climate. While it appears that both accessibility and demand are growing, the authors remind us that the physical constraints remain and will continue to influence the rate of resource development. A "resource rush" is not inevitable, but rather very much a function of world demand and related commodity prices. Transfer payments and traditional subsistence activities continue to be important to the Arctic economy, and we also see some diversification of the economy toward non-resource based industries and services.

A decision to merge the topics of political systems and geopolitics shapes Chapter 5. The chapter documents the multi-level governance of Arctic political life, notes the innovative approaches to governance developed in the Arctic, and discusses the benefits and challenges of devolutionary processes common to several Arctic sub-regions. It underscores the important role the Arctic plays in world politics. AHDR-II deliberately eschews a strong focus on military security in the Arctic, embracing a broader concept of "security." Food and water security are appraised in discussions of well-being (Chapter 8). The Arctic has certainly served as an area for state performances of sovereignty and national security assertions. As this report was being completed, we observed the beginning of a re-militarization of the Arctic, in the face of both a more accessible Arctic and geopolitical tensions among Arctic 
states. The topics of sovereignty and security, as important dimension of human development, may warrant more attention in future AHDRs.

Chapter 6, on Legal Systems, reviews the multiple legal orders of the Arctic (regional, national, global), then focuses on developments in Indigenous rights in the Arctic and on the current priority of delineating continental shelf boundaries in the Arctic Sea. It asserts that, despite occasional media coverage intimating acrimonious territorial conflicts, such geopolitical contests are being settled through diplomatic channels and cordially.

Through a set of cases that span different sub-regions, various scales, and numerous resources, Chapter 7, on Resource Governance, describes innovative ways developed by communities, government, industries and others, working together, to improve resource stewardship and benefits to all involved. Through several of the examples the chapter underscores how the scientific community has partnered with local resource users to incorporate traditional and local knowledge into governance structures, in an effort to make these more culturally appropriate and resilient.

Climate change as well as the increasing movement of people into the Arctic, are giving rise to new diseases in the Arctic. Chapter 8, on Health and Well-Being, describes this trend. It also draws attention to key challenges to well-being, such as chronic housing shortages throughout much of the North. The chapter also seeks to provide a more holistic picture of health and well-being by incorporating the indicators and findings from the Arctic Social Indicators project (ASI). It discusses how a set of Arctic-specific indicators as a collective helps us better understand the status of well-being of Arctic residents. Special attention is paid to different forms of violence, one of many contributors to significant health disparities between the Arctic and non-Arctic regions in the eight Arctic states.

This report's chapter on Education and Human Capital differs from the first $A H D R$ in that it pays more attention to non-formal types of education, and also to the broader concept of developing human capital in Arctic regions. The authors discuss the growth of creative capital in the North as a necessary precondition for economic and more general human development in the 21st century. The notable gender dimensions of educational attainment are highlighted.

While Chapters 2-9 address key domains of human development, two further chapters provide an integrative review of key processes at two scales. Chapter 10, Globalization, reviews global economic, political, cultural and social processes, referred to collectively as "globaliz- 
ing forces", that are affecting human development in the Arctic. In considering the impact of these forces, it attends to both regional differences and common trajectories. Chapter 11, on Community Viability and Adaptation provides a complementary synthesis of human development processes in the Arctic, focused at the local, community scale. Together, these two chapters serve as capstones to the discussion of changes in the Arctic over the past decade and their impacts on human well-being, and offer commentary on societal adaptation in this region.

In the concluding chapter we summarize the continuing and new trends in Arctic human development. Based on the findings of individual chapters we first highlight a number of cross cutting trends with relevance for policy makers and priority setting in governments and the Arctic Council. Next, we look at some of the key policy relevant conclusions specific to individual chapters. We then identify the most critical cross-cutting gaps in our knowledge that extend across various systems and thematic issues. While the current assessment of human development in the Arctic has shown that the region continues to face significant and even heightened challenges, there are many examples of Arctic success stories that can be told: we highlight a few of these. We conclude the report by offering a number of suggestions for follow-up activities.

\section{Reindeer Herding in Sakha Republic (Yakutia), Russian Federation}

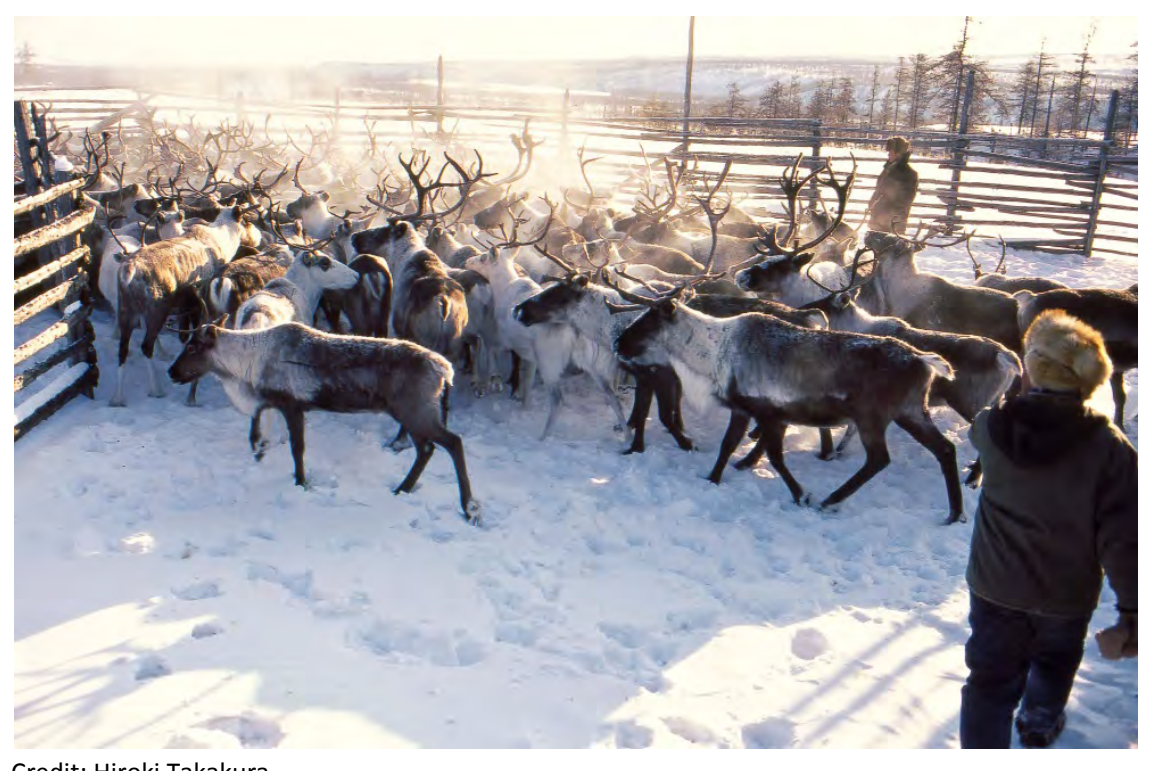

Credit: Hiroki Takakura. 


\subsection{Intended audiences}

AHDR-II was prepared with multiple audiences in mind. As with the first Arctic Human Development Report, we address the report to policy makers at all levels who focus on the Arctic. Many of these policy makers will work outside the Arctic region yet determine at least in part the course of human development within the region.

We also direct the report to students of the University of the Arctic and other students both at Northern post-secondary institutions and beyond. To begin to understand the many dimensions of human development in the Arctic is a critical step in developing future "human capital" dedicated to improving life in the Arctic, whether working in the Arctic or beyond its borders. Exposed to Arctic issues, even those individuals who do not pursue careers that intersect with Arctic affairs will nevertheless be more informed, and hopefully concerned, about global and regional action that affect the Arctic. An up-to-date assessment of human development in Sthe Arctic will also facilitate curricular development and educational initiatives focusing on this critical aspect of the Arctic.

In identifying gaps in knowledge we aim to inform priorities for future research. The report will contribute to the third International Conference on Arctic Research Planning's (ICARP-III) roadmap for research priorities and partnerships. We anticipate that the Arctic Council's Sustainable Development Working Group (SDWG) will utilize the report in its priority-setting exercises over the next decade. Other targeted audiences include the media, and non-governmental organizations that work, or aspire to work, in the Arctic. Tracking and understanding human development in the Arctic are important steps to informed, and hopefully compassionate, policy choices that will contribute to increasing the well-being of the Arctic's residents.

\subsection{References}

ACIA, 2005. Arctic Climate Impact Assessment. Cambridge University Press, Cambridge. AHDR, 2004. Arctic Human Development Report. Einarsson, N., J. N. Larsen, A. Nilsson and O. R. Young (eds.). Stefansson Arctic Institute, Akureyri.

AMAP, 2011. Snow, Water, Ice and Permafrost in the Arctic (SWIPA): Climate Change and the Cryosphere. Arctic Monitoring and Assessment Programme (AMAP), Oslo, Norway.

AMAP, 2014. Arctic Ocean Acidification 2013: An Overview. Arctic Monitoring and Assessment Programme (AMAP), Oslo, Norway.

Arctic Council, 2013a. Arctic Resilience Interim Report 2013. Stockholm: Stockholm Environment Institute \& Stockholm Resilience Centre. www.arctic-council.org/arr 
Arctic Council. 2013b. Kiruna Declaration. 15 May 2013. Kiruna, Sweden.

Arctic Council, 2011 Nuuk Declaration on the Occasion of the Seventh Ministerial Meeting of the Arctic Council, 12 May 2011, Nuuk.

ASI. 2014. Arctic Social Indicators II: Implementation. Larsen, J.N., P. Schweitzer, and

A. Petrov (eds.). TemaNord. Nordic Council of Ministers, Copenhagen.

ASI, 2010. Arctic Social Indicators - a follow-up to the Arctic Human Development Report. Larsen, J.N., P. Schweitzer, and G. Fondahl (eds.). TemaNord. Nordic Council of Ministers, Copenhagen.

CAFF, 2013. Arctic Biodiversity Assessment. Status and Trends in Arctic Biodiversity. Conservation of Arctic Fauna and Flora, Akureyri.

Chaturvedi, S., 2013. China and India in the 'receding' Arctic: rhetoric, routes and resources, Jadavpur Journal of International Relations, 17(1): 41-68.

Christensen, J. 2013. 'Our home, our way of life': spiritual homelessness and the socio-cultural dimension of homelessness in the Northwest Territories (NWT), Canada, Social \& Cultural Geography, 14(7): 804-828.

Conley, H., 2012. Arctic Economies in the 21st Century. The Benefits and Costs of Cold. Center for Strategic and International Studies, Washington, D.C.

CPAR, 2014. Ninth Conference of Parliamentarians of the Arctic Region, European Parliament, 13-15 September 2010. Conference Statement, Brussels. http://www. arcticparl.org/files/Conference\%20statement,\%20Final\%20draft(1).pdf

Dawson, J and L. Pizzolato, 2014. There's more behind Arctic shipping than climate change, The Globe and Mail, 3 Feb 2014.

Heinenen, L., H. Exner-Pirot, and J. Plouffe, 2013. From regional transition to global change: dualism in the Arctic, in: L. Heinenen, ed., Arctic Yearbook 2013, pp. 24-31. Northern Research Forum, Akureyri.

Krupnik, I., I. Allison, R. Bell. P Cutler, D. Hik, J. López-Martinez, V. Rachold, E. Srukhanian and C. Summerhayes, eds., 2011. Understanding Earth's Polar Challenges: International Polar Year 2007-2008. University of the Arctic, Rovaniemi, Finland/CCI Press, Edmonton, Alberta, Canada and ICSU/WMO Joint Committee for International Polar Year 2007-2008.

Lackenbauer, W., 2013. India's Arctic engagement perspectives. In: Heinenen, L. (ed.), Arctic Yearbook 2013, pp. 33-52. Northern Research Forum, Akureyri.

Larsen, J.N., O.A. Anisimov, A. Constable, A.B. Hollowed, N. Maynard, P. Prestrud, T.D. Prowse, and J.M.R. Stone, 2014: Polar regions. In: Barros,V.R., C.B. Field, D.J. Dokken, M.D. Mastrandrea, K.J. Mach, T.E. Bilir, M. Chatterjee, K.L. Ebi, Y.O. Estrada, R.C. Genova, B. Girma, E.S. Kissel, A.N. Levy, S. MacCracken, P.R. Mastrandrea, and L.L. White (eds.). Climate Change 2014: Impacts, Adaptation, and Vulnerability. Part B: Regional Aspects. Contribution of Working Group II to the Fifth Assessment Report of the Intergovernmental Panel on Climate Change, pp. 1567-1612. Cambridge University Press, Cambridge.

Mokhov, I.I. and V.C. Khon, 2008. Assessment of the Northern Sea Route perspectives under climate changes on the basis of simulations with the climate models ensemble. In: Kotlijakov, V.M. (ed.). Natural Processes in Polar Regions, Part 2 Vol. III of the Changes of Natural Environment and Climate: Natural and Possible Consequent Human-induced Catastrophes Series, pp. 20-27. The Institute of Geography (IG), Russian Academy of Sciences (RAS), IG RAS, Moscow.

O’Brien, K. and R. Leichenko, 2000. Double exposure: assessing the impacts of climate change within the context of economic globalization, Global Environmental Change 10: 221-232. 
PAME, 2011. Arctic Marine Shipping Assessment, http://www.pame.is (24 November 2014).

Preet, R., M. Nilsson, B. Schumann, and B. Evengård, 2010. The gender perspective in climate change and global health, Global Health Action, 3: 5720,

DOI:10.3402/gha.v310.5720

Sejersen, F., 2009. Resilience, human agency and climate change adaptation strategies in the Arctic, Kongelige Danske Videnskabernes Selskab. Historisk-Filosofiske Meddelelser, 106: 218-244.

Forbes, D.L. (ed.), 2011. State of the Arctic Coast 2010 - Scientific Review and Outlook. International Arctic Science Committee, Land-Ocean Interactions in the Coastal Zone, Arctic Monitoring and Assessment Programme, International Permafrost Association. Helmholtz-Zentrum, Geesthacht, Germany.

Stephenson, S.R., L.C. Smith, and J.A. Agnew, 2011. Divergent long-term trajectories of human access to the Arctic. Nature Climate Change, 1(3): 156-160.

Stewart, E., J. Dawson, S. Howell, M. Johnston, T. Pearce, and R. Lemelin, 2012. Sea ice change and cruise tourism in Arctic Canada's Northwest Passage: Implications for local communities. Polar Geography, 1 -20, DOI:10.1080/1088937X.2012.705352.

Stewart, E.J., S.E.L. Howell, J.D. Dawson, A. Tivy, and D. Draper, 2010. Cruise tourism and sea ice in Canada's Hudson Bay region. Arctic, 63: 57-66.

Xing, L. and R.G. Bertelsen, 2013. The drives of Chinese Arctic interests: political stability and energy transportation security. In: Heinenen, L. (ed.), Arctic Yearbook 2013, pp. 53-68. Northern Research Forum, Akureyri. 


\title{
2. Arctic Populations and Migration
}

\author{
Lead author \\ Timothy Heleniak, George Washington University, USA \\ Contributing author \\ Dimitry Bogoyavlensky (Higher School of Economics, Russian Federation)
}

\subsection{Introduction}

In 2013, there were 4,053,055 people living in the Arctic. This is a decline of 55,982 since 2000 , or $1.4 \%$. That the overall Arctic population has remained roughly the same over this period masks considerable regional variation in population change among Arctic countries, regions, and settlements. This chapter examines a number of key questions about the population of the Arctic including: factors influencing the number of children Arctic women are having, mortality levels and patterns, causes and implications of changes in the gender, age, and racial or ethnic composition of Arctic populations, the spatial distribution of the Arctic population and factors influencing population mobility across the Arctic, and the expected future size and composition of the Arctic population. It is appropriate that the first substantive chapter of a report on human development in the Arctic focuses on the people living in the Arctic.

In addition to describing population changes since the first $A H D R$, this chapter analyzes migration and its impact on Arctic populations. Because of the small population size of settlements in the Arctic, migration has the potential to have a significant impact on both origins and destinations. This chapter analyzes mobility and immobility, the role of climate change, urbanization, and international migration into and out of the Arctic. A final section examines projections of the Arctic population. To put population change in the Arctic in context, comparisons are made to the Arctic states and to global demographic trends, including separately to developed and developing countries 
as defined by the United Nations (UN Population Division, 2011). All Arctic states are classified as developed countries.

\subsection{Population change}

During the first decade of the 21st century, the global population continued to grow rapidly, increasing by $13 \%$ between 2000 and 2010 and reaching seven billion in October 2011 (Figure 2.1). Rapid population growth, along with technological advances and growing affluence of the global population, has contributed to anthropogenic climate change and warming, more so in the Arctic than other regions of the world. The world's population is expected to continue to grow, reaching 9.3 billion by mid-century and over 10 billion by the end of the century.

Figure 2.1: Population change in the Arctic, 2000-2010 (percent)

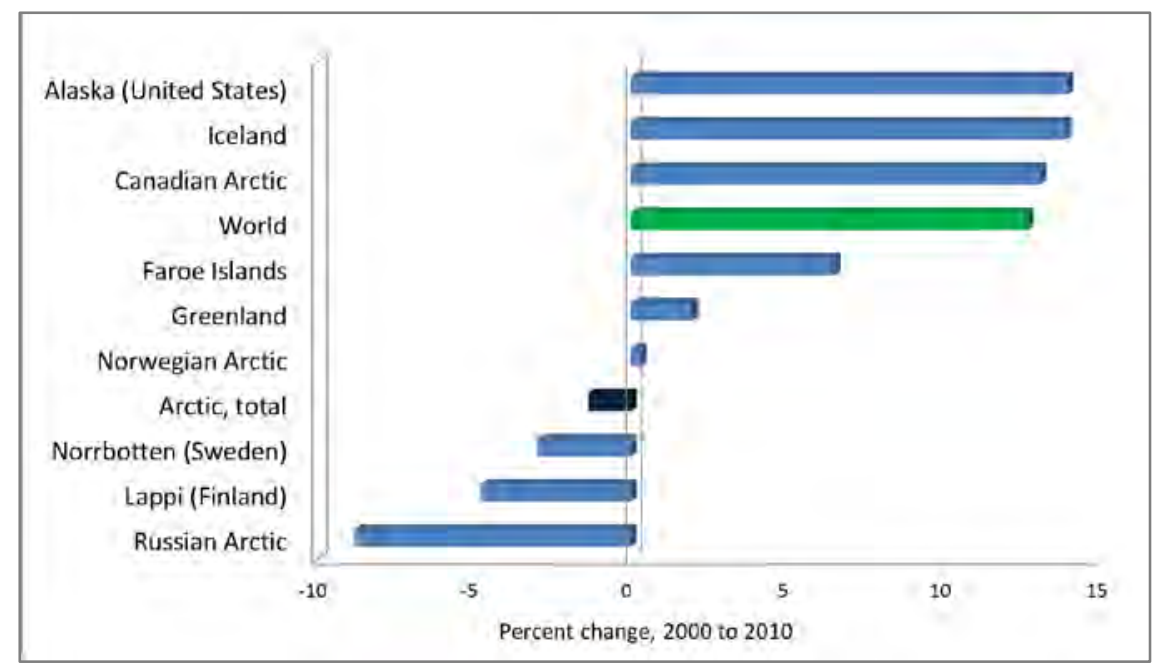

Source: National statistical offices and UN Population Division.

In contrast to rapid global population growth, the population of the Arctic has declined slightly since 2000 (historical data on the population of the Arctic extending before 2000 are contained in the chapter "Arctic Demography" in the first AHDR). This is largely due to the continued decline of the population of the Russia Arctic, which now contains less than half the Arctic population. Alaska, Iceland, and the Canadian Arctic all grew faster than the global rate. There was moderate population growth in the Faroe Islands. The populations of Greenland and the Nor- 
wegian Arctic remained roughly the same, while Lappi (Finland) and Norrbotten (Sweden) had moderate declines.

The populations of the Arctic regions in the United States and Canada both grew faster than those countries as a whole. The fastest growing region in the Arctic was Nunavut, which increased by nearly $20 \%$ since 2000. By contrast, the Arctic regions of Norway, Sweden, Finland, and Russia had slower population growth or faster decline than their respective countries. The Arctic regions with the largest declines were in Russia, which continued its post-Soviet contraction of economic activity. The cities of Vorkuta and Igarka, northern Yakutia, and Chukotka all had population declines of more than $20 \%$ in the 2000 s. By contrast, YamalNenets okrug had a population increase of $10 \%$ because its young age structure contributed to significant natural increase of the population.

\subsubsection{Population change from natural increase and net migration}

Population growth, or decline, for any settlement, region, or country can be disaggregated into natural increase or decrease and net migration. Natural increase is the difference between the number of births and the number of deaths while net migration is the difference between the number of in-migrants and out-migrants. The population of a region changes because of the combination of these two processes due to demographic and other factors. Demographic factors influencing natural increase or decrease include levels of fertility and mortality and the age and gender structure of a population. If the population of a region has relatively more young people, including many women in the childbearing ages, and fewer in older ages which have higher rates of mortality, then natural increase tends to be high. This is the case in many Arctic regions, including in many regions with high shares of Indigenous peoples. If the age structure of a population has relatively more older and fewer younger people, natural increase will be low or even show a decrease as has been the case in Russia over the past several decades.

Whether net migration is positive into a region (more people coming into a region than leaving) or negative (more people leaving the region than coming into it) has primarily to do with economic opportunities in the region versus opportunities elsewhere. Because migration tends to be age selective among people in the young-adult ages when mobility is highest, regions gaining people from migration tend to gain large numbers of people in the young-adult ages and their children, since the young-adult ages are also the prime child-bearing ages. Demographical- 
ly, this tends to keep the population of a region quite young (young people tend to be more productive) and, in addition offers other economic influences, such as being a boost to economic growth because these young people tend to be more productive. On the other hand, regions experiencing population declines from migration are losing large numbers of people in the young adult ages exacerbating population decline and serving to dampen economic dynamism. As will be illustrated, many Arctic regions and settlements are on the extremes of population change from high rates of in-migration or out-migration.

At a global level, the $13 \%$ increase in the population of the world is due to significantly more births than deaths (Figure 2.2). In spite of a slowdown in fertility across much of the developed and developing countries of the world, woman are still giving birth to more than two children on average, causing populations to grow. This is coupled with young populations across much of the developing world, where even if fertility were to decline, there are still large numbers of people in the child-bearing ages having children. All of the Arctic countries and regions are at more advanced stages demographically and have much older age structures and lower fertility rates than the developing countries of the world.

Figure 2.2: Population change from natural increase and net migration in selected Arctic regions, 2000 to present

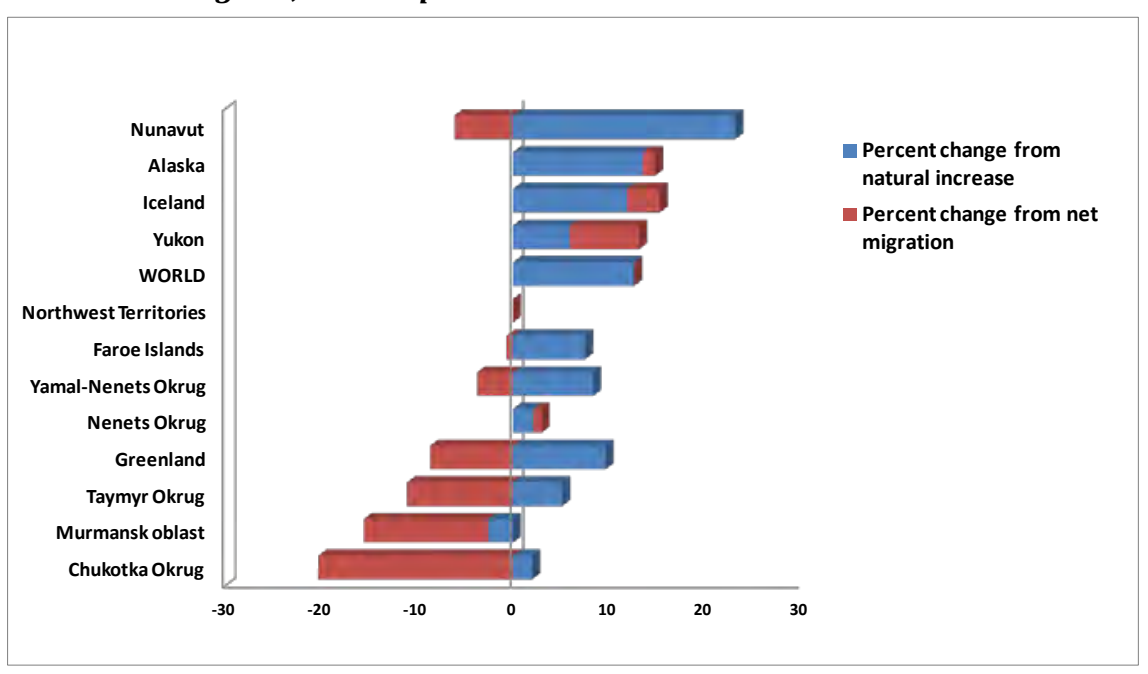

Sources: National and regional statistical offices. Data are in order by total population change.

Alaska's population growth is primarily due to more births than deaths, though there has been moderate net in-migration to the state over the period. In Yukon, migration is the largest driver of population change. 
With its young population, the major component of Nunavut's population change is natural increase combined with moderate out-migration.

Population growth in the Faroe Islands was driven largely by natural increase with only moderate out-migration. Greenland's population stayed about the same over the period because the natural increase of the country's young population was offset by the same amount of net out-migration. Iceland's population grew from having both natural increase and positive net migration into the country. Migration was quite significant into Iceland until the financial crisis of 2008.

In recent years, the regions of Arctic Norway have had both natural increase and net in-migration while those in Arctic Sweden and Finland have had either natural increase or decrease combined with net outmigration, resulting in population losses.

With one exception, all regions of the Russian Arctic had continued out-migration combined with natural increase. This was due to all having significant Indigenous populations who have higher birth rates than the national average or younger age structures due to recent waves of in-migrants, as in the case of Yamal-Nenets okrug.

\subsubsection{The demographic transition}

The demographic transition is the transition that most societies and countries go through from having high birth rates and death rates to having low birth rates and death rates (Weeks, 2008). Because death rates typically decline faster than birth rates, there is a period of rapid population growth when births exceed deaths by a considerable amount. Simultaneous with the demographic transition are a series of other transitions. These include a health and mortality transition where the major causes of death shift from being from infectious and communicable diseases to more being from degenerative and non-communicable causes. In the later stages of the health and mortality transition more people die from neoplasms and external causes - murder, suicides, and accidents. The underlying causes are often lifestyle factors such as smoking, obesity, and risk-taking behavior. The age and gender pattern of deaths also change from women and children dying first in the larger numbers to men having higher mortality rates.

There is also a fertility transition from high fertility rates, early marriage and first birth, and little birth control to lower fertility rates, later marriage and first birth, and increased birth control, especially modern, more effective methods. There are migration and urban transitions characterized by increased mobility and movement up the urban hierar- 
chy away from small settlements towards larger settlements and cities. There is also an age transition marked by a rise in the median age and a family and household transition away from large, multi-generation households towards smaller, two-generation households and an increased number of single-person households.

Most of the Arctic consists of economically advanced countries that are demographically at late stages of the transition. However, many of these states are home to Indigenous populations who are at earlier stages of the transition. These differentials can be seen when comparing data for Indigenous and non-Indigenous populations, or when examining data for Arctic regions that have predominantly Indigenous populations. Also, many Arctic Native populations have had somewhat interrupted transitions because of their encounters with outsiders, often marked by periods of high mortality from exposure to diseases from which they had little immunity (Textbox 2.1).

\section{Textbox 2.1}

\section{The unique demographic transition in the Arctic: the case of Greenland}

Societies composed primarily of Indigenous peoples who encounter Western peoples or other groups of outsiders may have unusual demographic transitions. The Inuit of Greenland are one such example. Demographic transitions in this population differ from the expected pattern in either their timing or pattern. The typical demographic transition is one from high birth and death rates to lower birth and death rates, a transition which demographers have divided into four stages. The decline in death rates precedes the decline in birth rates resulting in a period of rapid population growth.

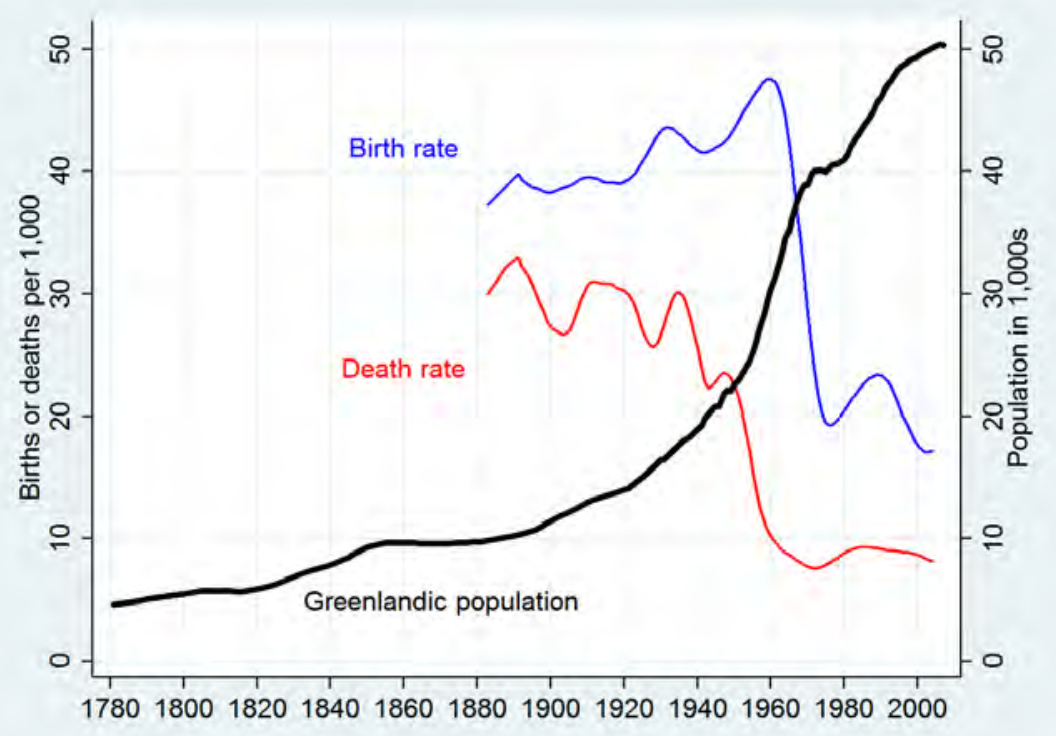


The figure shows the natural increase (difference between births and deaths) and population size among Greenlanders over the period from 1781-2007. Birth and death rates are for "Greenlanders." The deaths include only people born in Greenland and still living in Greenland two years later. For births, the definition is more complicated. It is possible to distinguish between persons as born "Greenlanders" with both parents born in Greenland and others having only one parent in Greenland.

The figure shows the natural increase (difference between births and deaths) and population size among Greenlanders over the period from 1781 to 2007, from the time of the start of the Royal Greenland Trade Company's monopoly to the present. Until 1781, the population size was small and population growth was slow. The economy was still largely based on the hunting of seals and whales, some land mammals, and fish and birds. Difficult climatic conditions and various epidemics resulted in a population decline from 8,000 to 6,000 during the 18th century.

Under colonial rule, the population doubled to 12,000 by 1900 . In the early 20th century, more than half of deaths were from tuberculosis and infectious diseases that caused the fluctuations in the death rate. A warmer climate reduced the seal population but increased cod supplies, giving rise to a commercial fishing industry. Population growth resulted in another doubling of the population in 30 years. After WWII, efforts were made to improve health conditions. In the 1960s, family planning efforts were introduced, resulting in a steep decline in fertility rates. The death rate fell and then leveled off in the 1970s. Births continue to exceed deaths by about 500 per year, placing Greenland at the third of four stages of the demographic transition. The total size of the population has stabilized at about 56,000 and is projected to remain at about this level because continued net outmigration, mainly to Denmark, is roughly equal to the amount of births exceeding amount of deaths.

Source: Hamilton and Rasmussen, 2010; figure reproduced by permission.

\subsubsection{Fertility}

The growth or decline of any country or region's population is especially sensitive to deviations from replacement-level fertility of 2.1 children per woman (the total fertility rate, often referred to simply as the fertility rate, is a synthetic measure which shows the number of children a woman would have if she passed through her childbearing years at the current age specific fertility levels). Over the long run, with a fertility rate of 2.1 children per woman, allowing for some mortality, a population will just replace itself; each generation will be the same size as the 
previous. Fertility rates above this level will lead to population growth as the current cohort will be replaced by larger cohorts. Rates below this lead to population decline as subsequent generations will be smaller. Global fertility rates continue to decline, going from 3.04 children per woman in 1990 to 2.45 in 2010. However, this level remains above replacement, thus contributing to continued significant population growth. Figure 2.3 shows the most recent fertility levels for the Arctic regions and includes comparisons to the global levels and those for developed and less developed countries (UN Population Division, 2011).

Figure 2.3: Total Fertility Rate in the Arctic, 2009-2012 (number of children per woman)

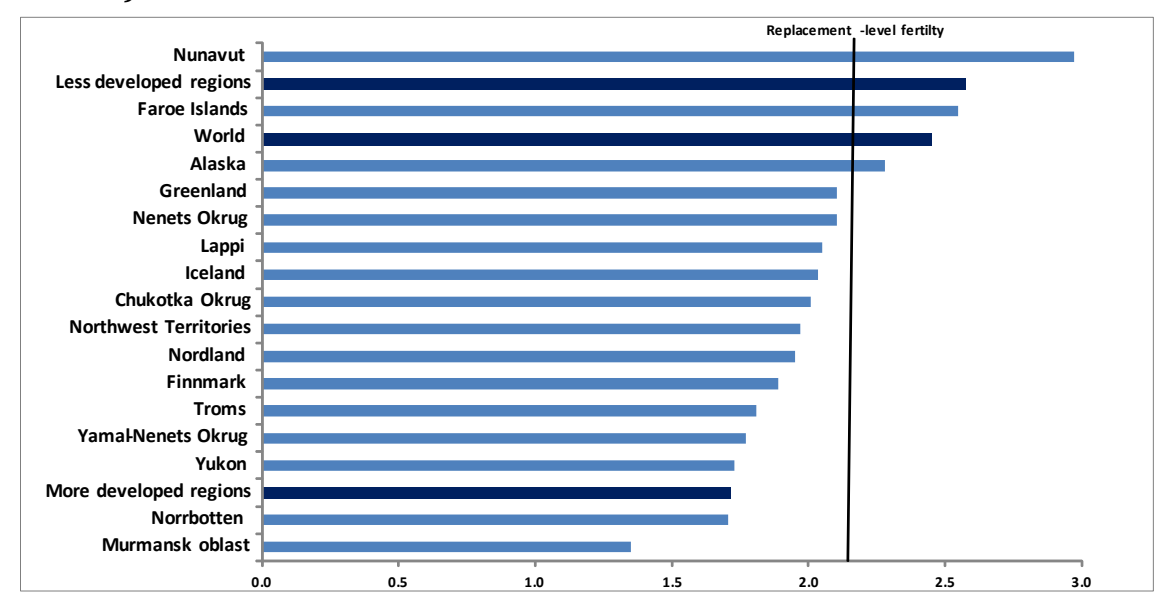

Sources and notes: Data are from national and regional statistical offices.

The United States has long had among the highest fertility rates among developed countries. This, and continued high levels of immigration, explain the higher levels of population growth in the United States. The total fertility rate in the United States has been about or just below replacement level for the past several decades. The fertility rate in Alaska has declined over the past two decades from 2.6 to 2.3 children per woman, though this level is still above replacement and above the national average. For the past several decades, Alaska has had among the highest fertility rates among U.S. states. There are significant differences in fertility rates between Natives and non-Natives with Alaska Natives having fertility rates of over 3 children per woman while non-Natives have less than 2.5 (Hunsinger and Sandberg, 2013).

Yukon and Northwest Territories (NWT) have fertility rates above the national average but below replacement level. Nunavut, with its majority Indigenous population, has a fertility rate of nearly 3 children per 
woman, which is above the level for less developed countries. This level, combined with a young age structure, will ensure continued high population growth in the region into the future.

The fertility rate in Iceland is among the highest in Europe at 2.04 children per woman in 2012. This high fertility rate persists in spite of a small decline from a peak of 2.2 just prior to the 2008 financial crisis. The fertility rate in Greenland, while declining, remains about the replacement level. The fertility rate in the Faroe Islands is also above replacement level.

The regions of the Norwegian Arctic have fertility rates of about 1.9 children per woman which is just below replacement level but slightly above the national average. Similar to the Arctic regions of Norway, the fertility rate in Lapland is just below replacement and slightly above the national level. Norrbotten in Sweden has recently had belowreplacement fertility and a rate below the national average.

During Russia's post-Soviet demographic crisis, fertility fell to unseen levels that demographers termed "lowest-low" fertility, which at its nadir fell to 1.157 births per woman (Figure 2.4) (Kohler, Billari, and Ortega, 2002). These low rates were also seen in the country's Arctic regions. The fertility rate in Murmansk has been lower than the national rate reaching a low of 1.015 in 2000 before increasing to 1.35 in 2009, though this is still far below replacement. The fertility rate in YamalNenets has been above the national average though below replacement while the rates in Chukotka and Yakutia have recently been about 2.0 children per women. These regions with large Indigenous populations have among the highest fertility rates in the country.

Figure 2.4: Total Fertility Rate in Russia and Selected Arctic Regions, 1989 to 2011

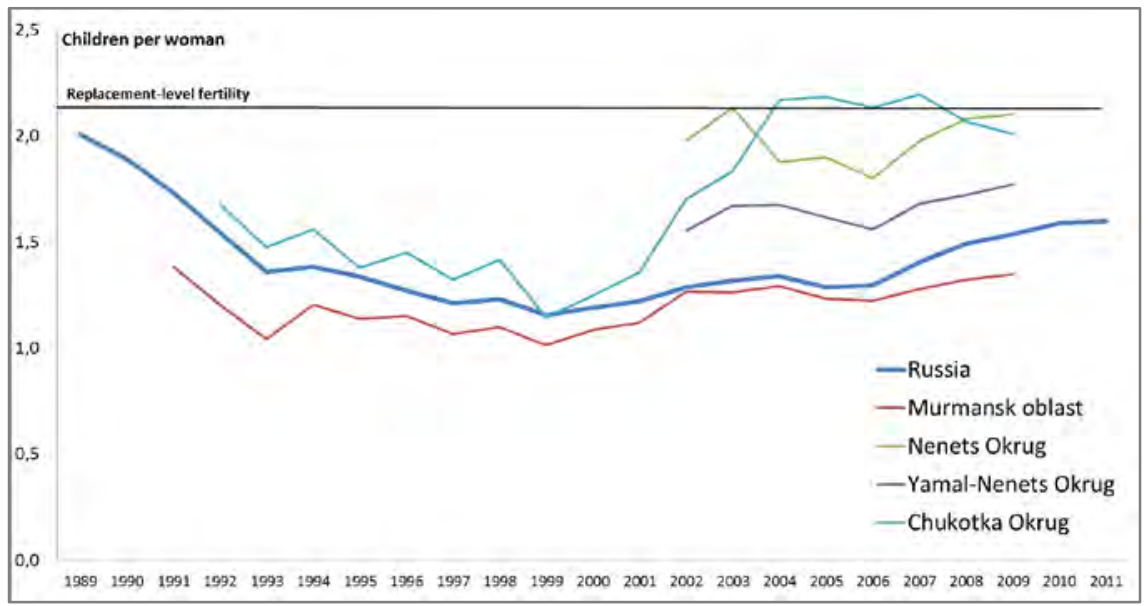

Source: Rosstat, Demographic Yearbook (annual editions). 
Differences in levels and the age pattern of fertility are shown in Figure 2.5 for Canada and the three northern territories. In 2011, Canada had a fertility rate of 1.6 while Yukon and NWT have slightly higher fertility rates, though still below replacement. Canada and Yukon had similar age pattern of fertility characteristic of societies at an advanced stage of the demographic transition with a later peak fertility among women ages 30 to 34. NWT had a pattern characteristic of a population at an earlier stage, with peak fertility among women ages 25 to 29 but moderately high levels from ages 20 to 39 . Women in Nunavut displayed a pattern of a population even earlier in the transition, with a peak among women ages 20 to 24 and continued high fertility in the late 20 s.

Figure 2.5: Age-specific fertilty rates for Canada and the northern territories, 2011

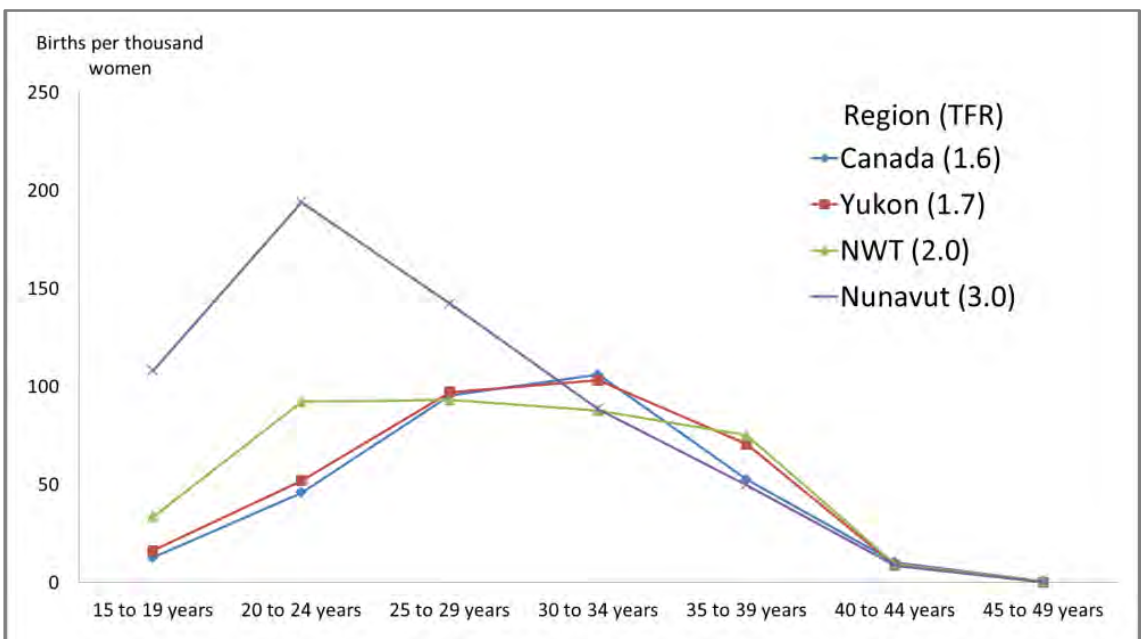

Source: Statistics Canada, CANSIM database (http://www.statcan.gc.ca/start-debut-eng.html).

As noted above, deviations from replacement-level fertility have a large impact on future population growth. With fertility rates above replacement level, and younger age structures, the populations of Nunavut, the Faroe Islands, and Alaska can expect to have significant population growth in the future. With a fertility rate of just 1.35, and an older age structure, the Murmansk oblast can expect to experience continued population decline. Most of the rest of the Arctic have fertility rates at or just below replacement level, indicating that the populations will decline but rather slowly. 


\subsubsection{Mortality}

Two indicators depict mortality trends among Arctic populations - life expectancy at birth and the infant mortality rate (more detail on the health status and trends in health among Arctic populations are in the health chapter). Gender differences and differences between Indigenous and non-Indigenous peoples are emphasized within these indicators. During the twentieth century and beyond, there have been significant improvements in health because of increased knowledge of infectious diseases and other causes of mortality, improved sanitation, food safety, and public health. Between 1950 and 2010, global life expectancy at birth has increased from 47.7 to 67.9 years (Figure 2.6), a remarkable increase of 22.2 years. Life expectancy gains have been greater in less developed countries than in developed countries as the populations in the latter approach what is believed to be the biological limits of human life span. Globally, the female life expectancy advantage has increased from 2.0 to 4.4 years between 1950 and 2010, as life-style causes of mortality, which affect males more than females, have become predominant.

Figure 2.6: Life expectancy in selected Arctic regions (circa 2010)

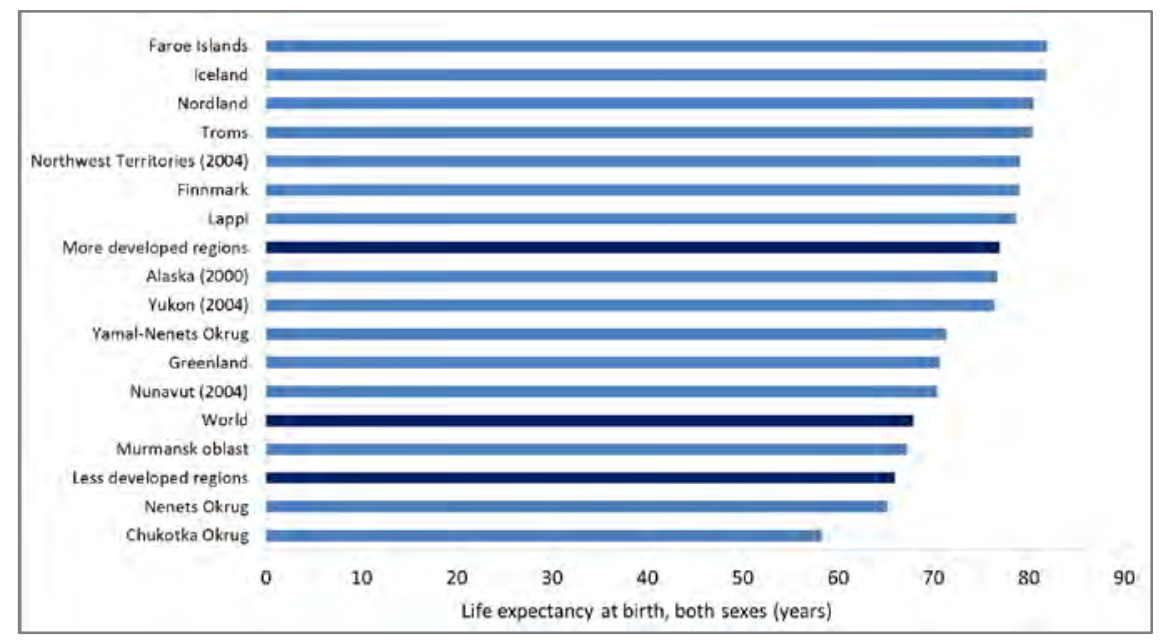

Sources: Data are from national and regional statisical offices.

\subsubsection{Life expectancy}

Based on data for 2000, life expectancy in Alaska does not differ from that for the United States. Life expectancy in the northern territories of Canada has consistently been below the national average with Nunavut having the lowest. In 2004, the last year for which data were available, 
life expectancy for both genders combined was 70.4, nearly 10 years less than the national average of 80.2.

Since 1990, life expectancy in Iceland and the Faroe Islands has risen by similar amounts to similar levels. For men in the Faroe Islands, life expectancy rose by 6 years in 1990 to 79.1 in 2011. For women in the Faroe Islands, life expectancy rose by 4.6 years to 84.9 . In Iceland, the life expectancy increase for women of 3.5 years was also smaller (to 83.6), while male life expectancy increased by 4.9 years from 75.0 to 79.9 years. Life expectancy levels in Greenland are significantly lower than in the other two North Atlantic northern countries but rose faster, especially for males. Male life expectancy in Greenland rose by 7.6 years to 68.3 in 2011, and for females by 4.6 years over the same period.

Of the Arctic regions of Norway, Nordland and Troms have levels of life expectancy close to the national average while Finnmark has generally had the lowest life expectancy of any of the twenty Norwegian regions at the NUTS3 level, though it is only 2.1 years below the national average for males and 1.3 for females. (NUTS, or Nomenclature of Territorial Units for Statistics, from the French nomenclature d'unités territoriales statistiques, is a system for referencing the administrative divisions of countries for statistical purposes). Life expectancy has increased for the entire country and for all regions, more so for males than females as the gender gap has narrowed. Life expectancy in Lapland is within a half year of the national average for both genders.

One of the consequences of Russia's post-Soviet transition away from a centrally-planned towards a market economy has been the steep increases in mortality and precipitous declines in life expectancy (Eberstadt, 2005; World Bank, 2005). The health status of the Russian population has never been good, and prior to the economic transition, levels of life expectancy were far below those expected based on the country's GDP and level of development. Life expectancy for men declined by 7.4 years from 64.9 in 1987, to 57.5 in 1994 before recovering somewhat to 64.0 in 2011. The male-female gap in life expectancy in Russia is the highest in the world with women currently outliving men by 12 years. The consensus is that the increase in mortality was due to extreme psychosocial stress brought about by the social upheaval of the transition period.

There are significant regional disparities in life expectancy in Russia, with many of the Arctic and Siberian regions having levels far below the already-low national average. In the early 1990s, life expectancy in Murmansk was about a year above the national average. By 1995, near the nadir of Russia's post-Soviet plunge in life expectancy, levels 
in Murmansk were less than the national average and in Chukotka they were two years less than the national average. In the 2002, the first year for which data for all of the okrugs were published, life expectancy in Yamal-Nenets okrug exceeded the national average, while that in Murmansk was less than the national level by 1.5 years. Life expectancy in Nenets okrug was 3.5 years below the national average and in Chukotka it was 7 years below the national average, at 58.0 years for both genders, or 63.9 for females and an absurdly low level of 53.3 years for males.

Social and economic dislocation explains Russia's steep decline in life expectancy. The largest increases in the causes of death were cardiovascular diseases and external causes including accidents, suicides, and murder. In these periphery Arctic regions, with the rapid shrinkage of local economies and high levels of out-migration, plunges in life expectancy were even steeper. In 2009, the last year for which data are available, the same rankings relative to the national average remained, with life expectancy in Chukotka falling even more relative to the Russian average. In that year, people in Chukotka could expect to live 10.5 years less than the Russian average, 68.7 years in Russia versus 58.2 years in Chukotka, the lowest level of any Russian region. The explanation for this low level can only partially be attributed to the Indigenous population as they only make up one-third of Chukotka's population.

Figure 2.6 summarizes data available on life expectancy for the Arctic regions showing the incredible diversity of life chances for Arctic residents of different regions. There is an enormous 24-year difference between the Faroe Islands and Chukotka. The Arctic regions of Russia have the lowest levels of life expectancy with Chukotka and the Nenets okrug having levels below those of less developed countries. Greenland and Nunavut also have relatively low levels, combining mortality patterns of both more and less developed countries, due in part to colonialism.

\subsubsection{Gender differences in life expectancy}

Globally, women outlive men by 4.4 years (Figure 2.7). The gap in developed countries, 7 years, is greater than in less developed countries where the female advantage is 3.5 years. The reason is that in developed countries, life-style factors, which disproportionally impact males, are the major causes of death. In less developed countries, factors contributing to major causes of death impact both genders more or less equally. In Arctic countries and regions there is correlation between large female advantages in life expectancy and low overall lev- 
els, as low levels of life expectancy by half the population would necessarily bring down the overall levels.

Three of the four Arctic regions of Russia stand out with women outliving men by more than ten years. The exception is the Yamal-Nenets region where women only outlive men by 4.2 years.

Nunavut and Lapland also have wide gaps though these are close to the level for more developed countries. At the other extreme are a number of Arctic regions that have female-males gaps in life expectancy close to or less than the global average.

\section{Figure 2.7: Female advantage in life expectancy (circa 2010)}

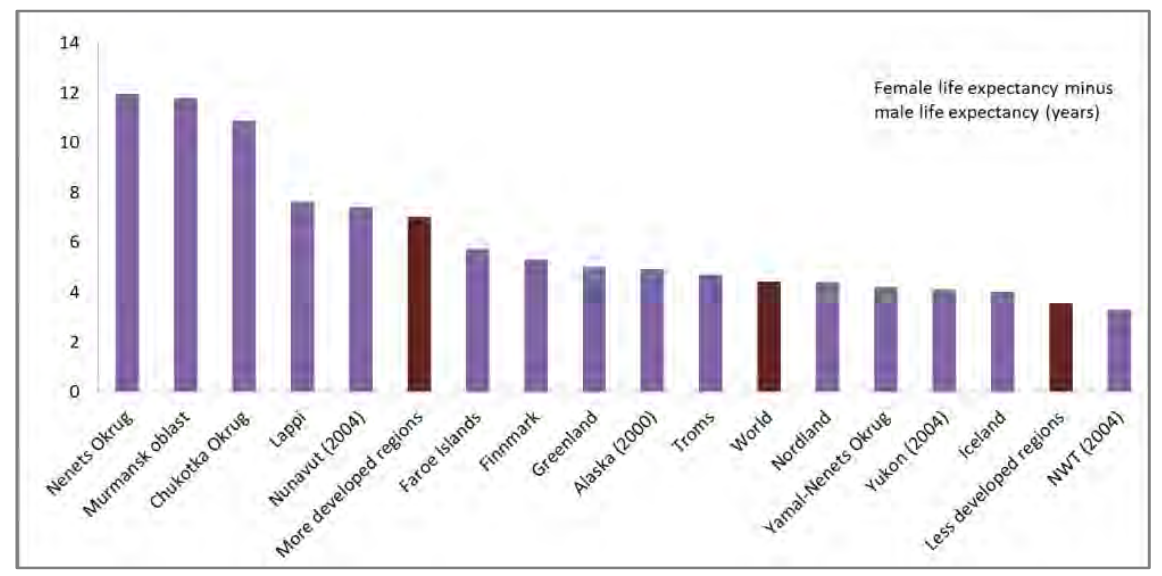

Sources: Data are from national and regional statistical offices.

\subsubsection{Infant mortality}

The infant mortality rate has been found to be a robust indicator of the level of development of a country or society. Large increases in life expectancy in the twentieth century are due in part to the steep declines in infant mortality. Globally, the infant mortality rate declined from 133 infant deaths per thousand births in 1950 to 46 in 2005. Among developed countries, there has been a decline from 60 to 6 . In some developed countries, the infant mortality rate is approaching the lowest practical limits. In less developed countries, the rate has fallen from 151 in 1950 to 50 in 2005. As with other indicators, the Arctic populations are combinations of more and less developed societies and the infant mortality rates reflect this.

Like the rest of the United States, the infant mortality rate in Alaska has steadily declined from 7.7 infant deaths per thousand births in 1994 to 6.7 in 2009 (though there are wide annual fluctuations for this indica- 
tor in Alaska and other Arctic regions because of small number of occurrences). The infant mortality rate for Alaska is close to the national average (Figure 2.8). However, the racial gaps in infant mortality in Alaska are wide and becoming wider. In 1997-1999, whites in Alaska had an infant mortality rate of 5.3 infant deaths per thousand births while Alaska Natives had a rate of 9.4 (Alaska Bureau of Vital Statistics, 2008). In 2007-2009, the rate for whites had declined to 4.0 while the rate for Natives had increased to 11.4.

Figure 2.8: Infant mortality in the Arctic

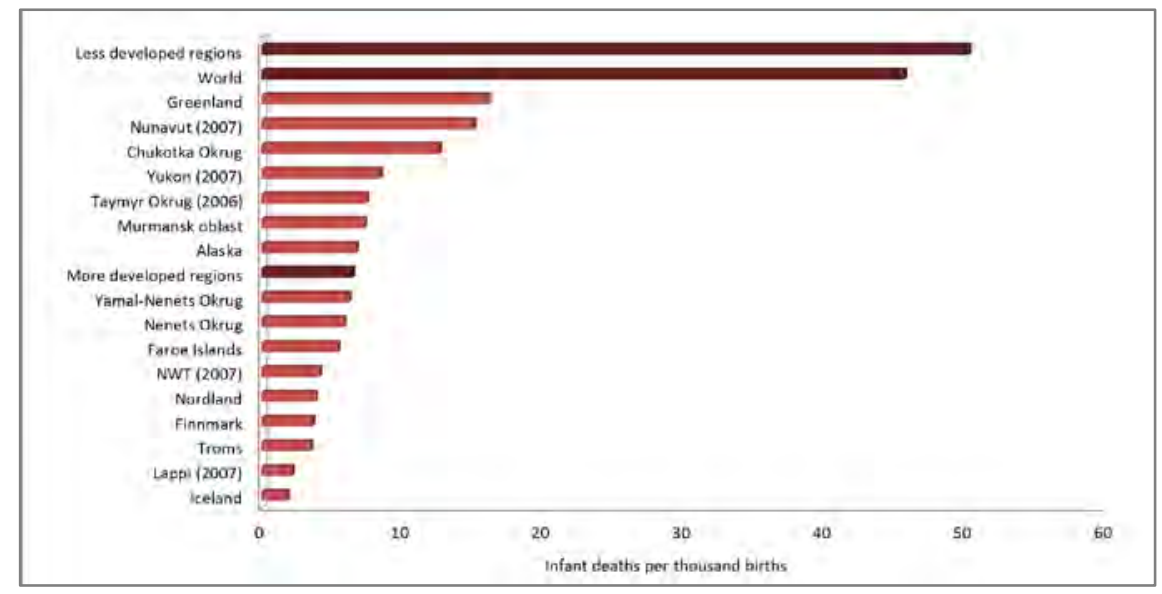

Sources and notes: Data are from national and regional statistical offices and UN Population Division. Data are for 2010 unless otherwise noted.

In Canada, the three northern territories have the highest infant mortality rates in the country with Nunavut consistently being the highest.

It is difficult to determine trends in infant mortality in the Faroe Islands, Greenland, and Iceland because of their rare occurrences in these small populations. Iceland has among the lowest rates in the world at 1.8. The rate in the Faroe Islands is also quite low at 5.4, while that in Greenland is higher at 16.4.

The infant mortality rate in Norway has declined from 7.9 infant deaths per thousand births in 1990 to 3.1 in 2009, placing it among the lowest rates in the world. As recently as 1990, the three Arctic regions of Norway had infant mortality rates among the highest in the country. While they still have rates above the national average, with rates between 3.5 and 3.8, they are quite low by international standards. The infant mortality rate in Finland and Lapland has declined to quite low levels and the rate in Lapland does not differ from the national average. 
Russia's recent demographic crisis affected working-age males the most and was not due to a collapse of the health care system, which would have had an effect on children and infants. The national infant mortality rate fell from 17.4 in 1990 to 8.1 in 2009. In 1990, most Arctic regions had infant mortality rates slightly above the national average. By 2000, the regional gaps had widened and some Arctic regions had rates significantly above the national average. For instance, the rate in Chukotka was 23.4 against the national average of 15.3. In 2009, most Arctic regions had rates below the national average except for Chukotka, which remained above with a rate of 12.6 while the national average was 8.1.

The Arctic countries and regions all have infant mortality rates well below the global average, with some such as Iceland and Norway having among the lowest rates in the world. Greenland, Nunavut, and Chukotka, all predominantly Indigenous regions, have the highest rates and are the only regions with rates above 10 infant deaths per thousand births - but these are also not particularly high by global standards. Most of the Arctic regions have rates around or less than the average for more developed countries. This includes the Arctic regions of Russia, where the mortality crisis has affected persons in the working ages more so than infants, children, or the elderly.

\section{AnnCatrin Blind, a young herder, and her son, Assar. Northern Sweden}

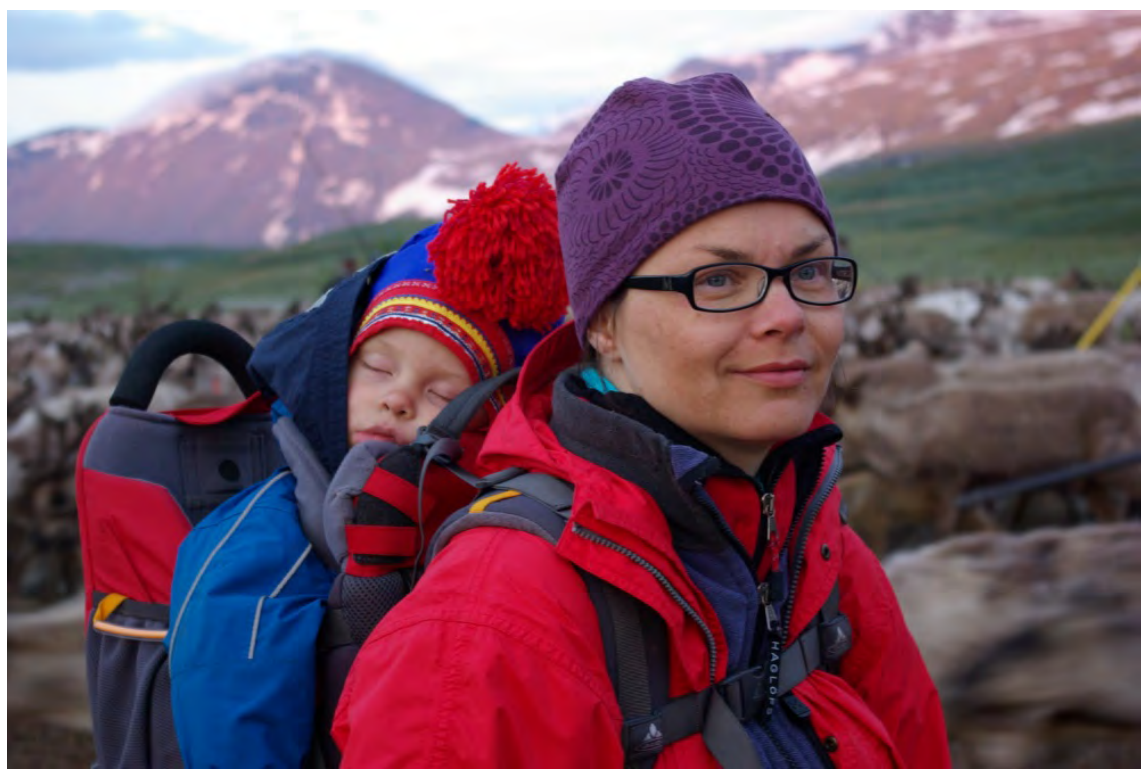

Credit: Hugh Beach. 


\subsection{Changing composition of the Arctic population}

The previous section examined overall changes in the population. This section turns to examination of changes in the gender, age, and ethnic composition of the population.

\subsubsection{Changing gender composition}

Because the Arctic economy is heavily based on resource extraction and fishing, the region has a relatively high male gender ratio. In the early days of outsider migration to the Arctic, the flows were predominantly male. For example, according to the 1900 census for Alaska, at the time of Alaska Gold Rush, there were 258 males per 100 females (Sandberg, 2013). The male gender ratio steadily declined as more families moved to the area but then increased again to 162 males per 100 females in 1950 with the migration of predominantly-male military personnel at the onset of the Cold War. Again, a pattern of more permanent settlement followed, including wives and children, which lowered the male gender ratio. Still, Alaska remains the state with the highest male gender ratio in the United States. A similar pattern of high male gender ratios in the period of initial contact and exploration took place across the Arctic. However, as the economies of Alaska and other Arctic regions have diversified the percentage males has declined, though in most the ratio of men-to-women remains above the national and global ratios. More recently, female outmigration has exceeded male outmigration from a number of Arctic regions, leading again to high male gender ratios, especially in some smaller settlements. For Greenland, this has been the case since the 1980s.

Globally, there are 102 males per 100 females. In developed countries, the male gender ratio is much lower, 95 males per 100 females, because of higher male mortality, whereas in less developed countries it is 103 males per 100 females. Analysis of gender ratios needs to also be compared to national gender ratios because of the wide diversity for the eight Arctic countries. Both Greenland, with 113 males per 100 females and the Faroe Islands with 108, have ratios which place them among the highest in the world. The reason for these high male gender ratios is due to recent high female out-migration (Hamilton and Rasmussen, 2010). Iceland has a male gender ratio of 101 males per 100 females, close to the global average. With one exception, most other Arctic regions have slightly lower male gender ratios of between 100 and 97 males per 100 females. Russia and other former Soviet states, with just 86 men per 100 
women, have the lowest male gender ratios in the world due to high male mortality and longer female life expectancy.

At the sub-national, and even more so at the settlement level, there are rather skewed male gender ratios, as shown in Figure 2.9. Arctic regions in darkest blue are those with the highest gender ratios relative to the national averages, which is the situation across most of the Arctic. Though some of the gender-based division of labor is disappearing, there is a new pattern emerging of higher female education in the Arctic resulting in higher rates of female out-migration, first from smaller to larger settlements and then out of the Arctic either to the metropoles of the Arctic states or abroad, in seek of work commensurate with their skill levels. While men also migrate away, they tend to do so on a temporary basis, while women tend to leave permanently. Only in some of the larger Arctic settlements with more diversified economies is there a surplus of women. At the other extreme are smaller settlements with a narrow economic base such as fishing, forestry, mining, construction, or military that are sectors dominated by males (Nordregio, 2011).

According to the 2010 U.S. census, in Alaska there were 109 males per 100 females, a slight increase since 2000 when there were 107 males per 100 females. However, even this slightly higher level is part of a long-term downward trend since World War II and the beginning of the Cold War when the male gender ratio peaked with the inflow of servicemen to the state. The inflow of military personnel at that time not only temporarily increased the gender ratio in Alaska but in other ways had a profound influence on the size and spatial pattern of settlement. All three northern territories of Canada have male gender ratios higher than the national average with Nunavut being the highest with 106 males per 100 females. All the Arctic regions of Fennoscandia have higher male gender ratios than the national averages by a few percentage points. 
Figure 2.9: Sex ratio of Arctic cities and regions

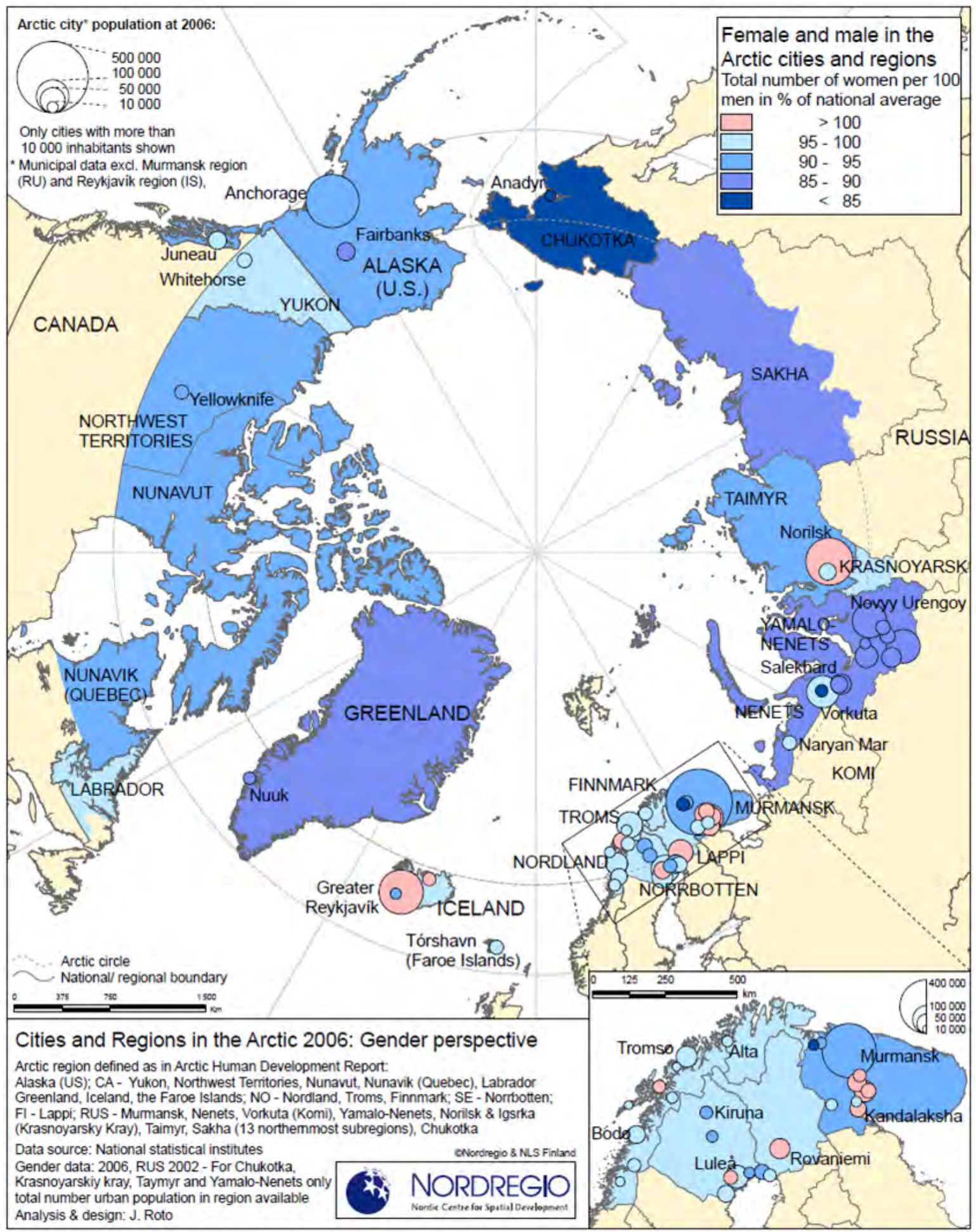


Similar to the situation in other Arctic countries, the male gender ratio in the Russian Arctic is higher than the country as a whole, 96 males per 100 females versus 86 for the entire country. However, the gender ratio in the Russian Arctic differs from other Arctic regions in two important respects (Heleniak, 2011). The first is that while male gender ratios in other Arctic regions have been stable or slightly increasing over the past decades, in the Russian Arctic, there has been a precipitous decline in the male gender ratio since the economic transition in Russia began in the early 1990s. In Murmansk, the ratio of males to females fell from 102 in 1990 to 94 in 2010, in Nenets okrug, from 96 to 94, in Yamal-Nenets from 109 to 98, in Taymyr okrug, from 100 to 93, and in Chukotka from 110 to 105. The second difference is that whereas levels and changes in the gender ratio elsewhere in the Arctic are driven largely by differential rates of migration between the genders, in the Russian Arctic, the steeps declines are the result of unusually large differences in rates of natural increase.

Figure 2.10 shows the percentage point change in the number of males per 100 females by region in Russia during the inter-census periods from 1989 to 2002 and from 2002 to 2010. It is the periphery regions in the Arctic and Siberia where the male gender ratios declined the most. The explanation is not primarily one of differential rates of migration. For a broader set of regions included in the Russian North, between 1990 and 2010, there was a net out-migration of 926 thousand for males and 827 thousand for females, a difference of 99 thousand. Over the same period, males had a natural decrease of 69 thousand while females had a natural increase of 209 thousand, a difference of 278 thousand. Thus, about threequarters of the explanation for the change in the gender ratios in these regions can be explained by the increased differentials in natural increase between men and women. The explanation for these differences lies in examination of causes of death in the Arctic. Death rates from external causes (murder, suicide, accidents) and cardiovascular diseases are much higher in the North than in the rest of Russia. These are factors that disproportionally affect men. Thus, the mortality crisis, which affected Russia during the past two decades as a result of the country's economic transition, had an even greater impact in the northern and Arctic regions where the social upheaval was even more pronounced. 
Fig 2.10a: Male-Female Change 1989-2002

Change in Sex Ratio by Region in Russia, 1989 to 2002

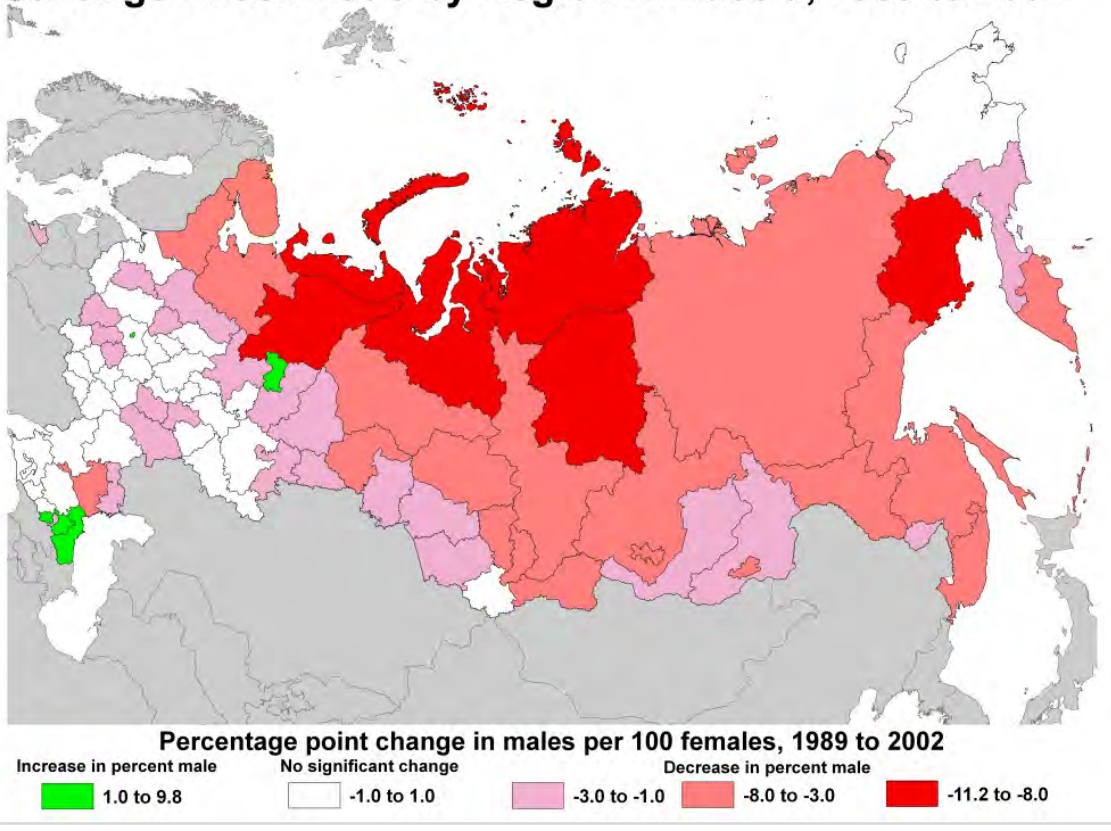

Fig 2.10b: Male-Female Change 2002-2010

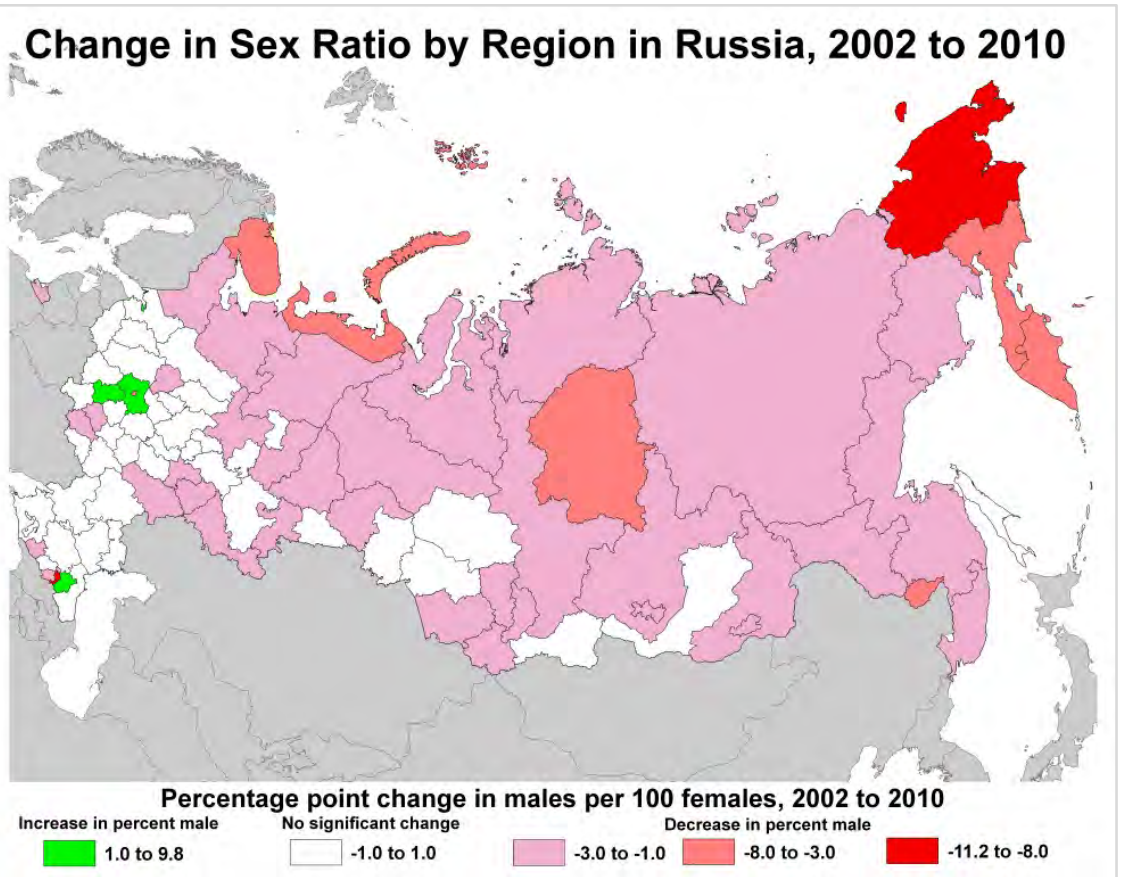


The wide diversity in the male-female gender ratios can be seen in Figure 2.11. With one exception, the region with the lowest male gender ratios are in the Russian Arctic but these need to be seen in the context of Russia which has among the lowest gender ratios in the world. The exception is Chukotka, which with a gender ratio of 105 males per 100, has nearly 20 more males per 100 females than the country as a whole. Aside from Russia, most Arctic regions have more males than females as expected by the occupational differences, which attract more men. At the high end are Greenland, Alaska, and the Faroe Islands.

Figure 2.11: Males per 100 females in the regions of the Arctic, 2010-2012

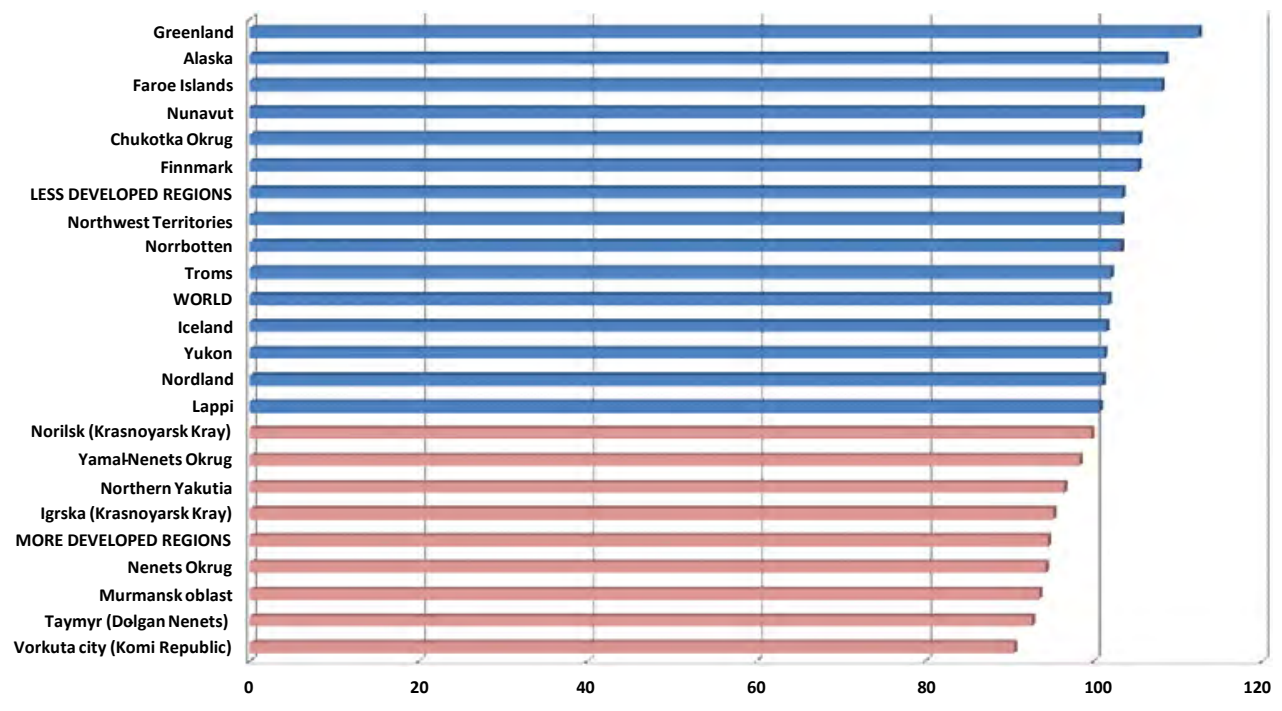

Source: UN Population Division, ArcticStat, and national statistical offices.

\subsubsection{Changes in the age composition}

The age structure of a population reflects past levels of fertility, mortality, and age-differential migration into and out of a region. The current age structure of a population also determines the parameters of future population growth or decline. All else being equal, a population that has a relatively young population will grow in the future because of factors built into its age structure, whereas an older population is likely to have no growth or a declining population. Several measures are used to evaluate the age structures of the Arctic regions - the median age of the population, the shares below, in, and above the working ages, and the age structure of the populations. Like elsewhere in the world, the population of the Arctic is aging which will have consequences for these societies. 
The median age is the age at which half the population is older and half the population is younger. In 2010, the median age of the global population was 29 years, in less developed countries 27 years, and in more developed countries 40 years, the latter reflecting unpredicted population aging. Among Arctic countries, Finland has the highest median age at 42 , only 3 years younger than Japan, the country with the oldest population in the world. Sweden is the next oldest Arctic country with a median age of 41 , followed by Canada at 40 years, Norway at 39 , the Faroe Islands and Russia at 38, the United States at 37, Iceland at 35, and Greenland at 32. In spite of Greenland having the youngest median age of all Arctic countries, it is still has an older population than the median age of the global population.

Among Arctic regions, Alaska has a median age three years younger than that for the United States. In Canada, Yukon has a median age just one year below that for Canada, while the median age for NWT is nearly 8 years younger. The median age for the predominantly Inuit population in Nunavut, at 24 years, is younger than the median age for less developed regions in the world. In Norway, Nordland has a median age roughly the same as the entire country while Troms and Finnmark are slightly younger. Both Norrbotten in Sweden and Lappi in Finland have median ages older than the national levels. In the Russian Arctic, the median age in Murmansk is 37 years, less than a year younger than the Russian average of 38 years. The other Arctic regions for which data are available, Nenets, Yamal-Nenets, and Chukotka okrugs have median ages about 33 years, or 5 years below the Russian level.

Population aging is nearly universal across the world due to declining birth rates and increasing longevity. Population aging can be depicted in several ways, using the median age, the shares of the population below, in, and above the working ages, or the dependency ratio the ratio of the number of persons outside the working ages to those in the working ages (to facilitate comparison across Arctic countries, a standard definition of working ages is used of persons between the ages of 15 and 64, even though countries differ in their official definitions of working ages). The dependency ratio can be sub-divided into a youth dependency ratio and an elderly dependency ratio. Differences in the sizes of dependency ratios call for differences in the mix of social spending (e.g. spending on health care and education). The size of the overall dependency ratio and the relative size of the workforce have implications for economic growth.

As with most demographic indicators, there are significant differences among Arctic regions in terms of the age structure as measured by 
the median age. The Arctic regions of Fennoscandia have the highest median ages between 38 and 45 years. These are regions in demographically advanced countries where the issue of how to deal with population aging has been part of the political discourse for a number of years (Megatrends, 2011). Yukon and the Faroe Islands are also in this group. A middle group consists of Alaska and Iceland with median ages of 34 to 35 years. Relatively high birth rates and continued in-migration into both contribute to the lower median age. A younger group is made up of the Siberian regions of the Russian Arctic, Greenland, NWT, and Nunavut with median ages of 34 years or younger. More so than other regions of the Arctic, the regions of the Russian Arctic were seen as places of temporary labor, from which people would return to the "mainland" or central Russia upon retirement.

The series of maps in Figure 2.12 show the relative portions of the populations in the youth category (age 15 and below), in the working ages (ages 15 to 64), and in the pension ages (64 and older), (though the actual pension ages differ among countries). The Arctic regions with the largest shares of persons in the youth ages are in Nunavut and the Russian Arctic (Figure 2.12a). The Arctic regions with the smallest youth shares are in Fennoscandia and Murmansk. This common trend among the Arctic regions of northern Fennoscandia (including the Murmansk oblast) shows up in a number of demographic indicators, even though they are from four different countries and two quite different economic systems. The difference between the relative youth shares also illustrates how different Murmansk is demographically from the rest of the Russian Arctic. The share of persons below age 15 now and in the future is crucial for determining the demand for education and training. 
Figure 2.12a: Population under 15 years in the Arctic regions

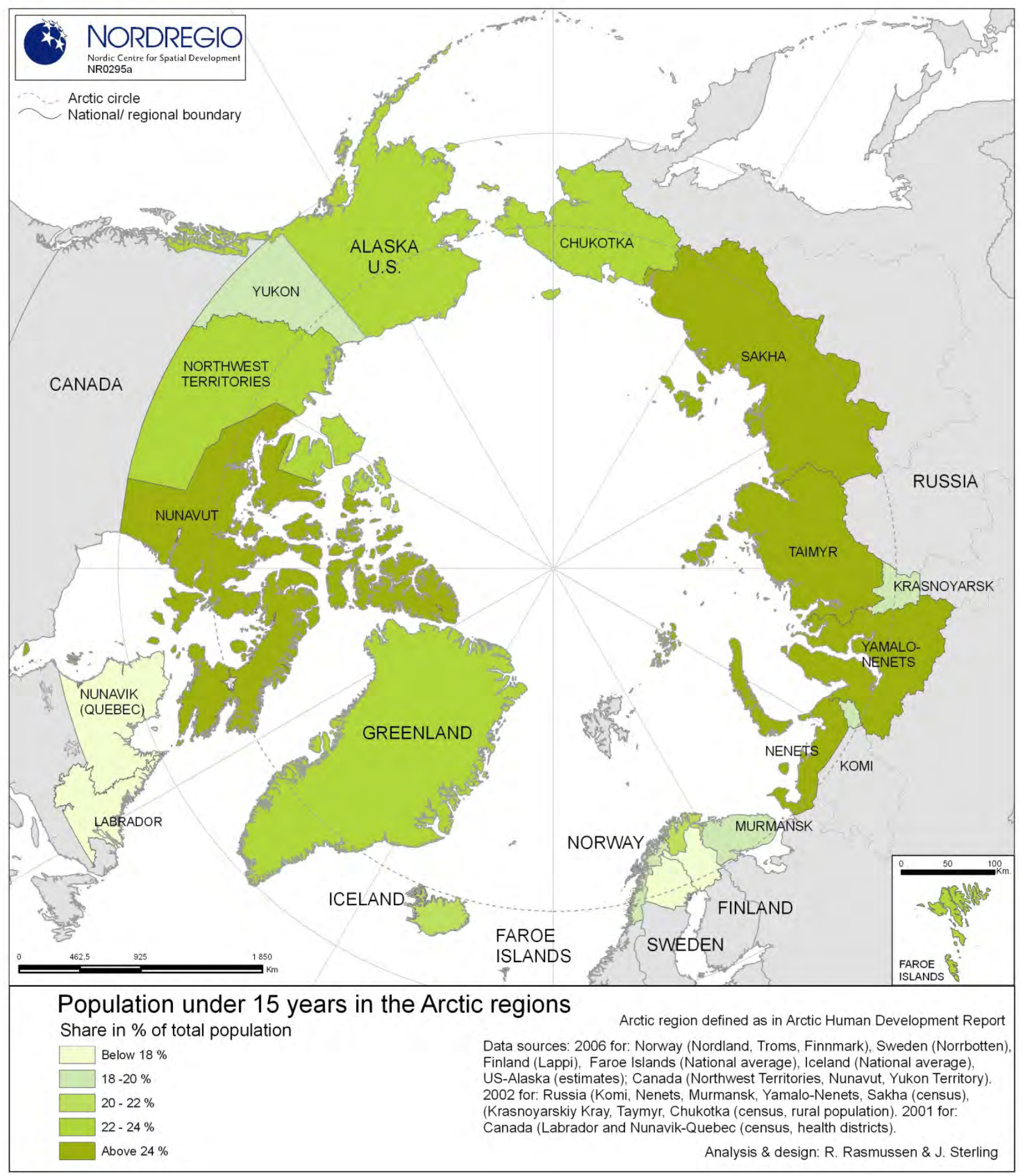


Figure 2.12b: Population 15-64 years (working-age)

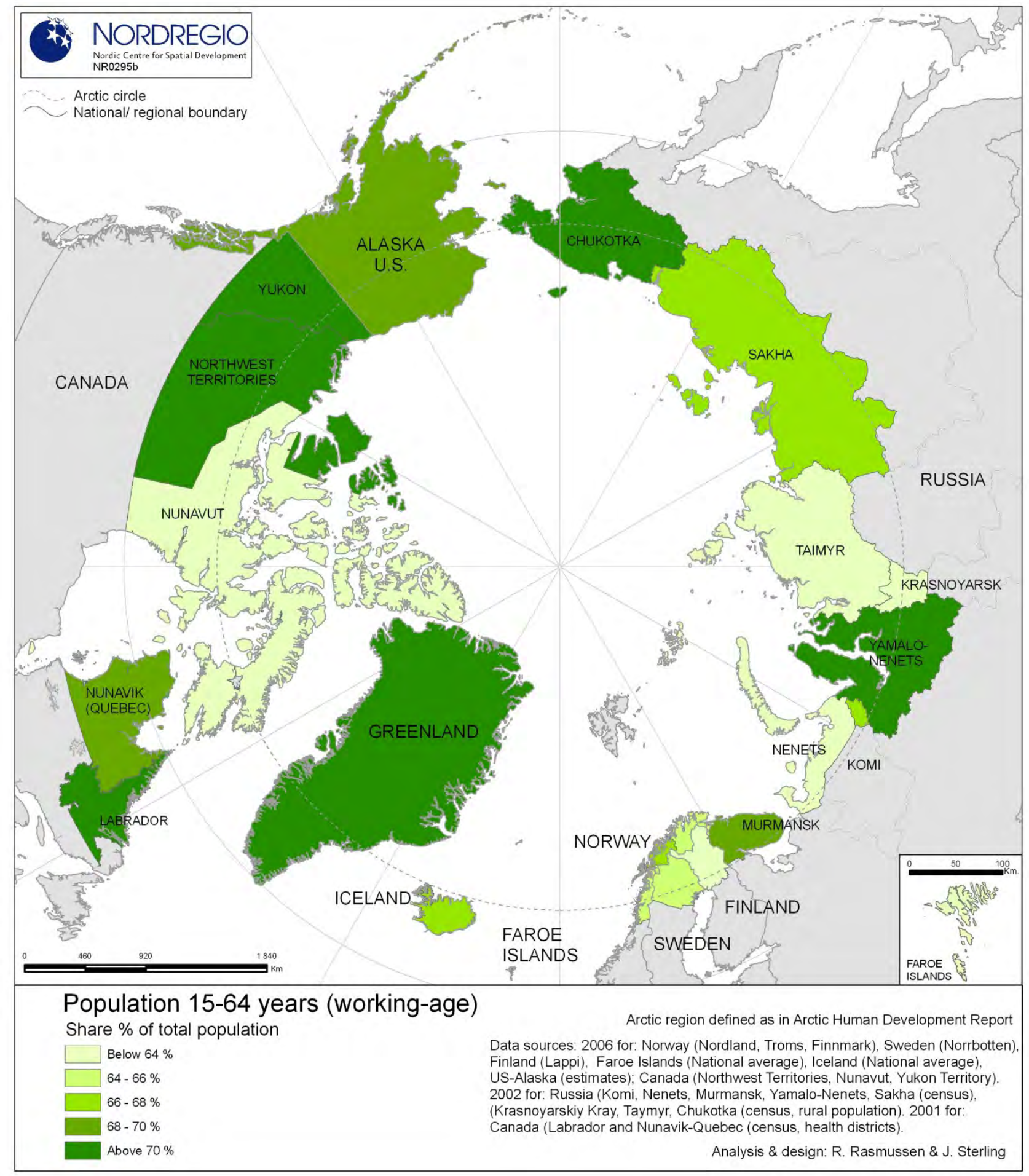


Figure 2.12c: Population over 64 years in the Arctic regions

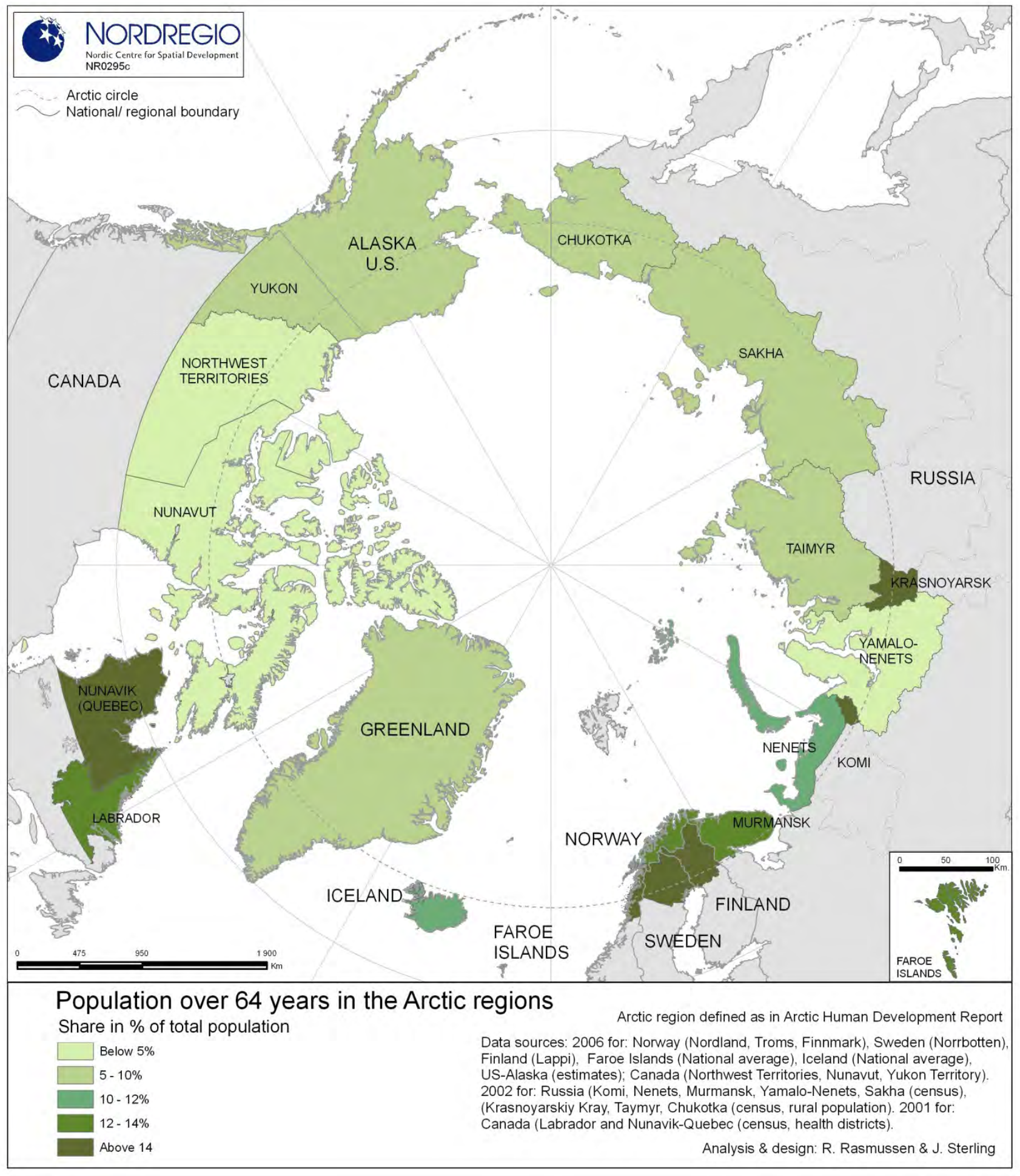


There is a diverse set of regions with low proportions in the working ages including Nunavut, the Arctic regions of Fennoscandia, the Nenets and Taymyr okrugs (Figure 2.12b). For Fennoscandia the explanation is the relatively high portions of pension-age persons and small youth populations while in the others, the explanation is the large youth shares. Declines in the working-age populations brought about by increases in life expectancy and out-migration is leading to a shortage of workers in some of these regions.

The Arctic regions of the Nordic countries as well as Murmansk, Nunavik and Labrador have the highest shares of pension-age persons (Figure 2.12c). Yamal-Nenets okrug and NWT have relatively small elderly shares because of recent in-migration of young persons due to growing resource economies and in Nunavut because of high fertility.

Indigenous and non-Indigenous populations have quite different age structures as most Indigenous populations are at an earlier stage of the demographic transition. As a result, most have much younger populations and are poised to have continued population growth into the future. Comparing the Alaska Native population and that of nonIndigenous peoples in Alaska shows some of these differences in the age structures (Figure 2.13) (the Indigenous population in Alaska includes American Indians and Alaska Natives). The Alaska Native population has a much younger population, as indicated by the much larger cohorts of persons under age 20; $39 \%$ of Alaska Natives are young as compared to $27 \%$ of non-Natives. The relative size of the non-Indigenous population becomes larger starting at age 20, in part because these are the most mobile age groups (at age 20 and higher), when young people begin to migrate to Alaska in large numbers. The non-Indigenous population in Alaska is much larger among ages 50 to 64 . The predominantly Inuit and Indigenous regions of Arctic Canada have similar age structures relative to the total population. In Nunavut, $42 \%$ of the population is below age 20 , whereas $23 \%$ of Canadians are below age 20 . 
Figure 2.13: Age-Sex Composition of Indigenous and Non-Indigenous Populations in Selected Arctic Regions, circa 2010

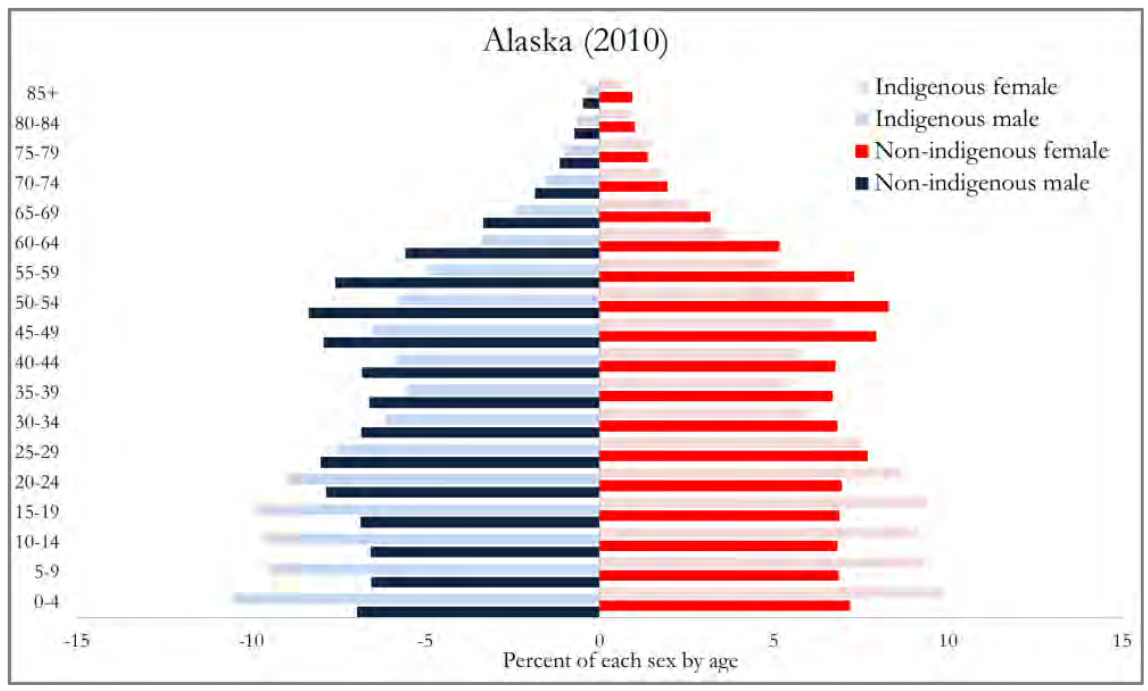

Sources: Alaska Department of Labor and Workforce Development, Research and Analysis Section; U.S. Census Bureau; and National Center for Health Statistics

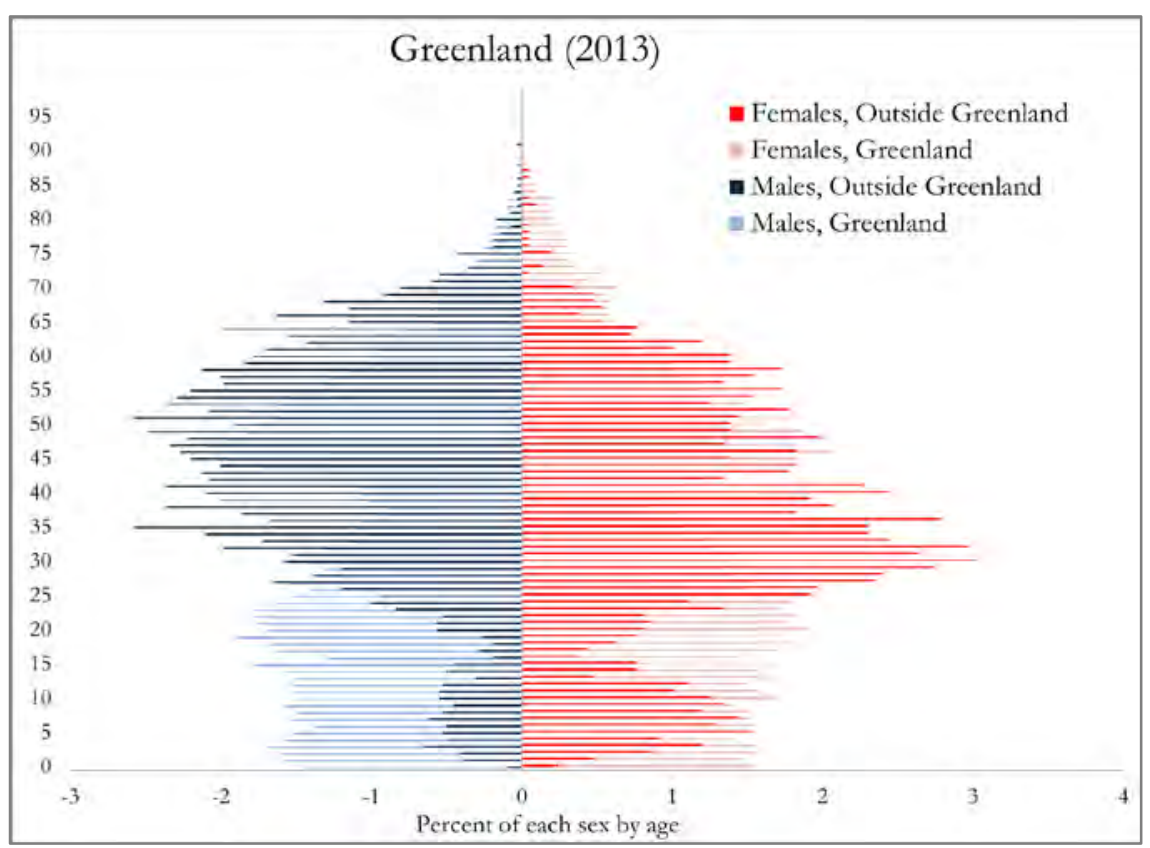

Source: Statistics Greenland. 


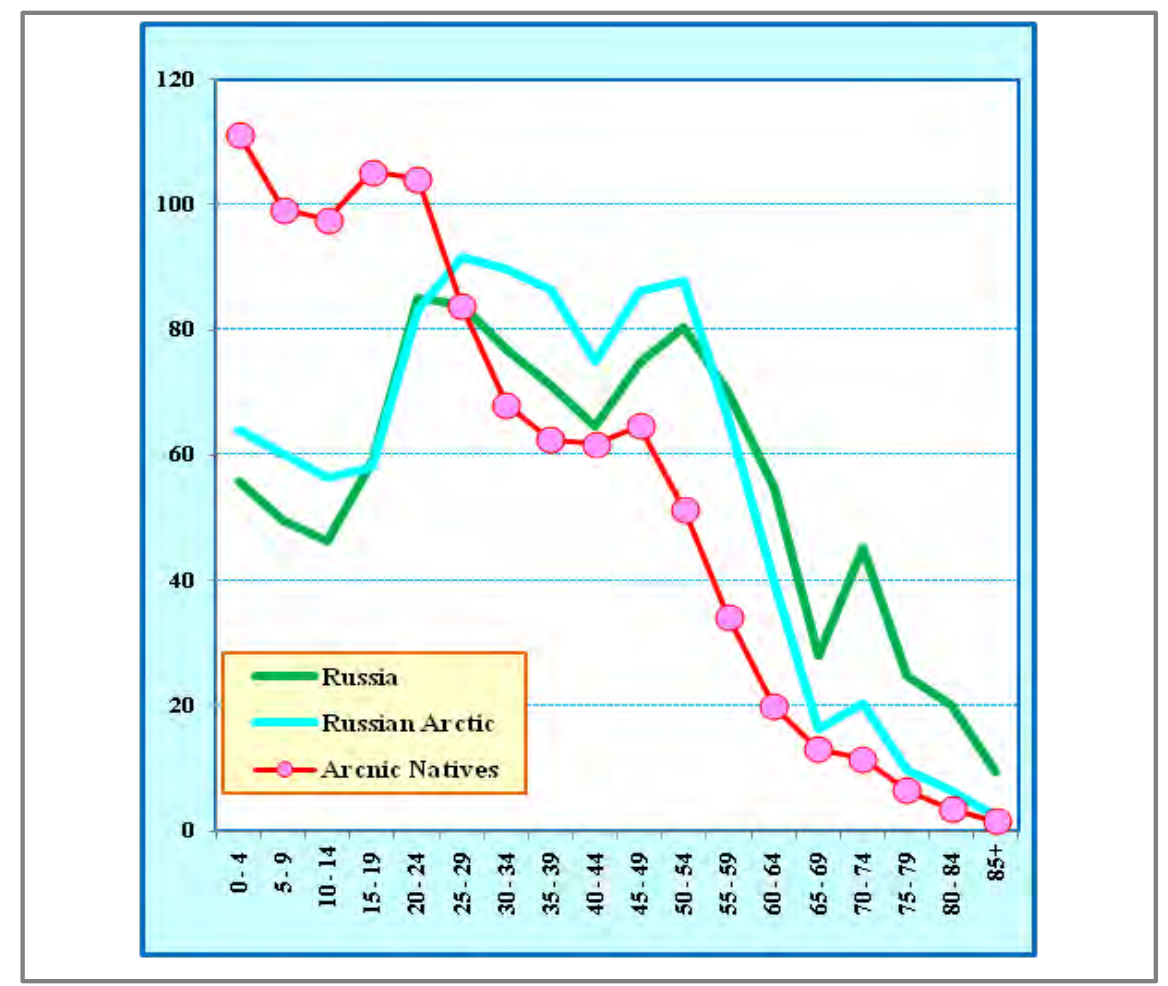

In Greenland, there are the same differences between the Indigenous population (those born in Greenland) and the non-Indigenous population (those born outside Greenland), where 31\% of those from Greenland are below 20 as distinct from the $12 \%$ of those born outside. For persons not from Greenland, it is obviously a place of work, as $80 \%$ of those born outside Greenland are in the working ages versus $61 \%$ of those born in Greenland. Further demonstrating that Greenland is a place of work for those from outside the country is that the male-female gender ratio is 200 males per 100 females for those born outside the country while it is a more expected 104 males per 100 females for those born inside Greenland. These differences are especially pronounced from age 38 and older as the gender ratio for those born outside the country is 3 or 4 males per female.

In the Russia Arctic, the mean age of the population (35.1 years) is slightly younger than the national average for all of Russia (39.0 years) For the numerically small peoples of the North, it is much younger (27.5 years). The Russian Arctic has a larger share of children (ages 0-15) than elsewhere in the country, $18.1 \%$ as opposed to $15.0 \%$. The proportions of adolescents and young adults (15-24 years) are almost equal: $14.1 \%$ in the Arctic and $14.4 \%$ outside. There are noticeably more adults (25-55 years of age) in the Arctic (51.7\%) than outside (45.0\%) and far fewer over 55 years of age, 
$16.0 \%$ in the Arctic versus $25.6 \%$ outside. These differences, in shares of adults and older, are a direct result of migration. Adults come to the Arctic regions and the elderly leave the Arctic. The reason for the high proportion of children is an indirect consequence of the same migration of adults arriving to the Arctic who marry and have children.

The Arctic Indigenous peoples are ethnically distinct from the rest of the inhabitants and have distinct demographic parameters. The Indigenous population has a much younger age structure. Of the total population of Arctic Indigenous peoples, the proportion of children under 15 years of age is more than $30 \%$, of adolescents and young people more than $20 \%$. The other age groups are much smaller than that of nonIndigenous people in the Arctic. In general, the age structure of the Indigenous population is entirely determined by the parameters of its natural movement: a relatively high birth rate, and ultra-high mortality rates, particularly in adulthood.

Russia aged during the inter-census period between 2002 and 2010. The entire population of Russia went from an average age of 37.8 years in 2002 to 39.0 in 2010, while the population of the entire Arctic went from 33.0 to 35.1 years over the same period. The Indigenous peoples in the Arctic had a slightly smaller increase, from 26.5 to 27.5 years.

\subsubsection{Changes in the ethnic composition}

The population of the Arctic is ethnically complex. It consists of various groups whose ancestors have resided in the region for millennia and who are considered Indigenous to the region, non-Indigenous groups who migrated to the region or whose ancestors migrated to the region from the non-Arctic regions of Arctic states to work in resource extraction, military, fishing, or government administration, people of mixed ethnicity, and more recently significant numbers of people who have migrated to the Arctic from non-Arctic states. Indigenous peoples have demographic structures and rates of change that are quite different from the non-Indigenous populations. The percent that the Indigenous populations make up of each Arctic region is shown in Figure 2.14. The highest shares, where the Arctic Indigenous groups are a majority are in Greenland, Nunavut, and Nunavik in northern Quebec. The lowest percent Indigenous are in Murmansk, Nenets okrug, and Yamal-Nenets okrug in Russia, all three the destinations of large numbers of Slavic migrants who moved to these regions to work in resource extraction and large-scale industry. However, the ethnic composition of the Arctic is much more nuanced and complex that what can be shown in a simple thematic map (see Textbox 2.2). 
Figure 2.14: Indigenous population in the Arctic regions

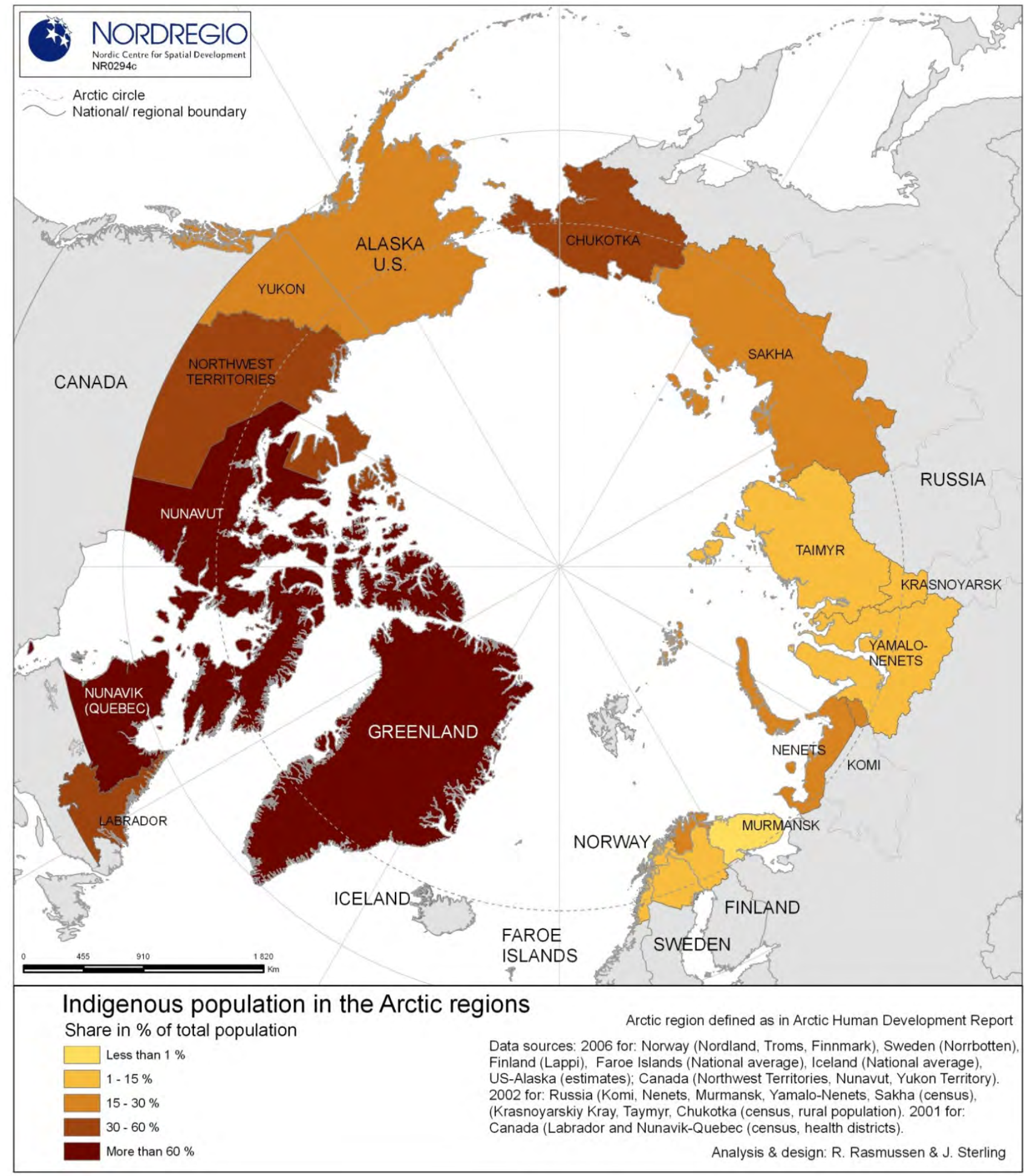




\section{Textbox 2.2}

\section{Categorizing Arctic peoples}

The Arctic states have adopted quite different approaches to the categorization of all peoples, including those in the Arctic. Among the provisions in the UN Declaration on the Rights of Indigenous Peoples (United Nations, 2007) are the rights to self-determination and that every Indigenous individual has the right to a nationality. How these rights are operationalized in statistical and legal terms varies across Arctic countries.

The United States classifies people based on race, a trait based mostly on phenotypes or observable characteristics, and since 1970, whether the person is Hispanic or not, which is considered their ethnicity. People of Hispanic ethnicity can be of any race. A category for American Indian was added in 1870 and Alaska Natives were included in this category. In 2000, the category was expanded to read 'American Indian or Alaska Native', though they are tabulated together as a combined group. People who check this category are asked to indicate their enrolled or principle tribe.

Canada classifies people based on ethnic origin. This includes three groups of aboriginal peoples - Inuit, Métis and First Nations. The first Canadian census to include the entire territory and all northern aboriginal peoples was in 1891 (Hamilton and Inwood, 2011).

Greenland categorizes people based on place of birth, the main distinction being in Greenland or outside Greenland. This distinction can be roughly thought to be Native Greenlanders or non-Greenlanders, or Inuit or non-Inuit. The Faroe Islands classifies people based on place of birth or citizenship (which they also refer to as nationality). Iceland also classifies people by place of birth and citizenship.

Norway, Sweden, and Finland are considered together because the Indigenous peoples in the northern regions are the same, the Sámi, and the treatment of them in censuses and statistical registers is similar. All these states ceased recording ethnicity in the censuses after World War II, in part because of the role that ethnicity played in some of the atrocities. The Sami people are spread across those three countries plus Russia, in the Sámi homeland called Sapmi.

When the Bolsheviks came to power after the 1917 revolution, they needed to make sense of the multi-national empire they were presiding over. They settled on the concept of natsionalnost' (nationality) as the term used to divide people into different groups, a concept still used in post-Soviet Russia (Hirsh, 1997: Hirsh, 2005). They used the results of the first all-union census after creation of Soviet Union in 1926 to demarcate ethnic homelands, including those of the Arctic and Siberian peoples. In the post-Soviet period, many of the various Arctic and Siberian groups are classified belonging to a legal category titled korennye malochislennye narody (numerically small Native peoples). Of these, 26 groups with populations less than 50,000 were designated as malochislenny 
narod Severa (numerically small peoples of the North), a number which has since grown to 37. In the 2010 census, 46 groups were classified as small numbered Indigenous peoples, of which 37 lived predominantly in Siberian or the Arctic. Thus, in the Arctic and Siberia, there are both numerically small peoples of the North and larger groups such as Yakuts, Komi, and Karelians.

Because of the different ways in which Arctic states categorize people based on their identity, no attempt is made to compare the percent Indigenous or Arctic Native across all countries. Rather, the ethnic or racial breakdown for each Arctic country and region is shown based on national definitions.

In the 2010 U.S. census, Whites make up $72 \%$ of the U.S. population. The combined category American Indians and Alaska Natives totaled 2.9 million people, or $0.9 \%$ of the total U.S. population. $44 \%$ of American Indians and Alaska Natives reported in combination with another race, the most common being "some other race". For Alaska, using the adjusted data, which apportions those identifying more than one race, the American Indian and Alaska Native population totaled 120,452, or $17.0 \%$ of the population of Alaska. This percentage has remained at about this level since 1960, though in absolute terms, this is the largest the Alaska Native population has ever been.

In the 2006 Canadian census, the Aboriginal population, consisting of First Nations people, Métis, and Inuit, exceeded one million for the first time when they totaled 1,172,785. Only small shares of First Nations people and Métis reside in the Canadian Arctic. In Yukon, Aboriginal peoples made up one-quarter of the population, the majority of these First Nations peoples. In NWT, Aboriginal peoples were half the population, with First Nations peoples being $31 \%$ of the total, Inuit $10 \%$, and Métis 9\%. In the Inuit homeland of Nunavut, Aboriginal peoples made up $85 \%$ of the population. The 24,635 Inuit in Nunavut comprise nearly half of the Inuit in Canada.

"Inuit Nunaat" is the Inuktitut expression for "Inuit homeland", an expanse comprising more than one-third of Canada's land-mass, extending from northern Labrador to Northwest Territories (Statistics Canada, 2006). Inuit Nunaat is comprised of four regions: Nunavut; Nunavik in northern Quebec (home to 9,565 Inuit, or 19\% of the total Inuit population); the Inuvialuit region in Northwest Territories (3,115 Inuit, or 6\% of all Inuit nationally); and Nunatsiavut, in northern Labrador (popula- 
tion of 2,160 Inuit, or 4\% of the total Inuit population). Four-fifths of all Inuit in Canada reside in Inuit Nunaat.

Greenland identifies people based on place of birth. In 2013, 11.1\% of the population was born outside Greenland, the majority born in Denmark. There has been a long-term decline in the percent born outside Greenland since it peaked at $19.2 \%$ in 1975 . The percent born outside Greenland fell below $12 \%$ in 2000 and has remained at about the same level since then. Though not perfect, the percent born in Greenland can be thought to be roughly equal to the percent Arctic Native or the percent Inuit. Because Greenland is a subject of Denmark, nearly all people, 98.6\%, were Danish citizens in 2011. Thai residents were the second largest group, though they only made up $0.3 \%$ of the population.

\section{Biking in Sisimiut, Greenland}

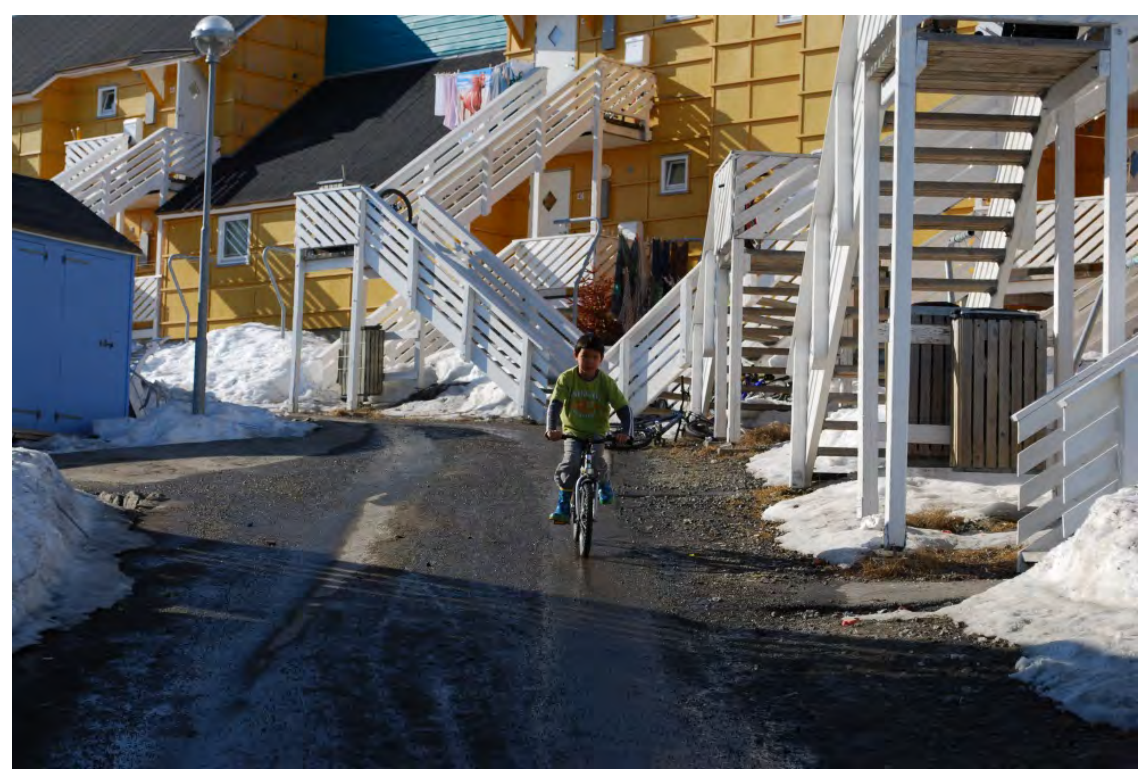

Credit: Rasmus Ole Rasmussen.

The Faroe Islands also classifies people based on place of birth, though unlike Greenland, there was no Indigenous population when Vikings from Norway first arrived more than a thousand years ago. In 2013, $85 \%$ of the population had been born in the Faroe Islands, $6 \%$ in Denmark, and $9 \%$ elsewhere outside the Faroe Islands. Now a subject of Denmark, the vast majority of the population - 97.4\% in 2013 - are Danish citizens. 
Iceland also had no Indigenous population when Norwegian Vikings first occupied the country in the 900s. Historically, Iceland is ethnically homogenous, but there has recently been increased immigration into the country as a result of economic prosperity. In $2013,11 \%$ of the population was foreign-born, up from $4.6 \%$ in 1995. In 2013, the countries of origin of the largest number of foreign-born were Poland $(2.6 \%$ of the population), Denmark (1.0\%), the United States (0.6\%), and Sweden $(0.6 \%)$. Non-citizens make up an increasing share of the population. In $2013,6.7 \%$ of the population did not have Icelandic citizenship, up from just $2.6 \%$ in 2000 . This is down slightly from 2008 , just prior to the financial crisis, when $7.4 \%$ of the population was foreign born. The noncitizens came primarily from Poland (2.9\% of the population) and Lithuania (0.5\%), both relatively recent EU accession countries that send large numbers of foreign workers abroad.

Because ethnicity has not been recorded in censuses in Norway, Sweden, or Finland in decades, estimates of the number of Sámi in this part of their traditional territory are quite wide, varying between 50,000 and 100,000 with 40,000 to 50,000 in Norway, 17,000 to 20,000 in Sweden, 7,000 to 8,000 in Finland, and 2,000 in Russia (Pettersen, 2011). The figures would be significantly higher if those of mixed ethnicity were included.

Ethnic Russians are obviously the dominant group in Russia, constituting $78 \%$ of the population. Tatars are the second largest ethnic group with $3.7 \%$ of the population followed by Ukrainians with $1.3 \%$. These groups are among the most highly educated and mobile and have a large presence in many Arctic regions. The large-numbered Arctic groups, Yakuts, Komi, and Karelians combined are only $0.5 \%$ of the population. The numerically small peoples of the North totaled 253,932 in the 2010 census that is just $0.2 \%$ of the national total. In the Russian Arctic, the total number of Indigenous peoples was 98,651 in 2010, which is 5.5\% of the total Arctic population, an increase over 2002 when they were $4.5 \%$.

The Murmansk oblast was very sparsely populated prior to Russian migration into the region and development. As a result, the Slavic population makes up $87 \%$ of the population. Nearly all of the Sámi in Russia $(1,599$ of 1,771$)$ reside in the Murmansk oblast.

In Nenets okrug, Russians make up $63 \%$ of the population, the titular Nenets, 18\%, and Komi, one of the larger Arctic groups, 9\% (the term titular refers to the group upon which the ethnic homeland is based).

Yamal-Nenets okrug is one of the few northern regions of Russia that actually had a population increase in the post-Soviet period as result of its growing economy from gas extraction. Ethnic Russians constitute $60 \%$ of 
the population followed by Ukrainians with 9\%. The Russian share is lower than in other northern regions because there are significant numbers of people from other parts of Russia and elsewhere in the former Soviet Union who have migrated to the region to work. Arctic Indigenous groups totaled 41,728 or $8 \%$ of the population. This includes 29,772 titular $\mathrm{Ne}-$ nets who make up $6 \%$ of the population of one of their ethnic homelands, 9,489 Khanty, and 1,968 Selkups.

According to estimates made for Taymyr (Dolgan-Nenets) okrug, the ethnic Russian was 59\% of the population, the Arctic Indigenous population primarily Dolgans and Nenets totaled 10,471 which is $29 \%$ of the population.

Following the mass outmigration of the past several decades, the ethnic Russian population in Chukotka now makes up less than half the population, down from 1989 when their percent was 66\%. Arctic Indigenous groups totaled 16,879, one-third of the population. Because of the out-migration of others, the Chukchi percent of the total population has increased from $7.3 \%$ in 1989 , to $24 \%$ in 2002, and 25\% in 2010 .

From the discussion above, it is apparent that the Arctic regions have quite diverse ethnic profiles, with some being composed predominantly of Indigenous peoples while others are made up mostly of migrants from outside the Arctic. As described elsewhere in this chapter, there are significant demographic differences among the various ethnic groups residing in the Arctic in terms of size, urban-rural residence, household size and composition, fertility levels, mortality levels and migration patterns. As described elsewhere in this report, there are also significant differences among the groups in terms of lifestyle, economic status, and political clout. Chapter 3, Cultures and Identities, provides a more nuanced and in-depth description of identity.

\subsubsection{Migration}

Since the time when the first humans crossed the Bering Land Bridge following the retreat of the last ice age, migration has played a large role in shaping the size, distribution, and composition of the Arctic population. The overall population of the Arctic is quite small and even the largest settlements are not very large compared to elsewhere in the world. Thus, the movement of people into or out of the settlements or regions in the Arctic has an enormous impact on the size and composition of the populations. This section provides an overview of trends and patterns of migration in the Arctic. 
Migration is referred to as investment in human capital across space - people migrate in order to improve their quality of life. People migrate to, from, and within the Arctic for the same reasons they migrate elsewhere in the world. There are a set of theories which explain how migration gets started and a separate set that explain how migration keeps going once it has started.

The neoclassical economic approach is the oldest theory of migration and holds that income differentials between regions are why people migration from low-income to high-income regions. An extension of this theory is the new household economics of migration, which uses the household as the unit of analysis, and holds that the goal is maximization of household income, where one member migrates, others remain behind to stabilize income. In dual labor market theory, there is one sector with well-educated persons and well paid and another sector with less well-educated and lower wages, often referred to as "immigrant jobs."

Network theory is one explanation of how migration is perpetuated once started. It states that ties created among migrants lower costs and risks and increase net returns. Related to this is the theory of cumulative causation, which explains that each act of migration makes the next act easier as information is gathered and a system has been created. Institutional theory explains that once migration has started, institutions develop to perpetuate migration flows. Incorporating climate change and the impact that it could play in migration in the Arctic is rather new (Textbox 2.3).

\section{Textbox 2.3}

\section{Climate change and migration in the Arctic}

According to recent IPCC (Intergovernmental Panel on Climate Change) and other reports, there is no doubt that the climate is changing, that much of the change is the result of human activity, and the Arctic is warming more rapidly than other parts of the planet. The warming of much of the Arctic will have impacts on the population at various geographic scales, which could impact settlement and migration patterns. These include reduced thickness of sea ice, which could lead to increased marine access and also to increased coastal erosion. Thawing permafrost could impact buildings in urban settlements. Terrestrial and marine ecosystems could shift causing changes in resource availability. These changes have both positive and negative effects on settlement patterns in the Arctic. 
While the climate in the Arctic is changing, in a number of studies of communities in the region, the impact of climate change on adaptation of communities to change is rather minimal compared to many other factors.

Migration is multi-causal: people move for many different reasons and it is often difficult to isolate just one cause. "Climigration" is a term that has recently been coined to refer to people who are forced or who choose to move because of changing climatic reasons. While there are certainly settlements that will be impacted from climate change, such as coastal villages in Alaska and larger urban settlements impacted by thawing permafrost in Russia, the overall impacts of climate change on migration and settlement patterns in the Arctic require more study.

Sources: Fay. et al., 2010; Ford and Smit, 2004;. Johansen and Skryzhevska, 2013; Bronen and Chapin, 2013.

\subsubsection{Mobility and immobility}

This section starts with analysis of mobility and immobility of Arctic populations. It then looks at and international migration and migration of Indigenous peoples away from the Arctic. The next set of sub-sections look at the changing urban-rural composition of the Arctic and population change in the largest Arctic cities.

Two measures of mobility are used to compare populations between Arctic and non-Arctic regions and across the Arctic. The first is annual mobility or another measure of short-term mobility, depending on data availability. The second is lifetime mobility measured by the percent of the population born outside each Arctic region. Globally, 3\% of the population resides outside their country of birth.

In the United States in 2010, $41 \%$ of the population was born outside the state where they are currently residing, including $13 \%$ who are foreign born (Ren, 2011). In Alaska, 61\% of the population was born outside of Alaska, including 7\% who were born abroad (Figure 2.15). Among U.S. states, only Arizona, Florida, and Nevada have higher shares born outside of these states. Whereas those states all have higher than average foreign-born populations, many Alaskans who were born outside the state come from elsewhere in the United States. In 2009, 22\% of the population of Alaska moved, which is well above the $15 \%$ of all Americans who moved in that year, reflecting higher mobility of the Alaskan population. 
Figure 2.15: Percent of population born outside region in selected Arctic regions, circa 2010

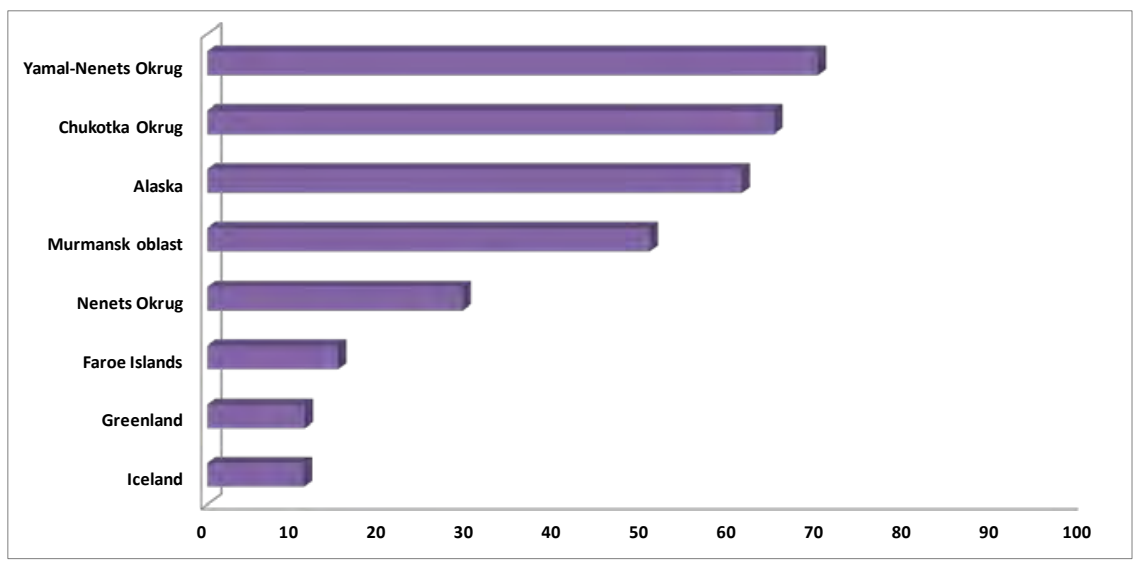

Source: National statistical offices.

According to data from the 2006 Canadian census, all three Arctic regions have had higher portions of their populations migrate over the previous five-year period than the national average.

The data for Greenland, Iceland, and the Faroe Islands show that percent of the population born outside of these countries is respectively 11 , 11 , and $15 \%$. In $2010,16 \%$ of the population of Greenland migrated either internationally or between towns or settlements. According to data for 2008, $13 \%$ of the Icelandic population migrated that year. In $2011,10.5 \%$ of the population of the Faroe Islands moved between the six districts or to or from abroad.

Like the other Arctic countries, Russia has a large foreign-born population. The 11.2 million foreign-born persons in Russia are the second-largest stock in the world after the United States. This consists of many who had migrated to the country from another republic when it was part of the Soviet Union and others who migrated since the breakup. The regions of the Russian Arctic have large numbers of people who were born either elsewhere in Russia or outside of Russia. Though the regions of the Russian Arctic continue to be made up largely of migrant populations, the shares have declined in the post-Soviet period, as it was those born elsewhere who had ties outside these regions who left these regions in the largest numbers during the steep economic decline of the post-Soviet period (Heleniak, 2009). Ukraine and Belarus were the sources of large numbers of people who migrated to live and work in the Arctic.

In 2010, for all of Russia, $10.5 \%$ of the population was born outside the region they were living in, of which $8 \%$ were foreign-born. In $\mathrm{Ne}-$ 
nets okrug, $29 \%$ of the population was born outside the okrug and $6 \%$ were foreign-born. In Murmansk, half the population was born outside the region and 13\% were born abroad. In Chukotka, 65\% were born outside the region, of which 15\% were foreign-born. In Yamal-Nenets, $70 \%$ were born outside the region, of which a quarter were born outside of Russia.

Along with Moscow and St. Petersburg, many of the periphery regions in the Arctic and Siberia have the highest rates of migration turnover. In these regions there are high levels of both in-migration and outmigration and there is a high correlation between the two indicating considerable migration turnover in the Arctic regions.

All of the Arctic regions have highly mobile populations and many people who have originated from outside the region, including many from abroad. This has implications for migration, should there be an economic downturn, as many retain ties to places outside the Arctic to where they could move.

\subsubsection{Changing urban-rural composition}

The percent of a country's population residing in urban areas is reflective of the structure of its economy. When larger shares are employed in mining, manufacturing, and the service sector than in agriculture, there are higher urban shares. Arctic regions tend to have rather small manufacturing sectors but many have large mining, forestry, or resource extraction sectors which tend to be concentrated in urban areas or which develop urban areas by their presence. Urban areas in the Arctic grow because of the in-migration of both outsiders and also Arctic Natives.

There are significant differences in lifestyle and amenities between urban and rural areas, which tend to perpetuate the pull of urban areas in the Arctic and elsewhere.

It is difficult enough to compare the percent urban across all of the world's countries let alone among Arctic countries. In preparing estimates and projections of the urban population, the United Nations relies on the data produced by national sources that reflect the definitions and criteria established by national authorities. It has long been recognized that, given the variety of situations in the countries of the world, it is not possible or desirable to adopt uniform criteria to distinguish urban areas from rural areas. Thus, including for Arctic countries, national definitions are used to define the percent urban.

Globally, the world's population crossed a threshold in 2008, when more than half of the world's population was defined as urban. This is 
a rather rapid rise in global urbanization, up from a $43 \%$ in 1990 . With their increasingly developed economies, more developed countries have long had more than half of their populations residing in urban areas, now 79\%, while less developed countries as a whole are nearing this mark with $49 \%$ currently living in urban areas. Of Arctic countries, all but the Faroe Islands have three-quarters or more of their populations residing in urban areas (Figure 2.16). The Faroe Islands, with a more dispersed economy based on fishing, is only $42 \%$ urban. Iceland, with much of the population residing in the capital of Reykjavik and a few other urban centers, has the highest percent urban of Arctic countries at $94 \%$, followed by Greenland and Sweden at $86 \%$. The percent urban in the Arctic countries continues to increase, albeit at a rather slow pace, as the shares are reaching their maximum possible without further and significant structural economic changes.

Figure 2.16: Percent urban in selected Arctic countries and regions, circa 2010

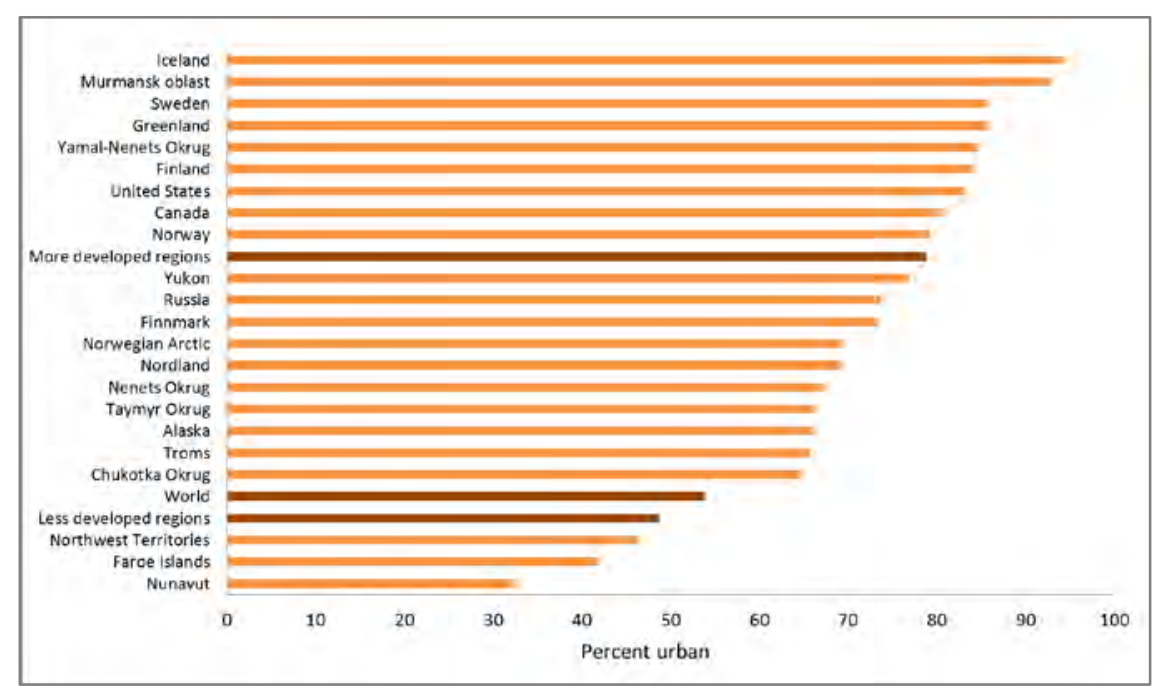

Sources: National statistical offices and UN Population Division.

The population of Alaska is becoming increasingly urbanized with inmigration to urban areas from outside the state as well as within. In 1920 , only $6 \%$ of the population resided in urban areas. With much of the economic growth concentrated in urban areas and the growth of new urban areas, by the 1970 census, more than half, 57\% of Alaska's population was residing in urban areas. However, the percent urban in Alaska has plateaued at about two-thirds of the population over the past three censuses, including the most recent 2010 census. This is far below 
the level of urbanization for the entire country but there are a number of U.S. states with smaller urban shares.

In northern Canada, only Yukon has a share of its population residing in urban areas (76\%) that is anywhere near that of Canada (81\%), while NWT (46\%) and Nunavut (32\%) remain predominantly rural territories. The percent of the population residing in urban areas of the Norwegian Arctic, 69\%, is less than the overall share in Norway, $79 \%$.

In all regions of the Russian Arctic, more than half the population resides in urban areas, with Murmansk (93\%) and Yamal-Nenets (85\%) having the highest shares. Much of the population of Murmansk oblast is concentrated in Murmansk city, the home of the North Sea Fleet, and in a number of other mining settlements, while much of the rest of the region is sparsely inhabited. In Yamal-Nenets, much of the population resides in a number of gas-producing and related transport settlements. Nenets, Taymyr, and Chukotka okrugs all have two-thirds of their populations in urban areas, which is less than the Russian average. Of Russian Arctic regions, Chukotka actually had a decline in the percent urban from 73 to $65 \%$ between the 1989 and 2010 censuses, as much of the contraction of economic activity took place in the large urban settlements. Overall, the sixteen regions defined as the Russian North have $80 \%$ of their populations residing in urban areas, reflecting the structure of their economies based on resource extraction and transport and small agricultural sectors.

There is obviously considerable diversity in each Arctic region's share of urban populations, reflecting differences in economic structures and spatial distributions. The percent urban also reflects the lifestyles and access to urban amenities that each region's population enjoys.

\subsubsection{Population change in largest cities}

Along with the general trend of increased urbanization taking place across the Arctic is increased concentration of the population in most Arctic regions into one or a few of the larger urban settlements. There are many ways to depict this trend but one simple way is to examine the share of the region's population in the largest settlement over time. For nearly all Arctic regions, this settlement serves as the economic, administrative, and transport hub. In many cases, there are far more amenities in these larger settlements, such as education, consumer goods, entertainment and leisure opportunities, as well as employment opportunities. The draw of the "bright lights and big city" will continue this trend well into the future. 
In 1960, 36\% of the population of Alaska lived in Anchorage. This share has steadily increased to about $42 \%$ currently (Figure 2.17). Anchorage's share has leveled off, though there has been increased population growth in the nearby Matanuska-Susitna Valley. Combined with Fairbanks, 55\% of Alaska's population resides in these two urban settlements. This reflects a confluence of factors, including migration of Alaska Natives to larger urban settlements. The share of Alaska Natives who reside in the five most-populous boroughs increased sharply from 42 to $49 \%$ between 2000 and 2010 (Sandberg 2013).

Figure 2.17: Largest city share of total population in selected Arctic regions, 1990-2013

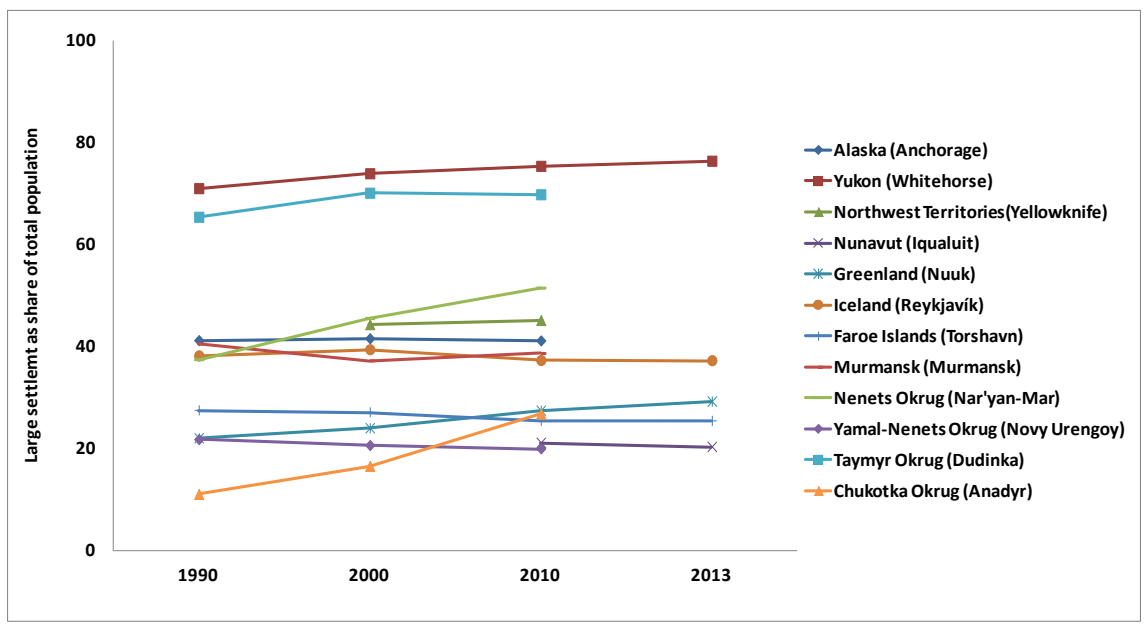

Sources: National and regional statistical offices.

The population of Yukon is extremely concentrated into the capital of Whitehorse and is becoming more so. In 1990, 71\% of the territory's population resided in Whitehorse, a figure that has risen to $76 \%$ in 2013. Between the 2001 and 2006 censuses, Yellowknife's share of NWT's population increased from 44.3 to $45.1 \%$. Because of a deliberate effort to decentralize jobs in Nunavut and higher natural increase in other settlements, Iqaluit's share of the territory's population has declined from $21.1 \%$ in 2006 to $20.3 \%$ in 2013.

Nuuk's share of Greenland's population has been steadily rising over time, from $17.2 \%$ in 1977 to $22.0 \%$ in 1990 to $29.2 \%$ currently. If not for housing shortages in Nuuk, the share might even be higher. Iceland is an extreme example of population concentration, with Reykjavik's population increasing from 8.5\% of Iceland's total in 1901 to $38.2 \%$ in 1990 (the larger capital region also increased from 10.5 to $57.1 \%$ over the 
same period). Reykjavik's share peaked in the early 2000 s at $39.4 \%$ and has declined slightly to $37.2 \%$ in 2013 , in part because of continued growth in the larger capital region. Tórshavn's share of the total population of the Faroe Islands has declined slightly from $27.5 \%$ in 1990 to $25.4 \%$ in 2013. However, with population numbers in Hoyvik and Argir included, Tórshavn's share of the total population of Faroe Islands has increased from 33\% in 1990 to $37 \%$ in 2013.

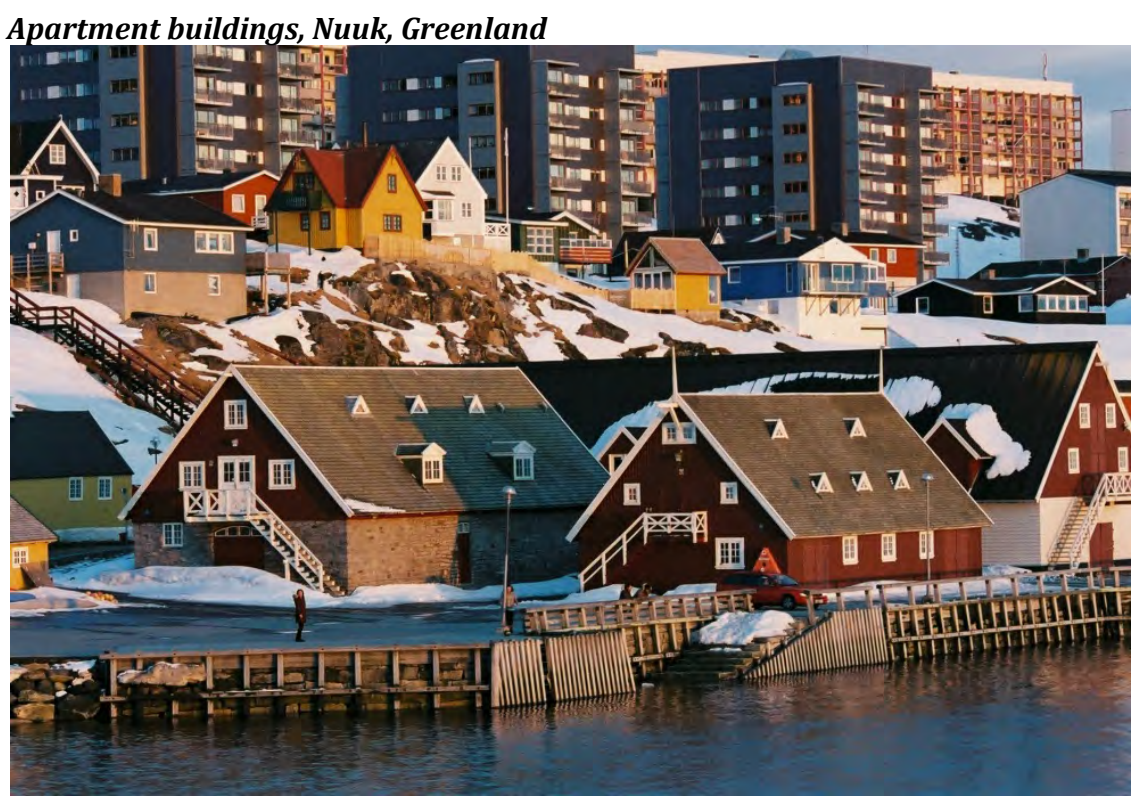

Credit: Harald Finkler.

The predominant trend across the Russia Arctic is absolute population decline in the oblast centers or largest settlements combined with increases in their shares of the regions' overall population. This obviously indicates significant depopulation of the areas outside of the largest settlements, including in many cases, complete closure of many smaller settlements. This trend is also taking place across a larger set of sixteen regions that comprise the Russian North. This trend is due to a confluence of factors, including greatly increased transport costs under market conditions in the Russian North and Arctic which have made both personal and freight transport extremely costly. This caused a significant contraction of the settlement structure in the Russian Arctic and the concentration of the population into a few of the largest settlements in each region. An example is the Anadyr in Chukotka okrug. Between 1989 and 2011, the population of the region shrank from 164,000 to just 50,000 , a decline of $70 \%$, as the true cost of living in this distant region 
became apparent with the withdrawal of many subsidies. However, while the population of Anadyr fell from 18,000 to 13,500 between the 1989 and 2002 censuses, its share of the region's population increased from 11 to $27 \%$.

Thus, with a few exceptions, the dominant trend has been high and increased population concentration into the urban areas and capital cities. This trend is expected to continue as economies of scale make jobs and life more attractive in the capitals than outside.

\subsection{Arctic populations in the future}

\subsubsection{Cohort-component methods of population projections}

The common method for projecting the population of a country or region into the future is the cohort-component method. The components of population change - fertility, mortality, and migration - are applied to the cohorts or the age-sex structure of a population to project how a population will change into the future. Assumptions are made about the future levels and age-sex structure of fertility, mortality, and migration based on the country's or region's recent past and demographic theory. Population projections are used by a variety of government agencies, businesses, and others in planning for the future. The statistical offices of the Arctic regions or national statistical offices make population projects for the Arctic regions. For any region or country, migration is the most difficult component of population change because of the exogenous factors affecting it, whereas future levels of fertility and mortality are bounded by the current age structure of a population. This is especially true in the Arctic because of small populations and the extreme volatility of migration.

This section examines projections between 2010 and 2030, or roughly one generation ahead. If there is more than one scenario, the median or baseline scenario is used. The world's population is projected to continue to grow and to increase by $29 \%$ between 2010 and 2040 , all obviously from there being more births than deaths. Global population growth is projected to continue through the end of the century, by which time, the world's population will exceed 10 billion. This enormous number of people has and will continue to impact the Arctic as this growing population will increasingly look to the region for natural resources. The impact of this growing, and increasingly affluent 
population will also contribute to anthropogenic climate change, which will affect the Arctic sooner and more severely than other regions.

\subsubsection{Projections of the population}

The population of Alaska is projected to increase by $28 \%$ to 915,211 by 2035 , most of this from natural increase. A low scenario with net outmigration from the state projects a moderate increase to 761,879 , while a high scenario with continued in-migration projects a population of over a million at 1,143,334. This projected rate of growth of the Alaskan population is higher than the rate for the United States, which is projected to increase by $15 \%$ to 370 million in 2035 . About $60 \%$ of the population increase for the United States will be from natural increase and $40 \%$ from migration.

Yukon is projected to grow by $19 \%$ to 41,698 over the next decade. Presumably this will be through a combination of natural increase and net in-migration, as the methodology states that the projections were based on demographic patterns during the reference period and that users of the data should determine how economic conditions will affect migration. Thus, the impact of resource development is not explicitly incorporated into these projections. The population of Yukon's capital of Whitehorse will increase faster than the rest of the territory, growing from 26,711 to 32,194 in 2021 when it will have over three-quarters of Yukon's population. The NWT is projected to have population growth of 19\% over the quarter-century from 2010 to 2035. Projections for the population of Nunavut are made by projecting the population of each of the region's twenty-seven communities and then aggregating them to the regional and territorial levels. The population of Nunavut is projected to increase by $39 \%$ by 2036 , when it will be 44,581 . This will occur through a rather significant natural increase of the population offset by projected net outmigration. The natural increase will occur because of the region's young age structure and continued above-replacement fertility level. The projected net out-migration could be detrimental to development in the region because it will likely be young persons in their twenties leaving in the largest numbers.

The population of Greenland reached 55,000 in 1990 and has stabilized within a few thousand of that amount over the past two decades. According to projections from Statistics Greenland, in 2040 the population will remain at about that same number. The population of Iceland is projected to increase by $36 \%$ by 2060 to 435,106 with two-thirds of this increase coming from natural increase and one-third from net immigra- 
tion. The population size of Faroe Islands is projected to be 53,000 in 2040 under the most optimistic scenario.

Under almost any scenario, there is projected to be continued immigration into Norway based on the assumption that income differentials between Norway and the rest of the world will remain wide (Statistics Norway, 2013). Under the medium scenario, the population of Norway is projected to increase by $31 \%$ by 2040 , while the three regions of the Norwegian Arctic will have much slower growth of just 9\%. Norrbotten, in northern Sweden is projected to continue to slowly lose population. About two-thirds of the projected growth will come from natural increase and one-third from in-migration. The population of Lapland is projected to remain roughly the same over the next three decades, while the population of Finland is projected to grow by $11 \%$.

The population of Russia is projected to continue to decline, albeit at a slower rate than over the past few decades. The total fertility rate for Russia is projected to stay below replacement level and life expectancy is projected to rise by nearly ten years, though there is little basis for such an optimistic projection as life expectancy for the country is currently lower than it was in 1964 and many of the Arctic regions have levels of life expectancy lower than the national average (Heleniak, 2010). Data for only four of the nine Arctic regions of Russia are available but some assumptions were made about the others based on recent population change. According to these projections, the population of the Russian Arctic will decline by 7\% between 2010 and 2030 and would be about 1.8 million, down from 2.1 million in 2000 and much lower than in 1990, at the beginning of the transition period, when it was 2.6 million. All regions are projected to show declines except for Nenets okrug, which will show a modest increase , and Yamal-Nenets okrug, which is projected to increase by a quarter. Regions with projected population increase will do so because of a combination of natural increase due to having younger age structures. Though data are not available for all regions, those with projected declines will do so because of large amounts of continued out-migration. For instance, the population of Chukotka is projected to decline by $26 \%$ to 35,400 in 2030 in spite of births projected to exceed deaths. If the projections hold, the population will be just one-fifth the size it was in 1989 when it had a population of 164,000.

Based on these different projections, the population of the Arctic will grow quite slowly from its current size of 4.050 million in 2010 to 4.127 million in 2020 and 4.197 million in 2030 (Figure 2.18). This is an increase of just under 150,000 or $4 \%$ over the next two decades, at a time when the global population is projected to increase by $29 \%$. The North 
American Arctic is projected to increase by nearly 200,000 persons. Greenland will have roughly the same population size. Iceland is projected to increase by 56,000 . The Arctic regions of Fennoscandia will only add 13,000 persons. It is the Russian Arctic where the largest loses will take place: that region is projected to lose another 124,000 persons. Already, less than half the Arctic population resides in Russia and by 2030, Russia's share of the Arctic population will be only $42 \%$.

Figure 2.18: Projected Population of the Arctic, 2010 to 2030

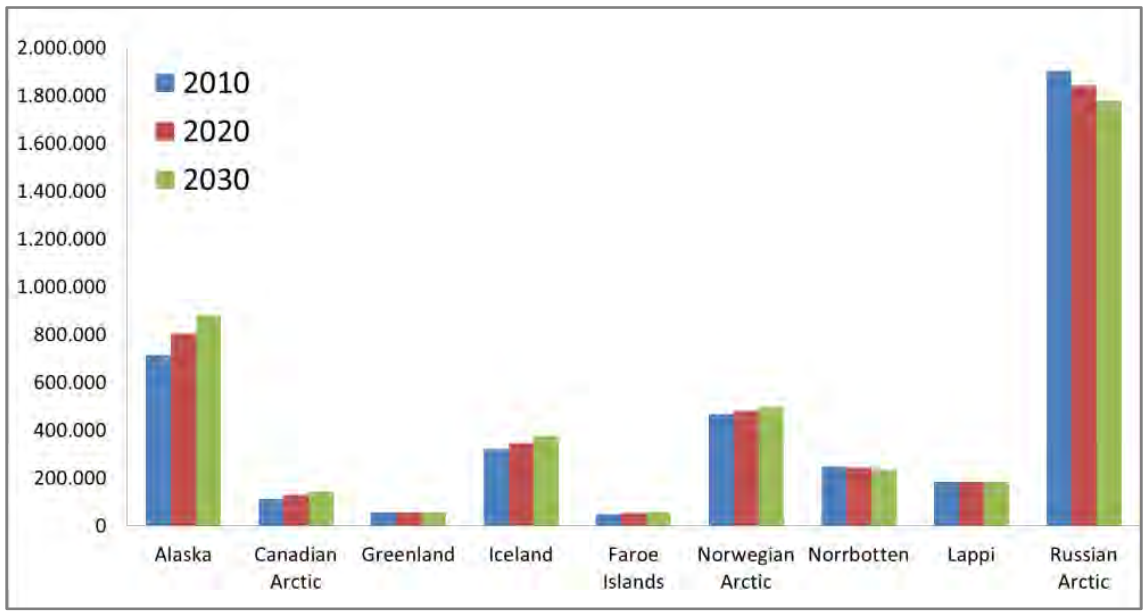

Sources and notes: National statistical offices.

\subsection{Summary of major trends}

- After decades of growth, the population of the Arctic has stabilized at just over 4 million. This is due to continued population decline in the Russian Arctic, which is offset by population growth in other Arctic regions.

- Most of the Arctic countries and regions are at a later stage in the demographic transition from high birth and death rates to low birth and death rates, meaning that population change from natural increase, the difference between birth and deaths, will be quite low or even negative. Exceptions to this are Arctic indigenous populations or regions with large indigenous populations, which are at earlier stages in the demographic transition and continue to have significant population growth because of younger age structures and higher fertility rates. The fertility rate in the Arctic ranges from 3.0 children per woman in Nunavut to 1.3 in Murmansk. 
- Across the Arctic, infant mortality is low by international standards, with some Arctic countries and regions having the lowest rates in the world. However, there is considerable diversity in overall life expectancy because of large differences in adult mortality. Life expectancy in several regions in the Russian Arctic is below the levels for less developed countries, while the levels in Iceland, the Faroe Islands, and several Arctic regions in Fennoscandia are among the highest in the world.

- There is a continued trend of increased urbanization and concentration of the populations in nearly all Arctic regions in the capital cities.

- In the future, the overall population of the Arctic is not projected to increase very much. Projected population increases in Alaska, the Canadian Arctic, Iceland and the Norwegian Arctic will be offset by declines in other Arctic regions, especially the Russian Arctic.

\subsection{Key conclusions and gaps in knowledge}

After decades of rather substantial population growth, the size of the population of the Arctic appears to have stabilized at just over four million. Projections of the future size do not foresee growth much above this level. The leveling off the overall size of the Arctic population is due to continued population declines in most of the Russian Arctic, combined with robust growth in some other Arctic regions. The booms and busts in the Arctic economy associated with the discovery and depletion of resources have always had and will continue to have a large influence on the size of the population.

This chapter has depicted the considerable demographic diversity among the different Arctic countries and regions. Some Arctic regions are made up largely of Arctic Indigenous peoples who continue to be engaged in traditional economic activities and have rural lifestyles. At the other extreme are urban settlements consisting almost entirely of people from outside the Arctic engaged in resource extraction or construction. This has led Arctic regions to differ in terms of their spatial settlement patterns. Some consist of a number of small, scattered villages while others have most of their populations concentrated into just a few larger urban settlements. The Arctic regions differ considerably in terms of fertility levels and patterns, mortality and morbidity patterns, age, gender, and ethnic composition. The role of migration also has a differential impact on Arctic regions and settlements. The difference in demographic levels and pat- 
terns between Arctic Indigenous peoples and outsiders is clearly evident. The role and influence of natural conditions also obviously differs across the Arctic, as does the influence of climate change on settlement and migration patterns. Thus, it is difficult to generalize about the Arctic population as this diversity needs to be considered.

The Arctic consists of countries and regions with very good statistical systems and much is known about the people living in the North. There are an increasing number of studies on various aspects of Arctic demographic patterns though, obviously, much more analysis and research could be done on specific demographic issues in the Arctic, including specific regions and explorations of the linkages between demographic and social, economic, and natural influences. This chapter has presented a broad overview of current and recent trends and patterns in the Arctic population as background for the chapters to follow on the human development in the region.

\subsection{References}

Alaska Bureau of Vital Statistics, 2008, Fetal, infant, and child deaths. http://dhss.alaska.gov/dph/VitalStats/Documents/PDFs/2008/2008_Fetal_Infant_ Child_web.pdf (24 February 2014).

Bronen, R. and F.S. Chapin III, 2013. Adaptive governance and institutional strategies for climate induced community relocations in Alaska, Proceedings of the National Academy of Sciences. http://www.pnas.org/content/early/2013/05/16/ 1210508110.full.pdf (23 February 2014).

Eberstadt, N., 2010. Russia's Peacetime Demographic Crisis: Dimensions, Causes, Implications. The National Bureau of Asian Research, NBR Project Report, Seattle. http://nbr.org/downloads/pdfs/psa/Russia_PR_May10.pdf (1 September 2014).

Fay, M., R.I. Block and J. Ebinger (eds.), 2010. Adapting to Climate change in Eastern Europe and Central Asia. The World Bank, Washington, D.C.

Ford, J.D., and B. Smit, 2004. A framework for assessing the vulnerability of communities in the Canadian Arctic to risks associated with climate change. Arctic, 57(4): 389-400.

Hamilton, L.C. and R.O. Rasmussen, 2010. Population, sex ratios and development in Greenland. Arctic, 63(1): 43-52.

Hamilton, M.A. and K Inwood, 2011. The Aboriginal population and the 1891 census of Canada. In: Axelsson, P. and P. Sköld (eds.). Indigenous Peoples and Demography: The Complex Relation between Identity and Statistics, pp. 95-115. Berghahn Books, New York and Oxford.

Heleniak, T., 2011. The changing gender composition of the Russian North, Presentation made at the Annual Meeting of the Association of American Geographers, Seattle, Washington, April 12-16.

Heleniak, T., 2010. Mortality trends in the former USSR. Geographische Rundschau, Special Issue on World Population, 62(3): 56-63. 
Heleniak, T., 2009. The role of attachment to place in migration decisions of the population of the Russian North. Polar Geography, 32(1-2): 31-60.

Hirsch, F., 2005. Empire of Nations: Ethnographic Knowledge and the Making of the Soviet Union, Cornell University Press, Ithaca.

Hirsch, F., 1997. The Soviet Union as a work in progress: ethnographers and the category Nationality in the 1926, 1937, and 1939 censuses. Slavic Review, 56(2): 251-78.

Hunsinger, E. and E. Sandberg, 2013. The Alaska Native population: steady growth for original Alaskans through years of change. Alaska Economic Trends, 33(4): 4-9. http://labor.state.ak.us/trends/apr13.pdf (1 September 2014).

Johansen, H.E. and Y.V. Skryzhevska, 2013. Adaptation priorities in Russia's High North: climate change vs. post-Soviet transition. Polar Geography, 36(4): 271-90;

Kohler, H., F.C. Billari, and J.A. Ortega, 2002. The emergence of lowest-low fertility in Europe during the 1990s. Population and Development Review, 28(4): 641-80.

Megatrends, 2011. Rasmussen, R.O. (ed). Nordic Council of Ministers, Copenhagen. http://www.norden.org/en/publications/publications/2011-527 (24 February 2014).

Pettersen, T. 2011. Out of the backwater? Prospects for contemporary Sámi demography in Norway. In: Axelsson, P. and P. Sköld (eds.) Indigenous Peoples and Demography: The Complex Relation between Identity and Statistics, pp. 185-96. Berghahn Books, New York and Oxford.

Ren, P., 2011. Lifetime mobility in the United States: 2010. American Community Survey Briefs, U.S. Census Bureau, Washington, D.C.

Sandberg, E. 2013. A history of Alaska population settlement, Alaska Department of Labor and Workforce Development. http://labor.alaska.gov/research/pop/ estimates/pub/pophistory.pdf (24 February 2014).

Statistics Canada, 2006 Census: Aboriginal Peoples in Canada in 2006: Inuit, Métis and First Nations. http://www12.statcan.ca/census-recensement/2006/as-sa/97558/p6-eng.cfm\#02 (24 February 2014).

Statistics Norway, Population projections, 2012-2100, release dated June 20, 2012. http://www.ssb.no/en/befolkning/statistikker/folkfram (19 April 2013).

UN Population Division, 2011. World Population Prospects: The 2010 Revision, Special Aggregates: list of groupings.

UNDRIP, 2007. United Nations Declaration on the Rights of Indigenous Peoples: resolution / adopted by the UN General Assembly, 2 October 2007, A/RES/61/295. http://www.refworld.org/docid/471355a82.html (24 February 2014).

Weeks, J.R., 2008. Population: An Introduction to Concepts and Issues, Tenth Edition, Thompson Wadsworth, Belmont, California.

World Bank, 2005. Dying Too Young: Addressing Premature Mortality and Ill Health Due to Non-Communicable Diseases and Injuries in the Russian Federation. World Bank, Washington, D.C. 


\title{
3. Cultures and Identities
}

\author{
Lead Authors \\ Peter Schweitzer, University of Vienna, Austria, Peter Sköld, Umeå Uni- \\ versity, Sweden and Olga Ulturgasheva, University of Manchester, UK
}

\section{Contributing Authors}

Shari Gearheard (University of Colorado at Boulder, USA); Aytalina Ivanova (North-Eastern Federal University, Russian Federation); Florian Stammler (University of Lapland, Finland); Aimar Ventsel (University of Tartu, Estonia)

\subsection{Introduction}

"Culture" and "identity" are two highly contested and debated notions, which permeate current public and political discourses throughout the Arctic. They also refer to features by which individuals, groups and peoples claim, negotiate or assert their differences in the context of national or ethnic frameworks. To share a culture or share an identity (national, ethnic, class or otherwise) implies that one shares certain features with members of that group. Since these features can be easily essentialized and rigidified in political and nationalist discourses, notions of "culture" and "identity" can lead to political exclusion, alienation, tension or conflict.

In order to avoid deterministic views of culture, we define it as a nonstatic, creative process that imbues people's actions with particular meanings, saturates their words with distinct sounds and frames their relations within certain logic. All the configurations of meanings, sounds, relations and logic change over time but what remains is their embeddedness in socio-economic environments and systems of adaptations to the Arctic landscape. We also accept the view that often people's identities are not fixed but are fluid and open to change.

This chapter sees itself as a successor to the first AHDR's on Societies and Cultures. The title change - to Cultures and Identities - indicates a shift of emphasis toward cultural dimensions of circumpolar life and away from its social aspects. Given the significance of culture in the realm of identity politics, we decided to focus on these two elements of 
Arctic human development. The topical field of societies is addressed in Chapter 11, Community Viability and Adaptation.

Discussions of identity, cultural identification and ethnic affiliation are often embedded in colonial discourses. In wider contexts where Indigenous and non-Indigenous communities overlap, Arctic Indigenous groups are often marginalized and labeled as suicidal, dysfunctional or dependent on the welfare state (Fienup-Riordan, 2000; Morrow, 1996; Vitebsky, 2005). Although these discussions demonstrate that culture and identity are highly contested and heavily influenced by the politicized world, they still play a crucial role and remain as critical components in the ways Arctic communities construe their relations with the outside world and position themselves in the global arena of human rights. Both "culture" and "identity" are often invoked when Indigenous peoples' groups try to establish and uphold legal frameworks for their rights. Therefore, in this chapter we find it necessary to present an updated view on what constitutes "culture" and "identity" for the people and peoples of the Arctic.

We proceed with a brief overview of the historical interactions of different groups of population in the Arctic, important to an understanding of the genesis of contemporary group identities and cultural practices. Such a historical overview was not provided in the first Arctic Human Development Report. Culture and identity are interdependent, but we will treat them in separate sections, distinguishing them for analytical purposes. The section on cultures addresses languages and language vitality, spirituality and worldview, and arts and sports as aspects of circumpolar cultural practices. Under the section on "Identities" we provide a theoretical discussion of facets of identity, followed by reflections on "Indigenous identities", "mixed identities" and "Arctic identity" as categories of identification in the North. We next offer a brief deliberation on "quantifying ethnicity", and then discuss subsistence, landscape and globalization in relation to identities. The final section of this chapter summarizes its findings and identifies the major trends regarding Arctic cultures and identities over the last decade. 


\subsection{Brief historical background to circumpolar cultures and identities}

The diversity of the Arctic is well illustrated by its spectrum of cultures and historical experiences. Recognizing the processes of historical change occurring across the Arctic regions is important for a proper understanding of the current situation. Contemporary cultures and identities are products of the past.

Human habitation of the Circumpolar North extends over several thousand years. In the north of Eurasia, the earliest traces of settlements of modern humans during the Paleolithic are in continental Siberia; modern humans expanded above the Arctic Circle between 12,000 and 7,000 years ago (Hoffecker, 2004). Starting with the earliest phases of human habitation of the Arctic, processes of interaction, exchange, and displacement among northern groups are recognizable. The direct presence of European colonial powers in the Arctic is a relatively recent phenomenon, but findings of iron and of other items that were not produced locally attest to long-standing connections with trade centers to the South. The territory of the Sámi has a history of more than 2,000 years of interaction with southern agriculturalists (Hansen and Olsen, 2014: 39-44), as the Norse pushed north along the western coast of contemporary Norway and Finnishspeakers moved into the southern and western parts of contemporary Finland. While the expansion of farming as well as economic exchange seemed to drive the process in northernmost Europe, the quest for marketable resources alone seemed to fuel European expansion into other parts of the North. From the 17th century onwards, the rich boreal forests of Siberia and Canada became staging areas for the fur trade. The areas north of the tree line were little affected by fur trapping prior to the 20th century, but the coastal areas of the Arctic close to the Atlantic and Pacific Oceans became important destinations for the Euro-American whaling industry in the 18th and 19th centuries.

\subsubsection{Alaska}

The Indigenous peoples of Alaska are generally divided into six major groupings: Unangan (Aleut), Sugpiaq (Alutiiq), Yupik (Central Yup'ik and Siberian Yupik), Iñupiaq (northwest Alaskan Inuit), Athabaskans (Interior Indians) and Tlingit and Haida (Southeast Coastal Indians). These peoples share linguistic and cultural similarities as a result of a long presence in different parts of the region. When Russian explorers en- 
tered Alaska in the mid-18th century, they found it occupied by approximately 80,000 Indigenous people (Langdon, 2002).

After several decades of uncoordinated exploitation of southern Alaskan human and natural resources by Russian fur traders, the incorporation in 1799 of the Russian American Company (RAC) - a statesponsored company that held a trade monopoly similar to the Hudson's Bay Company and the Royal Greenland Company - marked the beginning of a regulated colonial administration of the region. In 1867, the United States purchased Alaska from Russia, leading to the establishment of a new colonial order, which differed in its attitude toward religion and native languages (among other things) from the previous one. While Russian rule was in no way idyllic, U.S. American rule was characterized by even less tolerance toward Indigenous languages and belief systems. It took many decades for the territory of Alaska and its (Indigenous) inhabitants to receive full recognition of their citizens' rights and achieve political representation. For the non-Indigenous inhabitants of the region, Alaskan statehood in 1959 marked the major achievement along that way; for the Indigenous peoples, the passage of the Alaska Native Claims Settlement Act (ANCSA) in 1971 was the main event. ANCSA was triggered by the discovery of oil on Alaska's North Slope and addressed Indigenous land rights in an unusual way, by giving cash and land titles to newly formed "Native corporations" (on the regional and local level). ANCSA has been controversial since its passage, but Alaska's history of the last $40+$ years was impacted significantly by it (Berger, 1985; Mitchell, 2001).

\subsubsection{Canada}

Across present-day Canada, settlements and trade routes had been established by Indigenous peoples by 500 BCE - 1000 CE. Communities developed, each with its own culture, customs, and character. Cultures varied markedly across this expanse, from Tlingit and Athapaskan in the northwest to Innu and Abenaki on the Atlantic Coast. Various Inuit groups inhabited the Arctic coast (Damas, 1984).

After the short-lived settlement attempts by Vikings to establish a presence on the eastern shores of what is now Canada c.1000 CE, the next European exploration dated to the late 15th century, followed by settlements on the Atlantic coast and the St. Lawrence River in the 16th century. Northern and Arctic aboriginal groups, however, experienced European contact much later. This led to a very different dynamic between aboriginal groups and settlers in the North than in the South of 
Canada, a difference that continues to shape the North-South politics of Canada to this day (Freeman, 2000).

With few exceptions, the Inuit are coastal people. They are descendants of what anthropologists call the Thule culture, which emerged in western Alaska around $1000 \mathrm{CE}$ and spread eastward across the Arctic. Inuit who migrated east from Alaska possessed a sophisticated technology enabling them to hunt whales. They were highly adapted to the environment, and a rich material and spiritual culture. During the Little Ice Age (1600-1850) scarcity of game challenged the nomadic Inuit, who also experienced increased external pressure as the Canadian government began to put more focus on the Arctic (Creery, 1994).

From the late 18th century, European settlers in Canada pressured Aboriginals to assimilate into European, and later so-called "Canadian" culture. These attempts reached a climax in the late 19th and early 20th centuries with forced integration (Creery, 1994).

\subsubsection{Greenland}

Paleo-Eskimo groups of nomadic migrants arrived in Greenland about 4,500 years ago, from the Canadian High Arctic. They mainly hunted musk-ox, caribou, seals, whales and walrus. In $985 \mathrm{CE}$, the first European settlements were established in the southern parts of the island. These settlements lasted for 500 years, subsisting on farming and livestock husbandry (cattle, sheep and goats). At about the same time that the Norse arrived in Southern Greenland, a new influx of Arctic people from the west, the Late Dorset culture, entered the extreme northwest of Greenland.

Around 1200 members of the Thule Culture arrived from the west, having emerged 200 years earlier in Alaska. They settled south of the Late Dorset culture and ranged over vast areas of Greenland's west and east coasts. These ancestors of the modern Inuit engaged in the hunting of almost all animals on land and in the ocean, including big whales. They had dogs, which the Dorset did not, and used them as work animals to pull sleds. They also used bows and arrows, contrary to the Dorset. Increasingly settled, they stored large amounts of food to avoid winter famine. The early Thule avoided the highest latitudes, which only became populated again after renewed immigration from Canada in the 19th century. 
In 1721, two centuries after the Norse colonies disappeared from Greenland, the Norwegian-Danish priest Hans Egede arrived in Greenland and began missionary activities. Within a few decades, a colonial structure was established, and the presence of inspectors, traders and others had an overwhelming impact on the Inuit culture (Gad, 1973).

\subsubsection{Russia}

The Russian North has been occupied by different ethnic groups for several thousand years. Traditionally, they were either hunters and gatherers or reindeer herders. Before the 16th century they had no contact with Europeans. At this time the interest of the Russian state awoke. By the time of colonization, the Indigenous peoples of the North had already developed subsistence patterns in accordance with their natural environment, as well as regulations concerning social relations, mutual aid, public support of the disabled, orphans and widows, regulations of collective use of certain territories (hunting grounds, fishing sites, reindeer pastures), property relations and inheritance. Most of the Indigenous nations led a nomadic life and were organized in autonomous clans or territorial communities based on common law. Under these circumstances, the Tsarist policy towards the Indigenous population rested on indirect control through the traditional institutions of the community (Vakhtin, 1994).

Although military governors collected tribute, they interfered little with native Siberian customs and religions; while the smaller nomadic groups of hunters and reindeer herders (e.g., Chukchi, Koryak, Eveny, Evenki, Nenets, Khanty) formed ambiguous relations with Russians, larger stationary, cattle-breeding groups (Sakha, Buryat) submitted to Russians swiftly and were henceforth subjected to colonial relations with the Russian imperial center (Fisher, 1943; Slezkine, 1994). Siberian furs constituted an important source of wealth for Russia and figured prominently in Russian trade with Europe and China. These furs, along with customs duties levied on all Siberian raw materials acquired by Russian entrepreneurs, more than reimbursed the state for the costs of its Siberian acquisitions and administration (Forsyth, 1994). 
Russian settlement of Siberia on a large scale began only with the construction of the Trans-Siberian railroad between 1892-1905, after which the eastward migratory movement reached major proportions. Russia made efforts to reduce rural overpopulation in European Russia by encouraging Siberian colonization. The railroad's needs spurred the development of coal mining and the opening of repair shops. Before the Russian Revolution, however, Siberia contributed only a minute fraction of Russia's industrial output, mainly in the form of gold (Thompson, 2008).

\subsubsection{Fennoscandia}

The Sámi were traditionally hunters and gatherers in Fennoscandia and the Kola Peninsula. Historically they inhabited great parts of northern Russia and Finland. In Sweden and Norway, their traditional area was more extensive than the area used for reindeer pasture today. It is believed that since the Viking Age, the Sámi culture has been driven farther and farther north.

Along the Northern Norwegian coast, the Sámi culture came under pressure during the Iron Age by expanding Norse settlements and taxation from powerful Norse chieftains. In the early Middle Ages, the centralized Norwegian state broke the power of the chieftains. The fish trade in the 14th century triggered another wave of Norse settlement along the coast of Finnmark province (Hansen and Olsen, 2004).

In the mid-16th century, Swedish governmental interest in the northern region arose and it was not unusual that the Sámi paid taxes to three different kings, most commonly in furs and dried fish. There was an increasing demand for furs in Europe, so the Crown also traded these products. Dried fish was used as payment for the soldiers who were constantly involved in different wars. The Sámi also sold fish, and purchased food products such as butter and flour, which until then had been relatively rare in the Sámi culture. As a result of the improved nutritional supply, the population grew considerably. However, the fur market shifted and the lakes were soon almost depleted of fish, leading to a population crisis. Sámi society could not supply its increased population with sufficient foodstuffs when the import of butter and flour was stopped, and fish became scarce. It was around this time, in the early seventeenth century, that the Sámi became reindeer herders, which to a great extent can be seen as a response to the population crisis (Sköld, 1992).

At the end of the 17th century, the states of Fennoscandia encouraged settlers to move to the area, offering free land and generous condi- 
tions. This had, however, a limited impact on in-migration, a trend that did not shift until the mid-18th century. From this time, however, the process of colonization began, and during the next hundred years, the ethnic balance in Sápmi (the Sámi homeland) changed, turning the Sámi into a minority, except for some parts of northern Norway and smaller areas in Finland and Russia (Axelsson and Sköld, 2011).

\subsubsection{Iceland and the Faroe Islands}

Settlements by Viking explorers from the east, particularly Norway and the British Isles, in the late 9th century mark the beginning of Iceland as a nation. By 930, the chieftains had established a form of representative governance (Althing) that was one of the world's oldest parliaments. Towards the end of the 10th century, Christianity came to Iceland but the country remained independent. Internal conflict in the early $13^{\text {th }}$ century weakened Iceland, and resulted in subjugation to Norway. Norway in turn was united with Sweden (1319) and then Denmark (1376). A strict trade monopoly imposed by Denmark in the 17th and 18th centuries caused Iceland to fall into poverty. Additionally, natural disasters forced a population decline. Iceland remained part of Denmark, but in the 19th century an independence movement emerged as a part of the rising nationalism around Europe. In 1844, the Althing, which had been suspended in 1799, was restored. Iceland gained sovereignty after World War I, on 1 December 1918 (Karlsson, 2001).

Irish monks and Vikings had visited the Faroe Islands before stable settlements, Christianity and Norwegian rule and laws were established in the $11^{\text {th }}$ century. In 1380, the Faroe Islands followed Norway into the union with Denmark, and after the reformation, the Danish language and influence increased over time. Copenhagen monopolized and controlled foreign trade. Fishing and farming were the dominating industries. As a result of an increased movement of nationalism, Faroese developed into a written language in the mid-19th century. At this time, free trade opened and fishing was commercialized. The population numbered around 9,000 people. Danish was the official language until 1948, when extensive home rule was introduced, together with the position of a Faroese Prime Minister as head of a the government. The Faroe Islands is still part of the Danish Kingdom but together with Greenland, they often enjoy a special status within the Kingdom, and neither has joined the European Union (Wylie, 1987). 


\subsection{Aspects of circumpolar cultures}

Cultures in the Circumpolar North are complex and dynamic systems of meaning and identity. Given the encompassing definition of culture presented above, many facets of everyday life - from subsistence practices to religious beliefs, arts and music - could be presented under that rubric. In order to increase comparability with the first $A H D R$, we look at some of the same issue as in 2004 while adding some new components. We review language and language vitality, spirituality and worldview, and the arts and sports. As in the first $A H D R$, we begin with an overview of language.

\subsubsection{Language and language vitality}

Language is arguably one of the most important aspects of human culture(s) and a vital factor for the survival of culture. It is not just a means of communicating information, but also contributes to the preservation of memory, involves specific terminologies and the traditional knowledge encoded within, and articulates a world-view. Each language develops its own expressions that reflect areas that are important to the speakers (see Chapter 9, Education and Human Capital). In the Arctic, an area with great linguistic diversity (see below), colonial and assimilatory pressures against Indigenous language use and the small demographic numbers of many Indigenous groups have created difficult situations for many languages.

Attention to the dire prospects of Arctic languages has significantly increased in recent years. Ever since the Alaskan linguist Michael Krauss (1992: 10) urged his fellow language specialists toward a "serious rethinking of our priorities, lest linguistics go down in history as the only science that presided obliviously over the disappearance of $90 \%$ of the very field to which it is dedicated," the magnitude of the problem has been recognized by academics and practitioners alike. Thus, the Arctic Social Indicators report (ASI, 2010), which follows from the first AHDR, elevated "language retention" to the single best indicator of cultural vitality. Arctic Social Indicators II (ASI, 2014) addresses language retention in each of its case study applications.

Apart from the AHDR $(2004)$ and $A S I(2010,2014)$, it is notable that Arctic Council Working Groups are paying increased attention to the situation of Arctic languages. An example is the chapter on linguistic diversity in the Arctic Biodiversity Assessment, which provides a good treatment of the subject matter (Barry, 2013). 
Most importantly, Permanent Participants of the Arctic Council spearheaded two important initiatives on Arctic languages in recent years. The "Arctic Indigenous Languages Symposium" was organized by ICC Canada and held in Troms $\emptyset$, Norway, in October 2008 (ICC - Canada, 2008). ICC-Canada's Assessing, Monitoring and Promoting Arctic Indigenous Languages project was approved by the Arctic council's Sustainable Development Working Group in 2011 as a direct follow-up to the recommendations of the 2008 symposium. The Research Development Workshop "Assessing the Vitality of Arctic Indigenous Languages" was organized by the same organization and held in Ottawa in June 2012 (ICC - Canada, 2012), and a second Arctic Indigenous Languages Symposium is scheduled to take place in 2015.

Despite all this attention, the general tendency continues to be a reduction of the proportion of speakers of most languages of the North. One example of this trend is Alaska. As Figure 3.1 shows, all but one Alaska Native language had a lower proportion of speakers in 2007 than in 1997. At the same time, we see that most Alaskan languages - with the exception of Siberian Yupik and Central Yup'ik - had already a rather low retention during the 1990s. 
Figure 3.1: Language Retention Dynamic in Alaska, 1997-2007

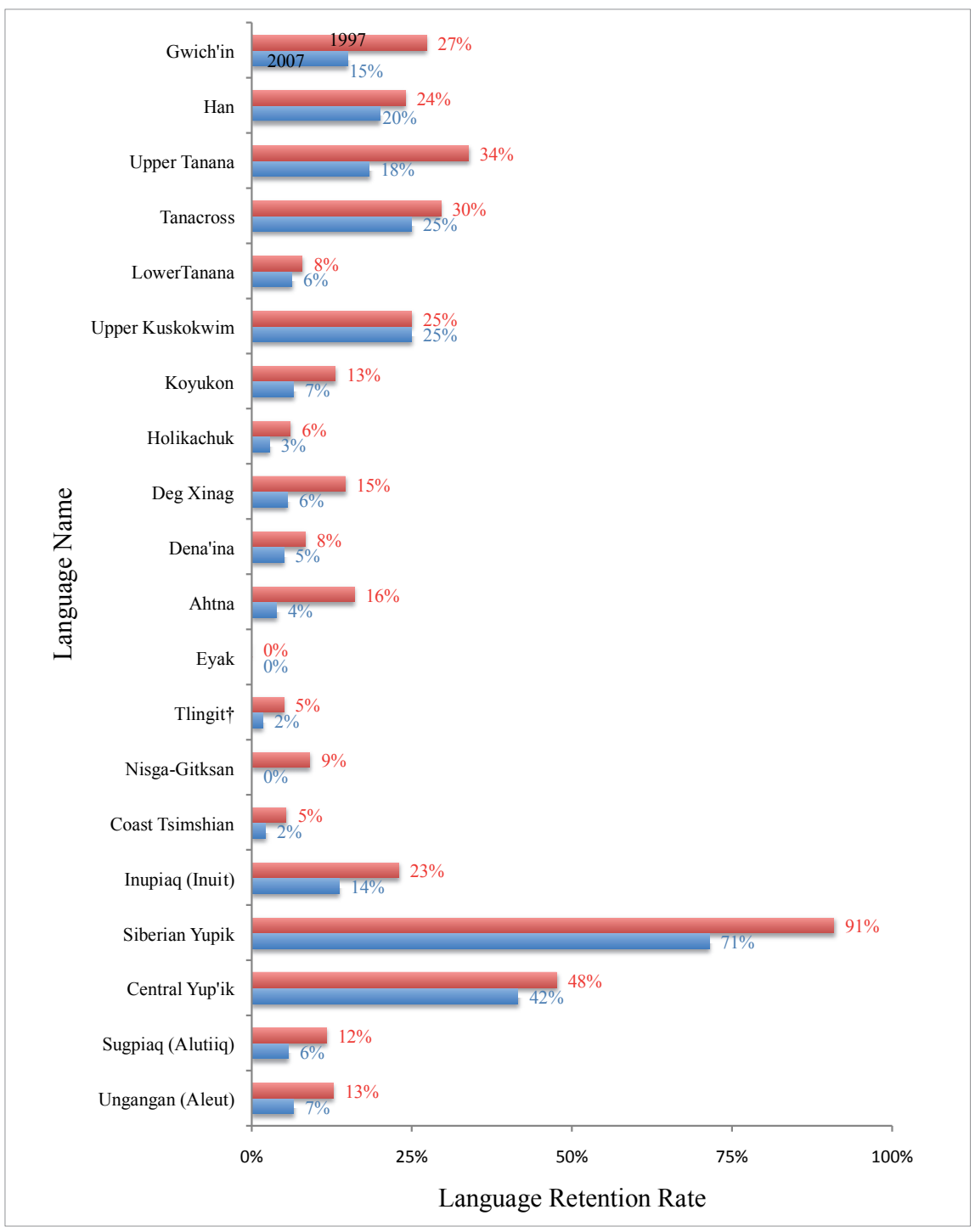

Source: Krauss, Michael E. 2007. Native languages of Alaska. In: The Vanishing Voices of the Pacific Rim, ed. by Osahito Miyaoko, Osamu Sakiyama, and Michael E. Krauss. Oxford: Oxford University Press. (Table 21.1, page 408). Michael E. Krauss (1997). The indigenous languages of the north: A report on their present state. Northern Minority Languages: Problems of Survival, ed. by Hiroshi Shoji \& Juha Janhunen, 1-34. (Senri Ethological Studies 44.) Osaka, Japan: National Museum of Ethnology.

The Icelandic language has changed very little from when the country was settled, and therefore a text from the 12th century is still understandable to Icelandic schoolchildren. The Icelandic language is considered a cornerstone of Icelandic culture, in large part due to a strong literary heritage. Icelandic does not usually adopt foreign words for new concepts, opting instead to coin new words, or give old words new meaning (Jóhannesson, 2007). 
The language of the Faroe Islands, Faroese, derives from the language of the Norsemen, who settled the islands some 1,200 years ago. Today, Faroese is spoken by approximately 75,000 to 80,000 people. It was established as a written language in 1854 , and accepted as a national language by the Danish authorities in 1948. The Faroese language is considered an important aspect of cultural identity and the Faroese are conscious of the need to preserve and develop their language in the face of global influences. Research and development of the Faroese language is thus a high political priority of the Faroese government (Nauerby, 1996).

There are other positive language-related developments elsewhere in the Arctic. For example, 50,000 of the 57,000 residents of Greenland speak Greenlandic (Lykke Thomsen, 2013); that is, almost 88\% of all people living in Greenland - whether of Inuit descent or not - speak this Inuit language. Such success is the result of decades of attention to language issues, crowned by declaring Greenlandic (Inuit) as the official language in the Greenland Self-Government Act of 2009. Inuit in the neighboring eastern Canadian Arctic also continue to keep native language retention at relatively high levels, while the western Canadian Arctic and the Subarctic are characterized by more substantial losses.

For many parts of the Arctic, data on language use and retention is absent or hard to access. However, the Russian Census provides figures regarding language retention in the northern parts of the Russian Federation. During the recent censuses of 2002 and 2010, people were asked about the languages they speak; at the same time, respondents selfidentified with a particular ethnic group. For example, 482 individuals declared themselves as Aleuts (Unangan) in 2010, while 45 respondents claimed to know the Aleut language. We can thus assume that we are dealing with a proportion of Aleut speakers of slightly fewer than $10 \%$. It should be pointed out, however, that we do not know with certainty whether the 45 speakers come from the group who identified themselves as Aleuts. Notwithstanding these and other issues of enumeration, a comparison of results for 2002 and 2010 shows a clear downward trend in the proportion of speakers: of 21 languages, only one (Siberian Yupik) claims a higher proportion of speakers in 2010 than in 2002.

The absence of enumeration of the population by ethnicity in Fennoscandian statistics makes the assessment of Sámi language retention difficult (see Chapter 2, Arctic Populations and Migration). In addition, the multitude of Sámi languages and dialects make generalizing statements on the vitality of language retention impossible. As depicted in Figure 3.2, Northern Sámi - spoken primarily in northern Norway, as well as in parts of northern Sweden and Finland - is the least vulnerable of all Sámi lan- 
guages, with most speakers and regular increases in the total number of speakers. Other Sámi languages, however, are in a much more difficult position, even where municipalities recognize them as official languages.

Figure 3.2: Sámi Languages and dialects

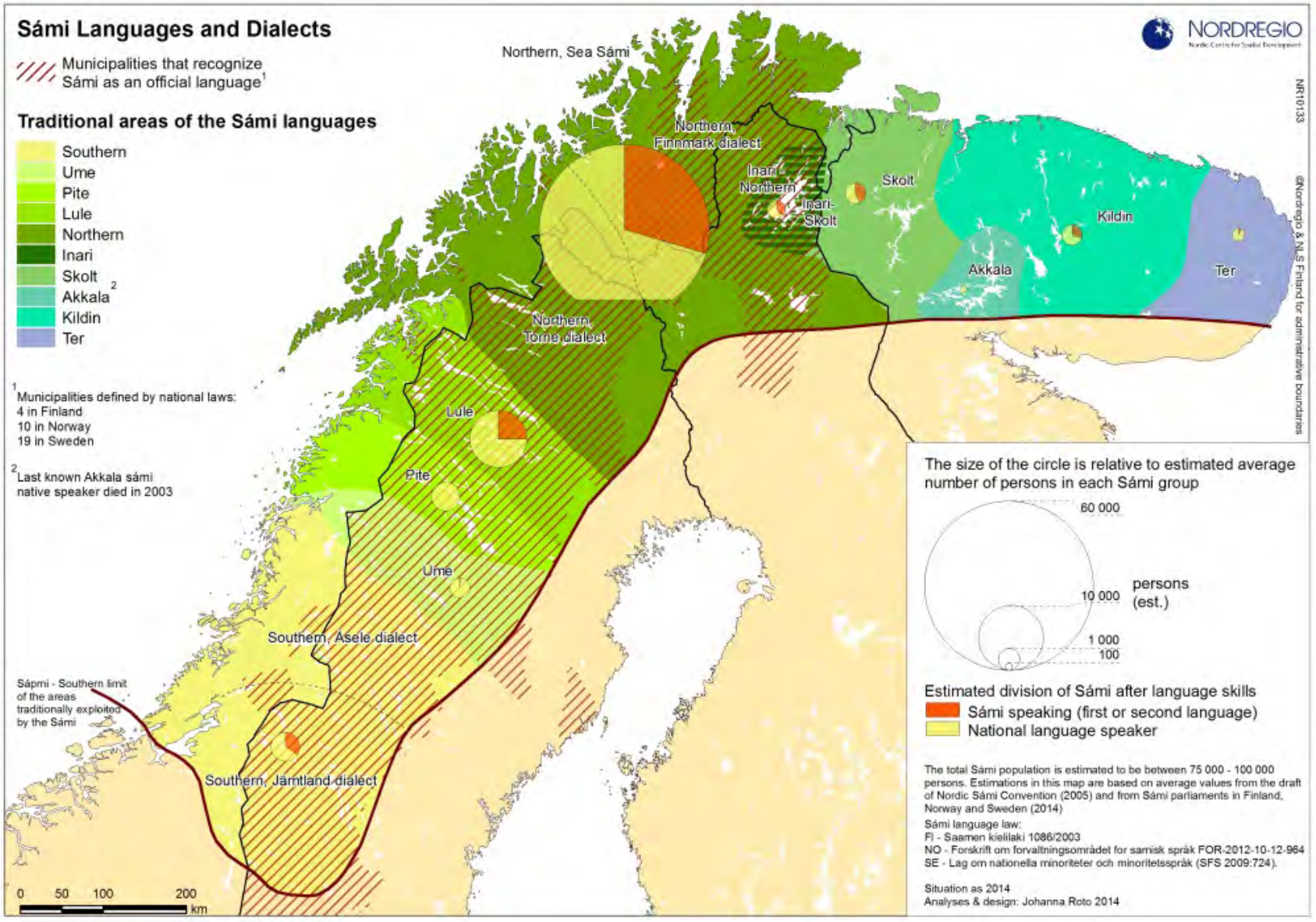

These ambiguous developments are best expressed by Figure 3.3, which we borrow from the Arctic Biodiversity Assessment (CAFF, 2013). It shows that most of the 46 languages captured in the graph experienced declines in the proportion of speakers between 1989 and 2006. Nine languages, however, saw an increase in the proportion of speakers during that same period. Thus, we can conclude that while the vitality of many Arctic languages is threatened some languages enjoy a stable position, and a few are even gaining in strength. Given that most parts of the Arctic saw the establishment of language revitalization programs in recent years, it is thus doubly important that such positive developments 
can be reported. It is to be hoped that these successes will stimulate the reversal of language loss in other parts of the Arctic as well.

Figure 3.3: Estimated change in proportion of speakers for 46 Arctic languages between 1989 and 2006

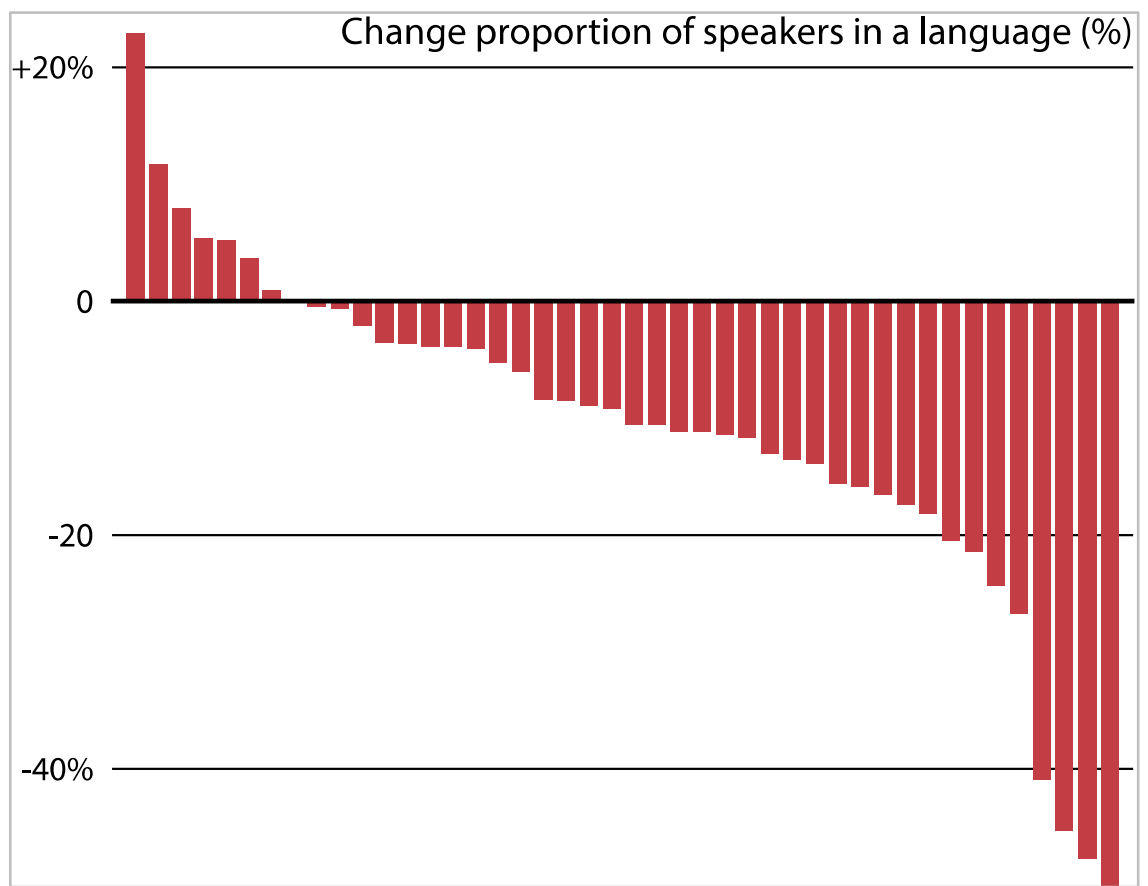

Source: Barry et al., 2013:549.

In recent years, new laws (e.g., in Fennoscandia) have given speakers of certain minority languages the right to use their language in public offices, in preschools and in geriatric care (Elenius, 2008). On one hand, this provides important recognition of the minority languages and cultures. On the other hand, not all minority languages are nationally protected and questions of which variant or dialect of a language is chosen as the standard adds further complications to language retention and vitality. 


\subsection{Spirituality and worldview}

"Traditional" forms of spirituality and worldview among Arctic Indigenous peoples need to be seen as part of their productive and reproductive relations with the land, its animals, and the spiritual and ancestral beings that affect the well-being of human communities in the Arctic region. Shaped in response to Arctic social and material ecologies, these systems involve complex and sophisticated religious forms, ideologies and practices that revolve around the activity of hunting. The concern over the necessity "to kill in order to live" expressed in the animist beliefs about return of animal souls and ancestor spirits in charge of souls of animals has remained a significant feature of the worldview of many Indigenous groups within the region (Willerslev, 2007). The practice of hunting which required intense human contact with the animal spirit world entails specific perceptions of "animals as persons" (Bird David, 2006; Scott, 2006; Descola, 2006). Personhood is attributed not only to humans but also to certain non-human beings, such as animals, plants and "things". Such concepts and practices form the basis of animist beliefs among Indigenous hunting groups in the Arctic.

Recent research on human-animal relations points to the common elements present in animist societies such as continuity between humans and non-humans, shamanist cosmology, egalitarian or semi-egalitarian ethos and unbounded potential for identification (Descola, 2006; Pedersen, 2001; Willerslev, 2007; Brightman, et al., 2012; Ulturgasheva, 2012). For example, bears are closely associated with shamans: they may be said to "be" shamans themselves, or to be able to transform themselves into humans. Shamans are likewise said to be able to transform themselves, or parts of themselves, into bears (Hallowell, 1926: 86; Kwon, 1999: 373-87). Perhaps most importantly, bears play key roles in the symbolic organization of society. As both powerful ancestors and significant actors, they participate in the reproduction of kinship patterns and in this mode of human-animal relations.

Among Iñupiat and Yup'ik of Alaska, and the Inuit of northern Canada the question about the identity of a child and a child's personhood cannot be considered without naming practices and Indigenous ideas about the circulation of souls between the living and the dead (Bodenhorn, 2000; Bodenhorn, 2004). The beliefs in reincarnation and rebirth among Inuit groups and some Siberian groups, e.g. Chukchi, Koryak and Itel'men, are most eloquently expressed in the system of naming children.

Shamanic and animistic practices, consistent with the everyday engagement with the animals and spirits of the land, persist, especially in 
rural areas, through small rituals, such as the feeding of the fire and the interpretation of dreams and omens practiced by the hunters and reindeer-herders in the Siberian forest (Vitebsky, 2005; Lavrillier, 2012). For those inhabiting the Siberian taiga and moving around vast areas of land, animistic worldview and the principles of sociality are rooted in legacies of accumulated ecological experience and interaction with the environment. For the urban nationalists, the current fad of a "shamanic renaissance" functions as a convenient and flexible metaphor for nationbuilding aspirations (Balzer, 2011; Vitebsky, 2005).

The first AHDR contained a box about sacred sites authored by RAIPON. If at the time, attention to the conservation of such sites was especially notable among Russian Indigenous peoples, over the past decade we have seen expansion of this interest. For example, the conference "Protecting the Sacred: Recognition of Sacred Sites of Indigenous Peoples for Sustaining Nature and Culture in Northern and Arctic Regions," held in Rovaniemi in September 2013, confirmed this increased interest. The conference approved a draft declaration that called for promotion of awareness of sacred sites, stronger mechanisms for consultation of Indigenous groups, and enforcement of effective measures to protect them from damage and destruction (Arctic Centre, 2013). A parallel movement deals with cultural heritage sites in the Arctic (Barr and Chaplin, 2004), which are overwhelmingly non-Indigenous in origin.

Another interesting development is the revitalization of certain aspects of shamanism in the Circumpolar North. This can happen at the grassroots level, in the form of northern residents actively trying to reengage with shamanism as a set of beliefs and practices. Even more powerful, however, is a situation in which there is encouragement and recognition from above, from state or regional authorities. In the Arctic, we are aware of two regions in which shamanism is an officially recognized religion: in the Sakha Republic (Russian Federation) it is a form of Tengreism (the Turkic version of shamanic beliefs with the god Tengri), which is mainly spread among urban Sakha, and the New Age form of shamanism emphasizing its healing aspect in the north Norwegian county of Tromsø (see Textbox 3.1). 


\section{Textbox 3.1}

\section{Shamanism as an officially recognized religion \\ Aytalina Ivanova and Florian Stammler}

Shamanism as a worldview of people living in close connection to the environment with all its animate features including spirits is gaining popularity among indigenous and settler populations in the Arctic. In the European and Russian Arctic, particularly in Siberia from where the word "shaman" originates, the revival of shamanism has had also an identity-forming influence for several societies in the Arctic. For that purpose of identity building there have been cases where shamanism has been recognized as a religion alongside other main religions such as Christianity, while some scholars refer to shamanism more as a worldview in animist societies (Vitebsky, 1995, 2012) than a religion. In the East Siberian Sakha Republic, for example, an officially recognized association of shamans (then called "people's healers" (народные целители)) has been active since 1990. In May 2014, shamanism was recognized by the Ministry of Justice of Russia in the Sakha Republic as a religion, after three local religious groups had united into a regional religious denomination (Ministry of Culture, 2014). The decision was made after an expert review in September 2011 (Ministry of Justice of the Russian Federation, 2011) had concluded that the specifics of Sakha Aar Ajyy (Аар Айыы, the official name of the religion) including 16 directions of the most influential rituals and prayers (Algysy-Dommy Алгысы-Доммы) qualify for official recognition as a religion according to point 1, Article 6 of the Russian Federal Law on the Freedom of Conscience and Religious Unions (Russian Federation, 1997). The expert statement specifically highlights the importance of the shaman as mediator between the spiritual and the material world. Such officially recognized religious practice qualifies for support by the state. Although there is no state salary for religious practitioners, through an especially established regional ministry for culture and spiritual development (министерство культуры и духовного развития), this support plays an important role in promoting not only the identity of the Sakha people, but also their well-being through connection to their land and its spirits, which is seen as a guarantor for a healthy lifestyle in an Arctic environment.

In the European Arctic, shamanism has also been recognized as a religion, where, like in Siberia, people can get ritual services such as marriage ceremonies, funerals, and baptisms. The first official decision in this respect was taken in the North Norwegian county of Troms in 2012, where the governor recognized the regional Shamanic Association as religious denomination (Shamanism, 2012). 


\subsection{The arts and sports}

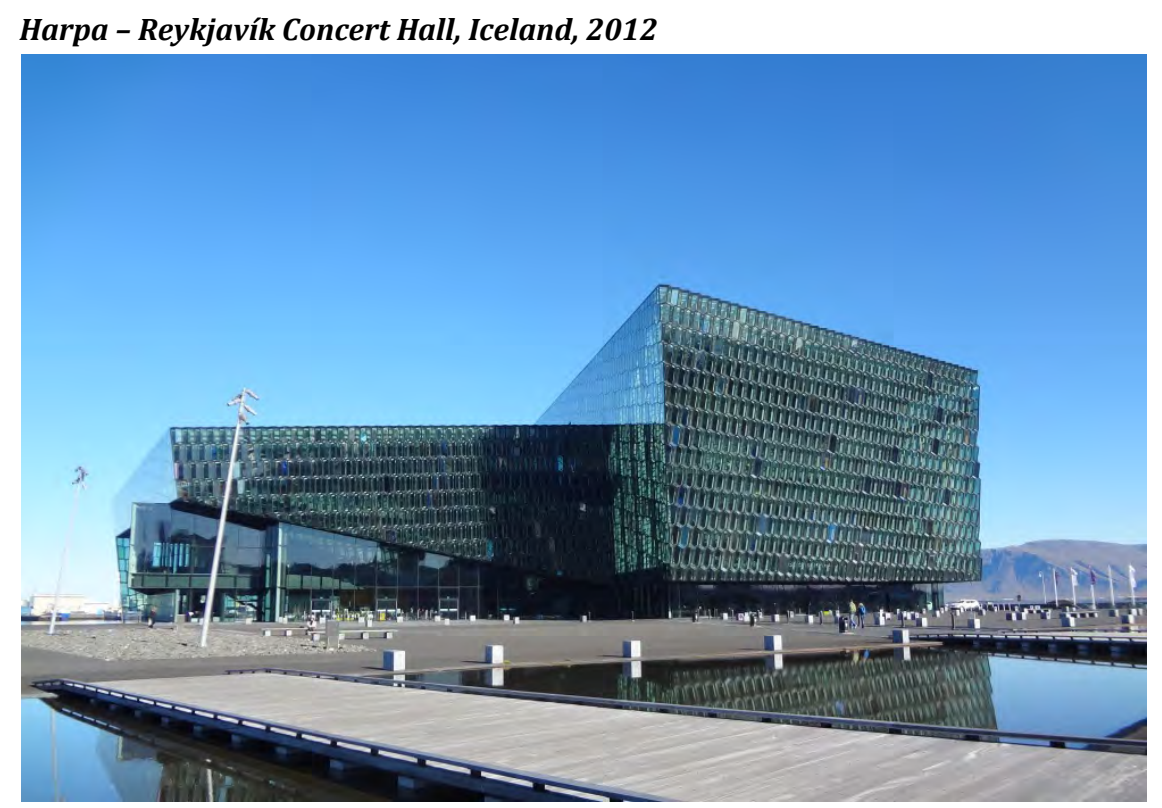

Credit: Joan Nymand Larsen

The arts are booming in the North. Be it a new concert hall in Reykjavík, the Greenland Eyes International Film festival, new choreography by the Yellowknife Dance Collective, or the opening of an art show in Anchorage or Arkhangelsk, the production, marketing, and consumption of northern art seems to be a growth market. Interestingly, both Indigenous and non-Indigenous art productions are sought after, as are creations consciously marking mixed identities. All art genres seem to be involved. While music, literature and fine arts have been prominent in the North for a while, Arctic film productions and film festivals are fairly new and successful phenomena.

The global language of hip-hop music, and related breakdancing found in almost any corner of the Arctic, provides an excellent example of adoption and adaptation of exogenous cultural forms for local purposes. We provide two examples of local applications of this music style (Textbox 3.2). As the cases illustrate, hip-hop is used to increase awareness of and pride in local cultures. Far from being a destructive aspect of globalization, locally produced hip-hop music appears to be a vehicle of cultural revitalization. 


\section{Textbox 3.2}

\section{Hip Hop in the Siberian and Canadian Norths: contributing to cultural vitality and physical well-being}

The Republic of Sakha (Yakutia), the largest republic of the Russian Federation, and famous for its diamond production, contains 140 different ethnic groups, including the dominant two: Sakha and Russian. Since the Russian Federation's declaration of sovereignty in 1991, the region has undergone several changes. The increase of the rural migration into cities, especially into the capital city Yakutsk, changed the ethnic and linguistic situation in urban centers. Post-Soviet transformations also initiated the emergence of new youth styles, such as hip hop.

There is an interesting correlation between growing rural-urban migration and evolution of local hip hop, including its language. The first hip hop artists or 'crews' appeared in Yakutsk at the end of the 1990s. They were mainly Russian speaking, urban Sakha. This was the period when the first substantial urban-born Sakha generation came into their teenage years and looked for cultural expressions that reflected their urban experience. Sakha pop and rock music was rooted in the village - the most popular songs romanticized rural life as the cradle of Sakha culture, and most of the musicians were of village origin. Hip hop thus embodied a counterpoint to the rural and manifested a new urban Sakha identity.

In contrast to this Russian speaking Sakha generation, by around 2005 a significant Sakha speaking urban youth community had developed; these were the children of village Sakha who moved to Yakutsk and sent their children to Sakhalanguage schools. This "youth market" was the target for Sakha-language youth radios, TV-shows and glossy magazines. As a result, Sakha-language media helped to promote Sakha hip hop, which developed as a distinct genre from local Russian-language hip hop. Currently Sakha-language hip hop enjoys support from media and state institutions, reflecting a general power shift in local politics that becomes increasingly dominated by ethnic Sakha.

In the last decade, hip hop has also found a prominent place in the Canadian Arctic. Hundreds of youth in dozens of communities across the Canadian North have connected to the music, history, art, and culture of hip hop, which is being used in a range of programs to promote health, prevent suicide, support youth leadership, and reduce crime. An active group in the movement has been Blueprint for Life (http://www.blueprintforlife.ca/), a southern-based organization offering social work and healing to youth through hip hop. In many cases, after introducing hip hop in northern communities, local schools and organizations have established clubs or programs to support the youth to continue. Through hip hop dance, art, and music youth have found a powerful outlet for expressing themselves. In many communities Elders have joined the activities, lending their support, and youth have used hip hop as a means to strengthen connections to their own culture, for example mixing traditional throat singing with "beat boxing", rapping in Inuktitut, or creating dance moves based on imitations of seal hunting, dog teaming, or Arctic animals. 
Spontaneous hiphop break dance event in the Ordzonikidze Square, Yakutsk, Russian Federation, during a summer youth festival (June 2013)

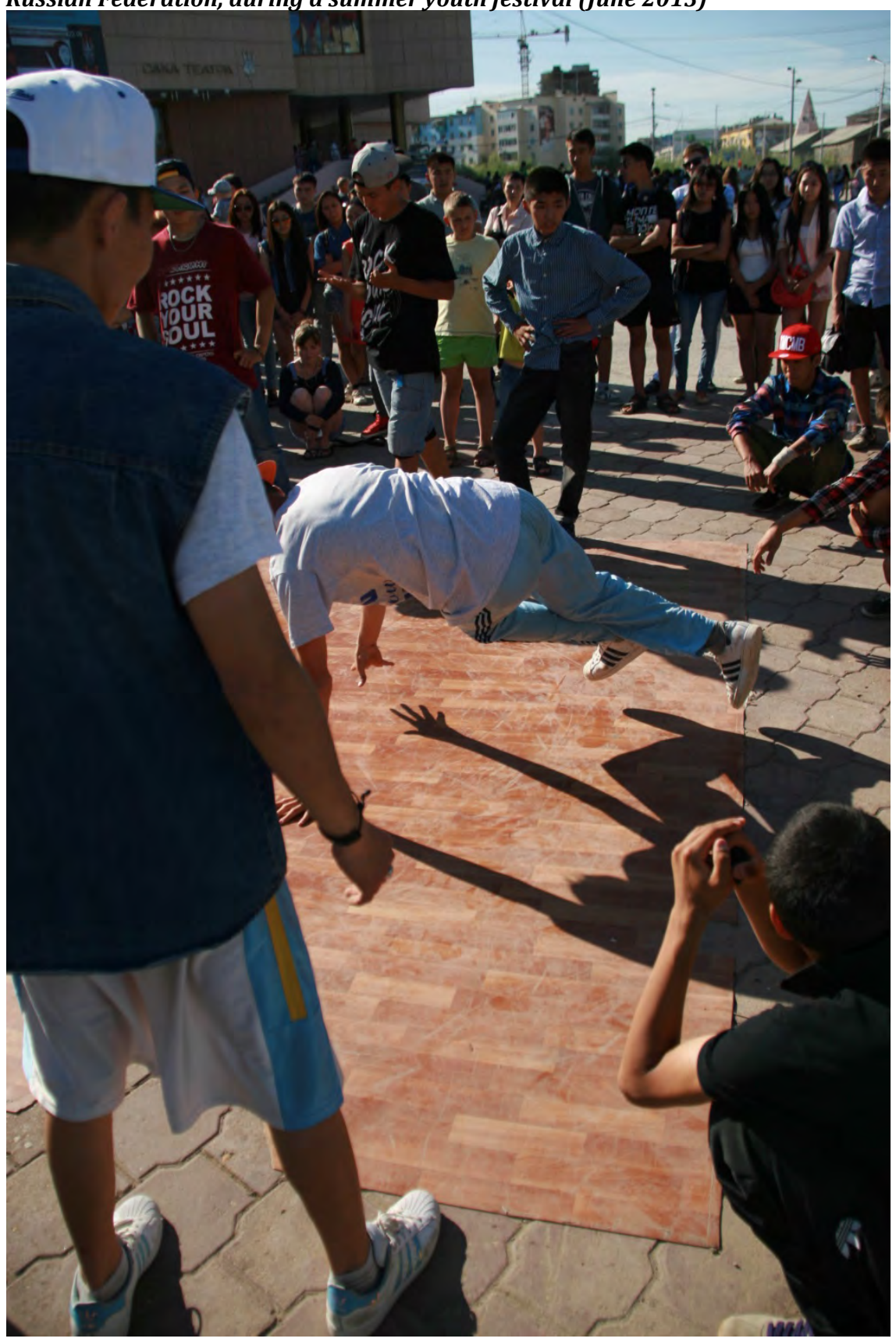

Credit: Aimar Ventsel (permission received) 
While singing, dancing and poetry are always recognized as part of culture, a sometimes less recognized component of culture is sport. Yet sports are cultural practices, and team sports are ideal vehicles for the expression of group identities.

When looking at Arctic sports, rather than be tempted to focus only on winter sports that seem to fit the Arctic's climatic conditions (such as skiing, ice hockey, etc.), we note that a number of other sports have recently become prevalent in the North. As one example, we cite the popularity of basketball in rural Alaska. Michael D'Orso (2007) followed a winning high school basketball team from Fort Yukon, Alaska for a season and provided evidence for the link between community resilience and team sports. Another example is the importance of football for the Faroe Islands. Admitted to the international football association FIFA in 1988, the Faroe Islands have since surprised a number of bigger football nations - such as Austria, which they beat in 1990. Likewise, handball has developed as a national sport in Iceland since the 1950s, and the team won silver medals in the 2008 Summer Olympics in Beijing. Greenland handball has been represented in the World Championships.

\section{Hockey Game in Makkovik, Canada}

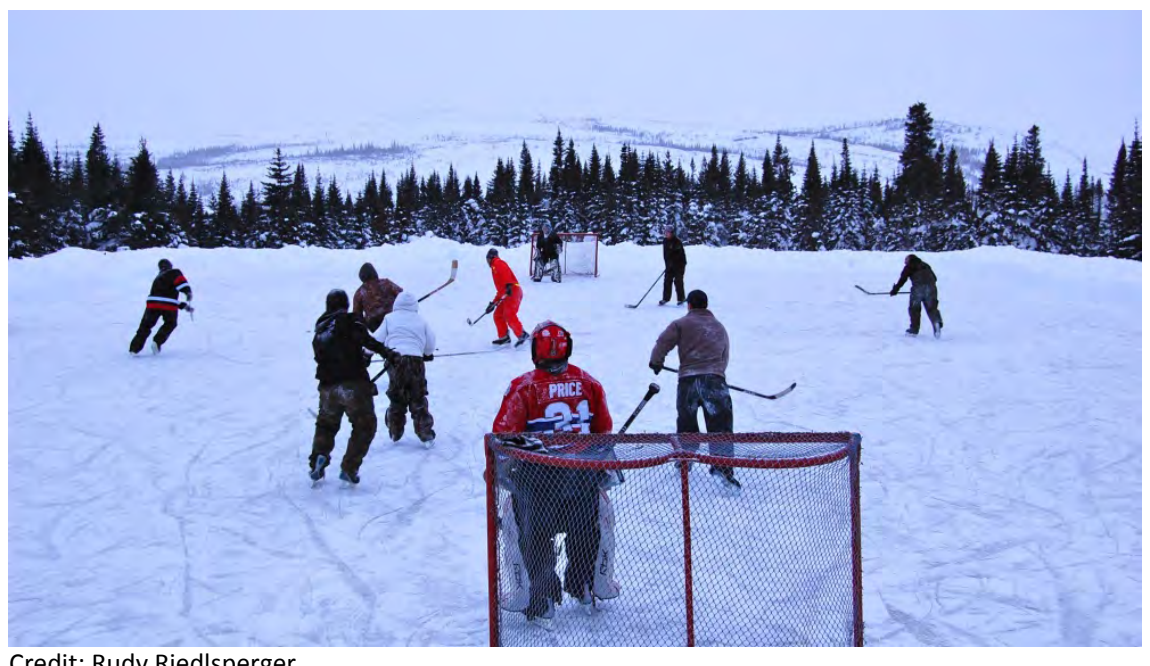

Canada and Russia are great winter sport nations with extensive records of internationally successful athletes from northern regions, with ice hockey and cross-country skiing as notable activities. The Arctic Winter Games (AWG) were established as collaboration between Yukon, Northwest Territories and Alaska, with the first games held in Yellowknife in 1970. Every two years, a Canadian or Alaskan city hosts the 
event, with the games traveling to Nuuk, Greenland in 2002. The AWG subscribe to the core values of "cultural awareness and understanding" (Arctic Winter Games, n.d.).

Sports not only encompass the more "western" activities noted above. The town of Fairbanks, Alaska, has been hosting the World Eskimo-Indian Olympics (WEIO) annually since the early 1960s. The event showcases a variety of competitions based on demonstrations of traditional skills and endurance, such as the "greased pole walk," the "kneel jump" and the "ear pull". In addition, WEIO includes dance performances, a Miss WEIO pageant, and a best-dressed baby contest. Held in the summer months, the event has become a major attraction for locals and tourists alike.

\section{High school basketball: the State playoffs in Anchorage, Alaska, USA}

(Girls' teams, 2011)

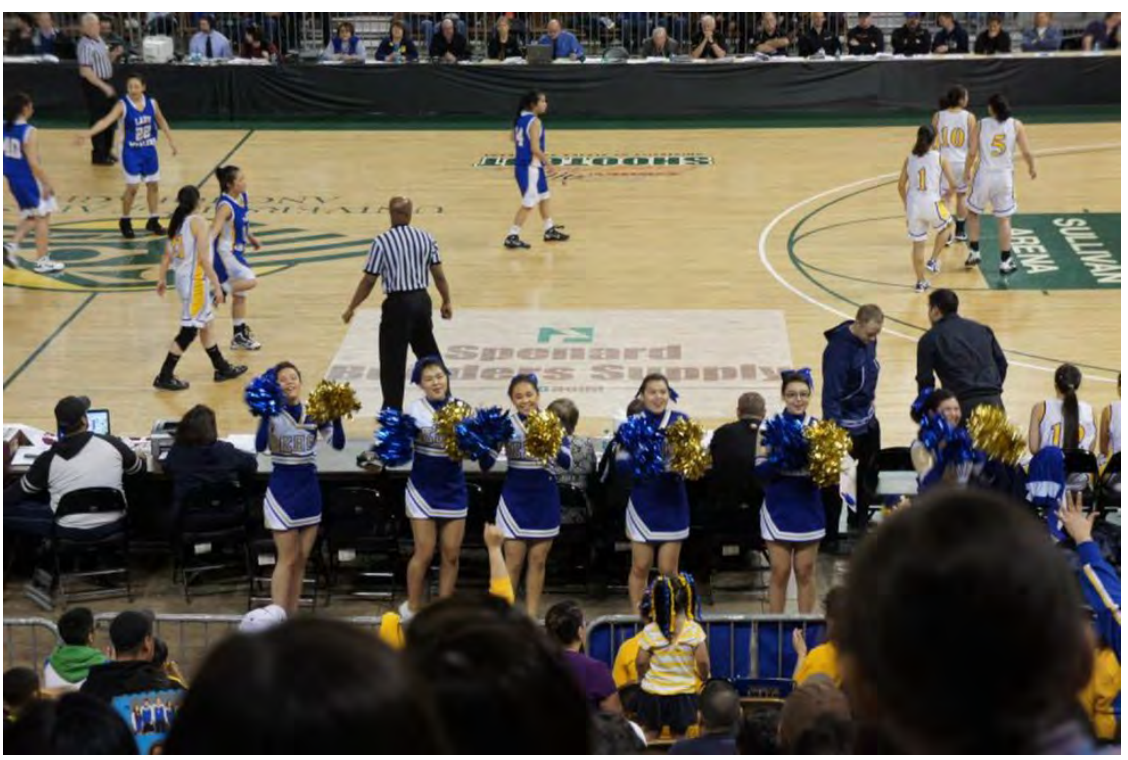

Credit: Hugh Beach.

The Sámi have several sports organizations and competitions that serve as important meeting places - not least for Sámi youth - and are important contributors to identity formation. The Sámi Championships include cross country skiing, lasso, and shooting. Reindeer sledge races are frequently held. Several Sámi have been internationally successful in alpine skiing, cross-country skiing, ice hockey and other less obviously northern sports, such as football. 


\subsection{Identities}

One is not born with an identity: rather it is socially constructed, and is formed and reformed over the course of one's lifetime. One's identity depends on one's experiences and one's relations with other members of society - people need to know and understand their position in society. As well, one's identity depends on one's calculated, strategic decisions. Identity is the conception and expression of both individuality and group affiliations. Social, cultural and ethnic identities emerge in any given situation where social interaction, political formation or cultural meetings take place. Thus, identities are not easily classified.

Identity can be self-ascribed, defined by others, or both (Patrick, 2005; Kishigami, 2002). Olofsson (2004) states that it is possible to both identify with the ethnic group of biological heritage without being socialized into it, or, conversely, to perceive identity with an ethnic group where socialization has taken place but where biological relations are missing. She introduces a model that illustrates the complexity of identity formation (Table 3.1).

Table 3.1: Models of Different Aspects of Identity

\begin{tabular}{lllll}
\hline & Ascribed & Experienced & Aspired & Recognized \\
\hline Self & $\begin{array}{l}\text { A person's ethnic } \\
\text { self-label }\end{array}$ & $\begin{array}{l}\text { How a person } \\
\text { experiences change } \\
\text { in ethnic self-identity } \\
\text { according to cultural } \\
\text { content and personal } \\
\text { circumstances }\end{array}$ & $\begin{array}{l}\text { A person's aspira- } \\
\text { tion for recognition } \\
\text { of ethnic self-label }\end{array}$ & $\begin{array}{l}\text { A person feels a } \\
\text { recognition of } \\
\text { ethnic self-label } \\
\text { from others }\end{array}$ \\
Other & $\begin{array}{l}\text { How others experi- } \\
\text { ence change in that } \\
\text { person's ethnic } \\
\text { identity according to } \\
\text { label of that } \\
\text { person }\end{array}$ & $\begin{array}{l}\text { Others' aspirations } \\
\text { for recognition of } \\
\text { ethnic self-label }\end{array}$ & $\begin{array}{l}\text { Others recognize } \\
\text { that person's } \\
\text { ethnic self-label }\end{array}$ \\
& $\begin{array}{l}\text { and personal circum- } \\
\text { stances }\end{array}$ & & \\
\hline
\end{tabular}

Source: Olofsson 2004: 364-265.

Individuals can belong to several collectives or have several identities. Identity choices can be strategic. A person can change identity over time, but it is also possible to experience and be associated with different identities at the same time. Moreover, identities often have varying degrees of status. Consequently, the identity of a person from an older generation can differ from that of a person from a younger generation of the same family. These complex circumstances highlight the striking differences that can occur within groups that at first glance seem homogenous 
(for a striking Evenki example of hybrid and complex identities see Chapter 5, Governance in the Arctic). Adequate data on ethnic identity (ethnicity) are often missing, and indeed hard to collect, in part due to the contextual and changing nature of a person's ethnic identity.

The social sciences and humanities have been using a relational notion of identity for some time; that is, identity as being created by the tension between Us and Them ("Other"). Still, it seems that modernization and globalization threaten the continuation of these distinctions (see Chapter 11, Community Viability and Adaptation). The Internet's rapid spread and the development of other communication systems present additional challenges to the adherence to cultural traditions. Nevertheless, these technological innovations have also contributed to the rapid spread of ethnopolitical mobilization (Southcott, 2005). Thus, globalization enables the development of new technologies and practices, which in turn offer opportunities to revitalize and strengthen cultures and identities in Arctic communities (see Chapter10, Globalization).

\subsubsection{Indigenous Arctic identities}

Colonial and post-colonial experiences continue to be central to the discussions of the postmodern identity creation in the Arctic. For example, official descriptions and enumerations of Indigenous peoples, including their numbers, the geographical extent of homelands, social structure, etc., sometimes inform Indigenous peoples' identity constructions - and sometimes clash with these identities, when they do not represent Indigenous realities. External definitions of who is Indigenous can result in exclusion of persons who otherwise would identify with a group, and their alienation from Indigenous culture. Acknowledging the negative effects that can be associated with exclusionary, ethnocentric perspectives, argues for an overwhelming need to integrate Indigenous perspectives when discussing "ethnic" categories based on demographic measures. Such perspectives provide a more culturally correct understanding and description of who is Indigenous.

Recent official definitions of "Indigenous peoples", such as those coming from the United Nations, stress historical continuity with a territory, the experience of colonization and self-identifications as being universal to all Indigenous peoples worldwide. The most used and cited definition has long been that of the Ecuadorian diplomat and at that time UN special rapporteur José Martinez Cobo, who in 1986 introduced a "working definition" of Indigenous peoples: 


\begin{abstract}
Indigenous communities, peoples and nations are those which, having a historical continuity with pre-invasion and pre-colonial societies that developed on their territories, consider themselves distinct from other sectors of the societies now prevailing in those territories, or parts of them. They form at present non-dominant sectors of society and are determined to preserve, develop and transmit to future generations their ancestral territories, and their ethnic identity, as the basis of their continued existence as peoples, in accordance with their own cultural patterns, social institutions and legal systems
\end{abstract}

(Martinez Cobo, 1987: paragraph 380).

Indigenous identities are more complex than Martinez Cobo's working definition and there are additional problems that occur when trying to implement it in the Arctic context. The United Nations, national governments, and academic institutions have different perspectives on defining or characterizing Indigenous peoples. Certainly, Indigenous peoples themselves best define how they wish to be viewed and identified. Nevertheless, self-identification has practical, and sometimes ideological challenges (Beach, 2007). More extensive definitions focus on historical processes and relationships, the aim being to remain sensitive to the circumstances of local Indigenous societies where central characteristics are small size, attachment to the land, value system and culture rooted in the environment, commitment to a sustainable lifestyle, positioning, mobility and cultural conservatism. The definition also includes shared experiences of economic and political domination by outsiders, selected integration/participation with non-Indigenous societies, limited or non-existent power within the nation state, and emerging involvement in a local or international process of decolonization (Coates, 2004; Loukacheva, 2009). More recently, Indigenous activists from across the globe have insisted on self-identification and oppose exogenous criteria that determine who qualifies as "Indigenous" and who does not (Hodgson, 2011).

Indigenous identity, status and rights have been under debate in most parts of the world during the last decades. Hundreds of thousands of individuals in the Arctic identify as Indigenous. The lack of a general or even regional definition of who is Indigenous is a challenge for both demographers and other researchers (Axelsson and Sköld, 2011; see also Chapter 2, Arctic Populations and Migration). In modern society, where identity becomes an increasingly personal project and belonging is important, considering oneself as a member of a recognized Indigenous group (e.g., I am Sámi, I am Nenets) can be tempting for individuals searching for such belonging. Indigenous societies can, however, some- 
times be exclusionary, valuing individuals differently, depending on heritage and levels of cultural competence (Åhrén, 2008).

\subsubsection{Mixed identities}

Most historical population records are based on rigid ideas of unchangeable cultural units. Prior to the 19th century many local peoples relied on very inclusive identity systems based on kinship, while governments increasingly relied on a race-based definition that focused on the individual's blood quantum. "Full-bloods", "half-castes" and "quadroons" and octoroons have all been official categories in the USA, Canada, Sweden and Norway, if different terminology is used in the different countries. This of course led to problems with "boundaries" (Olofsson, 2004). State definitions of whom to include as a member of a particular ethnic category, or national minority, were often based on certain stereotypes of cultural belonging or behavior that might have been well removed from the realities of life. "Pure" categories used for counting by necessity also created "mixed" categories, when enumerators discovered cases of "mixed marriage" or the accommodation of settler ways through the mastery of the languages of the incomers or their economic practices. In some cases the "mixed" cases cause indigenousness itself to disappear (as a case of assimilation). In other cases, entirely new peoples are created such as the Canadian Métis (ASI, 2010).

Studies on Indigenous societies in northern Sweden and Canada suggest that the ethnic formation of a person with mixed parentage can greatly differ from situations in which mixed parentage is not present or not problematized. Already in childhood there are different ways of managing the situation. Some children feel that they only belong to one ethnic group while others experience a more complex situation where they feel caught between two identities. Socialization including more persons than the parents is important for the self-experienced ethnic identity and the ethnic self-label, which nevertheless fluctuates and changes according to cultural context. A person can manage different self-representations attached to different cultural behaviors without getting confused in his or her personal identity (Olofsson, 2004).

Names and ethnic labels can have benefits and disadvantages depending on context. The descendants of the Old Settlers of Russian origin who married native women in northeast Siberia from the seventeenth century onward were never classified as a unique ethnic group and never received state recognition as a mixed group. Today, some of these groups have achieved Indigenous status with limited privileges, while 
most of them are left with ambiguous social status and ethnic identity (Schweitzer et al., 2013). Since the Arctic is being influenced by extensive processes of change, including internet access, migration, and globalization, the complexity of identities increase correspondingly. Overall, a slightly improved understanding of mixed or multiple identities is a trend since the first $A H D R$.

\subsection{An Arctic identity?}

There are reasons to wonder if an Arctic identity exists at the individual level, and whether such an identity is similarly understood in all eight Arctic (Council) countries (Keskitalo, 2009). Still, sometimes a broader and more general definition of an Arctic identity - stretching over nations and regions - is used. It denotes a sense of shared interests within a geographic region and is understood in terms of how members connect to their linguistic, cultural, and social similarities, and their perception of common interests and threats regardless of state boundaries. Regionalized Arctic identity is being claimed by institutions and sub-national groups to strengthen their political legitimacy and mobilize people for collective action. The identity is geographically bounded in the sense of referring to the "natural" or self-evident Arctic that makes up this circumpolar region, defined in part by latitude, climate, ecology, and even oceans (Berkman, 2012; Gerhardt et al., 2010). Arctic regional identity is, however, also an outcome of institutional and intergovernmental co-operation. It is partly created through language, policy, and political action. National identity draws on and stresses valued characteristics that include resourcefulness and hard work in using nature to secure material wealth and prosperity, resilience and adaptability in thriving in a cold climate, closeness with nature and the desire to explore it, and commitment to protecting the Arctic and its natural beauty. This Arctic identity points to transnational problems and interdependencies, the importance of non-state actors, and the multiple and often competing layers of identities that exist in the Arctic (Williams, 2011; Thisted, 2012). Nevertheless, our impression is that a general Arctic identity is still weak, while regional, local and Indigenous identities have developed.

Within each Arctic country, northern identity varies. While Greenland and Iceland have an indisputable northern geographic location, only parts of the remaining six countries fall within the Arctic. The label "Arctic" when used for northern Canada, Alaska, Greenland and northern 
Russia, is often understood in terms of "the frontier", including specific notions of the environment, Indigenous peoples, and forms of subsistence. In Norway, Sweden, Finland, and Iceland, nation states that have historically not been discussed as Arctic areas, such an "identity" seems less common. Keskitalo et al. (2013: 353) argue that "these countries have thus not necessarily been subject to processes associated with the term "Arctic", and in Sweden, domestic research and discourse regularly use other terms. Discussions of regional "norths" exist in the area, but the language used exhibits none of the very strong and specific characteristics related to the term Arctic." (See Textbox 3.3.).

The North has served as an important and enduring imaginative resource in the construction of Canadian national identity. Arnold (2012) argues that the North is more an idea, a discursive formation, as a social and political place that has been "nordicised". Canadian northerners feel largely ignored. They want to be recognized for their contribution to Canadian culture and identity, while remaining first and fundamentally northern (Robinson, 2012; England, 2000; Stephenson, 2012).

There is a complex interplay between Arctic identities and Arctic policy (see Textbox 3.4). The different countries' Arctic resources and exercises of sovereignty are part of the historic importance of the Arctic in producing distinct national identities. At the same time, the development of shared values, ideas and ambitions have been emphasized in the establishment of the Arctic Council, circumpolar organizations, and in the International Polar Year. One intention these initiatives share is to grow a circumpolar identity based on values, ideas, and interests that these countries have in common. These two identities (national and regional), and the policy options and directions that emerge from them, are in tension. They might converge into unique national circumpolar identities by pursuing a multi-level identity framework, in which post-colonial values and institutions compensate for the limitations of the national, and vice-versa (Williams, 2011). The eight member states of the Arctic Council all claim to have Arctic identities in one form or another. The five Arctic Ocean coastal states, however, have made even stronger Arctic identity claims. 


\section{Textbox 3.3}

\section{The Barents region}

Originating from an idea of the former Norwegian Foreign Minister Thorvald Stoltenberg the Kirkenes Declaration formally established the Barents EuroArctic Region in 1993, to further security objectives in the European North. In order to reach the superior goal of maintaining stability in the area, 'its declared aim to create a shared identity in the area. The common history of the populations in the area as well as their similar geographical frame of reference is emphasized as forces capable of enhancing such a process.

An urgent challenge for decision-makers lies in making the populations if the different states in the region feel that they have something in common with their neighbors across the border. To be more specific, the goal becomes one of urging a maximum number of people in the area to include Northerners from all the states in the region in their notion of a political self, that is to say, their identification of belonging to a group with specific common interests, prospects and threats, as opposed to other sub-groups in society. Region-building thus becomes a question of identity politics. This involves a view of identity as something flexible, a relation rather than a possession, a quality conditional to persons in different situations rather than categorical pertaining to persons as such. A person's identity may thus change over time, and he or she may at one point in time have, or be related to, several identities. Overlapping identities are not only considered possible, from a security point of view, they may even be regarded as desirable.

Official statements stress that people in the area all live in a region characterized by a harsh climate, a vulnerable nature, long distances to national centers, and a sparse population, which allegedly gives them some kind of common worldview and a mutual understanding of each other's situation, notwithstanding the state borders in the area.

However, in the cross-border region Karelia of Finland and Russia separation has been a more common historical state than community and interaction. Another factor that somewhat weakens the argument that a transnational Northern identity will easily develop - or is already in existence - is the fact that Northwestern Russia to a large degree is populated by "Southerners". Hönneland (1998) claims that the historical transnational identity outlined in the "Barents rhetoric" has never existed, and that the new northern identity is so far a castle in the air; the Barents citizen an illusion.

Many who live in the area have only a vague conception of the Barents region, others have never heard of it. It seems all the more appropriate to ask whether the new transnational northern identity relates only to a very limited group of entrepreneurial people with particular interests in developing contacts at the other side of the border. 


\subsection{Quantifying ethnicity}

The Arctic countries differ substantially in their efforts to collect and share data on ethnicity. It is often very difficult to trace ethnicity in both the historical records and in the present-day population statistics, even where statistics are collected. Official and self-determined definitions have varied extensively over time, and between countries (ASI, 2010). Looking into the future, the descendants of today's ethnic groups may or may not identify with the ethnic heritage of their ancestors. Cultural transition can threaten ethnic continuity, and thus Indigenous cultural persistence.

The incomplete inclusion and categorization of ethnicity in registers creates difficulties to estimate not only the population size and composition of different ethnic groups, but also specific features such as language spoken, educational attainment, occupations, and health status (Axelsson and Sköld, 2011) (see Chapter 2, Arctic Populations and Migration). The UN Special Rapporteur Paul Hunt has pointed out that it is practically impossible to improve the situation of Indigenous peoples if they are not visible through enumeration (Hunt, 2007). This was especially stressed for the Sámi in Sweden. All official estimations present a number of 20,000 Sámi living in Sweden. A genealogically based examination shows, however, that the number is probably almost three times as high (Hassler, 2005). This means that there are more or less 30,000 persons in Sweden with a Sámi background who are not aware of their ethnicity or for some reason have chosen not to identify with the Sámi culture. These persons can be said to represent transitions that threaten to undermine Indigenous identity: the threat is cultural rather than demographic (Axelsson and Sköld, 2006).

Language is one of those features of ethnicity closely tied to issues of identity and political rights. For example, in order to vote in the Sámi parliaments of Norway, Sweden and Finland, one must prove that either he or she speaks Sámi, or that it is spoken by a parent or grandparent (Keskitalo et al., 2013).

Overall, there is a great need for improving and integrating quantitative ethnic information at the individual level in official registers and statistics. This includes better coordination on the regional, national and international levels. Better information is a prerequisite for understanding the past and present situation, and thus for the long-term sustainability of Arctic cultures and identities. 


\subsection{Cultural identities and "traditional" activities}

"Connection with nature" is a defining feature of Arctic identity (ASI, 2010). Traditional activities - such as hunting, fishing and herding - represents both sources of monetary income and cultural values, and are strongly linked to northern identities (see Chapter 4, Economic Systems). In a time when Inuit were solely living on the land and constantly travelled between locations, the sharing of food was important. The move to settlements has brought about changes in Inuit notions of sharing food, and - indirectly - in ideas about identity (Gombay, 2005). While traditional subsistence economies and the right to possess and occupy the land of their ancestors are intrinsic to the cultural identity of Arctic Indigenous peoples (ASI, 2010: 136; MacLennan, 2008), non-Indigenous hunting and fishing practices often follow a different (sport or hobby) logic. Still, in some parts of the Arctic (e.g., in the Nordic countries) hunting and fishing are seen as a part of one's livelihood among non-Indigenous persons and are strongly related to one's identity (Keskitalo et al., 2013).

In the Faroe Islands, local forms of agriculture and hunting have enabled the Islanders to maintain a relatively high degree of self-sufficiency in local food production. Mountain grazing sheep provide up to $60 \%$ of all locally produced meat as well as wool, while some limited catching of sea birds such as puffins and fulmars, and the coastal hunting of the abundant and regularly available pilot whales provide meat and blubber. Enough dairy cattle are kept to satisfy all domestic milk needs, and small potato crops are carefully cultivated by private households for their own use. 


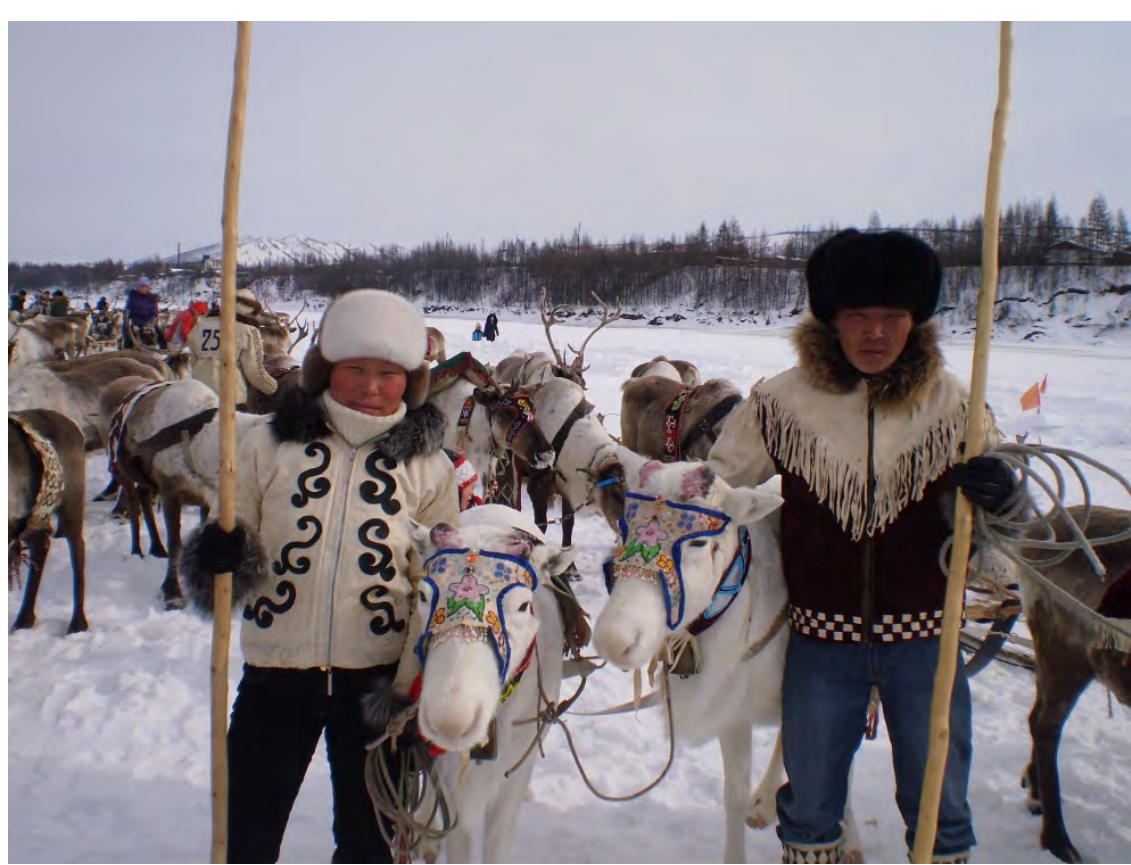

Credit: Olga Ulturgasheva.

Nomadic reindeer pastoralism for some Sámi and many Indigenous Siberian peoples is a key feature of their culture. Extensive reindeer pastoralism depends on large areas for pasturing the deer. Reindeer herding is under pressure from extractive industries and other infrastructural initiatives. In Fennoscandia, many rivers have been dammed and large areas of pasturage flooded as a result of extensive water regulation. Valuable riverside pasturage, natural pasture boundaries, and migration routes have disappeared. The pasture areas have been replaced with paddocks, the natural migration routes supplanted by road networks. Through clear-felling, soil scarification and road construction, forestry has broken up winter pasturage areas and reduced the supply of winter grazing for reindeer in northern Europe. Peat-harvesting activities also have an adverse impact on pastures. Today, less than 10 per cent of the Sámi population are connected to reindeer herding, which requires membership in a Sámi village. This keeps a major part of the Sámi population outside one of the traditional economic activities and today also outside hunting and fishing privileges, and in the end from the possibility of using the traditional areas at all. This has severe consequences for the ethnic and cultural identity among the Sámi (Sköld, 2011). 
A different, almost post-productionist, approach to reindeer herding can be observed in the Sakha Republic in Siberia (Aytalina Ivanova and Florian Stammler, personal communication, October 2014). There, a reindeer herding law passed in 1997 specifies the value of this livelihood as a "national property" that is worth of state support as such (Sakha Republic, 1997). Implementing this law, the region has introduced a guaranteed salary to herders of reindeer regardless of whether they are privately or publicly owned, paying them for just raising and keeping herds rather than producing meat or other agricultural output from these animals. The main condition for this is that they lead a nomadic way of life (Sakha Republic, 2002). Similar thoughts underlie support policies in other Arctic regions, such as in the West Siberian Yamal District, although there politics follow a double strategy of stimulating reindeer meat production alongside nomadic lifestyle (Stammler \& Ventsel, 2003).

Throughout the Arctic, subsistence activities such as fishing and hunting have suffered greatly under the impact of mining industries and the construction of hydro-electric power stations. For example, the Inuit in Nunavik and the Cree First Nations of James Bay Region have experienced a dramatic loss of hunting grounds through flooding for hydroelectric purposes. While mining in the Arctic has impacted traditional economies, resource exploitation can also form the basis for the (re)construction of identities, where the links to traditional economies have transformed into new occupation and settlement forms (see Chapter 11, Community Viability and Adaptation). Still, as a general tendency, conditions for traditionally important industries such as herding, hunting and fishing have been deteriorating due to mining and large-scale development, which have negative impacts on culture and identities. 


\section{Textbox 3.4}

\section{Arctic cultural representations in the interest of the nation}

Inuksuit are Inuit stone constructions stacked to resemble the human figure. They are strong cultural symbols embedded in songs, shamanism, myths, legends and stories. In April 2005 the Vancouver 2010 Olympic committee presented their logo, an Inuksuk named Ilanaaq, meaning friendship in Inuktitut. The decision was controversial to some, and logical to others (Arnold, 2010). A notion was expressed that the Inuksuk was a symbol for the entire Canada, and it has been claimed that it has also emerged as the unofficial symbol of the Arctic (Heyes, 2002).
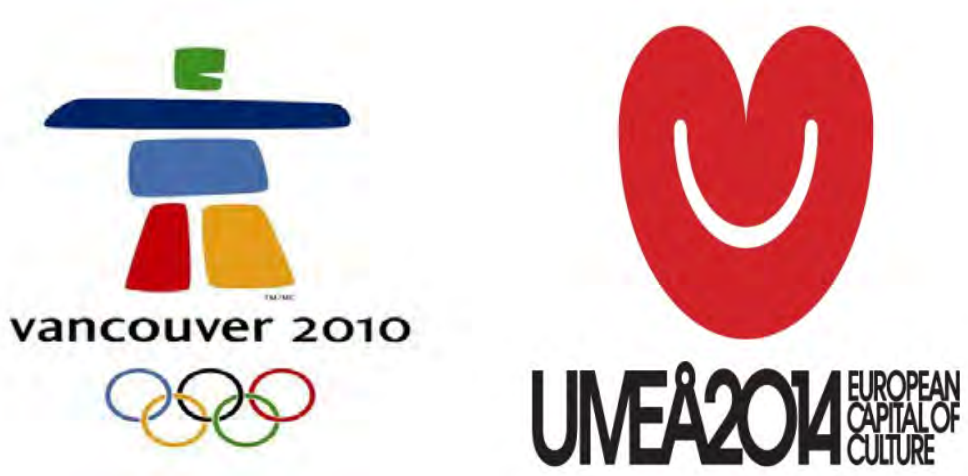

When the city of Umeå announced its candidacy for the European Capital of Culture 2014, the Indigenous culture of the Sámi was a prominent part of the application. The bid was said to be "an invitation to this magic and exciting borderless country, with the unique Sámi culture as one of its highlights." It was stated that historically, the outside world has often varied in its descriptions of this borderless northern place, seeing it either as a country of the future, or as completely marginalized. Umeå claimed a strong northern identity that understands the north through every fiber of its being; an essential attribute, as the northern region rises to the top of the European agenda. 2014 would offer fabulous opportunities for Sámi to show their art, music, handicraft and other expressions of culture to a wide and global audience, and to put to the attention of an extensive audience the too often problematic situation of Sámi and other Indigenous peoples in the world. There are also reasons to critically scrutinize how they were integrated, engaged and empowered during the process, and to see how others represented their culture (Rönnmark and Björinge, 2014). 


\subsection{Landscape and place}

Identity is certainly place-bound and time-bound, as well as relationally determined (Oosten and Remie, 1999). Relating to the landscape plays an important part in Arctic identities. The landscape is a source of collective (clan, family, national) history, which is captured through place names, monuments and oral tradition. It is on the land that the dynamics between humans, animals and the environment are experienced, and traditional skills learned and perfected (Anderson, 2014). Cultural traditions are most often communicated through physical activities on the land (and the sea). Through participation in these activities one learns how to behave properly. The capacity for deciphering and understanding landscapes can be developed over time (Patrick, 2005). Thus, respect for and knowledge of different places is an integral part of Arctic cultures and identities.

Place names (toponyms) for landscape features contribute to how a place is perceived. Place names may describe topographic features, draw attention to sites used by animals, provide directional/navigational references, or have religious/ritual significance (Collignon, 2006).

It is not economic motives that encourage many to continue with a traditional relation to nature, but the quality of life, the quasi-existential persuasion that the landscape contains the meaning of life. Or, as one Sámi put it to the historian Åsa Nordin:

Today it's a lifestyle. So long as we carry on reindeer herding the way we do, with extensive herding, migrations and guarding, it's a lifestyle. [...] Especially when reindeer herding doesn't bring in most money, you factor other Sami values into it, values which cannot be stated in money terms, and when you assimilate and experience those values as well, it's a lifestyle

(Nordin, 2007: 82).

Landscape is of pivotal importance to most Arctic communities and cultures. But Indigenous peoples are not alone in feeling strong ties to their home areas. One challenge facing tomorrow's land use systems in the Arctic will be that of devising forms of co-management of resources, both within communities and between groups living in the Arctic. Place identity is not a given but rather an outcome of a continuous process of place-making. Still, place identity is perhaps even more important for Indigenous peoples than for other populations. Where non-Indigenous populations have occupied their territories, Indigenous peoples must confront the impacts of disruptive incursions upon their land-based way of life and upon their sense of identity. 


\subsection{Identities in a changing Arctic}

The Arctic is constantly changing and so are the identities embedded in the region. Since identity is a way of relating with one's human, social and political surroundings, it is continually constructed, and should be seen as a process rather than as a category. Identity is enacted and reenacted every time one has to relate to someone else (Dorais, 2005). At the same time, the Arctic experiences a variety of change processes. Long-term socio-economic developments have major effects on cultural identities, not least among Indigenous peoples in the Circumpolar North (see Chapter 10, Globalization).

Climate change acts contributes to other factors of change that have far-reaching impacts. Changes to climate have a mostly negative effect on subsistence, travel on sea ice, reindeer pasture conditions, and thereby indirectly on cultural identities (ASI, 2010; Hovelsrud et al., 2011; Magraw et al., 2008). Community viability and a sense of common identity are challenged by changing environmental conditions and increasing competition over resources (see Chapter 11, Community Viability and Adaptation; Andrachuk and Smit, 2012; Keskitalo et al., 2013; Stepian et al., 2014). These challenges are especially keen among adolescents (Kvernmo and Heyerdahl, 2003; Caulfield, 2000). The loss of social identity resulting from these changes increases the risk for social alienation and destructive behaviours (ASI, 2010).

Urbanization has been a distinctive trend all over the Arctic during the past decades, involving Indigenous and non-Indigenous groups (Megatrends, 2011). For example, in the Canadian Arctic, recent demographic changes have made settlement patterns increasingly urban, with more and more people moving to larger communities. Despite these trends, Inuit cultural identity for many continues to be strongly connected to spending time out on the land, hunting, fishing, trapping and camping. Some Inuit indeed maintain a rather negative view of urban spaces in the Arctic, identifying them as places where non-Indigenous people have eclipsed Inuit values and practices (Searles, 2010). Many Inuit believe that living off the land creates intelligent and moral persons, and that individuals develop a capacity to think and reason through facing the elements of sea, snow, ice, and wind. On the other hand, an urban living has the potential to offer a new platform for identity building, where freedom, reflection and innovation are key elements (Kishigami, 2002; see Chapter 11, Community Viability and Adaptation). 
Graffiti protesting the appropriation of Sami culture during Umeå's celebration as European Capital of Culture (2014)

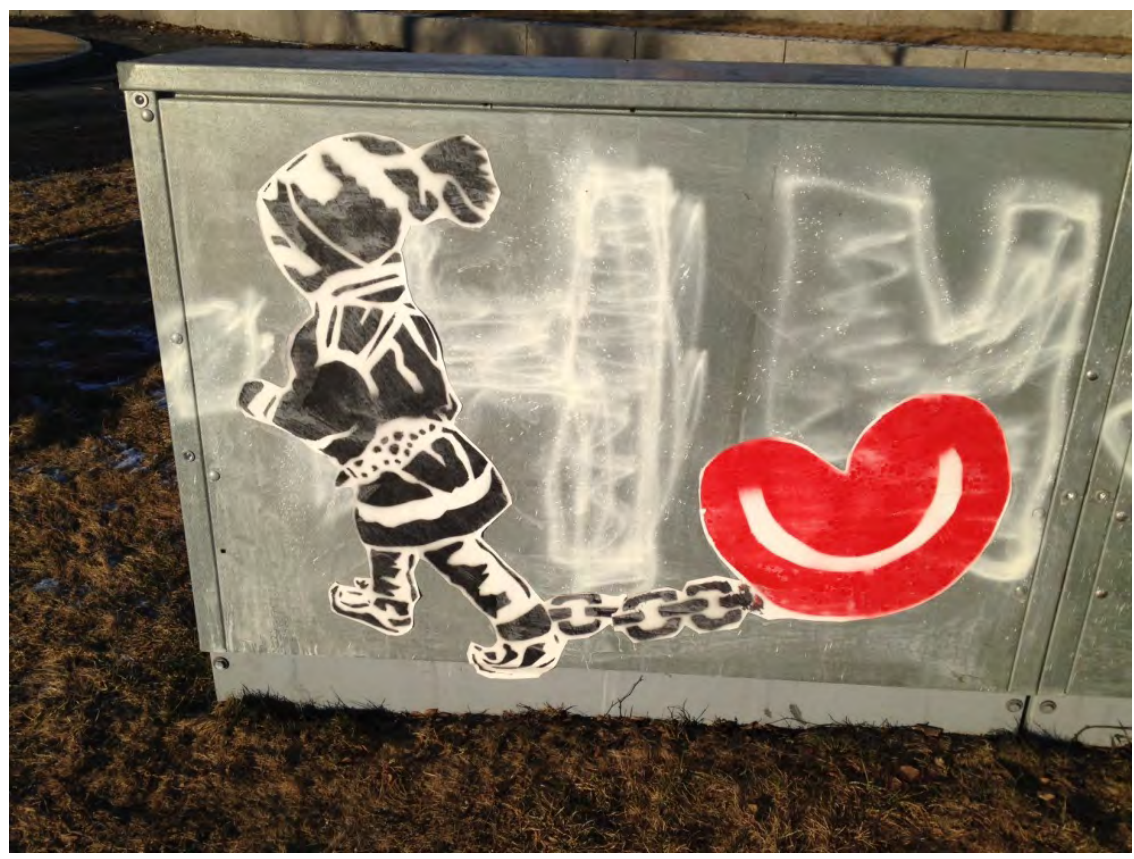

Credit: Gail Fondahl.

\subsection{Chapter summary and trends}

As was stated in the first $A H D R$, resilient cultures and modernity do not have to contradict each other. That is, contemporary Arctic Indigenous peoples understand that the challenge is not to choose between "modernity" and "unchanging tradition," but to find a livable combination of the two. Given the political sophistication of local communities, to work in the North has become a tremendously rewarding learning experience for scientists and other scholars. At the same time, there is an evolving self-consciousness in some areas of the North that goes beyond the (necessary) anti-colonial rhetoric of earlier years. There is also an increased awareness of "mixed" identities, and of the fact that many northerners have a mixed heritage. Still, these social realities are not always reflected in official policies and legislation, which tend to favor simplistic "black or white" identity categories.

There is an ongoing trend toward cultural and linguistic revitalization. Indigenous people are increasingly participating in academic and civil society discourses. This needs to be seen in the context of emerging northern identities and the positive valuation of regional perspectives. 
We must not forget, however, that there are enormous differences within the North regarding cultures and identities. While these differences must not be left out of the picture, a few general developments are nevertheless traceable.

We observe an increased focus on "culture." Culture, especially Indigenous culture in the North, has become more and more a resource, both in the sense of a commodity and in the sense of a tool that makes external recognition easier. Alongside this, there is a growing sense of the marketability of the North, which includes tourism but is not limited to it. So, it is not just "northern culture," but the North as a whole that becomes more and more reified, be it as commodity, experience, state of mind, wilderness, resource base, and homeland. This leads to a kind of strategic advantage of being in the North. What used to cause isolation and marginalization - that is, Arctic identities - can be an advantage in contemporary identity politics.

\section{Gwich'in Moccasins, Canada, 2014}

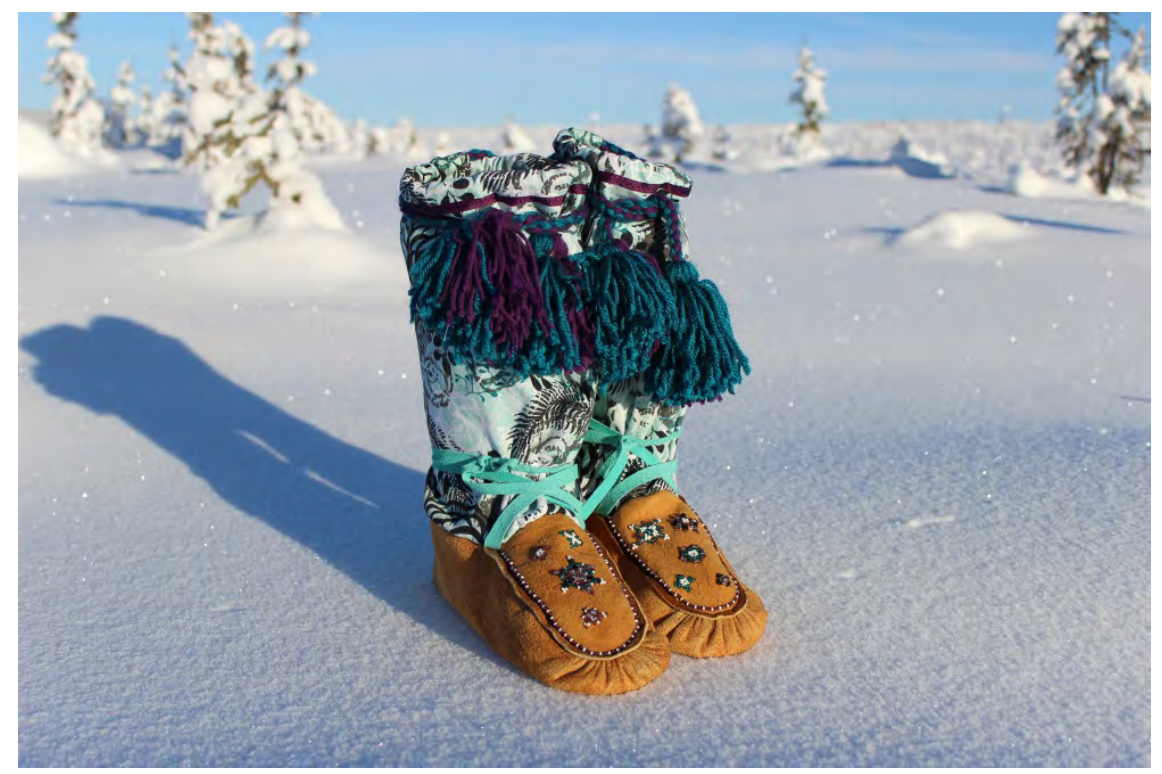

Photo credit: Christine Creyke.

Much has been written about climate change in the Arctic. It is obvious that the cultural impact of climate change across the Circumpolar North varies from place to place and region to region. Still, there has been an enormous increase in the overall amount of attention Arctic communities are receiving because of climate change. Thus, while climate change might or might not be a priority issue for a specific group of northerners, 
the (southern) discourse about climate change and its impact on northern cultures has changed dramatically since the first $A H D R$. This emphasizes a clash between inside and outside perspectives, and indicates that parts of the Arctic have acquired a new stereotypical position, namely that of victims of climate change.

In focusing on specific characteristics of Arctic cultures and identities, one is tempted to overemphasize the differences with the outside world. There are, however, multiple vectors of similarities that tie cultures and identities of the North to other parts of the globe. On the one hand, many metropoles of Arctic states, to varying degrees, are geographically and culturally located far south of the Arctic Circle. Given the historical and contemporary connections between southern political centers and Arctic peripheries, it is not surprising that cultures of the North share many features with their respective dominant (southern) societies. On the other hand, northern identities also share similarities with those of other peripheral parts of the world (Brightman et al., 2006; Ulturgasheva et al., 2010). The transnational Indigenous movement, in which Arctic organizations are very active participants, is an example of this development.

Even today, however, Arctic cultures and identities remain different from southern counterparts in many respects. For example, Arctic cultures and identities have been characterized by tight relations with the natural and social environment. Kinship ties and networks continue to be valuable, not least in the urban context.

Some argue that there is a more general circumpolar "Arctic" culture, as the different identities share common characteristics. The development of communication technologies linking different parts of North has increased this trend, while at the time encouraging globalizing tendencies.

Gender, age and ethnicity - and their interactions - are important aspects and parameters of Arctic livelihoods. Of these, indigeneity has received the most attention in the literature. Arctic cultures and identities have not been sufficiently investigated through the lens of gender. While we know that traditional circumpolar cultures were characterized by gender-specific realms of activities, we know too little about the gender dimensions of contemporary cultural practices. Since we know that urbanization and other aspects of modernity affect men and women in the Arctic differently, we can assume pronounced gender differentiation, especially in urban contexts. While "traditional" notions of culture and identity are often tied to rural areas, 21st century Arctic realities include significant rural to urban migration movements, which are often more 
difficult to align with male cultural practices and concepts than with those of women. The gender balance in the production of modern Indigenous cultural expressions is differentiated. Whether women or men are predominant in culturally important activities depends on a variety of local and regional factors. More gender-sensitive research on cultures and identities in the Arctic is much needed.

Likewise, the rapid changes of a globalized world are having and will have profound impacts on the younger generation (and future generations); thus, it is critical to understand the hopes and aspirations of Arctic youth. The future of Arctic cultures and identities will rest on the choices made by young people in the North today. These choices include - but are not limited to - decisions about where to live, which languages to learn and use, etc. Most important will be whether and to what extent Arctic Indigenous cultures and identities will be attractive for the younger generations. There are localized positive examples, as well as negative ones (Megatrends, 2011; Åhrén, 2008; Ulturgasheva et al., 2010).

There is great cultural variation and complexity in the Arctic, probably more so than recognized in the first AHDR. Even where forms of expression (e.g., drumming, story-telling, rituals) are similar, the underlying cultural ideologies can be different. Identities change over time and there are multiple identities, which further complicate a homogeneous understanding. There are, however, basic similarities in the worldviews and spiritualities of many Indigenous societies and cultures of the North.

So what other major trends concerning cultures and identities in the North can be found? To some extent, they seem contradictory. On the one hand, there is a trend toward revitalization of Indigenous languages and cultures and the strengthening of northern identities. On the other hand, there is growing threat to circumpolar cultures and identities through modernization, globalization and (urban) migration. Only northerners themselves can decide where they want to live and work. As indicated above, this is hardly an either/or question but a situation of where both revitalization and modernization are necessary. In these decisions, however, "fate control" as defined by AHDR (2004) and ASI (2010), seems to be a critical indicator of positive change.

Finally, we want to conclude with what we see as the major changes since 2004, the publication of the first AHDR. Given that certain layers of cultures and identities are "slow-changing" entities, it could be argued that not much has changed in terms of cultures and identities during the past ten years. Trends that started long before 2004 are visible in 2014 as well: increased connectedness with the non-Arctic, especially with 
national and international hubs and centers; decrease of local language use; and increase of cultural revitalization efforts.

However, there has been a marked growth in the global awareness of the Arctic between 2004 and 2014. This is primarily connected to the global climate change discourse and to anticipation of future economic development opportunities in the North. While these conversations typically originate in the South, northerners are active participants in them. The net costs and benefits for Arctic residents remain to be seen, not only in terms of economic development, but also whether the increased interest in Arctic cultures might cement old stereotypes rather than overcome them.

Still, it is clear that Arctic (Indigenous) "culture" has become an asset and a resource in many parts of the North. At the same time as indigeneity is being celebrated, cultural and social realities in the Arctic are increasingly characterized by "mixed" identities, whether or not they are socially and politically recognized. Parts of the Arctic show indications of increased strategic advantage of northern-ness and a growing marketability of symbols and things northern. Whether the interest in Arctic cultures and identities is a short-lived fad or not is a question for AHDR-III.

\subsection{References}

AHDR, 2004. Arctic Human Development Report. Einarsson, N., J. N. Larsen, A. Nilsson and O. R. Young (eds.). Stefansson Arctic Institute, Akureyri.

Åhrén, C., 2008. Är jag en riktig same? En etnologisk studie av unga samers identitetsarbete (Am I a Real Saami? An Ethnologic Study of Young Saami Identity Formation). Umeå: Umeå University.

Anderson, D., 2014. Cultures of reciprocity and cultures of control in the Circumpolar North. Journal of Northern Studies 9(2): 11-27.

Andrachuk, M. and B. Smit, 2012. Community-based vulnerability assessment of Tuktoyaktuk, NWT, Canada to environmental and socio-economic changes. Regional Environmental Change, 12:867-85.

Arctic Centre, 2013. Draft Conference Statement on Sacred Sites in the Arctic. http://www.arcticcentre.org/news/Sacred-sites-in-the-Arctic-need-protection$\%$ E2\%80\%93-First-International-declaration-drafted/pmtrvo5n/7a0574f4-4fc4443d-b892-598e8d768254 (23 November 2013).

Arctic Winter Games, nd. http://www.arcticwintergames.org/Role_Purpose_Values.html (28 October 2014).

Arnold, S., 2012. Constructing an Indigenous nordicity: The "new partnership" and Canada's northern agenda. International Studies Perspectives, 13:105-20.

Arnold, S., 2010. "The Men of the North" Redux: Nanook and Canadian National Unity. American Review of Canadian Studies, 40(4): 452-63. 
ASI, 2014. Arctic Social Indicators II: Implementation. Larsen, J.N., P. Schweitzer, and A. Petrov (eds.). TemaNord. Nordic Council of Ministers, Copenhagen.

ASI, 2010. Arctic Social Indicators - a follow-up to the Arctic Human Development Report. Larsen, J.N., P. Schweitzer, and G. Fondahl (eds.). TemaNord. Nordic Council of Ministers, Copenhagen.

Axelsson, P. and P. Sköld, 2011. Introduction. In: Axelsson, P. and P. Sköld (eds.). Indigenous Peoples and Demography. The Complex Relation Between Identity and Statistics, pp. 1-14. Berghahn Books, New York.

Axelsson, P. and P. Sköld, 2006.Indigenous populations and vulnerability. characterizing vulnerability in a Sami context. Annales de Demographie Historique, 111(1): 115-132.

Balzer, M. M., 2011. Shamans, Spirituality and Cultural Revitalization: Explorations in Siberia and Beyond. Palgrave Macmillan, New York.

Barr, S, and P. Chaplin, (eds.), 2004. Cultural heritage in the Arctic and Antarctic regions. ICOMOS IPHC.

Barry, T., L. Grenoble, and F. Fridriksson, 2013. Linguistic diversity. In: CAFF, 2013. Arctic Biodiversity Assessment. Status and Trends in Arctic Biodiversity. Conservation of Arctic Fauna and Flora, Akureyri.

Beach, H., 2007. Self-determining the self: aspects of Saami identity management in Sweden. Acta Borealia: A Nordic Journal of Circumpolar Societies, 24(1): 1-25.

Berger, T., 1985. Village Journey: The Report of the Alaska Native Review Commission. Hill and Wang, New York.

Berkman, P., 2012. Our common future in the Arctic Ocean. The round table. The Commonwealth Journal of International Affairs, 101(2): 123-135.

Bird-David, N., 2006. Animistic Epistemology: Why do some hunter-gatherers not depict animals? Ethnos 71(1): 33-50.

Bodenhorn, B., 2004. "He used to be my relative:" Exploring the bases of relatedness among Inupiat of Northern Alaska. In: Carsten. J. (ed.), Cultures of Relatedness: New Approaches to the Study of Kinship, pp. 128-48. Cambridge University Press, Cambridge.

Bodenhorn, B., 2000. It's good to know who your relatives are but we were taught to share with everybody: shares and sharing among Inupiaq households. Ethnological Studies 53: 27-60.

Brightman, M., V. Grotti, and O. Ulturgasheva, 2006. Introduction: rethinking the 'frontier' in Amazonia and Siberia: extractive economies, Indigenous politics and social transformations. Cambridge Anthropology, 26(2): 1-12.

Brightman, M., V. Grotti, and O. Ulturgasheva, 2012. Animism in Rainforest and Tundra: Personhood, Animals, Plants and Things in Contemporary Amazonia and Siberia. Berghahn Books, Oxford.

CAFF, 2013. Arctic Biodiversity Assessment. Status and Trends in Arctic Biodiversity. Conservation of Arctic Fauna and Flora, Akureyri.

Caulfield, R.A., 2000. The Kalaallit of West Greenland. In: M. Freeman (ed.). Endangered Peoples of the Arctic. Struggles to Survive and Thrive, pp. 167-186. Greenwood Press, Westport.

Coates, K.S., 2004. A Global History of Indigenous Peoples: Struggle and Survival. Houndmills, Palgrave Macmillan, Basingstoke.

Collignon, B. 2006. Knowing Places. The Inuinnait, Landscapes, and the Environment. Canadian Circumpolar Institute, Edmonton.

Creery, I., 1994. The Inuit of Canada. In: Minority Rights Group (ed.). Polar Peoples Self-Determination and Development, pp. 105-146. Minority Rights Publications, London. 
Damas, D., 1984. Arctic. In: Sturtevant, W.C. (ed.). Handbook of North American Indians, Vol. 5. Smithsonian Institution, Washington, D.C.

Descola, P., 2006. Beyond nature and culture. Proceedings of the British Academy, 139: 137-55.

Dorais, L., 2005. Comparing academic and aboriginal definitions of Arctic identities. Polar Record, 41(216): 1-10.

D’Orso, M., 2007. Eagle Blue: A Team, a Tribe, and a High School Basketball Season in Arctic Alaska. Bloomsbury Publishing, New York.

Elenius, L., 2008. Minority policy and postmodern ethnopolitical mobilisation at the North Calotte. In: Heininen, L. and K. Laine (eds.). The Borderless North: Publications of the Fourth Northern Research Forum, pp. 136-149. Oulu University, Oulu.

England, J.H., 2000. An urgent appeal to the Government of Canada to proclaim our Northern identity. Arctic, 53(2): 204-209.

Fienup-Riordan, A., 2000. The Yupiit of Western Alaska. In: Freeman, M. (ed). Endangered Peoples of the Arctic, pp. 247-266. Greenwood Press, Westport

Fisher, R.H., 1943. The Russian Fur Trade 1550-1700. University of California Press, California.

Forsyth, J., 1994. A History of the Peoples of Siberia. Russia's North Asian Colony 15811990. Cambridge University Press, Cambridge.

Freeman, M.M.R. (ed.), 2000. Endangered Peoples of the Arctic: Struggles to Survive and Thrive. Greenwood Press, Westport.

Gad, F., 1973. The History of Greenland II: 1700--1782. McGill-Queen's University Press, Montreal.

Gerhardt, H., P. Steinberg, J. Tasch, S. Fabiano, and R. Shields, 2010. Contested sovereignty in a changing Arctic. Annals of the Association of American Geographers, 100(4): 992-1002.

Gombay, N., 2005. Shifting identities in a shifting world: food, place, community, and the politics of scale in an Inuit settlement. Environment and Planning D: Society and Space, 23: 415-433.

Hallowell, A.I., 1926. Bear ceremonialism in the Northern Hemisphere. American Anthropologist, 28: 1-175.

Hansen, L. and B. Olsen, 2014. Hunters in Transition: An Outline of Early Sami History. Brill, Leiden.

Hansen, L. and B. Olsen, 2004. Samenes Historie fram til 1750 (Saami History until 1750). Cappelen Akademiske Forlag, Oslo.

Hassler, S., 2005. The Health Conditions in the Sami Population of Sweden, 1961-2002 - Causes of Death and Incidences of Cancer and Cardiovascular Diseases. Umeå University Medical Dissertations, Umeå.

Heyes, S., 2002. Protecting the authenticity and integrity of the Inuksuit with the Arctic milieu. Études Inuit/Inuit Studies, 26(2): 133-156.

Hodgson, D.L., 2011. Being Maasai, Becoming Indigenous: Postcolonial Politics in a Neoliberal World. Indiana University Press, Bloomington.

Hönneland, G., 1998. Identity formation in the Barents Euro-Arctic Region. Cooperation and Conflict, 33(3): 277-297.

Hoffecker, J.F., 2004. A Prehistory of the North: Human Settlement of the Higher Latitudes. Rutgers University Press, New Brunswick.

Hovelsrud, G.K., B. Poppel, B. van Oort, and J. Reist, 2011. Arctic societies, cultures, and peoples in a changing cryosphere. AMBIO, 40: 100-110. 
Hunt, P., 2007. Mission to Sweden: Report of the Special Rapporteur on the Right of Everyone to the Enjoyment of the Highest Attainable Standard of Physical and Mental Health. Report No. A/HRC/4/28/Add.2. United Nations General Assembly, Human Rights Council. New York.

Inuit Circumpolar Council Canada (ICC - Canada), 2012. Assessing the Vitality of Arctic Indigenous Languages Research Development Workshops. Ottawa, Canada, June 18-20.

Inuit Circumpolar Council Canada (ICC-Canada), 2008. Proceedings of the Arctic Indigenous Languages Symposium, October 19-21. Tromso, Norway.

Jóhannesson, J., 2007. The History of the Old Icelandic Commonwealth. The University of Manitoba Press, Winnipeg.

Karlsson, G., 2001. Iceland's 1100 Years: The History of a Marginal Society. C. Hurst \& Co. Publishers Ltd., London.

Keskitalo, C., 2009. 'The North' - is there such a thing? Deconstructing/contesting Northern and Arctic discourse. In: Hansson, H. and C. Norberg (eds.). Cold Matters. Cultural Perceptions of Snow, Ice and Cold, pp. 23-40. Umeå University, Umeå.

Keskitalo, C., G. Malmberg, K. Westin, U. Wiberg, D. Müller, and O. Pettersson, 2013. Contrasting Arctic and mainstream Swedish descriptions of Northern Sweden: The view from established domestic research. Arctic, 66(3): 351-365.

Kishigami, N., 2002. Inuit identities in Montreal, Canada. Études/Inuit/Studies, 26(1): 183-191.

Krauss, M., 1992. The world's languages in crisis. Language, 68(1): 4-10.

Kvernmo, S. and S. Heyerdahl, 2003. Acculturation strategies and ethnic identity as predictors of behavior problems in Arctic minority adolescents. Journal of the American Academy of Child and Adolescent Psychiatry, 42(1): 57-65.

Kwon, H., 1999. Play the bear: myth and ritual in East Siberia. History of Religions, 38 (4): 373-387.

Langdon, S.J., 2002. The Native People of Alaska. Traditional Living in a Northern Land. 4th edition. Greatland Graphics, Anchorage.

Lavrillier, A., 2012. 'Spirit-charged' animals in Siberia. In: Brightman, M., V.E. Grotti, \& O. Ulturgasheva (eds.) Animism in Rainforest and Tundra: Personhood, Animals, Plants and Things in Contemporary Amazonia and Siberia, pp. 113-129. Berghahn, Oxford.

Loukacheva, N., 2009. Arctic Indigenous peoples' internationalism: in search of a legal justification. Polar Record, 45(232): 51-58.

Lykke Thompson, M., 2013. Statement by Denmark-Greenland at UN PFII-12 on recommendations of the Permanent Forum - Culture.

http://fnnewyork.um.dk/en/statements/newsdisplaypage/?newsid=957121480534-4dcf-884d-cc439da43848 (2 November 2014)

MacLennan, A., 2008. Sewing and identity: Inuit skin sewing and seal hunting. In: Sejersen, F., K. Thisted and T. Thuesen (eds). The Urban Arctic. Living Communities: New Perspectives on Inuit Urban Life, pp. 131-138. University of Copenhagen, Copenhagen.

Magraw, D., S. Lalonde, B. Tuzmukhamedov, and D. Caron, 2008. Polar politics: Change in the Arctic. Proceedings of the Annual Meeting (American Society of International Law), 102: 151-160.

Martinez Cobo, J. R., 1987. Study of the Problem of Discrimination against Indigenous Populations. Vol. 5: Conclusions, Proposals and Recommendations. United Nations, New York.

Megatrends, 2011. Rasmussen, R.O. (ed). Nordic Council of Ministers, Copenhagen. 
Ministry of Culture of the Russian Federation, 2014. Drevnyaya religiya yakutov offitsial'no priznano v Rossii. [Ancient religions of the Yakuts recognized in Russia]. http://to14.minjust.ru/node/2579 (October 2014).

Ministry of Justice of the Russian Federation 2011. Ekspertnoe zaklyuchenie v otnoshenii mestnoy religioznoy organizatsii g. Yakutska Aar Ajyy ite5ele (religiya Aar Ajyy). [Expert opinion regarding the local religious organization of the city of Yakutsk Aar Ajyy ite5ele (religion Aar Ajyy)]. Upravlenie Ministerstva Yustitsii Rossiyskoy Federatsii po Respublike Sakha (Yakutiya). http://to14.minjust.ru/node/2579 (2 November 2014).

Mitchell, D., 2001. Take My Land, Take My Life: The Story of Congress's Historic Settlement of Alaska Native Land Claims, 1960-1971. University of Alaska Press, Faitrbanks.

Morrow, P., 1996. Yupik Eskimo agents and American legal agencies: perspectives on compliance and resistance. Journal of Royal Anthropological Institute, 2(3): 405-23.

Nauerby, T., 1996. No Nation is an Island. Language, Culture and National Identity in the Faroe Islands. Aarhus University Press, Aarhus.

Nordin, Å., 2007. Renskötseln är mitt liv. Analys av den samiska renskötselns ekonomiska anpassning [Reindeer Herding is My Life. Analysis of the Economic Adjustment of Saami Reindeer Herding]. Umeå University, Umeå.

Olofsson, E., 2004. In Search of a Fulfilling identity in a Modern World: Narratives of Indigenous Identities in Sweden and Canada. Department of Cultural Anthropology and Ethnology, Uppsala University, Uppsala.

Oosten, J. and C. Rieme, 1999. Introduction. In: Oosten, J. and C. Rieme (eds.). Arctic Identities. Continuity and Change in Inuit and Saami Societies, pp. 1-27. University of Leiden, Leiden.

Patrick, D., 2005. Language rights in Indigenous communities: the case of the Inuit of Arctic Quebec. Journal of Sociolinguistics, 9(3): 369-389.

Pedersen, M., 2001. Totemism, animism and north Asian Indigenous ontologies. Journal of the Royal Anthropological Institute, 7(3): 411-27.

Robinson, S., 2012. 'Take it from the top': northern conceptions about identity in the western Arctic and beyond. Polar Record, 48(246): 222-229.

Rönnmark, M. and J. Björinge (eds.), 2014. Curiosity and Passion - The Art of CoCreation. A Bid from Umeå to Become the EU's Northernmost European Capital of Culture Ever. Umeå.

Russian Federation, 1997. Federal'nyy zakon No. 124-F3 “O svobode sovesti i o religioznykh ob"edineniyakh." [Federal law No. 124-F3 “On Freedom of Conscience and Religious Associations"]. Sobranie zakonodatel'stva RF, September 26, 1997, No. 39, p. 4465.

Sakha Republic (Yakutia), 1997. Zakon ob olenevodstva [Law on reindeer husbandry], No. 179-I, 25 June 1997

Sakha Republic (Yakutia), 2002. Ob oplate truda i material'nom stimulirvoanii rabotnikov, zanyatykh $v$ oleneodstve [On wages and material stimulations for workers employed in reindeer husbandry], Postanovlenie pravitel'stva Respubliki Sakha (Yakutia) ot 24.10.2002 [Decree of the government of the Sakha Republic (Yakutia) of 24 October 2002] N.536. http://russia.bestpravo.ru/yakut/data02/tex13490.htm

Schweitzer, P., E. Golovko, and N. Vakhtin, 2013. Mixed communities in the Russian North; or, why are there no "Creoles" in Siberia? Ethnohistory, 60(3): 419-438.

Scott, C., 2006. Spirit and practical knowledge in the person of the bear among Wemindji Cree hunters. Ethnos, 71(1): 51-66.

Searles, E., 2010. Placing identity: town, land, and authenticity in Nunavut, Canada. Acta Borealia: A Nordic Journal of Circumpolar Societies, 27(2): 151-166. 
Sköld, P., 2011. Development, adjustment and conflict: the Sami and reindeer husbandry in Sweden in the light of political, social and economic changes. In: Antonsson, H., and U. Jansson (eds.). Agriculture and Forestry in Sweden since 1900, pp. 475-491. Royal Swedish Academy of Agriculture and Forestry, Stockholm.

Sköld, P., 1992. Samisk bosättning i Gällivare 1550-1750 [Saami Settlement in Gällivare 1550-1750]. Umeå University, Umeå.

Shamanism, 2012. Shamanism approved as a religion in Norway. The Nordic Page, March 15, 2012. http://www.tnp.no/norway/panorama/2792-shamanismapproved-as-a-religion-in-norway. (2 November 2014).

Slezkine, Y., 1994. Arctic mirrors: Russian and the small peoples of the North. Cornell University Press, Ithaca.

Southcott, C., 2005. Globalization, culture, and Northern identities: some considerations for a northern dimension foreign policy. Polar Geography, 29(2): 103-118.

Stammler, F., and A. Ventsel, 2003. Between Neoliberalism and Dirigisme: Approaches to reindeer herding in Yamal and Sakha. In: C. M. Hann (Ed.), The Postsocialist Agrarian Question: Property relations and the Rural Condition, pp. 321-362. Lit, Münster.

Stephenson, S., 2012. Collaborative infrastructures: a roadmap for international cooperation in the Arctic. In: Heininen, L. (ed.). Arctic Yearbook, pp. 300-321. Northern Research Forum, Akureyri.

Stepian, A., T. Koivurova, A. Gremsperger and H. Niemi. 2014. Arctic Indigenous peoples and the challenge of climate change. In: Tedsen, E., S. Cavalieri and R. A. Kraemer (eds.). Arctic Marine Governance. Opportunities for Transatlantic Cooperation, pp. 71-99. Springer, Berlin.

Thisted, K., 2012. Scales of attention in fieldwork: global connections and local concerns in the Arctic. Ethnography, 14(2): 145-164.

Thompson, N., 2008. Settlers on the Edge: Identity and Modernization on Russia's Arctic Frontier. UBC Press, Vancouver.

Ulturgasheva, O., V. Grotti, M. Brightman, 2010. Personhood and 'frontier' in contemporary Amazonia and Siberia. Laboratorium. Russian Review of Social Research. Thematic issue: Russia/CIS/Latin America: Comparative Studies in PostAuthoritarian Transformation, 2(3): 348-365.

Ulturgasheva, 0., 2012. Narrating the Future in Siberia: Childhood, Adolescence and Autobiography among the Eveny. Berghahn Books, Oxford, New York.

Vakhtin, N., 1994. Native peoples of the Russian North. In: Minority Rights Group (ed.). Polar Peoples - Self-Determination and Development, pp. 29-80. London, England.

Vitebsky, P., 2005. Reindeer People: Living with Animals and Spirits in Siberia. Harper Perennial, London.

Vitebsky, P., 1995. From cosmology to environmentalism: shamanism as local knowledge in a global setting. In: Fardon, R. (ed.), Counterworks: Managing the Diversity of Knowledge, pp. 199-201. Routledge, New York.

Willerslev, R., 2007. Soul Hunters: Hunting, Animism, and Personhood among the Siberian Yukaghirs. University of California Press, Berkeley, California.

Williams, L., 2011. Canada, the Arctic, and post-colonial identity in the Circumpolar world. The Northern Review, 33(Spring): 113-131.

Wylie, J., 1987. The Faroe Islands: Interpretations of History. University Press of Kentucky, Lexington. 


\title{
4. Economic Systems
}

\author{
Lead Authors \\ Lee Huskey, University of Alaska Anchorage, USA, Ilmo Mäenpää, Univer- \\ sity of Oulu, Finland and Alexander Pelyasov, Council of Productive Forc- \\ es, Russian Federation
}

\subsection{Introduction}

The Arctic economy encompasses a range of activities from the smallscale traditional production for community use to large-scale production and distribution of the region's natural resources to the international market. This economy includes the flow of rents and transfers into and out of the North. Because these economic activities define the material well-being of the region's residents, economic activity is a fundamental component of northern human development.

The Arctic economic region is not an integrated region, but rather a region of different economies with similar characteristics. The Arctic economic region shares economic and environmental conditions that shape the economy of any part of the region. But because the area crosses the boundaries of countries, the overall regional economy and its effect on human development is influenced by the variety of different histories, institutions and resources that affect economic performance. 
Arctic Gross Product. Circumpolar Arctic, regions and countries, 2010 (Millions of $\$ U S-P P P$ and \%)

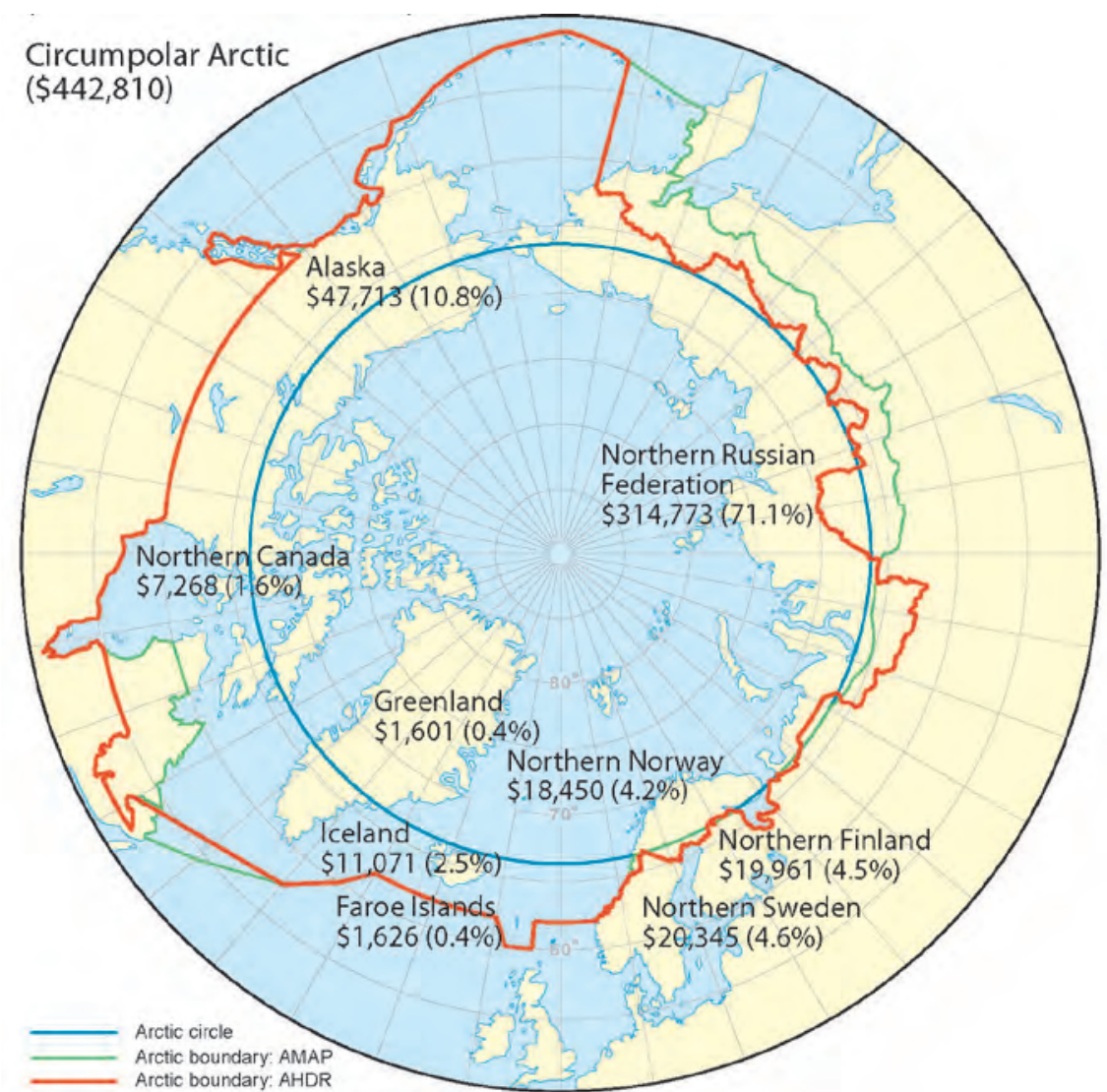

Source: Arctic map by W.K. Dallmann, Norwegian Polar Institute. Ilmo Mäenpää, Regional Economic Accounts of the Arctic, 2000-2010.

The Arctic economic region is not an integrated region, but rather a region of different economies with similar characteristics. The Arctic economic region shares economic and environmental conditions that shape the economy of any part of the region. But because the area crosses the boundaries of countries, the overall regional economy and its effect on human development is influenced by the variety of different histories, institutions and resources that affect economic performance. 


\subsection{Arctic regions}

In this chapter we define the Arctic as the northern regions of the eight Arctic nations, including:

- United States: Alaska

- Canada: Yukon, Northwest Territory, Nunavut

- Denmark: Greenland, Faroe Islands

- Iceland

- Norway: Finnmark, Troms, Nordland

- Sweden: Norrbotten, Västerbotten

- Finland: Lapland, Oulu, Kainuu

- Russia: Murmansk, Karelia, Arkhangelsk, Komi, Yamal-Nenets, KhantyMansi, Taimyr and Evenk, Sakha, Magadan, Koryak, Chukotka.

The Arctic economy as a whole responds to similar signals from world markets, but throughout the North the local response may differ from the response in other parts of the North. The different responses reflect differences in such things as the concentration of resources, the variation in the quality of deposits, and the level of transport infrastructure in northern regions (Glomsrød and Aslaksen, 2009).

The first Arctic Human Development Report described the northern economy by its structure of production and distribution of output (Duhaime, 2004). That Report presented two themes describing the Arctic economy. The Arctic economy was set apart from other economies by its structure combining large-scale natural resource production, small family-based production, and consumption supported by transfers from higher levels of government; and second by the variation in the level of economic activity across the region. The first AHDR also identified three important trends influencing the Arctic economy. First, the Circumpolar North continued its role as a natural resource reservoir for the world with successive waves of resources produced reflecting world markets. Second, the Report noted northern economic activity was increasingly privatized, as demonstrated by reduced federal subsidies and the increased importance of private companies and market decisions. Privatization affected economies throughout the Arctic but was especially important in the Russian North. Finally, the 2004 AHDR identified a general diversification or structural change in the North, as evidenced by a multi-level diversification with new activities (like tourism) and new actors (like local or regional associations) becoming part of the economy. 
This chapter describes the economy of the Circumpolar North and highlights significant changes since the last Arctic Human Development Report. It reviews the level of economic activity in the Arctic between 2000 and 2010. Over this decade, the Arctic has experienced dramatic expansion of its economy and increased expectations for its future (Graff, 2007). While there has been a change in the scale of economic activity in the Circumpolar North, the current economy of the Arctic region remains recognizable in structure and distribution to anyone familiar with the economy described in the earlier report. After examining the economy in terms of the themes and trends identified in the first $A H D R$, the report discusses the general causes of economic change in the Circumpolar North. A discussion of the variety of economic outcomes and the effects of the economy on Northerners is followed by a review of the performance of the components of the economy. The chapter concludes by discussing the region's likely future by identifying the trends that are likely to carry the economy into the future.

\subsection{General overview of the Arctic economy}

This section describes the aggregate Arctic economy. The Arctic economy is relatively large. In terms of output the economy ranks with countries of much greater population. Between 2000 and 2010 the Arctic region also experienced rapid economic growth, growing faster than the national economies of the eight Arctic nations. As an important part of the world resource economy, the pattern of change in the Arctic economy reflected changes in the world economy.

The Arctic economy, like all regional economies, serves two different markets. A wide range of resource products including diamonds, iron, gold, zinc, oil and natural gas, fish, and timber is produced for an international market (Glomsrød et al., 2009). A local market likewise exists to provide goods and services to the residents of local regions throughout the North. This local economy includes a significant public sector that provides income, jobs, and services to local residents. In some parts of the North, the local economy includes a traditional sector that provides for local consumption through fishing, hunting, herding, and gathering. The strength of the connections between these two parts of the local economy varies across the North.

The local and international economies often behave like two separate economies, occupying the same space, but with little in common. This difference exists, for example, in the structure of production. While the 
international economy supports modern large scale, capital-intensive production, local production often takes place in small groups, which mix modern and traditional methods of production. The two economies also have contrasting economic geographies. The international economy is concentrated while historic settlements in the local economy are small and scattered. These contrasts are less likely for those industries that have historical local roots in a region or those which may serve both local and international markets, like fishing and herding (HelanderRenvall, 2010; Crate, et al., 2010).

\section{Lukoil Offices, Salekhard, Russian Federation}

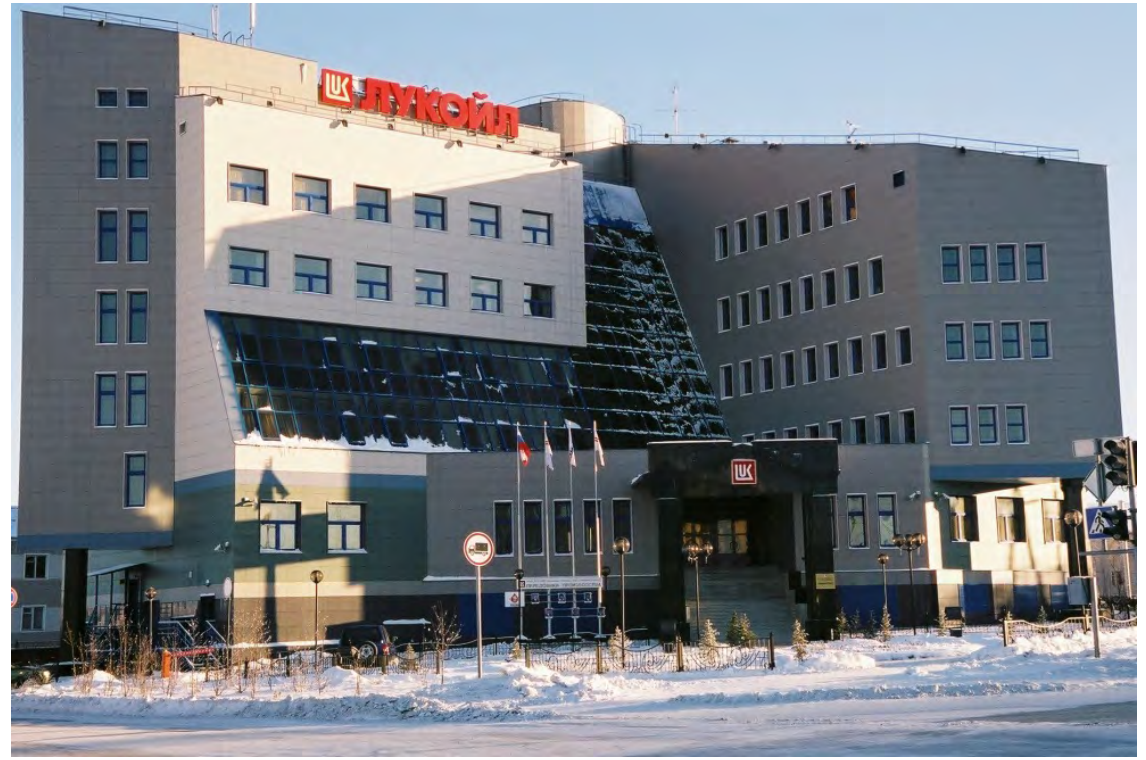

Credit: Harald Finkler.

This section uses Gross Regional Product (GRP) to describe the Arctic's economic production between 2000-2010. GRP is a monetary measure of the goods and services produced in a region in one year. GRP is the regional equivalent of national Gross Domestic Product, which is a widely accepted measure of production. Because GRP is a monetary measure of output, it describes real change in the amount produced as well as changes in the prices for the resources, goods, and services produced. 


\section{Textbox 4.1}

\section{The calculation of Arctic GRP}

The gross regional product, or GRP, of Arctic regions is defined throughout this section as the gross domestic product at market prices. The data were retrieved from the regional and national economic accounts web sites of the statistical offices of the Arctic nations. The original data were produced in local national currencies. Two nations, Norway and Russia, have published their GRP figures only at basic prices. The difference between market price and basic price valuation of GRP is the sum of accrued product taxes minus product subsidies. The basic price valued GRP figures of Norway and Russia has been converted into market values by the share of the product taxes minus subsidies in the GDP of the national accounts of mainland Norway (i.e., without off-shore activities) and Russian Federation. The difference of market valued GDP over basic price GDP was $17 \%$ in Norway and $16 \%$ in Russia in the year 2010.

In most countries, the GRP figures are provided by the statistical offices at both current and constant prices of a chosen reference year. The reference years differ by the country, however. The constant price time series has therefore been converted uniformly to the national price levels for the year 2010 . The GRP time series have, however, been published only at current prices in Norway and Faroe Islands. For Arctic regions of Norway the implicit price deflator of the GDP of the mainland Norway and for the Faroe Islands the implicit price deflator of the GDP of Greenland were used to convert the current price time series into constant price series.

Before adding up the GRP's of the Arctic regions of the different nations, the figures expressed at local currencies have to be converted into a uniform international monetary unit, United States dollar. For this conversion, the purchasing power parities (PPP) of each national currency were used. The purchasing power factors are based on a comprehensive work of the International Comparison Program of the main international economic organizations and retrieved here from the online databank of the OECD (OECD, 2012). One problem with using the PPP's is that they are national averages and using them to convert the local currencies of Arctic regions into uniform monetary unit implies that the price levels of Arctic regions correspond to their national averages.

Two price adjustments to the GRP data are made for each country. First, price changes over time are adjusted to reflect changes in real production. GRP is presented in 2010 prices by removing the effects of each country's general inflation from our estimate of output. Second, price differences across space are adjusted, because the Arctic region crosses 
the borders of eight countries and GRP in each part of the Arctic is calculated in home country currencies. US dollar purchasing power parity (PPP) is used to estimate GRP in each country in comparable units. Even with these adjustments, if the prices of resources, such as oil and gas, increase faster than the general price level, real GRP in those regions producing these resources will increase.

Arctic GRP provides a good estimate of the output in the money component of both the international and local sectors of the Arctic economies. However, it underestimates total production in some parts of the Arctic. When the national calculation of GRP ignores the non-market traditional or subsistence component of the economy, production is underestimated. The more important traditional activities are in a region of the Arctic, the greater the underestimation of total output. Goods that are not traded in a market are not counted as part of the GDP in some national accounts. This limit is more important in the North American Arctic than in northern Europe.

GRP has other limits as a measure of the material well-being for the region (Larsen and Huskey, 2010). For the North, GRP overestimates the material well-being because much of the income produced in the North leaves the region through rents, taxes, and wages paid to owners of resources in other regions. GRP underestimates well-being because it does not include transfers that do not create jobs; transfers that provide services or income directly are not part of the GRP. In parts of the North, services ranging from housing to health care to utilities are provided at subsidized rates underestimating their contribution to well-being.

The economy of the Arctic is larger than might be expected for a remote region of the world. Arctic GRP in 2010 was USD 442.8 billion (see Table 4.1). According to the World Bank (2013) the Arctic GRP was equivalent to the GDP of Malaysia and Columbia. The Arctic's contribution to world output was four times its share of population; the Arctic produced $0.6 \%$ of world GDP and had $0.15 \%$ of world population. On a per capita basis, Arctic GRP in 2010 was USD 45,360 per person. This was comparable to the United States and greater than most European countries. 
Table 4.1: Arctic GRP 2000-2010 (millions of 2010 USD and US PPP)

\begin{tabular}{llcrc}
\hline GRP Arctic Region & GRP Arctic Nation & $\begin{array}{c}\text { Arctic Region } \\
\text { Growth Rate }\end{array}$ & $\begin{array}{c}\text { Arctic Nation } \\
\text { Growth Rate }\end{array}$ \\
\hline 2000 & USD 311,036 & USD 16,345,663 & & \\
2001 & USD 329,729 & USD 16,626,132 & $6.0 \%$ & $1.7 \%$ \\
2002 & USD 340,444 & USD 16,979,638 & $3.2 \%$ & $2.1 \%$ \\
2003 & USD 357,991 & USD 17,435,960 & $5.2 \%$ & $2.7 \%$ \\
2004 & USD 378,967 & USD 18,095,244 & $5.9 \%$ & $3.8 \%$ \\
2005 & USD 399,748 & USD 18,683,039 & $5.5 \%$ & $3.2 \%$ \\
2006 & USD 421,678 & USD 19,326,038 & $5.5 \%$ & $3.4 \%$ \\
2007 & USD 435,637 & USD 19,862,329 & $3.3 \%$ & $2.8 \%$ \\
2008 & USD 446,983 & USD 19,923,946 & $2.6 \%$ & $0.3 \%$ \\
2009 & USD 428,364 & USD 19,055,081 & $-4.2 \%$ & $-4.4 \%$ \\
2010 & USD 442,810 & USD 19,685,256 & $3.4 \%$ & $3.3 \%$ \\
\hline
\end{tabular}

Source: Ilmo Mäenpää, 2012, Regional Economic Accounts of the Arctic 2000-2010, unpublished typescript in possession of the author.

The Arctic economy experienced relatively rapid growth during this decade. In real terms, Arctic GRP increased by $42.2 \%$ between 2000 and 2010. This was an annual average growth rate of $3.5 \%$. The growth rate of the Arctic economy was more than twice as fast as the growth of the eight Arctic nations. The Arctic nations' GRP grew $20.4 \%$ over this period, a yearly rate of $1.9 \%$. The performance of the Arctic economy reflected the world economic downturn in the later part of this period. As Table 4.1 shows, the Arctic Regional economy grew faster in the first part of the period than after 2006. Real Arctic GRP declined between 2008 and 2009.

\subsubsection{General causes of change in the Arctic economy}

Production for the international market in the Circumpolar North is determined by the world's demand for its resources and the cost of producing and moving the resources to market. In the past, governments may have promoted some northern resource development for strategic purposes, but as recognized in the first $A H D R$, the trend towards privatization means this is less likely (Hill and Gaddy, 2003). Modern resource developments must meet the market test: expected revenues must exceed expected costs. The long exploration and development time associated with mineral and petroleum development means that current economic activity in these industries is influenced by expectations of future costs and prices. Development and production for the international market in the Arctic economy will be driven by changes in expected prices and costs.

The cost side of this equation distinguishes the Arctic from other regions. The high costs affect the amount and type of resource production 
that takes place in the North (Budzik, 2009). These costs are partly a consequence of the region's harsh climate, along with sparse populations and remoteness of resource deposits from centers of consumption, production, and decision-making (Leven, 1986). The distance from markets and population centers increases the costs of shipping products to market and of bringing material, labor and other resources to production sites.

The level of development in the region also influences costs in the Arctic. The Arctic includes both frontier regions with limited development and mature development areas (Duhaime, 2004; Sugden, 1982). Greenland represents a frontier region for oil and gas development while Alaska's Prudhoe Bay is a mature region. In the frontier or undeveloped regions of the North, large scale or rich deposits are required to overcome the high costs of production (Huskey, 2010). Production in mature regions is less costly because the infrastructure, labor, and technology exist in place, while these must all be brought into frontier regions. Costs in the Arctic are path dependent and will be affected by the history of development in the area.

\section{Textbox 4.2}

\section{The Jack London hypothesis}

In his 1900 essay, "The economics of the Klondike", Jack London addressed the path dependent nature of northern development. When examining the balance sheet of the Klondike gold rush, London suggested that long run benefits needed to be considered. The often overlooked benefit of resource development is the change in the economics of the place that it brings. He described how this change in the economic structure of the place would change future development:

"The new Klondike, the Klondike of the future, will present remarkable contrasts with the Klondike of the past. Natural obstacles will be cleared away or surmounted, primitive methods abandoned, and hardship and toil and travel reduced to the smallest possible minimum. Explorations and transportation will be systematized. There will be no wasted energy, no harum-scarum carrying on of industry. The frontiersman will yield to the laborer, the prospector to the mining engineer, the dog-driver to the engine driver, the trader and speculator to the steady-going modern man of business; for these are the men in whose hands the destiny of the Klondike will be entrusted"

(London, J., 1900)

This dynamic is still at work lowering the cost of resource development in regions of existing and historic resource production. 
One of the themes of the first $A H D R$ was that the Arctic has long been considered a global storehouse for natural resource wealth that the environment makes difficult to access. For example, the USGS recently estimated that the Arctic holds almost a quarter of the world's undiscovered conventional oil and gas (Budzik, 2009). One reason people are optimistic about the future of Arctic resource production is climate change. Warming would allow longer ice-free travel in Arctic seas and lower the cost of access and development for northern resources. Improved access would lower the cost of delivering supplies and shipping the resources to markets. While the effects of warming on northern production are complex, lowering transport costs would increase the possibility of resource development in the Arctic.

\section{Agriculture in South Greenland. Qaqortoq Upernaviarsuk, October 2014}

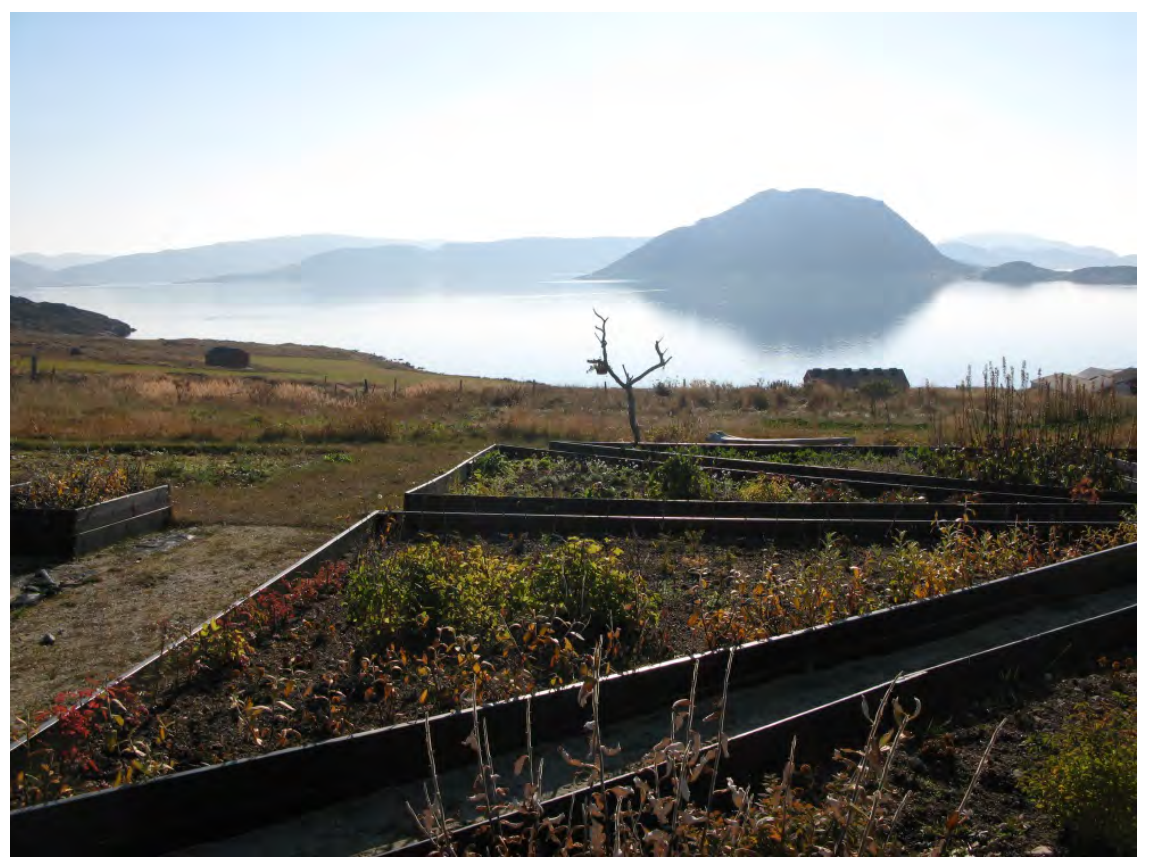

Credit: Jón Haukur Ingimundarson.

The potential for opening this vast northern storehouse of resources improved with the recent increases in commodity prices and the expectations that price increases will continue in the long run. The recent rise of commodity prices was driven primarily by the increasing demand for commodities resulting from the rapid expansion of the world's emerging economies, especially China (Kaufmann et al., 2008). These changes resulted in the expansion of resource activity in the resource producing 
regions of the world, including the Arctic (Sorenson, 2008; Doss, 2011). The expectation that the rise in commodity price is a long-term phenomenon is important because of the long time it takes to develop new resource projects.

\section{Textbox 4.3}

\section{Commodity prices over the decade}

The growth in demand from the emerging nations is one reason for the increase in prices of all commodities. China's appetite for natural resources has especially fired the imagination in Arctic nations. Prices of most commodities rose sharply between 2000 and 2011 and some argue that the likelihood of sharp reversals, especially in oil prices, is low (Kaufmann, et al., 2008).

The recent dramatic increase in commodity prices may not be a long run trend. The arguments supporting long-term price increases are based on the increasing resource demand resulting from the industrialization of the world's emerging economies. Recent analysis suggests that the current period is different from the past and that resource prices will diverge from past trends (O'Connor and Orsmond, 2009). However, the world has experienced dramatic expansion of economic output in the past. High and rapidly increasing resource prices have existed for periods in the past, but over the very long run most resource prices have fallen in real terms (Blackman and Baumol, 2008).

Historically, high prices for any resource provide the incentive for exploration to find more of the high price resource and innovation to find technology substitutes for the resource. The North has seen new technology replace existing northern production starting with petroleum replacing whales and continuing through the development of fish farming as a competing source of northern fish. For Arctic oil and gas, the development of techniques to produce oil and gas from unconventional sources provides a new substitute. These new techniques used in North America have resulted in what The Economist magazine called "An Unconventional Glut" describing the market for natural gas (2010). Technological innovations have also helped mineral producers produce more from existing deposits (Barta and Miller, 2013).

Together these effects suggest that high and rising prices of Arctic resources may not be a long-term phenomena. After almost tripling between 2000 and 2011, the International Monetary Fund's index of all commodity prices has recently declined about $12 \%$ from recent peaks (Barta and Miller, 2013). Arctic resources may face the long run pattern of resource prices declining in real terms. 
Throughout the Arctic, access to resources is also a public sector decision. The public sector may limit profitable resource production with taxes and regulation. Government may also prevent development by limiting access to the resources as in Alaska's Arctic National Wildlife Refuge. Environmental concerns and conflicts with other land uses may provide good reason for limiting access or removing some economic resources from potential production, but uncertainty in public decisions about access to resources will delay projects and increase the cost of development.

The trend of privatization, identified in the first $A H D R$, will change the discussions of access. For example, institutional changes have increased local self-government and ownership by local residents, thus offering the opportunity to control the development of local resources for an international market. This is especially the case in North America but also in Greenland and Norway (see Textbox 4.4) (Huskey, 2010).

The effect of production for the international market on the well-being of Northerners depends on the connections between Northern residents and resource production activities. These connections are often limited. The location of many northern settlements reflects historic or traditional economic activities and only by the accident of discovery will existing communities be located near modern resource discoveries. Scale and distance often result in enclave production with limited connections to local communities. The capital, technological, and labor needs of large scale resource production are often brought from outside the region, which means that much of the income generated by resource projects flows out of the region of production as payment to these resources.

Resource ownership and public resource revenue regimes also affect the flow of resource rents; control by non-local owners and governments reduces the economic contributions of resource production to the northern economy. Recent institutional changes in the North have increased the control and ownership of northern resources in some parts of the Arctic and have had an important effect on the relation between the international and local Arctic economies. Control by local governments or local organizations have increased the role of residents in making decisions about resource production and increased the share of resource rents and income that stays in the region. 


\section{Textbox 4.4}

\section{Institutional change in the Arctic}

In the last part of the 20th century there were significant changes in the institutional relationships between Arctic residents and resource producers. Changes were made in local control and ownership of natural resources, which will increase the economic effects of resource production in the North. Northern institutional changes have increased self-government and ownership by local residents (McBeath, 2010). Local, indigenous majority governments were established in Arctic Alaska, northern Canada, and Greenland and Finnmark in Norway, providing both opportunities and control for local residents. Ownership institutions have also been established through land claims settlements in Alaska and Canada. Ownership allows local residents both the ability to control and share the rewards of resource development. Finally, other types of ownershiplike arrangements have given residents more control. These include individual fishing quotas in Alaska, Iceland and the Faroe Islands. They also include more community ownership through mechanisms like Community Development Quota in the Alaska fisheries and the Alaska Eskimo Whaling Commission. These institutional changes give local residents more say in resource development, provide them with a certainty of rewards from development, and offer mechanisms for residents to be compensated for losses suffered from development. This may reduce conflict over future development of the Arctic's resources.

The importance of public sector to the local economies of the North means that the transfer policies of regional and national governments are important drivers for local economic change. The amount and type of transfers matter and changes in policies will have significant impact in local economies. Public transfers add to the economy by creating jobs and providing income and services for local residents. The size of this economic impact will both depend on and influence the size of the population of local areas.

In some parts of the remote Arctic, the economic well-being of residents continues to depend on traditional activities. This sector is also affected by production of resources for the international market. Production for the Arctic international market has two opposing effects on the traditional economy (Nutall, 2005). The negative impacts of development in the international economy of the North may damage ecosystems that support traditional economies as in the reindeer herding regions of the Russian North. However, income brought into the local economy because of activity in the international economy may also have positive impacts on the traditional economy. Cash may make the subsistence activities like hunting and fishing more productive. 


\subsection{The three pillars of the Arctic economy}

The first AHDR identified the well-known three part economic structure of northern economies (see also Knapp and Huskey, 1988). The Arctic economy was described as based on three separate sectors. The formal economy was primarily based on large-scale resource production. Traditional activities and small scale/family resource production provided a second support for the economy. Finally, transfers from higher levels of government supported much of the consumption through public sector jobs, direct payments to residents, and provision services. This economic organization continues to describe the Arctic and provides for its unique character.

\section{Textbox 4.5}

\section{Maturing resource industries and sustainable economies}

When Arctic development depends on production of a non-renewable resource such as petroleum or mineral production, sustainable development will be challenged as existing resource reserves are depleted. The decline of their economy's primary natural resource presents a challenge for both Northwest Territories (NWT) and Alaska. The diamond industry in Northwest Territories began with the opening of the first mine in 1998, and by the early 2000s, Canada had become the world's third largest diamond producer. The diamond mines are an important source of jobs for the NWT economy. A decline in diamond prices and the maturing of the region's original large mines led the Conference Board of Canada to forecast a near term decline in production as the early mines close up (Conference Board, 2013). In the short run, newer mines will fill the gap, but the Conference Board predicts the maturing resource will cause production to decline after 2020. The economy is likely to be rescued by the growth of other types of mining in the region.

The Alaska economy faces a similar challenge as petroleum production is in decline. From the beginning of production at Prudhoe Bay in 1977, Alaska's petroleum industry has significantly contributed to the Alaska economy. Oil has been the primary source of revenue for Alaska's general fund, accounting for about $90 \%$ of the state's revenue. Goldsmith (2011) has estimated that direct activity in the oil industry and the effect of the state's spending of oil revenue account for one-third of the jobs in the economy. But petroleum production peaked more than two decades ago and while new fields and new production technology have slowed the rate of decline, production continues to fall. Unlike NWT, Alaska's other natural resource industries are too small to fill the gap in the economy. Fortunately, though, Alaska has set up a number of savings funds of which Alaska's Permanent Fund is the largest. As Goldsmith (2011) has suggested these funds provide a source of revenue to mitigate the effect of the decline in petroleum production on the economy. 
Table 4.2 examines the distribution of Arctic production through the share of gross regional product found in the Arctic regions for industry groups in each of the eight Arctic nations for the year 2005. This distribution will not necessarily reflect the importance of each industry in the income of local residents. Some industries, such as the mining sector, use a significant amount of labor and capital from outside the region. For these industries, local income will be less than output.

The story told in Table 4.2 supports the theme of the first AHDR. The resource industries and public services accounted for more than half of Arctic's regional output in six of the Arctic nations. The share of GRP in the resources and public services is highest in Russia (72.3\%) and Alaska (62.8\%). The share of the resource industries and public services was lowest in Iceland, which still had more than one-third of its production in these industries. The resource sector includes both capturing and processing the resource. The public sector includes public administration, defense, utilities, and education, health, and social work.

Table 4.2 also suggests the differences may be as interesting as the similarities. The Russian Arctic presents one extreme, with over $60 \%$ of its output in resource production and only $9 \%$ in the public sector. Iceland may provide the other extreme, with over $55 \%$ of its output in 2005 coming from outside the natural resource and public services sectors (output in other service and the remainder). This distribution of output describes two general types of Arctic economies. Iceland, Finland, Norway, and Sweden may represent a more mature North with less than $20 \%$ of their output in natural resource production and more than $40 \%$ of their economy outside of resources and public sector. The Russian, North American, and Danish Arctic represent the frontier North, where resource production and the public sector remain more important to the economy.

Table 4.2: Distribution of GRP for Arctic regions, 2005 (percent)

\begin{tabular}{lrrrrrrrr}
\hline & United States & Canada & Finland & Iceland & Norway & Russia & Sweden & Denmark \\
\hline Fishing & 0.6 & 0 & 0.1 & 4.7 & 5 & 0.6 & 0 & 10.5 \\
Mining and petroleum & 33.2 & 27.7 & 0.8 & 0.1 & 1 & 56.9 & 7.5 & 3.2 \\
Other resource & 0.1 & 0.4 & 4.7 & 1.4 & 0.9 & 1.3 & 3 & 2.4 \\
Resource Processing & 2 & 0 & 13.3 & 4.8 & 5.2 & 4.4 & 7.2 & 5.8 \\
Construction & 5.4 & 8 & 6.8 & 9.6 & 6.9 & 5 & 5.4 & 7.2 \\
Public sector & 26.9 & 28.7 & 24.8 & 23.8 & 40.5 & 9.1 & 32.6 & 29.9 \\
Other services & 31.3 & 34.8 & 37.7 & 50.1 & 37.4 & 22.1 & 36.6 & 38.9 \\
Remainder & 0.5 & 0.4 & 11.8 & 5.5 & 3.1 & 0.6 & 7.7 & 2.1 \\
\hline
\end{tabular}

Source: Based on tables in Glomsrød, et al, 2009. 
Production outside of the resource and public sectors is significant throughout most of the Arctic. With the exception of the Russian Arctic, production outside of these industries accounted for more than $37 \%$ of output across all regions. These industries produce approximately half or more of the output in the Finnish, Norwegian, Swedish, and Danish Arctic and Iceland. Production in these industries includes production of goods and services for the region's resident population as well as export industries not connected to resource production.

\section{Small-scale Entrepreneurship: Pilu Arctic products from Greenland - tea and cosmetics}

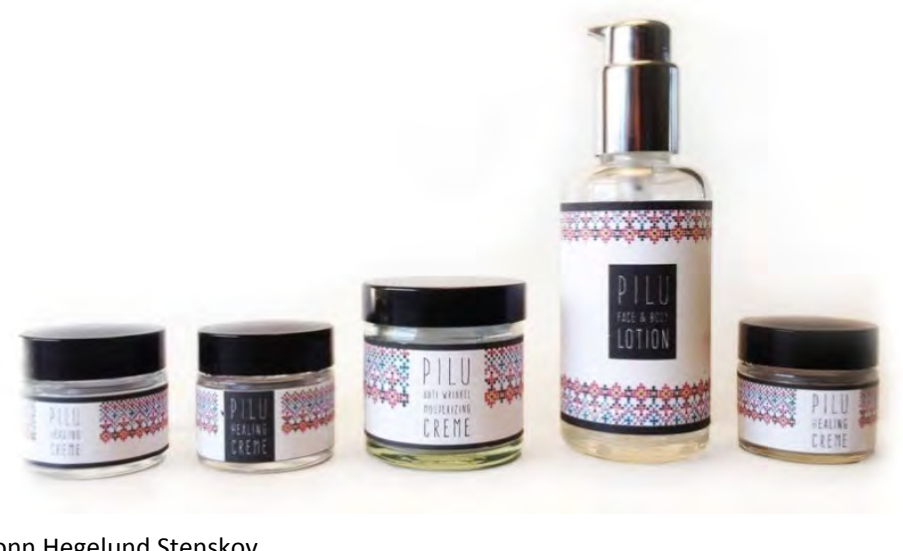

Credit: Ivonn Hegelund Stenskov.

The Arctic economy has been maturing while it grows. The Circumpolar North is comprised not just of small villages and resource enclaves, but also of towns and cities of significant size (Weber and Rasmussen, 2014). These urban economies provide the trade, personal, government, and business services and the manufacturing of products that have historically been supplied from outside the North. The growth of these agglomerations results in the growth of businesses that replace imports.

The rise of northern industries with no direct connection to extractive resources may be partly a result of the existence of these urban areas. Some of these new industries are connected to the region's resource base. The Arctic's natural beauty supports a growing tourism industry, and the aluminum smelting industry in Iceland is drawn by the low cost energy that country offers. Alaska's cargo operations grew because of the state's location and developed infrastructure. The electronics manufacturing industry in northern Finland is another important nontraditional industry. The growth of Iceland's financial industry in the 
first part of the decade provides another example of a non-traditional industry developed in the North.

\section{Svalbard dogsledding tourism, Norway}

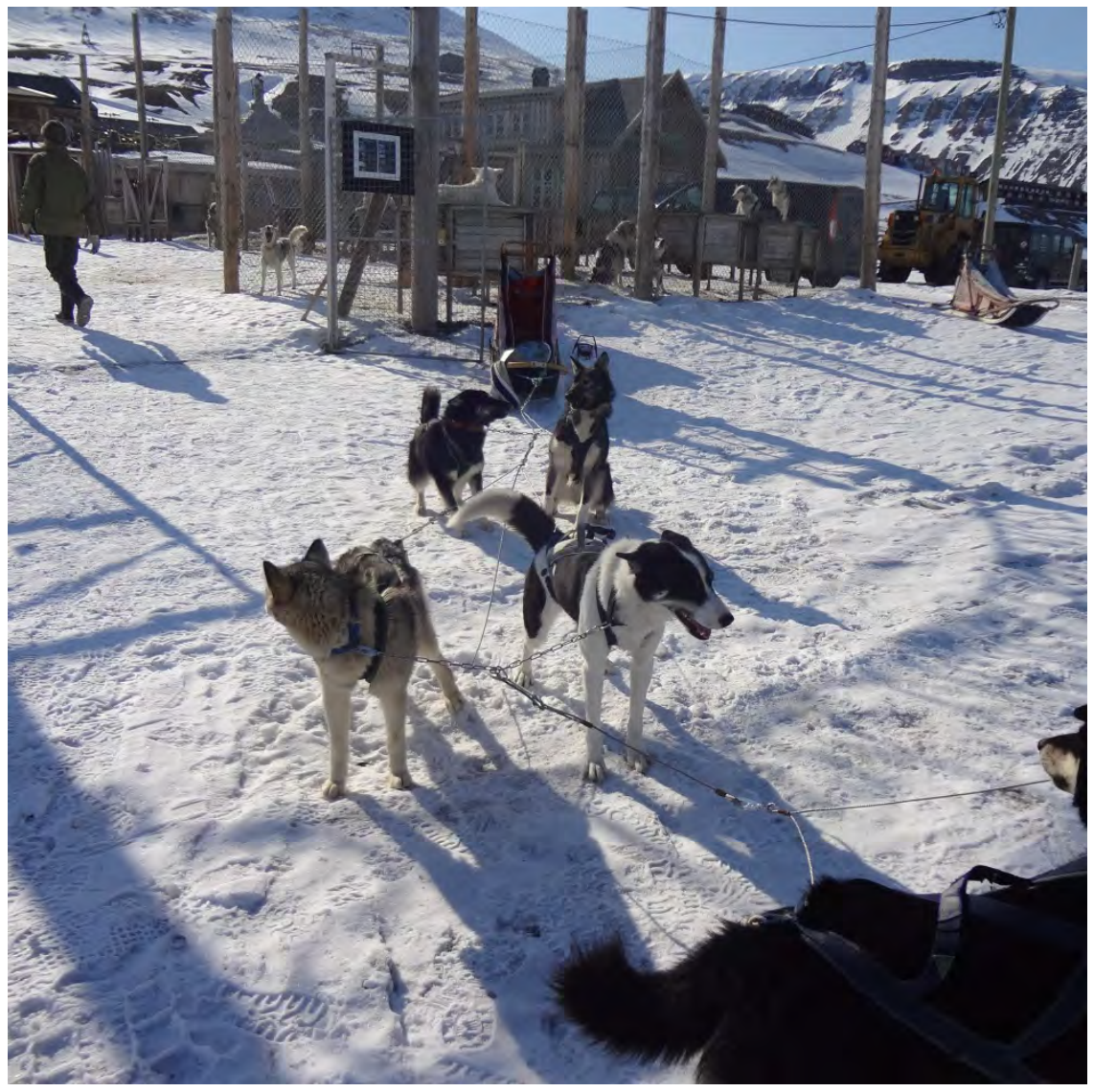

Credit: Joan Nymand Larsen.

Data on the third pillar of the northern economy, traditional harvesting or subsistence, is not universally collected but this sector remains important throughout the North. Hunting, herding, fishing, and gathering, like money income, support the material well-being or real income of the region's Indigenous population. These activities are also important for maintaining social relationships and cultural identity. Residents of remote regions often balance traditional and wage work to create real income. Surveys of traditional activity throughout the North find significant consumption of traditionally harvest foods, over 600 pounds per person in rural Alaska and the Canadian North. Subsistence provides at least half of the meat consumed by more than $40 \%$ of the Inuit population throughout the Arctic. Reindeer herding and meat production re- 
main important components of the economy in the Nenets Autonomous okrug (Aslaksen, et al., 2009).

\section{Textbox 4.6}

\section{Cultural economy of the Arctic}

Andrey Petrov

Elements of traditional knowledge, such as arts and crafts, are not only important components of Native culture, but are also commodities that can bring economic profit (this economic sector is known as 'cultural economy'). Artisans and handcrafters can provide incomes important to community well-being. The commercial production of arts and crafts from bone, ivory, soapstone, and hides has been important since the 1950s. According to the Survey of Living Conditions in the Arctic, $18 \%$ of Aboriginal residents of the Canadian Arctic manufactured crafts for sale (SLiCA, 2008, Table 1, p. 48). Almost one-third of all Aboriginal people reported receiving some income from selling pieces of traditional art. Involvement in commercial handcrafting and artisanship was the highest in Nunavut, especially in some communities, like Cape Dorset. In another survey, $30 \%$ of Inuit living in Nunavut reported deriving a part-time income from their sculpture, carving and print making. Interestingly, the region encompassing Baffin Island (including Iqaluit and Cape Dorset) has been the most creative rural area in Canada (Hill Strategies, 2010).

Commercial arts and crafts are a substantial and growing sector of northern economy. It is estimated that these activities contribute USD 30 million in earnings (Nordicity, 2010). Most of the purchases are made by tourists and collectors of Inuit art. Whereas they bring some sales earnings to Aboriginal communities, the challenge for local artists in the North is low incomes. Crafters and artists receive much smaller wages than their counterparts in other regions and than other workers in their own areas. The average earnings of an artist in Nunavut is just above USD 20,000, compared with an average wage of USD 38,000 for all workers in the Territory (Hill Strategies, 2009). In fact, this is below the official poverty line. As a result, arts and crafts are predominantly part-time activities for women: $40-80 \%$ of artisan products in Nunavut are done by Inuit women, many of whom are over 60 years old. Engaging younger people and men is an important strategy for furthering cultural economy in the North. Improving access to markets, business infrastructure and stimulating the demand for native arts are necessary to expand Nunavut's cultural economy (Nordicity, 2014) and increase the attractiveness of artisan occupations. 


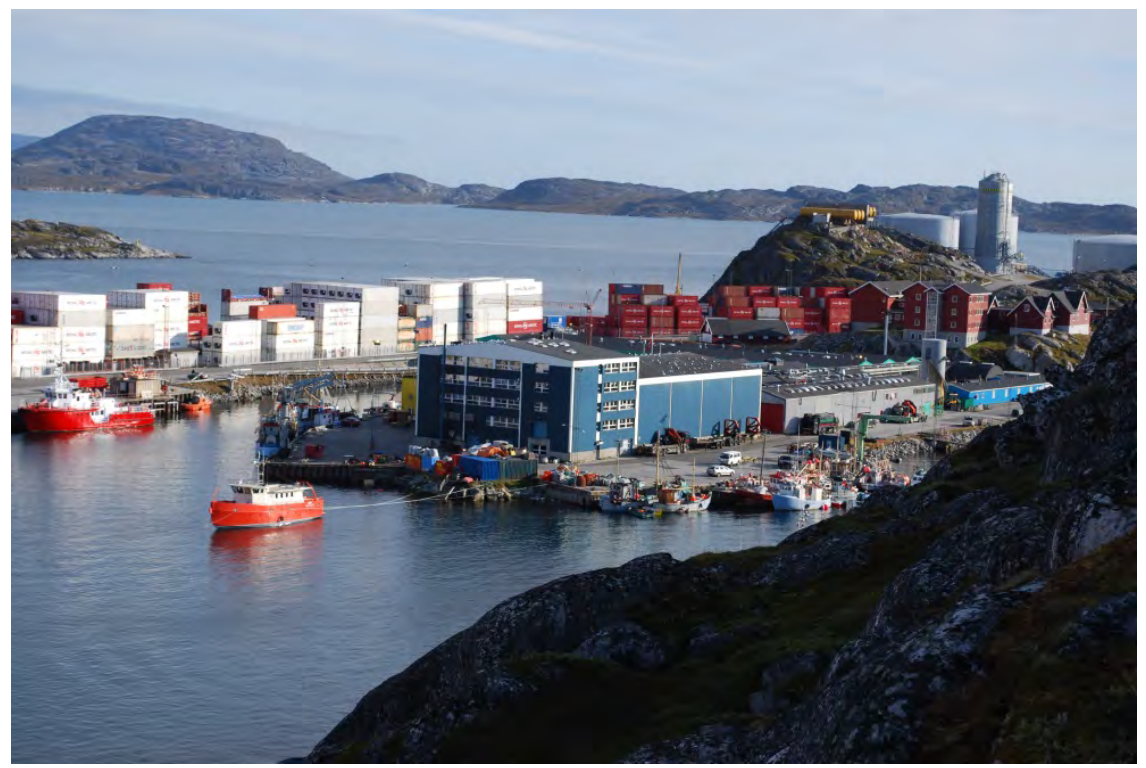

Credit: Joan Nymand Larsen.

\subsection{Variation in production around the North}

The significant variation in the size of the economy from one northern region to another identified in the first $A H D R$ continues to exist. Tables 4.3 and 4.4 describe the distribution of GRP by nation and by the Arctic subregions in each nation. Arctic output is not evenly distributed throughout the North, and varies significantly between and within nations.

Table 4.3: Arctic GRP by Arctic Nation, millions 2010 US dollar PPP

\begin{tabular}{lrrrrr}
\hline Year & $\begin{array}{r}\text { GRP Arctic } \\
\text { Region }\end{array}$ & GDP Arctic Nation & $\begin{array}{r}\text { Share Arctic } \\
\text { Region GRP }\end{array}$ & $\begin{array}{r}\text { Arctic } \\
\text { Share of } \\
\text { Nation GDP }\end{array}$ & $\begin{array}{r}\text { Arctic Share } \\
\text { of Nation } \\
\text { Population }\end{array}$ \\
\hline United States & USD 47,713.00 & USD 14,416,601.00 & $10.8 \%$ & $0.3 \%$ & $0.2 \%$ \\
Canada & USD 7,268.95 & USD 1,362,733.26 & $1.6 \%$ & $0.5 \%$ & $0.3 \%$ \\
Denmark & USD 3,226.51 & USD 221,243.88 & $0.7 \%$ & $1.5 \%$ & $1.8 \%$ \\
Iceland & USD 11,071.28 & USD 11,071.28 & $2.5 \%$ & $100.0 \%$ & $100 \%$ \\
Norway & USD 18,450.81 & USD 282,289.27 & $4.2 \%$ & $6.5 \%$ & $9.6 \%$ \\
Sweden & USD 20,345.05 & USD 369,320.86 & $4.6 \%$ & $5.5 \%$ & $5.4 \%$ \\
Finland & USD 19,961.05 & USD 194,994.45 & $4.5 \%$ & $10.2 \%$ & $12.3 \%$ \\
Russia & USD 314,773.41 & USD 2,827,002.00 & $71.1 \%$ & $11.1 \%$ & $4.8 \%$ \\
Arctic regions, & USD 442,810.05 & USD 19,685,256.00 & $100.0 \%$ & $2.2 \%$ & $1.9 \%$ \\
total & & & & & \\
\hline
\end{tabular}

Source: Ilmo Mäenpää, 2012, Regional Economic Accounts of the Arctic 2000-2010, unpublished typescript in possession of the author. 


\begin{tabular}{|c|c|c|c|c|}
\hline & & GRP by Locality & $\begin{array}{r}\text { Share of } \\
\text { National } \\
\text { Arctic GRP }\end{array}$ & $\begin{array}{r}\text { Share of } \\
\text { World Arctic } \\
\text { GRP }\end{array}$ \\
\hline United States & Alaska & USD 47,713 & $100.0 \%$ & $10.8 \%$ \\
\hline \multirow[t]{3}{*}{ Canada } & Yukon & USD 1,951 & $26.8 \%$ & $0.4 \%$ \\
\hline & Northwest Territories & USD 3,792 & $52.2 \%$ & $0.9 \%$ \\
\hline & Nunavut & USD 1,525 & $21.0 \%$ & $0.3 \%$ \\
\hline \multirow[t]{2}{*}{ Denmark } & Greenland & USD 1,601 & $49.6 \%$ & $0.4 \%$ \\
\hline & Faroe Islands & USD 1,626 & $50.4 \%$ & $0.4 \%$ \\
\hline Iceland & Iceland & USD 11,071 & $100.0 \%$ & $2.5 \%$ \\
\hline \multirow[t]{3}{*}{ Norway } & Finnmark & USD 2,876 & $15.6 \%$ & $0.6 \%$ \\
\hline & Troms & USD 6,154 & $33.4 \%$ & $1.4 \%$ \\
\hline & Nordland & USD 9,421 & $51.1 \%$ & $2.1 \%$ \\
\hline \multirow[t]{2}{*}{ Sweden } & Norrbotten & USD 11,226 & $55.2 \%$ & $2.5 \%$ \\
\hline & Västerbotten & USD 9,119 & $44.8 \%$ & $2.1 \%$ \\
\hline \multirow[t]{3}{*}{ Finland } & Lapland & USD 5,613 & $28.1 \%$ & $1.3 \%$ \\
\hline & Oulu & USD 12,239 & $61.3 \%$ & $2.8 \%$ \\
\hline & Kainuu & USD 2,109 & $10.6 \%$ & $0.5 \%$ \\
\hline \multirow[t]{11}{*}{ Russia } & Murmansk & USD 17,065 & $5.4 \%$ & $3.9 \%$ \\
\hline & Karelia & USD 9,290 & $3.0 \%$ & $2.1 \%$ \\
\hline & Arkhangelsk & USD 25,883 & $8.2 \%$ & $5.8 \%$ \\
\hline & Komi & USD 25,624 & $8.1 \%$ & $5.8 \%$ \\
\hline & Yamal-Nenets & USD 56,129 & $17.8 \%$ & $12.7 \%$ \\
\hline & Khanty-Mansi & USD 143,726 & $45.7 \%$ & $32.5 \%$ \\
\hline & Taimyr \& Evenk & USD 1,055 & $0.3 \%$ & $0.2 \%$ \\
\hline & Sakha & USD 27,980 & $8.9 \%$ & $6.3 \%$ \\
\hline & Magadan & USD 4,231 & $1.3 \%$ & $1.0 \%$ \\
\hline & Koryak & USD 737 & $0.2 \%$ & $0.2 \%$ \\
\hline & Chukotka & USD 3,053 & $1.0 \%$ & $0.7 \%$ \\
\hline \multicolumn{2}{|c|}{ Arctic regions, total } & USD 442,810 & & $100.0 \%$ \\
\hline
\end{tabular}

Source: Ilmo Mäenpää, 2012, Regional Economic Accounts of the Arctic 2000-2010, unpublished typescript in possession of the author.

As shown in Table 4.3, the Russian Arctic dominates Arctic production; it produces over 70\% of the Arctic's GRP. Russia's share of Arctic production is almost seven times the share of Alaska, the second largest economic region in the Arctic. Both regions are oil and gas areas and the high value of this resource during this period helps explain this dominance. The Arctic region contributes significantly (more than 5\%) to the national GDP in five of the Arctic nations. As a region, the Arctic accounts for a slightly greater share of national GDP than population. However, only the Russian Arctic contributes significantly more to the national economy than its population share.

Economic production is also concentrated within the Arctic regions of each Arctic nation. Table 4.4 shows the GRP in each of the sub-regions of each nation's Arctic. In all Arctic nations output is unevenly distributed between sub-regions (this cannot be observed for the US, Greenland, or Iceland). In Norway and Sweden, the difference in distribution likely reflects the location of major towns within particular sub-regions. In Finland, $60 \%$ of its Arctic GDP is in the Oulu region that includes the city 
of Oulu and its electronics industry. For Canada and Russia, the difference reflects the concentration of natural resources; diamonds in Canada and oil and gas in Russia. In Russia, over $45 \%$ of its Arctic output is concentrated in the Khanty-Mansi region of the country. Over $60 \%$ of Russia's Arctic GDP is produced in two regions - Khanty-Mansi and Yamal-Nenets.

There are two reasons to expect output to be concentrated in the North. First, the significance of natural resource production to the Arctic economy implies the spatial economy will reflect the geography of the region's natural resources. The Arctic is large and serves as a storehouse of natural resources but these resources are concentrated not spread evenly throughout the North. For example, a few sedimentary provinces in the Arctic account for most of the region's petroleum resources; $65 \%$ of the estimated reserves in the Arctic are in three provinces (Budzik, 2009). This results in the concentration of production in those places of rich or historic resource deposits. Second, the existence of an urban north also creates concentrations of economic production. Northern cities often serve as public administration, trade, transport, and health centers for large regions.

\subsection{The economy and the well-being of northern residents}

This section examines the well-being of northern residents in relation to the North's economic output. The benefits of economic production for residents of the Arctic depend on the residents' participation in the economy as owners of capital, resources, or labor, and the net flow of government revenues flowing to the region. In the North, natural resource production often separates local income from production. The scale and technology of Arctic resource production often makes resource industries importers of capital and labor. Income produced in the region will flow out of the region to pay for these imported factors of production.

The growth of employment faster than population would be consistent with Arctic residents increasing their share of available northern jobs. Table 4.5 compares employment and population growth in the Arctic regions of the eight Arctic nations between 2000 and 2010. Population declined during this period for the entire Arctic while employment increased. However, change in Russia drives this pattern since it dominates both Arctic population (70\% in 2010) and employment (72\% in 2010). 
Table 4.5: Employment Growth in the Arctic, 2000-2010

\begin{tabular}{lrrrrrr} 
& \multicolumn{3}{c}{ Employment (000s) } & \multicolumn{3}{c}{ Population (000s) } \\
& $\mathbf{2 0 0 0}$ & $\mathbf{2 0 1 0}$ & \% increase & $\mathbf{2 0 0 0}$ & $\mathbf{2 0 1 0}$ & \% increase \\
\hline United States & 392.37 & 446.77 & $13.9 \%$ & 627.96 & 714.15 & $13.7 \%$ \\
Canada & 47.24 & 57.29 & $21.3 \%$ & 98.41 & 111.50 & $13.3 \%$ \\
Denmark & 54.54 & 51.54 & $-5.5 \%$ & 101.95 & 105.08 & $3.1 \%$ \\
Iceland & 156.50 & 167.20 & $6.8 \%$ & 281.21 & 318.04 & $13.1 \%$ \\
Norway & 216.00 & 232.90 & $7.8 \%$ & 464.24 & 466.94 & $0.6 \%$ \\
Sweden & 224.50 & 233.10 & $3.8 \%$ & 513.00 & 508.00 & $-1.0 \%$ \\
Finland & 255.27 & 276.25 & $8.2 \%$ & 650.63 & 659.51 & $1.4 \%$ \\
Russia & 3577.20 & 3768.51 & $5.3 \%$ & 7263.10 & 6878.44 & $-5.3 \%$ \\
Arctic regions, total & 4923.63 & 5233.55 & $6.3 \%$ & 10000.50 & 9761.65 & $-2.4 \%$ \\
\hline
\end{tabular}

Source: Ilmo Mäenpää, 2012, Regional Economic Accounts of the Arctic 2000-2010, unpublished typescript in possession of the author.

For the non-Russian Arctic, both employment and population grew, with employment growing faster. Population and employment grew fastest in Alaska and the Canadian Arctic. Population grew slowest in the mature Arctic of Fennoscandia even though these regions had relatively healthy employment growth. Throughout the Arctic, population increased faster than employment in only Greenland/Faroe Islands and Iceland. The increased employment does not guarantee that residents increased their employment participation in the economy but it is consistent with that result.

The dependency rates shown in Table 4.6 provide another way of looking at the relative growth of employment and population. The dependency rate shows the population supported by each job in a region. The rate will be influenced by the age distribution of the population; a decline in the young and the old population will decrease the dependency rate. The dependency rate will also be lower if more of the resident population of working age is employed. Table 4.6 shows the dependency rate fell over our study period in five of the eight Arctic nations. It increased in only Iceland and the Danish Arctic. A declining dependency rate is consistent with increased employment for local residents. 


\begin{tabular}{lccc}
\hline & (Population /Employment) & & \\
& & & \\
& $\mathbf{2 0 0 0}$ & $\mathbf{2 0 0 5}$ & $\mathbf{2 0 1 0}$ \\
\hline United States & 1.60 & 1.57 & 1.60 \\
Canada & 2.08 & 2.07 & 1.95 \\
Denmark & 1.87 & 1.82 & 2.04 \\
Iceland & 1.80 & 1.84 & 1.90 \\
Norway & 2.15 & 2.10 & 2.00 \\
Sweden & 2.29 & 2.27 & 2.18 \\
Finland & 2.55 & 2.46 & 2.30 \\
Russia & 2.03 & 1.88 & 1.83 \\
The Arctic & 2.03 & 1.91 & 1.87 \\
\hline
\end{tabular}

Source: Ilmo Mäenpää, 2012, Regional Economic Accounts of the Arctic 2000-2010, unpublished typescript in possession of the author.

\section{Textbox 4.7}

\section{Long distance commuters in the Russian Arctic}

The long distance commuting worker is a significant component of the workforce used to develop the Northern oil and gas resources in Russia. This represents a change in northern development policy from Soviet times that encouraged settlement and the development of towns and cities in the North. The "Vakhtovy method" includes both commuters from within and outside of the northern region. Commuters live in work camps while at the work place. These workers experience shifts of intense work time away from home and time away from the workplace. Shift length depends on such things as workers' profession, travel time, and requirements of the operations. Common shifts are thirty days at work and 30 days off. This approach reduces the cost of resource production, since new towns and their infrastructure and services don't need to be created. It also impacts the sending regions through the incomes earned in the North by residents of these regions (Eilmsteiner-Saxinger, 2011).

Table 4.7 compares the most direct measure of resident well-being, per capita household disposable income, with per capita GRP for 2010. Household disposable income includes all income earned by households including net transfers from governments. Per capita household disposable income is a good measure of the household's material wellbeing (Larsen and Huskey, 2010). Table 4.7 shows the variation in per capita incomes across the Arctic and within each Arctic nation. The highest is Alaska's USD 40,600 which is more than three times the income in the poorest regions, Karelia in Arctic Russia. Three of the five regions with the highest per capita incomes are in North America while two are in Russia. Russia also illustrates the variation in per capita 
income. Two of the five highest per capita income regions are in Russia as well as two of the poorest.

Table 4.7: Arctic Per Capita Household Disposable Income and GRP, 2010

\begin{tabular}{|c|c|c|c|}
\hline & $\begin{array}{r}\text { Per Capita Disposable } \\
\text { Income (000s) }\end{array}$ & $\begin{array}{r}\text { Per Capita GRP } \\
(000 \mathrm{~s})\end{array}$ & $\begin{array}{l}\text { PC Income as a } \\
\text { share of PC GRP }\end{array}$ \\
\hline \multicolumn{4}{|l|}{ United States } \\
\hline Alaska & 40.6 & 66.8 & $60.8 \%$ \\
\hline \multicolumn{4}{|l|}{ Canada } \\
\hline Yukon & 32.3 & 56.5 & $57.1 \%$ \\
\hline Northwest Territories & 34.1 & 86.7 & $39.3 \%$ \\
\hline Nunavut & 24.6 & 45.9 & $53.7 \%$ \\
\hline \multicolumn{4}{|l|}{ Denmark } \\
\hline Greenland & 16.3 & 28.3 & $57.6 \%$ \\
\hline Faroe Islands & 16.2 & 33.5 & $48.4 \%$ \\
\hline Iceland & 15.0 & 34.8 & $43.1 \%$ \\
\hline \multicolumn{4}{|l|}{ Norway } \\
\hline Finnmark & 22.0 & 39.3 & $55.9 \%$ \\
\hline Troms & 21.9 & 39.2 & $55.8 \%$ \\
\hline Nordland & 21.5 & 39.8 & $54.0 \%$ \\
\hline \multicolumn{4}{|l|}{ Sweden } \\
\hline Norrbotten & 18.3 & 45.1 & $40.7 \%$ \\
\hline Västerbotten & 17.4 & 35.2 & $49.5 \%$ \\
\hline \multicolumn{4}{|l|}{ Finland } \\
\hline Lapland & 17.1 & 30.6 & $56.0 \%$ \\
\hline Oulu & 16.7 & 31.1 & $53.8 \%$ \\
\hline Kainuu & 17.0 & 25.6 & $66.4 \%$ \\
\hline \multicolumn{4}{|l|}{ Russia } \\
\hline Murmansk & 19.0 & 21.4 & $88.5 \%$ \\
\hline Karelia & 12.4 & 14.4 & $86.0 \%$ \\
\hline Arkhangelsk & 15.1 & 21.0 & $71.7 \%$ \\
\hline Komi & 18.1 & 28.3 & $64.1 \%$ \\
\hline Yamal-Nenets & 32.7 & 36.6 & $89.2 \%$ \\
\hline Khanty-Mansi & 24.4 & 274.9 & $8.9 \%$ \\
\hline Taimyr \& Evenk & 20.6 & 19.1 & $108.0 \%$ \\
\hline Sakha & 18.0 & 29.2 & $61.6 \%$ \\
\hline Magadan & 21.5 & 26.8 & $80.0 \%$ \\
\hline Koryak & 23.8 & 33.3 & $71.4 \%$ \\
\hline Chukotka & 29.2 & 60.1 & $48.6 \%$ \\
\hline Arctic & 21.9 & 45.4 & $48.3 \%$ \\
\hline
\end{tabular}

Source: IImo Mäenpää, 2012, Regional Economic Accounts of the Arctic 2000-2010, unpublished typescript in possession of the author.

Table 4.7 also compares each region's disposable income to it GRP. This ratio would be one (1) for a region that was entirely self-supporting, keeping all the resource rents and production costs and not relying on transfers from other governments. Ratios lower than one represent the flow of rents and spending to outside resource owners and governments. Of the five highest income regions, only Yamal-Nenets has a ratio of more than $60 \%$. Regions with higher ratios of income to output are the poorer regions, which may represent the importance of transfers to these economies. These ratios represent the connections between the regions of the arctic and other parts of the world. 
Table 4.8 compares the per capita disposable household income of each Arctic region with per capita income for its nation in 2010. The Arctic regions in FennoScandia have per capita incomes that are similar to national incomes. Per capita incomes in the Arctic regions are generally higher than the national per capita income in the US, Canada, and Russia. Although these results would seemingly lead to the conclusion that residents of the North experience similar levels of well-being to their counterparts in the rest of their respective countries, these results fail to account for the differences in costs of living in Northern communities. Since the North is a high cost region, these results likely overestimate the relative well-being of residents of the region.

\section{AIR NUNAVUT, Canada}

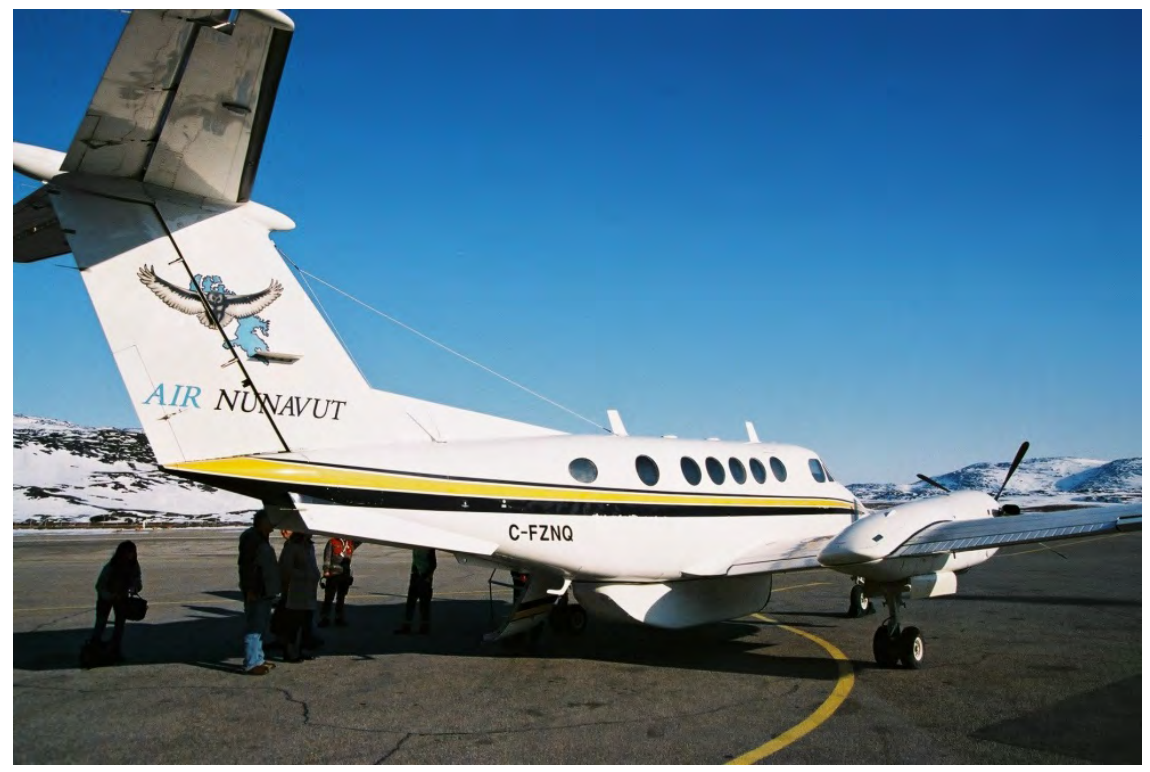

Credit: Harald Finkler. 
Table 4.8: Per Capita Disposable Household Income (PPP) in 2010 (100 US dollars)

\begin{tabular}{|c|c|c|c|}
\hline \multicolumn{4}{|c|}{ Per Capita Disposable Household Income (PPP) in 2010 (100 US dollars) } \\
\hline United States & USD 36.1 & Alaska & USD 40.6 \\
\hline \multirow[t]{3}{*}{ Canada } & USD 22.8 & Yukon Territories & USD 32.3 \\
\hline & & Northwest Territories & USD 34.1 \\
\hline & & Nunavut & USD 24.6 \\
\hline \multirow[t]{2}{*}{ Denmark } & USD 16.3 & Greenland & USD 16.3 \\
\hline & & Faroe Islands & USD 16.2 \\
\hline Iceland & USD 15.0 & Iceland & USD 15.0 \\
\hline \multirow[t]{3}{*}{ Norway } & USD 22.8 & Finnmark & USD 22.0 \\
\hline & & Troms & USD 21.9 \\
\hline & & Nordland & USD 21.5 \\
\hline \multirow[t]{2}{*}{ Sweden } & USD 18.8 & Norrbotten & USD 18.3 \\
\hline & & Västerbotten & USD 17.4 \\
\hline \multirow[t]{3}{*}{ Finland } & USD 18.7 & Lapland & USD 17.1 \\
\hline & & Oulu & USD 16.7 \\
\hline & & Kainuu & USD 17.0 \\
\hline \multirow[t]{11}{*}{ Russia } & USD 14.5 & Murmansk & USD 19.0 \\
\hline & & Karelia & USD 12.4 \\
\hline & & Arkhangelsk & USD 15.1 \\
\hline & & Komi & USD 18.1 \\
\hline & & Yamal-Nenets & USD 32.7 \\
\hline & & Khanty-Mansi & USD 24.4 \\
\hline & & Taimyr \& Evenk & USD 20.6 \\
\hline & & Sakha & USD 18.0 \\
\hline & & Magadan & USD 21.5 \\
\hline & & Koryak & USD 23.8 \\
\hline & & Chukotka & USD 29.2 \\
\hline
\end{tabular}

Source: Ilmo Mäenpää, 2012, Regional Economic Accounts of the Arctic 2000-2010, unpublished typescript in possession of the author.

\subsection{The future of the Arctic}

What will the future hold for the Arctic economy? The recent performance of the North's economy provides some clues to the future. This section describes a likely future for the economy in terms of five trends. These trends are extensions of important observations about recent economic change and its causes in the North.

\section{Trend 1}

Northern resources will be developed for the international resource markets only when market participants expect the development to be profitable. This relationship will determine the future pattern of Arctic resource development. The predicted changes in resource prices and development costs are encouraging signs for future development. Increased commodity prices and reduced costs from climate change will increase the profitability of northern resource development.

Unfortunately for the future of the Arctic economy, recent dramatic increases in commodity prices are not likely to be a long run trend. Re- 
cent high prices are based on the increasing resource demand resulting from the industrialization of the world's emerging economies. High and rapidly increasing resource prices have existed for periods in the past, but over the very long run most resource prices have fallen in real terms (Blackman and Baumol, 2008). Historically, high prices for any resource have provided the incentive for exploration to find more of the high price resource and innovation to find technology substitutes for the resource (Barta and Miller, 2013).

\section{Textbox 4.8}

\section{The Shtokman experience}

The glut of gas from American unconventional sources resulted in a slowdown in gas production in the High North. Development of the large Russian Shtokman gas field was originally scheduled to begin production in 2013. Many observers estimate the start date will be closer to 2020. Because of its northern location it is a difficult and costly project. The Shtokman project was affected by a decrease in demand from the recession but also by the availability of less expensive and more accessible gas affecting the profitability of this project (Nordregio, 2010; The Economist, 2010).

\section{Trend 2}

The Arctic will remain a high cost region. Costs are high because resources are far from markets and lie in a sparsely settled land and an inhospitable environment. Budzik (2009) identifies the reasons behind the high costs of development, including harsh winters requiring special designs; poor soil condition requiring additional site preparations; potentially damaging ice pack in Arctic seas; long supply lines which require large inventories of parts; limited transport access; and higher wages and salaries required to induce personnel to work in the region. Of these only the transport cost may be positively affected by climate change.

The effects of environmental changes in the Arctic will not always benefit economic development and there is evidence that climate change may make it more costly to develop resources. While warming may open the Arctic seas for transportation and the continental shelf for development, the sea ice will be unpredictable. The increase in flooding and the reduction in permafrost and snow cover will increase production costs even in areas with significant current resource activity; warming may shorten the period during which ice roads allow exploration and development activity on the tundra and thawing ground may destabilize existing systems of roads and pipelines and other industrial infrastructure in the North. These changes will impose costs 
on existing Arctic activity as well as increase the cost of future development (AHDR, 2004).

\section{Textbox 4.9}

\section{Prices and production from existing mines in northern Europe}

Including Northwest Russia there were 42 functioning mines in this region in 2009. High demand and resource prices have generated interest in the region's resource, which could result in the opening of as many as 68 mines, as well as increased production at existing mines. The geologic wealth of the Fennoscandian Shield provides both the history of mining and the reason for interest in the region. Significant exploration took place in Sweden, Norway and Finland from 2006-2007, costing the equivalent of EUR 55 million per year (Pietila, 2009).

\section{Trend 3}

Expectations of higher prices and lower costs for Arctic resources may be overly optimistic but natural resource production will continue as a driving force of this economy. Future resource production will likely be concentrated as it is today. Production is most likely to occur in regions of lower cost. These include places of existing production which allows the use of existing infrastructure; places near tidewater that reduce the cost of transportation; and places with low taxes and efficient regulation. Production will also be concentrated around extremely rich or bonanza resource deposits that provide the scale to overcome the high cost of Arctic development.

\section{Textbox 4.10}

\section{Greenland as a resource frontier}

Historically, Greenland's economy was based on fishing and transfers from Denmark. In 2009 self-governance came to Greenland. Its sub-surface mineral rights may become a source of further economic growth. In 2010 Cairn Energy, a British petroleum firm, found indications of worthwhile oil deposits off Greenland's west coast. The USGS suggested that the sea floor between Canada and Greenland holds major oil deposits. International mining companies are also interested in Greenland's mineral wealth. A recent change in policy opened the door for mining of valuable rare-earth minerals. Other mining interests include gold, molybdenum, and gems (The Economist, 2010; Areddy and Bomsdorf, 2013; Sorensen, 2008). 


\section{Trend 4}

There will be continual changes in the institutional relationships that define the connection between local residents and resource development. In the last part of the 20th century there have been significant changes in these institutions in the North. Changes occurred in local control and ownership and these should increase the economic benefits of resource development for local residents. These changes have allowed residents to participate in decision-making and to receive a greater share of the resource rents from development. Continued institutional change is likely to influence the future of the Arctic economy.

\section{Trend 5}

There will be significant economic activity outside the North's international resource economy. The traditional economy will remain important for the residents of the North. The public sector will remain important to the overall economic well-being of residents providing incomes, services, and goods. In the recent past northern economies have increased economic activities outside of the resource and public sectors in tourism, international air freight, electronics, and finance. These activities and import replacement activities are likely to accompany increases in the North's city population.

\section{Smelting of nickel ore in Monchegorsk, Kola Peninsula, Russian Federation}

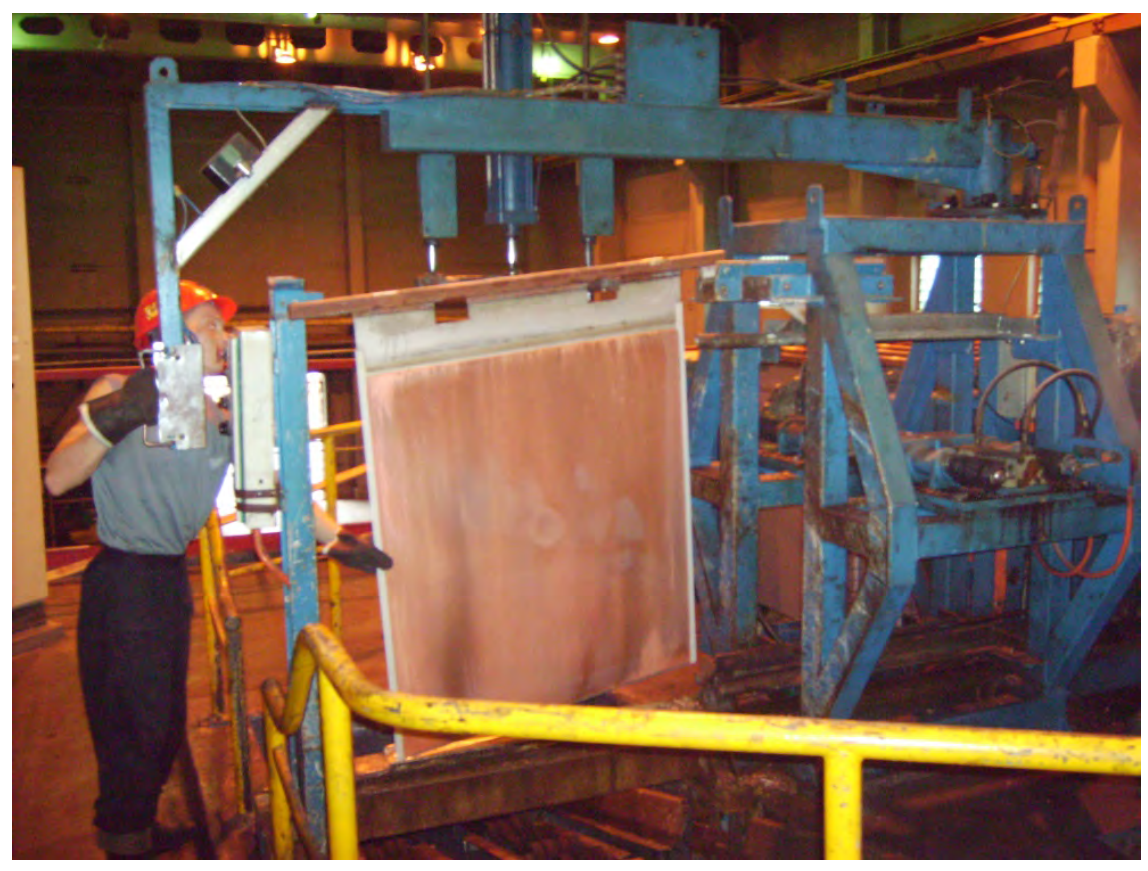

Credit: Rasmus Ole Rasmussen. 


\subsection{Summary}

The current Arctic economy looks much like the economy described in the first $A H D R$. The Arctic economy remains surprisingly large, especially when compared to its share of population. The economy experienced growth during the study period, and that growth slowed with the international economic slowdown. The economic output of the Arctic is highly concentrated in particular countries, Russia and the US. Output is also concentrated within regions in the Arctic nations. This concentration reflects both the concentration of resources and urbanization in the North. The Arctic economy as in the first $A H D R$ continues to rest on three pillars: large scale resource production for the international market, small-scale traditional production for local consumption, and transfers from higher levels of government. Traditional hunting, herding and fishing remain important to the economy of the Arctic. Finally, during the study period some of the diversification forecast by Duhaime in 2004 could be found in the non-resource based industries, like air cargo and electronics, found in the North.

\subsubsection{Gaps in knowledge}

This review has described the economy in the Circumpolar North. It has shown that the economy of the region is relatively large and experienced significant growth over the review period. The review has also shown that economic well-being is not evenly distributed across the North. This section presents some areas for research that will help to understand better the determinants of this economic pattern. Filling in these gaps in our knowledge of the Northern economy will also be helpful to policy makers charged with improving the economic well-being of the residents of the North.

\section{Gap 1}

What types of institutions work best to improve the economic wellbeing of northern residents? One of the most significant changes in the northern economy identified in this chapter is the introduction of a variety of new institutions. These provide residents of the North with more control over local resource projects and allow them to share more of the benefits of these projects. The variety of the types, history, and extent of these institutional changes across the North provides a natural experiment for developing an understanding of the institutional characteristics that promote the economic well-being of the residents. 


\section{Gap 2}

What is the economic role of large settlements in the economic growth of Northern regions? The North has a variety of settlement types from small villages to large cities. Cities and towns often serve as centers for providing services and hubs for transportation. The urban settlements may also help to generate new types of economic activity and change the cost of resource development. Better knowledge of the economies of these urban places in the North and their relationship to the economies of their surrounding regions will increase our understanding of the northern economy.

\section{Gap 3}

What is the role and significance of the traditional sector in the Northern economy? The traditional harvesting plays an important economic and cultural role throughout the North, but there are limited means of comparing this activity to activities that take place in the resource and public sectors in many countries. Developing measures of the extent and significance of this economic activity would allow a more complete picture of the Northern economy.

\subsection{References}

AHDR, 2004. Arctic Human Development Report. Einarsson, N., J. N. Larsen, A. Nilsson and O. R. Young (eds.). Stefansson Arctic Institute, Akureyri.

Areddy, J. and C. Bomsdorf. 2013. Greenland opens door to mining, Wall Street Journal, 25 October 2013.

Aslaksen, I., W. Dallmann, D. Holen, E. Hoydahl, J. Kruse, B. Poppel, M. Stapleton, and E.I. Turi, 2009. Interdependency of subsistence and market economies in the Arctic. In: Glomsrød, S. and I. Aslaksen (eds.). The Economy of the North, pp. 75-97. Statistics Norway, Oslo.

Barta, P. and J. Miller. 2013. Innovation and investment pop commodity price bubble, Wall Street Journal, 8 December 2013.

Blackman, S. and W. Baumol, 2008. Natural resources. The Concise Encyclopedia of Economics. http://www.econlib.org/library/Enc/NaturalResources.html (24 February 2014).

Budzik, P., 2009. Arctic oil and natural gas potential. U.S. Energy Information Administration. http://www.eia.gov/oiaf/analysispaper/arctic/pdf/arctic_oil.pdf (24 February 2014).

Conference Board of Canada, 2013. Economic Forecast: Territorial Outlook. Ottawa.

Crate, S., B. Forbes, L. King, and J. Kruse, 2010. Contact with nature. In: Larsen, J.N., P. Schweitzer, and G. Fondahl (eds.). ASI I, 2010. Arctic Social Indicators - a follow-up to the Arctic Human Development Report, pp. 109-125. Nordic Council of Ministers, Copenhagen. 
Doss, N. 2011. An iron rush above the Arctic Circle. Bloomberg Business Week. 31 January 2011.

Duhaime, G., 2004. Economic systems. In: AHDR, 2004. Arctic Human Development Report. Einarsson, N., J. N. Larsen, A. Nilsson and O. R. Young (eds.), pp. 69-84. Stefansson Arctic Institute, Akureyri.

Economist. 2010. An unconventional glut. The Economist, 11 March 2010.

Economist. 2010. Black stuff in a green land. The Economist, 28 August 2010.

Eilmsteiner-Saxinger, G., 2011. 'We feed the nation:' benefits and challenges of simultaneous use of resident and long-distance commuting labor in Russia's northern hydrocarbon industry. Journal of Contemporary Issues in Business and Government. 17(1): 53-67.

Glomsrød, S., I. Maenpaa, L. Lindholt, H. McDonald and S. Goldsmith, 2009. Arctic economies within the Arctic nations. In: Glomsrød, S. and I. Aslaksen (eds.). The Economy of the North, pp. 37-67. Statistics Norway, Oslo.

Goldsmith, S. 2011. Alaska after Prudhoe Bay: sustainability of an island economy, Annual Meeting of the Western Regional Science Association, Monterey, California. February, 2011.

Graff, J. 2007. Arctic: fight for the top of the world. Time, October, 2007.

Helander-Renvall, E., 2010. Globalization and Traditional Livelihoods. In: Heininen, L. and C. Southcott (eds.). Globalization and the Circumpolar North. University of Alaska Press, Fairbanks.

Hill, F. and C. Gaddy, 2003. The Siberian Curse: How Communist Planners Left Russia Out in the Cold. Brookings Institution Press, Washington, DC.

Hill Strategies Inc, 2009. Artists in Canada's Provinces and Territories Based on the 2006 Census, Statistic Insights on the Arts, Vol.7 No. 5.

http://www.hillstrategies.com/sites/default/files/Artists_provinces2006.pdf

Hill Strategies Inc. 2010. Artists in Small and Rural Communities in Canada. Statistical Insights on the Arts, Vol. 8 No. 2.

http://www.arts.on.ca/AssetFactory.aspx?did=5773

Huskey, L., 2010. Globalization and the economies of the North. In: Heininen, L. and C. Southcott (eds.). Globalization and the Circumpolar North, pp. 57-90. University of Alaska Press, Fairbanks.

Iglebaek, I., 2010. Gas slowdown in the High North. http://www.nordregio.se/en/Metameny/About-Nordregio/Journal-ofNordregio/Journal-of-Nordregio-2010/Journal-of-Nordregio-no-1-2010/Gasslowdown-in-the-High-North/ (24 February 2014).

Kaufmann, R., P. Karadeloglu, and F. di Mauro, 2008. Will oil price decline over the long run? European Central Bank Occasional Paper Series, No. 98.

Knapp, G. and L. Huskey, 1988. Effects of transfers on remote regional economies. Growth and Change, 19(2): 25-39.

Larsen, J.N. and L. Huskey, 2010. Material well-being in the Arctic. In: Larsen, J.N., P. Schweitzer, and G. Fondahl (eds.). ASI I, 2010. Arctic Social Indicators - a follow-up to the Arctic Human Development Report, pp. 47-66. Nordic Council of Ministers, Copenhagen.

Leven, C., 1986. A note on the economics of remoteness. In: Bylund, E. and U. Wiberg (eds.). Regional Dynamics of Socio-Economic Change: the Experiences and prospects of Sparsely Populated Areas. CERUM, Sweden.

London, J., 1900. The economics of the Klondike. The American Monthly Review of Reviews, 74. 
Mäenpää, I., 2012. Regional Economic Accounts of the Arctic 2000-2010, unpublished typescript in possession of the author.

McBeath, J., 2010. Changing forms of government in the North. In: Heininen, L. and C. Southcott (eds.). Globalization and the Circumpolar North, pp. 91-118. University of Alaska Press, Fairbanks.

Nordicity, 2010. Economic Impact Study: Nunavut Arts and Crafts. Report for the Department of Economic Development and Transportation, Government of Nunavut, Nordicity Group and Uqsiq Communications. http://assembly.nu.ca/library/GNedocs/2010/000056-e.pdf (30 October 2014).

Nordicity, 2014. Needs Assessment: Arts Administration Skills and Resources in Nunavut's Arts and Culture Sector. Report for the Canadian center for the Arts. Nordicity Group. http://canadacouncil.ca/council/research/findresearch/2014/needs-assessment (30 October 2014).

Nordregio, 2010. Gas slowdown in the High North. Journal of Nordregio, 1: 20-21. Nordregio, Stockholm.

Nuttall, M., 2005. Hunting, herding, fishing, and gathering: Indigenous peoples and renewable resource use in the Arctic. In: Symon, C., L. Arris, and B. Heal (eds.). Arctic Climate Impact Assessment, pp.650-690. Cambridge University Press. Cambridge.

O'Connor, J. and D. Orsmond, 2009. The recent rise in commodity prices: a long run perspective. Economic Analysis Department.

Pietila, R., 2009. Prices and geology will determine further developments in Fennoscandia. Journal of Nordregio. 3(9): 4-5.

Poppel, B., Kruse, J., Duhaime, G., Abryutina, L. 2007. Survey of Living Conditions in the Arctic: SLiCA Results. Anchorage: Institute of Social and Economic Research, University of Alaska Anchorage. http://www.arcticlivingconditions.org/.

Sorenson, F., 2008. Sub-surface and self-government. Journal of Nordregio, 3(8): 19-21.

Sugden, D.E., 1982. Arctic and Antarctic: a Modern Geographic Synthesis. Basil Blackwell, Oxford.

Weber, R. and R.O. Rasmussen (eds.), 2014. Sustainable Regions-Sustainable Local Communities. Nordregion Working Paper, 2014: 2. Nordregio: Stockholm.

World Bank, 2014. http://data.worldbank.org/indicator/NY.GDP.MKTP.CD (30 October 2014). 



\title{
5. Governance in the Arctic: Political Systems and Geopolitics
}

\author{
Lead Authors \\ Greg Poelzer, University of Saskatchewan, Canada and Gary N. Wilson, \\ University of Northern British Columbia, Canada

\section{Contributing Authors} \\ Else Grete Broderstad (Arctic University of Norway, Norway), Diane \\ Hirshberg (University of Alaska Anchorage, USA), Mara Kimmel (Institute \\ of the North - Anchorage, USA), Timo Koivurova (University of Lapland, \\ Finland), and Anatoly Sleptsov (Northeastern Federal University, Russian \\ Federation)
}

\subsection{Introduction}

Governance is fundamental to the future of the Arctic region, both for the people who make the Arctic their home and for the lands and resources that are of increasing global importance. For the purposes of this chapter, governance comprises political systems - the structures, processes, and actors involved in public decision-making for a political community, and geopolitics - the international relations among political communities. By international standards, the Arctic region has been a leader by constantly pushing the edges of governance innovation. Much of this innovation has been driven by the complexity of the issues facing the region and its peoples. The dramatic legal empowerment of Indigenous peoples over the past two decades has profoundly shaped new and emerging governance arrangements. At the same time, harsh climates, large geographic distances, persistent social challenges and limited organizational and administrative capacity, as well as new external drivers such as climate change and global energy demands, strain many subnational and local governments in the Arctic region in ways that are simply incomparable to southern metropoles. 
Part of the complexity of governance in the Arctic region is the multiple identities and political allegiances that Northern citizens hold. A person living in the village of Khatystyr in eastern Siberia, for example, may belong to a particular clan, but is also a citizen of the local municipality. He or she also shares a broader Evenk identity with other Evenk people, not only within Sakha Republic (Yakutia), but also with Evenk in other regions in Russia and, indeed, beyond the borders of Russia. That same person is also a citizen of the Sakha Republic (Yakutia) and a citizen of Russia. Accordingly, he or she may be a voting member of the local clan-based, selfgoverning economic collective (rodovaya obshchina), and a citizen with defined political rights and obligations toward the municipal, republic and federal governments. The individual may also be an active member of the local Evenk organization and the republic (regional) level Russian Association of the Indigenous Peoples of the North (RAIPON).

Sometimes the governments and organizations representing these different affiliations pursue common aims and hold common priorities; more often, they do not and are at odds with one another. A national priority to develop natural resources to export abroad may be at odds with local interests to preserve the environment. This does not even begin to speak of the complexity of governance at the international level within the Arctic. Northern residents, therefore, often have regional (province, state, or republic) citizenship, along with national citizenship rights and duties. Indigenous residents, in addition to regional and national citizenship rights, often have other political rights related to their cultural identity.

Today, multi-level governance is at the center of Arctic political life, reflecting these attachments to different political communities. Indeed, much of the governance innovation that has taken place in the Arctic centers on reconciling the interests of various political communities. What has emerged is a range of different governments, agencies and organizations. This range includes governments and government forums at the local, regional, national and even international levels. It also includes non-governmental organizations that contribute to the administration of this vast region. Multi-level governance involves interaction vertically (across local, regional, national and international bodies) and horizontally (among government and non-government actors on the same level). Accordingly, this chapter organizes its exploration of Arctic governance trends through a multi-level governance lens. 
Six trends in Arctic governance have evolved and/or have emerged since 2004: 1) devolution and tensions with centralization; 2) increasing Indigenous political and legal empowerment; 3) human capacity challenges; 4) fiscal capacity challenges; 5) challenges of defining political community and legitimate participation; 6) continued governance innovation. Although the circumstances - the particular combination of peoples, history, geography, and climate - that surround governance in the Arctic are unique to the region, many of the processes are not. Devolution and the ebb and flow of centralization versus decentralization have been a dominant theme in the recent history of Arctic governance. The devolution of authority from Denmark to Greenland, from the federal government in Canada to the three territorial governments, from the Russian federal government to regional subjects (as well as the recentralization of authority) are just some examples of these complex processes. It is important to stress that these processes are part of broader devolutionary trends globally.

Paralleling the devolution of authority from national to regional governments has been the waxing of Indigenous political rights and governance authorities (Coates and Poelzer, 2010a). This is profoundly shaping governance across the Arctic, from the North Slope of Alaska and Canada to Greenland and Finland. The number of Indigenous governance authorities grew rapidly in the Russian Federation in the 1990s, but the process of devolution has been caught up in the broader recentralization of power that has been taking place over the last decade.

Devolution and the political empowerment of Indigenous peoples have led to an increase in governance authorities at regional and local levels; this in turn has generated human and fiscal capacity challenges. Having governance authority is one matter, having the people and financial resources to effectively exercise that authority is another issue altogether. The Arctic is not exceptional in terms of the human and fiscal capacity challenges confronting regional and local governments. Whether or not the basic issues of governance can be addressed will determine, fundamentally, whether or not social issues can be tackled, economic opportunities can be seized, transportation, communication, and housing infrastructure can be delivered, and land and environments can be managed.

In regards to who should participate in governance, the Arctic Council has broken new ground from its very founding in terms of including Indigenous perspectives and voices through the involvement of the Permanent Participants, organizations that represent many of the Indigenous peoples of the Circumpolar North. As the Arctic has grown in sig- 
nificance globally and domestically, defining political community and legitimate participation in Arctic governance has become increasingly important. This issue has arisen on a global level in the Arctic Council, as non-Arctic states have sought a seat at the Arctic governance table. But this issue also occurs at the domestic level. In countries such as Canada and Russia, there are considerable variances in terms of which subArctic regions are included and when they are included in governance and decision-making processes. It is likely these latter governance issues will become increasingly salient over the coming years.

The final trend in governance is that innovation has not plateaued in the Arctic region. Political systems and geopolitics continue to evolve, placing the Arctic at the global forefront of finding solutions to accommodate both the vertical and horizontal dimensions of an increasingly complex multilevel governance system.

This chapter will examine the main institutional features that influence Arctic governance at the local, regional, national and international levels, with a particular focus on developments since the first Arctic $\mathrm{Hu}$ man Development Report (AHDR, 2004). The chapter will begin by reviewing the national political contexts in North America, the Nordic countries and Russia. The next section will explore developments and trends in local and regional or sub-national governance in specific countries, as well as provide a general synopsis of national development strategies of the Arctic states. The last section will look at geopolitical and international governance in the Arctic. The discussion that follows uses the terms Arctic and Circumpolar interchangeably.

\subsection{National political systems in comparative perspective}

Political systems and geopolitics operate within historical contexts and through institutions heavily shaped by the political cultures of particular regions and countries. For an overview of the context of contemporary political systems and geopolitical environments, please consult Chapters 4 and 12 of the Arctic Human Development Report (AHDR, 2004). This chapter outlines the current contexts. 


\subsubsection{North America: national majoritarian states and federal systems}

Governance regimes in North America (Canada and the United States) are generally characterized by two defining features: majoritarian democracy and federalism. In a majoritarian democracy, the person or party that wins the most votes or seats in an election is typically given the first opportunity to govern. In Canada, the political party that wins the most seats in an election forms the government, while the other parties become the opposition. In the United States, authority is divided between different branches of power, but control over any one branch is still based on the majoritarian principle.

While most national and regional (state, provincial and territorial) governments in Canada and the United States operate on the majoritarian principle, there are some important exceptions in the Canadian Arctic. The governments of Northwest Territories and Nunavut both operate according to a consensus system of democracy. This means that decisions are made with the consensus of those present, rather than by the more adversarial and competitive process that is common in other jurisdictions. This consensus-based system of decision-making is consistent with traditional governance methods of the Indigenous peoples who inhabit Northern Canada.

In a federal system of government, power is divided between different orders of government such as a national government and various regional governments. Under this system the responsibilities of these orders of government are outlined (and protected) in a constitution and these arrangements cannot be changed without consent of the orders of government affected by the changes. The Canadian federal system consists of a national or federal government and provincial and territorial governments. Of particular relevance to the Arctic are the territorial governments, which govern the regions north of the 60th parallel of latitude (there are small sections of the provinces of Quebec and Newfoundland and Labrador that extend north of the 60th parallel). Yukon Territory, Northwest Territories and Nunavut all have autonomy within the Canadian federal system, including their own legislatures and delegated authority. These territories, however, do not have the equivalent status of provinces within the Canadian federal system and are dependent on the federal government for much of their funding. Nevertheless, in recent years, the federal government has devolved province-like powers to Yukon (2003) and Northwest Territories (2013) and, in many respects, these territories operate like de facto provinces. 
In the United States, the federal system divides power between a national or federal government and state governments. The State of Alaska is the only American territory within the Arctic region. Alaska has a number of autonomous regions or boroughs, including the North Slope Borough (NSB) and the Northwest Arctic Borough (NAB). Like the regions of Nunavik and Nunatsiavut in Canada, the NSB and the NAB are nested within Alaska as political subdivisions of the state, and, therefore do not challenge the state's territorial integrity. Adding to the complexity of governance in America's only Arctic sub-national jurisdiction is the fact that although the United States has a constitutional obligation to deal with Indigenous tribes on a government-to-government basis, the state of Alaska does not have a similar relationship with the tribes within its borders. The disparity in relations between the tribes and the federal government, and the tribes and the state government, leads to tensions between governments at all levels (Kimmel, forthcoming).

While federalism supports the idea of regional autonomy because it recognizes that sovereignty can be divided between different governments within a single political system, this system of government does not always accommodate regional aspirations for autonomy. For example, large portions of the Canadian sub-Arctic, referred to as the provincial Norths, are constrained by federalism because they are located within the geographic boundaries of existing provinces. Although there are some examples of these Northern regions achieving greater autonomy within their respective provinces, for the most part, the provincial Norths are controlled politically and economically by their respective provinces. Communities situated in the provincial Norths have some limited autonomy through local government. In Canada, however, local government is not a recognized order of government and comes under the legal (constitutional) jurisdiction of the provincial governments.

\subsubsection{Nordic: consensus model of governance and unitary states}

The governance model of the Nordic countries of Northern Europe (Finland, Norway and Sweden, Denmark (Greenland) and Iceland) has been described as consensual democracy. It has been observed that the basis for consensual democracy is a high degree of political, economic and ideological unity within a particular country or society (Elder et al., 1998). For the most part, such unity existed in the Nordic countries for much of the post-war period. This model was reinforced by a robust social safety net called the Nordic Welfare Model (NWM) (Arter, 2001). 
In the past decade, however, the NWM and consensual democracy have been affected by globalization, changing ideological norms and, in the case of Finland and Sweden specifically, the process of European integration. Although membership in the European Union (EU) does weaken the control that individual member states exert over their respective political and economic systems, the EU's regional support and structural funds programs underlining principles of partnership and subsidiarity have worked together and even enhanced national regional policies in rural and northern areas of Sweden and Finland. The EU and its Nordic member states have also taken a leadership role in the Arctic through the Northern Dimension program, a policy that was initiated in 1999 and renewed in 2006. The policy aims to "promote dialogue and concrete cooperation, strengthen stability, well-being and intensified economic cooperation, and promote economic integration, competitiveness and sustainable development in Northern Europe" (European External Action Service, 2013).

The Nordic countries are also characterized by unitary systems of government. Unlike federal systems that constitutionally divide government into different levels of sub-national regions and territories, a standard unitary system centralizes power in a national government. Central ministries, therefore, administer issues relating to Northern and Arctic development. Depending on the issue, these ministries might be general in scope, such as health or education, or a specific ministry related to regional development. For example, in Norway, the Ministry of Local Government and Regional Development is responsible for development in all parts of the country, including its Arctic regions. The primary exceptions to the standard unitary model are the statuses of Greenland and the Faroe Islands within the Danish Kingdom, and Finland, where municipalities are constitutionally protected and have extensive administrative powers.

In unitary systems, national governments can also devolve power to regional and local governments, thereby allowing regions and communities a degree of autonomy. Unlike federal systems, however, those powers can always be reasserted by the national government. Throughout the Nordic countries, therefore, northern and remote regions do exercise a limited degree of autonomy. An example would be Norrbotten County in Sweden, which covers almost a quarter of the country's landmass. Norrbotten is governed by the County Administrative Board, which is headed by a centrally appointed Governor. 
With the exception of northwestern Russia, the Nordic countries contain all of the Arctic regions of Europe. In terms of regional governance, among the most important political features of Arctic Europe are the parliaments that represent the Sámi peoples of Sweden, Norway and Finland. These parliaments provide the Sámi, one of the Indigenous peoples of northwestern Europe, with limited levels of autonomy within their host states: the Sámi parliaments have the political autonomy to develop policy recommendations to their respective national parliaments, but are limited in that the recommendations are advisory and not legally binding.

\subsubsection{Russian Federation: managed democracy and federalism}

The Russian Federation (Russia) has undergone a series of profound and far-reaching transitions since the collapse of the Soviet Union in 1991. These transitions have political, economic and social dimensions. Politically, Russia moved away from the one-party rule of the Communist Party toward a multi-party democracy. During the 1990s, the authority of governing institutions waned with a parallel movement toward decentralization. Over the past decade, the Russian government has become a system of "managed" democracy in which the activities of opposition groups have become more limited. The political system was also centralized in an effort to increase the efficacy of governing institutions. As a consequence, the legislative branch of government, through the Parliament (Duma), and the federal system of territorial governance have both come under increasing control of the executive branch of government.

Russia has a hybrid system of presidential and parliamentary government. The executive and legislative branches of government are elected separately, but the President appoints the head of the Government (the Prime Minister). Under the presidency of Boris Yeltsin in the 1990s, the relationship between the Parliament and the President was much more balanced. Since Vladimir Putin came to power in 2000, the President has become the dominant political actor. One might even say that since 2000, power has become concentrated in the hands of Vladimir Putin. Even during the period of 2008-2012, when Dmitry Medvedev assumed the position of President, Putin, his appointed Prime Minister, was still widely perceived to be the most powerful politician in Russia (Remington, 2011). 
Russia's federal system of government has its roots in the Soviet federal system. For the most part, the territorial configurations that existed in the Soviet federal system were simply replicated in the post-Soviet system. This is particularly important for Northern Russia because the Soviet system provided de jure autonomy for many sparsely-populated, yet geographically large Northern regions. For example, Russia's autonomous okrugs (districts), most of which are located in the Northern and Arctic regions of the country, were remnants of the Soviet system. They were established in the 1930s to provide northern Indigenous peoples (malochislennyie narodi Severa) with a degree of administrative and cultural autonomy, albeit within the strict confines of a highly centralized authoritarian system of government (Wilson, 2003). In the postSoviet period, however, these regions gained real political and economic autonomy. In the 1990s, they were junior partners in the "parade of sovereignties" that challenged the power of the federal government.

During the Putin era, the Russian federal system has become much more centralized. Whereas in the 1990s, regional governments routinely flouted federal legislation in key areas such as natural resource development (Wilson, 2001), after 2000, the federal government systematically brought the regions into line. Reforms to Russia's federal system of government had particular consequences for the North. In the early 2000s, Putin introduced a system of federal districts as a means of controlling the regions and, in particular, making sure that they complied with federal legislation. Russia's northern regions, spread out across 11 time zones and thousands of kilometers of territory, were divided among several such federal districts, the capital cities of which are all in the South. The autonomy that these regions had enjoyed as a result of the weakness of the federal government and the chaos of the first decade of the post-Soviet transition, quickly dissipated as the regions were expected to conform to the dictates of the centrally-mandated federal district representatives.

Another, more gradual series of changes have affected the abovementioned autonomous okrugs, which comprise a large territorial portion of the Russian North and Arctic. Over the last decade, there has been pressure to merge these regions with larger, southern regions, as part of a general plan to consolidate the hold of those southern regions over the resource wealth of the North. Regional mergers, along with the creation of the federal districts, were also a way for the federal government to streamline the federation and reduce the number of regions (with over 80 regions, and six different types of regions, the Russian Federation is by far the most complicated federal state in the world). 


\subsection{Levels of Arctic governance: local, regional and national}

Sakha Republic (Yakutia) Parliament, Russian Federation, 2014

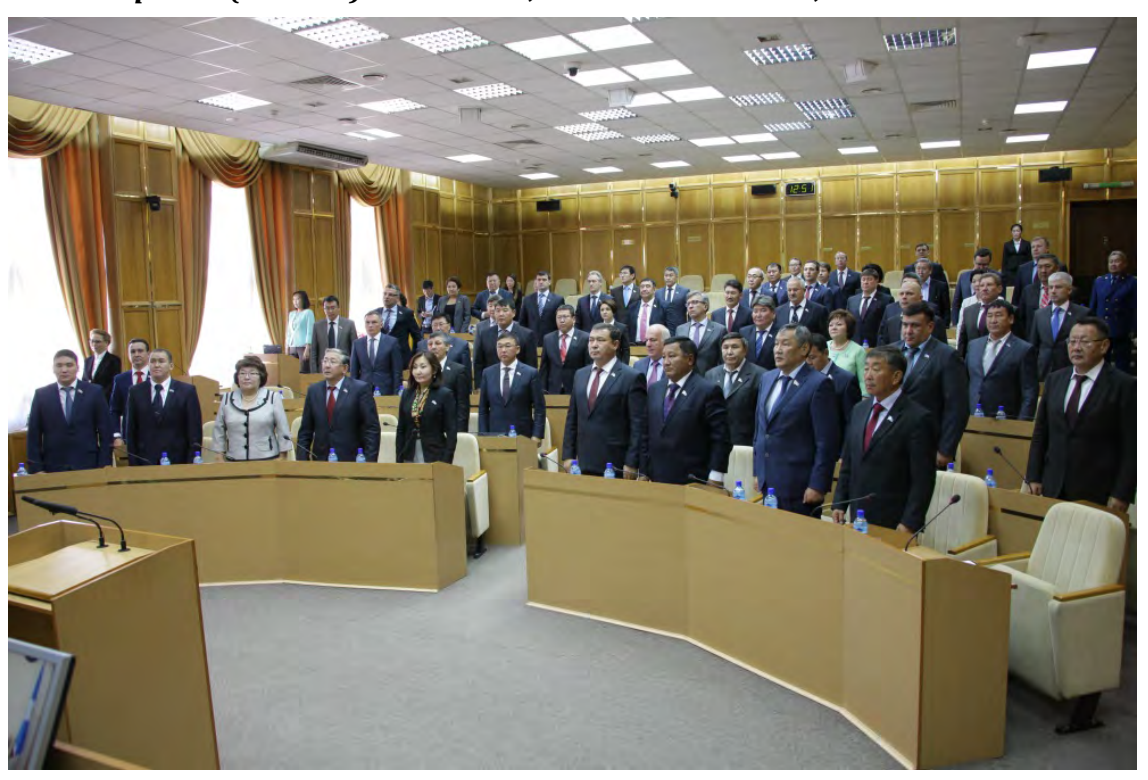

Credit: Vasiliy Kononov.

\subsubsection{Local level governance}

Local government is an important level of government because of the tasks it performs and its proximity to and interaction with the citizenry, relative to other levels of government. Local governments provide critical services that are essential to the proper functioning of communities, as well as a degree of local administrative control. This is particularly true in northern and remote regions where local government is often the only level of government that is controlled by and answerable to Northerners. While provincial/regional and national governments obviously play a key role in the administration of northern regions, local government is usually the point of first contact for northern residents.

As indicated in the first $A H D R$, over the past several decades, two distinct local governance models have emerged in the Circumpolar North: public and Indigenous. A public model of local governance exists when participation in the activities of local government, such as voting in elections and running for office, are open to all citizens, regardless of their status or ethnicity. An Indigenous model of local governance exists when only recognized members of a particular Indigenous group are allowed 
to participate in the activities of local government. The public governance model is more common in the Circumpolar North, but with the progress made towards Aboriginal self-government in some countries such as Canada, Indigenous forms of local and regional governance are becoming a reality.

\section{Norway}

Norway has a public model of local governance. As a unitary state, its central or national government (based in the capital Oslo) provides overarching political direction for the whole country. Below the central government, local administration is divided into two tiers: counties (fylker) and, located within the counties, municipalities (kommuner). There are 19 county authorities and 428 municipal authorities. The three Northern counties, encompassing the Northern half of the country are Finnmark, Troms and Nordland. Voters elect representatives to municipal and county councils, and the number of representatives present in municipal councils depends on the size of the municipality. For example, a municipality with a population under 5000 is expected to have at least 11 representatives on its municipal council (Norwegian Ministry of Local Government and Regional Development, 2008). By contrast, similar sized municipalities in Northern British Columbia in Canada only have 6 representatives.

The structure and responsibilities of local government are governed by the Local Government Act (1992), a centrally mandated piece of legislation. Over the last decade, there has been a great deal of discussion about expanding the responsibilities of local government in Norway. As one government publication notes: "Decentralisation of responsibilities and authority is a crucial step towards genuine local democracy and an efficient and user friendly public administration" (Norwegian Ministry of Local Government and Regional Development, 2008). Nowhere is this more relevant than in the North. Greater local autonomy provides Northerners with the capacity and authority to make decisions that are relevant to their particular circumstances.

\section{Canada}

Canada has both public and Indigenous models of local governance. Progress on Aboriginal self-government over the last decade has strengthened the legal capacity of Indigenous groups to manage their own affairs. A number of studies demonstrate the importance that Indigenous people place on local level government; the local community is often their primary political identity and attachment. This is demonstrated through higher voter turnout at the local level compared to regional and 
national level elections, a pattern that is opposite to the general population (Berdahl et al., 2012). At the same time, these groups face significant human and fiscal capacity challenges that prevent them from fully exercising their legal rights and responsibilities.

In Yukon Territory, the Umbrella Final Agreement was signed between the federal, territorial governments and the Council of Yukon Indians (now the Council of Yukon First Nations (CYFN)) in 1993. This agreement served as a template for the negotiation of separate agreements with individual First Nations in the territory. Eleven such final agreements have come into effect since 1993 (see, e.g., Yukon Government, 2012). In addition, nine self-government agreements have been concluded by members of the CYFN, as well as by the Kwanlin Dün First Nation and Vuntut Gwich'in First Nation, who are not members of CYFN at the present time.

\section{Textbox 5.1}

\section{The Tłįchǫ Agreement}

In the Northwest Territories (NWT), the precedent setting Tłįchọ Agreement came into effect on August 4, 2005. This tripartite agreement, concluded by the Dogrib Treaty 11 Council (Tłįchǫ), the Government of Canada and the Government of Northwest Territories is the first combined land, resources and selfgovernment agreement in NWT. The Agreement provides the Tłįcho with ownership of a single block of 39,000 square kilometers of land, including subsurface resources, adjacent to or surrounding the four Tłįchọ communities of Behchoko, Whatì, Gamètì, and Wekweètì. A Tłįchọ Community Services Agency, established under territorial legislation, is responsible for the management, administration and delivery of health, education and other social programs and services to all residents in the four Tłichọ communities and on Tłįchǫ lands, based on Tłįchǫ Laws and NWT Laws. Consistent with the Agreement, the Tłịchọ Community Governments (TCGs) of Behchoko, Whatì, Gamètì, and Wekweètì were established pursuant to territorial legislation on August 4, 2005. The TCGs replaced the Indian Act Bands and NWT municipal corporations that were in place prior to the effective date of the Agreement. The TCGs are responsible for municipal services and represent and serve all residents. The Agreement provides for guaranteed Tłichọ participation on the Wek'èezhìi Renewable Resources Board and the Wek'èezhìi Land and Water Board. This agreement underscores the increasing complexity of multilevel governance in the North.

While these agreements and that of NWT (see Textbox 5.1) represent a significant step forward in terms of self-government, many of these Indigenous groups are small and often lack the capacity to carry out the 
responsibilities that are outlined in the agreements (Dacks, 2004). Such capacity issues are replicated in other local jurisdictions in Northern Canada. It is important to note the different capacity challenges between public local governments and Aboriginal governments, especially for smaller communities. The responsibilities of public local governments in Canada are quite limited to areas such as municipal water and sanitation, parks and roads. By contrast, most Aboriginal governments have responsibilities akin to provincial and territorial governments, including areas such as health and education, above and beyond what public local governments administer. This reality exacerbates the capacity challenges that Aboriginal governments face.

Local level governments also confront fiscal capacity issues. Public local government in Northern Canada, as in other parts of Canada, falls under the constitutional jurisdiction of the provincial and territorial governments. The authority of local governments, therefore, is defined by provincial and territorial legislation. Over the past several decades, local governments have seen their range of activities expand, in large part due to the downloading of responsibilities by senior (provincial and federal) governments. The ability of local governments to raise revenue (in the form of taxes) is limited and often these downloaded responsibilities are not accompanied by new sources of revenue. Consequently, local governments are forced to do more with less or, at best, the same amount of revenue.

Indigenous and public governance arrangements address one set of representation issues. Another set is gender. Among municipal public governments, there were significant levels of participation by women in elected office. In 2013, the following percentages of mayors/council members for each territory were as follows: Yukon (50/33); Northwest Territories (20/43); Nunavut (20/34). Indigenous self-government and claims agreements have generated instruments of multilevel governance among Indigenous governments on one side, and local and regional level governments on the other. An important instrument is co-management. One study of the gender representation on co-management boards in Yukon Territory, Northwest Territories, and Nunavut found that among the total 210 members, 176 (84\%) were men and 34 (16\%) were women (Natcher, 2013). Among the 34 co-management boards, nine had entirely male members, and 18 boards, respectively, had only one female member. The reasons for this gender imbalance require further inquiry (Ibid.). 


\section{Russia}

Public local governance in Russia is characterized by many of the same challenges that are evident in other countries; namely, a lack of fiscal resources to pay for the myriad of programs and services for which local government are responsible. Like their counterparts in other parts of the Circumpolar North, northern local governments face particular challenges in terms of infrastructure, small population size (and, as a consequence, small revenue-generation bases) and large territories. Additionally, public local governments have also had to deal with the difficulties associated with the transition from central planning and communism to capitalism and democracy. The disintegration of the Soviet Union had a particularly egregious impact on small, remote, northern communities, as funding programs and other forms of economic support dried up with the collapse of the centralized state.

In the 2000s, the Putin government introduced a series of reforms to local government (Young and Wilson, 2007). The main impact of these reforms was to lessen the responsibilities of local governments and, at the same time, significantly increase the number of local governments. While the curtailing of local government responsibilities took some of the pressure off these governments to offer services and programs, such reforms did not bode well for local autonomy. Moreover, the increase in the number of local governments placed severe capacity strains on local officials (Poelzer, 2013). Not only did the costs of administration grow as new governments were forced to fill new posts and acquire new infrastructure, but democracy also suffered as local councils struggled to find citizens willing to serve as elected officials.

\section{United States}

The many layers of governance in Alaska complicate the ability of local communities to exercise effective governance. In addition to being subject to state and federal laws and regulations, each of the more than 220 communities scattered throughout the state may fall within the governance authority of a city, a borough, and/or a tribe. Because of the way Alaska Native land claims were settled, tribal governments no longer have regulatory or taxation authority over traditional lands conveyed under the Settlement Act, posing unique challenges to their abilities to raise revenues and govern their people. The lack of recognized authority and capacity has led to innovations in shared governance between federal, tribal and sometimes state government. There are many agreements to co-manage marine mammals such as the Alaska Eskimo Whaling Commission, and subsistence resources on federal lands are man- 
aged through a system of Regional Advisory Councils that integrate local, rural users into decision-making processes (Kimmel, forthcoming).

\section{Trends: Local-level governance}

One of the defining features of local level governance in the Circumpolar North is the degree of complexity compared to local governance in the southern regions of the eight Arctic states. Although local governance was caught in the general tide of recentralization in Russia, the trend toward increasing the authority of local government was evident in other Arctic countries such as Norway and Canada, including Indigenous governance in the latter case through the implementation of First Nations self-government agreements. In almost all cases, however, fiscal and human capacity challenges are felt the hardest at the local level (Poelzer, 2009). The enormous burden that local governments carry across the North needs to be underscored. In the south, residents have far greater access to regional and national government agencies where different respective program delivery responsibilities lie. In the North, local government is often the only government that residents encounter in their day-to-day lives (Coates and Poelzer, 2010b). As such, they are relied upon for meeting the needs of their residents, even though those responsibilities may not formally lie within their area of jurisdiction. Moreover, the general trend toward fiscal tightening by higher-level governments means there are fewer fiscal resources to meet these needs. On top of it all, local governments in the North are often engaged in intergovernmental relations on a more intense level than their southern counterparts because of environmental assessment processes and consultation requirements involving Indigenous rights, whether these be Indigenous governments or municipalities. Thus, the strain on the human capacity of local governments is even higher. This trend is likely to wax unless greater resources for fiscal and human capacity are made available (Poelzer, 2011).

\subsubsection{Regional governance}

Over the last several decades, Arctic countries have pioneered the development of many innovative regional governance models. Like local government, two different models characterize regional government in the Circumpolar North: public and Indigenous. One of the most important changes to occur in the last 40 years in the area of governance in the Circumpolar North has been the decentralization of authority to regional governments, both public and Indigenous. This trend towards decentralization has only intensified in the last decade. As noted previ- 
ously, this process of decentralization faces a number of challenges. While the decentralization of authority mainly involves the building of legal or legislative capacity, the more difficult tasks of building human and financial capacity to carry out the new responsibilities of decentralization take much longer to realize.

\section{Canada}

Regional governments in Canada have made considerable progress over the last decade in terms of expanding their autonomy. In 2003, the Yukon government completed the final phase of its devolution process, as it took over land and resource management responsibilities from the federal government. This was followed in 2013 by the signing of a comprehensive devolution agreement between Northwest Territories (NWT) and the federal government with implementation beginning in 2014. In 2008, Nunavut signed the Lands and Resources Devolution Negotiation Protocol between the Nunavut Tunngavik Incorporated, the Government of Nunavut and the Government of Canada. Devolution discussions between the three parties are ongoing. Nunavut is proceeding with devolution at a slower pace than Yukon and the NWT. This is not surprising given the greater capacity challenges and the fact that the Territory was only established in 1999 (White, 2009).

In contrast to the level of participation at the local level, the participation of women at the regional level as members of the territorial legislatures and cabinets (with one exception) is significantly lower. Women made up $19 \%$ of the elected members of the territorial legislatures $(11 \%$ of Northwest Territories, $16 \%$ of Nunavut, but $32 \%$ of Yukon). At the national level in Canada women constitute $21 \%$ of the House of Commons and $36 \%$ of the Senate. Within cabinet at the territorial level, fewer than $9 \%$ of ministers were women $(0 \%$ in Northwest Territories; $11 \%$ in Yukon; and, $14 \%$ in Nunavut). At the national level in Canada, $31 \%$ of ministers were women (Strapagiel, 2013).

In other parts of the Canadian Arctic, Indigenous peoples such as the Inuit have negotiated land claims and/or self-government agreements with other levels of government. In 2005, the Inuit of Nunatsiavut in Labrador signed a land claim and self-government agreement with the government of province of Newfoundland and Labrador, and the federal government. The self-government arrangement in place in Nunatsiavut is an example of Indigenous regional government because there are certain restrictions on who can participate in governance activities. Other Inuit communities, however, have chosen (or are contemplating) public governance models. The Territory of Nunavut in the eastern Arctic, for example, has a public government. Nunavik in Northern Quebec is also 
likely to follow this path. This region is currently in the process of negotiating self-government agreements with the Québec government and the federal government. Like Nunatsiavut (but unlike Nunavut), this agreement, if ratified, will create a new autonomous region and regional government that will be "nested" within an existing constituent unit of the Canadian federation (Wilson, 2008).

Some of the most fascinating features of Indigenous regional government in the Canadian Arctic are the development corporations that manage the funds obtained from land claim agreements on behalf of the Indigenous beneficiaries to those agreements (Wilson and Alcantara, 2012). Not only do development corporations, such as the Makivik Corporation in Nunavik, the Inuvialuit Regional Corporation in the Inuvialuit Settlement Region in the NWT and Nunavut Tunngavik Inc. in Nunavut, play an important economic role in their respective regions, creating employment and other opportunities. They have also served as representatives of their regions in intergovernmental negotiations on self-government. The involvement of Indigenous development corporations in public administration and intergovernmental relations is yet another example of the broad and innovative nature of governance in the Arctic.

\section{United States}

Over the past four decades, resource development (in particular oil, gas and minerals) has played a critical, yet controversial, role in the economy and politics of Alaska. Northeastern Alaska has been the site of intensive resource development since the 1970s. There are certainly concerns about the environmental consequences associated with such development and its impact on fragile Arctic ecosystems and the traditional livelihoods of Alaska Natives. At the same time, the economic benefits of resource development, both at the community and state level, are also apparent. As is the case in other resource producing countries and regions in the Circumpolar North, Alaska has established a Permanent Fund to invest a portion of the rents generated from resource development for future generations.

One of the most controversial issues in the Northern part of Alaska over the last decade has been the debate over drilling in the Arctic National Wildlife Refuge (ANWR), a protected area located within the North Slope Borough. The coastal areas of ANWR contain large oil deposits that proponents of drilling argue could provide the United States with greater energy security. Opponents contend that oil exploration will destroy the Refuge's fragile ecosystem. Moreover, they argue that the deposits in ANWR are not significant enough to provide the United 
States with long-term energy security. The same tensions have been seen more recently at the proposed Pebble Mine development in southwestern Alaska. In many respects, the debate about whether to drill or mine reveals the extent to which the economic and ecological destiny of the Northern boroughs is controlled by outside actors at the state and national levels.

Women from Alaska have played a prominent role in United States politics, as well as at the regional level through the bicameral legislative bodies of the State of Alaska. In 2013, women comprised 22\% of the House of Representatives and $20 \%$ of the Senate, in Alaska, which is higher than the percentage of seats held by women in the respective national level houses of the United States' Congress.

\section{Denmark}

Whereas most Arctic regions are content with having limited autonomy within an existing state structure, some aspire, or have achieved, either de facto or de jure statehood. In Denmark, there are two notable examples of this trend: Greenland and the Faroe Islands. Greenland was granted Home Rule from Denmark in 1979, a status that allowed it considerable autonomy (Nuttall, 2008). Thirty years later in 2009, it achieved Self-Rule, a higher level of autonomy that also included greater control over its natural resource revenues. Although Greenland is not fully independent from Denmark, Self-Rule provides it with a considerable degree of autonomy and many powers that are held by independent states. With the exception of Iceland, which gained independence from Denmark in 1944, it is the only example of an Arctic region that has achieved such wide-ranging autonomy in the post-war period. Greenland is still financially and militarily dependent on Denmark. Nevertheless, many Greenlanders support full independence.

Greenland has a public form of self-government, although, like other Inuit regions in Canada and the United States, the overwhelming majority of the population is Inuit and so it has a de facto Indigenous government. Since achieving Self-Rule, there have been a number of debates about the exploitation of Greenland's natural resource wealth (Wilson and Smith, 2011). The wealth is becoming more accessible due to climate change and the melting of Greenland's huge ice sheets. Some political leaders in Greenland feel that resource exploitation should be used as a means of providing greater autonomy and even independence from Denmark.

Self-Rule is major global accomplishment for Indigenous political representation; Self-Rule has also provided opportunities for meaningful political participation for women. In 2013, 36\% of the 31 seats in the 
Inatsisartut (Greenlandic Parliament) were held by women, as were $25 \%$ of the eight seats in the Naalakkersuisut (the cabinet or executive branch of government). The level of representation by women in the Greenlandic Parliament is only slightly lower than that in the Danish Parliament (Inter-Parliamentary Union, 2014; Inatsisartut, 2014).

Like Greenland, the Faroe Islands have political autonomy within the Danish Realm. They still receive financial subsidies from Denmark, although these have been reduced by approximately 50 per cent since 2001. Islanders and the political parties that represent them are divided on the issues of whether the Faroe Islands should become fully independent from Denmark. In 2011, the drafting of a Faroese constitution focused attention on the issue of independence. According to some observers, the draft constitution is not compatible with the Danish constitution, so if the constitution were adopted, the Faroe Islands would have to declare independence from Denmark (Denmark and Faroe Islands in Constitutional Clash, 2013).

\section{Russia}

As mentioned above, the regional government system in Russia has changed under the leadership of Vladimir Putin, with a greater emphasis on consolidation and amalgamation. Some Northern okrugs quickly acquiesced to this new arrangement, in part because the short-term economic rationale for doing so was so strong, but also because they came under intense political pressure from their host regions and the federal government. For example, in 2005, the Evenkiiskii and Dolgano-Nenetskii (Taimyr) autonomous okrugs were merged into the more powerful and populated Krasnoyarsk krai (territory), following successful referenda in all three regions (Mereu, 2005). Other okrugs, however, have resisted regional mergers. For instance, the Khanty-Mansiiskii and Yamalo-Nenetskii autonomous okrugs, the center of the Russian oil and gas industries, were politically, economically and demographically powerful enough to remain autonomous from their southerly neighbor, Tiumen oblast.

\section{Norway}

One of the most important developments in the area of regional governance in Norway in the past decade is the 2005 Finnmark Act. The Norwegian Government passed the Act: "to facilitate the management of land and natural resources in the county of Finnmark in a balanced and ecologically sustainable manner for the benefit of the residents of the county and particularly as a basis for Sami culture, reindeer husbandry, use of non-cultivated areas, commercial activity and social life" (Finnmark Act, 2005: Section 1). The transfer of territorial control mandated 
by this Act was a step forward in terms of local autonomy for the county of Finnmark and the Sámi Parliament. It also encourages greater collaboration across public and Indigenous governments in Norway.

\section{Trends: regional-level governance}

The single largest trend is that devolutionary pressures continue to be a defining feature of political systems in the Arctic. Greenland is increasingly taking steps to build greater self-reliance and strengthen its autonomy from Denmark through Self-Rule. The federal government in Canada continues to negotiate stronger authorities for the territorial governments. In Russia, the picture is far more mixed; however, some regions, especially those with greater fiscal wherewithal have had greater capacity to resist a wave of recentralization and regional consolidation. In spite of its unitary construction, Norway is finding creative solutions to reconciling political communities at the regional governance level through instruments such as the Finnmark Act. In a similar manner to local government (though not as acute), regional governments face real challenges in terms of both fiscal and human capacity. Indeed, the Berger Report (2006) outlined the challenges that building regional governments pose for other sectors. Often the only university educated local personnel are teachers, as teacher education programs are more readily available in Northern regions than any other university degree program. Graduates are often "poached" to staff administrative positions in regional governments and, as a consequence, do not end up in the classroom, undermining the ability to strengthen the school system. Yet, this poaching often does not even meet the staffing deficit in regional governments such as Nunavut (Poelzer, 2011). On top of human capacity challenges, regional governments face rapidly mounting fiscal challenges to provide the necessary infrastructure for businesses to be competitive regionally, let alone globally, for governments to meet complex environmental and other consultation and regulatory processes, and to maintain existing infrastructure - especially transportation and housing - in the face of climate change. These pressures on governing authorities are only going to increase in the future. 


\subsubsection{National level governance}

\section{National level strategies}

Since 2004, all of the eight Circumpolar states have developed formal, comprehensive Arctic policy frameworks, encompassing both domestic and international policy goals and positions. The development of these national Arctic policies into singular policy statements is new for a number of reasons. First, the development took place concurrently, with all states vitally aware that the other seven were working on policy statements. Second, the development of the respective positions anticipated the positions of both the seven other Arctic states and non-Arctic political actors. Third, the policy statements reflect shared core policy priorities that have emerged through inter-state cooperation, particularly through the Arctic Council, notwithstanding understandable expressions of national policy priorities particular to each respective state. Finally, each national policy framework integrates both international and domestic policy positions, recognizing their interdependence and, concomitantly, the multilevel governance reality, from the local to the global, that define Arctic governance in the 21st century.

The policy statements are outlined in the following documents:

- The Norwegian Government's High North Strategy (Norwegian Ministry of Foreign Affairs, 2006); and The High North: Visions and Strategies (Norwegian Ministry of Foreign Affairs, 2011).

- Foundations of State Policy of the Russian Federation in the Arctic to 2020 and Beyond (2008).

- Canada's Northern Strategy: Our North, Our Heritage, Our Future and Statement on Canada's Arctic Foreign Policy: Exercising Sovereignty and Promoting Canada's Northern Strategy Abroad (Government of Canada, 2010).

- Denmark, Greenland and the Faroe Islands: Kingdom of Denmark Strategy for the Arctic 2011-2020 (Kingdom of Denmark, 2011); The Faroe Islands - a nation in the Arctic, opportunities and challenges (Kingdom of Denmark, 2012).

- Sweden's Strategy for the Arctic Region (2011).

- A Parliamentary Resolution on Iceland's Arctic Policy (Iceland, 2011).

- Finland's Strategy for the Arctic Region (2013).

- The United States' National Strategy for the Arctic Region (Government of the United States, 2013). 
It is important to stress that none of the national policy statements were entirely new or simply reactions to the increasing global significance of the Arctic. Instead, each of these documents was typically either a revision of earlier policy statements or collations of previously disparate positions. For example, the Finnish statement builds on previous Finnish positions and includes emphases on domestic issues such as economic development, infrastructure, and Indigenous peoples. Notably, Finland places a priority on advancing Arctic interests not only through regional bodies such as Barents Euro-Arctic Council and the Barents Regional Council, but also through non-Arctic political bodies such as the European Union. Canada's strategy is similar in emphasis and scope, focusing specifically on domestic priorities such as devolution, social and economic development, environmental management and exercising Arctic sovereignty. At the same time it highlights Canada's commitment to multilateral engagement in the international arena. Russia places similar emphasis on the human dimensions of Arctic governance, but underscores the military and security dimensions to a far greater extent. Norway's strategy is broad and places greater attention on capacity-building than the other polities, while at the same time emphasizing the importance of relations with the Russian Federation. Sweden follows a similar course, but is notably critical of the approach taken by the five littoral states (the Arctic 5 - see Textbox 5.2).

\section{Textbox 5.2}

\section{Arctic Five}

The Arctic Five is comprised of representatives (foreign ministers) from the Arctic Ocean littoral states (Russia, the United States, Canada, Denmark (Greenland) and Norway). This group met in Greenland (2008) and in Canada (2010) In 2008, the group released the Ilulissat Declaration that emphasized the need for greater collaboration among the Arctic coastal states on issues not discussed in the Arctic Council, notably the management of the Arctic Ocean. This noninstitutionalized group has come under criticism from non-coastal Arctic states such as Sweden, who feel that pan-Arctic issues should be discussed by the wider Arctic Council. 


\section{Indigenous northern political organizations on the national level}

Across the Circumpolar North, there are a number of Indigenous political organizations that play an important role in governance issues at the national level. With the exception of Iceland, all Arctic countries have national level Indigenous organizations that advocate for Indigenous interests within their respective national political systems. In some cases, such as the Sámi Parliaments in the Nordic countries and Russia, these organizations are formal political institutions. In other cases, such as the Inuit Tapiriit Kanatami in Canada, they are nongovernmental organizations.

Indigenous governance in Norway is conducted through the Sámi Parliament, a national body that has political relations not only to the national government in Oslo, but also connections to municipal and county-level governments. The Sámi Parliament was established in 1989 and is currently comprised of 39 representatives from seven constituencies across Norway. The Parliament is based in Kárášjohka, a municipality in the northern county of Finnmark. Basically the responsibilities of the parliament are limited and connected mostly with matters pertaining to culture, language, education and support to small scale industries. Increasingly, however, consultations have replaced hearings as a contact mechanism between the Parliament and the Norwegian state authorities. The 2005 consultation agreement between the Sámi parliament and the Norwegian government has given the Sámi parliament a salient channel of influence on legislation, planning and concrete matters of importance for the Sámi population in Norway. Between 40 and 50 formalized consultations take place every year. Grounded in the International Labor Organization Convention No. 169 Concerning Indigenous populations and tribal people in independent nations, the consultations have to be conducted in good faith on the part of both parties, and with the objective of achieving an agreement. But challenges and setbacks are evident, as illustrated by the consultations on the bill for a new mining act where the parties failed to reach consensus. The Sámi parliament has also developed collaboration with public municipal and county-level governments (Josefsen, 2010).

Very significantly, however, since 2004, the Sámi Parliament had an historic breakthrough of global significance: women parliamentarians constituted an absolute majority of this elected body. The percentage of female Sámi parliamentarians jumped from 18\% (2001) to 51\% (2005). At the same time, the Sámi Parliament elected Aili Keskitalo as its President, also an historical first. 
In Canada, a parallel body is the Inuit Tapiriit Kanatami (ITK), which is the national organization representing the 50,000 Inuit across all of Canada (Inuvialuit (Western Arctic), Nunavut, Nunavik (Northern Quebec), and Nunatsiavut (Labrador)). ITK is governed by a president, who is elected for a three-year term at the general assembly, and a board of directors. The Board of Directors consists of the Presidents of the four regional Land Claims Organizations, Nunavut Tunngavik Incorporated (NTI), Makivik Corporation (Nunavik), Nunatsiavut Government, and the Inuvialuit Regional Corporation (Inuvialuit Settlement Region), who are all voting members, as well as (ex-officio) the presidents from the National Inuit Youth Council, the Pauktuutit Inuit Women of Canada and the Inuit Circumpolar Council (Canada). A pan-Canadian approach to policy development is reflected for instance in the National Strategy on Inuit Education, which was spearheaded by the ITK in 2011 (Inuit Tapiriit Kanatami, 2011).

\section{Representatives to the Sami Parliament (2013-2017) for Norwegian Sámi Asso- ciation for Southern Norway circuit (Anita Persdatter Ravna, Tor Gunnar Nystad and Kirsti Guvsám)}

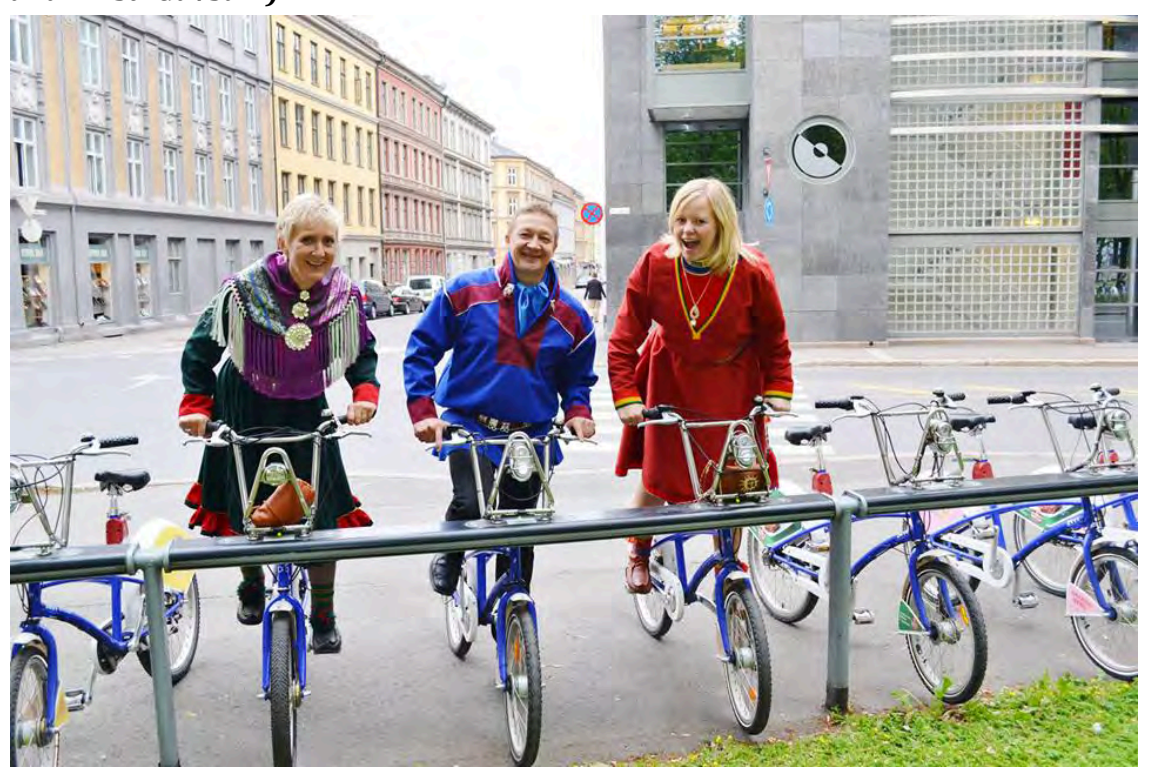

Credit: Kirsti Guvsám.

\section{Trends: regional governance}

Perhaps the single greatest trend at the national level is that northern issues have become mainstream and at or near the center of national policy agendas in almost all circumpolar countries. The drivers of global demand for natural resources, climate change, the possibilities for desti- 
nation and possibly even transit shipping mean that Arctic issues are likely to remain on the agendas of national governments for the foreseeable future. Another issue that is likely to emerge over the coming years is the question of which northern regions are included in the policy community of the Circumpolar North. For example, parts of Northern Russia and the Canadian provincial Norths are included in some forums and not in others. This non-inclusive approach is likely to inhibit Northern governance interests in the long run as the Northern political voice is far weaker than if it was more inclusive, and not based on historical accident of where a territorial boundary was drawn or a permafrost line once existed. Dene, Evenk, Inuit and other Indigenous communities, as well as the non-Indigenous residents of northern communities are often excluded from participating in decisions about their Arctic and subArctic futures, even though language, culture, history, and economies suggest they should be at the center of public decision-making processes about northern futures. In some respects, it is apparent that the nonArctic state actors have a much greater voice. It would not be surprising to see calls for more politically and culturally relevant criteria for defining which northern political communities should regularly be included in policy making on Arctic and sub-Arctic issues. As a consequence, such a development would likely to lead to more effective northern strategies domestically and a stronger Arctic voice internationally.

\subsection{Geopolitics and governance among Arctic political communities}

Geopolitics in the Arctic region is shaped first and foremost by the actors of the region themselves. Non-Arctic actors such as China, India or the EU (not including Sweden, Denmark, and Finland), have taken greater interest in the Arctic region, but the Arctic actors - both state and nonstate - have the dominant voice in the affairs of the region. Among the Arctic actors, one of the major trends is the emergence of three distinctive forms of managing geopolitical relations: a) bilateral, especially at the nation-state level; b) multilateral, but inclusive of actors from among a subset of Arctic countries; and c) pan-Arctic, aiming to include actors from all eight countries or at least all three geographic regions (North America, Russia, and Fennoscandia). Although the various forms of these relations among Arctic political communities create tensions from time to time, especially when some regions or actors feel excluded, the overall impact is to reinforce a pan-Arctic regional identity in the Arctic region. 


\subsubsection{Pan-Arctic region: multilateral governance}

The broadest level of governance in the Arctic region is at the pan-Arctic level. The multilateral governance bodies at this level encompass various political actors, including national and sub-national governments, Indigenous organizations, and non-governmental organizations. The number and scope of these organizations has changed significantly over the last decade, in response to the challenges facing the Arctic region, as well as the changing ideas about who should be involved in the governance of this region.

\section{Arctic Council}

The most important and inclusive pan-Arctic body is the Arctic Council. The establishment of the Arctic Council in 1996 as a forum for interstate cooperation among the eight Arctic states was remarkable for its time, particularly the inclusion of Indigenous organizations as Permanent Participants. There is no other inter-state body that has such a prominent role for Indigenous peoples in its decision-making.

As a governance body, the Arctic Council works through a series of multi-level meetings and consultations. Specifically, the Council operates in the following manner. A host country is appointed for a two-year period and convenes the major meetings of the Arctic Council. The host country operates the Council through a secretariat, which manages the administrative affairs of the Arctic Council. The host country also sets the policy direction and the priorities, which are agreed to at the previous Ministerial meeting. The Arctic Council operates through a multi-level series of meetings: a) Senior Arctic Officials (SAO) Meeting - senior representatives of the Member Nations and Permanent Participants; b) Ministerial Meeting - biannual high level meeting of the top governmental officials with either international relations or Arctic affairs responsibilities; and, c) Operational Activity - the Arctic Council conducts its detailed work through a number of specialized working groups and task forces.

The Arctic Council does not have any formal legislative or legal powers. It works through consensus, review/evaluation and public discussion of desired outcomes and progress toward established goals. The Arctic Council seeks to identify areas of concern, to develop practical, regionally appropriate solutions, and to mobilize governments to act accordingly. The Council uses moral political suasion rather than legislative authority to achieve its objectives. Given the complexity of the authority systems between nation-states and existing international organizations and legal regimes, it is hardly surprising that the Arctic Council has not operated, for the most part, as a formal decision-making body. 
Notably, however, the Arctic Council has engaged in formal decisionmaking in the recent case of Search and Rescue (SAR) (Poelzer, 2010) followed by the Agreement on Cooperation on Marine Oil Pollution Preparedness and Response in the Arctic (Arctic Council, 2013).

\section{Arctic Council Ministerial Meeting, Tromsö, Norway, 2009}

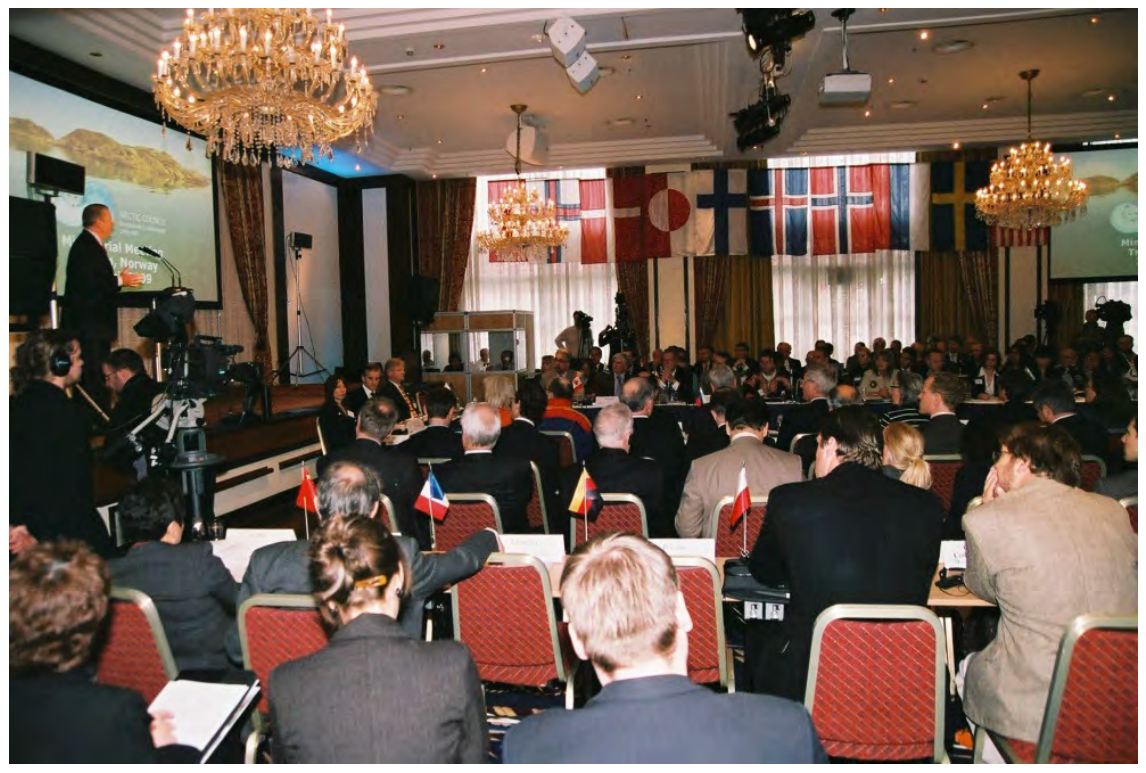

Credit: Harald Finkler.

One of the most important and, in many respects, unprecedented features of the Arctic Council is the role played by the Permanent Participants, organizations that represent many of the Indigenous peoples of the Arctic. The Arctic Council includes six Permanent Participant organizations, five of which represent Indigenous peoples in more than one country (the Sami Council, the Inuit Circumpolar Council (ICC), the Gwich'in Council International, the Arctic Athabaskan Council and the Aleut International Association). The Russian Association of Indigenous Peoples of the North (RAIPON) represents 41 different Indigenous peoples across the Russian Federation (Arctic Council, 2011). The work of the Permanent Participants in the Arctic Council is supported by the Indigenous Peoples Secretariat (IPS). The IPS was created in 1994 under the umbrella of the Arctic Environmental Protection Strategy and then became part of the governance framework of the Arctic Council when it was established in 1996.

Although they do not have voting status, the Permanent Participants play an active role in the deliberations of the Council. They sit at the 
Council alongside states, and take part in Council meetings and the various working groups that comprise the Council. Often they are able to exert influence through the state delegations of the voting members.

That being said, one of the on-going challenges in the operation of the Arctic Council is the capacity of the Permanent Participants to fulfill their roles as active members of the Council. Currently, fiscal support for Permanent Participants is highly uneven which makes meaningful participation by all Permanent Participant organizations very challenging. Moreover, Indigenous organizations often lack the capacity, in terms of personnel, to be fully engaged in all of the international and domestic files that are essential for their engagement. Progress on this front will require the Arctic Council as a whole, and the member states individually, to commit funds toward capacity building within Permanent Participant organizations, specifically in the area of Arctic policy development (Poelzer, 2010).

\section{Permanent Participants of the Arctic Council, SAO Meeting, Nuuk, Greenland, 2009}

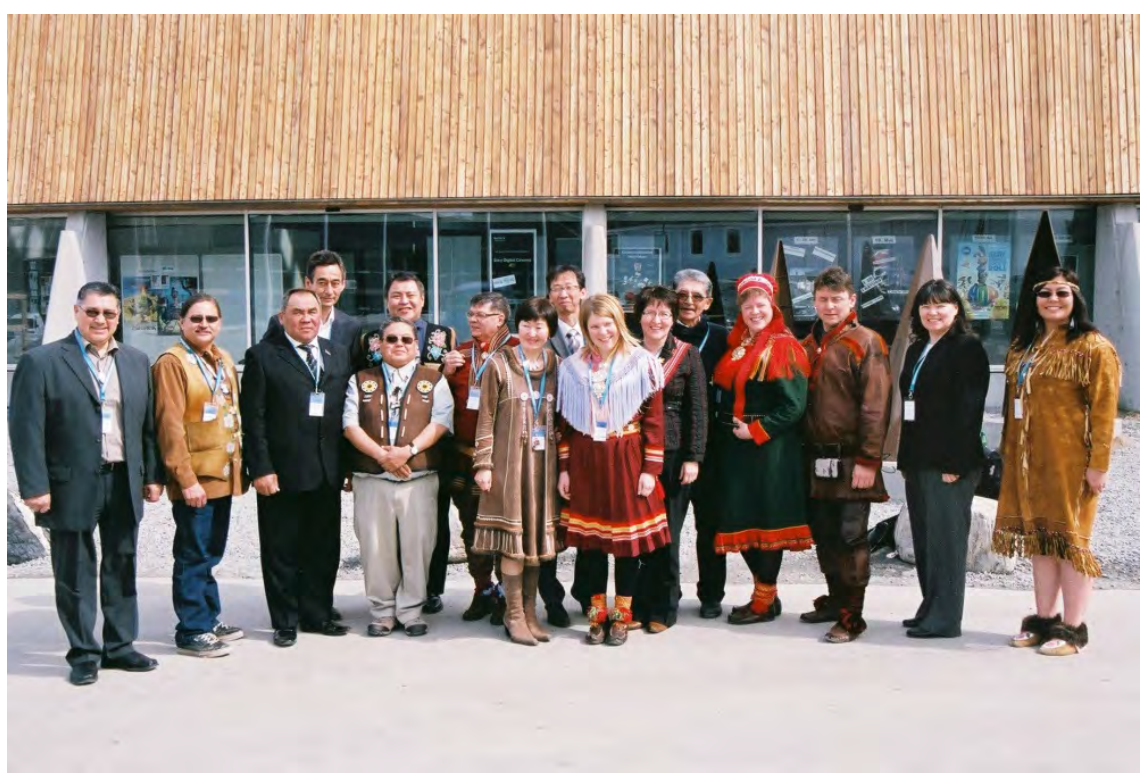

Credit: Harald Finkler.

The Arctic Council can - and does - raise the profile of Arctic issues, identify opportunities for collaborative action, and encourage rapid action on matters of pressing importance. It offers Indigenous Peoples' organizations and regional governments with unprecedented access to high-level international diplomatic conversations, providing a global model for Indigenous engagement in the process. Although the Arctic Council operates in the realm of soft power, rather than hard power, 
there is no denying the enormous impact that Indigenous people have played in shaping Arctic policy. This speaks to the effectiveness of moral suasion through the Arctic Council.

There has been some public debate about the effectiveness and presence of the Arctic Council. Many observers feel that the organization needs to be strengthened and member states need to attach sufficient or appropriate priority to its deliberations, if the Arctic Council is to play a leading governance and policy-making role for the Arctic region. Despite these perceived weaknesses, there have been several important developments since the first AHDR report. First, the Arctic Council made a very significant step forward in governance in the Arctic region by convening the eight Arctic Council States to negotiate the legally binding agreement on Aeronautical and Maritime Search and Rescue in the Arctic. The member states agreed that it is binding on all eight Arctic states. Even though the Arctic Council is not party to the agreement, it is difficult to overstate this precedent setting step. It has opened the possibility of the Arctic Council operating not only as a facilitator of public policy research, by developing guidelines and encouraging public implementation, but also as a governance body capable of convening Arctic States to conclude agreements that are binding on member states. Second, the creation of a Permanent Secretariat was an important step in institutionalizing the administrative capacity of the Arctic Council. The Permanent Secretariat is an important instrument to assist the Arctic Council in providing continuity and effectiveness in pursuing pan-Arctic policy initiatives at a time when global attention on the Arctic and its resources has never been stronger. Third, the formal adoption, at the Kiruna Ministerial Meeting in May 2013, of the Arctic Council Observer Manual For Subsidiary Bodies consolidates the rules and conditions around the participation of three categories of observers of the Arctic Council: (a) nonArctic States; (b) inter-governmental and inter-parliamentary organizations, global and regional; (c) non-governmental organizations. This arose out of increasing pressure from non-Arctic states to participate in the governance activities of the Arctic Council.

Another important contributor to the evolution of the Arctic Council is the granting of observer status to non-Arctic states and other entities. As of 2014, there are over 30 accredited observers including the following non-Arctic states: China, France, Germany, India, Italy, Japan, Republic of Korea, the Netherlands, Poland, Singapore, Spain, and the United Kingdom. Adding additional observers is contentious. On the one hand, proponents for expansion suggest that it is important to be proactive and bring non-Arctic state actors into the Arctic discussion, so that there 
is broader international support for policies aimed at benefitting the Arctic region. On the other hand, there are strong concerns that Northern voices, notably those of Indigenous peoples, will be marginalized as the interests of powerful, non-Arctic state actors begin to unduly influence the priorities and processes of the Arctic Council. Currently, all Arctic Council States and Permanent Participant Organizations agree that the participation of observer states in the activities of the Council should be strictly limited.

\section{Conference of Parliamentarians of the Arctic Region and Standing Committee of Parliamentarians of the Arctic Region}

Whereas the Arctic Council represents executive branches of the national governments of the Arctic states, the Conference of Parliamentarians of the Arctic Region is a forum for the legislative branches. The Conference is held on a biennial basis and has representatives from the legislative bodies of the eight Arctic Council states and the European Parliament. Representatives coming from the eight Arctic states are not necessarily from the Arctic regions of those countries, but represent the country as a whole. The Conference also engages the participation of Arctic permanent participants and observers, somewhat resembling the practices of the Arctic Council. The Standing Committee of Parliamentarians of the Arctic Region is responsible for the work of the Conference between sessions and meets several times annually. Although the Conference is able to influence debate on key Arctic issues, particularly those initiated and raised through the Arctic Council, the Arctic Council is undeniably the preeminent pan-Arctic political body.

\section{Indigenous diplomacy}

Indigenous organizations have a long history in geopolitics and governance at a pan-Arctic level and continue to play an influential role. The Inuit Circumpolar Council (ICC) has been one of the most active of all the Indigenous Arctic organizations. Founded in 1977, originally as the Inuit Circumpolar Conference, the ICC represents Inuit in four different Arctic Council member states: Russia (Chukotka); the United States (Alaska); Canada (Inuvialuit Settlement Region, Nunavut, Nunavik and Nunatsiavut) and Denmark (Greenland). Together with national-level Inuit organizations like the Inuit Tapiriit Kanatami (ITK) in Canada, the ICC provides a voice for Inuit on an international stage (Abele and Rodon, 2007). The ICC has been and remains an active participant in the Arctic Council. It has also been involved in various United Nations' forums, especially those concerning environmental issues such as Persistent Organic Pollutants. As a trans-national organization, it falls outside 
the realm of government and, like the Indigenous development corporations mentioned earlier, continues to push the boundaries of multi-level governance in the Arctic. It will be interesting to see how these transnational and national organizations evolve in the future, especially as Inuit governments in regions such as Greenland and Nunavut continue to develop and take on new responsibilities.

\section{The Northern Forum}

Pan-Arctic cooperation has not been limited to nation-states. One collaborative body for sub-national regions is the Northern Forum (NF). Like other regional and pan-Arctic organizations, the NF was established following the collapse of communism in Eastern Europe and the Soviet Union. Its membership consists of sub-national regions from Arctic states as well as non-Arctic states, such as Japan and the Republic of Korea. The mission of the NF is "to improve the quality of life of Northern peoples by providing Northern regional leaders a means to share their knowledge and experience in addressing common challenges (and) to support sustainable development and the implementation of cooperative socio-economic initiatives among Northern regions and international fora" (Northern Forum, 2013).

\subsection{Regional collaboration}

There are a number of examples of regional collaboration within the Arctic. Three in particular are the Nordic Council (NC), the West Nordic Council, and the Barents Euro-Arctic Council (BEAC). The NC was established 1952 as an inter-parliamentary forum for elected representatives from the Nordic countries of Denmark, Finland, Iceland, Norway and Sweden. The membership of this organization has expanded over the years to include the Faroe Islands, the Åland Islands and Greenland. The three Baltic states of Estonia, Latvia and Lithuania currently have observer status. Connected to the NC is the Nordic Council of Ministers (NCM), an intergovernmental forum for senior government officials. These organizations discuss matters of common interest, relating to trade, security and defense, the environment and, most recently, the future of the Nordic welfare state (Nordic Council, 2013).

The West Nordic Council (WNC) was established in 1985 to address issues of particular regional importance to the Faroe Islands, Greenland, and Iceland. The Council consists of six members of parliament from each of the respective members and the Annual General Meeting is the Council's highest authoritative body. Although the WNC addresses a 
broad spectrum of issues, fishery policy stands out as perhaps the most prominent. This is not surprising, given the important role that the fishing industry plays in the region's economy.

The BEAC is another intergovernmental forum that connects the countries of the Barents Sea region (Denmark, Norway, Sweden, Finland, Russia and the European Commission). The Council was established in 1993, mainly in response to the collapse of the Soviet Union: creating and maintaining pan-regional connections were particularly important as Russia began its transition to democracy. The BEAC also has a regional body called the Barents Regional Council (BRC), which consists of 13 member regions and an Indigenous representative from regional governments of Norway, Sweden, Finland and northwestern Denmark. At present, the focus of the BRC is on the development of natural resources and resource industries through the Barents 2010 Strategy (Barents Euro-Arctic Council, 2013).

Although the ICC is probably the only true pan-Arctic Indigenous organization, other Indigenous organizations have a very significant role on a regional level. Founded in 1956, the Sámi Council consists of fifteen plenary members drawn from nine Sámi associations among the four countries (Norway, Sweden, Finland, and Russia) that have Sámi populations. The Sámi Council participates not only in regional bodies such as BEAC, but also international bodies such as the Human Rights Committee of the United Nations and, of course, the Arctic Council. On the other side of the Atlantic, the Gwich'in Council International and the Arctic Athabaskan Council play a similar regional role, working across the borders of Alaska, Yukon, and the Northwest Territories.

\section{Bilateral issues}

Along with pan-Arctic relations, bilateral relations tend to capture much of the media attention because of the binary nature of some of the issues at stake. Border issues tend to dominate; however, cooperation in the areas of educational exchanges, scientific collaboration, and business development, though less prominent, have grown increasingly stronger and continue to cement the Circumpolar North as a region with its own set of policy concerns. Even in the area of challenging border issues, the trend since 2004 has been toward seeking resolution rather than exacerbating tensions.

In 2010, Norway and Russia concluded a treaty that resolved a decades old dispute over the maritime border between the two countries in the Barents Sea and the Arctic Ocean. The resolution of this dispute is critically important as the region is rich both in energy and fishery resources. At the same time, Canada and Denmark are working to resolve 
their dispute over Hans Island, a small island located between Greenland and Ellesmere Island. The extensive border between the two countries has been settled for some time, including up to the shores of the island. However, the island itself has been the subject of media attention and minor diplomatic disputes between the two countries. Between Canada and the United States, the wedge in the Beaufort Sea remains an unresolved, though not a particularly contentious, issue (Coates et al., 2010; see also Chapter 6, Legal Systems).

Bilateral issues are not restricted to nation-states; Indigenous governance bodies also play a significant role, infusing Indigenous values into policy processes. The Alaska-Yukon border is a case in point, where local communities are forging pathways around shared governance using science and traditional knowledge through the Yukon River Inter-Tribal Watershed Council (YRITWC), a consortium of 70 Alaska Native tribes and Canadian First Nations in neighboring Yukon Territory. The YRITWC was established in 1997 by an international accord, and its mission is to protect the Yukon River for future generations through clean-up and water quality monitoring projects. The YRITWC houses one of the largest environmental observing networks in the world, and is using the scientific data and traditional knowledge that they alone possess as a basis for asserting inherent governance rights over traditional lands and waters within the Yukon River watershed (Kimmel, forthcoming).

Despite the trend toward cooperation, traditional security issues, largely expressed as bilateral geopolitical issues, took on greater prominence over the past decade. The Canadian government has placed greater emphasis on exercising Arctic sovereignty, especially as it relates to the Northwest Passage. Russia has also emphasized the strategic significance of the Arctic, a region that had waned somewhat on the national policy priorities during the latter part of the twentieth century. Greater access to Arctic waterways resulting from climate change has heightened renewed interest in traditional security.

\section{Trends: Arctic geopolitics and governance}

There are several key trends in the geopolitics of the Arctic region. First, international relations operate from the local to the national levels and are likely to continue to do so, reinforcing a regional political community in the Circumpolar North. Second, Indigenous peoples and organizations are central actors in international forums and have a role unparalleled in other regions of the world in terms of their level of influence on geopolitical matters. Third, the pressure from non-Arctic state actors and nongovernmental organizations for inclusion on bodies and processes defining Arctic futures is almost certain to increase. This may have the conse- 
quence of weakening the role of Indigenous organizations and subnational actors from Arctic regions. Fourth, regional organizations such as the Nordic Council of Ministers and the Arctic Five are likely to be more prominent, especially as non-Arctic actors such as the European Union, China, and India play a larger role in the economic future of the Arctic.

\section{Textbox 5.3}

\section{Areas for Further Research}

One area that requires further research and consideration, both by scholars and governments, is the question of how the North is defined. In many Arctic countries, the boundary between north and south is often ambiguous and, at times, arbitrary. In Canada, for example, the North is often defined, politically and administratively, as the territory north of the 60 th parallel of latitude, when in fact, there are significant portions of Canadian territory below this line that exhibit the geographical, demographic and socio-economic features of northern regions. Indeed, the residents of these regions identify themselves as Northerners. Parallel situations occur across Russia and even parts of Fennoscandia. In many circumpolar states, arbitrary lines on maps, drawn by southern-based officials decades or even centuries ago, reinforce divisions within the North that are detrimental to the development of the region as a whole. Although some scholars have provided informed perspectives on the questions of what constitutes the North and how it should be defined (Coates, 1994; Coates and Morrison, 1992), more work needs to be done in comparative, circumpolar perspective. The current reality is that many Northerners are disenfranchised from governance processes and policy making about the North.

Another area for future research is gender and gender relations in the North. Compared to other areas such as Indigenous self-government and selfdetermination, regional and local government, economic development and public policy, there has been limited research on circumstances and challenges facing women throughout the Circumpolar North in the area of governance and geopolitics. This is surprising given the significant political strides made by women in the Arctic. Viewing the political development of the Arctic through the lens of gender will bring different perspectives and insights about the region and its people, as well as shed new light on important governance issues relating to political and economic participation, policy development and implementation, representation and international relations. 


\subsection{Key conclusions}

Upon closer inspection, the Arctic reveals a myriad of different and innovative governance structures. This multi-level system has both vertical and horizontal dimensions. Vertically, it extends from the local and community level to the realm of international politics. Horizontally, it encompasses typical government structures and institutions at the local, regional and national levels, as well as new, non-governmental actors and bodies. Over the last decade, this governance system has evolved and grown, in large part as a response to devolution and demands for greater regional autonomy. At the forefront of this movement towards greater autonomy are the Indigenous peoples of the Circumpolar North. Their efforts to secure self-determination and self-government are influencing Arctic governance in ways that will have a profound impact on the region and its inhabitants in the years to come.

In the last ten years, we have seen significant progress in terms of regional and local autonomy. New regions have come into being and older, more established regions have taken on new responsibilities. Although the devolution of authority brings great benefits to Northern regions and peoples, especially given the long history of colonization and control by southern-based governments, the transfer of authority also brings many challenges. Both the human and financial capacity of Northern governments is stretched as they attempt to take on the many new roles demanded by devolution. Building capacity in Northern regions is an ongoing priority, both now and in the future.

The capacity of Arctic regions and peoples to manage change is being stretched even more thinly by the geopolitical developments facing the Circumpolar North. Indigenous organizations and regional governments are playing a more active role in international forums such as the Arctic Council. Such activity certainly brings new perspectives and voices to the table; but it also places considerable pressure on already overburdened governments, politicians and Indigenous leaders. This pressure will likely mount now that new actors from outside the Arctic have started to play a more active role in the circumpolar governance bodies. The pressing issues that face the Arctic in the future will require a great deal of consideration and deliberation. Northern peoples and their governments continue to insist that they play a central role. 


\subsection{References}

Abele, F. and T. Rodon, 2007. Inuit diplomacy in the global era: the strengths of multilateral internationalism. Canadian Foreign Policy. 13(3) 45-65.

AHDR, 2004. Arctic Human Development Report. Einarsson, N., J. N. Larsen, A. Nilsson and O. R. Young (eds.). Stefansson Arctic Institute, Akureyri.

Arctic Council, 2013. Agreement on Cooperation on Marine Oil Pollution Preparedness and Response in the Arctic.

Arctic Council, 2011. Permanent Participants. http://www.arcticcouncil.org/index.php/en/about-us/permanent-participants/123resources/about/permanent-participants (15 September 2013).

Arter, D., 1999. Scandinavian Politics Today. Manchester University Press, Manchester.

Barents Euro-Arctic Council, 2013. http://www.beac.st/in-English/Barents-EuroArctic-Council (18 September 2013).

Berdahl, L., G. Poelzer and B. Beatty, 2012. Aboriginal voter turnout in Northern Saskatchewan. Aboriginal Policy Studies, 2(1): 26-41.

Berger, T.R., 2006. Conciliator's final report, March 1, 2006, The Nunavut Project. http://www.aadnc-aandc.gc.ca/DAM/DAM-INTER-HQ/STAGING/textetext/nlc_1100100030983_eng.pdf. (24 February 2014).

Coates, K. 1994. The rediscovery of the North: toward a conceptual framework for the study of the North/Northern Regions. The Northern Review. 12(13): 15-43.

Coates, K. and W. Morrison, 1992. The Forgotten North: A History of Canada's Provincial Norths. James Lorimer, Toronto.

Coates, K. and G. Poelzer, 2010a. Defining Indigenous space: the constitutional development of Aboriginal and resource rights in Canada. Georgetown Journal of International Affairs. 11(1): 17-26.

Coates, K. and G. Poelzer, 2010b. On the Front Lines of Canada's Northern Strategy, Federation of Canadian Municipalities.

Coates, K. S., P. W. Lackenbauer, W. R. Morrison and G. Poelzer, 2010. Arctic Front: Defending Canada in the Far North. Thomas Allen Publishers, Toronto.

Dacks, G., 2004. Implementing First Nations self-government in Yukon: lessons for Canada. Canadian Journal of Political Science. 37(3): 671-694.

Denmark and Faroe Islands in Constitutional Clash, 2013. Ice News: News from the Nordics. July 6, 2011). http://www.icenews.is/2011/07/06/denmark-and-faroeislands-in-constitutional-clash/ (4 November 2013).

Elder, N., A.H. Thomas and D. Arter, 1988. The Consensual Democracies? The Government and Politics of the Scandinavian States. Basil Blackwell, Oxford.

European External Action Service, Northern Dimension, 2013. http://eeas.europa.eu/North_dim/index_en.htm (20 November 2013).

Finland's Strategy for the Arctic Region, 2013. Prime Minister's Office Publications, Helsinki. http://vnk.fi/julkaisut/listaus/julkaisu/en.jsp?oid=395580 (20 November 2013).

Finnmark Act, 2005. Act No. 85 of June 17, 2005 relating to Legal Relations and Management of Land and Natural Resources in the County of Finnmark.

Government of Canada, 2010. Canada's Northern Strategy: Our North, Our Heritage, Our Future and Statement on Canada's Arctic Foreign Policy: Exercising Sovereignty and Promoting Canada's Northern Strategy Abroad. Government of Canada, Ottawa. http://www.international.gc.ca/arctic-

arctique/assets/pdfs/canada_arctic_foreign_policy-eng.pdf (20 November 2013). 
Government of the United States, 2013. National Strategy for the Arctic Region. http://www.whitehouse.gov/sites/default/files/docs/nat_arctic_strategy.pdf (12 March 2014).

Iceland, 2011. A Parliamentary Resolution on Iceland's Arctic Policy.

http://www.mfa.is/media/nordurlandaskrifstofa/A-Parliamentary-Resolution-onICE-Arctic-Policy-approved-by-Althingi.pdf (20 November 2013).

Inatsisartut, 2014. Members of the Inatsisartut. http://en.inatsisartut.gl/members-ofinatsisartut.aspx (16 February 2014).

Inter-Parliamentary Union, 2014. Women in National Parliaments. http://www.ipu.org/wmn-e/classif.htm (16 February 2014).

Inuit Tapiriit Kanatami, 2011. National Strategy on Inuit Education. https://www. itk.ca/publication/national-strategy-inuit-education (22 September 2011).

Josefsen, Eva, 2010. The Saami and the national parliaments: Channels for political influence. Mexico City: Inter-Parliamentary Union and United Nations Development Program.

Kimmel, M., forthcoming. Sovereigns, not stakeholders: an Alaskan study in fate control. Yearbook of Polar Law, Vol. 6.

Kingdom of Denmark, 2011. Denmark, Greenland and the Faroe Islands: Kingdom of Denmark Strategy for the Arctic 2011-2020. Copenhagen/Nuuk/Tórshavn: Ministry of Foreign Affairs/Department of Foreign Affairs/Ministry of Foreign Affairs. http://ec.europa.eu/enterprise/policies/raw-materials/files/docs/mssdenmark_en.pdf (20 November 2013).

Kingdom of Denmark, 2012. The Faroe Islands - a Nation in the Arctic, Opportunities and Challenges (in English): http://www.mfa.fo/Default.aspx?ID=13607 (10 March 2014).

Mereu, F., 2005. 3 Regions overwhelmingly agree to merge. Moscow Times. April 19, 2005.

Natcher, D.C., 2013. Gender and resource co-management in Northern Canada. Arctic 66/2: 218-221.

Nordic Council, 2013. http://www.norden.org/en/nordic-council (18 September 2013).

Northern Forum, 2013. http://www.Northernforum.org/servlet/content/ mission.html (18 September 2013).

Norwegian Ministry of Foreign Affairs, 2006. The Norwegian Government's High North Strategy.

Norwegian Ministry of Foreign Affairs, 2011. The High North: Visions and Strategies. Norwegian Ministry of Local Government and Regional Development, 2008. Local Government in Norway, 1-32.

Nuttall, M., 2008. Self-Rule in Greenland: towards the world's first independent Inuit state. Indigenous Affairs. 3-4: 64-70.

Osnovy gosudarstvennoi politiki Rossiiskoi Federatsii v Arktike na period do 2020 goda i dal'neishuyu perspective, 2008 [Foundations of the Russian Federation's State Policy in the Arctic Until 2020 and Beyond]. http://www.rg.ru/2009/03/30/arktika-osnovy-dok.html (18 July 2014).

Poelzer, G., 2013. Capacity building and sustainable development: comparative lessons and the Russian North, presentation at the Arctic Urban Sustainability Conference, The George Washington University, Washington, D.C., 31 May 2013.

Poelzer, G., 2011. Building the future of Nunavut, one student at a time. Our Schools, Centre for Policy Alternatives, 20(4): 15-30. 
Poelzer, G., 2010. Arctic Council: Recommendations for Greater Effectiveness and Ensuring Relevance in an Era of Global Change, Department of Indian Affairs and Northern Development.

Poelzer, G., 2009. Education: A critical foundation for a sustainable north. In: Northern Exposure, Abele, F., T.J. Courchene, Fl.L. Seidle and F. St. Hilaire (eds.), Northern Exposure. IRRP, Montreal.Remington, T., 2011. Politics in Russia. Longman, New York.

Strapagiel, Lauren, July 15, 2013. Harper appoints more women to cabinet than any other PM. http://o.canada.com/news/politics-and-the-nation/harper-cabinetshuffle-women-appointees/, (16 February 2014).

Sweden's Strategy for the Arctic Region, 2011. Government Offices of Sweden, Stockholm. http://www.government.se/content/1/c6/16/78/59/3baa039d.pdf (20 November 2013).

White, G., 2009. Governance in Nunavut: capacity vs. culture? Journal of Canadian Studies. 43(2): 57-81.

Wilson, G.N., 2008. Nested federalism in Arctic Quebec: a comparative perspective. Canadian Journal of Political Science. 41(1): 71-92.

Wilson, G.N., 2003. Abandoning the nest: regional mergers and their impact on the Russian North. Polar Geography. 26(3): 197-210.

Wilson, G.N., 2001. Matryoshka federalism and the case of the Khanty Mansiysk Autonomous Okrug. Post-Soviet Affairs. 17(2): 167-194.

Wilson, G.N. and C. Alcantara, 2012. Mixing politics and business in the Canadian Arctic: Inuit corporate governance in Nunavik and the Inuvialuit Settlement Region. Canadian Journal of Political Science. 45(4): 781-804.

Wilson, G.N. and H. Smith, 2011. The Inuit Circumpolar Council in an era of global and local change. International Journal. 64(4): 909-921.

Young, J.F. and G.N. Wilson, 2007. The view from below: local government and Putin's reforms. Europe-Asia Studies. 59(7): 1071-1088.

Yukon Government, 2012. The Umbrella Final Agreement, First Nation Final Agreements and Treaty Rights. http://www.eco.gov.yk.ca/landclaims/about.html (15 July 2014). 


\title{
6. Legal Systems
}

\author{
Lead authors \\ Nigel Bankes, University of Calgary, Canada and Timo Koivurova, Univer- \\ sity of Lapland, Finland
}

\section{Contributing authors}

Christina Allard (Luleå University, Sweden), Betsy Baker (Vermont Law School, USA), Sébastien Duyck (University of Lapland, Finland), Violet Ford (University of Lapland, Finland), Bent Ole Gram Mortensen (University of Southern Denmark, Denmark), Kristin Haraldsdóttir (University of Iceland, Iceland), Tore Henriksen (Arctic University of Norway, Norway), Inna Ignatyeva (Lomonosov Moscow State University, Russian Federation), Juha Joona (University of Lapland, Finland), Vladimir Khryazkov (National Research University - Higher School of Economics, Russian Federation), Mara Kimmel (Institute of the North, USA), David Leary (University of Technology, Australia), Natalia Loukacheva (University of Northern British Columbia, Canada), Øyvind Ravna (Arctic University of Norway, Norway), Rosemary Rayfuse (University of New South Wales, Australia), Thomas Trier Hansen (Nordic Law Group, Denmark), Adam Stepien (University of Lapland, Finland), and David VanderZwaag (Dalhousie University, Canada)

The formal legal systems of the Arctic comprise the legal systems of the eight nation states (including the Danish autonomous territories of Greenland and the Faroe Islands), of which three are federal states (with law-making powers devolved in varying degrees to their northern subunits) and five are unitary states. Greenland has a distinctive status, having opted for Self-Rule in 2009. Global and regional norms of international law are also significant, as are the norms of the European Union (EU) that bind Finland and Sweden as member states. In addition, as members of the European Economic Area (EEA) Agreement Norway and Iceland apply many EU norms. A more pluralist account of legal systems includes less formal arrangements such as the customary norms of Indigenous peoples (Watson Hamilton, 2013; Webber, 2013). There is a dynamic interaction between these different normative systems. 
The legal chapter of the first Arctic Human Development Report explored the diversity of the domestic legal systems of the Arctic states and their different legal cultures while emphasizing the importance of global norms, especially global human rights norms (Bankes et al., 2004). It also examined in some detail the status of Arctic Indigenous peoples in both national and international law. More specifically, it examined the extent to which each Arctic state provides explicit constitutional protection to the rights of Indigenous people and how each state protects the language rights, land rights and the governance rights of Indigenous peoples. The chapter concluded that the domestic laws of Arctic states offered increased recognition of language rights but observed that there was greater reluctance to recognize land ownership rights. The legal chapter also examined the property and resource regimes of the Arctic states and identified a trend within at least some Arctic states (e.g. USA \Alaska, Canada and Denmark in relation to Greenland and the Faroe Islands) for a transfer or devolution of authority from the center to the Arctic regions of these states (Bankes et al., 2004).

This chapter builds upon the legal chapter in the first AHDR. It continues to emphasize the importance of global norms (human rights norms, environmental norms and the law of the sea), but it also examines the extent to which regional and national laws have emerged that are specific to the Arctic region. The chapter follows two important trends that have taken place since the first $A H D R$ (2004). First, given the important role of Indigenous people throughout the Arctic (with the exception of Iceland), this chapter identifies the continuing elaboration of international instruments that address the rights of Indigenous peoples, including the adoption of the United Nations Declaration on the Rights of Indigenous Peoples. Second, the chapter explores the practices of Arctic states in delimiting their maritime boundaries with neighboring and opposite states, and in relation to extended continental shelf entitlements. While the media have tended to portray the Arctic as an area of conflict, the practices described here emphasize cooperation between Arctic states and the progressive settlement of maritime boundary delimitations. The chapter concludes that these trends add to legal certainty in this region, thereby facilitating Arctic human development. 


\section{Textbox 6.1}

\section{Abbreviations}

- AAC: Arctic Athabaskan Council.

- ACIA: Arctic Climate Impact Assessment.

- ACPB: Agreement on the Conservation of Polar Bears.

- CITES: Convention on International Trade in Endangered Species of Flora and Fauna.

- ECE: Economic Commission for Europe.

- EEA: European Economic Area (Agreement on).

- EU: European Union.

- ICESR: International Covenant on Economic, Social and Cultural Rights.

- ICCPR: International Covenant on Civil and Political Rights.

- ICRW: International Convention for the Regulation of Whaling.

- ILO: International Labor Organization.

- IMO: International Maritime Organization.

- IWC: International Whaling Commission.

- LOSC: Law of the Sea Convention.

- LRTAP: Long-range Transboundary Air Pollution (Convention on).

- NAMMCO: North Atlantic Marine Mammal Commission.

- NORDREG: Northern Canada Vessel Traffic Services Zone.

- NORSEL: Nordic Research Network for Sami and Environmental Law.

- NORSIL: Nordic Research Network for Sámi and Indigenous Peoples' Law.

- OAS: Organization of American States.

- POPs: Persistent Organic Pollutants.

- SAR: Search and Rescue (Agreement on).

- UNFCCC: United Nations Framework Convention on Climate Change.

- WTO: World Trade Organization.

\subsection{The multiple legal orders of the Arctic}

The Arctic is subject to a variety of legal norms that govern law and policy in the Circumpolar North. As described in the first $A H D R$, global norms and domestic laws of general application continue to provide the primary sources of law for the region. However, the Arctic is also subject to regional norms as a result of the membership of Arctic states in different regional organizations. We note that the number of Arctic specific norms has increased since the first $A H D R$. 


\subsubsection{The importance of global norms in the Arctic}

The Arctic is mostly governed by domestic laws and policies of general application, and by global international treaties and customary norms. These global norms include the Law of the Sea Convention (LOSC), the United Nations Framework Convention on Climate Change (UNFCCC) and the international human rights covenants described in the first AHDR (Bankes, 2004). When the Arctic Ocean was mostly ice-covered there was little need to consider the practical implications of the LOSC applying to the Arctic Ocean but the loss of sea ice caused the Central Arctic Ocean coastal states to emphasize the importance and applicability of LOSC with the adoption of Ilulissat Declaration in 2008. In that Declaration, the United States, Russia Canada, Norway and Denmark confirmed that the existing international legal framework provided "a solid foundation for responsible management by the five [Central Arctic Ocean] coastal states and other users of the Arctic Ocean through national implementation and application of relevant provisions." The parties confirmed that there is "no need to develop a new comprehensive legal regime to govern the Arctic Ocean". The Central Arctic Ocean coastal states took a similar position at a later meeting in Chelsea, Quebec specifically in the context of shipping and fisheries (DFAIT, 2010). While some, including non-governmental organizations such as the World Wildlife Fund (Koivurova and Molenaar, 2009) and Arctic Parliamentarians (2012), continue to urge the negotiation of a comprehensive legally binding treaty for the Arctic, others urge a more functional, problem solving approach that will focus on particular issues in developing a "governance or regime complex" (Rayfuse, 2007; Rayfuse, 2008; Rayfuse 2009; Young, 2011). The EU Parliament initially advocated an Antarctic treaty approach for the Arctic in its 2008 resolution (European Parliament, 2008), but now recognizes that there is a plurality of relevant instruments for the Arctic and thus "concludes that the Arctic region is not to be regarded as a legal vacuum, but as an area with well-developed tools for governance; nevertheless ... due to the challenges of climate change and increasing economic development, those existing rules need to be further developed, strengthened and implemented by all parties concerned" (European Parliament, 2011, para. 42).

The global nature of the environmental problems that face the Arctic such as climate change and ozone depletion requires states and other actors to consider how Arctic concerns can be addressed through other levels of governance (the problem of scale). In some cases, Arctic states have taken complementary measures to reinforce global developments. The Arctic Climate Impact Assessment is a prominent example (ACIA, 
2005). In other cases, Arctic states have been able to use regional fora such as the UN Economic Commission for Europe (UN ECE) to pursue Arctic goals (the ECE includes all Arctic states, although Canada, the USA, and Russia are less involved in some of the ECE's initiatives). One example was the elaboration of the Persistent Organic Pollutants (POPs) Protocol to the Convention on Long-range Transboundary Air Pollution (LRTAP) that was subsequently scaled up globally with the adoption of the Stockholm Convention on Persistent Organic Pollutants (Downie and Fenge, 2003). A more recent example is the effort to consider revisions to the 1999 Gothenburg Protocol to Abate Acidification, Eutrophication and Ground-level Ozone in order to regulate emissions of black carbon (UN ECE, 2010; Boone, 2012). However, some commentators have argued that the Arctic Council needs to step up its policy development work to adequately address the regional implications of global developments (French and Scott, 2009; Duyck, 2012).

\section{The treatment of Arctic issues within multilateral treaty regimes}

There are examples of multilateral regimes taking up Arctic issues. For example, there has been increased interest in Arctic species among the parties to the Convention on International Trade in Endangered Species (CITES) and the Convention on Migratory Species (the Bonn Convention) (Bankes, 2013). The United States has proposed uplisting polar bear (Ursus maritimus) from Appendix II to Appendix I at the last two meetings of the CITES Conference of the Parties (Parsons and Cornick, 2011; Clark et al., 2013). The proposals failed: those opposed argued that the species did not meet the criteria to warrant an Appendix I listing. Similarly, the EU has tried to focus attention on narwhal within the context of the "significant trade review" procedure of CITES (Fitzmaurice, 2009; Bankes, 2013). This procedure is triggered when a state identifies conservation concerns with a species listed on Appendix II. Once the procedure is triggered, range states for the species (in this case, Greenland and Canada) must justify why trade in the listed species should be allowed to continue. Such efforts to deal with Arctic issues at a global level are often contentious. If polar bear uplisting provides one example, another, that of the International Convention for the Regulation of Whaling (ICRW), offers an even more compelling illustration (Gillespie, 2002). Some Arctic states have responded to the ongoing moratorium under the ICRW on the taking of large whales with various strategies including catching under the category of Aboriginal Subsistence Whaling (Kingdom of Denmark and the United States), withdrawal from the Convention (Canada), taking reservations (Norway and Iceland), and creating an organization, the North Atlantic Marine Mammal Commission (NAM- 
MCO), that is more responsive to the needs and values of Arctic states (Gillespie, 2002; Caron, 1995). Some Arctic states have followed similar strategies under CITES and the Bonn Convention (Bankes, 2013).

\subsubsection{The application of different regional norms in the Arctic}

Regional norms introduce new concepts and ideas to the Arctic, even when these norms only bind some Arctic states. For example, the European Union is an important source of new normative ideas in such diverse areas as energy market liberalization (Johnston and Block, 2012), networks of protected areas (EU, 1992) and marine environmental policy (EU, 2008; Long, 2011). Similarly, the Convention for the Protection of the Marine Environment of the North-East Atlantic (OSPAR Convention) provides critical guidance in relation to dumping activities and more generally in relation to offshore oil and gas activities. The Council of Europe and the broad range of treaties adopted under its auspices deal with such diverse topics as minority rights and the conservation of flora and fauna. In addition, the membership of Canada and the United States in the Organization of American States (OAS) gives Arctic relevance to the jurisprudence of the Inter American Court of Human Rights and the Inter American Commission on Human Rights. While neither Canada nor the United States is party to the American Convention on Human Rights (and therefore not bound by the rulings of the Court with respect to the Convention) it is clear that the decisions of the Court influence the Commission in its deliberations and in its interpretation of the cognate provisions of the American Declaration of the Rights and Duties of Man. Both the United States and Canada are subject to the jurisdiction of the Commission with respect to the Declaration by virtue of their membership in the OAS.

In sum, there are various regional regimes operating at different scales which serve to introduce new ideas and concepts which are relevant to human development in the Arctic. 


\section{Textbox 6.2}

\section{European Union Law, international trade law, human rights law and the Arctic seal hunt}

A good example of how various legal orders are relevant in the Arctic is EU Regulation (EC) No. 1007/2009 of the European Parliament and of the Council of 16 September 2009 on trade in seal products (the Seal Regulation). This regulation has a strong impact on Arctic livelihoods and has led to serious legal and political controversies. The Regulation was adopted due to public concerns over animal welfare as a result of the hunting methods used in the commercial seal hunt, in particular in Atlantic Canada. Notwithstanding the fact that the Regulation contains an exemption for seal products "from hunts traditionally conducted by Inuit and other Indigenous communities and which contribute to their subsistence," the adoption of the Regulation has triggered various disputes and legal action by both Inuit and non-Indigenous hunters, organizations and seal products processors before the EU's General Court (GC) and by Canada and Norway in the World Trade Organization (WTO).

In the GC action the Inuit argued (unsuccessfully) that the seal products ban seriously decreased the demand for seal products irrespective of the hunting methods and origins of the seal products. In its argument the EU referenced the UN Declaration on the Rights of Indigenous Peoples and the importance of the Inuit hunt as an integral part of their culture and identity, to justify exempting from the Regulation products from seals harvested by Indigenous people.

The case demonstrates the complexity of modern day regulation. For the EU, the matter is framed as a matter of market harmonization - a measure aiming to improve conditions for the establishment and functioning of the internal market - given that two of its member states had already banned trade in seal products while others were in the process of doing so. For Canada and Norway the Regulation breaches the EU's legal obligations under the WTO agreements. For the Inuit, it is a matter of human rights.

The seal products ban affected the EU's application for observer status to the Arctic Council. Using the slogan "No Seal, No Deal" Inuit organizations tried to convince the Canadian government not to accord the EU observer status in the Council. The Council deferred acting on the EU's application at the Kiruna ministerial meeting in May 2013. 
The WTO panel issued its report in November 2013 (WTO, 2013). The Panel concluded that while the EU Regulation violated some basic disciplines under the Agreement on Technical Barrier to Trade (TBT) and the General Agreement on Tariffs and Trade (GATT), those violations were in principle justified as necessary to protect public morals. In so concluding the Panel, unlike the EU General Court, found that the dominant purpose of the Regulation was the protection of public morals and not the proper functioning of the internal market. The Panel also found that the EU had not properly justified the exceptions in the Regulation and in particular an exception for seals placed on the market taken in a management harvest in the EU Baltic states and the Indigenous community harvest exception. The Panel Report directs the EU to bring these non-conforming aspects of the Regulation into conformity with the agreements. This requires adjustments to the exceptions rather than to the basic approach underlying the Regulation. Both Canada and the EU have appealed the Panel Report to the WTO’s Appellate Body.

\section{Heading out for Seal Hunt, Greenland}

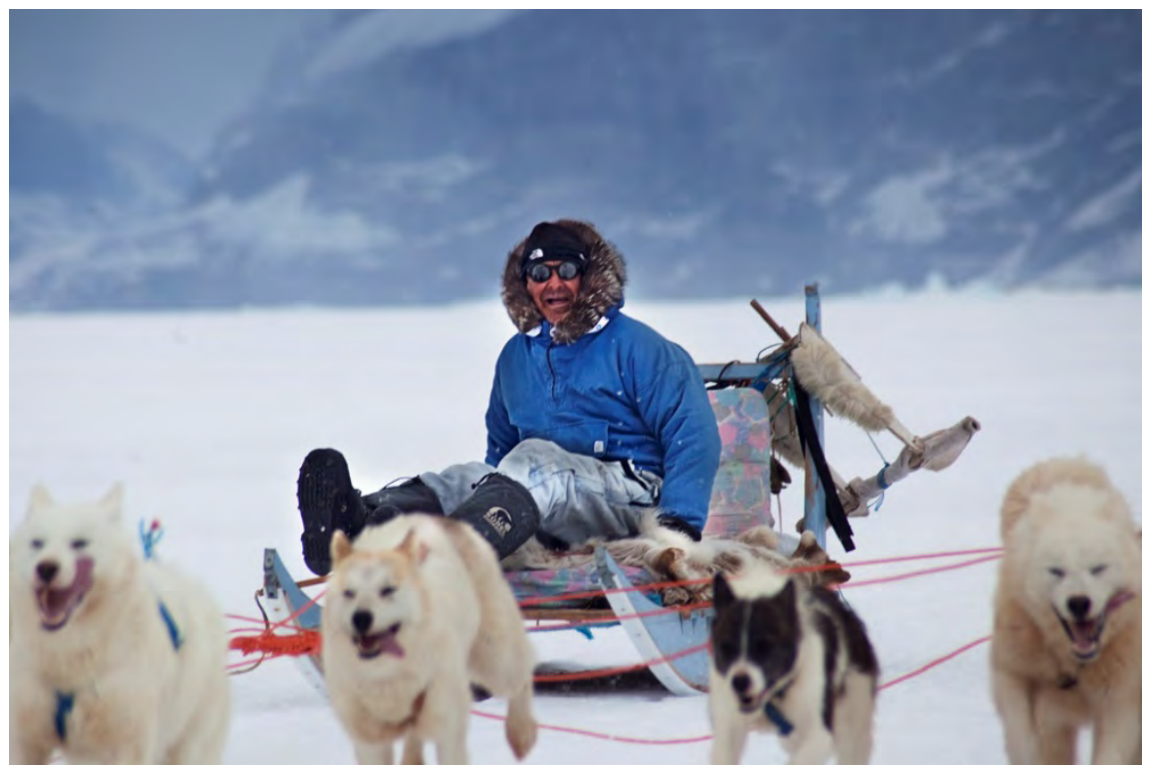

Source: GridArendal; photo by Lawrence Hislop. 


\subsubsection{Arctic specific norms}

While most of the norms that apply in the Arctic are general (i.e. national laws that are of general application and global norms) there is a limited amount of law, both domestic and international, that is specific to the Arctic. Some of these specific norms have a relatively long history. For example, there is the agreement relating to the creation of the international ice patrol (dating back to 1913 immediately following the sinking of the SS Titanic, and currently based on the 1956 Agreement Regarding Financial Support of the North Atlantic Ice Patrol), the unique Spitsbergen Treaty of 1920, the 1973 Agreement on Conservation of Polar Bears (ACPB), and the 1992 agreement establishing the North Atlantic Marine Mammal Commission (NAMMCO). And there is one article in the LOSC (Article 234) that addresses the rights of coastal states to adopt and enforce pollution laws in relation to ice-covered areas (McRae and Goundry, 1982; Bartenstein, 2011b).

The Arctic Council is the most important institutional exception, but, importantly, the Council was established by a political accord rather than by a treaty (Bloom, 1999; Koivurova and Vanderzwaag, 2007). Nevertheless the Arctic Council (and its precursor, the 1991 Arctic Environmental Protection Strategy) has been responsible for the creation of guidelines, manuals and other soft-law instruments that are specific to Arctic conditions.

Seen in this light, the adoption by the eight Arctic States of the Agreement on Cooperation on Aeronautical and Maritime Search and Rescue in the Arctic in 2011 at the Nuuk Ministerial Meeting of the Arctic Council (the SAR Agreement) is important. As the first international treaty to come out of the Arctic Council, it establishes that even if the Arctic Council cannot enact binding decisions for its members, it can act as a forum for negotiating Arctic-specific treaties (Vasiliev, 2013). An additional legally binding agreement on oil spill preparedness and response was adopted by the eight Arctic States at the Arctic Council's Kiruna Ministerial Meeting in May 2013. Both agreements are firmly anchored in existing global agreements (the 1979 International Convention on Maritime Search and Rescue, the 1944 Convention on International Civil Aviation and the 1990 International Convention on Oil Pollution Preparedness, Response and Cooperation) and implement these conventions in the Arctic setting. There is also growing interest in the negotiation of an Arctic Ocean fisheries agreement, initially proposed by the United States Congress in 2008. The scientific community and non-governmental organizations are pressing for the adoption of such an agreement and preliminary discussions have commenced. 
Hilary Clinton signing Arctic Council's Nuuk Declaration, Greenland, May 2009

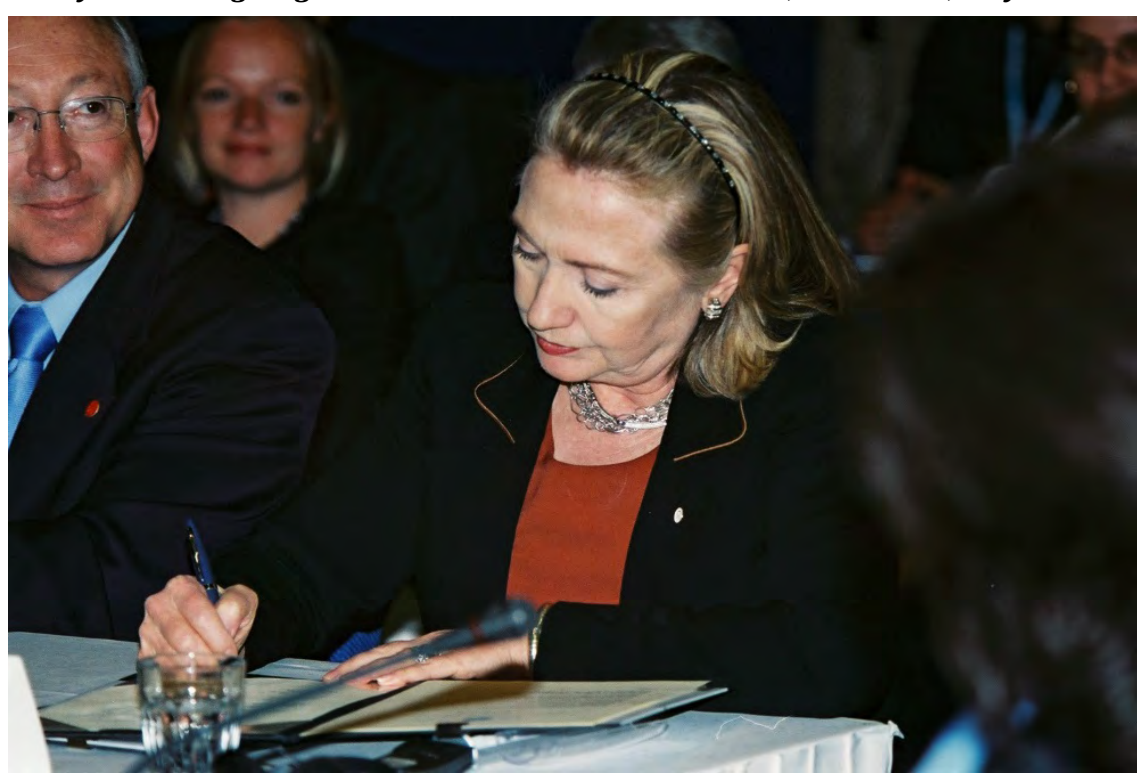

Credit: Harald Finkler.

Another example of the development of an Arctic or polar specific legal regime is the process within the International Maritime Organization (IMO) to adopt a legally binding Code to make Arctic navigation safer and environmentally more sustainable in an increasingly ice-free ocean (Lalonde, 2013). This would replace the current non-legally binding 2009 Polar Code (Guidelines for Ships Operating in Polar Waters, IMO 2009) (Jensen, 2008; Boone, 2012).

There are also efforts within existing Arctic instruments to deepen cooperation in response to external threats, especially environmental threats. The ACPB, for example, has been revitalized as a result of the decision of the Parties (in this case the five range states) to convene regular formal meetings and to develop management approaches for polar bear in response to the loss of polar bear habitat (sea ice) caused by climate change (Tromsø, 2009 and Iqaluit, 2011 and Federation of Russia, 2013). 


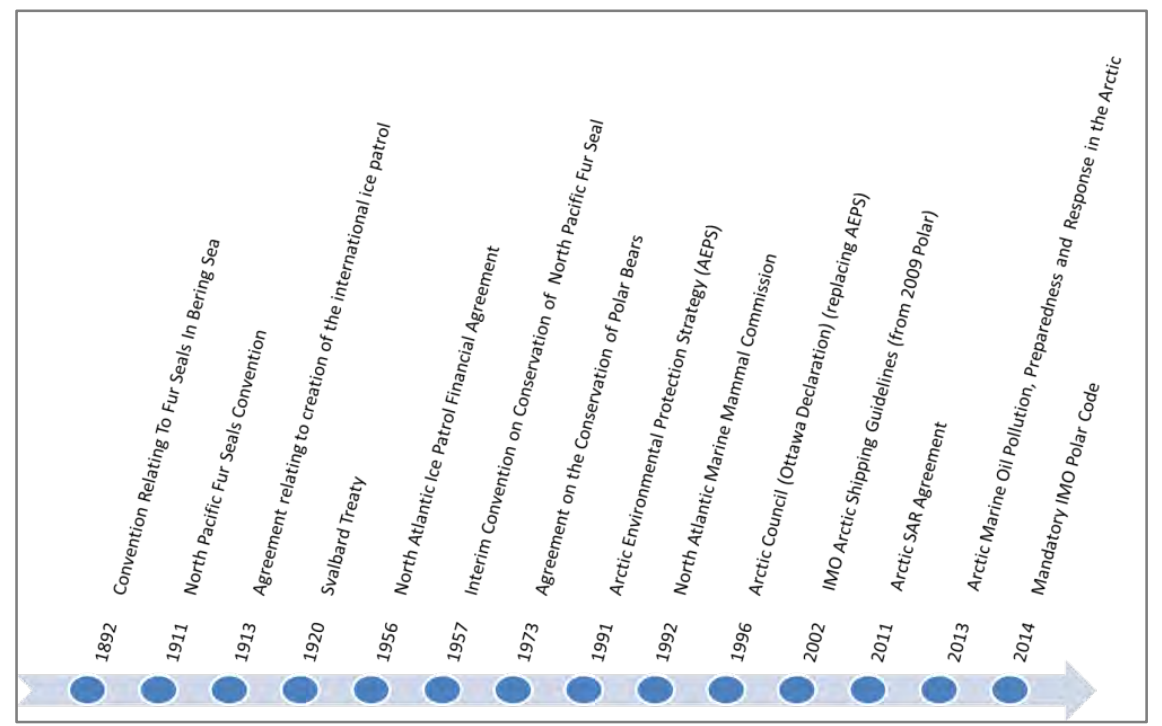

\section{Arctic specific norms in domestic law}

Arctic states have also developed their own domestic Arctic-specific regulations, especially in relation to marine areas. For example, in July 2012 the Russian President signed new legislation for the Northern Sea Route (Federal Law No. 132) while Canada made its Northern Canada Vessel Traffic Services Regulations (NORDREG) mandatory as of July 2010 (Bartenstein, 2011a; McDorman, 2012). Likewise, the decision of the US North Pacific Fisheries Management Council (2009) to ban all new industrial fisheries in its waters north of the Bering Strait, including the Chukchi and Beaufort Seas evidences an Arctic specific focus. Similarly, there are laws that recognize self-governance or co-management for Indigenous peoples in the Arctic, such as Norway's Finnmark Act (2005), Greenland's SelfGovernance Act (2009) and various land claims agreements and selfgovernance arrangements in Canada. Other initiatives include the decision of the United States to list polar bear as threatened (2008) under its Endangered Species Act and the proposal of 2011 for uplisting of ringed seal and bearded seal.

\subsubsection{Emerging research and scholarship on Arctic and polar law}

Developments in Arctic-specific governance arrangements are also reflected within the growing interests of the community of legal scholars that conceptualize the Arctic as a distinct region worthy of study. Notable advances over the past decade in this context include the launch of 
two new legal journals, one with an Arctic focus (Arctic Review on Law and Politics) since 2010 (Ravna, 2010) and one with a polar focus (Yearbook of Polar Law) since 2009. Integral to the Yearbook of Polar Law is the annual symposium on polar law. The crystallization of the Arctic within the legal imagination has fostered the development of Arctic and Polar law graduate programs, curriculum, and teaching materials, notably the Polar Law Textbooks (Loukacheva, 2010; Loukacheva, 2013). Of equal note is the Thematic Network on Arctic Law, an initiative led by the Northern Institute for Environmental and Minority Law at the University of Lapland and launched in 2010 under the auspices of the University of the Arctic. Regional initiatives such as the Nordic Research Network for Sámi and Indigenous Peoples' Law (NORSIL) program based at the University of Tromsø complement these broader pan-Arctic and polar programs.

\subsubsection{Summary: legal orders and norms}

The legal norms that apply in the Arctic are principally global norms and national laws of general application. Regional norms are also important in the Arctic although the "region" in question is frequently not the Arctic itself but some other regional grouping (e.g. the EU) which includes some but not all Arctic states. However, there is also evidence of the increasing importance of Arctic specific norms. While there have long been Arctic specific treaties, such as the Spitsbergen Treaty, the number of Arcticspecific instruments is growing and comprises both soft-law arrangements of the Arctic Council and Arctic-specific treaties such as the SAR Agreement and the Agreement on Oil Spill Preparedness and Response.

\subsection{Developments in international law pertaining to Indigenous peoples}

As outlined in $A H D R$ (2004), there are two distinct approaches to developing international human rights law to protect the rights of Indigenous peoples. One approach is to elaborate specific norms recognizing the rights of Indigenous peoples. Examples of this approach include the International Labor Organization's Convention concerning Indigenous and Tribal Peoples in Independent Countries (ILO Convention 169) (Swepston, 2011; Yupsanis, 2011), the UN Declaration on the Rights of Indigenous Peoples, and the proposed American Declaration on the Rights of Indigenous Peoples within the OAS. The second ap- 
proach is to recognize that the rights of Indigenous peoples may be protected through the application of general human rights norms. Examples of this approach include the application of the minorities' provision of the ICCPR (Article 27) (Möller, 2011) and the property rights protections offered by regional human rights instruments (Pentassuglia 2011; Bankes, 2011a; Bankes, 2011b; Kovler, 2012). Both approaches continue to evolve.

\subsubsection{The Indigenous-specific approach}

\section{The UN Declaration on the Rights of Indigenous Peoples}

The Indigenous-specific approach culminated in the adoption of the UN Declaration on the Rights of Indigenous Peoples by the General Assembly (UN DRIP, 2007). Initially, two Arctic states voted against adoption (USA and Canada) and one abstained (Russia). However, both the USA and Canada have since endorsed the Declaration but expressed concerns (Sweden did likewise at the time of adoption). Russia has yet to endorse the Declaration but has indicated that it will take the fundamental principles underlying the Declaration into account in developing new legislation provided that those principles do not conflict with the Constitution of the Russian Federation (Andrichenko, 2011).

Figure 6.2: UN Declaration: the position of Arctic States on the UN Declaration on the Rights of Indigenous Peoples

\begin{tabular}{ll}
\hline Country & Stance on the declaration \\
\hline Canada & $\begin{array}{l}\text { Voted against, then endorsed with reservations (10 November 2010; http://www.aadnc- } \\
\text { aandc.gc.ca/eng/1292354321165), noting that the Declaration is aspirational and not } \\
\text { legally binding and expressing "concerns with various provisions ... including provisions } \\
\text { dealing with lands, territories and resources; free, prior and informed consent when used } \\
\text { as a veto; self-government without recognition of the importance of negotiations; intellec- } \\
\text { tual property; military issues; and the need to achieve an appropriate balance between } \\
\text { the rights and obligations of Indigenous peoples, States and third parties." "These con- } \\
\text { cerns ... remain." } \\
\text { Denmark voted in favor with no explanation of its vote. } \\
\text { Denmark } \\
\text { (Greenland) }\end{array} \begin{array}{l}\text { Voted in favor emphasizing that the adoption of the Declaration "would strengthen the } \\
\text { rights of Indigenous peoples worldwide and serve as a comprehensive framework for } \\
\text { cooperation in implementing new minimal international standards for Indigenous people's } \\
\text { rights." }\end{array}$ \\
$\begin{array}{l}\text { No explanation but voted in favor (no Indigenous peoples in the country). } \\
\text { Iceland }\end{array}$ \\
$\begin{array}{l}\text { Voted in favor and explained that the Declaration "set the standard of achievement to be } \\
\text { pursued in a spirit of cooperation" but emphasized that "several articles in the Declaration } \\
\text { dealt with the exercise of self-determination and stipulated that such rights should be } \\
\text { exercised in the framework of international law." }\end{array}$
\end{tabular}




\begin{tabular}{|c|c|}
\hline Country & Stance on the declaration \\
\hline $\begin{array}{l}\text { The Russian } \\
\text { Federation }\end{array}$ & $\begin{array}{l}\text { Abstained from voting and has yet to endorse the Declaration. Russia explained that, } \\
\text { overall, it "supported the rights of Indigenous people and the development of internation- } \\
\text { al standards in that regard" but that the process to produce the Declaration was non- } \\
\text { transparent and did not enjoy consensus. Russia also argued that the Declaration "was not } \\
\text { a truly balanced document, in particular regarding land and natural resources or the } \\
\text { procedures for compensation and redress" but that it would still continue to "foster } \\
\text { cooperation in order to protect and promote the rights of Indigenous peoples." }\end{array}$ \\
\hline Sweden & $\begin{array}{l}\text { Voted for but with serious reservations as to the existence of group rights. Sweden stated } \\
\text { that the declaration includes several references to collective rights and explained that "While } \\
\text { the Swedish Government had no difficulty in recognizing such rights outside the framework } \\
\text { of international law, it was of the firm opinion that individual human rights prevailed over the } \\
\text { collective rights mentioned in the Declaration." }\end{array}$ \\
\hline $\begin{array}{l}\text { The United } \\
\text { States }\end{array}$ & $\begin{array}{l}\text { Voted against and then endorsed with a full explanation ( } 15 \text { December } 2010 \text { ) of its under- } \\
\text { standing (http://www.state.gov/documents/organization/184099.pdf) of what the Decla- } \\
\text { ration requires, also noting that the Declaration is not legally binding, but has moral and } \\
\text { political force. On the basis of a thorough review, the USA explains, e.g. that the Declara- } \\
\text { tion advances a distinct international concept of self-determination specific to Indigenous } \\
\text { peoples, which "promote[s] the development of a concept of self-determination for } \\
\text { Indigenous peoples that is different from the existing right of self-determination in inter- } \\
\text { national law. In addition, the USA supports the strong articles of the declaration as regards } \\
\text { Indigenous after peoples' rights over their lands, territories, and natural resources: United } \\
\text { States stresses the importance of the lands, territories, resources and redress provisions } \\
\text { of the Declaration in calling on all States to recognize the rights of Indigenous peoples to } \\
\text { their lands, territories, and natural resources. Consistent with its understanding of the } \\
\text { intention of the States that negotiated and adopted the Declaration, the United States } \\
\text { understands these provisions to call for the existence of national laws and mechanisms for } \\
\text { the full legal recognition of the lands, territories, and natural resources Indigenous peo- } \\
\text { ples currently possess by reason of traditional ownership, occupation, or use as well as } \\
\text { those that they have otherwise acquired." }\end{array}$ \\
\hline
\end{tabular}

Main source:http://www.un.org/News/Press/docs/2007/ga10612.doc.htm

The Declaration has been referred to in domestic court proceedings and even in international arbitral practice (see for example Grand River Enterprises Six Nations Ltd et al. v. United States). Domestically, there are several Canadian cases in which the petitioner has relied upon the Declaration in framing its case (see, for example, Hupacasath First Nation $v$. Canada, 2013 FC 900) and the Supreme Arbitration Court of the Russian Federation has also referred to the Declaration (December 3, 2012, No. VAS-12173/12).

Similarly, the Declaration is cited in international debates within the International Whaling Commission (IWC) in an Arctic-specific context. For many years, the ICRW parties recognized that aboriginal subsistence whaling should be permitted to continue notwithstanding the 1982 general moratorium on the taking of large whales. However, it is still necessary for the IWC to approve the necessary amendments to permit this to occur. For example, in 2010 at the 62nd Meeting of the IWC, Denmark renewed an application to permit a Greenlandic harvest of humpback whales and an adjustment to the quota for minke whales. Speaking in support of the application, the Greenlandic minister referred to the UN 
Declaration but so also did many other states, who "spoke in support of the rights of Indigenous people, the need for aboriginal subsistence whaling quotas and the requirement for the IWC to act in the spirit of the UN Declaration" (IWC, 2010, 19-20). Similar sentiments were expressed a year later by Sweden and other states at the 63rd meeting in the context of establishing an Aboriginal Subsistence Whaling Working Group (IWC, 2011, 23-25).

\section{Other Indigenous-specific instruments}

Another important development is the proposal of the Nordic states and the Sámi peoples of Norway, Finland and Sweden to produce a Nordic Sámi Convention. The parties released a Draft of the Convention in 2005 (Sámi Council, 2005) and negotiations are currently underway to finalize an international treaty on the basis of the draft (Åhrén, 2007; Koivurova 2006/7; Koivurova, 2008; Bankes and Koivurova, 2013).

The only modern Indigenous-specific convention currently in force is ILO Convention 169. Of the seven Arctic states with Indigenous peoples in their territory, only Denmark (Greenland) and Norway have ratified the Convention. Finland and Sweden are exploring the possibility of doing so and ratification of the Convention is an explicit goal of the present Government of Finland and written into its plan for its term of office. Ratification is not on the agenda for any of the three federal states in the Arctic: Canada, Russia and the USA.

\subsubsection{The general human rights approach}

\section{Global norms}

The general human rights approach - especially the work in the treatymonitoring bodies of UN human rights treaties - has also increasingly addressed the rights of Indigenous peoples, including Arctic Indigenous peoples. One discernible trend is that the human rights treaty monitoring bodies associated with the International Covenant on Civil and Political Rights (ICCPR) and the International Covenant on Economic, Social and Cultural Rights (ICESCR) have commenced a consistent practice of asking states to address the position of Indigenous peoples under common Article 1 (the right of all peoples to self-determination) in their regular reports. In doing so, both the Human Rights Committee and the Committee on Economic, Social and Cultural Rights take the view that Indigenous peoples are peoples within the meaning of Common Article 1 to the ICCPR and the ICESCR. The monitoring body for the latter has asked Russia to report on its Indigenous peoples under common Article 1 and the former 
has asked USA, Canada, Norway, Sweden and Finland to do the same for their respective Indigenous peoples. These monitoring bodies have concluded that Indigenous peoples possess collective rights enshrined in Common Article 1. Both committees have also started to view their treaties in light of the UN Declaration on the Rights of Indigenous Peoples. We identify this as an important development for two reasons. First, it helps "mainstream" the significance of this instrument in the broader normative human rights framework, and, second, it helps to confirm the normative significance and status of the Declaration, which some prefer to view as a purely aspirational rather than declaratory document.

\section{Regional human rights norms}

The Inter-American system has been very active in protecting the land and resource rights of Indigenous peoples in Central and South America in cases before the Inter-American Court, such as Awas Tingni $v$ Nicaragua (2001), Saramaka v Suriname (2007), three cases involving Paraguay (2005, 2006, 2010), Kichwa Indigenous People of Sarayaku v Ecuador (2012) and several decisions of the Inter American Commission including Maya Communities v. Belize (2003) (Bankes, 2011b). These decisions support the proposition that the State parties to the Inter American Convention on Human Rights are obliged to delimit, demarcate and title lands that Indigenous communities occupy in accordance with their customary norms. However, while Canada and the USA are members of the OAS, they are not State Parties to the Inter American Convention on Human Rights and are thus not subject to the jurisdiction of the Inter American Court.

In the first Arctic specific petition made to the Commission, the Inuit alleged that the USA could be legally responsible for the human rights violations of the Inuit as a result of climate change. The Commission ultimately took the view that the petition was not admissible, because the information it provided was insufficient for it to make a determination. While unsuccessful, the petition did provoke a lot of discussion on the relationship between human rights and climate change (Osofsky, 2009; Heinämäki, 2009). On April 23, 2013, the Arctic Athabaskan Council (AAC) filed a similar petition with the Commission, this time targeting Canada and asking the Commission to declare that Canada is undermining the petitioners' human rights by not adequately regulating emissions of black carbon (soot), a short-lived climate pollutant which contributes to Arctic warming and melting (AAC, 2013).

Indigenous peoples have been less successful in raising their issues, especially land rights issues, within the European regional human rights system (Koivurova, 2011). Some suggest that this is because the European system is based firmly on protecting individual rights, not group 
rights, while others point to the systemic problems associated with prosecuting land rights issues before the European Court of Human Rights and in particular the requirement that the petitioner must first exhaust its local remedies in a state-based system that is designed to protect settler titles and land uses rather than Indigenous titles and uses (Bankes, 2011a). Judge Ziemele drew attention to this systemic problem in her dissenting opinion in Handölsdalen Sami Village $v$ Sweden (2010), a case dealing with Sámi reindeer grazing rights.

One possible European forum for dealing with Indigenous peoples is the Framework Convention for the Protection of National Minorities (a Council of Europe agreement) which has the capacity to deal with Indigenous rights in Sweden, Finland, Norway, the Russian Federation and Sweden (Council of Europe, 2004). The monitoring body of the Convention, the Advisory Committee on National Minorities, issues opinions, provides comments on state reports and produces thematic commentaries including those concerning Indigenous minorities. For instance, in its 2008 thematic commentary on the Effective Participation of Persons belonging to National Minorities in Cultural, Social and Economic Life and in Public Affairs, the Committee underlined the participation rights of representatives of Indigenous peoples in any decision-making affecting the use of land in their traditional areas of residency because their economic situation is closely connected to land (Commentary, 2008).

\section{Textbox 6.3}

\section{On the treatment of gender in international instruments pertaining to Indigenous peoples}

Human rights instruments pertaining to Indigenous peoples sometimes, but not always, specifically address gender. At the national level, Canada's constitution specifies that the protection of aboriginal and treaty rights is "guaranteed equally to male and female persons." At the international level Article 44 of the UN Declaration on the Rights of Indigenous Peoples makes it clear that the rights and freedoms recognized in the Declaration are "equally guaranteed to male and female Indigenous individuals" while Article 22 calls upon States to pay particular attention "to the special needs of Indigenous elders, women, youth, children and persons with disabilities" and to take special measures "to ensure that Indigenous women and children enjoy the full protection and guarantees against all forms of violence and discrimination." Similarly, Article 3 of ILO Convention 169 provides that "The provisions of the Convention shall be applied without discrimination to male and female members of these peoples." By contrast, there is no specific non-discrimination clause in the operative text of the Draft Nordic Saami Convention (Koshan, 2013). 


\subsubsection{The implications of international norms for domestic law}

While we can identify a clear trend in the consolidation of Indigenous rights in international law, this trend has only penetrated the domestic legal systems of the Arctic states in a limited fashion. This is particularly the case in the three large federal Arctic states, the USA, Canada and the Russian Federation, although as noted above there has been some limited engagement with the Declaration within the judicial systems of at least Canada and Russia. The four Nordic states with Indigenous peoples living in their territory (Denmark, Norway, Sweden, Finland), and especially Norway, have been more open to influences from international law (Graver and Ulfstein, 2004). Each of these states is party to the two international covenants and Norway and Denmark are both parties to the ILO Convention 169. Denmark has enacted a new self-governance act, which entitles the Inuit of Greenland to self-determination as a people (Kleist, 2010).

\section{The Greenland flag in the capital Nuuk, Greenland}

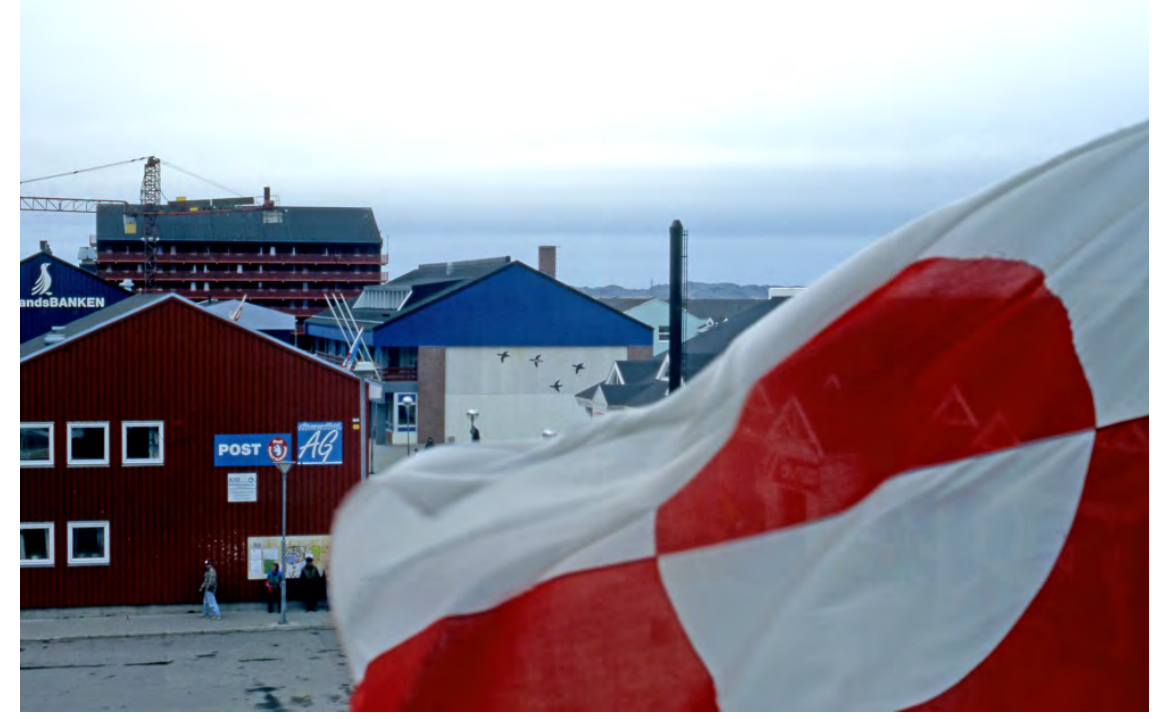

Source: GridArendal; photo by Peter Prokosh.

Norway has implemented ILO Convention 169 in part through the Finnmark Act, by which Norway accords 95\% of Finnmark (northernmost county of Norway) to the so-called Estate, consisting of an equal number representatives of Sámi parliament and other residents of Finnmark (Ravna, 2011). Even if the Finnmark Act has been criticized for not implementing the ILO 169 properly (including the criticism that it has not 
been implemented in marine and other Sámi regions), it concretely consolidates Norway's obligation to identify and recognize Sámi lands (Special Rapporteur, 2011; Ravna, 2013).

One relevant way to track domestic legal developments in the area of Indigenous peoples' rights is through the Arctic Social Indicators reports. The first ASI report identified two domains of human development indicators that correspond particularly well to the evolution of international norms pertaining to Indigenous people (ASI, 2010). First, cultural well-being and vitality are concepts enshrined in the UN Declaration on the Rights of Indigenous People, and operationalized within the $A S I$. The $A S I$ identifies cultural indicators such as rights to language use and retention as a way of documenting "state recognition for fostering . . cultural development" (ASI, 2010: 99). Similarly, the extent to which a state uses Indigenous languages "in government and legal proceedings . . . and official documents" likewise indicates support for Indigenous cultures and is also consistent with provisions of the Declaration. Cultural well-being indicators also identify state recognition of cultural and ethnic institutions as indicative of governmental support of the legal rights to culture, a concept consistent with principles contained within the Declaration.

The second relevant set of indicators contained in the first ASI report is the notion of fate control. Defined as the ability for people to control their own destiny, "fate control" parallels the right to self-determination identified in the Declaration (ASI, 2010: 130). Notably, the ASI developed indicators necessary to measure fate control at a collective as well as individual level, making this approach relevant to Indigenous communities where collective identity is critical to cultural values. This indicator measures legally enforceable rights to land and sea resources as a key component of fate control, as well as the ability to "effectively govern the use of the resources" (ASI, 2010: 136, 137). Finally, fate control measures in the ASI use "recognition of human rights" as an indicator of human development (ASI, 2010: 142, 144, Tables 7.1 and 7.3).

Overall, despite important developments in the international law relating to Indigenous rights since the first $A D H R$, these developments, except in the cases of Norway and Denmark $\backslash$ Greenland, have not had a significant impact on the domestic legislation of the Arctic states. Even if most Arctic states see themselves as strong supporters and promoters of Indigenous and human rights around the world, they have not done much to advance Indigenous rights on the basis of international law standards at home. Similarly, even though Arctic states have accorded Indigenous peoples a unique standing in the Arctic Council as permanent 
participants, this status has generally not helped these Indigenous peoples to convince their governments to incorporate and implement international law guaranteeing Indigenous rights in domestic law (Koivurova and Stepien, 2011; Bankes, 2009b). This does not mean that Indigenous rights are not protected in these countries since clearly there are important protections in their domestic legal systems, but it does mean that most Arctic states are reluctant to have their domestic laws measured against international standards in a rigorous and authoritative way.

\subsubsection{Summary: international law and Indigenous peoples}

There have been important developments in international human rights law as it pertains to the rights of Indigenous peoples since the first $A H D R$. These developments include Indigenous-specific norms and general human rights law, both global norms and regional norms. The most important development was the adoption of the UN Declaration. The work of the Inter American Court of Human Rights in developing a jurisprudence on the Indigenous right to property is also very significant. We anticipate that both Indigenous-specific and general human rights norms will continue to develop as part of the general decolonization of international law as it pertains to Indigenous peoples.

\subsection{The delimitation of continental shelf boundaries between Arctic states and the extended continental shelf entitlements of Arctic states}

The law of the sea has become a prominent object of discussion, in academic, non-governmental and governmental policy-making contexts within the Arctic. This is mainly due to the retreat of sea ice, driven by climate change (ACIA, 2005). The significant decrease in sea-ice in the summer of 2007 and the almost simultaneous planting of the Russian flag on the sea-bed underneath the North Pole provoked the media, and led some international relations scholars (BBC, 2007) to argue that there is a scramble for Arctic Ocean resources between Central Arctic Ocean coastal states. In the eyes of these observers, the Arctic Ocean had suddenly become a place for a scramble for resources, perhaps even triggering traditional military security challenges (Borgerson, 2008; since qualified in Borgerson, 2013). As the 2008 Paper from the High Representative and the European Commission to the European Council argued, "the increased accessibility of the enormous hydrocarbon resources in the 
Arctic region is changing the geo-strategic dynamics of the region with potential consequences for international stability and European security interests" (European Commission, 2008 a, section 6).

The actual record however does not support these claims. Rather, there is steady and peaceful progress between the Arctic states in delimiting overlapping maritime boundaries, thereby enabling investments in resource development in these areas. Moreover, the delineation of the outermost limits of the continental shelves has progressed in the Arctic Ocean in the manner required by the LOSC and by the law of the sea in general.

\subsubsection{Settlement of maritime boundaries between Arctic coastal states}

Since land is the basis of all claims to maritime zones it is hardly surprising that in the Arctic, where the continents converge, there are many overlapping claims. These overlapping claims must be resolved either by agreement or international litigation or arbitration. This is an area in which we have seen important developments in the Arctic since the first AHDR. Arctic states have continued to resolve their disputes in accordance with international law, including Article 83 of LOSC for the delimitation of continental shelf boundaries between adjacent and opposite states and Article 76, which prescribes criteria and a process for defining the outer limits of an extended continental shelf (Byers, 2013). The LOSC delimitation rules are generally considered to have codified customary law and as such are binding on the United States even though the United States has yet to accede to the Convention.

Figure 6.3 describes the status of delimitations as between Arctic states. 
Figure 6.3: Maritime delimitation arrangements between Arctic States

\begin{tabular}{|c|c|c|}
\hline Description & Status & Basis of arrangement \\
\hline $\begin{array}{l}\text { CanadalUnited States, Beau- } \\
\text { fort Sea }\end{array}$ & No agreement & \\
\hline Canada\Denmark, Davis Strait & $\begin{array}{l}\text { Settled (except for Hans } \\
\text { Island, and Lincoln Sea) }\end{array}$ & $\begin{array}{l}\text { Agreement of } 17 \text { December } 1973 \text { delimit- } \\
\text { ing the Continental Shelf (CS) }\end{array}$ \\
\hline Canada\Denmark, Lincoln Sea & Tentative agreement & $\begin{array}{l}\text { Tentative agreement, November } 28,2012 \text {, } \\
\text { will extend the current equidistance line to } \\
200 \text { NM from the coasts of Ellesmere Is and } \\
\text { Greenland }\end{array}$ \\
\hline $\begin{array}{l}\text { United States \Russia Ber- } \\
\text { ing\Chukchi Sea }\end{array}$ & $\begin{array}{l}\text { Treaty signed, entered into } \\
\text { force provisionally, but not } \\
\text { yet ratified by Russia }\end{array}$ & $\begin{array}{l}\text { Agreement settling the maritime boundary, } \\
1 \text { June } 1990\end{array}$ \\
\hline Denmark \& Greenland \Iceland & Settled & $\begin{array}{l}\text { Agreement between Denmark and the } \\
\text { Home Rule Government and Iceland } \\
\text { delimiting the CS and Fishing Zone (FZ), } 11 \\
\text { November } 1997 \text { (1) }\end{array}$ \\
\hline Denmark \& Faroe Islands & Settled & $\begin{array}{l}\text { Agreement on the Maritime Border be- } \\
\text { tween the Faroe Islands and Iceland, } 2 \\
\text { February } 2007\end{array}$ \\
\hline $\begin{array}{l}\text { Denmark \& Greenland \Iceland, } \\
\text { CS Area beyond } 200 \text { NM, } \\
\text { Irminger Sea (Kunoy, 2013) }\end{array}$ & $\begin{array}{l}\text { Agreed Minutes (not } \\
\text { formally binding) }\end{array}$ & $\begin{array}{l}\text { Agreed Minutes on the Delimitation of the } \\
\text { CS beyond 200NM, Irminger Sea } 16 \text { Janu- } \\
\text { ary } 2013\end{array}$ \\
\hline $\begin{array}{l}\text { Denmark and Norway re Faroe } \\
\text { Islands }\end{array}$ & Settled & $\begin{array}{l}\text { Agreement of } 15 \text { June } 1979 \text { delimiting the } \\
\text { CS and FZ }\end{array}$ \\
\hline $\begin{array}{l}\text { Faroe Islands, Iceland and } \\
\text { Norway, Southern Part of the } \\
\text { Banana Hole, NE Atlantic, Area } \\
\text { beyond 200NM (Kunoy, 2013) }\end{array}$ & $\begin{array}{l}\text { Agreed Minutes (not } \\
\text { formally binding) }\end{array}$ & $\begin{array}{l}\text { Agreed Minutes, between Faroe Islands, } \\
\text { Iceland and Norway, Southern Part of the } \\
\text { Banana Hole, NE Atlantic, Area beyond } \\
\text { 200NM, } 20 \text { September } 2006\end{array}$ \\
\hline $\begin{array}{l}\text { Denmark and Norway re. Jan } \\
\text { Mayen and Greenland }\end{array}$ & Settled, 1993 ICJ Judgment & $\begin{array}{l}\text { Agreement of } 18 \text { December } 1995 \text { delimit- } \\
\text { ing the CS and FZ and Protocol of 1997(1) }\end{array}$ \\
\hline $\begin{array}{l}\text { Denmark \& Greenland \Norway } \\
\text { re Svalbard }\end{array}$ & Settled & $\begin{array}{l}\text { Agreement of } 20 \text { February } 2006 \text { delimiting } \\
\text { CS and FZ }\end{array}$ \\
\hline $\begin{array}{l}\text { Iceland and Norway (Jan } \\
\text { Mayen) }\end{array}$ & $\begin{array}{l}\text { Settled. Conciliation Com- } \\
\text { mission Report of } 1981\end{array}$ & $\begin{array}{l}\text { Agreement of } 22 \text { October } 1981 \text { on the CS } \\
\text { and Protocol of } 1997 \text { (1) }\end{array}$ \\
\hline Norway and Russia & Settled & $\begin{array}{l}\text { Agreements of } 15 \text { February } 1957 \text { re Va- } \\
\text { rangerfjord, } 11 \text { July } 2007 \text { Varangerfjord } \\
\text { area and } 15 \text { September } 2010 \text { re Barents } \\
\text { Sea and Arctic Ocean. The latter contains } \\
\text { two important annexes dealing with } \\
\text { fisheries matters and transboundary } \\
\text { hydrocarbon deposits }\end{array}$ \\
\hline
\end{tabular}

Notes:

1. These agreements followed consultations between Norway, Iceland and Denmark concerning the final delimitation of the maritime waters between Jan Mayen, Iceland and Greenland where the delimitation lines of the three states intersect.

2. The delimitation of the continental shelf in the Hatton Rockall area between Iceland, UK, Ireland and Denmark Faroe Islands remains unsettled. 
In the period since the first AHDR, the most significant development in Arctic delimitation practice has been the conclusion of the Treaty between Russia and Norway concerning Maritime Delimitation and Cooperation in the Barents Sea and the Arctic Ocean in 2010 (Henriksen and Ulfstein, 2011; Ørebech, 2012). The treaty brings to an end a forty-year process and supports orderly governance within the region at a time when the loss of sea-ice will lead to increased navigation and marine transport (Henriksen and Ulfstein, 2011).

The general picture that emerges of Arctic delimitation practice is that Arctic states have made steady progress towards resolving their maritime boundary issues. In one case (Norway $\backslash$ Denmark regarding Jan Mayen) the International Court of Justice effected the delimitation and in another case (Iceland \Norway) an International Conciliation Commission helped the parties reach a mutually satisfactory conclusion. Most delimitation disputes have been resolved by negotiations.

In some cases there remain minor outstanding matters to be resolved (e.g., Hans Island, between Canada and Greenland), and at least one important boundary (between the United States and Canada in the Beaufort Sea) still needs to be delimited. Canada has traditionally taken the position that the maritime boundary should follow the 141st line of longitude (which is the land boundary between Yukon and Alaska) while the United States prefers an equidistance line. More recently, Baker and Byers (2012) note that Canada may have reason to re-think its position in the context of an extended shelf claim insofar as an extended shelf equidistance line will trend northwest and ultimately cross back over the 141st longitude line. There is another set of outstanding issues in relation to Svalbard, especially as regards the legal status of surrounding waters (Churchill and Ulfstein, 2010; Anderson, 2009).

\subsubsection{Claims to an extended continental shelf: a scramble for resources or evidence of cooperation?}

The procedures for establishing an extended continental shelf are prescribed in the LOSC and as such apply to the parties to the convention. The United States has also been participating cooperatively (especially with Canada in the Beaufort Sea area) in the scientific work that is required in order to justify an extended shelf entitlement. Article 76 of LOSC stipulates that the continental shelf that accrues to the coastal state comprises the seabed and subsoil beyond the territorial sea throughout the natural prolongation of its land territory to the outer edge of the continental margin (or 200 nautical miles, whichever is the greater), subject to some 
overall limits prescribed by the technical language of this Article. States are to delineate the outer limits of their shelf entitlement by working through the Commission on the Limits of the Continental Shelf. Thus, LOSC contemplates that a party to the Convention shall submit information about the outer limits of its continental shelf to the Commission within ten years of becoming party to the Convention. This deadline has been relaxed by virtue of decisions taken by the Meeting of States Parties to the Law of the Sea (SPLOS). The Commission shall then examine the submission and "make recommendations" "to the coastal state with respect to the outer limits of the shelf". It is the coastal state itself that ultimately establishes the outer limits of its shelf. The Convention provides that limits established "on the basis of these recommendations shall be final and binding" (Elferink, 2001; Macnab, 2004; McDorman, 2013).

Russia, Norway, Iceland, Denmark and Canada have all made submissions to the Commission. Russia was the first to make a submission in 2001 but the Commission asked for further information relating to the Central Arctic Ocean basin, data that Russia is still collecting. The Government of Denmark together with the Government of the Faroe Islands made two partial submissions one in respect of the area north of the Faroe Islands, and one in respect of the Faroe-Rockall Plateau Region. The Government of Denmark together with the Government of Greenland also made two partial submissions; one in respect of the Southern Continental Shelf of Greenland and one in respect of the area northeast of Greenland. Still outstanding is a submission in respect of areas north and northeast of Greenland. Iceland made a partial submission as regards Ægir Basin area in 2009 and in the southern and western parts of the Reykjanes Ridge, but not including the Hatton-Rockall area, which is the subject of overlapping claims by other states. In December 2013, Canada filed a partial submission in respect of the outer limits of its continental shelf in the Atlantic Ocean and preliminary information in respect of the outer limits of its continental shelf in the Arctic Ocean.

There has been a remarkable degree of collaboration between Arctic states in completing the scientific work required to support the various submissions. For example, Canada has conducted four joint surveys with the USA in the Beaufort Sea as well as a number of joint surveys with Denmark, and has also cooperated with Russia. A similarly cooperative approach has been displayed by states in allowing the Commission to consider applications notwithstanding the possibility of a dispute between adjacent or opposite states.

This co-operative practice and the statement in the 2008 Ilulissat Declaration to the effect that the Central Arctic Ocean coastal states "re- 
main committed to this legal framework [law of the sea] and to the orderly settlement of any possible overlapping claims" together make it clear that suggestions in the media that there is a political power game between the Central Arctic Ocean coastal states as to who gets to occupy seabed resources are exaggerated (Ilulissat Declaration, 2008).

\section{Figure 6.4: International Maritime Boundaries in the Arctic}

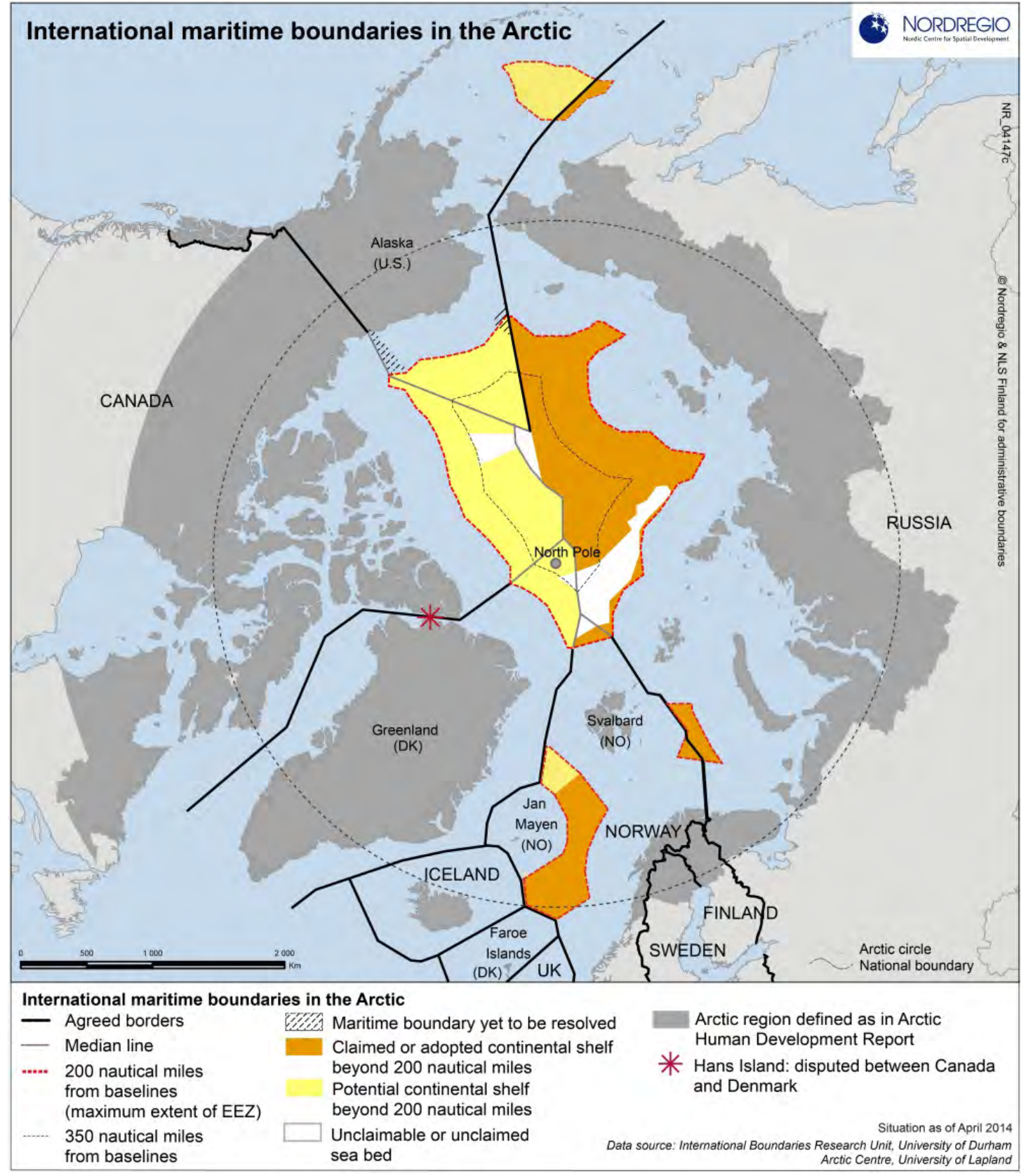




\subsubsection{Summary: marine boundary delimitation issues}

Arctic coastal states have made steady progress in delimiting their maritime boundaries and in completing their extended continental shelf filings with the Commission on the Limits of the Continental Shelf. The most notable delimitation agreement is that between Norway and Russia. Both activities establish that the coastal states are committed to continuing cooperation in the Arctic Ocean and are committed to applying the global norms of the law of the sea in their relationships. We anticipate that this trend will continue and that Arctic states will amicably resolve remaining delimitation issues in relation to their maritime boundaries.

\subsection{Chapter conclusions and gaps in knowledge}

This chapter has dealt with three main topics. The first was the interplay between Arctic specific norms and global norms. Both play important roles and while we can discern a trend towards the increased adoption of Arctic specific norms, global norms will always play an important role. In part this is because of the issue of scale. A problem such as climate change cannot be solved locally or even regionally. Globalization in all its forms (economic, cultural, communication, etc.) also requires global norms to deal with the associated regulatory challenges.

The second main topic dealt with was the development of international human rights law in relation to Indigenous peoples. Here, much of the interplay continues to be between general human rights norms (e.g. the protection of property) and the Indigenous specific norms of the UN Declaration and ILO Convention 169. While there is some evidence of the development of more specialized regional norms, such regional processes (within the Nordic states and within the Americas) have yet to result in the adoption of final instruments. The interplay between international law and domestic law is more limited, especially in the three federal Arctic states. However, as the language of the UN Declaration (in particular the language of free, prior, informed consent) is increasingly mainstreamed in a variety of fora from the International Whaling Commission to the European Court of Justice we may see increased interplay between the two normative orders.

The third main topic was the delimitation of maritime boundaries and extended continental shelf delineation. Here the main message is that notwithstanding media accounts of conflict, Arctic coastal states are 
going about both exercises peacefully, cooperatively and strictly in accordance with LOSC and general international law.

As was the case with the legal chapter in the first $A H D R$, this chapter has necessarily been selective. It has also operated, like the first $A H D R$ at the apex of the legal hierarchy, concerning itself with international and constitutional norms rather than detailed regulations and interactions between legal rules and the daily lives of Arctic residents. The legal chapter in the first AHDR (2004) called for a more rigorous and systematic comparative law of the Arctic; it also called for studies of actual practice in relation to the implementation of laws and the interaction between state norms and folk norms. There continue to be gaps in our understanding of the relevance of law for the attainment of sustainable development and a greater emphasis on these issues and laws of human relationships (e.g., criminal and family law) rather than issues of resource governance might usefully be taken up and assume more prominence in a legal systems chapter in a third edition of the Arctic Human Development Report.

\subsection{References}

AAC (Arctic Athabaskan Council), 2013. Petition To The Inter-American Commission On Human Rights Seeking Relief From Violations of The Rights of Arctic Athabaskan Peoples Resulting From Rapid Arctic Warming And Melting Caused By Emissions Of Black Carbon By Canada. http://www.ecojustice.ca/files/aac-petitionapril-2013/at_download/file (23 February 2014).

ACIA, 2005. Arctic Climate Impact Assessment. Cambridge University Press, Cambridge.

Åhrén, M., 2007. The Saami Convention. Journal of Indigenous Peoples Rights, 3: 8-39.

Anderson, H. D., 2009. The status under international law of the maritime areas around Svalbard. Ocean Development and International Law, 40:373.

Andrichenko, L. V., 2011. Kommentariy k stat'ye 69 konstitutsii Rossiyskoy Federatsii [Comment to Article 69 of the Constitution of the Russian Federation]. In: Zorkin, V.D. (ed.). Kommnetariy k Konstitutsii Rossiskoy Federatsii (postateynyy), 2nd edition, revised. M.: Norma, Infra-M.

Arctic Parliamentarians, 2012. Parliamentarians of the Arctic Region, Conference Statement (September 5-7, 2012). http://www.arcticparl.org/files/conferencestatement $\% 2 \mathrm{C}$-final-draft1-2.pdf (23 October 2013).

ASI, 2010. Arctic Social Indicators - a follow-up to the Arctic Human Development Report. Larsen, J.N., P. Schweitzer, and G. Fondahl (eds.). TemaNord. Nordic Council of Ministers, Copenhagen.

Baker, J. and M. Byers, 2012. Crossed lines: the curious case of the Beaufort Sea maritime boundary dispute. Ocean Development and International Law, 43: 70-95.

Bankes, N., 2004. Legal systems. In: Arctic Human Development Report, pp. 101-118. Akureyri: Stefansson Arctic Institute. 
Bankes, N., 2009a. Climate change and the regime for the conservation of Polar bears. In: T. Koivurova, E. Carina, H. Keskitalo and N. Bankes (eds.), Climate Governance in the Arctic, pp. 351-382. Springer, Berlin.

Bankes, N., 2009b. Land claim agreements in Arctic Canada in light of international human rights norms. Yearbook of Polar Law, 1: 175.

Bankes, N., 2011a. The protection of the rights of Indigenous peoples to territory through the property rights provisions of international regional human rights instruments. Yearbook of Polar Law, 3: 57-112.

Bankes, N., 2011b. Indigenous land and resource rights in the jurisprudence of the Inter-American Court of Human Rights: comparisons with the draft Nordic Saami Convention. German Yearbook of International Law, 54: 233-80.

Bankes, N., 2013. The conservation and utilization of marine mammals in the Arctic region. In: Molenaar, E., A. Elferink and D. Rothwell (eds.). The Law of the Sea and the Polar Regions: Interactions Between Global and Regional Norms, pp. 293-321. Martinus Nijhoff, Leiden, Boston.

Bankes, N. and T. Koivurova (eds.), 2013. The Proposed Nordic Saami convention: National and International Dimensions of Indigenous Property Rights. Hart Publishing, Oxford.

Bartenstein, K., 2011a. Navigating the Arctic: the Canadian NORDREG, the International Polar Code and regional cooperation. German Yearbook of International Law, 54: 77-124.

Bartenstein, K., 2011b. The "Arctic Exception" in the Law of the Sea Convention: a contribution to safer navigation in the Northwest Passage? Ocean Development and International Law, 42: 22-52.

BBC 2007, Russia plants flag under N Pole (2 August 2007), http://news.bbc.co.uk/2/hi/europe/6927395.stm (23 February 2014).

Bloom, E.T., 1999. Establishment of the Arctic Council. American Journal of International Law 93(3): 712-22.

Boone, L., 2012. Development of an environmental chapter in the Polar Code: introducing a new player - black carbon. Yearbook of Polar Law, 4: 541-60.

Borgerson, S., 2008. Arctic meltdown; The economic and security implications of global warming. Foreign Affairs, March/April, 63-77.

Borgerson, S., 2013. The coming Arctic boom. As the ice melts, the region heats up. Foreign Affairs, July/August, 76-89.

Byers, M., 2013. International Law and the Arctic. Cambridge University Press, Cambridge.

Caron, D., 1995. The International Whaling Commission and the North Atlantic Marine Mammal Commission. American Journal of International Law, 89: 154.

Churchill, R. and G. Ulfstein, 2010. The disputed maritime zones around Svalbard. In: Nordquist, M., J. Norton Moore and T. Heidar (eds.). Changes in the Arctic Environment and Law of the Sea, pp. 551-593. Martinus Nijhoff, Leiden, Boston.

Clarke, D., C. Meek, J. Cheechoo, S. Clark, A Foote, D. Lee and G. York, 2013. Polar bears and CITES: A rejoinder to Parsons and Cornick. Marine Policy, 38: 365-368.

Commentary, 2008. Commentary on the effective participation of persons belonging to national minorities in cultural, social and economic life and in public affairs. Advisory Committee on the Framework Convention for the Protection of National Minorities (27 February 2008).

http://www.coe.int/t/dghl/monitoring/minorities/3_fcnmdocs/PDF_Commentary Participation_en.pdf (24 February 2014). 
Council of Europe, (ed.), 2004. Filling the Frame: Five Years of Monitoring the Framework Convention for the Protection of National Minorities. Council of Europe Publishing, Strasbourg.

DFAIT, 2010. Chair's Summary, Chelsea Meeting, 29 March 2010, http://www.international.gc.ca/media/aff/news-communiques/2010/120.aspx (26 October 2013)

Downie D. and T. Fenge (eds.), 2003. Northern lights against POPs: combatting toxic threats in the Arctic. McGill-Queen's University Press, Montreal and Kingston.

Duyck, S., 2012. Which canary in the coalmine? The Arctic in the international climate change regime. Yearbook of Polar Law, 4: 583-617.

Elferink, A. G. O., 2001. The Outer Continental Shelf in the Arctic: the application of Article 76 to the LOS Convention in a regional context. In: Elferink, A.G.O. and D. R. Rothwell (eds.). The Law of the Sea and Polar Maritime Delimitation and Jurisdiction. Martinus Nijhoff, The Hague.

EU, 1992. Council Directive 92/43/EEC on the conservation of natural habitats and of wild fauna and flora (21 May 1992).

EU, 2008. Marine Strategy Framework Directive, Directive 2008/56/EC 17 June 2008).

European Commission, 2008. Climate change and international security. Paper from the High Representative and the European Commission to the European Council (14 March 2008, S113/08),

http://www.consilium.europa.eu/uedocs/cms_data/docs/pressdata/en/reports/9 9387.pdf (24 February 2014).

European Parliament, 2008. European Parliament resolution on Arctic governance ( 9 October 2008). http://www.europarl.europa.eu/sides/getDoc.do?type= TA\&reference=P6-TA-2008-0474\&language=EN (24 February 2014).

European Parliament, 2011. European Parliament resolution on a sustainable EU policy for the High North (of 20 January 2011).

http://www.europarl.europa.eu/sides/getDoc.do?pubRef=-//EP//TEXT+TA+P7TA-2011-0024+0+DOC+XML+V0//EN (24 February 2014).

Fitzmaurice, M., 2009. So much law so little protection! A case study of the protection of the narwhal. Yearbook of Polar Law, 1: 21-54.

French, D. and K. Scott, 2009. International legal implications of climate change for the Polar regions: too much, too little, too late. Melbourne Journal of International Law, 10: 631-54.

Gillespie, A., 2002. Forum shopping in international environmental law: the IWC, CITES and the management of cetaceans. Ocean Development and International Law, 3: 17.

Graver, H. and G. Ulfstein, 2004. The Sámi People's right to land in Norway. International Journal on Minority and Group Rights, 11: 337.

Heinämäki, L., 2009. Rethinking the status of Indigenous Peoples in international environmental decision-making: pondering the role of Arctic Indigenous Peoples and the challenge of climate change. In: Koivurova, T., E.C.H. Keskitalo and N. Bankes (eds.). Climate Governance in the Arctic, pp. 207-262. Springer, Germany.

Henriksen, T. and G. Ulfstein, 2011. Maritime delimitation in the Arctic: the Barents Sea treaty. Ocean Development and International Law, 42: 1.

Ilulissat Declaration 2008. 28 May 2008, http://www.oceanlaw.org/downloads/arctic/Ilulissat_Declaration.pdf (24 February 2014).

IMO, 2009. Guidelines for Ships Operating in Polar Waters ( 2 December 2009). IMO Res A.1024(26) (18 January 2010, Annex). 
IWC, 2010. 62 $2^{\text {nd }}$ Meeting of the International Whaling Commission Chair's Report. http://iwcoffice.org/chairs-reports (23 October 2013).

IWC, 2011. 63 ${ }^{\text {rd }}$ Meeting of the International Whaling Commission Chair's report. http://iwcoffice.org/chairs-reports (23 October 2013).

Jensen, Ø., 2008. Arctic shipping guidelines: towards a legal regime for navigation safety and environmental protection. Polar Record, 44 (229): 107-114.

Johnston, A. and G. Block, 2012. EU Energy Law. Oxford University Press, Oxford.

Kleist, K., 2010. Greenland's self-government. In: Loukacheva, N. (ed.). Polar Law Textbook, pp. 171-196. Nordic Council of Ministers, Copenhagen.

Koivurova, T., 2006/7. Draft for a Nordic Saami Convention. European Yearbook of Minority Issues, 6: 103-136.

Koivurova, T., 2008. Draft for a Nordic Saami convention: nations working together. International Community Law Review, 10: 279-293.

Koivurova, T., 2011. Jurisprudence of the European Court of human rights regarding Indigenous peoples: retrospect and prospect. International Journal on Minority and Group Rights, 18: 1-37.

Koivurova, T. and E. Molenaar, 2009. International governance and regulation of the marine Arctic. Three reports produced for the WWF Arctic international programme, Oslo, 2009. http://awsassets.panda.org/downloads/3in1_final.pdf (7 January 2014).

Koivurova, T. and A. Stepien, 2011. How international law has influenced the national policy and law related to Indigenous peoples in the Arctic. Waikato Law Review, 19: 123-143.

Koivurova, T. and D. VanderZwaag, 2007. The Arctic Council at 10 Years: retrospect and prospects. University of British Columbia Law Review, 40(1): 121-194.

Koshan, J., 2013. The Nordic Saami Convention and the rights of Saami women: lessons from Canada. In: Bankes, N. and T. Koivurova (eds.), 2013. The Proposed Nordic Saami Convention: National and International Dimensions of Indigenous Property Rights, pp. 379-398. Hart Publishing, Oxford.

Kovler, A., 2012. International protection mechanism of Indigenous people. Yearbook of Polar Law, 4: 205-225.

Kunoy, B., 2013. Agreed minutes on the delimitation of the Continental Shelf beyond 200 nautical miles between Greenland and Iceland in the Irminger Sea. Chinese Journal of International Law, 12: 124-42.

Lalonde, S., 2013. The Arctic exception and the IMO's PSSA mechanism: assessing their value as sources of protection for the Northwest Passage. International Journal of Maritime and Coastal Law, 28(3): 401-432.

Long, R., 2011. The Marine Strategy Framework Directive: a new European approach to the regulation of the marine environment, marine natural resources and marine ecological services. Journal of Energy and Natural Resources Law, 29: 1-44.

Loukacheva, N. (ed.), 2010. Polar Law Textbook. Nordic Council of Ministers, Copenhagen.

Loukacheva, N. (ed.), 2013. Polar Law Textbook II. Nordic Council of Ministers, Copenhagen.

Macnab, R., 2004. The outer limit of the continental shelf in the Arctic Ocean. In: Nordquist, M.H., J. N. Moore and T. H. Heidar (eds.). Legal and Scientific Aspects of Continental Shelf Limits, pp. 304-05. Martinus Nijhoff, Leiden.

McDorman, T. L., 2012. National measures for the safety of navigation in Arctic waters: NORDREG, Article 234 and Canada. In: Nordquist, M.H., J. N. Moore, A.H.A. 
Soons and Kak-so Kim (eds.). The Law of the Sea Convention: US Accession and Globalization, pp. 409-24. Martinus Nijhoff, Leiden.

McDorman, T. L., 2013. The international legal regime of the Continental Shelf with special reference to the polar regions. In: Loukacheva, N. (ed.). Polar Law Textbook II, pp. 77-93. Nordic Council of Ministers, Copenhagen.

McRae, D.M. and D.J. Goundrey, 1983. Environmental jurisdiction in the Arctic: the extent of Article 234. University of British Columbia Law Review, 16: 197-228.

Möller, J.T., 2011. Case law of the UN Human Rights Committee relevant to members of Minorities and Peoples in the Arctic Region. Yearbook of Polar Law, 3: 27-56.

North Pacific Fisheries Management Council, (2009). Arctic Fishery Management Plan for Fish Resources of the Arctic Management Area (August 2009).

http://www.fakr.noaa.gov/npfmc/fmp/arctic/ArcticFMP.pdf (23 October 2013).

Ørebech, P., 2012. The Barents Sea 2010 Norway-Russia border: the triumph of the negotiation principle at the expense of the median- and sector line pretentions. Yearbook of Polar Law, 4: 505-517.

Osofsky, H., 2009. The Inuit petition as a bridge? Beyond dialectics of climate change and Indigenous peoples' rights. In: Burns, W. and H. Osofsky (eds.). Adjudicating Climate Change: State, National and International Approaches, pp. 272-292. Cambridge University Press, Cambridge.

Parsons, E. and L. Cornick, 2011. Sweeping scientific data under a polar bear skin rug: the IUCN and the proposed listing of polar bears under CITES Appendix I. Marine Policy 35(5): 729-31.

Pentassuglia, G., 2011. Towards a jurisprudential articulation of Indigenous land rights. European Journal of International Law, 22: 165.

Ravna, Ø., 2010. A new academic journal is born. Arctic Review on Law and Politics 1: 1-3.

Ravna, Ø., 2011. The process of identifying land rights in parts of northern Norway: does the Finnmark Act prescribe an adequate protection procedure within the national law? Yearbook of Polar Law, 3: 423.

Ravna, Ø., 2013. The first investigation report of the Norwegian Finnmark Commission. International Journal on Minority and Group Rights, 20: 443-57.

Rayfuse, R., 2007. Melting moments: the future of polar oceans governance in a warming world. Review of European Community and International Environmental Law, 16(2): 196-216.

Rayfuse, R., 2008. Protecting marine biodiversity in polar areas beyond national jurisdiction. Review of European Community and International Environmental Law, 17(1): 3-13.

Rayfuse, R., 2009. Warm waters and cold shoulders: jostling for jurisdiction in polar oceans. Yearbook of Polar Law. 1: 465-76.

Saami Council, 2005. Nordic Saami Convention [Draft]. http://www.galdu.org/govat/doc/samekoneng_nett.pdf (January 3, 2014).

Special Rapporteur, 2011. Report of the Special Rapporteur on the rights of Indigenous peoples, the situation of the Sámi people in the Sápmi region of Norway, Sweden and Finland. http://unsr.jamesanaya.org/docs/countries/2011-report-sapmia-hrc-18-35-add2_en.pdf (23 October 2013).

Swepston, L., 2011. Good governance and Indigenous and tribal peoples. Yearbook of Polar Law, 3: 423.

UN ECE, 2010. Black Carbon, Report by the Co-Chairs of the Ad Hoc Expert Group on Black Carbon (30 September 2010), ECE/EB.AIR/2010/7. 
UN DRIP, 2007. United Nations Declaration on the Rights of Indigenous Peoples: resolution / adopted by the UN General Assembly, 2 October 2007, A/RES/61/295

Vasilev, A., 2013. The agreement on cooperation on aeronautical and maritime search and rescue in the Arctic - a new chapter in polar law. In: Loukacheva, N., (ed.). Polar Law Textbook II, pp. 53-65. Nordic Council of Ministers, Copenhagen.

Watson Hamilton, J., 2013. Acknowledging and accommodating legal pluralism: an application to the draft Nordic Saami Convention. In: Bankes, N. and T. Koivurova (eds.). The Proposed Nordic Saami Convention: National and International Dimensions of Indigenous Property Rights, pp. 45-77. Hart Publishing, Oxford,

Webber, J., 2013. The public-law dimension of Indigenous property rights. In: Bankes, N. and T. Koivurova (eds.). The Proposed Nordic Saami Convention: National and International Dimensions of Indigenous Property Rights, pp.79-102 Hart Publishing, Oxford.

WTO, 2013. World Trade Organization Panel Report, European Communities Measures Prohibiting the Importation and Marketing of Seal Products, 25 November 2013, http://www.wto.org/english/tratop_e/dispu_e/cases_e/ds400_e.htm (5 January 2014)

Young, O., 2011. If an Arctic Ocean treaty is not the solution, what is the alternative? Polar Record, 47 (243): 327-34.

Yupsanis, A. 2011. The International Labour Organization and its contribution to the protection of Indigenous peoples. Canadian Yearbook of International Law, 49: 117-76. 


\section{Resource Governance}

\section{Lead authors}

Bruce C. Forbes, University of Lapland, Finland and Gary Kofinas, University of Alaska Fairbanks, USA

\section{Contributing authors}

Hugh Beach (Uppsala University, Sweden), Camilla Brattland (Norwegian Institute for Cultural Heritage Research, Norway), Paula Kankaanpää (University of Lapland, Finland), E. C. H. Keskitalo (Umeå University, Sweden), Mitdlarak Lennert (University of Greenland, Greenland), Chanda L. Meek (University of Alaska Fairbanks, USA), Vera K. Metcalf (Eskimo Walrus Commission, Kawerak, Inc., USA), Martin D. Robards (Wildlife Conservation Society, USA), Oran R. Young (University of California Santa Barbara, USA)

\subsection{Introduction}

Resource governance plays a critical role in human responses to conditions of rapid change in the Arctic (Young, 2005; Young, 2012; Arctic Council, 2013). The importance reflects the special relationship Arctic peoples have to land and resources and the role of the Arctic in global affairs (Huntington et al., 2005; Klein et al., 2005; Nuttall et al., 2005; Hovelsrud and Smit, 2010). Interest in the North is intensifying as a source of renewable and non-renewable resources for southern markets, and because of the strong climate signal observed across the region. The options for humans to respond to social, economic and environmental changes, whether through mitigation, adaptation, or transformation, are best achieved through effective systems of resource governance (Young, 1996; Berkes et al., 2005; Biermann, 2007; Young, 2009a; Armitage and Plummer, 2010).

Resource governance refers to the collective efforts of society to define and achieve societal goals related to human interactions with the environment (Dietz et al., 2003; Folke et al., 2005; Young et al., 2008; Kofinas, 2009). Governance is a device to navigate and address societal challenges. It is the process by which citizen groups, non-governmental 
organizations (NGOs), government agencies, businesses, local communities and partnerships of individuals and organizations interact in resource stewardship, a process that may or may not involve government (Young et al., 2008; Kofinas, 2009). Resource governance is shaped both by formal and informal rules, policies, norms, and conventions that determine power relationships among groups, provide certainty in social interactions, and potentially restrict or enhance human choice (Ostrom, 2009). In the context of human development in the Arctic, resource governance addresses problems of maintaining natural and social assets that are important to sustaining ecosystem services - the benefits to humans derived from ecosystems (Chapin et al., 2006; Young, 2012).

The Arctic has historically been bountiful in renewable and nonrenewable resources derived from its terrestrial, aquatic, and marine systems (AHDR, 2004). For millennia, northern Indigenous peoples co-evolved with their ecosystems. Today the human footprint in the Arctic environment is significant (Nellemann et al., 2001; AMAP, 2010), with actors within resource regimes ranging from individuals and families, to nation states, to some of the largest and most profitable global multinational corporations. For many Arctic residents, continued access to Arctic resources is linked closely to livelihoods, long-term economic development, and overall wellbeing (Anderson et al., 2002; ASI, 2010). For Indigenous peoples of the North, cultural survival is closely tied to access to living resources and a meaningful role in resource governance (Nuttall, 1998).

The first Arctic Human Development Report (AHDR) focused on resource governance as supporting Arctic livelihoods and issues of power relations, including property rights and the state of Indigenous peoples securing rights in governance, the legitimacy and utility of traditional knowledge, and devolution and co-management arrangements (Caulfield et al., 2004). The issues of power and power sharing associated with Arctic resource governance continue to be of critical importance. However, since the publication of the first $A H D R$, the challenges of resource governance have expanded to consider changes in climate, land use, and economies (Arctic Council, 2013). These conditions suggest that to meet the challenges of sustainability, adaptive approaches to governance must be implemented (Berkes et al., 2005; Armitage et al., 2007; Young, 2012). While adaptive resource governance in the Arctic includes a power dimension, it also demands flexibility in decision making, high levels of responsiveness, decision making in conditions of high uncertainty, on-going and systematic monitoring of social and ecological systems, the active integration of knowledge systems, and development of decision-support systems that are accessible to a wide range of stake- 
holders across multiple levels (Armitage et al., 2007; Young, 2009b). Realizing these objectives will require considerable transformations in today's paradigms of resource management, social networks, and political institutions (Chapin et al., 2009; Arctic Council, 2013).

Arctic resource governance has emerged as an array of polycentric, multi-scaled and novel arrangements that in some cases provide for Indigenous self-governance, or for shared governance between many layers of governments, ranging from Indigenous to state to international. Resource governance in the Arctic occupies a unique international environment that includes co-management arrangements for wildlife and land resources, Indigenous land claims agreements, national research programs recognizing the value of traditional knowledge, and the transactions of the Arctic Council (Young, 2005; Arctic Council, 2009; Koivurova et al., 2009). Yet in spite of significant changes in Arctic governance, colonial legacies, institutional deficiencies, and power imbalances continue to challenge resource governance especially in the absence of funding and community capacity. This is especially the case in northern Europe and Arctic Russia where co-management and recognition of land and resource rights are mostly absent (Forbes, 2008; Hovelsrud et al., 2010).

Climate change, increased resource development, and social and economic change add layers of unpredictability in what is already a stressful milieu for the players involved, particularly those who possess less economic or political power. These rapid and dramatic changes in the Arctic up the ante of what is required to steward resources, and challenge the capacity of current governance systems to address power inequities while being responsive to social-ecological change and innovative in problem solving.

The emerging need for adaptive governance in the North recognizes that sustainability goals should consider how social-ecological systems are buffered from the trajectories and shocks of new conditions. The seven case studies in this chapter illustrate principles of adaptive governance in resource stewardship, and highlight the challenges that result when unresolved issues such as on-going colonialist attitudes hamper the capacity of institutions to respond.

How these tensions are manifesting and when and how they are being resolved across the Circumpolar North are the foci of this chapter. The chapter presents a set of crosscutting issues related to resource governance in a changing North, followed by case studies that illustrate points made in the general text. The chapter identifies best practices as well as shortcomings in resource governance, with a view to improving 
present conditions toward more desired outcomes for both Arctic peoples and Arctic rim nation-states.

\subsection{Putting Arctic resource governance in a global context}

Arctic resource governance is front and center on a world stage of attention. There is increasing awareness that the fate of Arctic resources affects the well-being of people in distant regions. On the other hand, the lack of action on climate change by southern governments has potential negative effects on northern peoples and raises questions about the extent to which the North bears the burden for southern consumption. The emergence of technology, the Internet and global communication networks, along with greater political skill, provides increased opportunities for Arctic residents to influence the future of the North.

\subsection{Local resource management systems}

Local resource governance systems are fundamental to sustaining important living resources and cultural integrity of northern peoples. In many cases, local resource management functions through informal institutions based on kinship and community interactions independent of regional and national scale arenas. Formal bureaucratic systems often overlay local informal systems, creating conflict with local cultures and constraining local response capability. In other cases, formal local resource management systems have emerged in innovative ways that display greater capacity, particularly where funding is available to support these systems. In those cases, many First Nations have developed highly active research and monitoring capabilities, such as GIS mapping and video production studies (Berkes and Jolly, 2002; Nemes et al., 2007; Gearheard et al., 2011; Bali and Kofinas, 2014). However, the lack of financial and human resources in some areas means that limitations to organizational capacity and development persist. 


\subsection{From co-management to adaptive co-management?}

The first AHDR highlighted formal "co-management" arrangements. Comanagement emerged in the 1970s and 1980s in North America as a way of sharing power between governments and resource users to reduce conflicts over common resources and achieve sustainability. Since that time, variants of these types of power sharing arrangements have proliferated in many northern regions (Berkes, 2009; Clark and Slocombe, 2011). For Indigenous peoples these arrangements increasingly provide greater authority in resource decision-making, and for governments, these agreements improve compliance with regulations and policies. Several new international arrangements have been negotiated among Indigenous organizations, with limited to no national government involvement (Meek et al., 2008). In many situations, these arrangements have evolved from co-management structures to adaptive co-management, a dynamic process where institutions and understandings of ecological change are constantly tested and revised to reflect complexities of the particular socio-ecological systems (Berkes and Armitage, 2010; Berkes, 2012). While there has been considerable innovation in co-management in many regions, such as Canada, Alaska, and parts of Fennoscandia, in other cases, such as in many parts of Russia, there is little to no local decision-making authority.

\subsubsection{Non-renewable resource extraction in the Arctic}

The "development" (exploration, extraction, and delivery) of northern non-renewable resources is controversial and has both direct and indirect social and environmental impacts on northern residents (Walker et al., 1987; Chapin et al., 2006; AMAP, 2010). Today, Arctic resource extraction activities are extensive, operating with varying governance systems shaping environmental quality and human well-being (AMAP, 2010). The distribution of benefits to residents from these activities also varies (Haley, 2004; Forbes, 2008). In some cases, local resident glean considerable financial and employment benefits, and in others, local residents bear the burden of responding to changes caused by extractive industries, while receiving limited remunerations. The global need for oil and gas resources has pushed exploration offshore where jurisdictional boundaries may not be well defined, and the risk of oil spills and other potentially devastating consequences is significant. 


\subsection{Responding to climate change}

Since the completion of the Arctic Climate Impact Assessment (ACIA) in 2004-2005, climate change has become the predominant narrative in defining drivers of Arctic change. This narrative often describes northern residents as passive actors rather than agents in their own regard. This view overlooks the importance of existing and developing local and regional institutions in complementing and facilitating adaptation. It is also important to understand climate change as one of many factors and drivers challenging people and governance institutions in the future.

The rapid retreat and thinning of Arctic sea ice at rates faster than those predicted by the ACIA (2005) and IPCC (Anisimov et al., 2007) is among the most dramatic changes since the first $A H D R$. These changes have major implications for marine mammal management, access to high latitude pelagic fisheries, and stores of oil and gas. With the retreat of ice, it is expected that Arctic shipping will increase exponentially (Robards, 2013). Climate change creates additional challenges, such as increases in boreal and tundra wildfires, thawing of permafrost and its implications to ecosystems and human infrastructure, and changes in plant communities and their effects of grazing systems such as caribou, reindeer, and musk ox. The possible cumulative effects of these interacting forces are not well understood, and suggest that without concerted efforts by all stakeholders, future resource management may be reactive rather than proactive. For example, in Alaska, the combination of decreases in sea ice and thawing permafrost has resulted in coastal erosion and the need to relocate several villages. While many recognize these problems, there are significant institutional deficiencies that hamper the ability to adequately address them. Despite the absence of financial resources and formal protocols for village relocation, some communities are proactively responding by addressing local needs and creating new institutions with limited government support (Bronen, 2011; Kates et al., 2012; Bronen and Chapin, 2013).

\subsection{Integrating knowledge systems in Arctic resource governance}

In the early to mid 1980s, when many of the first northern comanagement arrangements were established, few decision makers made explicit reference to "Indigenous," "local" or "traditional knowledge." In the ensuing three decades, the concept gained considerable traction 
(Berkes, 2012), both as rhetoric and in practice. The concept has since been applied in many ways, including to assert a role for local resource users in various resource management functions and to suggest that local knowledge provides opportunities for more complete understanding of Arctic change (Huntington, 2000; Krupnik and Jolly, 2002; Krupnik et al., 2010). Since the first $A H D R$, a proliferation of studies have documented local and traditional knowledge, and in some cases, sought to "integrate" this knowledge (Huntington et al., 2005; Kumpula et al., 2010; Gaup Eira et al., 2013). Calls for funding proposals now commonly require that the knowledge and involvement of local residents be included in study designs. New "observation systems" that include Indigenous and local knowledge have also been implemented, some experimenting with GPS technologies that capture real time observations of local users (Gearheard et al., 2011; Galginaitis, 2012; Kumpula et al., 2012). Most advances have occurred in North America, some in Fennoscandia, and few in Russia. However, there continues to be conceptual confusion, with many scientists and agency resource managers viewing traditional knowledge as a data source separate from its underlying worldview and day-to-day utility in practice (e.g., how to harvest). Evidence also shows how in some cases there are problems with ignoring the cultural underpinnings of knowledge. Yet in spite of both a growing interest and a deep misunderstanding, the increased legitimacy of local knowledge bodes well for making resource governance in the Arctic more adaptive.

\title{
7.7 Case studies
}

\subsubsection{Partnering across the Bering Strait to support resilient walrus-human relations}

\author{
By Chanda L. Meek, Martin D. Robards and Vera K. Metcalf
}

This case study illustrates the evolution of adaptive governance in an international setting. The Pacific walrus population ranges between the United States and Russian Federation, necessitating coordinated actions by both countries to conserve walrus at a range-wide scale. The U.S. and Russia have formal collaborations, including the 1972 Bilateral Agreement on Cooperation in the Field of Environmental Protection and the 2009 U.S.-Russia Presidential Bilateral Commission. These collaborations are enriched and made more resilient by an active Alaska Native- 
to-Chukotka Native relationship (Meek et al., 2008). This case study illustrates the value of informal, international networking institutions in building adaptive governance through sharing strategies to reducing disturbance to subsistence resources and building the adaptive governance capacity of managers and hunters through joint learning and coordinated collective action.

Government management of Pacific walrus hunting in Alaska did not begin until after the commercial exploitation of walrus had decimated their herds in the nineteenth century, leading to starvation in some Indigenous communities along the Bering Sea coast (Fay 1982). Environmental conditions and federal protections supported a population rebound in the 1970s and 1980s, which was so successful that they were in danger of exceeding the ecosystem's carrying capacity. This rebound led to fears of another decline, which, in conjunction with continued uncertainty over management authority, led to several Alaskan communities coming together in 1978 to create the Eskimo Walrus Commission (EWC). The EWC provided a mechanism for Alaska Native hunters to participate in Pacific Walrus management (Langdon, 1989). In 1994, the U.S. Congress amended the Marine Mammal Protection Act to formalize comanagement arrangements between federal agencies and Alaska Native organizations like the EWC. The subsequent co-management agreements for Pacific Walrus have strengthened and expanded harvest monitoring programs in Alaska (and Chukotka), documented Indigenous knowledge and best hunting practices, and enhanced efforts to develop locally based subsistence hunting ordinances.

The collapse of the Soviet Union in the early 1990s precipitated a decline in economic vitality for Chukotka, necessitating a return to subsistence-based diets that includes walrus (Metcalf and Robards, 2008). As a result, even in the midst of this political transformation, or perhaps because of it, transborder walrus management collaboration intensified and led to a series of international and interlocal (i.e. transboundary community-tocommunity relationships) discussions about bilateral walrus management (Meek et al. 2008). The interlocal relationships established between communities eventually gave rise to a series of international frameworks to share information and management ideas.

During the early 2000s, with the cooperation of Alaska Native groups, bilateral monitoring agreements were developed to ensure the longterm health of the walrus population through the National Park Service Beringian Heritage Program (a U.S. federal agency) and the Pacific Walrus Conservation Fund (a non-governmental organization (NGO)). These agreements have primarily focused on monitoring of Alaska walrus 
hunts, but have also facilitated data collection on Chukotkan haul-outs and traditional knowledge. In recent years, Alaska and Chukotka Natives have begun to formalize stewardship activities in relation to hunting and the protection of haul-outs. Communities and partner agencies on both sides of the Bering Strait (e.g., U.S. Fish and Wildlife Service and ChukotTINRO) are rapidly adapting to new conditions. Communication connections across the Strait have facilitated rapid uptake of traditional knowledge-tested practices used in Russia for the establishment of community-led emergency measures (e.g., media management, fly-over restrictions, disturbance restrictions, etc.) in areas where walruses have hauled out en masse. In Alaska, hunter-based organizations such as the Eskimo Walrus Commission, have shared findings from communities, scientists and regulators evaluating the impact of industrial development on walruses to evaluate Russian oil and gas development plans.

Through an ongoing history of governance interactions, walrus and subsistence users alike have benefited from protections that reduce disturbance, minimize mortality of walrus, and directly support the continued use of walrus for subsistence. Interlocal institutions have allowed partners to continually share strategies across the Strait and evaluate the success of both models. Nevertheless, funding remains a difficult challenge, as US agencies (e.g., the National Park Service and Fish and Wildlife Service), charitable foundations, and NGOs (e.g., World Wildlife Fund and the Wildlife Conservation Society) absorb much of the costs for conservation work on both sides of the border.

Community-to-community relationships contribute to upholding the rights and interests of the Indigenous peoples and to ensuring greater food security and other inter-related economic, social, and cultural rights. Recently, unfavorable ice conditions and low levels of hunting success are testing community resilience, but communities, agencies and NGOs involved in governing the social-ecological system within the Bering Strait have undoubtedly strengthened resilience through strong, adaptive partnerships. However, the addition of more formal channels of cooperation between tribal governments and federal agencies may also be needed to adaptively govern the region as a whole. 


\title{
7.7.2 Nordic reindeer governance in the context of contemporary protected areas and species
}

\author{
By Hugh Beach
}

In an increasingly interconnected world of growing demands on limited physical resources challenged by rapid climatic change, how can the rights and protective clauses be forged to sustain Indigenous livelihoods and protect cultures? To what extent might livelihoods improve rather than deteriorate in the face of these changes? The case of Nordic reindeer governance in Sweden provides insights into these questions, illustrating how on-going conflicts and the failure to recognize fully Indigenous people's rights can lead to a degradation of conditions.

Indigenous peoples are often accused of wanting "special rights" over land and resources, which non-Indigenous peoples, many with long generational depth of permanent local residency, do not share. The degree to which this question is considered by law (e.g., through treaties or courtroom precedent) or political policies varies among and within northern nations over time. The last half of the last century witnessed the birth of the Sámi parliaments and the revision of Sámi herding laws. Of course, such proclamations and land-claims settlements do not erase the ongoing debates and struggles over resources in the North. In Sweden, court cases contesting the immemorial right of Sámi herders to utilize winter grazing on lands in forested areas owned by settlers have become frequent, since the state has thus far refused to pass general legislation on Sámi resource rights (as opposed to the rights of herders).

Sweden has not ratified the International Labor Organization (ILO) Convention 169, concerning Indigenous Peoples, and, in at least one instance, has acted against the principles contained in the Convention (ILO 169). Article 14 of the ILO Convention calls for the strengthening of Sámi ownership claims to land, but in 1993, Sweden confiscated Sámi small-game hunting rights, without due process or just compensation, claiming that game was in sufficient abundance for the state to assert a parallel hunting right and to sell its own licenses. Similarly, the UN Declaration on the Rights of Indigenous Peoples has held little added value for the Sámi, whose attainments in a broader social and economic setting are due primarily to policies resulting from liberal welfare ideologies (UN DRIP, 2007).

Currently, reindeer herding, the main idiom of Sámi culture and repository of Sámi rights, struggles for survival in Sweden in light of the massive damages inflicted by inadequate predator protection policies, rampant destructive mineral prospecting and mining, and the estab- 
lishment of huge so-called "wind parks." In its quest to meet clean energy goals as part of its commitment to international environmental responsibilities and in the wake of the Fukushima disaster, Sweden has installed thousands of wind-power turbines on reindeer ranges. The Sámi have fared far better in Norway during the last decades, with the acceptance in 2005 of the Finnmarksloven, the Finnmark Law, in which the state specified Sámi resource rights while it simultaneously secured rights for non-Sámi in the Finnmark area (Svensson, 2005; Beach, 2012).

The ongoing domestication of the northern Fennoscandian wild lands is analogous to the domestication of wild animals like the wolf when their every movement, their reproduction, and survival, become matters of human legislation. The compensation that provided the herders for their lost reindeer is often conceived as a subsidy to reindeer herding. It would be more logical to class it as a subsidy to wolf ranching (Beach, 2004), for under Swedish wolf governance, the destruction caused by wolves is no longer an uncontrollable act of nature, but a consequence of human legal construction. The combination of herding law, taxation policy, predator policy, and other regulatory constraints on the one hand, along with new technological developments and government catastrophe aid for starving reindeer on the other, shape the variable responses of herders when it comes to labor investment and animal-handling techniques. On the collective sameby (a Sámi social and territorial grazing unit) level, the gradually advancing implementation of the wage system funded by membership herding fees results in greater labor efficiency, but also alters settlement patterns and on occasion, increases herding extensivity (less control over and contact with the reindeer), loss of skills, and often reduces internal sameby solidarity.

There has been a strong trend recently towards acknowledging cognitive rather than physical human-land relations, together with the international harmonization of policies. The dramatic proliferation of World Heritage Sites, such as northern Sweden's Laponia, bestowing protected status on areas of unique natural and cultural value, is one example.

The increasing scope of human power, heated by population growth and rapid technological change - occurring at a rate far faster than that of rapid climatic change - gives humankind the potential to have an unprecedented environmental impact. Regardless of how this human power is actually exercised - even were specific regions removed from human influence by creating parks, nature reserves, or World Heritage Sites, - it signifies a form of human colonization of the world. As such, the world becomes increasingly governed by the exercise of human choice. Even when the choice taken is one for environmental preserva- 
tion, either with a "hands off" policy (forcibly removing people from their homelands) or with policies of active repair, wilderness becomes tamed. It becomes our nature preserve, our environment (Beach, 2012).

The Nordic countries illustrate a difficult problem. Improvements in living conditions for Indigenous individuals who adopt and benefit from the technologies and comforts of the increasingly globalized ambient society, cause them to lose the necessary sympathy of the democratically controlling majority for whom "indigeneity" might be supported, but only when possible to regard it as vulnerability in need of corrective welfare. Consideration for Indigenous rights over limited resources tends to wane rapidly along with the equalizing of observable prosperity. As this case demonstrates, such rights are revoked or reduced to privileges that can in turn be reduced to insignificance under the noble banner of equal justice for all, regardless of ethnicity.

\section{Path marker, informing hikers of reindeer corral ahead, Swedish Sámiland}

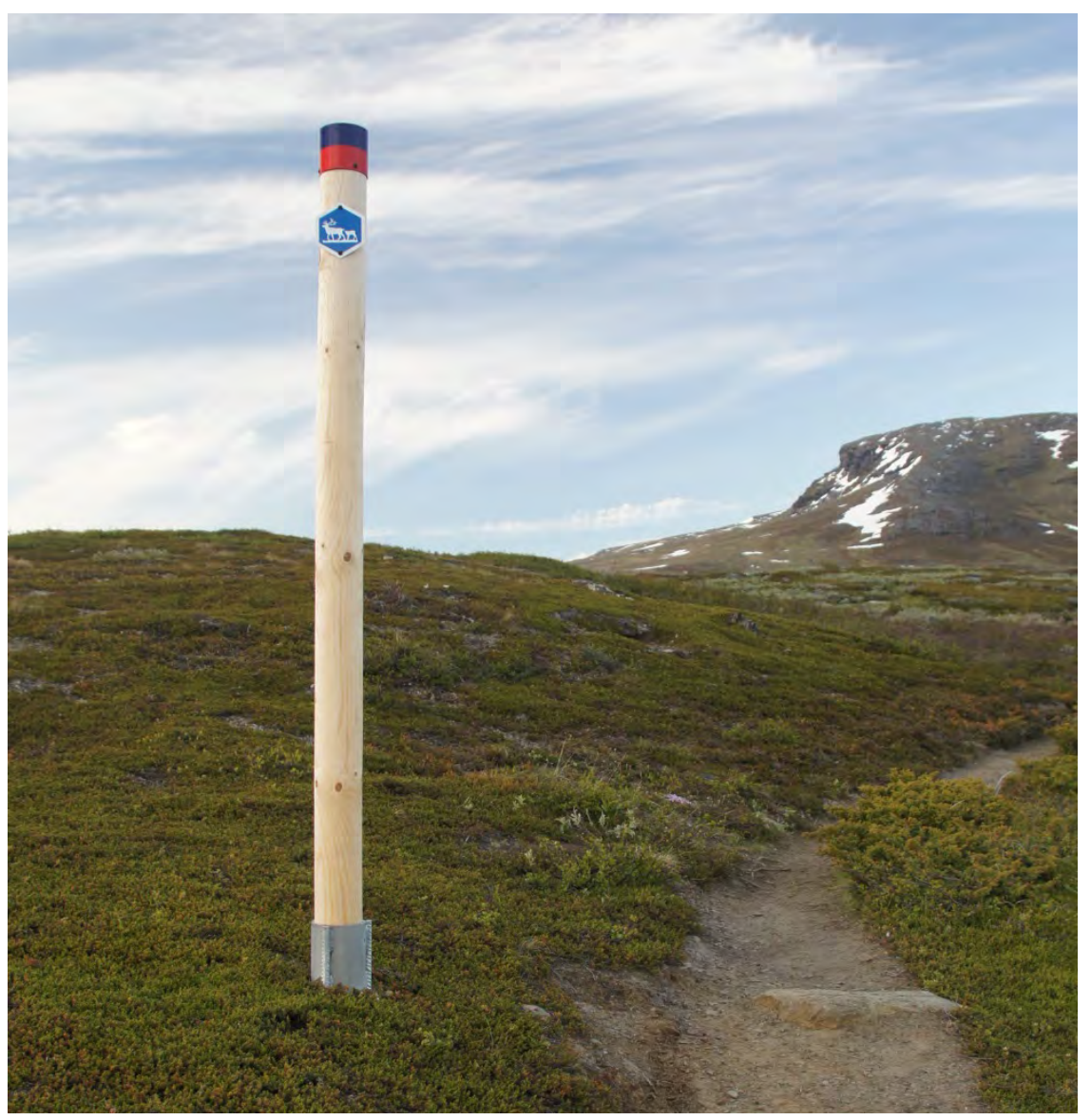

Credit: Hugh Beach. 


\title{
7.7.3 Sámi fishing rights and Indigenous participation in Norwegian fisheries governance
}

\author{
By Camilla Brattland
}

Although the Sámi Parliament has grown in power and currently has a central role in co-managing marine resources in Norway, the Norwegian state has not recognized the historical fishing rights of the Sámi people. This is happening at a time when fish stocks are moving north with the warming climate, traditional capture fisheries are met with competition from a globalized fisheries industry, and northern coastal communities are searching for appropriate and effective mechanisms for coping with and responding to change. Broderstad and Eythórsson (2014) argue that northern Norwegian fishing communities' capacity to respond to change is largely dependent on political and institutional reforms as well as ecological variables. This case study illustrates the extent of current political recognition and implementation of Sámi fishing rights and Indigenous participation in resource governance, as well as its limitations.

When the Norwegian Sámi Parliament was established in 1989, it represented a variety of Indigenous Sámi communities, individuals and institutions. With limited but increasing power and responsibilities, the Sámi Parliament interacts with the Norwegian state in matters of importance to Sámi culture, livelihoods, and cultural survival. Fisheries management for small-scale commercial fisheries is one area of active involvement. Although the rights to access marine resources are not exclusively Indigenous, fisheries governance affects Sámi traditional livelihoods and opportunities to participate in and benefit from commercial fisheries.

Before 1990, Sámi fishers constituted a marginalized group in the Norwegian fisheries management system (Eythórsson, 2003). The local fish stocks upon which many Sámi communities relied were not sufficiently recognized and managed, and overfishing and conflicts between locals and foreign fishers on limited fish stocks in inshore areas were frequent. The fisheries closure and introduction of a vessel quota system in 1990 meant that many Sámi fishers were excluded from accessing the increasingly licensed coastal fishery. However, Sámi fishers emerged as an important new stakeholder in fisheries management with the creation of the Sámi Parliament in 1989. Shortly thereafter, the Ministry of Fisheries implemented measures for strengthening Sámi fisheries within the existing regulatory framework, and the Sámi Parliament began to participate in annual quota negotiations and regulatory meetings (Sámi Parliament, 2004). This co-management framework facilitated coopera- 
tion between the Sámi Parliament and the fisheries authorities resulting in a new governance policy for all fishers within a geographically defined management area.

Since the Sámi management measures were applied universally for all ethnic groups, they have come to encompass large parts of the northern Norwegian small-scale fishing fleet. The area has grown from a handful of municipalities in 1990 to 26 municipalities and 20\% (1,200 out of approximately 5,800) of the northern fishers (Norwegian Directorate of Fisheries, 2013). However, the funding available to the Sámi Parliament from the Norwegian Government has not correspondingly increased, either for small-scale fisheries, or for Sámi fishers in particular. Similarly, some of the special management measures for protecting Sámi culture implemented by the Ministry of Fisheries are covering more and more fishers. What used to be a distinct management policy for a minority of Sámi fishers has changed into an integrated, Norwegian-Sámi small-scale industry policy for a growing group of northern people. This is advantageous in that Sámi fishers are no longer excluded, but it has become difficult for the Sámi Parliament to represent only the Sámi people in marine resource governance. This new challenge is even more evident as new legislation has further cemented the Sámi fisheries management area as a priority area for small-scale fishers in general.

This challenge became clear in the 2005 Consultation Agreement between the Sámi Parliament and the Norwegian government. This Agreement changed the governance relations between the two parties and elevated the Sámi Parliament from one among many stakeholders to a body that was to be consulted separately and exercise decision-making power through consenting or withholding consent for policy proposals pertaining to Sámi matters. Despite this change, the Norwegian Parliament (Storting) did not acknowledge the historical fishing rights of the Sámi people, instead offering to legislate the fishing rights of small-scale fishers in the Sámi fisheries management area and increase the maximum quota for this group of fishers. The Sámi Parliament made it clear that the question of the historical fishing rights of the Sámi had not been settled, but consented to the rest of the proposals, and in 2011, the new Fishing Rights Agreement came to be.

With the new consultation procedures and the new, integrated smallscale fishery policy where the Sámi Parliament has a central role, the political influence of the Sámi Parliament in marine resource governance has grown considerably. However, the current solution has its limitations. As the 2011 Fishing Rights Agreement illustrates, recognition of Indigenous fishing rights is subject to political 
negotiations and consensus among the majority population, and ultimately by the Norwegian Parliament. The issue of the historical fishing rights of the Sámi people as an Indigenous group in northern Norway remains an issue due to opposition from the National Fisheries Union and a majority of the Norwegian political parties, who do not see the utility of recognizing rights as long as the material basis is secured through quota allocations (Jentoft and Brattland, 2011).

In 2014, the Sámi Parliament and the three County Councils (Fjordfiskenemnda) created a new advisory board for northern inshore (fjord) fisheries. The board's mandate is to propose measures for strengthening coastal fisheries with special regard for Sámi communities. In a situation where cod stocks are moving north and the abundance of cod in the Barents Sea reached a record high (2014) while the ability to improve utilization of market demand is low, any improvements in the ability of coastal communities to benefit are welcome. In terms of the capacity to respond to rapid change on the local level, the new board may be more adept than the conventional governance system. With limited powers of decision-making, however, it remains to be seen whether the new mechanisms will have real effects on not only the legitimacy of Indigenous participation in resource governance, but on relations between Sámi fjord fishers, coastal communities, and markets.

The new fishing rights regime moves towards an increasingly userregulated resource governance regime in Arctic Norway. Decision-making power is increasingly devolved to stakeholders like the Sámi Parliament and others. Brattland (2012) argued that the increasing integration of Sámi rights and policies into resource governance is creating a new form of ethno-ecological governance regime. This regime does not necessarily mean that Indigenous peoples have increased their power as stakeholders and are co-governors of resource governance systems, but that states have translated Indigenous rights and policies into their own language and established co-governance structures and consultation. Relative to the larger Arctic context where Indigenous groups are emerging as increasingly instutionalized stakeholders and co-managers in resource governance, the Norwegian integration of Sámi policies in the overall resource governance structure may illustrate the limits to the growing power of current Arctic Indigenous institutions. While not an ideal arrangement in terms of full recognition of Indigenous fishing rights, management measures aimed at protecting Indigenous fisheries are actively discussed, even if not agreed to, among the majority population as a legitimate part of national resource governance. 
Ansgar Hansen tending his nets during the cod fishing season in Kåfjord, Norway, 2010

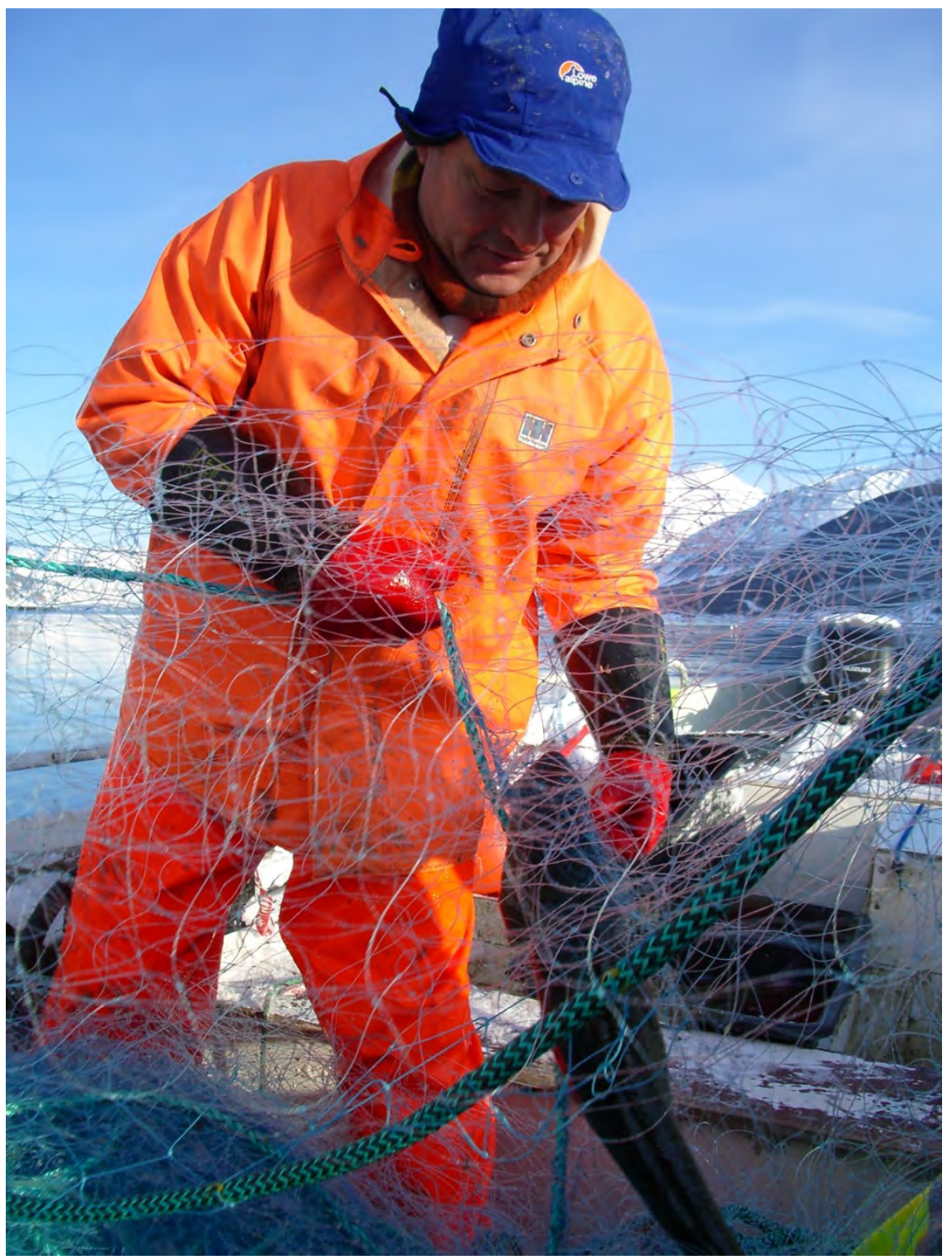

Photo Credit: Camilla Brattland. 


\title{
7.7.4 Indigenous-industrial relations in the tundra zone of Russia's Timan-Pechora and West Siberian Basins
}

\author{
By Bruce C. Forbes
}

As Russia increases its share of hydrocarbon exports to Europe and North America, it steadily becomes more of a de facto international actor, even as the enterprises producing the oil and gas are functionally state-held monopolies with few genuine western shareholders. How do the current and foreseen governance regimes account for the fact that current and proposed future oil and fields of the Nenets Autonomous okrug (NAO) and Yamal-Nenets Autonomous okrug (YNAO) overlap territories relied upon by Indigenous nomads for their livelihoods (reindeer herding, hunting, fishing, gathering)? This case study reviews the extent to which contemporary governance regimes in these regions either facilitate or hinder adaptation by Nenets nomads of the tundra zone.

The Timan-Pechora and West Siberian Basins are neighboring regions where both reindeer management regimes and the extent of engagement with the hydrocarbon extraction industry differ substantially (Stammler and Wilson, 2006; Stammler and Peskov, 2008; Kumpula et al., 2011, 2012). Whereas the linked social-ecological system of Yamal's tundra Nenets has demonstrated a remarkable level of overall resilience in recent decades, the Nenets in the NAO just west of the Ural Mountains have fared less well by comparison (Forbes et al., 2009; Kumpula et al., 2012; Forbes, 2013). On the other hand, the level of direct dialogue between Indigenous, state, and industrial stakeholders in NAO has generally exceeded that demonstrated so far in the Yamal region (Stammler and Wilson, 2006; Stammler and Peskov, 2008; Stammler and Forbes, 2009). At the local level, joint efforts to provide for mutual coexistence between nomadic herders and the oil and gas industry are impressive. This coexistence is possible as long as experienced people from industry are present on the ground and have authority commensurate with the tasks at hand, such as facilitating migration through rapidly expanding infrastructure (Stammler and Forbes, 2009; Kumpula et al., 2012). A key factor related to the coastal geography of Russian Arctic hydrocarbon development is the overlap between the major onshore deposits of TimanPechora and West Siberia with extensive areas used by Indigenous peoples for reindeer herding, hunting, fishing and gathering (Forbes et al., 2009; Stammler and Forbes, 2009; Kumpula et al., 2011, 2012). 
The post-Soviet climate for foreign investment in the oil industry has been unstable and risky, so international integration to date has been minimal (Locatelli, 2006; Andreyeva and Kryukov, 2008). Issues concerning rights to territories and resources are neither legislatively nor administratively resolved, resulting in uncertainties for stakeholders and conflicts between groups of Indigenous peoples and industrial companies (Andreyeva and Kryukov, 2008; Stammler and Forbes, 2009; Stammler, 2011). As in the West, the strength and extent of environmental governance in the Russian North has been highly variable in the post WWII era, fluctuating according to political and economic winds. At the same time, state governmental structures encourage implementation of sustainable development principles regarding exploration of hydrocarbon and renewable resources (Andreyeva and Kryukov, 2008).

To date, almost all of the developed Russian oil and gas fields are located onshore, and many of the most important giant fields are north of the Arctic Circle (Bishop et al., 2011). As these new sources come online they are expected to more than offset the declines of older fields farther south (Offerdal, 2007). Coastal provenance translates into a greater degree of freedom in terms of the mode and direction of product delivery, especially for liquid hydrocarbons (Andreyeva and Kryukov, 2008). As of Spring 2013, serious attention is being paid to the potential for hydraulic fracturing, or "fracking," within West Siberia's massive Bazhenov shale deposit, which is seen as cheaper to exploit and carrying far less risk than the Arctic seabed (Chazan, 2013). In a sign of the shifting outlook, in late summer 2013, Novatek - Russia's second largest gas producer after Gazprom - announced that it was looking to sell a major stake in its Yamal liquefied natural gas development (Shiraevskaya, 2013). Shell halted exploration in Alaska's Beaufort Sea in 2013 while outlining plans for shale oil development with Gazprom in the Pechora, East Siberian, and Chukchi Seas (Quinn, 2013). At the same time, North America's shale boom is making it less dependent on foreign sources of gas, freeing up liquefied natural gas for European customers (Anonymous, 2013).

One of the biggest challenges both offshore and onshore is the potential hazard associated with drilling in permafrost (Bishop et al., 2011). For onshore deposits, resource governance under the rubric of "best practices" is generally designed to avoid chemical and mechanical damage to permafrost terrain that, even under low intensity disturbance, can subside and quickly incapacitate costly infrastructure as well as cause lasting harm to frozen soils, tundra vegetation, freshwater systems and wildlife populations (Crandall et al., 2010; Bishop et al., 2011; Kumpula et al., 2011, Kumpula et al., 2012; OGP, 2013). Offshore guidelines tend 
to be general and pose obligations of intent on the national states, but few of them are binding (Offerdal, 2007; PAME, 2009). Petroleum industry groups have also established International Best Practices for onshore discharges, emissions, and wastes commonly generated during normal operations. Yet the individual operational standards of most petroleum companies are often much more stringent than International Best Practices or the performance standards set by individual countries. Russian company performance standards, at least on paper, are some of the most stringent in the world (Crandall and Thurston, 2010).

In general, difficulties associated with extractive industries operating in the Arctic may arise if exploration and production strategies conflict with existing plans for protection or development of the region (OGP, 2013). A major ongoing problem is the inequitable distribution of the wealth within the main oil and gas bearing regions. The hydrocarbon industry has raised the income and living standards for the specialized high wage earners who have migrated to YNAO and NAO. However, little of the money reaches the villages, let alone nomadic households on the tundra (Baffrey et al., 2010; Stammler, 2011), and barter remains common practice in and around active gas fields (Forbes, 2013). In practice, the distribution of revenues is hotly contested and there is fierce competition between Moscow, the regional governments, and the oil and gas companies over their respective shares of the profits (Stammler and Wilson, 2006). As the federal government and industry accrue ever-larger proportions of the income, the net result of this competition is reduced investment in social services at the okrug level (Baffrey and Thurston, 2010).

From the perspective of full-time tundra dwellers, employment in this emerging cash economy cannot easily replace hunting or herding since traditional foods are not interchangeable with imported foods, and cash will not compensate for loss of services outside the market economy. Herders and hunters often lack the skills and experience needed to participate in the wage employment provided by industrial development, and often these activities conflict with traditional cultural practices (Baffrey et al., 2010).

Likewise, tundra disturbances cause conflicts for traditional practices. Arctic Council guidelines assert that "physical disturbance due to [offshore] exploration activities has declined over the last two decades due both to a reduction in activities and due to improvements in technology and best practices" (PAME 2009, p. 7). Impacts linking the offshore to the onshore are recognized and addressed specifically in Gazprom's strategy for its Yamal megaproject (Gazprom, 2013). In its draft 
Program for the Comprehensive Development of Fields on the Yamal Peninsula and in the Adjacent Offshore Areas, Gazprom's goals have been to minimize environmental impacts to lands and waters relied upon by Indigenous populations. These guidelines are detailed in English and Russian on their website and include: a) sustained environmental monitoring during field pre-development and operation periods; b) planning of technological and special-purpose activities mitigating negative impacts on the surface air; c) utilization of water recycling systems that prevent pollution of surface reservoirs and soils; d) application of special technologies for reducing thermal and mechanical impacts on frozen ground; e) utilization of technical solutions to decrease the extent of land withdrawal areas, coupled with reclamation; f) prohibiting construction and installation activities during the spring bird nesting season; g ) water intake through fish protection systems; and viii) arranging for an unobstructed migration of reindeer herds through infrastructure.

Nomadic Nenets reindeer herders camp during summer migration through the Bovanenkovo gas field on Yamal Peninsula, Northwest Siberia, Russian Federation

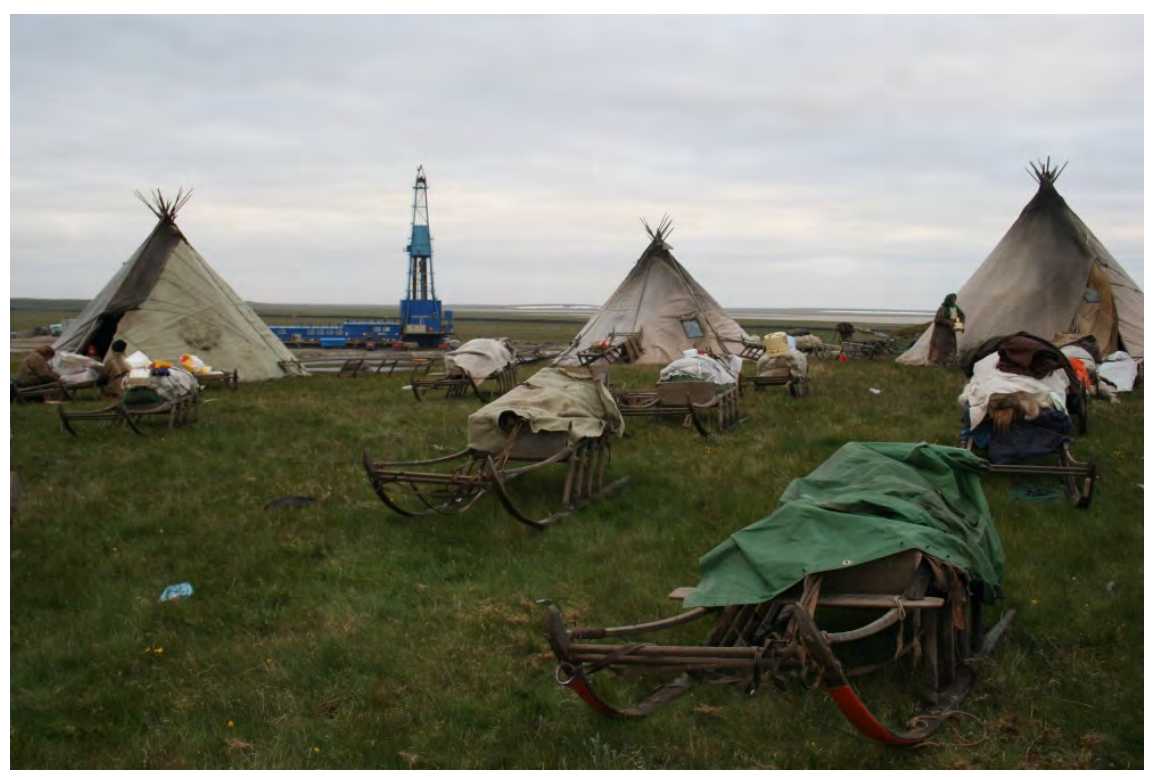

Photo Credit: Bruce Forbes.

Gazprom's socio-economic goals are to minimize impacts to and conflicts with Indigenous populations and include: a) protection of traditional areas of economic activities, archaeological and cultural sites of the indigenous population; b) utilization of advanced pre-development and operation technologies for oil and gas fields to minimize environ- 
mental risks and damage to the economic activities of the nomadic tundra population; c) reclamation of lands disturbed during oil and gas exploration in the 1980s to increase forage acreage for reindeer herding; d) construction of reindeer meat and fish processing complexes to supply shift personnel with high quality products, and employment of the Native population at such complexes; and e) ensuring efficient interaction between subsurface users and reindeer herders (Gazprom, 2013). A useful model can be drawn from Timan-Pechora Basin of NAO. There, older residents in the tundra zone have excellent knowledge of best practices dating from the early post-Soviet years at the Ardalin oil field (ConocoPhillips, 2013; Forbes, 2013). Mitigation measures at the field, operated by the Polar Lights Company since 1992, have been strictly adhered to, including bans on alcohol, drugs, firearms, fishing nets, and pets. The company is being careful to minimize the footprint on the ground rendered by infrastructure and associated terrain disturbance. Meanwhile on Yamal, from 1989 to 1996, one of the big producers, Amoco Eurasia, invested heavily in cultural heritage, financing archaeological work in areas slated for infrastructure development (Fitzhugh, 2004), as well as research on reindeer rangelands (Martens et al., 1996) and revegetation of disturbed tundra (Martens, 1995). Tundra nomads in both regions took close notice, and began to hope that the language of mutual coexistence introduced broadly across the country under Soviet-era resource governance would shift from rhetoric into concrete actions (Fitzhugh, 2004; Stammler and Forbes, 2009; Stammler, 2011; Kumpula et al., 2011; Forbes, 2013).

Since the withdrawal of Amoco Eurasia from the Yamal Peninsula in the mid-1990s, no other Western major company has stepped in to take its place. Although Statoil has had some serious discussions about partnering with Novatek in recent years (Nilsen, 2011), Gazprom remains the dominant force in resource governance of the Yamal tundra. Notwithstanding relatively small-scale production via joint ventures, such as that between ConocoPhillips and Rosneft, in NAO the main players continue to be Russian majors like Lukoil. Formal governance is now less haphazard than during late Soviet and early post-Soviet times, yet still the onus for adaptation - much of it informal - has been on those Nenets who remain resident with their herds much or all of the year within the tundra zone. Key segments of resource governance, including the pasture compensation and free right of passage through active extraction fields, have become highly institutionalized and function as well as can be expected at least in the high traffic areas which are closely monitored, such as the Bovanenkovo gas field (Kumpula et al., 2012; 
Forbes, 2013). The current feeling is that tundra nomads can live with the unavoidable but manageable damage to the terrestrial and aquatic ecosystems, to which people have decades of experience adapting.

Still, the anticipated underwater pipeline from Taz Peninsula to Yamal is considered by some to be a risky venture, with the potential for methane leaks that would degrade the highly prized Ob river and delta fisheries (Florian Stammler, Personal Communication, 1 July 2013).

A warming climate may present significant challenges in the near future if, for example, autumn/winter/spring reindeer pastures become more frequently and intensely ice-encrusted (Bartsch et al., 2010). Encroaching infrastructure, such as new roads and railways, may impede timely movement of large numbers of animals unless proper consultation leads to adequately planned and provisioned crossing points (Forbes et al., 2009; Kumpula et al., 2011; Kumpula et al., 2012). For now, herders place a higher priority, relative to climate-related issues, on managing relations with the various stakeholders surrounding hydrocarbon extraction (Forbes and Stammler, 2009). The decades-long coexistence of herders with the gas industry in the south of YNAO has shown that in the long run, both ways of using the land can survive in close proximity to each other, even though some of the adaptations were painful for herders who had to change and abandon traditional migration routes (Stammler, 2011; Kumpula et al., 2012). The sheer existence of laws and rules on paper is not sufficient to address the diversity of problems that arise during the practice of coexistence. The need for more intensive consultation will increase in urgency as the tundra zone in both NAO and YNAO becomes more crowded (Stammler and Forbes, 2009). In the end, climate is just one of many drivers with the potential to challenge the adaptation of tundra dwellers to hydrocarbon extraction activities that will almost certainly continue for several decades (Stammler and Wilson, 2006; Stammler and Forbes, 2009; Kumpula et al., 2012).

\subsubsection{Greenland's legal framework for non-renewable resource exploitation: The challenges of creating transparent public consultation processes}

\section{By Mitdlarak Lennert}

Despite Greenland's wealth of resources, the level of resource exploitation has been modest compared to other Arctic regions. With great potential for subsurface mineral exploitation, Greenland today may be on the threshold of becoming a mining nation. The challenges associated 
with governance and sustainability in relation to these activities will be to ensure sovereignty over these resources in and around Greenland, to ensure that the society benefits economically by creating jobs and education possibilities, and to avoid potential environmental problems.

Greenland became a Danish colony in 1721. In 1953, Greenland was recognized as a county within the Danish realm. Subsequently, the legal framework for non-renewable resource management was based on the Danish legislation. In 1960, a Danish-Greenlandic Commission was created to consider the management of future mineral exploitation activities. Consequently, the first law on non-renewable resources in Greenland was passed by the Danish parliament in 1965 (Sørensen, 1983; BMP, 1999).

The question of rights to minerals and energy resources was an important part of the Commission's negotiations prior to the establishment of Home Rule in 1979. The Greenlanders in the Commission demanded full rights to all subsurface resources, but the Danish Government only accepted the Greenlanders' rights to the renewable resources (Skydsbjerg, 1999). The compromise was to recognize that Greenlanders hold fundamental rights over the natural resources and that a joint decisionmaking body consisting of representatives of the Danish state and the Government of Greenland, the Joint Committee on Mineral Resources in Greenland should be established. This Committee consisted of five Danish and five Greenlandic members, appointed respectively by the Folketing (the Danish Parliament) and the Landsting (the Greenlandic Parliament). The Danish Queen appointed the chairman for periods of four years and the Committee held meetings alternating in Denmark and Greenland. The main tasks of the Joint Committee were to assess the mineral resources in Greenland and make recommendations to the Greenland Home Rule Government and the Danish Government on fundamental issues related to mineral exploration (BMP, 1999)

In 1998, negotiations between the Government of Greenland and the Danish Ministry of Environment and Energy resulted in an agreement to transfer the administration of mineral resources to the Government of Greenland. Greenland then held sole responsibility for managing the administration of all mineral and energy resource activities, as well as promoting Greenland's mineral potential to the international mining industry (BMP, 1998). With the introduction of self-governance in Greenland in 2009 and the passage of the Mineral Resources Act in 2010, the Greenlandic Government assumed responsibility over all aspects of mineral resources and the Joint Committee was abolished. Since January of 2010, the Greenlandic authorities have held responsibility for the non-renewable resources and subsurface rights. 
The Danish state still assumes responsibility for a number of activities in Greenland and Greenland receives an annual block grant from the Danish state to cover the expenses associated with the activities for which the Greenlandic authorities have taken over responsibility. If the Greenland government acquires revenue from mineral resource activities, the block grant will be reduced by an amount equal to half of the revenue beyond a set annual amount. Further negotiations on the future economic relations between the Danish state and the Greenland selfgovernment will be held when the Danish state's contribution to the Greenland government is reduced to zero.

With the warming climate, the melting of the Arctic sea ice and the increasing global need for a variety of minerals over the past years, there is a rising interest in non-renewable resources in Greenland. While the total number of exclusive exploration licenses in 2002 was 17, the number had increased to 94 licenses in 2011 (BMP, 2011). In addition, the Greenland government is eager to see renewable resource development become a bigger component of economic development. The 2012 report from the Greenlandic Economic Council argued that there is a need for a more diversified economy to maintain Greenland's standard of living. The development of non-renewable and energy resources is seen as a means to create more "pillars" to the Greenlandic economy, reduce the dependency on the annual block grant from Denmark and ultimately to become economically selfsufficient (BMP, 1997; Grønlands Økonomisk Råd, 2012)

Since the introduction of self-governance in 2009, and the assumption of authority over the mineral resources in 2010, the Greenland government has established a number of structures to facilitate public participation throughout the process of mineral exploration and extraction. The Mineral Resources Act came into effect in 2010, and is the legal foundation for granting licenses. Furthermore, the Act also aims to ensure that activities conform to safety, health, and environment standards, use proper consultation practices, and incorporate best international practices. The Mineral Resources Act requires all applicants to undergo an Environmental Impact Assessment (EIA) and a Social Sustainability Assessment (SSA). These processes require companies to send the reports for public consultation to project stakeholders.

The Mineral Resources Act places formal responsibility for the public process and citizen involvement with the Greenland Government and the Mineral License and Safety Authority (formerly the Bureau of Minerals and Petroleum (BMP)). The main part of the public process occurs in the context of corporate EIA and SSA reports and the organization of 
public meetings. The guidelines require, as a minimum, that the mining companies organize and hold a public information meeting prior to the commencement of the EIA and SIA and hold another public meeting to present the results (BMP, 2013).

In practice, the public process is about information flowing from oil and mining companies to the public and the transfer of responsibility for public participation from the government to the mining companies is problematic (Aaen, 2012). The purpose of transferring responsibility for the process to the government, in a situation of power asymmetry, is to ensure that it will not be possible for the strongest stakeholder to take over the process and thus control the debate. By leaving parts of the process to the companies that are already the most powerful stakeholders, there is a risk of exclusion of stakeholders who do not share interests with the company.

Problems with the public participation process are illustrated within one of the largest proposed development projects in Greenland. The UKbased London Mining Company is planning to develop an iron mine at the edge of the ice cap at the bottom of the Nuuk Fjord, with an estimated 1.1 billion tons of iron. In addition to the prospects of a much-needed boost to the Greenlandic economy, the Isua Project would support up to 450 local jobs with the potential of more after training programs start (London Mining, 2013).

Reviewing the public consultations meetings concerning the Isua Project, one can identify a number of trends and challenges. Over the course of four public meetings arranged by the Government of Greenland and facilitated by a private consultancy, the discussions centered more around the process, or lack thereof, of involving the public, than on a comprehensive discussion of the Isua project. The structure and location of the public meetings were far from ideal, as the first three meetings were held outside the city in Ilimmarfik (the university campus), and the last meeting in the cultural center Katuaq. Invitations to the consultation meetings were sent out late, and resulted in between 50 and 160 participants, with officials comprising the majority in the first meetings. The meetings consisted of presentations from representatives of London Mining Company and the government, followed by group sessions and discussions. The duration of the meetings was critiqued, and there was a concern that members of the public often did not receive answers to questions right away, that there was no real dialogue, and criticisms were not taken seriously. Finally, the fact that answers and debates often had to be handled in two, and often three, languages provided a set of problems unique to Greenland. 
The lack of transparency on decision-making processes by the government, as well as London Mining, in addition to information regarding the project being unavailable or inadequate, raised questions as to who controls the agenda and an expressed concern among the population to become spectators in their own country. The need for independent expert opponents to analyze and assess the information being given was stressed by both the public and NGOs. A comprehensive report on the democratic legitimacy of the consultation processes in Greenland criticized the information meetings for being conducted by the companies, rather than the government or neutral parties, as well as noting that the period for public comments and discussions was too short and a belief was held by the vast majority of Greenlanders that their opinion didn't matter (Aaen, 2012).

More education, among other initiatives, and more people actively participating in debates and consultations, are essential to empowering Indigenous peoples in Greenland. To balance the needs of a company that has to answer to investors, time and money, and the needs of a country and a population that face some of the largest industrial projects in their history of their homeland, continuous, informed and seriously conducted consultations and a constructive dialogue with the public is of the utmost importance.

\subsubsection{Transformation and restructuring in forestry: examples from Sweden}

By E. C. H, Keskitalo

This case study reviews the ways in which forestry is currently changing and being restructured in Sweden, with a focus on impacts at the local level. While this section reviews the case of Sweden, similar processes are also ongoing in other regions of Fennoscandia. Largely a result of globalization, the structure of large-scale land use sectors is changing, including a shift towards internationalization and higher international competition. Changes in traditional uses of pulp and paper with the decline in print media result in less demand and require re-organization and development of new products in forest industry. Forestry related employment has also declined due to far-reaching technological shifts that substitute labor with technology. Local logging teams have been replaced by hired contractors who serve multiple sites. The historical role of forestry in supporting employment in rural 
areas is thereby also shifting while retaining the economic output of forestry (Keskitalo, 2008).

In Sweden, products from forestry and forest industry make up about 3\% of GDP and about $10 \%$ of export value. Much of this value comes from the boreal forest, which is subject to multiple competing uses (Swedish Forest Agency, 2013a; Swedish Forest Agency, 2013b). Given its strong economic and historical importance in Sweden, forestry is well institutionalized and can be seen as given primacy in certain cases, both legislatively and in relation to other sectors. For instance, the key legislation of the Forest Act of Sweden lies separate from the Environmental Code, thereby fundamentally separating forest management from environmental issues in general. The Forest Act is also a "framework act" rather than detailed law, and therefore fundamentally leaves forestry managers open to select the ways in which legal requirements are fulfilled. Forest management is similarly not part of the otherwise strong municipal planning monopoly at the local level (Stjernström et al., 2013).

This situation may also result in conflicts with other land uses. For instance, forest owners (including the state, large multinational corporations, small-scale forest farms and other private owners) in northern Sweden hold ownership rights to the same lands on which reindeer herders possess parallel user rights. While forestry is in many areas required to undertake consultations with reindeer husbandry, these consultations cannot negatively influence what is considered ongoing forest use (in most cases active forestry). Conflicts may thus arise between different user and interest groups. These are further complicated by the need to consider biodiversity and conservation within forestry management. As a result, forest certification as a voluntary, marketbased mechanism has become one important additional means to support environmental biodiversity and social considerations in forestry (Keskitalo, 2008; Keskitalo et al., 2009).

While forestry has traditionally gained strong local support as a source of local employment, increasing internationalization today limits the number of people directly employed in forestry at a local level. Urbanization and changes in resource use (including technology shifts) result in increasing out-migration from rural areas, and forest owners are increasingly less dependent on profits from their forest holdings. Many forest owners concurrently maintain other employment and may increasingly embrace urban values that may include an increasing focus on conservation. Forest owners today also often receive land due to inheritance and today comprise a larger number of women, which may 
result in changes both in demography and values of forest owners as well as in activity within forest management. These new forest owners may thus include a focus on conservation, or on other land uses than forestry, and/or may express less active or direct involvement in forest management (Nordlund and Westin, 2011).

The decrease in employment in forest-related occupations in rural areas has further resulted in discussions of new options for rural employment, with some focus being placed for instance on tourism and in the present also mining development. The general intensification in resource use in society is resulting in increased pressures on resource use and thereby in increased discussion of the potential benefit of resource extraction at large to local areas. As a result the distribution of costs and benefits in forestry and mining, as well as the role of local planning, are currently being re-considered in Sweden (Keskitalo, 2008; Stjernström et al., 2013). Some similarities to this situation with regard to restructuring in forest management can be seen for instance in Finland (cf. Keskitalo et al., 2009).

\subsubsection{The role of the Arctic Council in resource governance}

By Oran Young and Paula Kankaanpää

Is there a role for a regional body like the Arctic Council (AC) in resource governance? If so, is this role likely to grow or develop in new directions during the coming years? To address these questions, we need to start with some observations about the nature of the Arctic Council and the development of its place in Arctic affairs over time.

The 1996 Ottawa Declaration on the Establishment of the Arctic Council launched the Council as a high level forum for "promoting cooperation, coordination and interaction among the Arctic States with the involvement of the Arctic indigenous communities and other Arctic inhabitants on common Arctic issues, in particular issues of sustainable development and environmental protection" (Arctic Council, 1996). Several features of the declaration make it clear that those who crafted its provisions intended a limited role for the Council in resource governance issues. The Council is a soft-law arrangement; it lacks the authority to make decisions that its members are required to accept, much less obligated to implement within their own jurisdictions. The Council's focus, moreover, is on regional issues requiring "intergovernmental consideration." It is not designed to address domestic concerns like estab- 
lishing and operating co-management regimes governing humanenvironment interactions on a local or sub-regional scale.

Yet the Arctic Council also has constitutive features that provide openings for addressing issues of resource governance. Unlike its predecessor, the Arctic Environmental Protection Strategy (AEPS), the Council is authorized explicitly to address "issues of sustainable development" and environmental protection. It therefore has a mandate to consider economic and social or cultural matters, rather than sticking to the biophysical issues that are central to environmental protection. Unlike the AEPS, moreover, the Council includes an explicit role for Permanent Participants (PPs) "... to provide for active participation and full consultation with the Arctic Indigenous representatives within the Arctic Council" (Arctic Council, 1996). Because the use of natural resources is a central feature of the economic, cultural and spiritual systems of the Arctic's Indigenous peoples, the practice of including the PPs in all the Council's activities ensures that issues relating to humanenvironment interactions and the effectiveness of governance systems designed to manage these interactions will surface on a regular basis in Council deliberations.

Despite the limits imposed by the Ottawa Declaration, the Council has emerged as a significant force in Arctic affairs. Its most striking successes to date involve what is described in policymaking literature as agenda setting (Kingdon, 1995) and, more specifically, the identification of emerging issues, the framing of these issues for consideration in public settings, and the promotion of selected issues to prominent places on the Arctic agenda. The most significant cases center on assessments carried out under the auspices of the AEPS and the Council, including the AMAP report on Arctic pollution issues (AMAP, 1998), the Arctic Climate Impact Assessment (ACIA, 2004; ACIA, 2005), and the Arctic Marine Shipping Assessment (Arctic Council, 2009). In each case, the Council's work drew attention to the relevant issues and helped set the terms of the subsequent debate about how to come to terms with them. In some instances, these assessments have influenced the course of Arctic affairs and the treatment of issues in global forums. The contributions of the Arctic Council regarding the impacts of persistent organic pollutants on human health, for example, are widely credited with influencing the negotiation of the 2001 Stockholm Convention on Persistent Organic Pollutants (Downie and Fenge, 2003). The ACIA documented the onset of climate change in the Arctic. In the process, it played a role in shifting the global debate about climate change from a modeling exercise to an examination of observable impacts (Nilsson, 2009). The importance of 
co-management through stakeholder participation was acknowledged already in 1997 as part of the Guidelines for Environmental Impact Assessment in the Arctic (AEPS, 1997). Today, these guidelines have more relevance than they did at the time of their adoption.

In recent years, as transformative changes bring the Arctic to the attention of policymakers worldwide, the Arctic Council's role has expanded to include policymaking as well as policy-shaping (Kankaanpää and Young, 2012). The Agreement on Cooperation in Aeronautical and Maritime Search and Rescue in the Arctic, negotiated under the auspices of the Council and signed at the 2011 Ministerial Meeting, exemplifies this development. The Agreement on Cooperation on Marine Oil Pollution, Preparedness and Response in the Arctic, signed at the 2013 Ministerial Meeting, continues this trend. The terms of the Ottawa Declaration impose clear limits on the development of the Council's role in policymaking. But the sharp rise of human activities in the Arctic arising from commercial shipping, oil and gas development, ship-based tourism, and (potentially) commercial fishing have brought an array of policy concerns into focus and generated pressures to address them sooner rather than later. It would be a mistake to expect the members of the Arctic Council to revise and expand the Council formally to provide it with the authority to make legally binding decisions on a range of emerging policy issues. But the practices of the Council are likely to continue to evolve towards resource governance and co-management policy making. The 2013 Kiruna Declaration (Arctic Council, 2013) highlights the importance of sustainable use of resources and economic development as well as the role of business and corporate social responsibility.

The substantive work of the Arctic Council is generally conducted through working groups (Koivurova and VanderZwaag, 2007). The Ottawa Declaration speaks of "... sustainable development and environmental protection" as parallel concerns, implying that they should generate distinct and roughly comparable programmatic activities. Thus, the Council is to "... oversee and coordinate the programs established under the AEPS" and "... adopt terms of reference for, and oversee and coordinate a sustainable development program" (Arctic Council, 1996). On the strength of this mandate, the Council transformed the AEPS Task Force into the Sustainable Development Working Group and charged this working group with the function of defining the place of sustainable development within the overall system of international cooperation in the Arctic.

Defining sustainable development, however, is easier said than done. To begin, the process of devising terms of reference for the SDWG produced protracted debates pitting issues of human-environment relations 
directly against concerns about creeping international jurisdiction that could interfere with domestic policies of member states (Koivurova and VanderZwaag, 2007; Kankaanpää and Young, 2012). Equally complex has been clarifying the relationship between sustainable development and environmental protection in the practices of the Council. In some cases, the pursuit of sustainable development necessitates tradeoffs among ecological, economic, and social considerations. In the case of resource governance, such trade offs often surface in tensions between the needs of subsistence users and the recommendations of ecologists and population biologists.

Within the Arctic Council there are roadblocks to organizing programmatic activities along these lines. The Ottawa Declaration did not mandate any restructuring of the programmatic activities inherited from the AEPS. The environmental protection working groups (e.g. the Arctic Monitoring and Assessment Programme, the Working Group on the Conservation of Arctic Flora and Fauna) were well-established by the time the SDWG came along. They were not about to place themselves under the umbrella of sustainable development and, in the process, to subordinate their efforts to the vision and priorities articulated within the SDWG.

The difficulties experienced by all who have sought to translate the overarching idea of sustainable development into a set of criteria that are sufficiently precise and operational to be used to guide a coherent work program have also hampered the efforts of the SDWG (Parris and Kates, 2003). The SDWG has become a vessel containing a disparate collection of projects that have appealed to various constituencies in a position to mobilize the resources needed to carry them out. Some of these projects (e.g., the project on reindeer herding and adaptation to climate change) clearly have implications for resource governance. Others seek to generate empirical data relevant to sustainable development (e.g., the Survey of Living Conditions in the Arctic), to draw attention to socially important concerns (e.g. the Arctic Human Health Initiative), to bring intellectual capital produced in other settings to bear on Arctic issues (e.g., the Arctic Resilience Report), or to address emerging issues now coming into focus as a result of the transformative changes occurring in the Arctic (e.g., the project on Adaptation Actions for a Changing Arctic).

One initiative that has helped to frame the agenda of the SDWG is the first Arctic Human Development Report (AHDR, 2004). Completed in 2004, the AHDR formulated a set of social indicators designed to supplement those included in the UN Human Development Index with indi- 
cators that are particularly appropriate to the assessment of human well-being in the Arctic. Of particular importance regarding resource governance is the follow-up effort to develop empirical indicators useful in assessing performance relating to ongoing contact with nature, social cohesion, and fate control (ASI, 2010).

The decision to revisit these issues in a second volume of the $A H D R$ provides a critical opportunity to identify trends, noting both areas in which progress is being made and areas where problems have arisen or become more severe since the completion of the first AHDR. Over time, these reports can play an increasingly important role in providing the data needed not only to evaluate the success of the SDWG's programmatic activities but also, and particularly relevant to the focus of this chapter, to allow the Arctic Council to play a useful role in assessing the performance of resource regimes and offering suggestions for improving their performance over time.

To sharpen their ability to perform this assessment function, those operating under the auspices of the Arctic Council could endeavor to join together general knowledge about resource regimes (Young, 1982), including methods of environmental impact assessment and permit processes, and in-depth assessments of resource governance systems in place in the Arctic (case studies this chapter). The goal of this effort would be to take advantage of the development of a large body of knowledge dealing with matters like the processes involved in creating resource regimes and the conditions that determine the effectiveness of these arrangements, while simultaneously paying attention to the specific conditions prevailing in the Arctic and even contributing to our general understanding of resource governance through a detailed examination of Arctic experience (Young and Osherenko, 1993). In cases involving highly migratory species (e.g., birds and marine mammals), the Arctic Council might well be able to initiate a constructive dialogue with those seeking to deal with matters of resource governance regarding specific species (e.g., grey whales, brant geese) during the stages of their migratory cycles when they are located outside the Arctic.

The resultant activities would be politically sensitive, requiring the Council to take an interest in activities occurring within the jurisdiction of individual states (e.g., the harvesting of bowhead whales by Alaska Natives within the Exclusive Economic Zone of the United States). Handled with care, this should not present an insurmountable problem. The value added from the Council's efforts stems from bringing together more general knowledge pertaining to resource governance with an indepth understanding of the issues surrounding resource governance 
under the conditions prevailing in the Arctic and from forging bonds among those concerned with the management of highly migratory species. The application of the resultant insights would be advisory; it would not entail any adjustment in the existing structure of authority regarding such matters. But the result could be an improvement in the effectiveness of resource governance in a variety of settings.

\section{Arctic Council, Salekhard, Russian Federation, 2006}

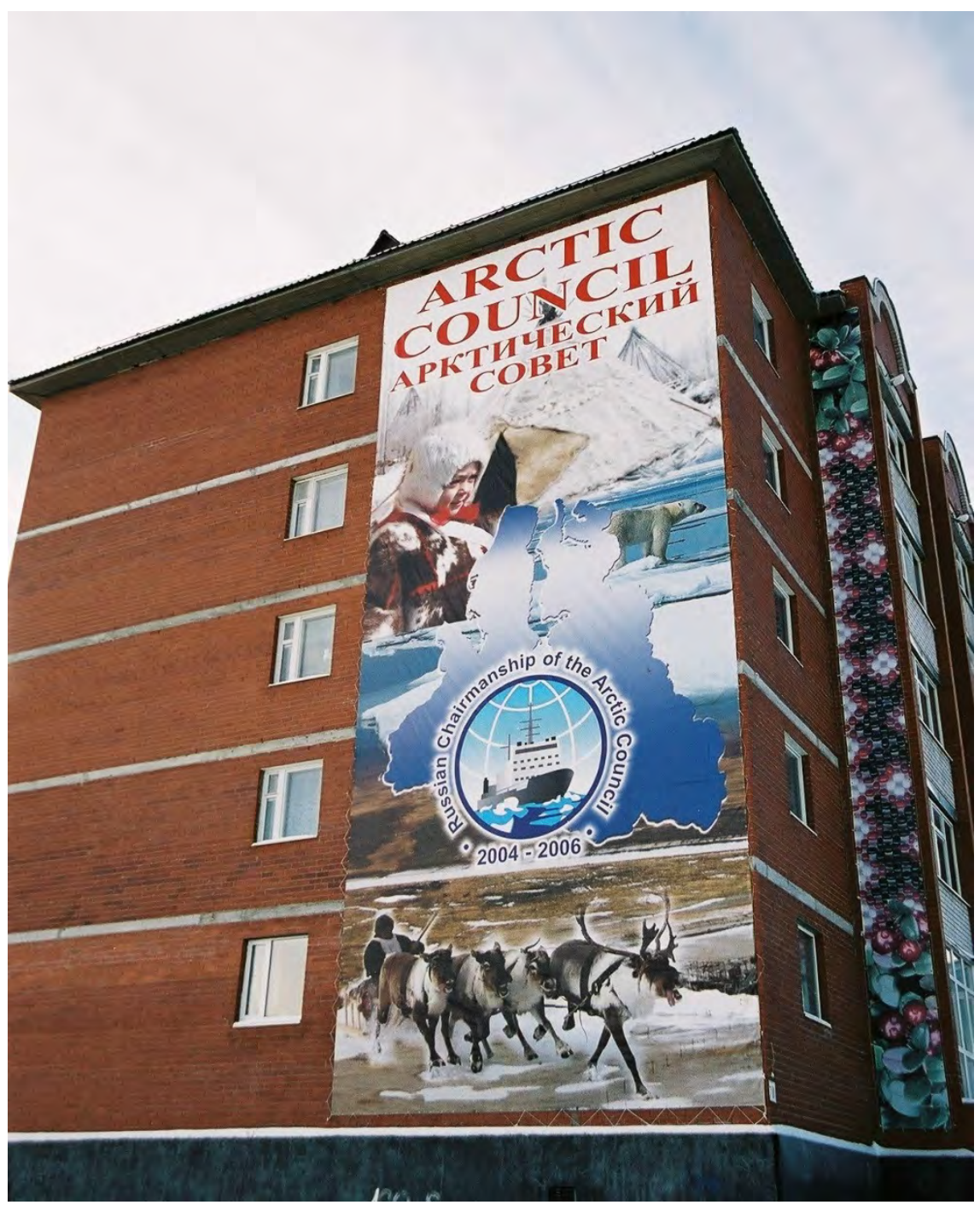

Credit: Harald Finkler 


\subsection{Conclusion}

In light of the rapid pace of environmental change and the strong push to extract both renewable and non-renewable resources from the Arctic, understanding the interrelationships between Arctic peoples and resource governance is more critical than ever. With or without additional climate warming, certain sectors of the Arctic are bound to experience significant new resource development in the coming years, as is described in many of the cases highlighted in this chapter.

\subsubsection{Insights from case studies}

Our case studies encompass both terrestrial and marine systems, and a mix of regional, national and international resource regimes. Together they represent a cross section of some of the most important and widespread renewable and non-renewable resources subject to governance in today's Arctic: reindeer herding, fisheries, marine mammal hunting, forestry, mining and hydrocarbon extraction.

These cases identify three important trends in resource governance with major implications for long-term sustainability and selfdetermination. The first trend is the increasing complexity of Arctic resource governance, as illustrated by Sámi fisheries and Nordic forestry and reindeer herding. The second trend is the increasing acknowledgment of cognitive rather than physical human-land relations. A third trend is the increasing adoption of best practices, for example within the Russian oil and gas fields.

In terms of outcomes, the new and rapidly evolving co-management in Sámi fisheries offers positive lessons for how such a regime might be implemented in Eurasian terrestrial systems. The case studies make clear that Nordic reindeer and forestry management, in particular, face serious challenges in developing legitimate management regimes with user participation (see also Ulvevadet, 2008, 2011). Informal institutions at the regional and international levels can be the basis for highly flexible and responsive governance systems such as in Bering Strait Walrus management and Russian oil and gas. An informal, interlocal relationship persists as the key locus for international Bering Strait walrus management coordination. Yet in spite of emerging informal networks, there is clearly a need for formal rights and specified laws with meaningful enforcement, instead of a system allowing industry to select the ways in which legal requirements are fulfilled, such as in Nordic reindeer/forestry governance and Russian oil and gas. Establishing Arctic 
World Heritage Sites, as in Sweden, represents international harmonization of policies, but also raises questions regarding the future of Indigenous land use rights. Several cases point to the increasing need for meaningful consultation processes to replace those that vary widely in terms of their intent, efficacy and outcomes. As seen in Greenlandic and Russian non-renewable resource exploitation, it takes more than legislation to ensure effective public participation. Operationalizing effective public involvement is a multi-level and social learning enterprise, involving education systems, communities, and those managing resources and their exploitation. Greenland is an interesting contrast with Russia, as Greenland is typically considered a haven for Indigenous selfgovernance, and Russia as home to a more paternal governance regime, so it is particularly illuminating to see both myths challenged and outcomes contrary to what some may expect.

Finally, in the Arctic Council we see a challenge to Arctic rim countries to aim higher than acting as advisors. For example, the oil and gas guidelines discussed in the Russian case study are based on obligations of intent, rather than binding. Despite this limitation, it can be argued that the Council has demonstrated influence not only within but also beyond the Arctic. In terms of the latter, examples of positive outcomes cited include negotiation of the Stockholm Convention on Persistent Organic Pollutants and the global examination of climate impacts triggered in part by the increased awareness following the Arctic Climate Impact Assessment.

Northern residents perceive "contact with nature" as essential to their well-being (ASI, 2010). How well people with resource based livelihoods navigate this need within contemporary governance regimes bears strongly on their capacity to effectively respond within a world characterized by rapidly changing socio-economic, political and environmental drivers. In some case studies presented here, informal arrangements are already working reasonably well without regulatory enforcement. Documenting these experiences in more detail is needed so their basic lessons can be transferred within and among different legal systems and proper consultation procedures can be implemented more broadly. Russia, comprising nearly half of the Arctic geographically and home to a large number of Indigenous cultures, is typically an outlier in political and resource regimes. Despite its occasionally fierce geopolitical stance, it must be noted that Russian practices directed toward coexistence between Arctic Indigenous peoples and resource development in at least some areas have improved in recent years. In light of this positive outcome, longer-term engagement based on truly participa- 
tory science with policy relevance is more likely to have an impact than short-term environmental activism designed to completely halt Arctic resource development. Maintaining a constructive resource governance dialogue at the circumpolar scale requires including Russia.

\subsubsection{Outstanding questions}

While the case studies and general observations of this chapter highlight common patterns and conditions in resource governance across the Arctic, they also raise many questions, pointing up the need for future study. The Walrus and Yamal case studies suggest the need to understand better the capacity of informal institutions to complement formal institutions in ways that insure high sensitivity and responsiveness in resource management across scales. The Swedish cases on reindeer herding and fisheries raise complex questions about how well Indigenous peoples who pursue traditional livelihoods can work alongside other local non-Indigenous interest groups. The Greenland case suggests the need to cultivate and enhance public participation and poses the question of the extent to which public participation can shape sustainable policies in the face of rapid large-scale and highly profitable resource development. The Yamal study raises the questions of how groups access funds to support involvement in resource governance and what are the consequences for groups that are marginalized and excluded.

Likewise, broader questions remain. To what extent will limited funding for community involvement, social and ecological monitoring, and research in resource management leave resource managers (and comanagers) with the information they need to respond to the inevitable changes that are to occur? On a broader scale is the question of whether established "power sharing" or co-management relationships can be transformed to systems of governance that facilitate social learning and adaptation. Finally, the international scale transactions of the Arctic Council have proven successful in a number of areas in spite of holding limited formal authority. The extent to which the Arctic Council and its groups, like the SDWG, can continue and perhaps broaden its initiatives is also worthy of on-going inquiry.

These questions underscore the highly dynamic nature of resource governance in the Arctic, and the need for a greater awareness of the critical role of institutions, formal and informal, in supporting future human development in the North. 


\subsection{References}

Aaen, S. B., 2012. Democratic legitimacy in consultation processes associated with large scale projects in Greenland. Employer's Association in Greenland.

ACIA, 2005. Arctic Climate Impact Assessment - Scientific Report. Cambridge University Press, Cambridge.

ACIA, 2004. Arctic Climate Impact Assessment. Cambridge University Press, Cambridge.

Act on Greenland Self-Government, 2009. http://www.stm.dk/multimedia/GR_SelfGovernment_UK.doc. (27 May 2014).

AEPS, 1997. Arctic Environmental Protection Strategy. http://library.arcticportal.org/1271/ (9 September 2014).

AHDR, 2004. Arctic Human Development Report. Einarsson, N., J. N. Larsen, A. Nilsson and 0. R. Young (eds.). Stefansson Arctic Institute, Akureyri.

AMAP, 2010. Assessment 2007: Oil and Gas Activities in the Arctic - Effects and Potential Effects, Vols. 1 and 2. Arctic Monitoring and Assessment Program, Oslo.

AMAP, 1998. AMAP Assessment Report: Arctic Pollution Issues. Arctic Monitoring and Assessment Programme, Oslo.

Anderson, T., J. Kruse and B. Poppel, 2002. Survey of Living Conditions in the Arctic: Inuit, Saami and Indigenous Peoples of Chukotka (SLiCA). Arctic, 55(3): 310-317.

Andreyeva, E. N. and V. A. Krukov, 2008. The Russian model: merging profit and sustainability. In: Mikkelsen, A. and O. Langhelle (eds.). Arctic Oil and Gas: Sustainability at Risk? pp. 240-287. Routledge, London.

Anisimov, Oleg A., D. G. Vaughan, T. Callaghan, et al. 2007. Polar regions (Arctic \& Antarctic). In Climate Change 2007: Impacts, adaptation and vulnerability: Contribution of Working Group II to the fourth assessment report of the Intergovernmental Panel on Climate Change. M. L. Parry, O. F. Canziani, J. P. Palutikof, P. J. van der Linden, and C. E. Hanson (eds.), pp. 653-685. Cambridge University Press, Cambridge.

Anonymous, 2013. Gazprom: Russia's wounded giant. The Economist. http://www.economist.com/news/business/21573975-worlds-biggest-gasproducer-ailing-it-should-be-broken-up-russias-wounded-giant (17 May 2013).

AMSA, 2009. Arctic Marine Shipping Assessment Report. Ellis, B. and L. Brigham (eds.). Arctic Council.

Arctic Council, 2013. Arctic Resilience Interim Report. Stockholm Environment Institute and Stockholm Resilience Centre, Stockholm.

Arctic Council, 1996. Declaration on the Establishment of the Arctic Council. Ottawa, September 19, 1996. www.arctic-council.org/ (9 September 2014).

Armitage, D. and R. Plummer (eds.), 2010. Adaptive Capacity and Environmental Governance. Springer, Berlin.

Armitage, D., F. Berkes, and N. Doubleday, (eds.), 2007. Adaptive Co-Management. Collaboration, Learning and Multi-Level Governance. UBC Press, Vancouver.

ASI, 2010. Arctic Social Indicators - a follow-up to the Arctic Human Development Report. Larsen, J.N., P. Schweitzer, and G. Fondahl (eds.). TemaNord. Nordic Council of Ministers, Copenhagen.

Baffrey, M., H. P. Huntington, F. Stammler, T. Tuisku, S. Haley, T. Stubbs, W. Greenall, A. H. Hoel, and O. L. Pedersen, 2010. Social and economic effects of oil and gas activities in the Arctic. In: Assessment 2007: Oil and gas activities in the Arctic - effects and potential effects, pp. 3_1-3_71. Vol. I. AMAP, Oslo. 
Bali, A., and G. P. Kofinas, 2014. Voices of the Caribou People: a participatory videography method to document and share local knowledge from the North American human-rangifer systems. Ecology and Society, 19(2): 16. http://dx.doi.org/10.5751/ES-06327-190216 (9 September 2014).

Bartsch, A., T. Kumpula, B. C. Forbes and F. Stammler, 2010. Detection of snow surface thawing and refreezing using QuikSCAT: implications for reindeer herding. Ecological Applications 20: 2346-2358.

Beach, H., 2012. Nordic reflections on northern social research. In: Kockel, U., M.N. Craith, and J. Frykman (eds.). A Companion to the Anthropology of Europe, first edition, pp. 33050. Blackwell Publishing Ltd., Sussex.

Beach, H., 2004. Political ecology in Swedish Saamiland. In: Anderson, D. and M. Nuttall, (eds.). Cultivating Arctic Landscapes: Knowing and Managing Animals in the Circumpolar North, pp. 110-123. Berghahn, Oxford.

Berkes, F., 2012. Sacred Ecology: Traditional Ecological Knowledge and Resource Management. Taylor and Francis, Philadelphia.

Berkes, F., 2009. Evolution of co-management: role of knowledge generation, bridging organizations and social learning. Journal of Environmental Management, 90(5): 1692-1702.

Berkes, F. and D. Armitage, 2010. Co-management institutions, knowledge and learning: adapting to change in the Arctic. Etudes/Inuit/Studies, 34(1): 109-131.

Berkes, F. and D. Jolly, 2002. Adapting to climate change: social-ecological resilience in a Canadian western Arctic community. Conservation Ecology, 5(2): 18.

Berkes, F., N. Bankes, M. Marschke, D. Armitage, and D. Clark, 2005. Cross-scale institutions \& building resilience is the Canadian North. In: Berkes, F., R. Huebert, H. Fast, M. Maneau and A. Diduck. Breaking Ice, pp. 225-247. University of Calgary Press, Calgary.

Biermann, F., 2007. 'Earth system governance' as a crosscutting theme of global change research. Global Environmental Change, 17: 326-337.

Bishop, A., C. Bremner, A. Laake, C. Strobbia, P. Parno and G. Utskot, 2011. Petroleum potential of the Arctic: challenges and solutions. Oilfield Review, 22(4): 36-49.

BMP (Bureau of Minerals and Energy), 2013. Application procedures and standard terms for exploration and prospecting licenses for minerals in Greenland. Government of Greenland.

BMP (Bureau of Minerals and Energy), 2011. Greenland mineral exploration brief in figures - A short overview of statistics in the mineral resource exploration industry in Greenland 2002 to 2011." Government of Greenland.

BMP (Bureau of Minerals and Energy), 1999. Annual Report on Mineral and Petroleum Activities in Greenland 1999. Government of Greenland and Joint Committee on Mineral Resources in Greenland.

BMP (Bureau of Minerals and Energy), 1998. Annual Report on Mineral and Petroleum Activities in Greenland 1998. Government of Greenland.

BMP (Bureau of Minerals and Energy), 1997. Report on socio economic aspects of hydrocarbon exploration and exploitation in Greenland. A preliminary survey and suggestions for further work. Report of the Committee on Socio-economic impacts of oil and gas and mineral industry. Government of Greenland, 1997.

Brattland, C., 2012. Making Sámi seascapes matter: ethno-ecological governance in coastal Norway. Unpublished PhD dissertation, Faculty of Biosciences, Fisheries and Economics, University of Tromsø, Tromsø. 
Broderstad, E. G., and E. Eythórsson. 2014. Resilient communities? Collapse and recovery of a social-ecological system in Arctic Norway. Ecology and Society 19(3): 1. http://dx.doi.org/10.5751/ES-06533-190301 (9 September 2014).

Bronen, R., 2011. Climate-induced community relocations: creating an adaptive governance framework based in human rights doctrine. New York University Review of Law and Social Change, 35(2): 356-406.

Bronen, R. and F. S. Chapin III, 2013. Adaptive governance and institutional strategies for climate-induced community relocations in Alaska. Proceedings of the $\mathrm{Na}$ tional Academy of Sciences, 110(23): 9320-9325.

Caulfield, R., S. Haley, A. Hakon Hoel, G. Hovelsrud-Broda, A. Jessen, C. Johnson and K. Klokov, 2004. Resource Governance. In: AHDR, 2004. Arctic Human Development Report. Einarsson, N., J. N. Larsen, A. Nilsson and O. R. Young (eds.). Stefansson Arctic Institute, Akureyri.

Chapin, F. S., III, M. Berman, T.V. Callaghan, P. Convey, A.-S. Crepin, K. Danell, H. Ducklow, B., G. Kofinas, A. D. McGuire, R. V. M. Nuttall, O. Young and S. Zimov, 2006. Polar Systems. Millennium Assessment - Conditions and Trends.

http://www.unep.org/maweb/documents/document.294.aspx.pdf (9 September 2014).

Chapin, F. S., III, S.R. Carpenter, G. P. Kofinas, C. Folke, N. Abel, W. C. Clark, P. Olsson, D. M. Stafford Smith, B. Walker, O. R. Young, F. Berkes, R. Biggs, J. M. Grove, R. L. Naylor, E. Pinkerton, W. Steffen, and F. J. Swanson, 2009. Ecosystem stewardship: sustainability strategies for a rapidly changing planet. Trends in Ecology and Evolution, 25(4): 241-249.

Chapin, F. S., III, M. Hoel, S. R. Carpenter, J. Lubchenco, B. Walker, T. V. Callaghan, C. Folke, S. Levin, K. G. Mäler, C. Nilsson, S. Barrett, F. Berkes, A. S. Crépin, K. Danell, T. Rosswall, D. Starrett, T. Xepapadeas and S. A. Zimov, 2006. Building resilience and adaptation to manage Arctic change. Ambio, 35: 198-202.

Chazan, G., 2013. 'Red Lenin' leads Russia's oil revolution. Financial Times, 31 March 2013. http://www.ft.com/intl/cms/s/0/39c32d90-9247-11e2-851f00144feabdc0.html\#axzz2QnPPyOMh (18 April 2013).

Clark, D. A. and S. Slocombe, 2011. Adaptive co-management and grizzly bear-human conflicts in two Northern Canadian Aboriginal communities. Human Ecology, 39(5): 627-640.

ConocoPhillips, 2013. Projects in Russia: Polar Lights Company. http://www.conocophillips.ru/EN/project-russia/polar_lights/Pages/index.aspx (9 September 2014).

Crandall, R. and D. Thurston, 2010. Oil and gas activities in the Arctic. In: AMAP, 2010. Assessment 2007: Oil and Gas Activities in the Arctic - Effects and Potential Effects, Vols. 1 and 2. Arctic Monitoring and Assessment Program, Oslo.

Dietz, T., E. Ostrom and P. C. Stern, 2003. The struggle to govern the commons. Science, 302(12): 1907-1912.

Downie, D. L. and T. Fenge, 2003. Northern Lights against POPs: Toxic Threats in the Arctic. McGill-Queen's University Press, Montreal and Kingston.

Eythórsson, E., 2003. The coastal Sámi: a 'pariah caste' of the Norwegian fisheries? A reflection on ethnicity and power in Norwegian resource management. In: Jentoft, S., H. Minde, R. Nilsen, E. Uitgeverij (eds.). Indigenous Peoples: Resource Management and Global Rights, pp. 149-162. Eburon Academic, Delft, The Netherlands.

Fay F. H., 1982. Ecology and biology of the Pacific walrus, Odobenus rosmarus divergens. United States Department of the Interior, Washington, D.C. 
Fitzhugh, W., 2004. The Living Yamal. Arctic Studies Center, Museum of Natural History, Smithsonian Institution, Washington, DC. http://www.mnh.si.edu/arctic/html/yamal.html (31 July 2014).

Folke, C., T. Hahn, P. Olsson, and J. Norberg, 2005. Adaptive governance of socialecological systems. Annual Review of Environmental Resources, 30: 441-473.

Forbes, B. C., 2013. Cultural resilience of social-ecological systems in the Nenets and Yamal-Nenets Autonomous okrugs, Russia: a focus on reindeer nomads of the tundra. Ecology and Society, 18(4).

Forbes, B. C., 2008. Equity, vulnerability and resilience in social-ecological systems: A contemporary example from the Russian Arctic. Research in Social Problems and Public Policy, 15: 203-236.

Forbes, B. C. and F. Stammler, 2009. Arctic climate change discourse: the contrasting politics of research agendas in the West and Russia. Polar Research, 28: 28-42.

Forbes, B. C., F. Stammler, T. Kumpula, N. Meschtyb, A. Pajunen, and E. Kaarlejärvi, 2009. High resilience in the Yamal-Nenets social-ecological system, West Siberian Arctic, Russia. Proceedings of the National Academy of Sciences, 106: 22041-22048.

Galginaitis, M., 2012. Inupiat Fall whaling and climate change - observations form Cross Island. 28th Lowell Wakefield Fisheries Symposium, Anchorage, Alaska.

Gaup Eira, I. M., C. Jaedicke, O. H. Magga, N. G. Maynard, D. Vikhamar-Schuler, S. D. Mathiesen, 2013. Traditional Sámi snow terminology and physical snow classification-Two ways of knowing, Cold Regions Science and Technology, 85: 117-130.

Gazprom, 2013. Yamal Megaproject (brochure). http://www.gazprom.com/f/posts/25/697739/book_my_eng_1.pdf (9 September 2014).

Gearheard, S., C. Aporta, G. Aipellee and K. O'Keefe, 2011. The Igliniit project: Inuit hunters document life on the trail to map and monitor Arctic change. Canadian Geographer / Le Géographe Canadien, 55(1): 42-55.

Grønlands Økonomisk Råd, 2012. Økonomisk Råds Rapport 2012 - naturressourcer som vækststrategi. [Economic Council Report 2012 - natural resources as growth strategy]. Greenland Economic Council, Nuuk.

Haley, S., 2004. Local control and impacts of oil and gas development: Nuiqsut case study. Unpublished report. Institute of Social and Economic Research. University of Alaska Anchorage, Anchorage.

Hovelsrud G. K. and B. Smit (eds.), 2010. Community Adaptation and Vulnerability in the Arctic Regions. Springer, Berlin.

Hovelsrud, G. K., White J. L., Andrachuk M. and Smit D., 2010. Community adaptation and vulnerability integrated. In: Hovelsrud G.K. and Smit B. Community Adaptation and Vulnerability in the Arctic Regions, pp. 335-349. Springer Publishers, Dordrecht.

Huntington, H. P., 2000. Using traditional ecological knowledge in science: methods and applications. Ecological Applications, 10(5): 1270-1274.

Huntington, H. P., F. Birkes. S. Fox and I. Krupnik, et al., 2005. The changing Arctic: Indigenous perspectives. In: ACIA, 2005. Arctic Climate Impact Assessment, pp. 6198. Cambridge University Press, Cambridge.

International Labor Organization 169 (ILO 169), Indigenous and Tribal Peoples Convention, C169, 27 June 1989, C169.

http://www.refworld.org/docid/3ddb6d514.html (9 September 2014).

Jentoft, S. and C. Brattland, 2011. Mot en samisk fiskeriforvaltning? [Towards a Sámi fisheries management regime?] In: Jentoft, S., Nergård, J.I., and K. A. Røvik (eds.) Hvor går Nord-Norge? Tidsbilder fra en landsdel i forandring. [Where is northern 
Norway headed? Images from a region in transition]. Orkana Akademisk, Stamsund.

Kankaanpää, P. and O. R. Young (2012), The effectiveness of the Arctic Council, Polar Research, 31: 17176.

http://www.polarresearch.net/index.php/polar/article/view/17176 (9 September 2014).

Kates, R. W., W. R. Travis and T. J. Wilbanks, 2012. Transformational adaptation when incremental adaptations to climate change are insufficient. Proceedings of the National Academy of Sciences, 109(19): 7156-7161.

Keskitalo, E. C. H., 2008. Climate Change and Globalization in the Arctic: An Integrated Approach to Vulnerability Assessment. Earthscan Publications, London.

Keskitalo, E. C. H., C. Sandström, M. Tysiachniouk, and J. Johansson, 2009. Local consequences of applying international norms: differences in the application of forest certification in northern Sweden, northern Finland, and northwest Russia. Ecology and Society 14(2): 1. http://www.ecologyandsociety.org/vol14/iss2/art1/ (9 September 2014).

Kingdon, J. W., 1995. Agendas, Alternatives, and Public Policies, 2nd ed. Harper Collins, New York.

Klein, D. R., L. M. Baskin, L. S. Bogoslovskaya, K. Danell, A. Gunn, D. B. Irons, G. P. Kofinas, K. M. Kovacs, M. Magomedova, R. H. Meehan, D. E. Russell and P. Valkenburg, 2005. Management and conservation of wildlife in a changing Arctic environment. In: ACIA, 2005. Arctic Climate Impact Assessment. Pp. 597-648. Cambridge University Press, Cambridge.

Kofinas, G. P., 2009. Adaptive co-management in social-ecological governance. In: Chapin, F. S. III, G.P. Kofinas, and C. Folke. Principles of Ecosystem Stewardship: Resilience-Based Natural Resource Management in a Changing World, pp. 77-102. Cambridge University Press, Cambridge.

Koivurova, T., E. Keskitalo and N. Bankes (eds.), 2009. Climate governance in the Arctic. Environment \& Policy, Vol. 50, Springer, New York.

Koivurova, T. and D. VanderZwaag, 2007. The Arctic Council at 10 years: retrospect and prospects. University of British Columbia Law Review, 40(1): 121-194.

Krupnik, I., C. Aporta, S. Gearheard, G. J. Laidler and L. K. Holm (eds.), 2010. SIKU: Knowing Our Ice. Springer, New York.

Krupnik, I. and D. Jolly, 2002. The Earth is Faster Now: Indigenous Observations of Arctic Environmental Change. ARCUS, Fairbanks.

Kumpula, T., B. C. Forbes, and F. Stammler, 2010. Detection of snow surface thawing and refreezing in the Eurasian Arctic using QuikSCAT: Implications for reindeer herding. Ecological Applications, 20: 2346-2358.

Kumpula, T., A. Pajunen, E. M. Kaarlejärvi, B. C. Forbes, and F. Stammler, 2011. Land use and land cover change in Arctic Russia: ecological and social implications of industrial development. Global Environmental Change, 21: 550-562.

Kumpula, T., B. C. Forbes, F. Stammler and N. Meschtyb, 2012. Dynamics of a coupled system: multi-resolution remote sensing in assessing social-ecological responses during 25 years of gas field development in Arctic Russia. Remote Sensing, 4: 10461068

Langdon S., 1989. Prospects for co-management of marine animals in Alaska. In: Pinkerton E. (ed.). Co-operative Management of Local Fisheries: New Directions for Improved Management and Community Development, pp. 154-169. University of British Columbia Press, Vancouver. 
Locatelli, C., 2006. The Russian oil industry between public and private governance: obstacles to international oil companies' investment strategies. Energy Policy, 34: 1075-1085.

London Mining, 2013. Isua Project in Nuuk http://www.londonmining.com/operations/greenland/ (27 May 2014).

Martens, H., 1995. Revegetation research Western Siberia: Year 4. Report prepared for Amoco Eurasia Production Co., Harvey Martens \& Assoc., Inc., Calgary.

Martens, H., M. Magomedova and L. Morozova, 1996. Rangeland studies in the Bovanenkovo proposed development area: Year 3. Report prepared for Amoco Eurasia Production Co., Harvey Martens \& Assoc., Ekaterinburg Institute of Flora and Fauna, Calgary.

Meek, C. L., A. L. Lovecraft, M. D. Robards and G. P. Kofinas, 2008. Building resilience through interlocal relations: case studies of polar bear and walrus management in the Bering Strait. Marine Policy, 32(6): 1080-1089.

Metcalf, V. and M. Robards, 2008. Sustaining a healthy human-walrus (Odobenus rosmarus divergens) relationship in a changing environment: challenges for comanagement. Ecological Applications, 18(2): S148-156.

Nellemann, C., I. Vistnes, B. C. Forbes, T. Forseman, E. Husby, G.P. Kofinas, B. P. Kaltenborn, J. Rouaud, M. Magomedova, R. Bobiwash, C. Lambrechts, P. J. Schei, S. Tveitdal, O. Gron, and T. S. Larens, 2001. GLOBIO - Global Method for Mapping Human Impacts on the Biosphere. UNEP Report.

Nemes, G., C. High, N. Shafer and R. Goldsmith, 2007. Using participatory video to evaluate community development. Presentation at the XXII European Congress of Rural Sociology, Wageningen, Netherlands.

Nilsen, T., 2011. Novatek considers Statoil in Yamal partnership. Barents Observer, 18 March 2011. http://barentsobserver.com/en/sections/business/novatekconsiders-statoil-yamal-partnership (27 May 2014).

Nilsson, A. E., 2009. A Changing Arctic Climate: Science and Policy in the Arctic Climate Impact Assessment. In: Koivurova, T., C. Keskitalo, and N. Bankes (eds.). Climate Governance in the Arctic, pp. 77-95. Springer, New York.

Nordlund, A. and K. Westin, 2011. Forest Values and Forest Management Attitudes among Private Forest Owners in Sweden. Forests, 2011, 2: 30-50.

Norwegian Directorate of Fisheries, 2013. Statistics - fishers. www.fiskeridir.no (1 February 2013).

Nuttall, M., 1998. Protecting the Arctic: Indigenous Peoples and Cultural Survival, Harwood Academic Publishers, Newark.

Nuttall, M., F. Berkes, B. C. Forbes, G. Kofinas, T. Vlassova and G. Wenzel, 2005. Hunting, herding, fishing and gathering: Indigenous peoples and renewable resource use in the Arctic. In: ACIA, 2005. Arctic Climate Impact Assessment, pp. 649-690. Cambridge University Press, Cambridge.

Offerdal, K. 2007. Oil, gas and the environment. In: Stokke, O.S. and G. Hønneland (eds.). International cooperation and Arctic governance: regime effectiveness and northern region building, pp. 138-163. Routledge, London.

OGP, 2013. Environmental Management in Arctic Oil and Gas Operations: Good Practice Guide. Report No. 449. International Association of Oil \& Gas Producers, London, UK.

Ostrom, E., 2009. A general framework for analyzing sustainability of socialecological systems. Science, 325(5939): 419-422.

PAME (Protection of the Arctic Marine Environment), 2009. Arctic Offshore Oil and Gas Guidelines. Arctic Council. http://www.pame.is (23 November 2014). 
Parris, T.M. and R.W. Kates, 2003. Characterizing and Measuring Sustainable Development. Annual Review Environmental Resources, 28: 1-13.

Quinn, E., 2013. Shell signs agreement with Russia to seek oil in Arctic. Alaska Dispatch, 16 April 2013. http://www.alaskadispatch.com/article/20130416/shellsigns-agreement-russia-seek-oil-Arctic (18 April 2013).

Robards, M., 2013. Resilience of international policies to changing social-ecological systems: Arctic shipping in the Bering Strait. Arctic Resilience Interim Report 2013. Arctic Council. Stockholm, Stockholm Environment Institute and Stockholm Resilience Centre: 100-103.

Sámi Parliament, 2004. Sámisk fiskerimelding. White paper on Sámi fisheries. Sámi Parliament, Karasjok.

Shiraevskaya, A., 2013. Novatek in 'advanced' talks to sell Yamal LNG stake. Bloomberg, 14 August 2013. http://www.bloomberg.com/news/2013-08-13/novatek-inadvanced-talks-to-sell-additional-yamal-lng-stake.html (27 May 2014).

Skydsbjerg, H., 1999. Grønland - 20 år med hjemmestyre [Greenland - 20 years of home rule]. Atuagkat, Nuuk.

Sørensen, A. K., 1983. Danmark-Grønland i det 20. århundrede - en historisk oversigt [Denmark-Greenland in the 20th Century - an overview]. Nyt Nordisk Forlag Arnold Busck.

Stammler, F., 2011. Oil without conflict? The anthropology of industrialization in northern Russia. In: A. Behrends, S.R. Reyna and G. Schlee (eds.) Crude Domination: An Anthropology of Oil, pp. 243-269. Berghahn Books, Oxford.

Stammler, F., and V. Peskov, 2008. Building a 'culture of dialogue' among stakeholders in North-West Russian oil extraction. Europe-Asia Studies, 5:831-849.

Stammler, F. and E. Wilson, 2006. Dialogue for development: an exploration of relations between oil and gas companies, communities and the state. Sibirica, 5(2): 1-42.

Stammler, F., and B. C. Forbes, 2009. "Ilebts" declaration on coexistence of oil and gas activities and Indigenous communities on Nenets and other territories in the Russian North. Arctic Centre, University of Lapland, Rovaniemi.

Stjernström, O., S. Karsson, Ö. Pettersson, 2013. Skogen och den kommunala planeringen [The forest and municipal planning]. PLAN 1: 42-45

Svensson, T. G., 2005. Interlegality, a process for strengthening Indigenous peoples' autonomy: the case of the Sámi in Norway. The Journal of Legal Pluralism and Unofficial Law, 37(51): 51-77.

Swedish Forest Agency, 2013a. Utrikeshandel [Foreign trade]. http://www.skogsstyrelsen.se/expimp. Swedish Forest Agency, Jönköping.

Swedish Forest Agency, 2013b. Virkets användning och ekonomiska betydelse [Wood use and economic importance]. http://www.skogsstyrelsen.se/Upptackskogen/Skog-i-Sverige/Fakta-om-skogen/Virket-fran-skogen/. Swedish Forest Agency, Jönköping.

Ulvevadet, B., 2011. Problems and challenges for user participation: the system of representation in reindeer husbandry in Norway. Rangifer, 31(1): 161-182.

Ulvevadet, B., 2008. Management of reindeer husbandry in Norway - power-sharing and participation. Rangifer, 28(1): 53-78.

UN DRIP, 2007. United Nations Declaration on the Rights of Indigenous Peoples: resolution / adopted by the UN General Assembly, 2 October 2007, A/RES/61/295.

Walker, D. A., P. J. Webber, E. F. Binnian, K. R. Everett, N. D. Lederer, E. A. Nordstrand and M. D. Walker, 1987. Cumulative impacts of oil fields on northern Alaskan landscapes. Science, 238(4828): 757-761. 
Young, O.R., 2012. Arctic tipping points: governance in turbulent times. Ambio, 41: 75-84.

Young, O. R., 2009a. Whither the Arctic? Conflict or cooperation in the Circumpolar North. Polar Record, 45: 73-82.

Young, O. R., 2009b. Arctic in play: governance in a time of rapid change. International Journal of Marine and Coastal Law, 24: 423.

Young, O. R., 2005. Governing the Arctic: from Cold War theater to mosaic of cooperation. Global Governance, 11(1): 9-15.

Young, O. R., 1996. Institutional linkages in international society: polar perspectives. Global Governance 2(1): 1-23.

Young, O. R., 1982. Resource Regimes: Natural Resources and Social Institutions. University of California Press, Berkeley.

Young, O. R. and G. Osherenko (eds.), 1993. Polar Politics: Creating International Environmental Regimes. Cornell University Press, Ithaca.

Young, O. R., H. Schroeder, and L. A. King (eds.), 2008. Institutions and Environmental Change: Principal Finds, Applications, and Research Frontiers. MIT Press, Boston. 


\title{
8. Human Health and Well-Being
}

\author{
Lead Authors \\ Arja Rautio, University of Oulu, Finland, Birger Poppel, Ilisimatusarfik, \\ Greenland and Kue Young, University of Alberta, Canada \\ Contributing authors \\ Anastasia Emelyanova (University of Oulu, Finland; Northern (Arctic) \\ Federal University, Russia), Sandra Juutilainen (University of Oulu, Fin- \\ land), Vappu Sunnari (University of Oulu, Finland)
}

\subsection{Introduction}

Several international organizations promote health and well-being across Arctic countries and regions. A variety of monitoring programs and research networks collect and share a variety of health data. In recent years, Arctic health research is increasingly focusing on the health and well-being of Indigenous peoples of the North. This priority recognizes the major changes experienced by Indigenous peoples in terms of their living conditions. Indigenous people in some regions continue to suffer poorer health status compared to the majority populations of the Arctic nations and redressing such disparities requires the attention of policy makers and health service agencies. The increasing interest in the development of the Arctic has resulted in several initiatives, such as the Arctic Social Indicators project (ASI, 2010; ASI, 2014) and the first Arctic Human Development Report (AHDR, 2004).

Among emerging threats for human health and well-being are the effects of a warming climate resulting in worsening food and water security; changes in the pattern of infectious diseases, and impact on health care infrastructure; and mental health problems including suicide, accidents and domestic violence. Since the publication of the first AHDR in 2004, much has changed in the health and well-being of the diverse populations in the Arctic. Improvements continue to be made, while certain problems remain intractable. The major trends and patterns will be presented in this chapter. 


\subsection{Health and well-being in the Circumpolar North}

The development of international collaborative efforts in data collection, management and dissemination facilitates monitoring the health of Circumpolar peoples. Notable examples include Arctic Council projects such as the Survey of Living Conditions in the Arctic (SLiCA), a circumpolar review involving interviews of more than 7,000 Indigenous people in the Arctic; the Arctic Monitoring and Assessment Programme (AMAP) which reported on human health assessments that have collected and analyzed data on the effects of environmental contaminants since 1991; the International Circumpolar Surveillance of Emerging Infectious Diseases (ICS), a collaboration of public health departments and laboratories from several circumpolar countries that collects information on invasive bacterial diseases and tuberculosis; the Inuit Health in Transition Study; and the Circumpolar Health Observatory (CircHOB), a webbased, interactive resource which presents freely downloadable tables and maps of a series of health indicators for all northern regions, the majority of which are derived from publicly available sources (Young et al., 2010). Major surveys like the Aboriginal Peoples Survey and Inuit Health Survey in Canada and SAMINOR in Norway have focused on the conditions of Indigenous peoples. There is also some information about health and well-being of the non-Indigenous peoples in northern regions as part of national health surveys.

Several international organizations promote health and well-being across Arctic countries and regions. The International Union of Circumpolar Health was formed in 1981, a federation of national scientific and professional organizations in Canada, the Nordic countries, United States, and the Soviet Union (later the Russian Federation). It organizes a triennial international circumpolar health congress, an important forum for the exchange of information and interactions among health care professionals, researchers and policy-makers. The Circumpolar Health Research Network (CirchNet, 2013) is an organization that focuses specifically on health research and supports academic exchanges, organizes summer institutes in health research, and publishes the International Journal of Circumpolar Health. In 2008, the Arctic Council's Sustainable Development Working Group created a subsidiary, the Arctic Human Health Expert Group (AHHEG), to provide expert advice on health matters.

The training of health care providers and researchers with a focus on the North occurs in several centers, linked together through the University of the Arctic (UArctic) (see Chapter 9, Education and Human Capital). The thematic networks of the UArctic form a natural framework for the devel- 
opment of education and research to increase and share knowledge across the North. The Health and Wellbeing in the Arctic network was established in 2005, and now member universities and institutes within the network offer international Masters (since 2009) and PhD programs (since 2012). Future activities of this thematic network include increasing the exchange of researchers and teachers and developing courses for health staff about the special features of Arctic health.

Health is also a major concern of Indigenous peoples' organizations. For example, the Inuit Circumpolar Council proposed the Inuit health strategy in 2009 to improve Inuit health and wellness. It aims to influence international, national, and regional health and social policies and programs, improve awareness of the Inuit's situation in the Arctic among health professionals, and promote research on health and wellness that reflects the communities' priorities.

It is clear that many institutions, projects, and groups are now actively engaged in monitoring, understanding, and improving the health and well-being of Circumpolar peoples. This chapter reviews the current state of health and well-being around the Arctic to assist organizations in determining priorities and identifying promising practices.

\section{Alaska Native Medical Centre, Anchorage, Alaska, USA}

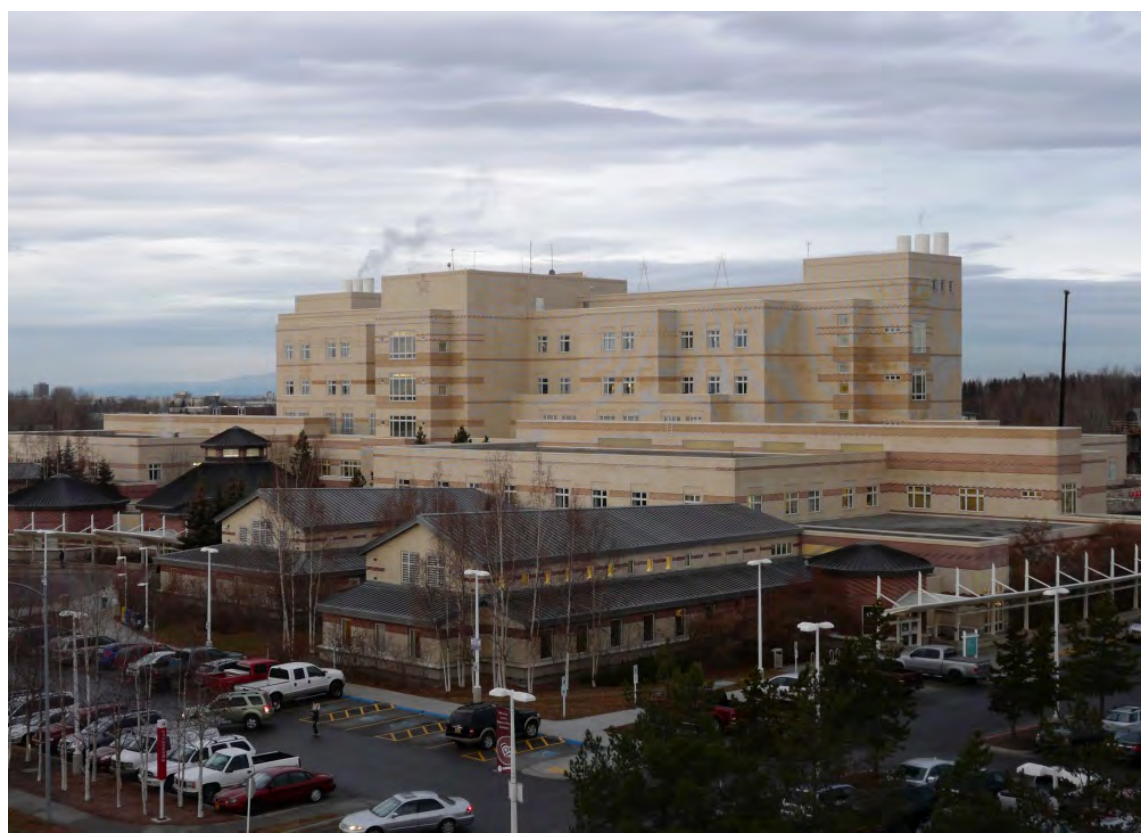

Credit: Diane Hirshberg. 


\subsection{Continuing health disparities}

The people in the Circumpolar North do not all enjoy the same health. There are substantial disparities among countries and regions, and within regions among population subgroups, particularly between Indigenous and non-Indigenous people.

At the level of the eight Arctic States, there are clear inter-country disparities in the overall index of well-being such as the United Nations Human Development Index, a composite index of health, education and living standards. In 2012, Russia ranked behind the others, at 55th, whereas the other Arctic States were all within the top 20 countries in the world (UN HDI, n.d.).

In general terms, circumpolar regions basically fall into four groups in terms of their health status:

- The Nordic countries - these rank the highest in every health indicator, and there is generally little difference between north and south, or between Indigenous and non-Indigenous people.

- Alaska, Yukon, and Northwest Territories - health status in these jurisdictions is comparable to, or even better than, the national average of the United States and Canada; however, within these regions, there are significant disparities between Indigenous and non-Indigenous people.

- Greenland and Nunavut - with over $85 \%$ of the population Indigenous, there is a wide gap in health status between these regions and Denmark and Canada.

- The Russian Arctic - while the regions in the European North tend to fare better than those in Siberia, for almost any health indicator, the Arctic regions of Russia tend toward the lower end of the spectrum.

During the period between 2005-2009, the regions with the greatest health disparities experienced lower life expectancy (see Chapter 2, Arctic Population and Migration), higher infant mortality, and higher rates of tuberculosis, sexually transmitted diseases, and some cancers. Injury, homicide and suicide rates also tend to be higher in the Circumpolar North. Each is discussed in brief below. 


\subsubsection{Infant mortality rate (IMR)}

IMR varies from less than 5 per 1,000 live births in the Nordic countries (as low as 2 in Iceland) to over 10 in Nunavut and Greenland, and over 20 in some Russian regions, and is as high as 28 in the Koryak okrug. There is little regional difference (between the northern and southern regions) in Nordic countries (Figure 8.1). Greenland is about thirty years behind Denmark in IMR rates, i.e., the rate for Greenland today is similar to that observed in Denmark 30 years ago (Young and Bjerregaard, 2008:32).

Figur 8.1: Infant mortality rates among circumpolar regions

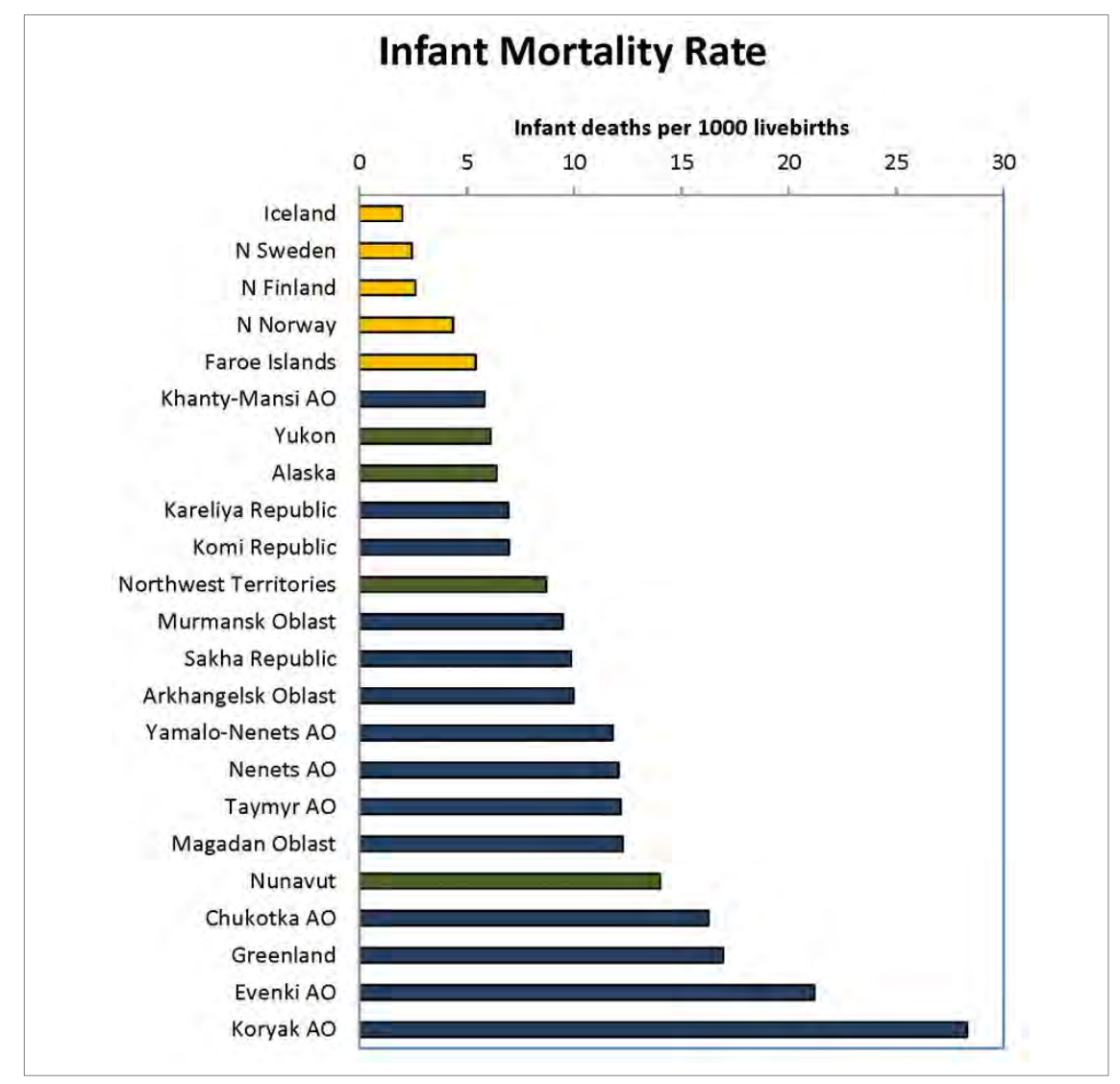

Note: Mean of 2005-09, rates expressed as infant deaths per 1,000 live births.

AO - autonomous okrug; the Evenkia, Koryak, and Taymyr AO were dissolved in 2007 although some data were continued to be collected and reported for these regions:

Nordic countries

USA and Canada

Russia

Source: Circumpolar Health Observatory [http://circhob.circumpolarhealth.org], based on data as reported by national statistical agencies. 


\subsubsection{Tuberculosis}

The mean national incidence rates for tuberculosis in the Nordic countries, Canada and the United States are less than $10 / 100,000$. The rates for Greenland $(130 / 100,000)$ and Nunavut $(150 / 100,000)$ are more than 10 times higher. Meanwhile, the mean rate of the northern Russian regions is about $80 / 100,000$, among them is Koryak okrug, with a rate as high as $450 / 100,000$, almost 100 times the rate of Iceland and Faroe Islands. TB affects the Inuit in Canada, Alaska and Greenland disproportionately, where its incidence reached its peak in the 1950s. The decline in incidence of the disease has been impressive (Figure 8.2), although a substantial gap still exists between the Indigenous and non-Indigenous populations.

Fig. 8.2: Trend in incidence of tuberculosis in selected circumpolar populations

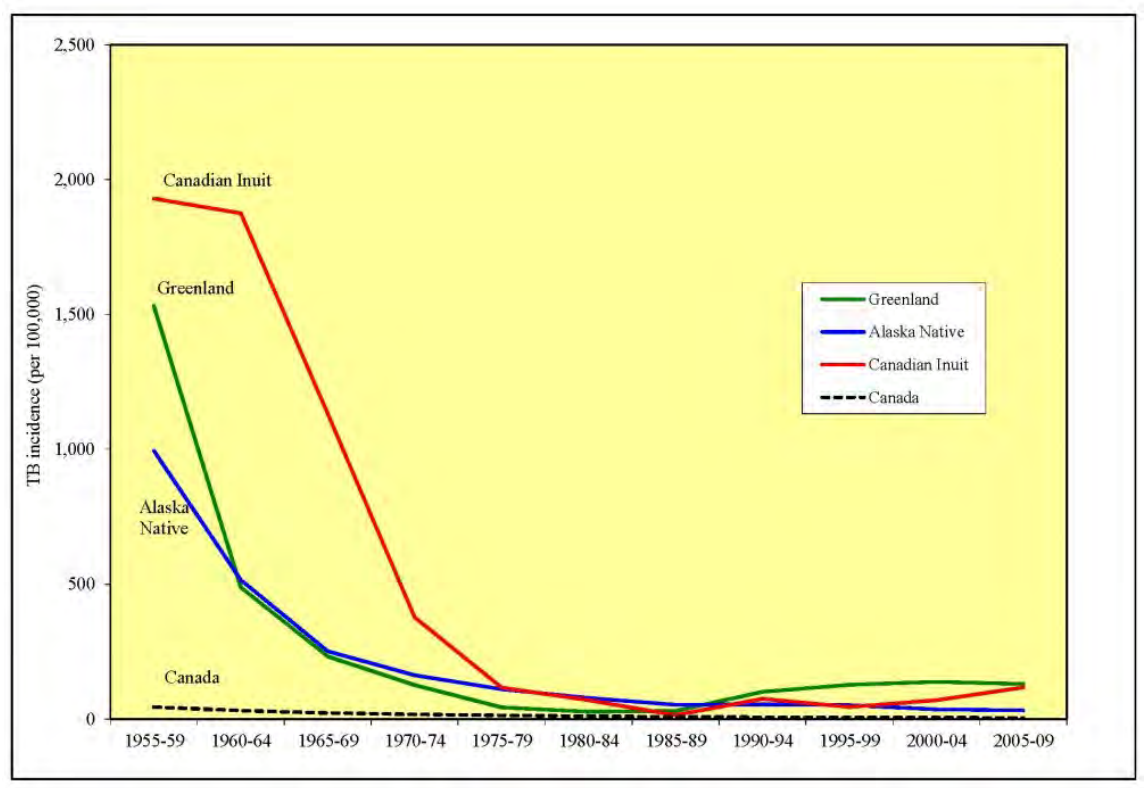

Source: Reproduced by permission from Circumpolar Health Atlas, p.96.

\subsubsection{Sexually transmitted diseases (STDs)}

Other infectious diseases also vary substantially across circumpolar regions, a notable example being gonorrhoea (Figure 8.3). Greenland has a high number of cases of gonorrhoea. Its incidence increased steadily during the 1950s and 1960s. A systematic intervention strategy that included partner tracing and treatment had resulted in an impressive decline, but the current level is still over 200 times higher than in Denmark. The pattern of chlamydia infection tends to parallel that of gonor- 
rhoea, with Greenland, Nunavut, Northwest Territories, and Alaska reporting the highest rates.

Given the high rates of STDs, there was much fear of the human immunodeficiency virus (HIV) spreading to the Arctic when the epidemic began in the 1980s. This has not happened, but HIV/AIDS nonetheless represents a major public health threat. The distribution of risk groups men having sex with men, heterosexual contacts and injection drug users - vary across regions. There is a long incubation period between HIV infection and the onset of the clinical symptoms of AIDS. Most jurisdictions report these HIV and AIDS separately, but given that the extent of HIV testing in the population varies, cross-national comparisons should only be done with caution.

\section{Fig. 8.3: Map showing incidence of gonorrhea in circumpolar regions}

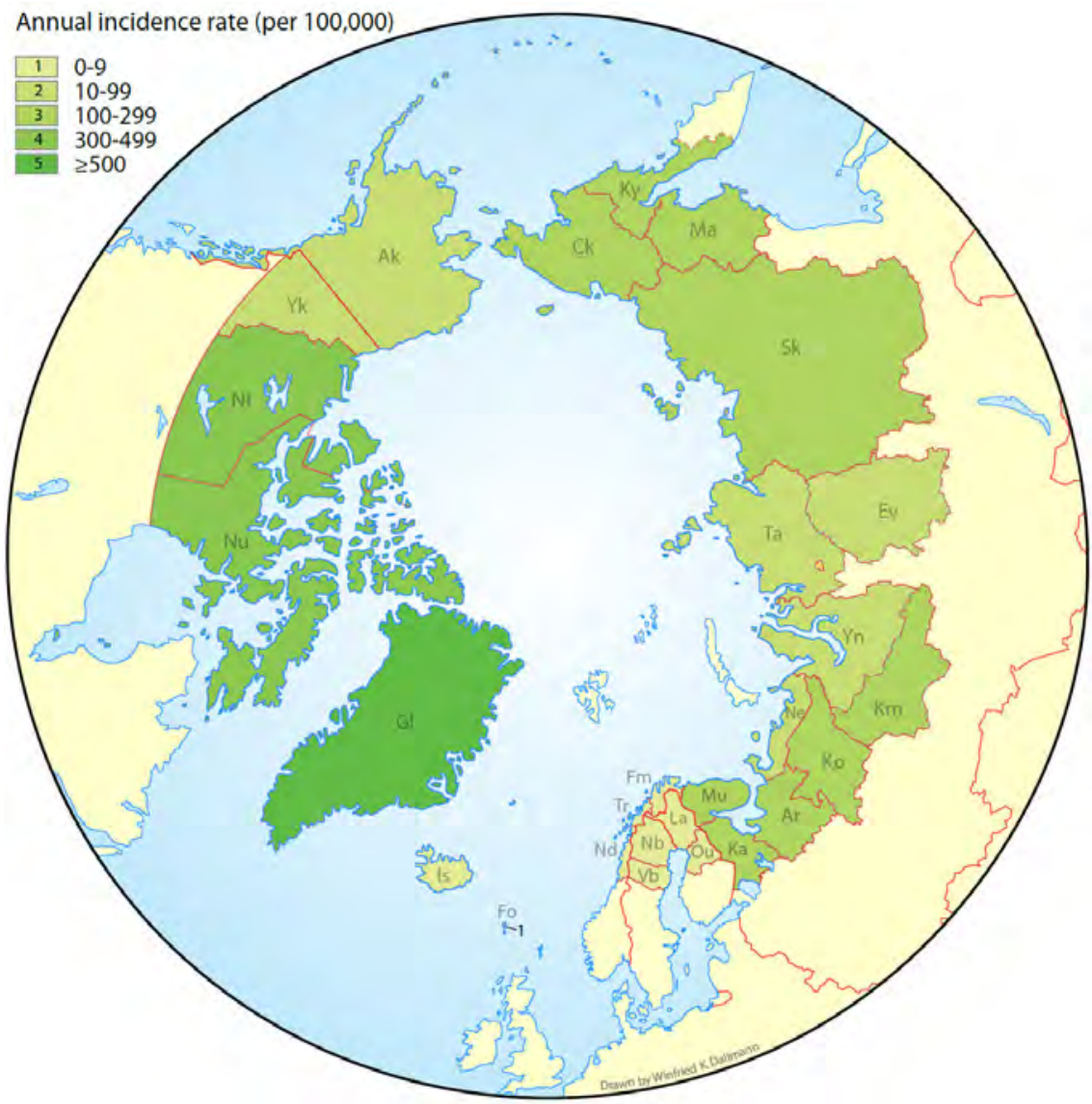

Source: Reproduced by permission from Circumpolar Health Atlas, p.98. 
Cancers: Cancer incidence also varies across regions. Among the most common cancers is lung cancer: the highest incidence can be found in Nunavut, Greenland, and some Russian regions (Figure 8.4). While rates tend to be higher among males than females, the sex difference is particularly pronounced in Russian North, where the male rate may be as high as 10 times the female one, reflecting the very low smoking prevalence among Russian women.

Fig. 8.4: Incidence of lung cancer in Arctic States and their northern regions

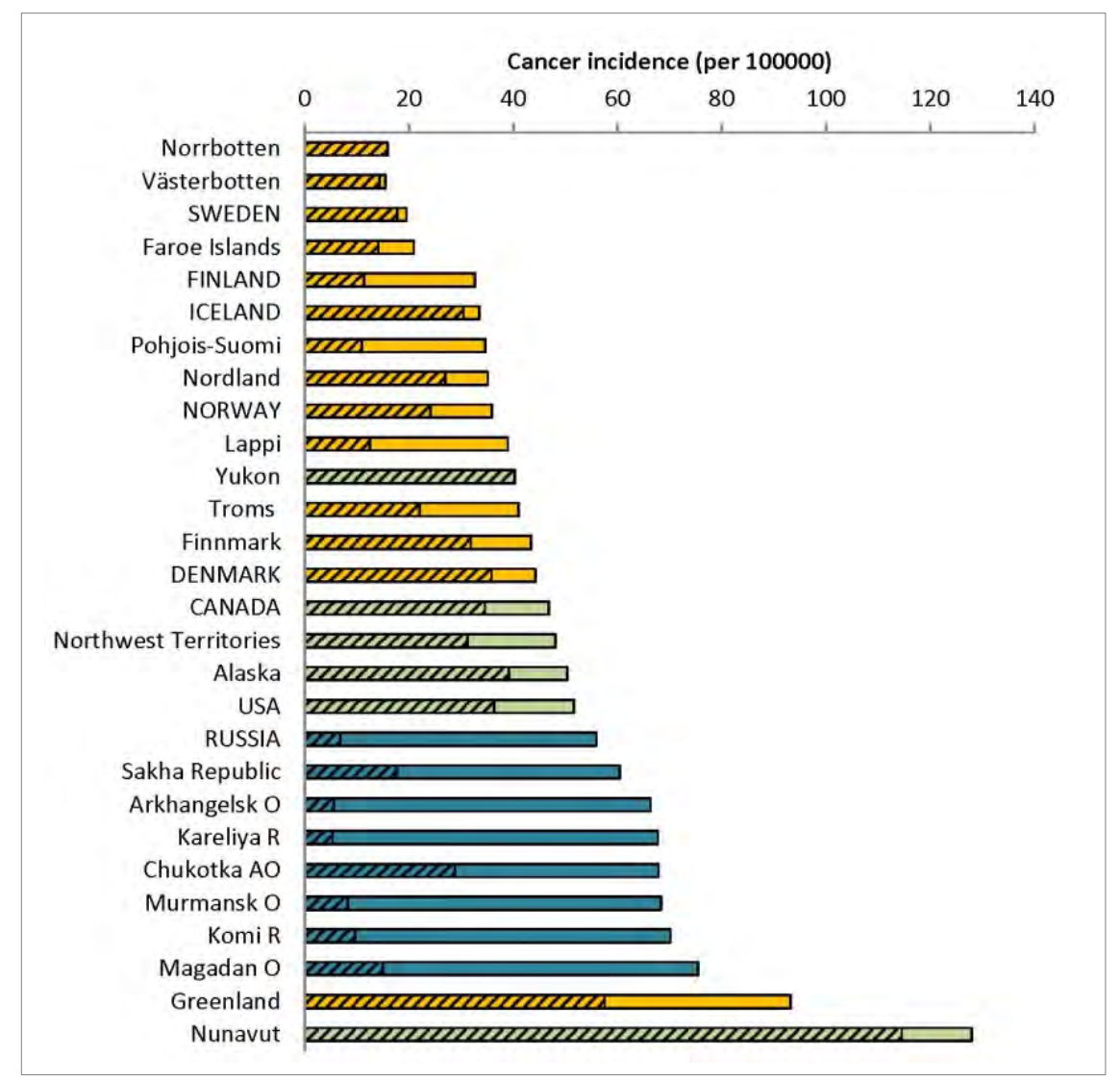

Note: Mean of 2005-09; AO - autonomous okrug, O - Oblast, R - Republic; cross-hatched area in bars represents the female rate, total length of bar represents the male rate:
Nordic countries
USA and Canada
Russia

Source: Circumpolar Health Observatory [http://circhob.circumpolarhealth.org], based on data as reported by national statistical agencies. 
An international review of cancer among the Inuit reveals that cancer (all combined) has increased in all Inuit regions over a 35-year period from 1969-2003. The increase is particularly marked for lung and colorectal cancers, while cervical cancer has declined. For the 1989-2003 period, the overall risk of cancer among Inuit men and women was not in excess of that among non-Inuit. Inuit continue to be at extreme high risk for certain so-called "traditional" cancers such as nasopharyngeal and salivary gland cancer. However, Inuit today also have the world's highest incidence rate of lung cancer. The likeliest cause is the exceedingly high smoking rate, approaching 60\% among adults (Circumpolar Inuit Cancer Review Working Group, 2008).

\subsubsection{Cardiovascular disease}

Across Russia, cardiovascular disease mortality rates are much higher than in the other regions. Within Scandinavia, northern regions have higher rates than the national averages. Among Alaska Natives, mortality from ischemic heart diseases increased during the 1980s, and began to decline during the mid-1990s. At the same time, rates have declined dramatically among Americans nationally. The Alaska Native rate is still lower than that of the state or national all-race rates.

\section{"Alaska Natives are the Healthiest People in the World"}

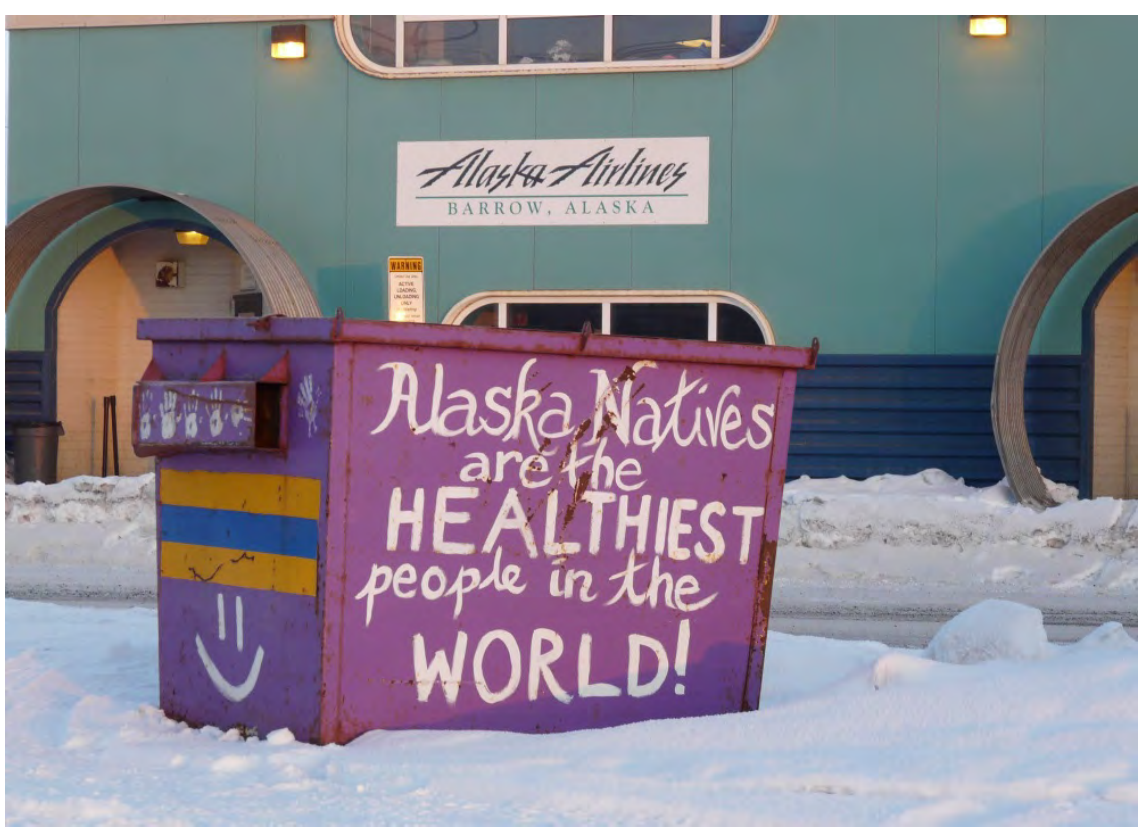

Credit: Diane Hirshberg. 


\subsubsection{Injury, homicide and suicide}

Injury is an important indicator of health, but also of social well-being, as it comprises both the unintentional ("accidents") and intentional (including suicide and violence). Russia and its regions have the highest mortality rates from injuries (Figure 8.5). In Alaska and Northern Canada, the age-standardized mortality rate for injury is two to three times higher than the respective national rates. Greenland's rate is more than four times that of Denmark. In northern Fennoscandia, the rates are little different from the respective national rates. In all jurisdictions, injury mortality is higher among men than women. Within the North, there is also substantial difference between Indigenous and non-Indigenous people. In Alaska, the rate for all injuries among Alaska Natives is three times that of the USA all-races rate.

\section{Fig. 8.5: Map showing injury mortality rate in circumpolar regions}

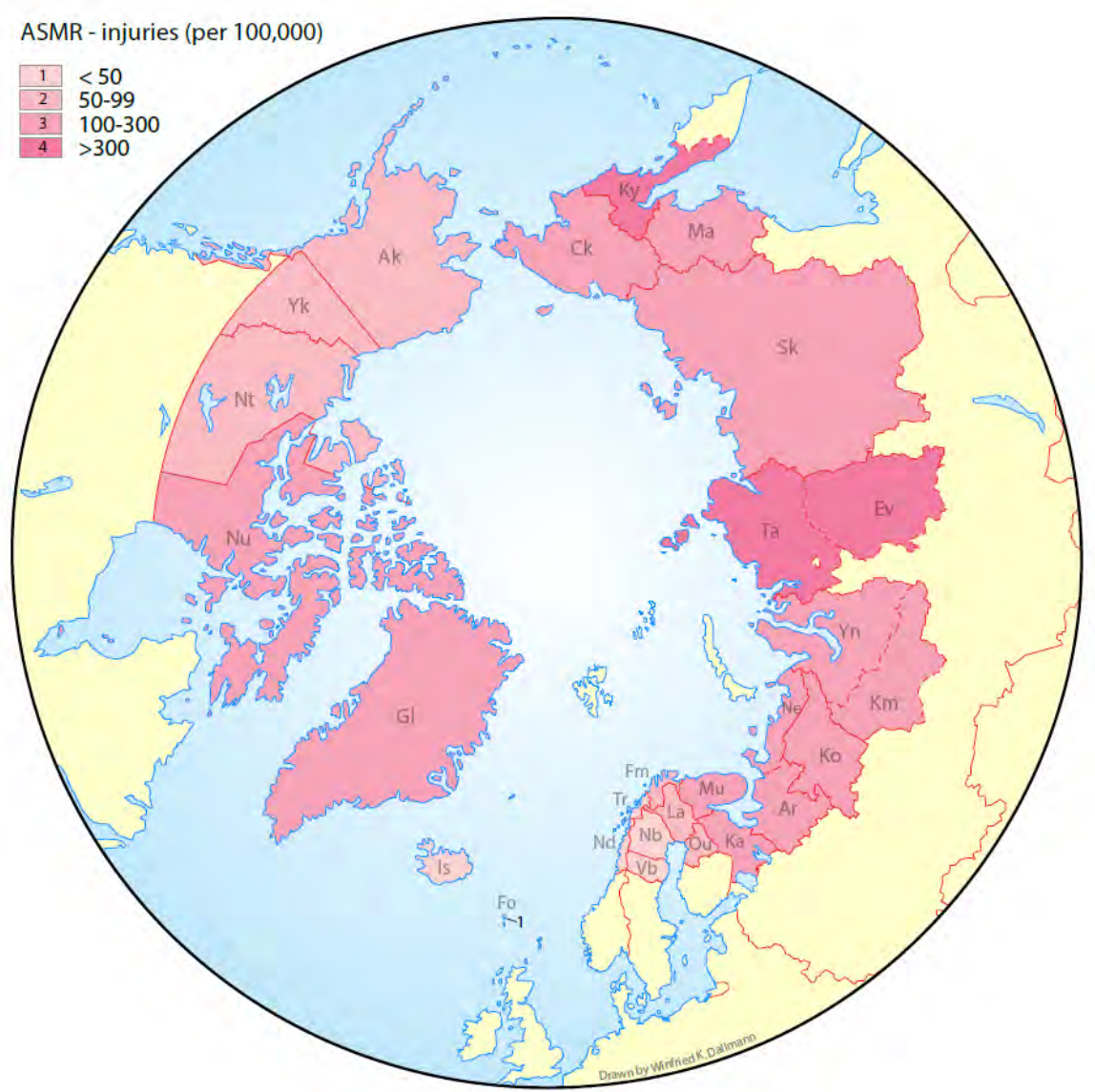

Note: Mortality rate (per 100,000) age-standardized to the European standard population. Source: Reproduced with permission from Circumpolar Health Atlas, p.111. 
The northern regions of Fennoscandia generally do not show an excess of homicides, compared to rates in their respective countries as a whole. The highest rates of homicide are found among Alaska Natives and Greenlanders. Greenland's rate exceeds 10 times that of Denmark. While the rate for Alaska Natives is only twice that of the USA-all races rate, this is due to high homicide rate in the United States nationally, which exceeds all other circumpolar countries, and is comparable to those of the northern territories of Canada.

Fig. 8.6: Evolution of the suicide epidemic in three circumpolar indigenous populations

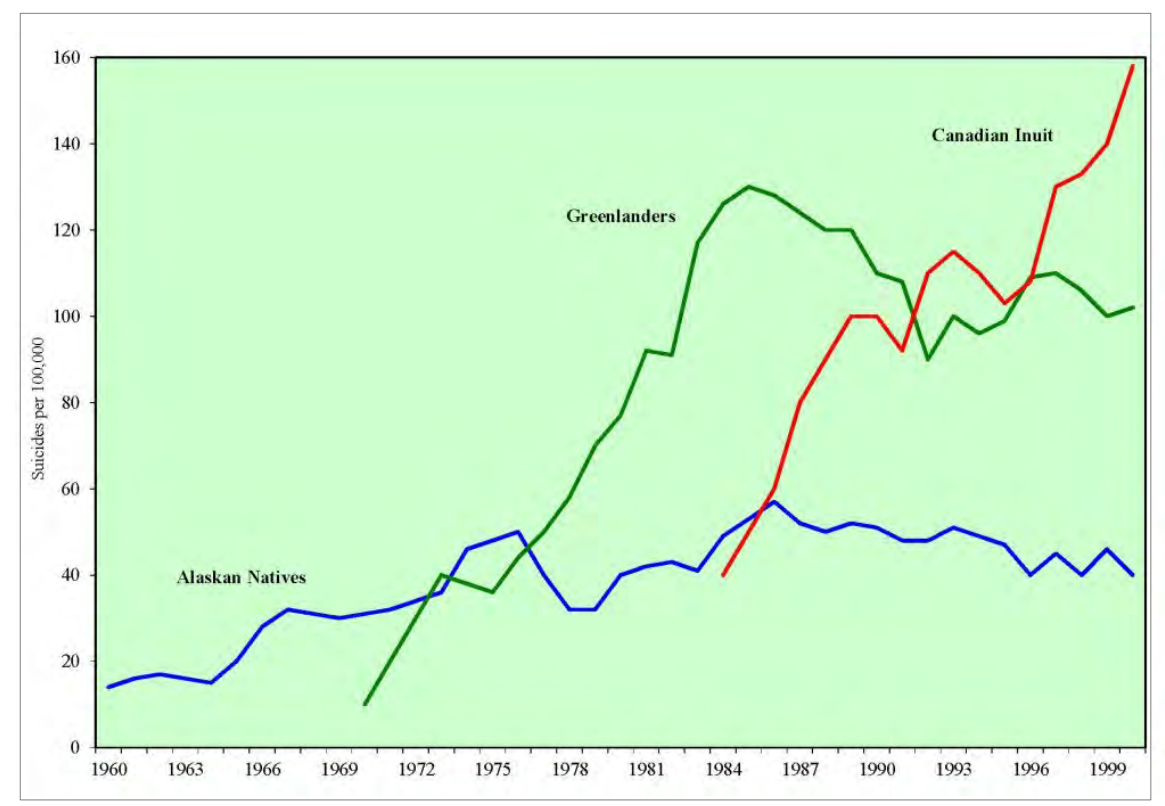

Source: Data from Jack Hicks, as presented to the Hope and Resilience: Suicide Prevention in the Arctic Conference in Nuuk, November 2009.

Suicide among youth is particularly high in Greenland and Nunavut, a phenomenon observed also among Alaska Natives. Suicide is not as common in the non-Indigenous populations in most northern regions. Among the Inuit, the increase in suicide rates occurred first in Alaska, later in Greenland, and still later in Canada, with each later epidemic more severe than the preceding one (Figure 8.6). One characteristic of youth suicides is their tendency to occur in clusters, with devastating impacts on small communities. Suicide statistics are only the tip of the iceberg - for every suicide there are many more suicide attempts and individuals harboring suicidal thoughts. In an effort to help curb the high rates of suicide, the Government of Nunavut has created a Suicide Prevention Strategy (Nu- 
navut Suicide Prevention Strategy Action Plan, 2011). Surveys among Indigenous people in several regions such as SLiCA provide some measure of the extent of suicidal ideation, as well as people's perception of suicide as a community problem (Larsen et al., 2010).

\subsection{Emerging issues}

Human health and well-being are the result of complex interactions among genetic, economic, social, cultural, political and environmental factors. Due to globalization and climate change, there are many potential new challenges and threats for individuals and communities in the Arctic (see Megatrends, 2011 and Chapter 10, Globalization). The Arctic is already and will continue to be less isolated than in the past. The pace of natural resources development such as oil and gas and mining, and the scale of human activities such as shipping and tourism have accelerated. Traditional livelihoods have come into conflict with new ones in many areas. Socioeconomic and environmental changes have created new groups at risk of marginalization.

As with the rest of the world, the Arctic is facing increasing urbanization, which brings with it challenges for human health and well-being. Large cities with populations close to or exceeding 300,000 can be found in Russia (such as Murmansk, Arkhangelsk and Yakutsk), and Alaska (Anchorage); cities with population around 100,000 can be found in the Nordic countries (e.g., Umeå, Oulu), whereas in Canada's North and Greenland, no cities have populations exceeding 30,000 people.

\subsubsection{Food and water security}

A major factor in the deteriorating food and water security situation in the Arctic is the changing climate, characterized by degradation of permafrost, reduced ice cover, and extreme weather conditions (such as floods and storms). These changes impact the harvest from fishing and hunting, affect the health of animals and the safe storage of food, and the nutritional status of Indigenous people who depend on traditional foods for subsistence (Nilsson and Evengård, 2013).

Health care can also be affected by the changing climate. It has been documented that in Arctic Russia, the reindeer herding Nenets people are "stuck" in the tundra for longer periods of time waiting for snow and have to leave their villages earlier before the snow melts in order to reach medical care (Fedotov et al., 2011). The frequency of fishing and 
hunting accidents and drownings in Northwest Alaska (Brubaker et al., 2011) and among the Nenets (Davydov and Mikhailova, 2011) has increased due to thin ice or changes in snow cover.

Surveys in northern Canada have found food insecurity affecting over $60 \%$ of Inuit households (Huet et al., 2012). The situation in the Russian Arctic has also deteriorated (Dudarev et al., 2013). Decreased access to safe food and water is associated with an increase in infections such as gastroenteritis, respiratory infections and vector-borne diseases. The warming climate may introduce new host species and pathogens into new habitats that may lead to infections in humans. Increases in the morbidity rates of zoonotic infectious diseases (such as tick-borne encephalitis, tularaemia, brucellosis, rabies and anthrax) among humans, domestic animals and wildlife in the Russian Arctic (Revich et al., 2012) and Alaska (Hueffer et al., 2013) have been reported. Climate change in the Russian Arctic and Alaska has been more pronounced than in the other parts of these countries. However, there are also some positive effects of warming in terms of food security in the Circumpolar North, for example, an increasing potential for agriculture during the longer growing seasons (AMAP, 2011).

\section{Textbox 8.1}

Indicators of food and water security in an Arctic health context

- Healthy weight (BMI, ratio $>30$, also for children).

- Self-estimated proportion of traditional food in diet.

- Non-monetary food accessibility.

- Monetary food accessibility.

- Food-related contaminants.

- Food-borne diseases.

- Per capita renewable water.

- Accessibility of running water.

- Waterborne diseases.

- Drinking water contaminants.

- Authorized water quality assurance.

- Water safety plans.

These 12 indicators suggested are based on existing WHO and FAO indicators, and they will be a starting point for monitoring both Indigenous and nonIndigenous populations in rural and urban areas in the Arctic.

Sources: Nilsson et al., 2013a, Nilsson et al., 2013b. 
During the Swedish chairmanship of the Arctic Council (2011-2013), food and water security was declared one of its priority issues, and a joint project was initiated, aimed at providing a basis for indicator selection that is relevant for food and water security in the circumpolar areas and which could be used in international collaborations of surveillance in the Arctic (Nilsson and Evengård, 2013). An attempt was made to identify key indicators to follow changes in food and water security in Arctic populations. Such indicators are recommended to be genderbased, since food and water insecurity affect men and women differently. The results of an extensive literature search and critical review were presented to a joint workshop of the Sustainable Development Working Group's (SDWG) Arctic Human Health Expert Group and the Arctic Monitoring and Assessment Program's (AMAP) Human Health Assessment Group, which selected 12 candidate indicators for future monitoring (Nilsson et al., 2013a; Nilsson et al., 2013b).

\subsubsection{Environmental contaminants}

For more than 20 years, AMAP has monitored the levels of persistent organic pollutants and heavy metals in circumpolar countries. AMAP has regularly published reports on human health and the trends of environmental contaminants such as mercury and lead in traditional/local foods and their body burden in humans (AMAP, 1998; AMAP, 2003; AMAP, 2009; AMAP, 2011; see Textbox 8.2). Future research is needed that would combine bio-monitoring of contaminants with dietary surveys in order to provide more accurate estimates of exposure and better dietary advice. Several joint research and educational projects have been launched, such as the multidisciplinary EU-funded ArcRisk project led by AMAP (ArcRisk, 2013), which aims to determine how climate-mediated changes in the environmental fate of contaminants affect the exposure of human populations via the food-web, both now and in the future. The levels of the old persistent organic contaminants will decrease in the environment and in humans in the future but there will be differences among regions and among contaminants (Nost et al., 2013). There are now "hot spots" in industrial areas in the Russian North. Increased amounts of persistent environmental contaminants and heavy metals, especially mercury, are being released from the frozen soil and contaminate drinking water (Dudarev, 2012; Dudarev et al., 2013). Increased traffic and tourism, together with oil and gas and mining activities, will bring contaminants closer to people in new ways. Previously, most contaminants entered the Arctic 
through long-distance transport. Such changes will present new challenges to the health and well-being of all Arctic residents.

\section{Textbox 8.2}

\section{Relevant key findings of Arctic Monitoring and Assessment Programme}

- Levels of legacy POPs in human tissues are declining in many regions of the Circumpolar Arctic. New sources and patterns are being seen in Arctic Russia.

- Levels of mercury in human tissues are declining in several Arctic regions. Inuit continue to have the highest exposure levels of mercury in the Arctic and most often exceed blood guidelines.

- Traditional foods are an important source of nutrients for many Arctic residents. These foods are also the main source of exposure to contaminants.

- New evidence indicates that POPs, mercury, and lead can affect the health of people and especially children at lower levels of exposure than previously thought.

- Climate change may increase the mobilization of POPs and mercury, and lead to higher releases of contaminants within the Arctic.

The 2011 AMAP Assessment "Mercury in the Arctic" concludes: "It has been shown that exposure to mercury at the current levels in the Arctic can have adverse impacts on human health, particularly for the developing fetus and children, although further research is required to determine if the subtle effects of Hg on human health are persistent." (AMAP 2011: 168).

Sources: AMAP, 2009; AMAP, 2011.

\subsubsection{Dietary transition}

The change from "traditional" foods based on hunting and fishing to a "western" type of diet has steadily progressed in many Indigenous communities around the Arctic. These changes have led to increased rates of modern diseases such as obesity, diabetes and cardiovascular diseases. Various circumpolar countries and regions have promoted dietary recommendations to ensure a balanced and nutritious diet (Jeppesen et al., 2011). However, achieving this balance is difficult. Many remote Arctic communities are only accessible by air or marine transport, although some have road access. Transportation costs are high, which result in high food prices that may be beyond the reach of many people. Even those who engage in harvesting fish and game animals require expensive hunting equipment, fuel and vehicles. Long delays in shipments due to weather 
conditions often result in spoiling of perishable items. Those who rely mainly on market foods become increasingly dependent on cheaper, unhealthy choices, further affecting food security, nutritional status, and overall health (Jeppesen et al., 2011; Bjerregaard and Mulvad, 2012).

\subsubsection{The aging population}

Societal values in many countries consider the younger generation as particularly vulnerable and requiring special attention as the pace of Arctic development increases. The working force or middle-aged generation is considered a key factor in shaping the social and economic agenda of the region. However, the "grey" population - elderly people, many of whom are retired - is often neglected in many kinds of analyses of Arctic populations. This "grey" population is a viable resource for regional capacity building. The phenomenon of the aging population manifests itself differently across the Arctic, due to the varied contributions of declining fertility, mortality and immigration (Emelyanova and Rautio, 2013; Lewis, 2013; Nordic Population Aging, 2013).

Despite common concerns associated with the growing size of older population, such as strains on the pension and health care systems (especially the cost of long-term care), senior residents of the Arctic make important contributions to the local community: late age labor participation, experience in decision-making, unpaid "volunteering" and the intergenerational transmission of cultural values and practices.

There are urgent questions for policy makers in the Arctic related to this sector of the population. How will Arctic societies adapt to the aging population and what impact will it have on not only the health of seniors, but on the entire population? What kind of political will is needed to make policy adjustments, for example, in working life, pension reforms, living arrangements, and health services? It is often not recognized that the elderly person is a powerful but underutilized resource for Arctic development.

The process of population aging can be expected to continue in the coming decades. For policy responses to be effective in optimizing societal well-being, they must be based on scientific understanding of the critical dynamics associated with population aging (Emelyanova and Rautio, 2013). Both conventional and prospective methodologies provide such evidence. "Conventional" tools such as the United Nations indicators on aging (World Population Aging 1950-2050, 2007) are based on the concept of "chronological" age. More recently, "prospective" indices have emerged based on the concept of prospective age and remain- 
ing life expectancy (Sanderson and Scherbov, 2008). Generally, a slower speed of aging is observed when changes in longevity over time (prospective approach) are taken into consideration. Clearly, a group of very aged Scandinavian countries is a forerunner in population aging within the Arctic (Emelyanova and Rautio, 2012).

More evidence is required to understand the complexity of aging in Arctic populations and to support the development of a comprehensive Arctic strategy to address the health and well-being of older people. Qualitative research methods are needed to investigate both the needs of older people and available capacities of regional policy-makers to respond to those needs. Large gaps in our understanding of Arctic demography remain, for example, variations in movement (in- and outmigration), gender (male and female aging), patterns of settlement (urban vs. rural), and nomadic patterns (Indigenous vs. non-Indigenous) in the cross-territorial context. Access to vital statistical data at the regional and municipal levels likewise remains limited.

\subsection{Changing quality of life}

The term "development" appears in the title of both this report and the first AHDR (AHDR, 2004). There has been a paradigm shift in how this concept is operationalized and measured. Whether Gross Domestic Product (GDP) can adequately assess development in a broader sense has been discussed for quite some time. Globally, considerable attention has been given to developing alternative measures for development to supplement or even substitute GDP because of concerns that GDP is often misleading when used to quantify subjective well-being, quality of life or happiness. Within the last decades, a large number of initiatives have been launched to better grasp the complexity embedded in the terms "individual wellbeing", "quality of life" and "happiness". These initiatives include: the United Nations Human Development Index (UN HDI, n.d.), the European Quality of Life Index (Eurofound, 2012); the European System of Social Indicators (GESIS, ZUMA) (ZSI, nd), the Happy Planet Index (HPI, 2014), the Bhutanese "Gross National Happiness Index" (GNHI, 2014), the OECD Better Life Index (OECD, n.d.), the so-called Sarkozy initiative that resulted in a number of recommendations from a group of prominent scholars on measuring human development (CMEPSP, n.d.), and most recently, "Wellbeing and Policy" (O'Donnel et al., 2014).

The terms "subjective well-being", "quality of life" and "happiness" increasingly appear in a variety of assessments, surveys and analyses in 
the Arctic. Recent examples include the Arctic Human Development Report (AHDR, 2004), the Arctic Social Indicators project (ASI, 2010; ASI, 2014), the Survey of Living Conditions in the Arctic (SLiCA) (Andersen and Poppel, 2002; Kruse et al., 2008; Poppel, 2014), chapters focusing on well-being in the Arctic Climate Impact Assessment (Nuttall et al., 2005), and in the Snow, Water, Ice and Permafrost in the Arctic Report (SWIPA) (Hovelsrud et al., 2011). This trend reflects discourses about human development, not only in the Arctic, but also on a global scale in both political and academic fora. In discussions related to Arctic human development, this might be seen as an indication of an increasing demand from Indigenous and other Arctic residents to be acknowledged as active participants in developing the Arctic, and not passive recipients of the consequences of rapid social and economic changes.

\section{"Be Smoke Free" - Anti-smoking logo from Nunavut, Canada}

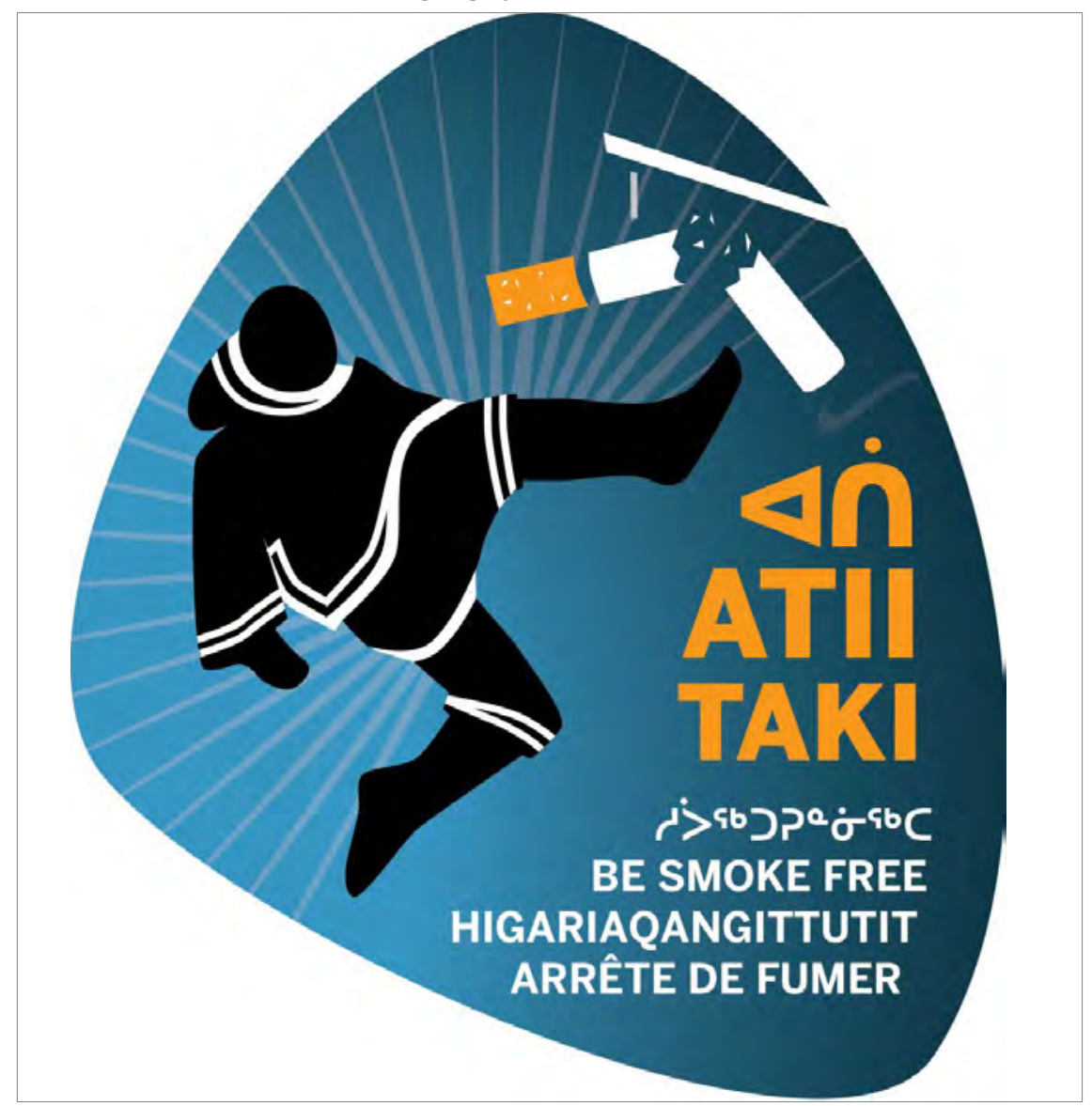

Credit: Public campaign. 


\subsubsection{The Arctic Human Development Report (AHDR)}

The first Arctic Human Development Report (AHDR, 2004) focused on well-being in the "Human Health and Well-being"-chapter. Well-being was considered in close relation to and determined by a number of different health aspects as well as some more overall living conditions dimensions like "local control" and "spiritual values" (AHDR, 2004:157). Furthermore, the first $A H D R$ stated:

“' [c]ommunity cohesion' and individual and community 'resilience' are important for well-being, but these factors are seldom reflected in health statistics. We need to find ways to quantify these factors and their role in quality of life in a way that makes it possible to compare the situation across the Arctic nations."

(AHDR, 2004: 166)

The AHDR then concluded, "[r]esidents of the Arctic - settlers as well as Indigenous peoples - regularly emphasize the importance of at least three dimensions of human development over and above those included in the HDI:

- Fate Control (controlling one's own destiny).

- Maintaining cultural identity.

- Living close to nature (AHDR, 2004: 240).

The AHDR argued that "it would be a mistake to ignore perspectives on human development, especially in areas of the world like the Arctic where distinctive cultures remain influential" (AHDR, 2004: 241) and recommended that "[t]he Sustainable Development Working Group should organize a workshop to begin the process of devising a small number of tractable indicators to be used in tracking changes in key elements of human development in the Arctic over time" (AHDR, 2004: 242).

This section assesses research and statistics on well-being and Arctic human development measured by different social indicators, and assesses the trends that might potentially be apparent since the first $A H D R$. 


\subsection{Application of the United Nations' Human Development Index (HDI) to the Circumpolar Arctic}

Following the original intention of the first $A H D R$, the following paragraphs attempt to apply the UN HDI to the Arctic regions.

The United Nations' Human Development Index, HDI, is a composite index combining indicators on health (infant mortality and life expectancy), education (measured by educational attainment) and income (GDP per capita). Whereas the UN HDI is computed at a state level, a regional approach is needed to compare human development in the Circumpolar region. The increased focus on human development in the Arctic has initiated a number of efforts to gather and organize data to facilitate inter- and intraregional comparisons of human development and socioeconomic conditions in the Arctic. The sources of the data in this section are primarily ArcticStat (n.d.) and the Circumpolar Health Observatory (CircHOB, 2012) and websites of national and regional statistical institutes as well as UN, OECD and IMF, to add to and substantiate these data archives. Furthermore, a number of initiatives like Sustainable Arctic Observing Network (SAON), Arctic Observing Network (AON) and Arctic Observing Network - Social Indicator Project (AONSIP) (see Kruse et al., 2011) have been developed in the Arctic to ensure standardization and thus comparability of Arctic socio-economic data (the initiatives are often collaborative efforts with the natural sciences). These efforts combined with data from national and regional statistical institutes make it possible to compare indicators.

As noted above, the Arctic falls into four groups (ranked according to a number of health indicators):

1. The Nordic countries.

2. Alaska, Yukon and Northwest Territories.

3. Greenland and Nunavut.

4. The Russian Arctic.

Two of the health indicators applied in the HDI, infant mortality and life expectancy, both substantiate this conclusion. Comparing the 2004-2009 average to the 2000-2004 average, it is important to note that the regions ranking lowest (primarily regions of the Russian Arctic with a few exceptions: the Koryak okrug, the (former) Evenki okrug and Magadan oblast not only experienced a decrease in infant mortality and an increase in life 
expectancy, but also made significant progress in these important health issues compared to most other Arctic regions (see Figure 8.1; also, Chapter 2, Arctic Populations and Migration, Figures 2.6 and 2.8).

Regional educational attainment levels reflect not only the selfinitiative of young people and residents desirous of an education, but also the demand for an educated work force in both the public and private sector. Often, this demand is met by immigrant laborers who move to the region on either a short or long term basis. The five-year average for 2000-2004 shows major differences for educational attainment: under $20 \%$ for Greenland and some of the Russian regions, to a high of about one-third in Alaska, Yukon and Northwest Territories. Data were not available for the Russian Arctic for the period 2005-2009. Comparing the five-year average attainment level for the period 2005-2009 for the other Arctic regions shows that averages have increased in all regions (except Alaska), and education levels for women have increased everywhere more than that of the male population. This development is not distinct to the Arctic region but seems to follow a trend that we see in most of the Arctic states.

The third dimension of the Human Development Index, income, is measured by "Gross Domestic Product (GDP) per capita" (UN HDI). GDP is measured in domestic currencies of each country and each country experiences different consumer patterns, price levels and inflation rates. Comparing GDP per capita thus presupposes a procedure that converts GDP (in basic prices) in domestic currencies to a common currency (USD) and creates a common "consumer basket" via the so-called purchasing power parities, or "PPP". The geographical delimitation used to categorize and rank the Arctic regions according to the health indicators does not directly reflect the ranking according to income. There are parallels, though. In 2000, the per capita GDP in most of the Russian regions (except Yamalo-Nenets Autonomous okrug (AO), Khanti-Mansy AO and Nenets AO) were the lowest in the Circumpolar region, and below USD PPP 15,000. The regions with the highest GDP per capita (USD 25,000 and above) included Alaska and the three Canadian Arctic regions as well as the Arctic regions of Finland and Norrbotten (northern Sweden). GDP data for the last year of the first decade of the 21st century have not been available for all regions and GDP developments for these regions have thus been measured for the period including the last year where data are available. The GDP time series for the three Finnish Arctic regions are only available for the period 2000-2005, and for three of the Russian Arctic regions (former Evenki AO, former Taymyr AO and former Koryak AO) GDP data are only available for the period 2000-2007. 
The more significant changes in the GDP indicator (GDP USD PPP in fixed prices) include:

- The decrease of more than $20 \%$ in the Icelandic GDP from 2000 to 2009 (due to the huge impacts of the financial crisis - see, e.g. Matthiasson, 2010).

- The substantial decrease from 2000 to 2005 in the GDP of former Koryak AO and former Taymyr AO.

- The decrease (Oulu/Pohjois-Pohjanmaa) and relatively low percentage increase in the Finnish Arctic region respectively from 2000 to 2007.

- The generally high percentage increases in most Russian Arctic regions - especially Nenets AO (more than doubling of GDP from 2000 to 2009) and Chukotka (more than trebling of GDP from 2000 to 2009).

In Figure 8.7, two of the UN human development indicators are illustrated: "green" indicates a better situation during the first decade of the 21st millennium, "yellow" indicates status quo, and red indicates a retrograde step.

As 2009 was the first whole calendar year after the beginning of the financial crises, it is not possible to predict the outcome for the regions where data are lacking. Subject to these deficiencies, though, it appears that the overall distribution of the 28 Arctic regions by GDP is much the same at the end of the decade as in the beginning. Eight out of ten of the regions with highest GDP per capita in the period 2005-2009 were also among the top-ten in 2000, and nine out of ten were among the regions with lowest GDP in both 2000 and in the period 2005-2009. It seems to be justified to compare the average GDP of the lowest ranking regions and the highest-ranking 10 regions in 2000 and in the period 20052009. The overall conclusion based on these averages is that the difference between the countries with the lowest and the highest GDP per capita has narrowed. In 2000, the GDP per capita average of the ten lowest ranking regions was roughly one fifth of the ten highest ranking. 
Figure 8.7 Development of two indicators of the UN HDI: Life Expectancy and Gross Domestic Product, GDP in the first decade of the 21st century. Percent changes

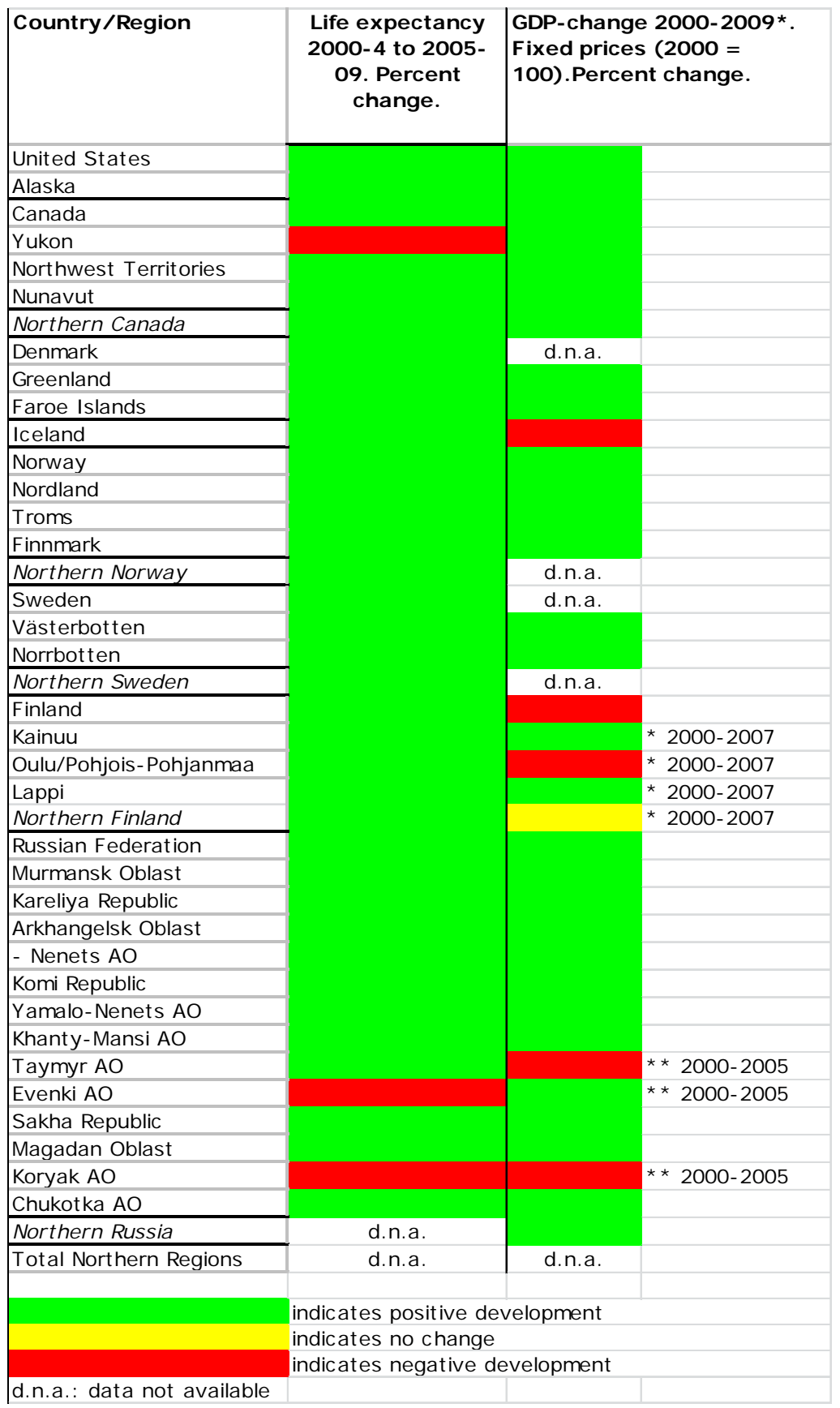

Sources: ArcticStat (http://www.arcticstat.org/) and The Circumpolar Health Observatory (CircHOB) (http://circhob.circumpolarhealth.org/), validated, substatiated and with additions from websites of national and regional statistical institutes as well as UN, OECD and IMF. 
The data from 2009 - and for some regions the 2005 and 2007 figures respectively - indicates a narrowing to one fourth, and thus a somewhat decreased disparity between the Arctic regions measured by the gross production value of the regions. The background for the decreased disparity between Arctic regions is an economic growth - primarily in the Russian Arctic - based on oil, gas and mineral resource exploitation.

It should be noted, however, that GDP only tells us about the total added value of production (e.g., Goldsmith, 2009: 34-35) and gives no indications about potential negative externalities (environmental problems, for example) or about the distribution of income. Resource development might have regional economic spin-offs and generate income locally, but major extractive activities often are decoupled from both local and regional economic activities and therefore contribute more to economic activities and to large corporations and economic centers outside the Arctic (e.g., Duhaime, 2004). Furthermore, it is important to understand that the non-renewable resources that have been extracted for the region represent a loss of wealth for future generations (Mäenpää, 2009).

In sum, almost all Arctic regions have experienced positive developments in the indicators constituting the overall UN Human Development Index. The inequalities and disparities between the Arctic regions of Arctic states and the Arctic states, between the Arctic regions and also within the Arctic regions still exist. But when it comes to child mortality, life expectancy and GDP per capita, the data substantiates the perception that these inequalities narrow. Koryak okrug seems to be the most significant exception to this overall statement.

\subsection{Including additional dimensions in the assessment of human development}

The first Arctic Human Development Report (AHDR, 2004) recommended developing measurable indicators within six domains; the three domains of the UN HDI as well as "fate control"; "maintaining cultural identity"; "living close to nature". The Arctic Social Indicators report (ASI, 2010: 157; see Chapter 1, Introduction), further elaborated on the six dimensions in an Arctic context and developed a number of indicators to assess well-being in the North:

- Infant Mortality (Domain: Health/Population).

- Net-migration (Domains: Health/Population and Material Well-being). 
- Consumption/harvest of local foods (Domains: Closeness to Nature and Material Well-being).

- Per capita household income (Domain: Material Well-being).

- Ratio of students successfully completing post-secondary education (Domain: Education).

- Language retention (Domain: Cultural Well-being).

- Fate Control Index (Domain: Fate Control).

Data are not available - either from statistical institutes/associations or collaborative research projects - to make circumpolar wide comparisons including all regions and applying the indicators for the three additional AHDR-/ASI-dimensions: "closeness to nature" (consumption/harvest of local foods); "cultural well-being" (language retention); "fate control" (an index composed by indicators for: political control, control over land/resources, economic control, control over knowledge construction). There are regional and national examples, though, that might be considered "prototypes" for developing comparable data. Examples include the annual updates "Subsistence in Alaska" that the State of Alaska has produced since 1990 (Alaska Department of Fish and Game, 2014), and the assessments that Statistics Canada has conducted on language abilities (Statistic Canada, n.d.)

\subsection{What can we learn about human development from different community, regional, national, circumpolar studies?}

A number of assessments and research projects focusing on Arctic human development have been conducted in recent years. The assessments and research projects fall into three main categories:

- Studies assessing Arctic human development by a number of key social indicators. Examples are: the Arctic Social Indicators project (ASI, 2010; ASI, 2014); Aboriginal Peoples Survey (APS) (Wallace, 2014) SAMINOR (Brustad et al., 2014); and the Inuvialuit Baseline Indicators (IBI).

- Studies assessing Arctic human development by measuring subjective or community well-being/quality of life either by constructing composite indices or by people's self-evaluation. Examples are Survey of Living Conditions in the Arctic (Poppel et al., 
2007); (Rethinking the Top of the World (Canada Centre for Global Security Studies, 2014); Aboriginal Quality of Life (NAEDB, 2012); The Community Well-Being Index (CWB) (Aboriginal Affairs and Northern Development Canada, 2012); The North Slope Social Indicator Survey (NSSIS).

- Both types of studies can be carried out at the community, regional, national or international level and can be based on the total population or focussing on groups of the residents - most often the Indigenous peoples.

- A third category of data collection of importance, primarily for future research and assessments, are data archives (like Arctic Stat) and databases that are being constructed to contain a variety of socioeconomic and other key indicators. Examples are: Arctic Observing Network (AON), Arctic Observing Network - Social Indicator Project (AON-SIP) and the Sustaining Arctic Observing Network (SAON).

Only few of the abovementioned studies make it possible to do time series studies, but they may nevertheless provide useful insight. Some of the studies will be presented in brief. The findings presented will primarily be from North American studies as few surveys and social indicator studies are conducted in Fennoscandia (the focus has primarily been on health studies) and the Russian Arctic (an increased focus on human development has resulted in a number of studies).

\subsection{Findings from studies assessing Arctic Human Development by a number of key social indicators}

\subsubsection{Arctic Social Indicators Project (ASI) II}

The recommendation to develop indicators reflecting different dimensions and thus measuring human development more holistically became the point of departure for the Arctic Social Indicators (ASI) Project, a direct follow-up to the first AHDR. In the first phase of the ASI project the working group focused on elaborating and developing indicators within the three United Nations Human Development Index domains and for three additional domains recommended in the first AHDR (ASI, 2010). 
Relaxing near a corral during calf-marking, Sweden

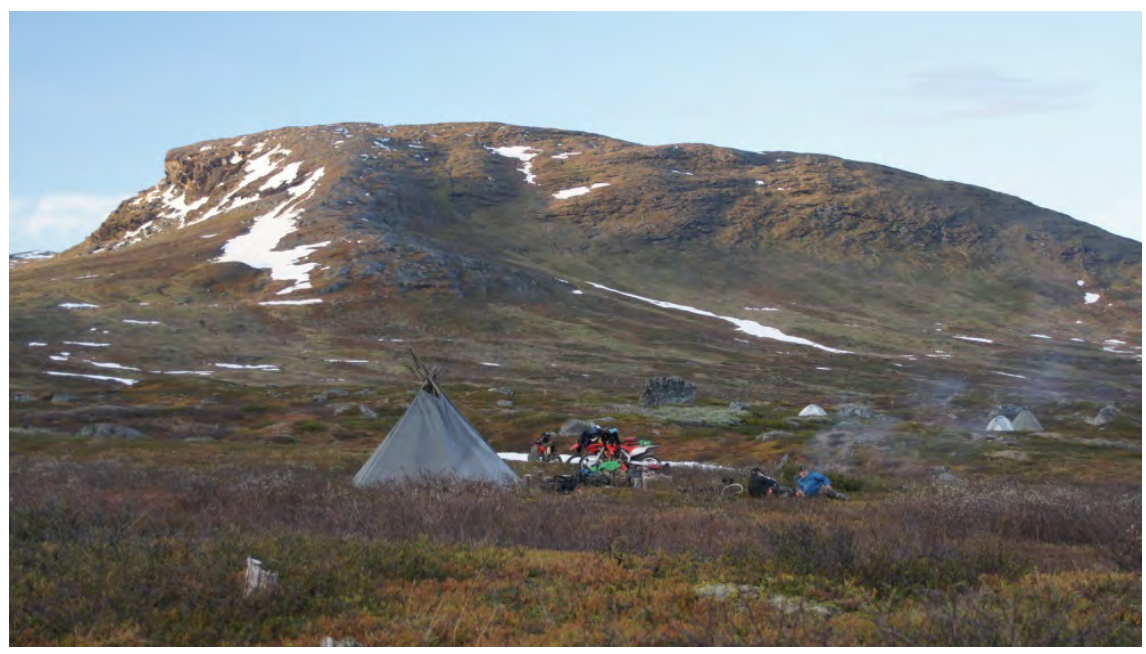

Credit: Hugh Beach.

The second report of the Arctic Social Indicators project selected four regions as pilot study regions for further analyses, applying the selected social indicators:

1. Sakha - Yakutia, Russian Federation.

2. Northwest Territories, Canada.

3. West-Nordic region.

4. Alaska Inuit, USA.

Generally, the second ASI report's conclusion about human development in the four regions is that the results suggest a general improvement, but that considerable differences exist among regions, and between rural and urban areas in the Arctic (ASI, 2014).

The study of Northwest Territories is an example of this overall conclusion. It examines the 15-year period from 1991 to 2006 and states that whereas a general improvement in human development has taken place there are major regional discrepancies especially when it comes to material well-being (Chapter 3 of ASI, 2014). This leads Petrov and King to distinguish between a small group of communities/cities that are "haves" and a larger and more remote group that are "have-nots" (Ibid.). When measured by "language retention" (cultural well-being) and harvest and consumption of local food (contact with nature), the smaller 
and more remote Indigenous communities are better off, whereas language retention for Northwest Territories as a whole has declined.

The Sakha Republic (Yakutia) in the Russian Federation is another example of a regional application of the ASI indicators, with a different context and development than Northwest Territories but with overall results that are much the same: a general improvement in human development with major rural-urban differences, but for areas inhabited by numerically small peoples most of the indicators show lower-thanaverage attainment (Chapter 2 of ASI, 2014). The results also suggest different developments in the different indicators: a substantial increase in material well-being and positive developments in the education level and increased time spent on the land ("contact with nature" indicator) whereas the ASI-indicators for "cultural vitality" and "contact with nature" are ambiguous (Ibid.).

\subsection{Studies assessing Arctic human development by measuring subjective or community well- being/quality of life}

\subsubsection{Canadian Community Well-Being (CWB) Index}

Aboriginal Affairs and Northern Development Canada has developed and published The Community Well-Being (CWB) Index for 1981, 1991, 1996, 2001, and 2006 (Penney et al., 2012). The index is based on Statistics Canada's population census data and composed by indicators on income (income per capita), education (high school and university completion rates), housing (housing quantity and quality), and labor force activity (employment and labor force participation rates). A special study has been conducted comparing the communities in the four Inuit settlement regions (including both Inuit and non-Inuit residents), Inuit Nunangat: Nunatsiavut (Northern Labrador); Nunavik (Northern Quebec); the territory of Nunavut; the Inuvialuit Settlement Region (Northwest Territories) with First Nations communities and with non-Indigenous communities.

Figure 8.8 shows that, whereas all indicators have increased in the quarter of a century since 1981, for all four Inuit regions, the scores for "Education" and the "Labor Force Activity" declined from 2001 to 2006.ii 
Figure 8.8. Canadian Community Well-Being Index: Component scores over time, Inuit Nunangat, 1981-2006

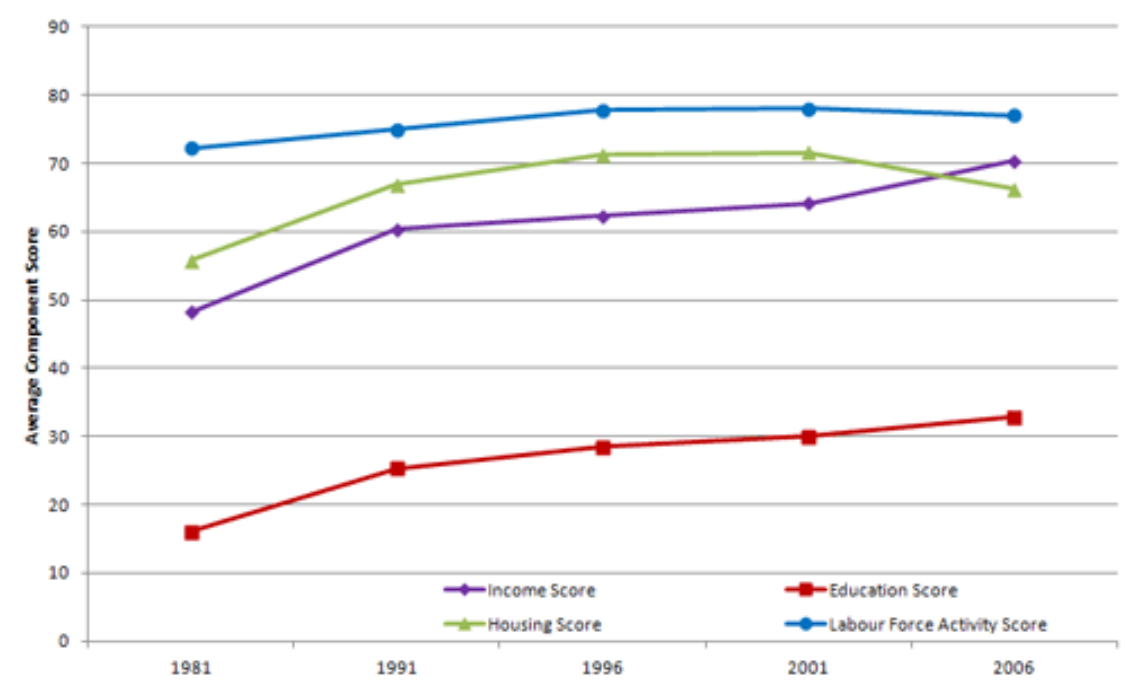

Source: Penney et al. , p. 10, 2012.

Figure 8.9. Canadian Community Well-Being Index. Average scores over time, Nunangat, 1981-2006

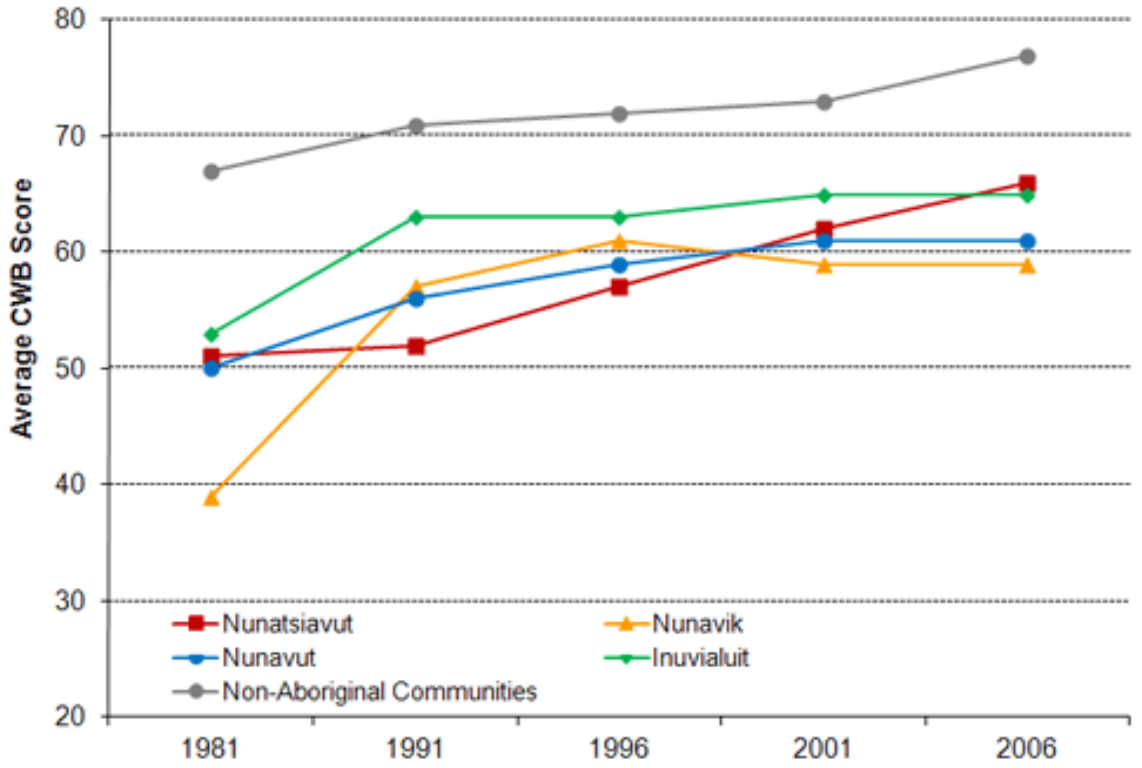

Source: Penney et al., p. 11, 2012.

All four Inuit regions are better off in 2006 than in 1981, measured by the CWB Index, but only Nunatsiavut has better scores in 2006 than in 2001 (Figure 8.9). As the increase in the CWB Index for non-Aboriginal 
communities continued a positive development ever since 1981, it means that the gap between most Inuit regions and non-Aboriginal communities has widened since 2001.iii

\subsubsection{The Aboriginal Economic Benchmark Report}

In 2012, the National Aboriginal Economic Development Board, (NAEDB) published The Aboriginal Economic Benchmark Report (AEBR). The National Aboriginal Economic Development Board was established in 1990 and is "appointed by Order-in-Council to provide policy and program advice to the federal government on Aboriginal economic development" (NAEDB, 2012: 2). The 2012 report focuses on the economic progress of First Nations, Inuit, and Métis in Canada and builds on three core indicators: "employment", "income" and "wealth and wellbeing" as well as five underlying indicators: "education", "entrepreneurship and business development", "governance", "lands and resources" and "infrastructure" and builds on official statistics from Statistics Canada as well as a number of other sources to establish baseline indicators. In total, more than 100 indicators are used to assess the economic development of Indigenous peoples in Canada and to compare the findings with the development for non-Indigenous Canadians (NAEDB, 2012).

The NAEDB Report compares socio-economic conditions among the different aboriginal and non-aboriginal groups and the community wellbeing index is calculated according to this typology. As all Inuit communities are located in the Canadian North, some results concerning Inuit and Inuit communities are briefly introduced. It should be noted that "Inuit communities" also include non-Inuit residents of these communities. The report's conclusions, in brief, about Inuit and Inuit communities state:

"Inuit have generally seen little improvement in key indicators over the period 1996 to 2006. For example, Inuit have the lowest education completion rates of any Aboriginal heritage group. As a result, there are sizeable gaps between the economic outcomes of Inuit and non-Aboriginal Canadians living in the same regions."

(NAEDB, 2012: 9)

Based on the CWB developed by Aboriginal Affairs and Northern Development Canada, the AEBR further concludes, "Inuit communities have a CWB score 15.1 points (on a 100 point scale - author) below other Canadian communities" (NAEDB, 2012: 37) and that "70\% of Inuit communities had stable or increasing CWB scores between 2001 to 2006, as compared to $90 \%$ of other Canadian communities" (NAEDB, 2012: 17). 


\subsection{Findings from studies assessing Arctic human development by measuring subjective or community well-being/quality of life}

A number of measures have been developed to better express the individuals' subjective perception of their life situation. "Happiness" is one such measure. The question often asked in international surveys, in order to identify "the happiest countries in the world", is: "How satisfied are you with your life as whole?" This question was also part of the questionnaire applied in the Survey of Living Conditions in the Arctic, SLiCA and in "Rethinking the Top of the World: Arctic Security Public Opinion Survey" and some key findings will be presented below.

\subsubsection{Quality of life among Inuit and Sámi and Indigenous peoples of Chukotka and the Kola Peninsula - SLiCA}

The Survey of Living Conditions in the Arctic, SLiCA, is an attempt to measure quality of life among Inuit, Sámi and the Indigenous peoples of Chukotka and the Kola Peninsula (see Textbox 8.3) both by addressing a variety of living conditions and by asking about people's self-evaluation. The "quality of life as a whole" question was asked in three SLiCA survey regions (Alaska, Greenland and Sweden) whereas a question asking about "quality of life in this community" was asked in all SLiCA survey regions.

\section{Textbox 8.3}

\section{The Survey of Living Conditions in the Arctic (SLiCA)}

The SLiCA project was launched at a workshop in 1998 and the SLiCA core questionnaire was developed in partnership with representatives of the respondents (the Inuit, Sami and Indigenous peoples of Chukotka and the Kola Peninsula) until 2000. Eight thousand face-to-face interviews were conducted in different periods in the different survey regions until 2008 (see e.g. Andersen and Poppel, 2002; Kruse et al., 2008; Eliassen et al., 2012; Poppel, 2014). Only in Greenland were non-Indigenous residents included in the survey.

The reason to develop a new research design to investigate living conditions, subjective well-being and quality of life of Indigenous peoples of the Arctic was the assumption that conventional social indicators do not fully reflect the welfare priorities of the Indigenous peoples (Andersen and Poppel, 2002). 
Individual well-being was defined as covering all aspects of living including the individuals' perception of their resources and overall satisfaction with the quality of their lives. The SLiCA questionnaire (ca. 200 questions) made it possible to analyze a number of dimensions: Communication and Technology, Community viability, Discrimination, Education, Employment/Harvest, Environ-ment/Resource management, Family relations and social networks, Health, Household economy, Housing, Identity management, Justice/Safety, Language, Mobility, Political resources, Religion/Spirituality, and Work/Leisure (Andersen and Poppel, 2002). Furthermore it is possible to analyze the correlation between a number of living conditions indicators, for example, "satisfaction with quality of life" and "self-rated health".

The first SLiCA results from the Inuit settlement regions were published in 2007 and almost 600 tables were organized in six domains according to the AHDR recommendations (Poppel et al., 2007). In 2011, the first article based on data from all SLiCA survey regions was published, including the results of the Inuit settlement regions, Chukotka and the Sámi in Northern Norway, Northern Sweden and the Kola Peninsula (Poppel, 2011).

\section{Figure 8.10}

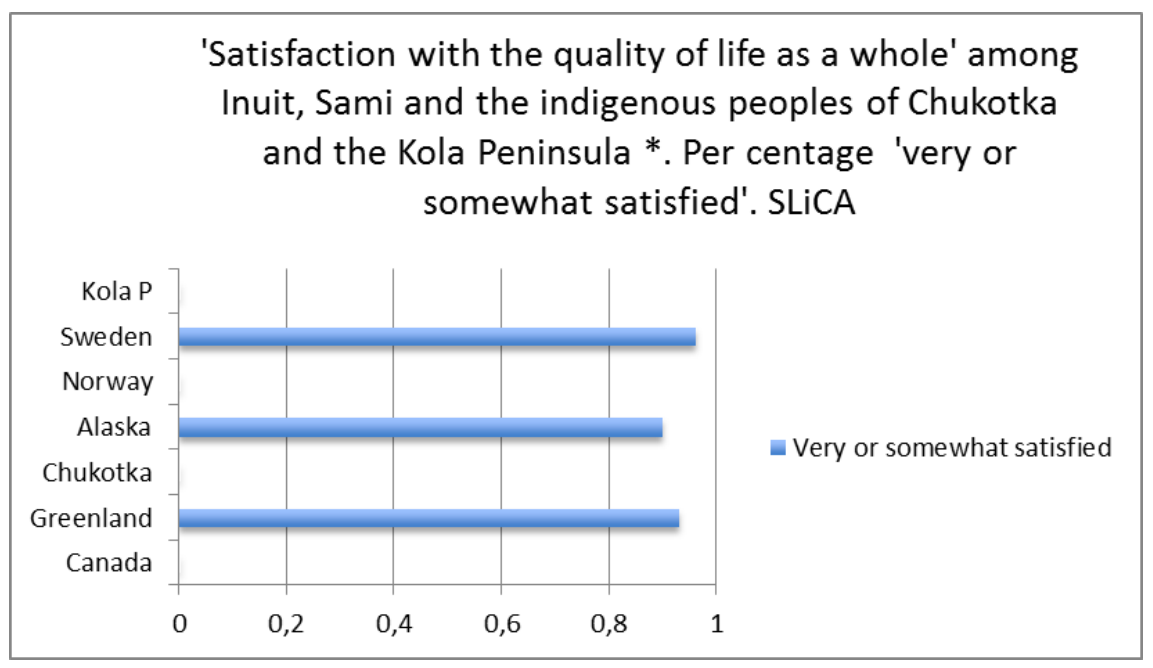

* Data Not Available for Canada, Chukotka, Norway and the Kola Peninsula.

** Source: Poppel et al., 2007 and Poppel, forthcoming.

Among Indigenous people in Greenland, Northern Alaska and Northern Sweden, nine-in-ten are "somewhat" or "very satisfied" with their life as a whole (see Figure 8.10). Taking into account that living standards are 
typically lower among the Indigenous peoples of the Arctic than among non-Indigenous peoples, these findings support the assumptions that other than material factors influence subjective well-being.

Analysis of the data for Greenland and Alaska combined concluded that satisfaction with an individual's actual job or outcome of actual fishing and hunting activities were less important to overall satisfaction with life compared to job opportunities and the amount of fish and game locally available. This means that "availability" and "accessibility" seem to mean more to the quality of life of Inuit than satisfaction with the actual job and actual catch. Also important in explaining overall wellbeing is the combination of market and non-market activities (hunting and fishing, for example) and the influence people have over natural resources and the environment (Kruse et al., 2008; Poppel and Kruse, 2009; Poppel, forthcoming).

Figure 8.11

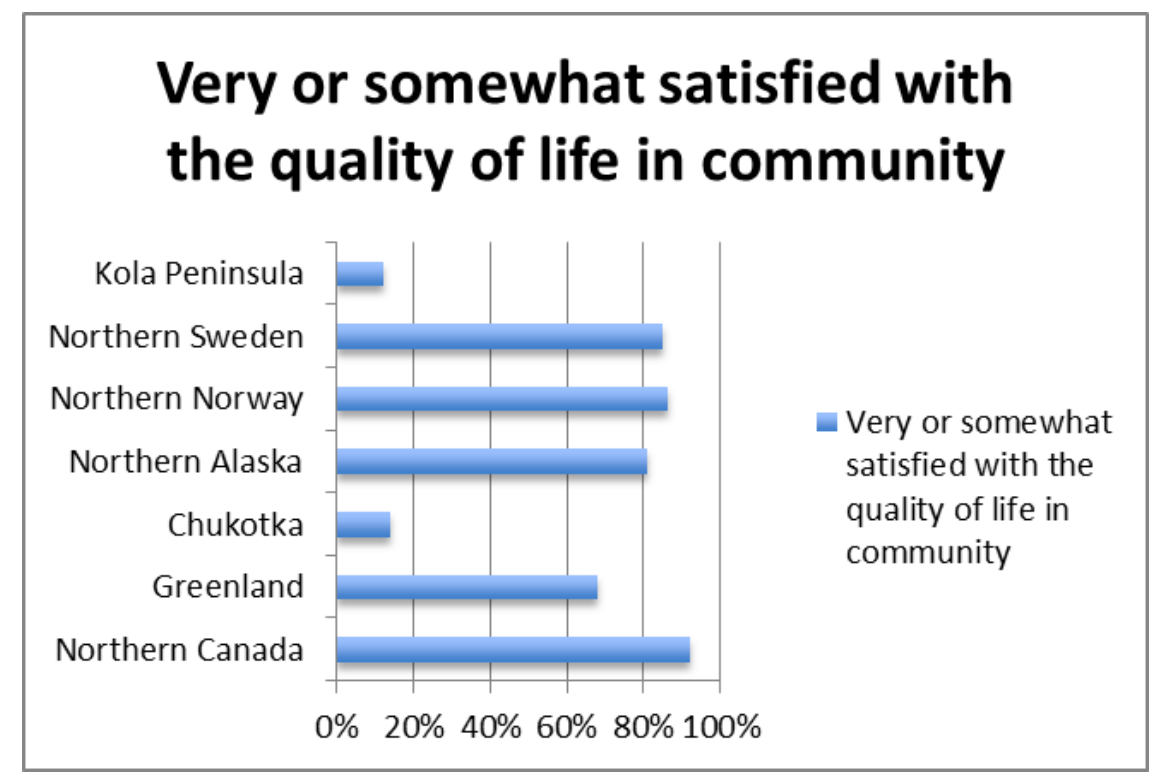

* Data Not Available for Canada, Chukotka, Norway and the Kola Peninsula.

** Source: Poppel et al., 2007 and Poppel, forthcoming.

Figure 8.11 refers to "satisfaction with quality of life in this community", i.e., the perceived quality of "community life" where the respondent lives, including the quality of life of the respondent. Interviews were conducted in different periods in the different regions/countries due to lack of coordination between funding agencies: Northern Canada (2001); Alaska (2002-03); Greenland (2004-06); Chukotka (2004-06); 
Northern Norway (2006-08); Northern Sweden (2006-08) and Kola Peninsula (2006-08). Of those interviewed, more than three out of four Sámi living in northern Sweden and northern Norway and Inuit in northern Alaska were somewhat or very satisfied with the quality of life in their community. The corresponding figure in Greenland was seven out of ten. A significantly smaller proportion - only one out of seven - of the Indigenous people of Chukotka and the Kola Peninsula were somewhat or very satisfied with the quality of life in their community. Other SLiCA findings suggest a correlation between a low rating of satisfaction with quality of life in respondent's community, different kinds of social problems such as unemployment, domestic violence and suicide, lower self rated health, and poor health care among Indigenous people in the two Arctic Russian survey regions (Poppel, forthcoming).

\subsubsection{Quality of Life in Northern Canada -"Rethinking the Top of the World: Arctic Security Public Opinion Survey"}

In a report, Rethinking the Top of the World: Arctic Security Public Opinion Survey, a number of living conditions, perceptions and attitudes were analysed. The analyses were based on an international comparative study (9,000 interviews). Almost 3,000 people were interviewed in Canada (of which 744 were in Northwest Territories, Yukon and Nunavut). The analysis compared responses in northern and southern Canada and reached the following conclusions:

- Northerners are dissatisfied with the human and environmental infrastructure in their region. It is therefore surprising to find that, in terms of how they rate their lives and their health, residents of the North are modestly more likely to see themselves as healthy. Northern residents are significantly more likely to rate their quality of life as excellent than those in the South. Northerners appear to be very optimistic about future well-being. This resilience and optimism was one of the more surprising study findings (EKOS Research Associates, 2011: vi).

- Sociocultural identity and preservation of traditional ways of life (language and culture) are deeply entrenched and are a very important priority for the North (EKOS Research Associates, 2011: vii).

- Respondents from Northern Canada are more likely to rate their quality of life as good (75\%), compared to respondents from Southern Canada (67\%) (EKOS Research Associates, 2011: xiii). 
- In Northern Canada, Yukon residents provide the most positive assessment of their quality of life ( $84 \%$ say good), while residents of Nunavut are less upbeat (65\%). Perceived quality of life increases progressively with income ( $91 \%$ of those with a household income of over USD 120,000 , compared to just $54 \%$ of those earning less than USD 40,000 ), education ( $87 \%$ of university graduates, compared to $56 \%$ of those limited to a high school education), and age ( $82 \%$ of those over 60 compared to $56 \%$ of youth) (EKOS Research Associates, 2011: 3).

\section{Textbox 8.4: Examples on studies of well-being in the Arctic}

Arctic

The Arctic Observing Network, AON, Social Indicators project was initiated to develop datasets relevant to the scientific community and identify gaps in existing observation systems. It also makes recommendations on appropriate actions to fill those gaps (Kruse et al., 2011), for example, in the fields of climate-human interactions, subsistence hunting, tourism, marine transportation and commercial fishing (Ibid.).

\section{Alaska}

North Slope Social Indicator Survey: This project looks at the well-being of North Slope residents. It is important because their well-being may be affected by offshore oil and gas exploration and development. Monitoring well-being will help identify and mitigate impacts. The North Slope Social Indicators Project is designed to build on the work of previous studies by including the best indicators from them and comparing the well-being of residents today with those in 2003, 1988, and even as far back as 1977 . It will also be possible to monitor changes in well-being in the future.

\section{Canada}

In the report Rethinking the Top of the World: Arctic Security Public Opinion Survey different views on living conditions, perceptions and attitudes were analyzed in Northwest Territories, Yukon and Nunavut. The analysis compared responses in northern and southern Canada and showed that residents of the North feel themselves more likely to be healthy in the spite of the dissatisfaction of Northerners with the human and environmental infrastructure in their region. Northerners are significantly more likely to rate their quality of life as excellent than those in the South. Northerners appear to be very optimistic about their future well-being. This resilience and optimism was one of the more surprising study findings (see EKOS Research Associates, 2011).

The Community Well-being (CWB) Index was developed to help measure the quality of life of First Nations and Inuit communities in Canada relative to other communities and over time. CWB Index scores are derived from Canada's Censuses of Population, which are conducted every five years. Scores have been 
calculated for 1981, 1991, 1996, 2001 and 2006. A community's CWB index score is a single number that is composed of data on income, education, housing conditions and labor force activity (INAC, 2010).

The Inuvialuit Baseline Indicators (IBI) project is a collaborative effort among researchers associated with the Resources and Sustainable Development in the Arctic (ReSDA) and the Arctic Social Indicators (ASI) projects with the Inuvialuit Regional Corporation (IRC). The goal is to develop a set of measurable, reliable and accessible indicators to monitor socio-economic conditions in the Inuvialuit Settlement Region (ISR) with an emphasis on tracking impacts of resource development. This effort is focused on creating a framework to be used by local actors to collect, manage and analyse community-based data.

The Institute for Research on Public Policy (IRPP, founded in 1972) is an independent, national, nonprofit organization, which has launched a new research program on the quality of life of Aboriginal people in Canada, like the broad issues related to the quality of life and well-being of Aboriginal people, some of the innovations that are ameliorating their living conditions and the linkages between quality of life and governance in Aboriginal communities. The paper also identifies the areas in which further exploration might be needed, and proposes new directions for policy-relevant research (IRPP, 2014).

\section{Russia}

The strategy of development of the Russian Federation Arctic zone until 2020 states, "there is a low quality of life among Indigenous peoples of the North" (Strategy, 2013).

The emphasis on "quality of life" in the Russian Arctic strategy follows an emerging interest in recent years among Russian researchers studying quality of life in the Russian Arctic. The following references are a few prominent examples

Shats, Shevchenko, Zhideleva and Kaptsein have studied quality of life of populations in the Russian North, focusing on migrant workers' health problems, acclimatization as well as identification of the ways to raise the general quality of life (Zhideleva and Kapstein, 2006; Shevchenko, 2009; Shats, 2010).

Naberejnaya assessed and modelled quality of life with an economic focus based on studies from Sakha-Yakutia republic (Naberezhnaya, 2007).

Whereas the abovementioned authors focused on quality of life of all population groups, other Russian researchers have investigated Indigenous peoples' health and well-being related to a number of impacting factors and indicators of quality of life, particularly among Nenets, Yamalo-Nenets and Sakhalin minorities (Dregalo and Ulyanovskiy, 2007; Belyaeva, 2009; Kholodilova, 2010; Novikova and Stepanov, 2010), but also within the European, Siberian and Far Eastern North (Kozlov et. al, 2012). Others have focused on specific population groups such as for example the elderly people in the Yamalo-Nenets autonomous area and the Arkhangelsk region (Kabanov, 2009; Golubeva, 2012) or children and students in the European North (e.g., Chesnakova and Gribanov, 2012). 


\subsection{Gaps in knowledge}

\subsubsection{Violence in families}

Violence in families is a specific and largely silent health, security and human rights problem. It ranges in a continuum from harassment and psychological abuse to murder, and it happens all over the world (WHO, 2005; UN Women, 2011). In addition to silence, it has been - and still is - deeply associated with shame and normalization (WHO, 2005; EC, 2010). Historically, many of the forms of violence in families were not recognized at all, and those that were, were seldom treated as a serious crime (EC, 2010). Prevalence studies are limited. Some data are available from Arctic Canada and Greenland (see Megatrends, 2011), and useful data comes out of the Alaska Victimization Survey (2014). In many communities, the topic is highly sensitive. There are historical, cultural, social and psychological factors that influence how people conceptualize violence, and its occurrence is often considered an inevitable burden of life, or purely a private secret. Violence is a known risk factor for suicide, mental disorders and substance abuse in Arctic communities (Allen et al., 2011; Gone and Trimble, 2012).

One of the first global reports on violence in interpersonal relationships, specifically violence in families, is the World Report on Violence and Health (WHO, 2002). It argues that the home is one of the most common settings for violence worldwide. While women and children are particularly at risk, the elderly also experiences violence. Typically, victims know the perpetrators and abuse takes place in a familiar location. People who experience family violence are often emotionally involved with and dependent on the person(s) who victimize them. This strengthens the possibility that being unsafe becomes a life context for the victim (WHO, 2002; Johnson et al., 2008; EC, 2010).

The Arctic is no exception. Rates of violence women experience at home are high in Iceland, Greenland (Curtis et al., 2002; Poppel, 2006,), Finland (Piispa et al., 2006) and Russia (UN Women, 2011). Further, the data also reveal very high rates of violence against Indigenous women (Megatrends, 2011; Alaska Victimization Survey, 2014). For example, in the United States, the rate of violence in families against Alaska Native and American Indian women are nearly three times higher than those of white women (Bohn, 2003). Some researchers attribute this to historical trauma induced by colonization, cultural assimilation, and forced attendance at boarding schools, which have been internalized and passed on from generation to generation. The power imbalance between women and men that exists in many cultures is a further contributing factor (Smith, 2005). 


\section{Textbox 8.5}

\section{Sexual harassment}

The culturally maintained status difference between men and women is often gendered, and sexualized, and learned indeed starting from childhood. This was discussed in Vappu Sunnari's research that focused on physical sexual harassment experienced by 11-12 year old children at school (Sunnari, 2010). There were 1,738 children from northern Finnish and northwest Russian school classes who answered a group of questions concerning their experiences on physical sexual harassment at school or on the way to school. The results of the research inform that every fifth of the northern Finnish and every fourth of the northwestern Russian girls experienced physical sexual harassment at school or on the way to school. Every tenth of the Russian boys and every twentieth of the Finnish boys had partly corresponding experiences. A girl was groped in nine cases out of ten by a male classmate in both the Russian and Finnish data. If the harassed child mentioned more than one perpetrator, which was quite common for the Russian children, at least one of the harassers had been a boy. But, the boys' harassers were not commonly girls. Typically Russian victims of physical sexual harassment did not want to tell the details of what had happened. The most common reason they mentioned was shame.

Source: Sunnari, 2010.

The UN Secretary-General's World Report on Violence Against Children demonstrates that violence against children is widespread (Pinheiro, 2006) and it constitutes serious violations of children's rights. The violence against children in families can be even more complex than that experienced by women (EC, 2010). The exact extent and severity of such a complex health and security problem is difficult to estimate. In addition to experiencing violence directly, children often witness violence at home, which is a serious mental health issue in its own right (Curtis et al., 2002).

\subsubsection{Discrimination and racism}

Scientific knowledge regarding Sámi health and living conditions in Norway, Sweden, Finland and Russia is limited compared to the detailed data on the health and socio-economic situations of Indigenous peoples in North America and Greenland. The SAMINOR study in Norway is a rare example of a large-scale population-based survey of health and living conditions in areas with both Sámi and Norwegian populations. Data was collected during 2003-2004 in 24 municipalities, in which at least $5 \%$ of the residents were of Sámi ethnicity. All together 16, 865 
people (Sámi and non-Sámi) participated in the survey, which included questionnaires, a clinical examination and analyses of blood samples (Lund et al., 2007). An important finding from this study was that the prevalence of self-perceived ethnic discrimination and bullying among Sámi was twice as high as that for the majority population. Those who experienced ethnic discrimination were more likely to also report inferior self-perceived health (Hansen, 2010). A second phase of the SAMINOR, initiated in 2013, focuses on gender based violence, ethnicity and health, SAMINOR II will be the first study to investigate these issues in the Sámi population (Eriksen et al., 2012).

While the severity of health disparities between the Indigenous peoples and the rest of the population varies from country to country, it is clear that such health inequalities exist, even in highly developed countries such as the Arctic States. But why? Health disparities, measured for decades, have been attributed to social determinants of health. More recently, it has been proposed that structural racism and its intergenerational impacts may provide new understanding into the causes of health disparities experienced by Indigenous peoples worldwide. Structural racism is defined as the macro-level systems, social forces, institutions, ideologies, and processes that interact with one another to generate and reinforce inequities among racial and ethnic groups (Powell, 2008). By looking at the residential school and boarding school experiences of Indigenous peoples through the lens of racism at the structural level, these experiences from different countries can provide a deeper understanding of their present day impact on Indigenous people's health and well-being.

Within the confines of residential schools and boarding schools, Indigenous children from a very young age were taught that their own culture and heritage was "less than" that of the dominant society. How does this affect Indigenous identity, which is at the very core of health and well-being? And what are the intergenerational impacts of such treatment? Recent studies have documented that individuals who report experiencing racism have greater rates of illnesses (Williams and Mohammed, 2009; Chae et al., 2011). While this type of research is important to give a greater understanding of root causes of health inequities, it still places the experiences of racism at the individual level (Williams and Mohammed, 2009; Gee and Ford, 2011; Paradies and Cunningham, 2012). Gee and Ford suggest that studies of disparities should more seriously consider the multiple dimensions of racism as fundamental causes of health disparities, particularly structural racism. In the Canadian context, residential schools resulted in stigmatization, marginalization, loss of cultural identity, and a health status that falls 
below that of mainstream Canadians (Health Canada, 2002). A review of the literature of colonization in Canada's Far North establishes the position that colonization is a determinant of health (Moffit, 2004). Residential school experiences in Canada paralleled with emerging discussions of boarding school experiences in the Nordic countries can add to the discourse about racism at the structural level and its link to the present health inequalities experienced by Indigenous peoples (see Textbox 8.6).

\section{Textbox 8.6}

\section{Residential school and boarding school systems}

Residential school and boarding school experiences were studied in Canada (Six Nations of the Grand River) and Finland (Inari municipality). Results from Canada and Finland found that negative impacts to Indigenous identity included language and cultural loss, fractured identity, and negative self-worth, all of which have far reaching impacts to health and well-being. Despite the negative impacts that require on-going healing, it was also emphasized that there was power at the personal level to stop generational abuses and the resilience of Indigenous peoples to keep their languages and culture alive and retain a strong Indigenous identity (Juutilainen et al., 2014). There are very few studies in the Nordic countries about boarding school attendance and its impact on health and well-being of Sámi. Further studies about Indigenous identity, resilience and impact of racism at the structural level may provide valuable insight into health disparities experienced by Indigenous peoples worldwide.

Source: Juutilainen et al., 2014.

\subsubsection{Well-being indicators}

Major knowledge gaps remain, especially concerning the "nonconventional" (AHDR/ASI) indicators measuring closeness to nature, cultural well-being and fate control. The challenge is not merely gathering data on these dimensions, but also developing concepts, agreeing on definitions and standardized measures, and ensuring that data are stored in ways that both ensures confidentiality and accessibility. To minimize the response burden, available administrative registers (i.e., regional and national wildlife harvest and health registers) will have to be used wherever possible. Circumpolar collaborative initiatives on data gathering and data management like Arctic Observing Network (AON), Arctic Observing Network - Social Indicator Project (AON-SIP) and the Sustaining Arctic Observing Network (SAON) will help to facilitate future assessments on Arctic human development. If the ultimate goal of examining 
different aspects of living conditions and developing social indicators is to assess and compare subjective well-being and quality of life over time and between regions and population groups, it will be necessary to conduct representative surveys regularly (e.g., every five or ten years) to capture different aspects of human development.

The main gap in knowledge includes the health and well-being of both Indigenous and non-Indigenous peoples in the Russian Arctic (especially those living in cities); the health and well-being of Sámi in the Nordic countries, and accidents and domestic violence across the Circumpolar North. These gaps in knowledge can only be filled through a joint and orchestrated effort in creating and maintaining national and regional health statistical databases, which require collaboration between health authorities, the research community, and Indigenous peoples' organizations. It is important that vital statistics and disease registry data be supplemented by regular health and social surveys that capture the diverse aspects of human development, subjective well-being and quality of life.

\subsection{Key conclusions}

The people in the Circumpolar North do not all enjoy the same health. There are substantial disparities among countries and regions, and within regions among population subgroups, particularly between Indigenous and non-Indigenous peoples. Efforts are needed to integrate older people in Arctic societal life, and to appreciate their productive participation. As climate and environmental change affect living conditions, these changes also impact health, subjective well-being, and quality of life.

There is a need for health promotion programs, disease prevention strategies and redressing social inequalities. Indicators of food and water security should be used together with surveillance and monitoring programs in all circumpolar countries. Health care reform, education and research are also major challenges in the future.

Generally, the change in human development measured by indicators included in the UN Human Development Index (health, income and education) has been positive since the beginning of the 21st century. At the same time, there is a lot of variation between rural-urban regions. The indicators originating from the specific AHDR/ASI dimensions "closeness to nature", "cultural well-being" and "fate control" create a more complete picture. "Language retention" (as a measure of cultural well-being), for instance, is increasing (or at a stable high level) in some 
regions like Greenland and some of the Canadian Arctic regions, whereas a decline has been experinced in the Inuit region of Alaska. Furthermore, comparisons are often complicated because of lack of data or because data can be difficult to interpret.

People's self-evaluation of well-being and quality of life has been the focus of two surveys and the findings in both studies - one comparing Canadian Arctic residents with Canadians living in the south and the other one focusing on Inuit, Sámi and Indigenous peoples of Chukotka and the Kola Peninsula - are intriguing and puzzling, as "satisfaction with life" (and thus subjective well-being) is rated high among the residents of the Arctic despite high rates of unemployment and low household income. The Indigenous peoples of Chukotka and the Kola Peninsula is an exception to this overall finding.

\subsection{References}

ACIA (2005). Arctic Climate Impact Assessment. Cambridge University Press, Cambridge. AHDR (2004). Arctic Human Development Report. Einarsson, N., J. N. Larsen, A. Nilsson and O. R. Young (eds.). Stefansson Arctic Institute, Akureyri.

Alaska Department of Fish and Game (2014). Subsistence in Alaska. http://www.adfg.alaska.gov/index.cfm?adfg=subsistence.main (30th August 2014).

Alaska Victimization Survey (2014). Research on Violence Against Women in Alaska http://justice.uaa.alaska.edu/avs/. (30th August 2014).

Allen, J., M. Levintova and G. Mohatt (2011). Suicide and alcohol-related disorders in the U.S. Arctic: boosting research to address a primary determinant of health disparities. International Journal of Circumpolar Health, 70(5): 473-487. http://dx.doi.org/10.3402/ijch.v70i5.17847

AMAP (2011). Snow, Water, Ice and Permafrost in the Arctic (SWIPA): Climate Change and the Cryosphere. Arctic Monitoring and Assessment Program, Oslo.

AMAP (2009). Assessment 2009: Human Health in the Arctic. Arctic Monitoring and Assessment Program, Oslo.

AMAP (2003). Assessment 2002: Human Health in the Arctic. Arctic Monitoring and Assessment Program, Oslo.

AMAP (1998). Assessment Report: Arctic Pollution Issues. Arctic Monitoring and Assessment Program, Oslo.

Andersen, T. and B. Poppel (2002). Living conditions in the Arctic. In: Hagerty, M.R., J. Vogel, and V. Møller (eds), 2002. Assessing Quality of Life and Living Conditions to Guide National Policy. The State of the Art, pp. 193-216. Social Indicators Research Series, Vol. 11. Kluwer Academic Publishers, Dordrecht.

ArcRisk (2013). Arctic Health Risks: Impacts on Health in the Arctic and Europe Owing to Climate-Induced Changes in Contaminant Cycling. http://www.arcrisk.eu/ (30 August 2014).

ASI (2014). Arctic Social Indicators II: Implementation. Larsen, J.N., P. Schweitzer, and A. Petrov (eds.). TemaNord. Nordic Council of Ministers, Copenhagen. http://dx.doi.org/10.6027/TN2014-568 
ASI (2010). Arctic Social Indicators - a follow-up to the Arctic Human Development Report. Larsen, J.N., P. Schweitzer, and G. Fondahl (eds.). TemaNord. Nordic Council of Ministers, Copenhagen.

ArcticStat. nd. ArcticState Socioeconomic Circumpolar Data Base. Université Laval . http://www.arcticstat.org (30 August 2014).

Belyaeva, L.A. (2009). Uroven' i kachestvo zhizni: problem ismereniya i interpretatsii [Standards and quality of life: measurement problems and interpretations]. Sotsiologicheskie issledovaniya, 1:33-42.

Bjerregaard, P., G. Mulvad (2012). The best of two worlds: how the Greenland Board of Nutrition has handled conflicting evidence about diet and health. International Journal of Circumpolar Health. http://www.ncbi.nlm.nih.gov/pmc/ articles/PMC3417583/ (30 August 2014). http://dx.doi.org/10.3402/ ijch.v71i0.18588

Bohn, D. (2003). Lifetime physical and sexual abuse, substance abuse, and suicide attempts among Native American women. Issues in Mental Health Nursing, 24(3): pp. 333-352. http://dx.doi.org/10.1080/01612840305277

Brubaker, M., J. Berner and J. Warren (2011). Climate change and health effects in Northwest Alaska. Global Health Action, 4. http://www.globalhealthaction.net/ index.php/gha/article/view/8445 (24th June 2014). http://dx.doi.org/10.3402/gha.v4i0.8445

Brustad, M., K. L. Hansen, A. R. Broderstad,S. Hansen and M. Melhus (2014). A population-based study on health and living conditions in areas with mixed Sami and Norwegian settlements the SAMINOR 2 questionnaire study. International Journal of Circumpolar Health, 2014, 73: 23147. http://dx.doi.org/10.3402/ijch.v73.23147

Centre for Bhutan Studies and Gross National Happiness Research (CBS) (2014). Gross National Happiness. Development With Values.

http://www.grossnationalhappiness.com/ (13th November 2014).

Chae, D.H., A.M. Nuru-Jeter, K.D. Lincoln and D.D. Francis (2011). Conceptualizing racial inequalities in health. Du Bois Review, 8(1): pp. 63-77. http://dx.doi.org/10.1017/S1742058X11000166

Chesnokova, V.N. and Gribanov, A.V. (2012). Izmenenie genodinamiki u studentov v usloviyakh severnogo regiona $v$ techenie uchebnogo goda [Changes in hemodynamics of adolescents under the conditions of life in a northern region during the academic year]. Sovremennye problem nauk i obrazovanie1. http://www.scienceeducation.ru/pdf/2012/1/6.pdf (6th September 2014).

Circumpolar Health Observatory (CircHOB) (2012).

http://circhob.circumpolarhealth.org/ (30th August 2014).

Circumpolar Health Research Network (2013). http://circhnet.org/ (30th August 2014).

Circumpolar Inuit Cancer Review Working Group (2008). Cancer among the circumpolar Inuit, 1989-2003. II. Patterns and trends. International Journal of Circumpolar Health, 67: pp. 408-420.

Commission on the Measurement of Economic Performance and Social Progress (CMEPSP), nd. Commission on the Measurement of Economic Performance and Social Progress website. http://www.stiglitz-sen-fitoussi.fr/en/index.htm (30th August 2014).

Curtis, T., F.B. Larsen, K. Helweg-Larsen and P. Bjerregaard (2002). Violence, sexual abuse and health in Greenland. International Journal of Circumpolar Health, 6: pp. 110-122. 
Davydov, A.N. and G.V. Mikhailova (2011). Climate change and consequences in the Arctic: perception of climate change by the Nenets people of Vaigach Island. Global Health Action, 4: 8436. http://dx.doi.org/10.3402/gha.v4i0.8436

Dregalo, A. A and V.I. Ulyanovskiy, V. (2007). Usloviya i kachestvo zhizni evropeyskikh nentsev $v$ monoetnicheskom poselke [Conditions and quality of life of the European Nenets in a mono-ethnic village]. Vestnik Severnogo (Arkticheskogo) federal'nogo universiteta. Seriya: Gumanitarnye i sotsial'nye nauki, 2(12): pp. 54-58.

Dudarev, A.A. (2012). Dietary exposure to persistent organic pollutants and metals among Inuit and Chukchi in Russian Arctic Chukotka. International Journal of Circumpolar Health, 71: 18592. http://dx.doi.org/10.3402/ijch.v71i0.18592

Dudarev, A.A., P.R. Alloyarov, V.S. Shupakhin, E.V. Dushkina, Y.N. Sladkova, V.M. Dorofeyev, T.A. Kolesnikova, K.B. Fridman, L.M. Nilsson, and B. Evengård (2013). Food and water security issues in Russia I: food security in the general population of the Russian Arctic, Siberia and the Far East, 2000-11. International Journal of Circumpolar Health, 72.:21848. http://dx.doi.org/10.3402/ijch.v72i0.21848. eCollection 2013.

Duhaime, G. (2004). Economic systems. In: AHDR, 2004. Arctic Human Development Report. Einarsson, N., J. N. Larsen, A. Nilsson and O. R. Young (eds.), pp. 69-84. Stefansson Arctic Institute, Akureyri.

Eliassen, B.M., M. Melhus, J. Kruse, B. Poppel and A.R. Broderstad (2012). Design and methods in a survey of living conditions in the Arctic - the SLiCA study. International Journal of Circumpolar Health, 71: 17229. http://dx.doi.org/10.3402/IJCH.v71i0.17229

Emelyanova, A. and A. Rautio (2013). Perspectives for population aging in the Russian North. Journal of Population Aging, 6(3): 161-187. http://dx.doi.org/10.1007/s12062-013-9086-z

Emelyanova, A. and A. Rautio (2012). Aging population in the Barents Euro-Arctic Region. Journal of European Geriatric Medicine, 3(3): 167-173. http://dx.doi.org/10.1016/j.eurger.2012.03.005

EKOS Research Associates (2011). Rethinking the Top of the World: Arctic Security Public Opinion Survey. Final Report. Submitted to: The Walter and Duncan Gordon Foundation and The Canada Centre for Global Security Studies at the Munk School of Global Affairs. Ottawa and Toronto. http://www.ekospolitics.com/articles/201101-25ArcticSecurityReport.pdf

Eriksen, A, K.L. Hansen, C. Jáve, and B. Schei (2012). Poster presentation at ICCH 15: SAMINOR 2, Gender Based Violence, Ethnicity and Health. A Subproject of SAMINOR II, Fairbanks, Alaska.

Eurofound (2012) European Quality of Life Surveys, http://www.eurofound.europa.eu/ surveys/eqls/ (30th August 2014).

EC (2010). European Commission, Feasibility study to assess the possibilities, opportunities and needs to standardize national legislation on violence against women, violence against children and sexual orientation violence. Publications Office of the European Union, Luxemburg.

EKOS Research Associates (2011). Rethinking the Top of the World: Arctic Security Public Opinion Survey. Final Report. Submitted to: The Walter and Duncan Gordon Foundation And The Canada Centre for Global Security Studies at the Munk School of Global Affairs. Ottawa and Toronto. http://www.ekospolitics.com/articles/201101-25ArcticSecurityReport.pdf (13th November 2014).

Fedotov, D.M., L.A. Zubov, and A.A. Nazarov (2011). Vozdeystvie izmeneniy klimata na traditionnyy uklad zhizni korennogo naseleniya i usloviya okazaniya meditsinskoy pomoshchi v Nenetskom Avtonomnom okruge [The impact of climate change on the 
traditional way of life of the Indigenous population and the conditions of the provision of medical care in Nenets autonomous okrug]. Proceeding of All-Russian Scienceand-Practice Conference with international participation "Circumpolar Medicine: Impact of Environmental Factors on Human Health Formation", 27th-29th June 2011, pp. 294-297. Arkhangelsk, Russian Federation.

INAC (Indian and Northern Affairs Canada) (2010). First Nation and Inuit community well-being: describing historical trends (1981-2006) Ottawa: INAC Strategic Research and Analysis Directorate. http://www.aadnc-aandc.gc.ca/DAM/DAM-INTERHQ/STAGING/texte-text/cwbdck_1100100016601_eng.pdf (13th November 2013).

Gee, G. and C. Ford (2011). Structural racism and health inequalities. Du Bois Review, 8(1): pp. 115-132. http://dx.doi.org/10.1017/S1742058X11000130

Goldsmith, S. (2009). Notes on gross domestic product and value added comparisons across Arctic regions. In: Glomsråd, S. and I. Aslaksen (eds.) The Economy of the North 2008, pp. 34-35. Statistisk Sentralbyrå/Statistics Norway, Oslo-Kongsvinger.

Golubeva, E.Y. (2012). Kachestvo zhizni naseleniya pozhilogo i starcheskogo vozrasta v Rossiyskoy chasti Barents-Evroarkticheskogo rayona (na primere Arkhagel'skoy oblasti): puti sovershenstvovaniya organizatsii medico-sotsial'nogo obsluzhivniya) [Quality of life among elderly and very aged people in the Russian part of the Barents Euro-Arctic region (a case of the Arkhangelsk oblast: ways of improvements in health- and social care organization)]. Dissertation abstract for the degree of Doctor of biological sciences, St. Petersburg Institute of Bioregulation and Gerontology, Russian Federation.

Gone, J.P. and J.E. Trimble (2012). American Indian and Alaska Native mental health: diverse perspectives on enduring disparities. Annual Review of Clinical Psychology, 8: pp. 131-60. http://dx.doi.org/10.1146/annurev-clinpsy-032511-143127

Government of Greenland (2010). Hope and Resilience: Suicide in the Arctic, November 7-8, 2009. Conference Report. Nuuk. http://www.peqqik.gl/ /media/Files/ Publikationer/Rapporter_og_redegoerelser/2010/ ConferenceReport_HopeResilience_2010.ashx (11th November 2104).

Gross National Happiness Index (GNHI) (2014).

http://www.grossnationalhappiness.com/ (30th August 2014).

Hansen, K.L. (2010). Ethnic Discrimination and Bullying in Relation to Self-Reported Physical and Mental Health in Sami Settlement Areas in Norway. The Saminor study. Unpublished PhD Thesis, University of Tromso, Troms $\varnothing$.

Happy Planet Index (HPI) (2014). http://www.happyplanetindex.org (30th August 2014).

Health Canada (2002). Healthy Canadians: A federal report on comparable health indicators. www.hc-sc.gc.ca/iacb-dgiac/arad-draa/English/accountability/ printindicators.html (21st August 2013).

Hovelsrud, G. K., B. Poppel, B. van Oort, and J. D. Reist (2011). Arctic societies, cultures, and peoples in a changing cryosphere. AMBIO, 40(1): pp. 100-110. http://dx.doi.org/10.1007/s13280-011-0219-4

Hueffer, K., A.J. Parkinson, R. Gerlach and J. Berner (2013). Zoonotic infections in Alaska: disease prevalence, potential impact of climate change and recommended actions for earlier disease detection, research, prevention and control. International Journal of Circumpolar Health, 72. http://dx.doi.org/10.3402/ijch.v72i0.19562

Huet, C., R. Rosol and G.M. Egeland (2012). The prevalence of food insecurity is high and the diet quality poor in Inuit communities. Journal of Nutrition, 142(3): pp. 541-547. http://dx.doi.org/10.3945/jn.111.149278 
International Journal of Circumpolar Health.

http://www.circumpolarhealthjournal.net/ (30th August 2014).

INAC (Indian and Northern Affairs Canada) (2010). First Nation and Inuit community well-being: describing historical trends (1981-2006). INAC Strategic Research and Analysis Directorate, Ottawa. http://www.aadnc-aandc.gc.ca/DAM/DAM-INTERHQ/STAGING/texte-text/cwbdck_1100100016601_eng.pdf (13th November 2013). IRPP (2014). The Institute for Research on Public Policy research publications. http://www.irpp.org/en/research/ (30th August 2014).

Jeppesen, C., P. Bjerregaard and K. Young (2011). Food-based dietary guidelines in circumpolar regions. International Journal of Circumpolar Health, Supplement 8.

Johnson, H., N. Ollus and S. Nevala (2008). Violence against Women. An International Perspective. Springer, New York.

Juutilainen, S., R. Miller, L. Heikkilä and A. Rautio (2014). Structural racism and Indigenous health: what Indigenous perspectives of boarding school and residential school tell us. A case study of Finland and Canada. The International Indigenous Policy Journal 5(3). http://ir.lib.uwo.ca/iipj/vol5/iss3/3

Kabanov, A.O. (2009). Upravlenie kachestvom zhizni naseleniya pensionnogo vozrasta $v$ severnom regione [Managing the quality of life of the retired population in the northern region]. Dissertation for the degree of candidate of sociological sciences. Tyumen, Russian Federation.

Kholodilova, K.A. (2010). Kachestva zhizni naselenie neftogazopromyslovogo regiona na Kraynem Severe [The Quality of Life of the Population in an Oil- and Gas-Production Region of the Far North]. Unpublished PhD Thesis. Tyumen, Russian Federation.

Kozlov, A.I., M.A. Kozlova, G.G. Vershybskaya, and A.B. Shilov (2012). Zdorov'e korennogo naseleniya Severa RF: na grani vekov $i$ kul'tur [The health of the Indigenous population of the North of the Russian Federation: at the edge of centuries and cultures]. Perm, Russian Federation.

Kruse, J., B. Poppel, L. Abryutina, G. Duhaime, S. Martin, M. Poppel, M. Kruse, E. Ward, P. Cochran and V. Hanna, 2008. Survey of Living Conditions in the Arctic, SLiCA. In: Møller, V., D. Huschka and A.C. Michalos (eds.), Barometers of Quality of Life Around the Globe, pp. 107-134. Springer Social Indicators Research Series. Springer Verlag, Berlin.

Kruse, J., M. Lowe, S. Haley, G. Fay, L. Hamilton and M. Berman (2011). Arctic observing network social indicators project: overview. Polar Geography, 34(1-2): pp. 1-8. http://dx.doi.org/10.1080/1088937X.2011.584446

Larsen, C.V.L., C.P. Pedersen, S.W. Berthelsen and C. Chew (2010). Hope and Resilience: Suicide Prevention in the Arctic, Conference Report. Arctic Council, Sustainable Development Working Group (SDWG). http://library.arcticportal.org/1480/1/ Hope_and_resilience.pdf (24th June 2013).

Lewis, J. (2013). The future of successful aging in Alaska. International Journal of Circumpolar Health, 72: 21186. http://dx.doi.org/10.3402/ijch.v72i0.21186

Lund, E., M. Melhus, K.L. Hansen, T. Nystad, A.R. Broderstad, R. Selmer and P.G. LundLarsen (2007). Population based study of health and living conditions in areas with both Sámi and Norwegian populations. The Saminor Study. International Journal of Circumpolar Health, 66(2): pp. 113-128.

http://dx.doi.org/10.3402/ijch.v66i2.18241

Mäenpää, I. (2009). Comparative analysis of Arctic economies at macro level In: Glomsråd, S. and I. Aslaksen (eds.) The Economy of the North 2008, pp. 27-36. Statistisk Sentralbyrå/Statistics Norway, Oslo-Kongsvinger.

Megatrends (2011). Rasmussen, R.O. (ed). Nordic Council of Ministers, Copenhagen. 
Moffit, P. (2004). Colonialization: A health determinant for pregnant Dogrib women. Journal of Transcultural Nursing, 15: 323-330. http://dx.doi.org/10.1177/ 1043659604268959

Naberezhnaya, A.T. (2007). Modelirovanie i otsenka urovnya zhizhni naseleniya regiona. [Modelling and Appraisal of Standards of Living among the Population of the Region]. YaNTs So RAN,Yakutsk.

National Aboriginal Economic Development Board (NAEDB) (2012). Aboriginal Economic Benchmark Report. National Aboriginal Economic Development Board, NAEDB. http://www.naedb-cndea.com/wp-content/uploads/ TheAboriginalEconomicBenchmarkingReport2.pdf

Nilsson L.M., G. Destonuni, J. Berner, A. Dudarev, G. Mulvad, J.O. Odland, A. Rautio, C. Tikhonov and B. Evengård (2013a). A call for urgent monitoring of food and water security based on relevant indicators for the Arctic. AMBIO, 42: pp. 816-22. http://dx.doi.org/10.1007/s13280-013-0427-1

Nilsson L.M., J. Berner, A. Dudarev, G. Mulvad, J.O. Odland, A. Rautio, C. Tikhonov and B. Evengård, (2013b). Selecting indicators of food and water security in the Arctic health context - results from an international workshop discussion. International Journal of Circumpolar Health, 72: 21530. http://dx.doi.org/10.3402/ijch.v72i0.21530

Nilsson, L.M. and B. Evengård (2013). Food and water security indicators in an Arctic health context. A report by the AHHEG/SDWG, and the AMAP/HHAG during the Swedish chairmanship of the Arctic Council 2011-2013. Arctic Research Centre (Arcum), Umeå. http://umu.diva-portal.org/ (15th August 2013).

Nordic population ageing - challenge and opportunity (2013). Nordregio News Issue 3, Oslo, Norway. http://www.nordregio.se/en/Metameny/Nordregio-News/2013/ Nordic-Population-Ageing--Challenge-and-Opportunity/ (21st October 2013).

Nost, T.H., K. Breivik, O.M. Fuskevåg, E. Nieboer, J.O. Odland and T.M. Sandanger (2013). Persistent organic pollutants in Norwegian men from 1979 to 2007: Intraindividual changes, age-period-cohort effects, and model predictions. Environmental Health Perspective, 121(11-12): 1292-8.

Novikova, N.I. and V.V. Stepanov (2010). Indikatory kachestva zhizni korennykh malochislennykh narodov Severa Sakhalinskoy oblasti [Indicators of the quality of life of Indigenous peoples of the north of the Sakhalinsk oblast]. Issledovaniia po prikladnoi i neotlozhnoi etnologi, 217:1-46.

Nunavut Suicide Prevention Strategy Action Plan, 2011. http://www.tunngavik.com/ files/2011/09/nsps-action-plan-eng4.pdf/

Nuttall, M., F. Berkes, B. Forbes, G. Kofinas, T. Vlassova, and G. Wenzel (2005). Hunting, Herding, Fishing and Gathering: Indigenous peoples and renewable resource use in the Arctic. In: ACIA, 2005. Arctic Climate Impact Assessment. Cambridge University Press, Cambridge.

OECD, n.d. "Better Life Index". http://www.oecdbetterlifeindex.org/ (30th August 2014).

O'Donnell, G., A. Deaton, M. Durand, D. Halpern and R. Layard (2014). Wellbeing and Policy. Legatum Institute. http://www.li.com/docs/default-source/commission-onwellbeing-and-policy/commission-on-wellbeing-and-policy-report---march-2014pdf.pdf?sfvrsn=2 (13th November 2014).

Paradies, Y. and J. Cunningham (2012). The Druid study: racism and self-assessed health status in and Indigenous population. BMC Public Health, 12: 131.

http://dx.doi.org/10.1186/1471-2458-12-131 
Penney, C., E. O'Sullivan, and S. Senécal (2012). The Community Well-Being Index (CWB): Examining Well-Being in Inuit Communities, 1981-2006. Unpublished report. Aboriginal Affairs and Northern Development Canada.

Piispa, M., M. Heiskanen, J. Kääriäinen and R. Sirèn (2006). Violence against women in Finland. (Summary). United Nations European Institute for Crime Prevention and Control Publication Series 51 and National Research Institute of Legal Policy Publication 225, Helsinki, Finland.

Pinheiro, P. (2006). The World Report on Violence Against Children. United Nations, Geneva, Switzerland.

Poppel, B., forthcoming. Living Conditions, Subjective Well-being, Quality of Life among Indigenous Peoples in the Arctic. In: Glatzer, W. (ed.): Global Handbook of Subjective Well-being and Quality of Life. Springer, Dordrecht.

Poppel, B. (2014). SLiCA, Survey of Living Conditions in the Arctic. In: Michalos, A. C. (Ed.). Encyclopedia of Quality of Life and Well-Being Research, pp. 5993-6003. Springer, Dordrecht.

Poppel, B. (ed.) (2011). Arctic living conditions. Survey of Living Conditions in the Arctic. Major SLiCA findings presented to the Arctic Council Ministerial meeting. 6 pages. Nuuk.

Poppel, B. (2010). Some data sources on people, peoples, communities, regions and human activities in Greenland. In: Winther, G. (ed.). The Political Economy of Northern Regional Development - Yearbook 2008, pp. 254-282. TemaNord, Nordic Council of Ministers.

Poppel, B. and J. Kruse (2009). The importance of a mixed cash- and harvest herding based economy to living in the Arctic - an analysis based on Survey of Living Conditions in the Arctic (SLiCA) In: Møller, V. and D. Huscka (eds.): Quality of Life and the Millennium Challenge: Advances in Quality-of-Life Studies, Theory and Research, pp. 27-43. Social Indicators Research Series. Springer Science+Business Media B.V., Dordrecht.

Poppel, B., J. Kruse, G. Duhaime and L. Abryutina (2007). Survey of Living Conditions in the Arctic: Results. Institute of Social and Economic Research, University of Alaska Anchorage Anchorage. http://www.arcticlivingconditions.org (11th September 2013).

Poppel, M. (2006). Mænds vold mod kvinder i Grønland i nordisk og arktisk perspektiv. [Men's violence against women in Greenland and in the Nordic and Arctic perspective] In: H. Petersen (ed.). Grønland i Verdenssamfundet. Forlaget Atuagkat, pp. 83-93. Nuuk.

Powell, J. (2008). Structural racism: Building upon the insights of John Calmore. North Carolina Law Review, 86: 791-816.

Revich, B., B. Tokarevich, and A.J. Parkinson (2012). Climate change and zoonotic infections in the Russian Arctic. International Journal of Circumpolar Health, 71: 18792. http://dx.doi.org/10.3402/ijch.v71i0.18792

Sanderson, W. and S. Scherbov (2008). Rethinking age and aging. Population Bulletin, 63(4): pp. 1-16.

Shats, M.M. (2010). Esli zhit' na Severe neobkhodimo... [If it is necessary to live in the North.... Ekologiya i zhizn' 9: 82-85.

Shevchenko, S.I. (2009). Kachestvo zhizni naseleniya regionov Severa Rossii: sostoyanie i puti povysheniya [The quality of life of the population in the Russian North: current situation and the ways for improving it]. Dissertation for the degree of a candidate of economical sciences. Moscow, All-Russian Centre of Standard of Living, Russian Federation.

Smith, A. (2005). Conquest: Sexual violence and American Indian genocide. South End Press, Boston, United States. 
Statistics Canada, n.d. Aboriginal Languages. http://www.statcan.gc.ca/pub/89-645x/2010001/languages-langues-eng.htm. (30th August 2013).

Strategy (2013). Stratgiya razvitiya Arkticheskoy zony Rossiyskoy Federatsii i obespecheniya natsional'noy bezopastnosti na period do 2020 goda [Strategy for the development of the Arctic zone of the Russian Federation and ensuring its national security zone to 2020]. Approved by the President V.V. Putin 08.02.2013, Order No. 232.

Sunnari, V. (2010). "I cannot speak about it". Physical Sexual Harassment as Experienced by Children at School in Northern Finland and Northwest Russia. VDM Verlag Dr. Müller, Saarbrücken.

UN HDI. Human Development Index. United Nations Development Program. http://hdr.undp.org/en/data/ (30th August 2014).

UN Women (2011). Violence Against Women. Prevalence Data: Surveys by Country. Compiled by UN Women. http://www.unifem.org/attachments/gender_issues/ violence_against_women/vaw-prevalence-matrix-2011.pdf (5th May 2013).

Wallace, S. (2014). Inuit health: Selected findings from the 2012 Aboriginal Peoples Survey. Cat. No. 89-653-X, Statistics Canada, Ottawa.

WHO (2002). World Report on Violence and Health. World Health Organization (WHO). http://www.who.int/violence_injury_prevention/violence/world_report/en/ (25th May 2013).

WHO (2005). Multi-Country Study on Women's Health and Domestic Violence against Women. World Health Organization (WHO). http://who.int/gender/violence/whomulticountry-study/summary-report/en/index.htm/ (15th June 2013).

Williams, D. and S. Mohammed (2009). Discrimination and racial disparities in health: evidence and needed research. Journal of Behavioral Medicine, 32(1): pp 20-41. http://dx.doi.org/10.1007/s10865-008-9185-0

World Population Aging 1950-2050 (2007). Population Division, Department of Economic and Social Affairs, United Nations, http://www.un.org/esa/population/ publications/worldaging19502050/pdf/95annexi.pdf (06 April 2013).

Young, K. (ed.) (2012). Circumpolar Health Atlas. University of Toronto Press, Toronto.

Young, T.K. and P. Bjerregaard (2008). Health Transitions in Arctic Populations. University of Toronto Press, Toronto.

Young, T.K., S. Chatwood and R. Rawat (2010). Data brief from the Circumpolar Health Observatory: introduction and population. International Journal of Circumpolar Health, 69: 404-408.

Zhideleva, V.V. and Y.N. Kapstein (2006). Ob issledovanii problem kachestva zhizni cheloveka na Severe) [On a study of human quality of life challenges in the Russian North]. Modern Science Intensive Technologies, 3: pp. 90-92.

ZSI (Social Indicators Research Center). European System of Social Indicators, http://www.gesis.org/en/social-indicators/home/ (13th November 2014). 


\title{
Endnotes for chapter 8
}

\begin{abstract}
i) During the decade under consideration by this report, the Koryak Autonomous okrug was merged with the Kamchatka oblast to form the Kamchatka Territory (kray): the Koryak okrug now forms an administrative division of the Kamchatka territory. The Evenki Autonomous okrug and Taymyr Autonomous okrug were merged into the Krasnoyarsk Territory, as administrative districts (rayony).

ii) In an e-mail of 17th November 2014 from Aboriginal Affairs and Northern Development Canada, AANDC it is stated that following a verification of their historic CWB data, the federal government department decided that two communities included in the 1981 calculations should have been excluded due to missing data. One of these was an Inuit community, in the region of Nunavik. The corrections have not been completed, but based on existing information, the CWB averages for 1981, particularly the housing component, and the Nunavik regional scores, will probably be a little lower than the published averages. Furthermore AANDC informs that a planned release of updated 2011 data is meant to include corrections.

iii) See endnote 2 .
\end{abstract}




\section{Education and Human Capital}

\section{Lead authors}

Diane Hirshberg, University of Alaska Anchorage, USA and Andrey N. Petrov, University of Northern Iowa, USA

\section{Contributing Authors}

Ray Barnhardt (University of Alaska Fairbanks, USA), Philip Cavin (University of Northern Iowa, USA), Shari Gearheard (University of Colorado, USA), Andrew Hodgkins (University of Alberta, Canada), Jan Henry Keskitalo (Sámi University College, Norway), Gisèle Maheux (Université du Québec en Abitibi-Témiscamingue, Canada), Heather E. McGregor (University of British Columbia, Canada), Paartoq Karl Kristian Olsen (Ilisimatusarfik, Greenland), Véronique Paul (Université du Québec en Abitibi-Témiscamingue), Rasmus Ole Rasmussen (Nordregio, Sweden), Arja Rautio (University of Oulu, Finland), Anna Rönkä (University of Oulu, Finland), and Elisapi Uitangak (Ikaarvik School, Puvirnituq, Canada)

\subsection{Introduction}

Since the publication of the first Arctic Human Development Report (2004), there have been significant changes in formal education systems throughout the Circumpolar North. In some countries, for example, Russia, the federal government has introduced major reforms while in others, for example, USA/Alaska, local efforts are changing the look of schooling. In this chapter, we look at the major trends in education across the North. The first part of the chapter focuses on the unique challenges facing primary and secondary education systems in the Arctic. It updates the state of $\mathrm{K}-12$ education since the first $A H D R$, and expands into countries and regions that were not addressed in the first report, specifically Greenland, the far north in Canada, and the Faroe Islands. The second part of the chapter assesses human capital and knowledge production as it pertains to human development and wellbeing of Arctic societies. Human capital is the stock of knowledge and skills embodied in a human population. This section discusses patterns and trends in postsecondary educational attainment and attendance in 
Arctic regions and related formal and informal education. It provides an assessment of human capital and knowledge production in the Arctic as it pertains to human development and well-being of Arctic societies.

In most formal school systems, education is typically classified into three major levels: primary, secondary and post-secondary. Primary, or elementary, education follows pre-school and generally includes students from around age 6 to 12, or in grades 1-6. Secondary education (also known as upper secondary or high school) generally refers to schooling for students age 13 to 18 , and culminates with a diploma. Postsecondary education (also called higher education or tertiary education) is a third stage education that includes undergraduate and postgraduate education, and vocational and professional training. Post-secondary academic education is distinct from post-secondary career and technical education. Adult education refers to a formal and informal learning that takes place after leaving initial education and training, and that most often is not linked to pursuit of a degree or certificate. Adult education programs are offered in a broad array of settings, including folk schools, community organizations and institutions of higher education.

\section{University of the Arctic class}

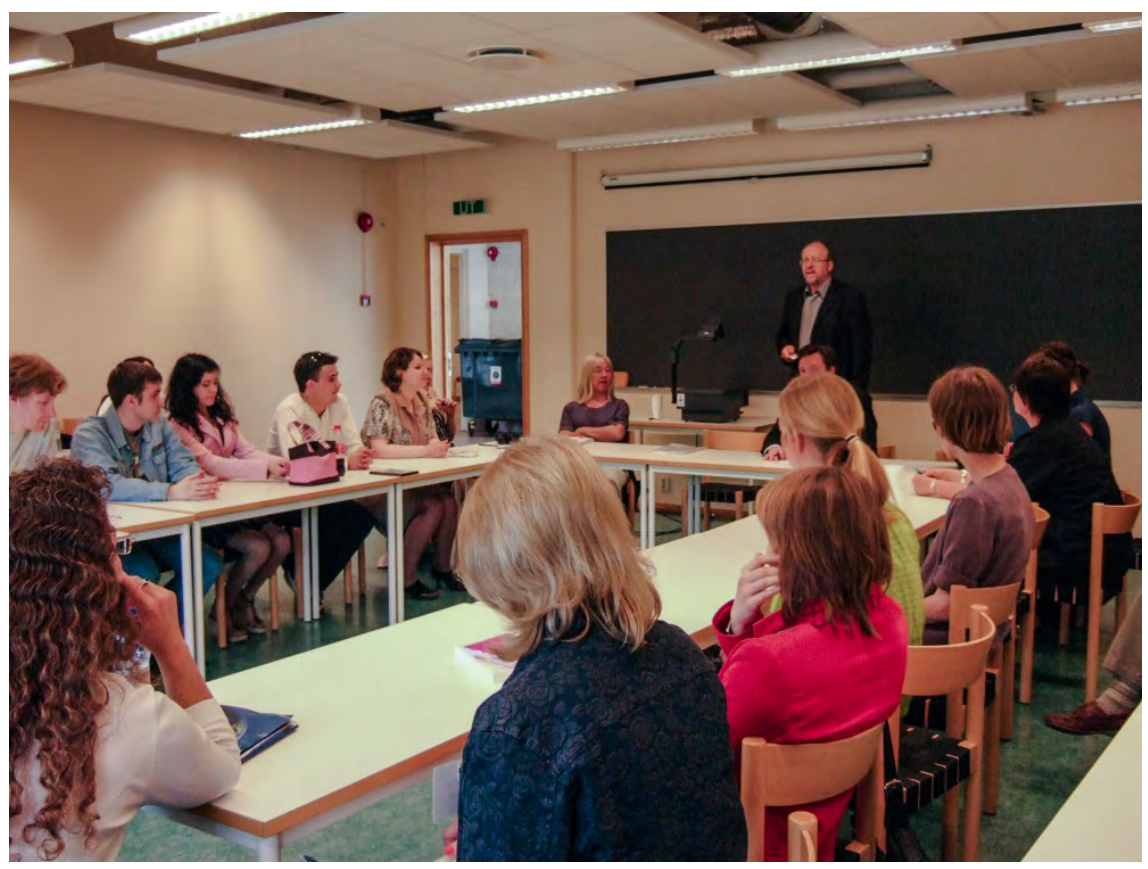

Credit: Amanda Graham. 


\subsection{Education and educational systems in the Arctic}

\subsubsection{Primary and secondary education in the Circumpolar North}

In this section, we discuss the challenges inherent in circumpolar education due to the remote nature of many northern communities, mentioned but not explicated in the first AHDR. These challenges include consolidation and closing of small, remote schools, providing sufficiently comprehensive education opportunities to keep students in small schools, and the recruiting and retaining of teachers for remote communities. We address issues of language of instruction in schools in terms of efforts to retain heritage languages and to ensure that students acquire the national languages of commerce and higher education. We also address Indigenous education, focusing on efforts to increase Indigenous control over education and new ways to use and transmit that Indigenous knowledge and ways of teaching and learning, whether within or external to the formal school systems. And finally we look at student achievement issues, focusing especially on the gender gap between females and males and on the continuing underperformance of Indigenous students across much of the North.

We present data on student learning outcomes. In the first $A H D R$, the authors expressed a desire for education indicators allowing comparisons of education functions and outcomes, such as graduation rates, per capita spending, educator recruitment and retention and so on (Johansson et al., 2004). The lack of consistent primary and secondary education data across circumpolar regions continues to be a problem; the Arctic Social Indicators project only includes post-secondary indicators, which is symptomatic of this problem (Rasmussen et al., 2010). Our hope is that there is a movement among circumpolar nations to collect and disseminate consistent and comparable data on primary and secondary education before another decade passes.

\section{Defining education}

In the first $A H D R$, the authors defined education as contributing to the development of human capital, a non-neutral "promotion of skills, values, history, languages, and ways of thinking and behaving" and a formalized process by which nations "perpetuate their values and beliefs from one generation to the next" (Johansson et al., 2004: 170). While we agree that this definition describes typical government-imposed systems of formal schooling, this definition does not accurately describe the edu- 
cation envisioned by many parents, community leaders and educators seeking to create systems that reflect the diverse cultures of the North. Across much of the Circumpolar North, Indigenous peoples had common experiences within education systems based on "Western" ways of teaching, learning and knowledge and operating with the intent of assimilation. Now, across the North and elsewhere, such as New Zealand and Hawaii, Indigenous peoples are working to create Indigenouscontrolled education systems based not on the Western form of schooling imposed on them for the past century (or longer) but rather based on Indigenous epistemologies and worldviews. In some circumpolar regions, these efforts are happening alongside the dominant Western education system, while in others, the Western systems are being replaced entirely.

\section{Textbox 9.1}

\section{Self-determination in education}

The efforts we describe in this chapter are being made against a backdrop of an increasing push for self-determination in education among many Indigenous peoples. Self-determination in education is recognized internationally as a human right. Article 14 of the United Nations Declaration on the Rights of Indigenous Peoples (2007) states, "Indigenous peoples have the right to establish and control their educational systems and institutions providing education in their own languages, in a manner appropriate to their cultural methods of teaching and learning" (UN DRIP, 2007).

But self-governance is also a key factor in creating systems that improve educational outcomes for Indigenous students, not just in the north, but across the globe (Smith, 2003; Hill et al., 2012).

In some places, such as Greenland, this effort is occurring alongside the move from colony to home rule, though education reform efforts in the country still face resistance from many within the country and in Denmark. In other places, such as Alaska, this work is happening within a state-run education system that is not necessarily supportive of such efforts, under a state government that does not recognize the legal sovereignty of tribes, and in a federal context of accountability that has pushed many schools to narrow mainstream curricular offerings in the quest to improve student standardized test scores. In Canada, the level of self-determination varies from province to province, with First Nations peoples in southern provinces just winning some of the rights that Inuit in the northern regions have had for several decades. 
As one Indigenous leader noted:

"The success of Native education is directly related to the amount of community control and involvement there is in the school system. Only when Native people feel a part of that system, that they have a stake in it, will they assume responsibility in a meaningful way and become committed to its success."

Mary Simon, President, Inuit Circumpolar Conference, 1989, (Darnell and Hoem, 1996: 149-150)

Moreover, while many distinguish between formal and informal education in the transmission of traditional knowledge, some scholars argue for re-thinking these definitions. For example, Christensen (2012: n.p.) contends "there is a need to reframe the question of schooling and how we think about education to get beyond notions of formal and informal learning." In studying the learning young people do during hunting, she found it possible "to rethink education from within a different social context than the institutionalized and state-based one, namely the social context of hunting. The activity of hunting and/or land-based practices organizes individuals relative to hunting as a culturally specific way of living and of being in the world. To view hunting as a way of being in the world brings out more profound perspectives than viewing hunting as in informal or traditional technique." Some of the education reforms underway in northern nations ground formal schooling in the Inuit or Inupiaq ways of living and being, and thus make fluid the relationship between "formal" education and "traditional" learning, so that in Barrow, Alaska whaling can be both the backbone of the community and a foundation on which learning is built in school. This rethinking of the definition also allows for inclusion of differing educational goals for parents and students, whether it is enabling young people to continue on in formal schooling and pursue a professional career in a location away from their home community, or developing the skills that enable them to stay in their home and be a successful subsistence hunter or herder. 
And so we look at education as including both formal and informal processes that prepare students for future success in their communities, further education and the workplace, in whatever they choose to do and wherever they choose to live. These processes ground students in their own culture, values and beliefs and enable them to successfully negotiate the socio-political and economic world beyond their own communities. We also acknowledge the importance in Indigenous communities of instruction in heritage languages as well as in western languages.

\subsubsection{Trends in education across the Circumpolar North}

Across the North we see a number of trends in education. While these may not be occurring in every circumpolar nation, they are affecting the majority. These include challenges in delivering education in rural and remote locations, including school consolidations and closures, providing access specifically for secondary education, and difficulties in recruiting and retaining teachers. There also are challenges around educating in and maintaining heritage languages. Low achievement and high dropout rates among Indigenous populations also continue to be problematic. The gender gap between males and females continues, with females generally achieving at a higher level than males and also going on to obtain more formal schooling. Other trends include the increased use of Indigenous knowledge and ways of teaching and learning in some formal school systems. All are discussed below.

\subsubsection{Challenges of delivering education in rural and remote locations}

\section{School closure and consolidation}

Rural communities are losing their local schools across the North. The reasons behind school closure vary, from outmigration and falling school age populations leading to too few students to keep a school open (see Chapter 2, Arctic Populations and Migration), to funding issues, to parents opting to send their children to larger schools (often boarding schools) in order to have access to a broader, more comprehensive education. This can have a number of impacts on students and communities. When students are educated away from their home, there often is a lack of connection between the school and the home and between the school and the culture of the home community. Across North America, boarding or residential schools contributed to language and culture loss for several generations of Indigenous students. But perhaps more dramatically, it 
can affect the sustainability of a community as a whole. First, parents in communities without a school may choose to move to a different community with a school rather than sending their child away. Second, students who leave home for school may choose not to return to their home community once graduated. Both outcomes can lead to entire communities dying, as has happened in Alaska.

The Russian Federation is experiencing falling school age populations, resulting in many school closures, especially in rural communities where the number of schools has decreased by almost $25 \%$ in past decade (Nikolaev and Chugunov, 2012). Operating schools is especially hard in northern herding communities. There are some "nomadic schools" in Siberia where the teachers follow the reindeer herders and also sometimes utilize educated parents alongside regular teachers. Small taiga schools also offer education in small rural communities, not far from where the herders work. However, keeping these alternatives to boarding schools open has proved difficult (Lavrillier, 2013).

On top of this, the 2007 "Priority National Project 'Education'” Russian federal reform mandated that schools be financed based on the size of the student population, which encourages school consolidation and the closure of smaller schools (Chevalier, 2012). However, a new national education law, "On Education in the Russian Federation", which became effective as of 1st September 2013, prohibits the closing of rural schools "without consulting the residents of the rural community" (Russian Federation, 2012: Chapter 3, Article 22,\#12). It is too soon to know whether this law will slow the rate of school closures in the Russian North.

In the Faroe Island, national education policy is that every child be able to attend lower primary school in their home community, no matter the size. Some schools are as small as one teacher with fewer than ten students. School closures are an issue in small rural communities, especially when parents choose to take their children out of the local school and either home school them or move to a larger community. Once a school closes, it cannot be reopened without permission of the municipality.

School closure threatens the viability of small rural communities across Norway, Sweden and Finland. In all three countries the proportion of small rural schools is high, approximately one in three (Hargreaves et al., 2009). In Norway, low student numbers and the municipal economies are among the most frequent reasons for closure or amalgamation of schools (Utdanningsdirektoratet, 2010). Consolidation means longer school transportation distances, creating a new challenge. Communities in the three northernmost counties in Norway all have experienced school closures recently. Since traditionally the Sámi 
homeland consists of small rural communities within municipalities with less income, school closure due to economic reasons is not uncommon.

In Alaska, the state currently supports schools in any community with at least 10 students. Students in communities with too few students to support a school can opt for home schooling, participate in a correspondence school program, or attend one of three secondary public boarding schools in the state, if their family does not want to relocate. Since the legislature increased the minimum number of students for a school to receive funding from eight to ten, twenty-seven rural Alaska schools have shut down (DeMarban, 2012).

\subsubsection{Accessibility of secondary education}

In many circumpolar regions, students can attend primary school in their home community but must go away to a residential program to attend upper secondary or high school. This is true in Greenland and the Faroe Islands, as well as for parts of Iceland and the Russian Far North. This situation can affect graduation rates, as not all secondary students have the maturity or skills to manage living away from home, and other family demands sometimes require that students return to their family.

The Faroe Islands have about 120 settlements but only nine upper secondary schools. There are no boarding schools in the Faroe Islands. Students who do not live in or near one of the larger communities with a secondary school must stay with relatives or live by themselves (e.g., in student housing). In Iceland, while many small compulsory schools are located outside of Reykjavik, including some as small as 10 students, not every community has one. Students in some rural communities sometimes have to attend secondary programs away from home, and live in boarding facilities (Rønning and Wiborg, 2008). In Siberia, the secondary school options for most students are either boarding schools or schools in little settlements, which don't provide the same quality as the boarding schools (Lavrillier, 2013), leading to many parents opting for boarding schools for their children, as noted above. However, dropout rates from boarding schools are high, and compulsory attendance is not enforced (Dudeck, 2013).

\section{Problems recruiting and retaining teachers}

A common problem across northern schools is recruiting and retaining well-prepared teachers. In many regions, there are too few educators who are from the North and used to the challenging living conditions or proficient in the local languages. In Nunavut, there are multiple chal- 
lenges around staffing schools successfully. These include a shortage of available and interested trained Inuit educators to deliver Inuit language instruction, high turnover among non-Inuit teachers, a lack of orientation and training in culturally responsive curriculum and pedagogy for non-Inuit teachers, and geographic isolation from support networks including infrastructure, administrative, and program support staff (Berger and Epp, 2007, Berger et al., 2006). Likewise in Nunavik, nonInuit teachers from the southern parts of Québec or elsewhere in Canada are hired to teach in grades three and up. Generally, they have not received specialized training to work in this particular cultural context, and are unable to teach in the local language. Many stay only a few years in the remote Nunavik schools.

Greenland has also faced difficulties in hiring qualified teachers. In 2005-2006, fully one third of teachers did not have teaching qualifications, and many of these were teaching in the remote settlements (Wyatt, 2012).

In Alaska, rural and remote schools face difficulties in hiring and retaining teachers. While the student population in rural Alaska is primarily Indigenous, the educators in rural schools are overwhelmingly nonNative - less than $5 \%$ of certificated teachers are Indigenous people, and fewer yet are administrators. Most are also from outside Alaska; the University of Alaska system prepares only $20 \%$ of the teachers hired by districts each year. Average teacher turnover rates in rural school districts vary tremendously, from a low of $7 \%$ to a high of $52 \%$; ten out of 53 have turnover rates over $30 \%$, and as a whole rural districts average 20\% turnover per year (Hill and Hirshberg, 2013). In Siberia, finding teachers for the schools serving nomadic communities is difficult; nonIndigenous teachers are not prepared for the lifestyle challenges and teacher turnover rates can be high in taiga schools (Lavrillier, 2013; Dudeck, 2013).

\section{Teaching in heritage languages}

In the first Arctic Social Indicators report (ASI, 2010), cultural well-being and vitality was measured in part by the use of Indigenous languages in both the home and in formal schooling, and the retention of language among heritage language speakers (Schweitzer et al., 2010). The concept of Fate Control in the first ASI also includes language retention within the category of Knowledge Construction (Dahl et al., 2010). And yet, the use of Indigenous languages in circumpolar schools serving Indigenous students varies considerably, due both to formal policies and challenges around finding certified educators who speak heritage languages and educational materials in those languages. 
The Nunavut Education Act (GN, 2008) requires that by the year 2019-2020, the school system must deliver programs that are fully bilingual from kindergarten through grade 12 . In most schools, language instruction will follow a model of high Inuit language instruction in the elementary years, moving towards $50 \%$ of instructional time split between Inuit language and English or French at the secondary level. This goal is currently being implemented, but is challenged by the need for more educators who have high quality Inuit language and instructional skills at all grade levels and in all courses, as well as access to appropriate teaching resources and learning materials (Aylward, 2010). Challenges arise from the extra time involved in building a bilingual education context and the day-to-day operations associated with working in at least two languages (McGregor, 2010).

The Kativik School Board (KSB), which governs the whole educational system in the Nunavik region, has chosen to educate the pupils in their mother tongue, Inuktitut, from kindergarten to grade three (KSB, 2013). When children get to grade two, families must decide in which of the two official languages of the province (French or English) they want their children to be educated. After grade three, the children pursue their schooling in either French or English, while continuing to learn Inuktitut language and culture, since a few courses are integrated into their program (KSB, 2013). An important educational priority shared among the people and the KSB is the maintenance and development of the Inuktituk language and culture. Educational instruction in the learner's home language in the first years of schooling has been identified as a relevant means to develop a solid educational foundation for youth. Therefore, competent Inuktitut-speaking teachers are required to work with the pupils. When KSB was created, a teacher-training program was developed and implemented, according to the training needs in this particular context, by KSB in collaboration with McGill University (de Krom et al., 2011; Cram, 1987).

The Sámi languages are vital to the Sámi people across the Nordic countries. The language is central to Sámi identity, and is considered essential to their survival as a people (Gaski, 1998). Finland, Norway and Sweden have all ratified the European Charter for Regional and Minority Languages (CETS 148, 1992). Accordingly, there is emphasis in the education systems to respond to the requirements; however there are gaps to be filled. The existing international and national regulations regarding Sámi language and education rights are implemented in different ways in each of the nations. 
While the principal language of instruction in Russian Federation schools is Russian, citizens have the right to be educated in their native language in basic general education (grades 5-9). Those operating the schools choose the language of instruction (NIC ARM, n.d.). However, the federal curriculum only allots two hours per week to the study of local languages (Chevalier, 2012). The loss of heritage languages in Russia's northern communities is a significant problem to which education contributes, although education can also be the means for preserving the languages (Dudeck, 2013; Lavrillier, 2013; Chevalier, 2012). In Siberia, "national" schools are secondary schools that offer instruction in Indigenous languages. Many of these schools are reducing the amount of instruction in Indigenous languages, due largely to the Unified State Examination (Chevalier, 2013). Passing the Russian language and mathematics portions of the Unified State Examination is required for graduation, and these exams are entirely in Russian. In some cases, falling levels of Russian language proficiency among rural children have led families to move from rural areas served by "national" schools into urban areas.

The new national education law in the Russian Federation reinforces the idea that while students have a right to study their heritage languages, that right has limits. On the one hand, the law states:

Citizens of the Russian Federation shall have the right to pre-school, primary and general basic general education in the mother tongue of the number of languages of the peoples of the Russian Federation, as well as the right to study their native language among the languages of the Russian Federation within the possibilities offered by the system of education, in accordance with the legislation on education.

(Russian Federation, 2012: Chapter 1, Article 14 \#4)

On the other hand, it also stipulates that "teaching and learning of the official languages of the republics of the Russian Federation shall not be to the detriment of teaching and learning the state language of the Russian Federation" (Russian Federation, 2012: Chapter 1, Article 14 \#3). It is too soon to know the impact of the new law on education across the Russian Federation, and in the North specifically, but the loss of heritage languages is not likely to be stemmed.

Greenland's situation is quite different from other Arctic regions; Greenlanders do not have to revitalize the native language as it is the language of instruction in schools (Wyatt, 2012). Students study in both Greenlandic and Danish starting in the early years, and then add English and, if desired, another foreign language in later years. The challenge Greenland students face is developing sufficient proficiency in Danish 
and English so that they can pursue a post-secondary education, because even within Greenland most of the high school and post-secondary education offerings are in Danish, not in Greenlandic (Boolsen, 2009; EU Commission, 2013).

\section{Low achievement and graduation rates}

Across the North, student achievement as measured by secondary graduation and dropout rates continues to be problematic in many regions, especially for Indigenous students. In Nunavut, graduation rates (calculated by dividing the number of graduates by the number of estimated 17 and 18 year olds) ranged between $32 \%$ and 38\% over the past four years (Nunavut Bureau of Statistics, 2012). Students continue to miss between a third and a quarter of instructional time, and in the context of the other complexities of education in Nunavut outlined here, educators, parents and community members all continue to express concerns about the standard of achievement amongst students and to what extent they are on par with other Canadian students.

In Nunavik, as in Nunavut, the high school diploma achievement rate of Inuit youth is very low compared to the overall national rates. In Québec, fewer than one student in five successfully completes Secondary V (the final year of secondary school). Very few Inuit students attend postsecondary studies (Gouvernement du Québec, 2011).

Greenland also has problems with students dropping out from academic high schools. There are only four of these, all located in towns, which means that students from villages have to leave home to complete their high school education. Between 2005 and 2008, the number of students in high school increased by $17 \%$, and the number of graduates went up by $37 \%$, but the number of dropouts also increased by $34 \%$. These numbers are very different from vocational training outcomes, where the number of dropouts has decreased by $3 \%$ even as the total number of students has increased by $31 \%$, the number of apprentices has gone up by $20 \%$, and the number of graduates has risen by $25 \%$ (Boolsen, 2009).

In Alaska, while non-Native student achievement mirrors or even exceeds national averages, Alaska Native student achievement is generally poor, particularly in small villages. Alaska Native students today drop out at rates triple the national average, and most who attend college need remedial work (Martin and Hill, 2009, McDiarmid and Hill, 2010). The gap between Alaska Native and non-Native students in Alaska is wide. In 2012-13, Alaska Natives made up $22.7 \%$ of students in grades $7-12$, but $34.7 \%$ of the dropouts from those grades. They had a dropout rate of $6.2 \%$, compared with $4.0 \%$ for all students in those grades (AK EED, 2013). The high-school graduation rate for all Alaska students in 2012- 
2013 was $71.8 \%$, but just $57.1 \%$ among Alaska Native students - the lowest among all racial and ethnic groups in the state (AK EED, 2013).

Upper secondary completion rates in Iceland are not very high. Among students starting upper secondary in 2003, only $44 \%$ had completed their program after four years, and that number increased to only $62 \%$ seven years after starting. Still, these numbers represent a significant increase over the educational attainment of earlier generations of Icelanders; the rate of upper secondary completion amongst Icelanders age $25-64$ in 2011 was only $36.7 \%$ (Statistics Iceland, 2013).

\section{Barrow High School, Alaska, USA}

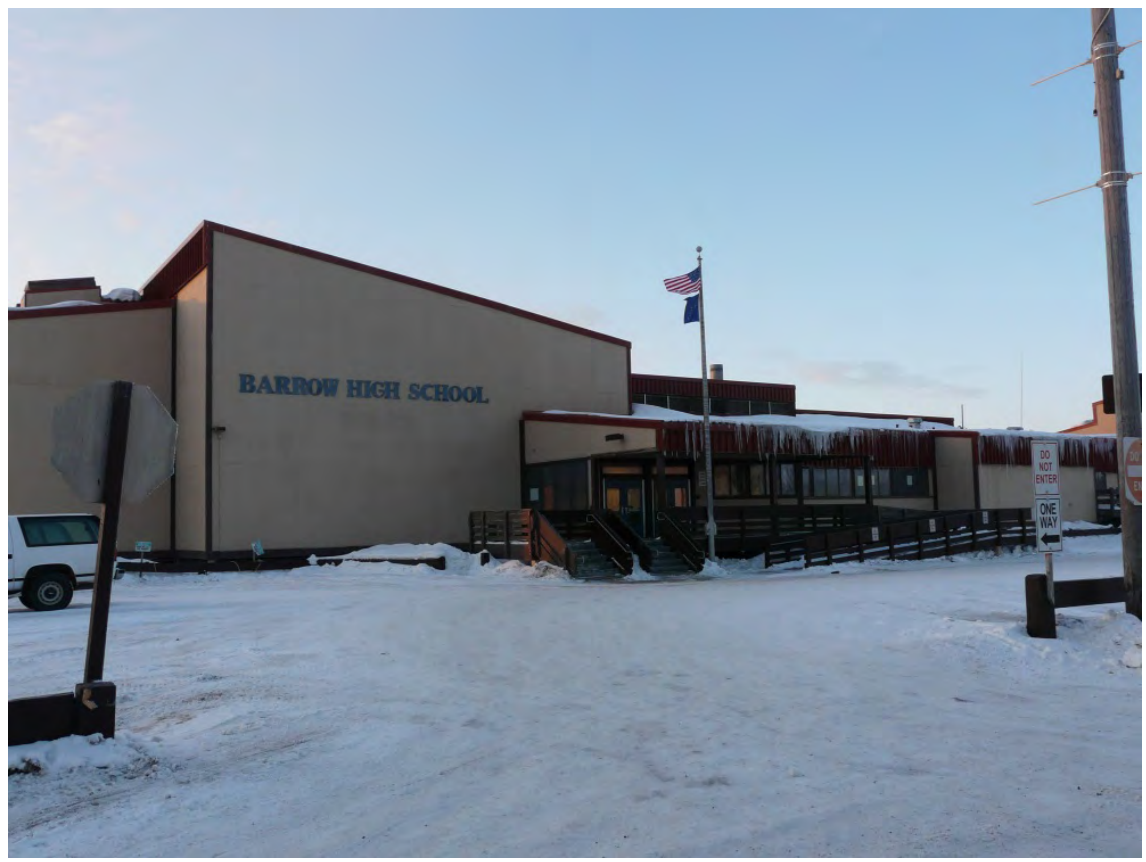

Credit: Diane Hirshberg.

\subsubsection{Indigenizing education}

Many Indigenous communities in the North are moving toward creating educational models within the formal school system that are based on traditional ways of teaching and learning. Many of these efforts are part of government driven or sanctioned school reform efforts, and others occur despite prevailing policies and trends.

In Alaska, the education model in most schools is still very western, even in communities where the great majority of students are Indigenous. In Spring 2012 the Alaska Board of Education adopted new guide- 
lines for implementing the "Alaska Cultural Standards Educators", and is focusing on disseminating these widely (AK EED, 2012; Chris Simon, personal communication, 20th February 2013). However, this has not yet led to widespread change around the state. That said, there are places where Indigenous education models are in place or being implemented. In the Ayaprun Elitnaurvik Yupik Immersion School in Bethel, an elementary school, students learn fully in Yupik in grades K-2 and then are introduced to English starting in grade three. Pedagogical approaches mix traditional Yupik ways of teaching and learning with more Western styles. Students in the school generally outperform district averages on standardized tests, and graduates have gone on to be valedictorians in their high school class.

The North Slope Borough School District in Alaska begins its mission statement by saying "Learning in our schools is rooted in the values, history and language of the Iñupiat." The district has developed the Iñupiaq Learning Framework and is now developing curriculum and pedagogical approaches to create an Iñupiaq education system based in local epistemologies but also preparing students to succeed in the Western system. The school board has driven this reform, and hired a superintendent who is implementing its vision. The reform effort is based on extensive work with elders, educators, and community members across all borough villages, to determine what children should know when they graduate, based in Iñupiaq culture, values and beliefs rather than in the system imposed by external Western educators.

In Greenland, the 2002 School Act states that schooling: "shall create the basis for the pupil's development of his/her knowledge about and understanding of his/her own social identity, culture and values" (Greenland Government, Chapter 2, §5). The education reform effort that emerged from this Act, known as "Atuarfitsialak", focused on creating a culturally compatible education model in pre-K through higher education, and included restructuring teacher education and professional development. While the Act did not explicitly mention strengthening Greenlandic identity and culture, the reformers set that as a goal for the reform effort. The effort differed from other northern school reform efforts because it did not focus on language revitalization, and indeed explicitly supported strengthening Danish, while also trying to strengthen the use of the Greenlandic culture in the schools.

In Nunavut, the 2008 Education Act lays out "Fundamental Principles for Education", which start with "The public education system in $\mathrm{Nu}-$ navut shall be based on Inuit societal values and the principles and concepts of Inuit Qaujimajatuqangit" (Nunavut Government, 2008, Chapter 
$15, \S 1(1))$. Nunavut is the only jurisdiction in Canada in which the entire public education system (kindergarten to grade 12) is legally required to address the educational needs and desires of an Indigenous (Inuit) population (McGregor, 2012b). Education staff and administrators in $\mathrm{Nu}-$ navut are also required to ensure that all aspects of schooling reflect and reference the values, principles and approaches of Inuit knowledge. This has necessitated a great deal of research with Elders and start-fromscratch program development by Nunavut educators and curriculum support staff (Aylward, 2009; Aylward, 2012; McGregor, 2012a). Nunavut aims to develop entirely culturally-responsive and locally-relevant curriculum, programs, materials and assessments that also ensure their students have access to post-secondary and university entrance requirements (Nunavut Department of Education, 2007). For example, the former grade 12 standardized social studies examination has been replaced by a project-based assessment model developed in Nunavut to facilitate student assessment that combines local topics, cultural content as well as 21st century skills.

There are Sámi schools in each of the national areas of northern Fennoscandia, under the respective national school systems operating under special regulations/mandate and/or funding models. The concept of Sámi education differs throughout the area. On the Norwegian side there are two national curricula for the basic education 1-13, the Norwegian and the Sámi. The Sámi school concept for grades 1-10 reflects groups, classes or schools which are municipally owned and which follow the Sámi National Curriculum, several specialized Sámi schools, and for the upper secondary level two state operated schools. Also, all students are required to learn about Sámi themes according to syllabi grades 1-13.

In Sweden, five schools operate at levels 1-6 under a separate state unit, the Sámi School Board. Elsewhere the Sámi language is integrated into mainstream schools as a minority language. There is a Sámi Education Center in Jokkmokk. In addition, the Lapplands gymnasium, the northernmost upper secondary school in Sweden, organizes a national level program with a Sámi focus including the study of reindeer herding.

In Finland, there is no actual Sámi school, but Sámi education refers to classes/groups where the Sámi language is the language of instruction; Sámi language programs otherwise are delivered within regular public schools. The municipal schools in the Sámi Domicile Area offer Sámi language programs, and one municipality organizes a Sámi program. Daycare in Sámi language is also offered in Northern Finland: for example the city of Oulu has a daycare service in Sámi language in one center for children age 1-6 who comes from Sámi- 
speaking families. The daycare centers also address cultural issues and support for Sámi identity. The Oulu center also offers courses in the Sámi language for Sámi children in grades 1-9 in cooperation with a local elementary school.

Norway implemented the "Knowledge Promotion 2006", a comprehensive reform, with a partly revised and partly new national curriculum along with a national Sámi curriculum. The Sámi parliament was responsible for developing the Sámi language syllabi and the Sámi traditional handicraft (duodji) syllabi, and otherwise was a consulting partner in the process. The implementation of the Sámi curriculum faced challenges such as a lack of teaching materials and teachers. The situation is worse in the lule- and south Sámi area and in marginal north-Sámi areas. The Norwegian state also operates two upper-secondary Sámi schools with a special focus on Sámi education, including integrated or specialized traditional knowledge programs (arts/craft/reindeer herding/music), and there are multiple county upper-secondary schools throughout the country delivering Sámi language programs.

\subsubsection{Gendergap}

In general, across the globe girls outperform boys on measures of learning such as international and national tests and measures of overall achievement, including grades and graduation rates. While boys outperform girls on a few individual subjects, overall girls are doing better in terms of formal schooling. This holds true across the Circumpolar North.

In 2012, males across Alaska graduated at a rate of $66.3 \%$ while females had a much higher graduation rate of 73.1\% (AK EED, 2013). The gaps between males and females differ considerably by race; the highest gap between males and females was for African American students, with almost $12.5 \%$ more females graduating. The next largest gap was among Alaska Native students, with almost $11 \%$ more Alaska Native females graduating than males. Alaska Native males had the lowest graduation rate $-47.7 \%$ - of any ethnic/gender group (Brian Laurent, personal communication, 3rd November 2013). In Nunavut, in the past decade women graduated high school at a higher rate than men in all but one year, though both had relatively low rates, between $42 \%$ and $50 \%$ for males and between $46 \%$ and $58 \%$ for females $(\mathrm{Nu}-$ navut Bureau of Statistics, 2012).

In Russia, while information specific to the North is not available, data from the Programme for International Student Assessment (PISA) 
exam shows nationwide a gap between female and male achievement, with girls outperforming boys to a greater extent than in other Organization for Economic Co-operation and Development (OECD) countries. Across almost all OECD countries, females are more likely than males to graduate from upper secondary education, including Norway, Denmark and Finland, though Finland has the lowest gap between males and females in terms of dropout rates (OECD, 2011).

\subsection{Human capital and knowledge in the Arctic}

The Arctic is often viewed as a treasure chest of natural resources. Although natural resources have a great importance, the true treasure of the Arctic is its people. Since pre-historic times Arctic residents have developed skills and knowledge that enable them to survive and thrive in harsh conditions. It has been observed that northerners have a good grasp of matters important for their livelihoods and possess unique and diversified knowledge on how to ensure their well-being (Megatrends, 2011). At the same time, the ability of Arctic societies to benefit from standardized codified knowledge and formal education has been rather limited. As a result, Arctic regions demonstrate substantial gaps in terms of development of local human capital.

The first AHDR (2004) does not explicitly discuss human capital issues. However, it addresses conditions associated with schooling and their impact on Arctic communities (Johansson et al., 2004). The report concludes that the most critical concerns regard control, relevance, and access to education. It argues that education systems and learning practices need to adapt education services to fit local needs and conditions. The report advocates a shift from viewing knowledge as a standardized commodity to seeing it as a distributed resource that has led to pressures for decentralization of control and decision-making, local adaptations, and increased use of technology to access knowledge. The attainment of education (whether formal or not) is an investment in human capital. The outcome of this investment is knowledge production and transfer that ensures the livelihoods and prosperity of Arctic communities. This section extends the discussion of the relevance of formal and informal education and knowledge in human development in the Arctic. We provide an assessment of human capital and knowledge production in the Arctic as it pertains to human development and well-being of Arctic societies. 


\subsubsection{The need for knowledge and human capital}

Human capital can be defined as the stock of knowledge and skills embodied in a human population that has economic value. It most generally refers to formal and tacit knowledge and skills, which can be deployed to general economic returns. Human capital incorporates three components: general skills (literacy), specific skills (related to particulate technologies and operations), and technical and scientific knowledge (mastery of specific bodies of knowledge at advanced levels). There is a broad agreement in the literature that human capital is closely related to both individual and aggregated labor outcomes: higher individual wages and enhanced employability on one hand and greater productivity and accelerated technological progress on the other (De la Fuente and Ciccone, 2002).

All three components of human capital are closely tied to schooling. The general skills (functional literacy) level is typically achieved at the primary education level. Specific skills are acquired during secondary education and advanced scientific and technical knowledge obtained through higher education. However, formal education is not a sole source of human capital. In fact, recent studies suggest that in the Arctic human capital is less related to formal levels of schooling than it is in the south (Petrov, 2008; Petrov and Cavin, 2013). This difference is very important and is attributable to the human capital associated with traditional skills and knowledge, not obtained through attending educational institutions. In this chapter we discuss both human capital accumulated through formal schooling and human capital based on local and Indigenous knowledge (LIK). Certainly, measuring the latter is a more challenging task given the difficulty of quantifying the value and scope of LIK. It is worth noting that the lack of one (e.g., formal education) may be partially compensated, albeit indirectly, by another (LIK) in terms of human development.

Human capital is a crucial factor of regional economic growth and development and a key attribute of a modern post-industrial economy. First, human capital is the most important ingredient in the "knowledge sector", which includes technologically advanced industries and services (e.g. information technology, high tech manufacturing, financial services, etc.) the most intensively growing elements of modern economy that define the overall success and competitiveness of regional economic systems in a globalizing world. Second, knowledge underpins "old" industries including the whole array of primary (e.g., extractive industries, agriculture), secondary (manufacturing) and tertiary (services) sectors. In fact, $50 \%$ of GDP in OECD countries is based on knowledge production and utilization 
(Megatrends, 2011). In other words, the modern economy is an economy heavily based on knowledge (Bell, 1973; Jacobs, 1984).

Just as other regions of the world, the Arctic is touched by globalization and the emergence of the knowledge economy (see Chapter 10, Globalization). While ties between Arctic economies and resource and government sectors are persistent (see Chapter 4, Economic Systems), globalization has brought new opportunities and challenges that create a competitive edge for Arctic communities in the new knowledge-driven world economy. Northern regions can become "learning" regions (Morgan, 1997) that adopt and adapt innovations while developing their own body of economically relevant knowledge and skills based on local experiences and traditions.

In this respect, a development strategy based on enabling local human capacities to advance economic development is appealing. However, the Arctic faces formidable challenges to become such a region: "endogenous growth" is inhibited by limited local capacities (institutional, financial and infrastructural) and, most importantly, by the shortage of human capital (Petrov, 2011). Evidence from northern success stories suggests that human capital's economic returns tend to be more connected to local economies. This connectedness is partially determined by the endogenous nature of the knowledge-based economy in general, but also by a tight relation of human capital with other forms of societal capital in the periphery (Aarsæther, 2004). In addition, communities can capitalize on Indigenous knowledge and tradition and facilitate institution building and formation of civic society.

\subsubsection{Human capital and human development}

The accumulation of human capital is necessary to maintain a stable and expanding economic base and ensure human well-being. Education is an integral part of human development as identified both by the UN and Arctic Social Indicators project (ASI, 2010). Both the first AHDR and ASI emphasize the role of education in ensuring economic well-being, fate control (empowerment), and cultural continuity, especially if standard schooling practices are intertwined with local and traditional contexts. ASI (2010) identified education as one of the six key domains of human development in the Arctic.

Studies show that in the EU, for each additional year of schooling, an individual gains $6.5 \%$ in wages. The figure is even higher in North America (De la Fuente and Ciccone, 2002; Psacharopoulos and Patrinos, 2004). Post-secondary education provides even more substantial gains: for example, the average private return to post-secondary education for Euro- 
pean men is estimated at $12 \%$ (OECD, 2011). This is even higher for Indigenous residents: in Saskatchewan, Canada the lifetime earnings of an Indigenous male were found to increase by $38 \%$ if a university degree was completed, and for Indigenous women this increased by 59\% (Howe, 2011). Higher education levels are also associated with greater labor force participation and lower unemployment. Schooling has a role in reducing poverty and providing means for economically disadvantaged groups to improve their standard of living (ASI, 2010). In Canada, studies show that completion of university education is the most financially rewarding in terms of improving earnings of Aboriginal Canadians, followed by the completion of non-university post-secondary education (Hossain and Lamb, 2012). In addition to benefitting an individual, investment in education also increases overall productivity by as much as $5 \%$ per additional year of schooling (De la Fuente and Ciccone, 2002) and competitiveness of national and regional economies. There are other positive outcomes of education that are felt both in households and society at large, such as the increased ability of educated women to manage their lives and financial situations through reproductive control (Oxaal, 1997).

The link between education and empowerment is key. One aspect is the empowerment of the individual where a growing proportion of students are able to continue past primary through secondary school and further on to post-secondary institutions. The empowerment gains are especially significant for women. Individuals may be empowered to fulfill important functions in the community and their education may provide them with a reason for staying. On the other hand, educational attainment may also empower them to start looking for other opportunities, including leaving the Arctic.

In this context it is clear that empowerment is a multi-dimensional concept with often divergent consequences. ASI (2010) offered a new way to conceptualize empowerment - through the notion of fate control, i.e. ability of individuals and communities to define their own destiny. According to the ASI I report, education is an integral part of fate control. Stronger fate control may strengthen communities, making them able to resist the pressure from the outside, at least for a while. It may, however, be less responsive to the marked differences in both gender and generational foci in relation to the development process, which may eventually result in unexpected and unwanted out-migration patterns, and thus the loss of the new human capital gained, for example, through the expansion of educational opportunities. Another possible consequence of formal education is the erosion of cultural identity and loss of contact with nature (ASI, 2014; Battiste, 2000). 
A common problem of non-metropolitan, peripheral regions is the "flight" of human capital (see Chapter 2, Arctic Populations and Migration). With an increased level of education the ability (and desire) of local residents to find employment or new educational opportunities elsewhere grows as well. An increasing number of northerners, especially women, move away from the Arctic to receive or use their education. At the same time, many Arctic regions are attracting human capital from the south as skilled professionals take advantage of high earnings in certain Arctic sectors (mining, oil, etc.). Unfortunately, however, most of them stay in the Arctic only for a limited time, and, as surveys indicate, remain fairly detached from each other (Voswinkel, 2012). Departing educated Native northerners and returning migrants create a "brain drain" from the Arctic (Handland, 2004; Petrov and Vlasova, 2010). This brain drain in many regions also coincides with a "brain turnover" (intensive in- and outmigration of human capital) and "brain waves" (surges and dips of human capital associated with the boom-and-bust economic cycle (Heleniak, 2010)). As a result, the study of human capital mobility may illuminate the ways in which human capital can be retained in place and/or attracted (back) to the Arctic. With the growing access to education for Arctic residents in their regions and elsewhere, the issue of retention becomes even more critical (Megatrends, 2011; Petrov, 2010).

\section{Arctic Design Show, Rovaniemi, Finland, 2014}

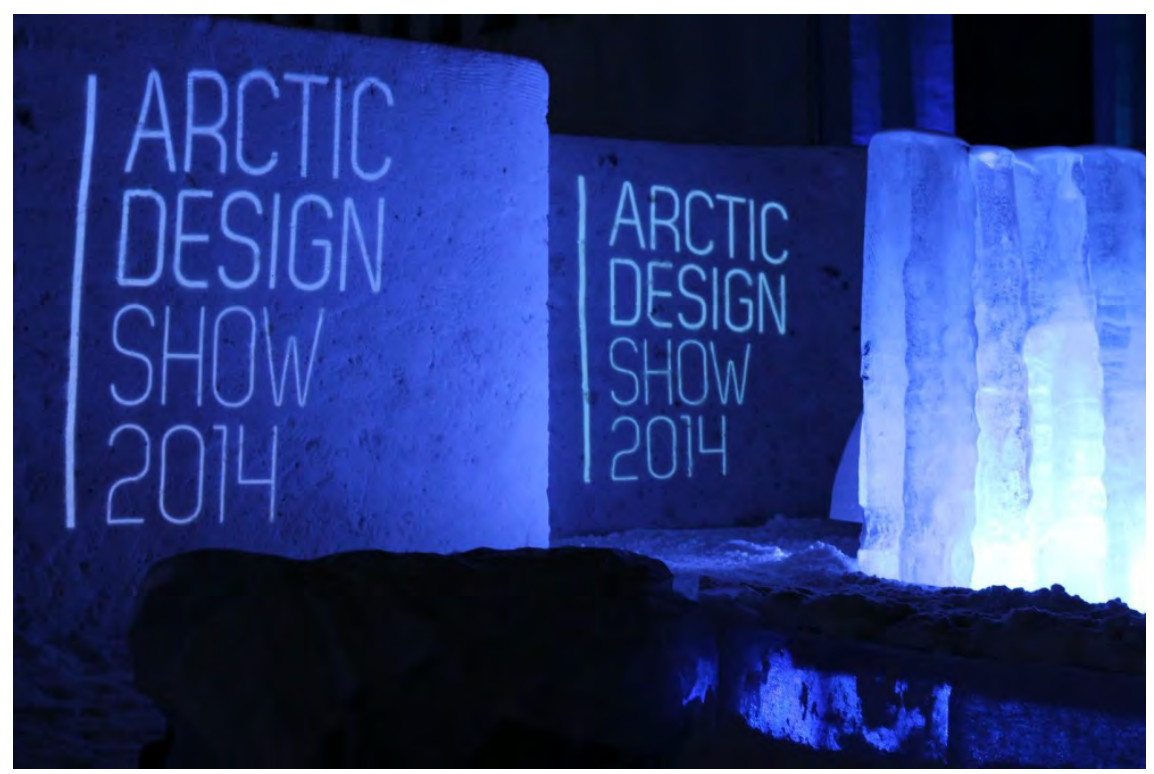

Credit: Kamil Jagodzinski. 


\subsubsection{Educational attainment and attendance statistics in the Arctic}

The most typical indicators utilized to measure human capital deal with levels of educational attainment - the highest level of education that an individual has completed. More advanced education is an investment in someone's human capital. A higher level of schooling is considered to indicate greater human capital. Although there are many levels of educational attainment (from primary to tertiary), the most common indicator used deals either with high school, post-secondary or university education. ASI makes a strong argument for including this measure to assess human development:

If we are to make a snapshot assessment of the contribution of education to the well-being of a community, it must include taking a look at the highest levels of educational attainment that people are pursuing; anything short of that would present an incomplete picture.

(Rasmussen et al., 2010: 82)

The main problems with educational attainment as an indicator are the availability of data and difference in definitions of schooling levels. Relevant data are largely collected from censusses since in most jurisdictions individuals are the only source of such information. Consequently, the existing data only coincide with census years (every 5th or 10th year), and definitions used in subsequent censuses may not be the same. Discrepancy in defining educational attainment is also marked among Arctic countries, which use diverging models of higher education (see section 1). Direct comparisons are therefore difficult.

Another relevant notion is educational attendance, i.e., enrollment in an educational institution. The advantage of this measure is that most educational establishments produce these data based on enrollments in each respective level of education. For that reason, ASI (2010) recommends using educational attendance and completion rates statistics in addition to educational attainment to measure the education domain of human development. The Report also suggests as an indicator the retention rates of post-secondary education graduates who stay in northern communities 10 years after graduation. All these measures are related to human capital and important for estimating well-being in Arctic communities. Given high mobility of human capital, retention rates can be especially informative. Unfortunately, the data on retention are not available in most Arctic regions. 
In this report we consider the key indicator: the proportion of residents with post-secondary education, which includes all levels of education following secondary school. Post-secondary education corresponds to acquisition of advanced specific skills and technical and scientific knowledge. In other words, people who completed post-secondary education have the highest human capital (its formal schooling component). In the following section, we analyze regional differences and temporal dynamics of post-secondary education in general as well as its components: university degree and tertiary education. Note that only formally obtained education is included in this analysis.

\subsubsection{Growing access to post-secondary education in the Arctic and educational attendance}

In the recent decades, Arctic regions saw an increase in the number and capacity of post-secondary institutions. Colleges and universities exist in all major Arctic jurisdictions, although there are more in the Russian and Nordic sectors (Figures 9.1). Umeå University, the University of Alaska system, Luleå University, University of Oulu, University of Iceland, Arctic University of Norway, and Murmansk State Technical University are the leading Arctic institutions by enrollment. All of these universities are associated with larger cities, although some have branch campuses in rural areas. Remote regions are served to a much lesser degree. In most Arctic colleges and universities, female students constitute the majority of enrollment (see below for further discussion). Another continuing trend is the growing level of control over post-secondary education by Indigenous people (Stonechild, 2006). Higher education institutions serving indigenous populations exist in various jurisdictions, e.g., Sámi University College (Norway), Taimyr College (Russia), Nunavut Arctic College (Canada), and Ilisagvik College (Alaska). 


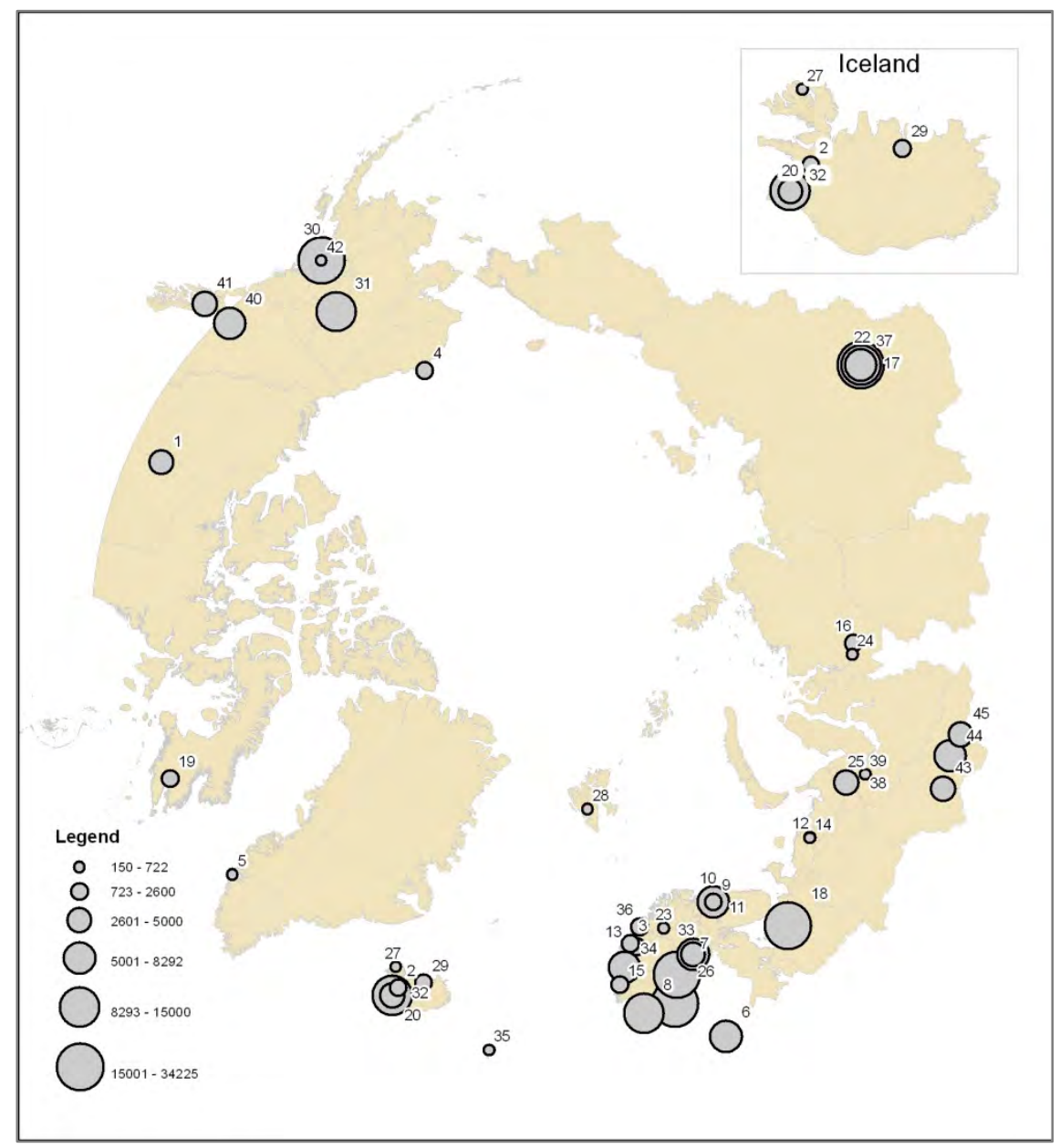

The Russian North has the most extensive system of post-secondary education of all the Arctic nations. As of 2011, in the nine northern regions there were 136 institutions of higher education, including 25 flagship universities and 111 branch campuses. The overwhelming majority are state institutions. While these are impressive numbers compared to most other Arctic jurisdictions, the level of attendance is relatively modest. The number of students per 10,000 residents in all northern regions is below the national benchmark. Not surprisingly, the proportion of highly educated individuals (any form of post-secondary education) in the Russian North is generally lower than country's average. At the same time the trend is positive, and in the last decade most northern territories experienced gains in student attendance and educational attainment (Figure 9.2). Perhaps, the most spectacular increase has been observed in Yamal-Nenets region, although it still lacks a flagship university. 
Figure 9.2. Enrollment in post-secondary institutions in the Russian North

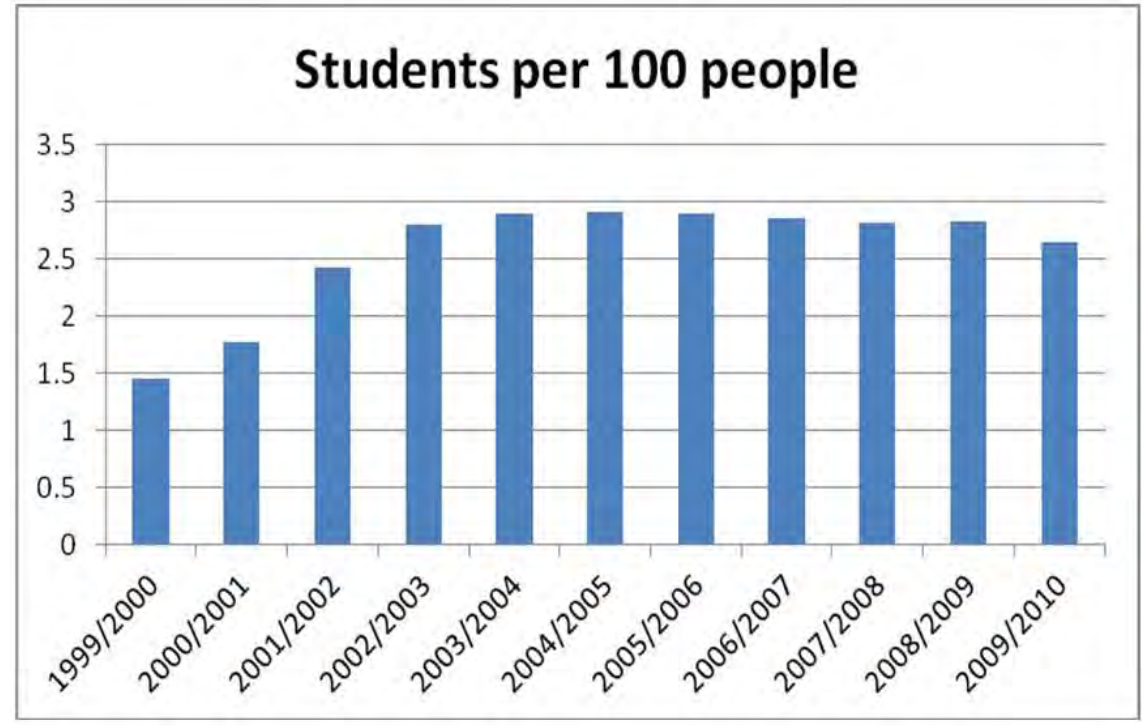

In northern Fennoscandia, the university and college level offerings are quite substantial with many institutions, delivery sites and programs including distance education. There are both larger universities and smaller university colleges. Northern Norway has two universities and four university colleges. The tendency has been consolidation towards larger entities with university colleges merging with University of Tromsø. Northern Sweden has two universities as does northern Finland. Finland also organizes Universities of Applied Sciences, including three in northern Finland.

Iceland has seven nationally accredited higher education institutions. The state runs four and private entities run three (with some state support). Of the seven, two are agricultural institutions, one is an art academy, and four are comprehensive universities. There are also numerous adult education learning opportunities in Iceland, including institutions that specifically target the $35 \%$ of the Icelandic workforce who did not complete upper secondary education. Continuing and adult education are offered across a broad array of institutions, from upper secondary institutions to higher education institutions to centers and colleges for continuing education to private company and association offerings.

For those who want to pursue a higher education in Greenland there is one university and a College for Social Pedagogy. The University of Greenland, Ilisimatusarfik, is located in Nuuk, the capital, and College for Social Pedagogy is located in Ilulissat. The Government of Greenland runs these institutions. The language of instruction is Greenlandic, Danish or English, depending on the instructor and curricula. The university 
and college together had about 665 active students in the year 2012. In this same year, 406 Greenlandic students attended colleges and universities in Denmark, and 17 in other countries.

\section{University of Akureyri, Iceland. Year 2014. This northern university has been growing significantly in size both in terms of student population and physical capacity over the past 10 years}

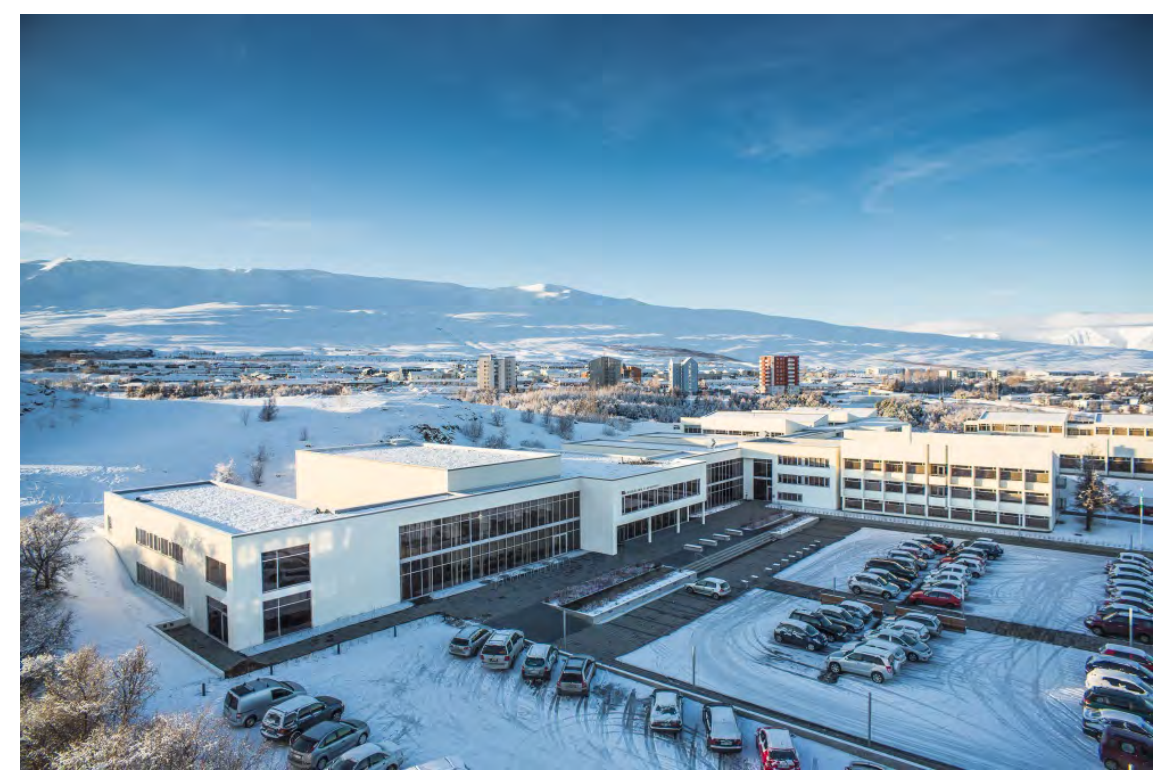

Photo credit: Daníel Starrason.

The University of the Faroe Islands, the only university in the Faroe Islands, is a small, state-run institution, consisting of two faculties in "Humanities, Social Sciences and Education" and "Natural and Health Sciences." Located in Tórshavn, the capital, it enrolls about 800 students. The language of instruction is Faroese. Vocational education is also available through a vocational college. Otherwise, many students attend college in Denmark or other countries.

In North America, the contrast is considerable between Alaska and the Canadian Arctic. Canada is the only circumpolar country to lack a university north of the 60th parallel. Canada's principle adult and post-secondary institutions in Nunavut, Yukon, and Northwest Territories remain community colleges. The territorial college system began in the 1960 s as vocational and adult education centers located in a handful of communities. Since then it has grown to include regional campuses and community learning centers. In Nunavut and Northwest Territories the majority of students are Indigenous, whereas in Yukon, Indigenous students comprise about one-third of the post-secondary students. Significant progress in 
delivery of post-secondary education in northern Canada has been made in a relatively short period of time. Yet access to education remains an ongoing challenge, especially as it relates to geographical access. Still, change is imminent; on 30th June 2011, Canada's three territorial governments announced that they had signed a Memorandum of Understanding (MOU) to further explore university models. The MOU establishes a tri-territorial committee, which will develop options on how the three territories can advance university development in Canada's North.

Access to post-secondary education in Alaska is fairly widespread. The largest provider of post-secondary education is the state-funded University of Alaska (UA) system. The UA system has three main campuses in Fairbanks (UAF), Anchorage (UAA) and Juneau (Southeast, or UAS), and 11 community campuses, in hub communities ranging from Kotzebue to Dillingham to Valdez. Alaska is also host to a few small, private post-secondary institutions. For instance, Alaska Pacific University, a small, private, liberal arts institution, has operated in Anchorage since 1960. There are also several career and technical training institutes. The state's only tribal college, Ilisagvik College, is based in Barrow. A part of the North Slope Borough, Ilisagvik offers two-year degrees and vocational and technical certificates.

In addition to national systems of post-secondary education, the University of the Arctic (UArctic) has built an international and interinstitutional higher education framework. UArctic is a key player in promoting, standardizing and facilitating educational exchange in the Arctic. It is also an umbrella for innovative teaching, distance learning and collaborative research (see Textbox 9.2).

\section{Textbox 9.2}

\section{Higher education spotlights}

The University of the Arctic (UArctic) was founded in 2001 and is a cooperative network of universities, colleges, and other organizations committed to higher education in the North. In 2013 UArctic membership totals 157 higher education institutions and research centers, Indigenous peoples organizations, and others as well as associate members from outside the Arctic region.

The overall goal is to create a strong, sustainable circumpolar region by empowering peoples of the north in general, and Indigenous peoples in particular. This is done through organizing members' collaboration and synergy within thematic based multilevel networking of several kinds in education and research; and through training; sharing of experiences, expertise and facilities; and organizing mobility programs for students and faculty. UArctic aims, through its members' collaboration and networking, to generate knowledge and improve access to relevant post-secondary education. UArctic respects the Indigenous 
peoples' integral role in northern education by engaging Indigenous organizations and institutions in UArctic's governance system, and seeks to engage Indigenous organizations and institutions, in a reciprocal manner, in different activities and in developing understanding of traditional knowledge as one among different learning systems. UArctic also aims at reaching out with experience distribution and learning options in cooperation with and for the peoples and the communities of the North through programs, conferences, publications and other outreach activities.

UArctic works in close partnership with the Arctic Council, the Arctic Parliamentarians, regional scientific organizations, national authorities and Indigenous peoples' organizations. The ultimate goal is to contribute to a sustainable North in an interdependent world by reflecting the diversity of peoples and cultures and knowledge systems of the Arctic.

State Polar Academy is an institution of higher education located in St. Petersburg and designated as Russia's "university for peoples of traditional cultures." The Academy was established in 1991 and has since graduated over 2,000 students. The student body includes representatives of 57 Indigenous groups from Russia and abroad, mostly from the Arctic. The academy awards bachelor, master and $\mathrm{PhD}$ degrees. Many students return to home regions where they pursue careers in administration, business and other sectors (State Polar Academy, n.d.)

\subsubsection{Post-secondary education in the Arctic: patterns and trends}

\section{Regional differences}

The Circumpolar North has different historical legacies of postsecondary education, leading to regional differences in levels of post secondary educational achievement. Russia and the Nordic countries have a relatively high proportion of the population with post-secondary education, while parts of northern Canada and Alaska show a relatively low level. While post-secondary education has been emphasized as an important tool in both regional and minority development in Russia and in the Nordic countries, a similar trend has been missing or been entered into quite recently in substantial parts of the North American continent. Regional differences in the percentage of people in the North with postsecondary education are illustrated in Figure 9.3. 
Figure 9.3a: Proportion of people with post-secondary education

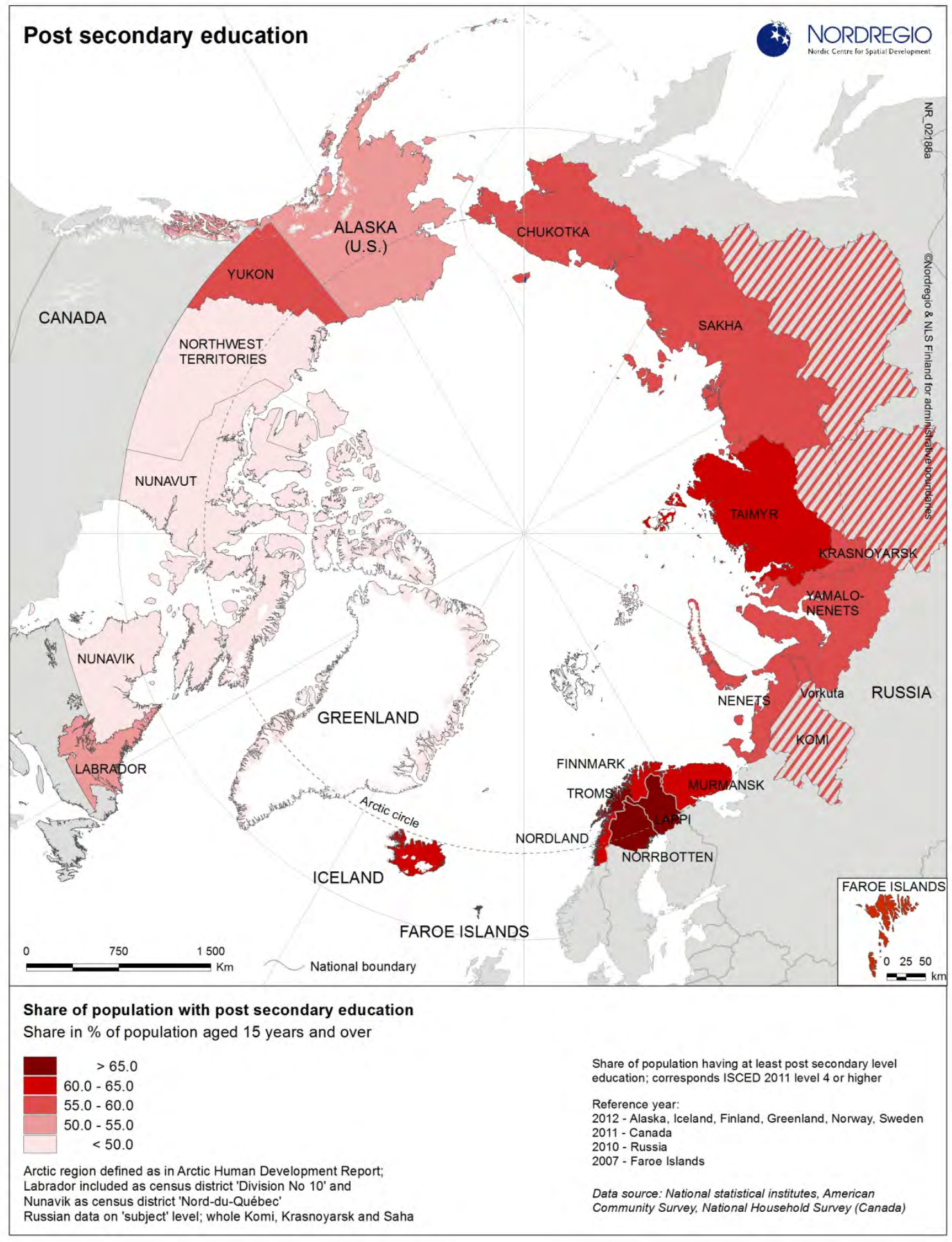


Figure 9.3b: Proportion of people with tertiary education

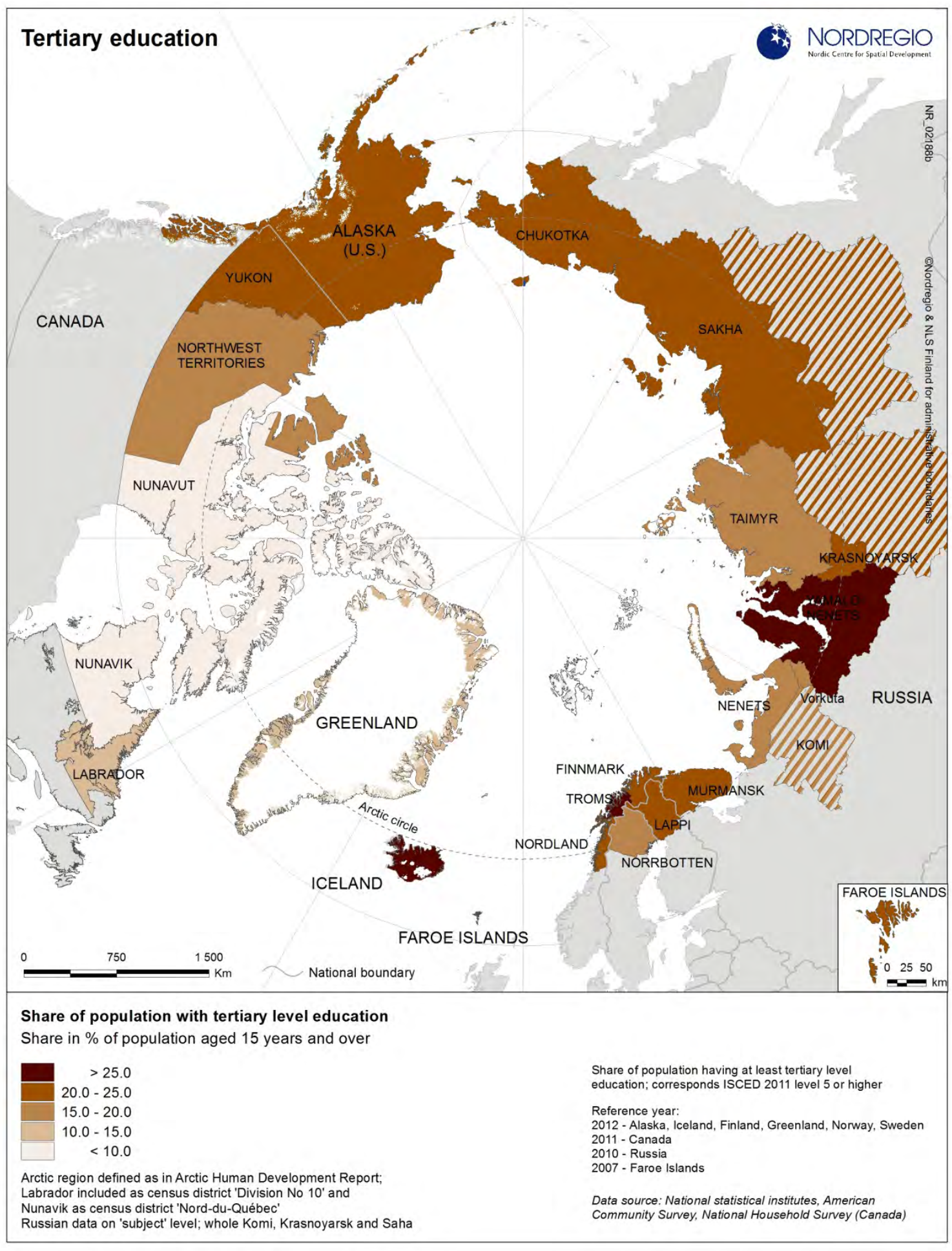


The differences in post secondary education policies make it difficult to compare jurisdictions. However, we can see patterns emerge that supersede national boundaries and historical legacies. Witness the high level of education in major resource regions, such as Yamal-Nenets district in Russia, and in more diversified regional economies, such as Yukon in Canada. Such high levels are also seen in the more urbanized parts of the Arctic, including Murmansk region of Russia, northern Scandinavia, and Iceland. Studies indicate that the bulk of human capital there are "newcomers" or non-Indigenous residents (although there are some exceptions) mostly working in resource sectors and public administration (Megatrends, 2011; Voswinkel, 2012). Human capital is also heavily concentrated in urban settlements, e.g. Troms $\varnothing$ in northern Norway, Rovaniemi in Finland, Murmansk, Salekhard, Magadan, and Noril'sk in northern Russia, Whitehorse and Yellowknife in Canada and Anchorage, Fairbanks and Juneau in Alaska.

To provide a more revealing picture of post-secondary education attainment in the Arctic and avoid definitional and historical discrepancies among Arctic regions, we can analyze the location quotient (LQ) of people with a university degree, sometimes referred to as the Talent Index (TI) (Florida, 2002). Since LQ measures relative proportion of educated people in the total population, it allows comparing regional figures in the Arctic with a baseline, which in this case is represented by a respective country, illustrating human capital accumulation relative to country's average. Northern regions of Canada, especially Yukon, have high concentration of educated professionals (in fact, in 2006 Yukon had the largest share of adult population with post-secondary accreditation among Canadian provinces and territories - 53.6\% (YBS, 2008). For example, Anadyr (the capital of Chukotka) has a LQ 1.72, compared to Moscow with an LQ of 1.79. More urbanized and resource-rich YamalNenets and Murmansk regions have higher proportion of residents with post-secondary education compared to the rest of Russia, so do several Alaskan boroughs and Nuuk in Greenland.

In fact, many Arctic cities demonstrate relatively high LQ of people with a university education. Very high LQs are also observed in other regional (and national) capitals both in Russia and across the Arctic, including Salekhard, Yakutsk, Umeå, Magadan, Juneau, Yellowknife, Tromsø, Reykjavik and Nuuk. Another large cluster of highly educated labor force is observed in the Yamal-Nenets region of Russia. In addition to its capital, Salekhard, Novy Urengoy and Nadym have LQs above 1.4. This may reflect the influx of educated labor migrants in the last decade as LQs in these cities grew substantially between 2002 and 2010 . 
At the same time, the majority of Arctic territories lag behind their southern metropoles in formal education levels. This gap is especially evident in areas with relatively high Indigenous populations, such as Nunavut, rural Alaska, and the Nenets, Koryak and Taimyr regions of Russia. Small remote urban communities, such as Iqaluit, Dudinka, Tura, and Susuman, also have low levels of educational attainment. Slightly higher, but still a relatively low Talent Index is observed in the "old" industrial cities of the Russian North: (e.g. Noril'sk, Apatity, Olenegorsk, Monchegorsk, and Vorkuta).

Tertiary education (beyond first post-secondary degree) is skewed by the differences in educational systems. North American jurisdictions to have high levels of tertiary education (post-baccalaureate), while the Scandinavian and Russian North demonstrate a lower prevalence of tertiary degrees because post-secondary education frequently ends with a masters-equivalent degree. Important differences are found within countries: for instance, the gap between Yukon, NWT and Nunavut, Nunavik and Labrador in Canada, or between affluent regions of western Siberia and the rest of the Russian North (Figure 9.3b).

This analysis demonstrates two gaps in Arctic post-secondary education: the gap between the Arctic and southern regions and the gap between urban/industrial Arctic territories and the rest of the Arctic. Generally, the pattern of less formal post-secondary education in the North is prevalent.

Whereas human capital in the Arctic is typically viewed as underdeveloped, such a view fails to reflect the variability and diversity of Arctic regions. Recent research points to substantial levels of creativity based on non-codified informal knowledge, which might not conform the conventional notion of human capital (Aarsæther, 2004; Copus and Skuras, 2006; Petrov 2007; Petrov, 2008; Petrov and Cavin, 2013). Creative human capital is critical for economic development and socio-economic transformation in the Arctic, as it often becomes the engine of economic reinvention and revitalization of a region (see Textbox 4.6 in Chapter 4, Economic Systems). 
Salekhard Pedagogical College, Russian Federation

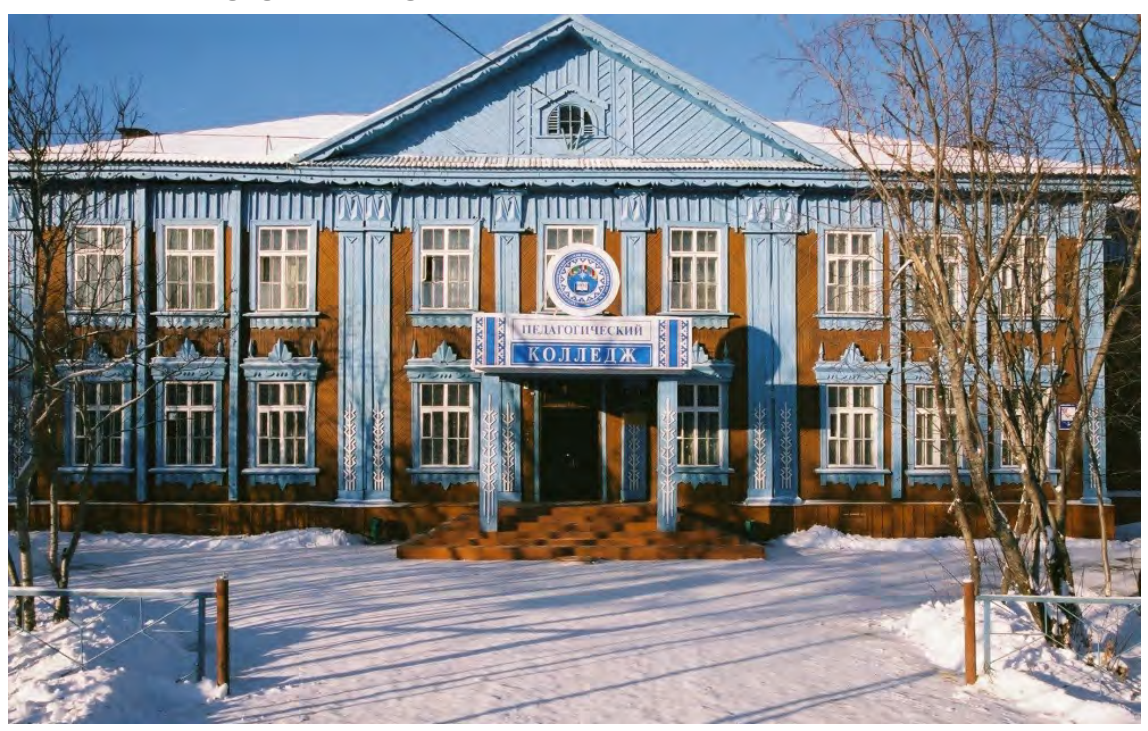

Credit: Harald Finkler.

Changes in post secondary education accessibility and attainment

Access to post-secondary education is a key component in harnessing human capital. The first AHDR concluded that educational opportunities for Arctic residents are improving. Today, there are more educated people in the Arctic than a decade ago, and options for post-secondary training in the regions have increased. Almost every jurisdiction has a university or a college. However, regional differences within the Arctic persist: postsecondary educational opportunities are still more extensive in northern Europe and Russia than in the far north of Canada and the United States. There is a relatively lower proportion of people with post-secondary education in the Canadian Territories and Alaska. In addition, the ability of Indigenous people to access institutions of higher learning, while improving, is still problematic (Stonechild, 2006; Preston, 2008; Megatrends, 2011) While opportunities are improving, many Arctic residents are still compelled to pursue education elsewhere, and their return to the home region after completing their education is far from guaranteed (Textbox 9.3). 


\section{Textbox 9.3}

\section{Human capital dynamics and turnover: successes and challenges in the Russian Arctic}

Migration plays a key role in regulating human capital accumulation in urban communities. Faced with the collapse of the Soviet economy and/or bust-andboom cycles of the resource sector many educated residents leave the Arctic (Petrov, 2006; Petrov, 2010). At the same time industrialization and attractive labor compensation brings an influx of human capital to the North during favorable times. Both processes create considerable volatility and turnover in human capital in the region. The most effective mitigation strategy for this problem is education and retention of local youth. In the recent years many northern cities increased their own educational capacities. Yukon Territory heavily invested in Yukon College, Greenland developed its own university, and some Russian cities increased the number of institutions of higher education. In fact, the number of students pursuing higher education in Russia's Territories of the Extreme North and Equated Areas more than doubled between 1999 and 2009 (Rosstat, 2012). Nonetheless, out-migration of college students is one of the primary problems for many northern regions. In a pilot survey conducted by the University of Northern Iowa and State Polar Academy in St. Petersburg, Russia (Van Drasek, 2012), most student-northerners (90\% Indigenous people) who left the Arctic in order to receive education in St. Petersburg indicated that there were few or no opportunities for higher education in their respective home regions. Prospects for professional success were also generally considered lower at home and students felt compelled to pursue opportunities elsewhere. At the same time when asked about plans to return to their home regions, the vast majority of participants replied that jobs and career prospects were the most important factors. Many students also indicated that they may not return to the same town, village, or community, but would be interested in relocating to a larger city within their home region. Many, but not most, also indicated that they would have stayed to pursue their studies and careers in the Arctic if the similar professional and educational and educational opportunities existed in their home region. When asked what could be done to improve opportunities for young people to pursue creative careers in their home regions most students suggested an increase in government spending on institutions of higher education, related infrastructure, and university instructors. Investment in skilled trades and jobs in Northern regions was also cited as a possibility to train and employ local residents rather than lose them to other regions. 
These results are indicative of two things. One is that Russian northern cities are not well positioned to educate and retain human capital. Another is that young northerners are willing to consider being educated and living in their home regions, if educational and job opportunities are good. Investment in education in Arctic cities in Russia and in other Arctic counties is an important priority that can assist in sustaining a long-term economic viability of Arctic cities.

Gains in post-secondary education in the last decade have been observed in many Arctic regions. For example, in 1999, in NWT 46.5\% of the population over the age of 15 had a certificate, diploma or degree beyond high school. This increased to $47.6 \%$ in 2009 (NWTBS, 2009). However, in the same period, the percent of population with a university degree jumped from $14.0 \%$ to $19.3 \%$. Likewise, in 2009 there were 3.3 times more Aboriginal people with university education than in 1999 (NWTBS, 2009). Whereas Indigenous residents still had a dramatic university education gap compared to non-Indigenous residents (4.9\% vs. $32.3 \%$ ), the gap has been slowly closing. In addition, higher education institutions still struggle to retain and graduate Indigenous students. For example, in 2010-2011, the retention rate for Indigenous students at the University of Alaska was 59\% compared with $75 \%$ for all students, and the 6 -year graduation rate was $12 \%$ compared with $28 \%$ overall. Special programs, such as the Post-Secondary Student Support Program in Canada, have been established in many Arctic countries to provide direct support to Indigenous students to complete post-secondary degrees (Usher, 2006). For example the U.S. government launched the Initiative on American Indian and Alaska Native Education that seeks to expand post-secondary education opportunities and improving education outcomes for all American Indian and Alaska Native students (WH, 2011). 
Figure 9.4a: Gender distribution of Arctic residents with post-secondary education

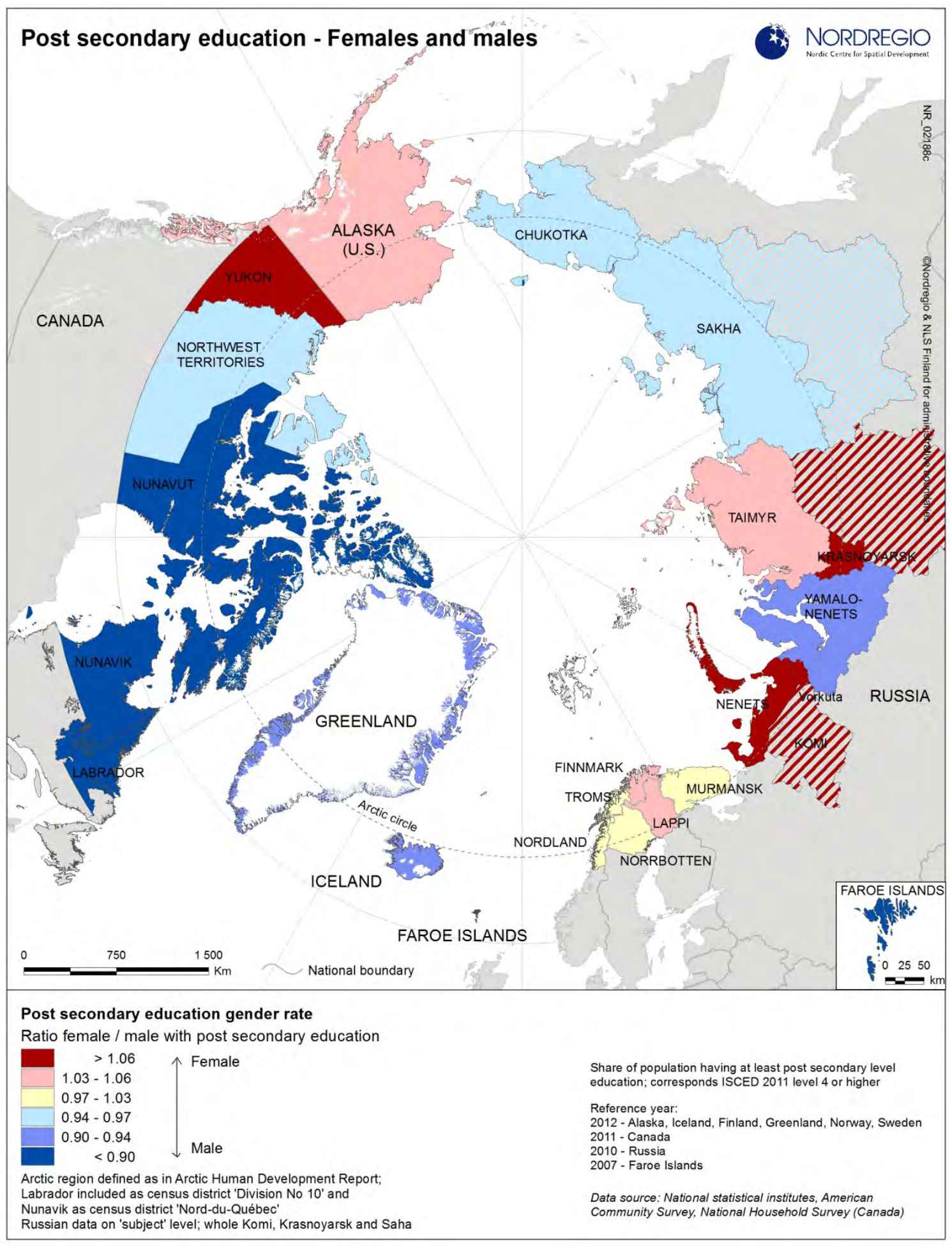




\subsubsection{Gender: feminization of human capital}

Marked changes in gender patterns of education took place during the last 10-15 years. Figure 9.4 illustrates how women dominate the realm of education in most of the Arctic. In the 1990s, women had become the majority group in relation to higher education in several countries, and by the late 1990s, this occurred throughout most regions in the Arctic. Everywhere in the Arctic, with the exception of the Faroe Islands, there are more women with tertiary education than there are men. Northern Scandinavia, Russia and Alaska are three areas with the most feminized human capital. Some resource regions in Canada and Russia, as well as Greenland, Iceland and Faroe Islands show a dominance of males in relation to post-secondary education, a situation attributable to both women's departure to pursue educational opportunities and an influx of educated male labor force attracted by the resource sector.

Many young women tend to leave the region to pursue their educational goals. This situation is prevalent in the Faroe Islands where more than $60 \%$ of students, especially women, leave home to study, predominantly in Denmark (Megatrends, 2011). There they pursue careers where they are able to take advantage of their acquired skills. This has also been the pattern in Greenland, due to the limited level of social acceptance traditionally afforded to highly qualified women. However, in recent years, women have assumed an increasing number of positions requiring higher education.

The feminization of human capital is a rather new phenomenon. In Greenland, males dominated the educational system until around 1990, with $5-10 \%$ more boys than girls finishing a secondary or postsecondary diploma or degree. However, during the 1990s and the 2000s, between 10 and $20 \%$ more girls than boys finished an education, and since 2003 more than $60 \%$ of the persons finishing an education have been girls (Rasmussen et al., 2010). Differences are also clearly seen in educational choices. Among the boys registered as active students, almost $70 \%$ are in vocational training and $30 \%$ in short and long term academic programs. Among the girls, however, there is an almost equal division between vocational and academic training (53\% and $47 \%$ respectively (Megatrends, 2011)). 
Figure 9.4b: Gender distribution of Arctic residents with tertiary education

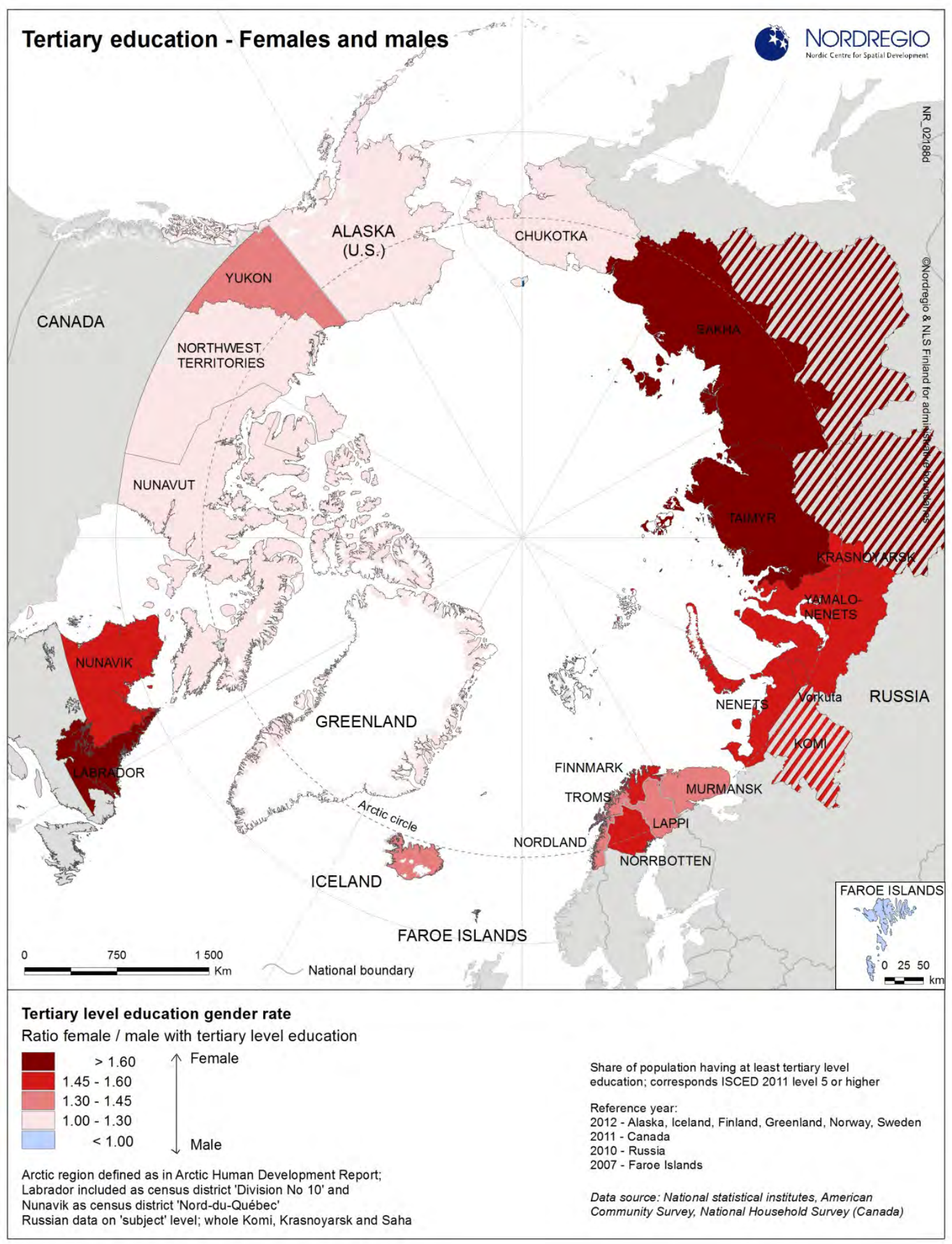


Alaska is another region where feminization of human capital over the last decade has led to an increase in women with post-secondary education (52.3\% as compared to $47.7 \%$ among men). Alaskan women are also more educated: $29.8 \%$ of female Alaskans have post-secondary education as opposed to $25.2 \%$ of males. At the same time, men lead among adult residents with post-baccalaureate degrees (especially in professional and doctorate degrees, where the male to female ratio is 1.5:1) (U.S. Census Bureau, 2012).

Data from NWT show that while there are still more men with postsecondary education, women experienced steady gains in the last 10 years and by 2009 constituted $46 \%$ of post-secondary educated population over the age of 15 (NWTBS, 2009). Women are traditionally better represented in certain fields of study (e.g. healthcare, education and training, humanities) and less represented in others (engineering, applied sciences). However, in recent decades women gained considerable ground in some male-dominated fields such as engineering, applied sciences, mathematics, computer and physical sciences. In the Russian Arctic, women dominate among residents with higher (postsecondary) education. The gap between men and women is fairly substantial: for example, $24.5 \%$ of adult female residents of Murmansk oblast' have completed a post-secondary degree, compared to $20.8 \%$ of male residents. In Yamal-Nenets okrug, a region with quite different population structure and settlement history, the gender gap is even more pronounced: $31.1 \%$ of women attain post-secondary education and $22.8 \%$ of men. However, in rural areas the gap is less significant or men comprise a larger percent of university graduates than women (e.g. in Murmansk oblast). The Yamal-Nenets data also indicate that in the last decade the gender gap in favor of women was widening, particularly in urban areas (Rosstat, 2013).

\subsubsection{Indigenous vs. non-Indigenous human capital: closing the education gap}

The levels of engagement in post-secondary education among Indigenous and non-Indigenous people clearly illustrate an "education gap". Closing this gap is not only beneficial for Indigenous individuals in terms of lifetime earnings (Howe, 2011; Hull, 2005), but will likely inject millions in the economy (Sharpe et al., 2007). It is dramatic in some regions, and less evident (but still present) in others. NWT is the case in point as a region with a drastic education differential: only $4.9 \%$ of NWT Aboriginal residents held university degrees in 2009 compared to $32.3 \%$ of 
non-Aboriginal persons (NWTBS, 2009). Although Indigenous people in NWT made formidable gains in the last decades (only 1.8\% had university degrees in 1999), the gap is still wide (NWTBS, 2009).

In Russia, the discrepancy between Indigenous and non-Indigenous population can be approximated using statistics for urban (predominantly non-Indigenous) and rural (heavily Indigenous) areas. The gap in percent of formally educated individuals with a post-secondary degree varies from region to region between 5 and 20\%. For example, only $14.3 \%$ of rural residents in Yamal-Nenets okrug have attained the postsecondary level compared to $29.2 \%$ of urban dwellers. Similarly, in Chukotka higher education is reported by $7.6 \%$ of adults living in rural areas in contrast to $28.4 \%$ of adults living in urban settings. Only $5.9 \%$ of rural men in Chukotka (mostly Indigenous people) have a post-secondary degree, reflecting both the deep urban-rural and Indigenous - nonIndigenous education gaps (Rosstat, 2012).

The differences in education attainment likely arise from a variety of factors including differences in entrance requirements, varying levels of individual academic and social integration, conflict between educational activities and needs of traditional economy and the interplay between the institution and the surrounding community (Barnhardt and Kawagley, 2004; Darnell and Hoëm, 1996; Keskitalo, 1998; Rasmussen et al., 2010). Mainstream (western) education systems are not always adjusted to consider local context, community needs and aspirations of young Indigenous people. As noted above, pursuit of post-secondary education often requires a departure from northern communities, relocation within their region or to outside southern cities. Many students felt that detachment from families and native land were difficult tradeoffs of their decision (Van Drasek, 2012). A major concern is a drop-out rate from both school and higher education institutions (Gilmore, 2010; Berger, 2006; see Part 1 of this chapter).

Difficulties in the secondary education system faced by Indigenous students and low graduation rates shrink the number of northerners who pursue post-secondary, including trade certificates, college diplomas or university degrees. The problem is exacerbated because skills acquired by northerners in high school (including literacy/proficiency in English or other language used in the post-secondary system) are not always at a level acceptable to many post secondary institutions. Northerners (both Indigenous and non-Indigenous) may also be disadvantaged by the lack of appropriate courses and resources in their local schools, and by the shortage of qualified teachers. Finally, a pursuit of 
post-secondary, especially university education, most often requires leaving home community or even relocate outside the Arctic.

Literature on Indigenous education strongly argues that a more effective education system in the Arctic must be based on systemic integration of a western science, knowledge and traditional (local and Indigenous) knowledge (Barnhardt, 2005; Berger, 2006). The integrated curriculum aims to connect both cultural and instrumental needs and aspirations, and provide balanced training to develop skills and personal values commensurable with local culture (Rasmussen et al., 2010). The emphasis on practical skills may also curtail dropout rates, decrease absenteeism and enhance student performance by making studies more relevant and culturally valuable. These principles, for example, are embedded by the new Nunavut high school curriculum developed in cooperation between educators and Inuit elders (CBC News, 2012).

\subsubsection{Human capital and local and Indigenous knowledge (LIK)}

Northern communities have informal, highly adaptive traditional knowledge systems that have ensured the survival of many generations in harsh environment conditions (Cruikshank, 2005). These systems, known here as Local Indigenous Knowledge (LIK) systems, include technologies, "know-how" skills, practices and beliefs that have been transferred between generations in Arctic societies. LIK is a set of experiences generated by Indigenous people developed in order to adapt to local environments. It is embedded in community practices, institutions, and rituals (Berkes, 1993; UNESCO, 2013).

The limited role of LIK in formal education, and the lack of recognition afforded to LIK as a component of human capital capacity of Arctic regions continues to curtail competitiveness and well-being of northern communities and societies. The ASI recognized the need to shift this practice, and more fully incorporate LIK into Arctic educational and economic systems as a way of ensuring prosperity, improving material wellbeing, and advancing human development (ASI, 2010).

Integrating LIK requires reversing a historic trend. Throughout the Arctic, western educational systems have historically marginalized and rejected LIK. Modernization, acculturation and assimilation went hand in hand with the erosion of LIK. Indigenous languages, one key component of LIK, have been under siege by education systems that use and impose the "colonial" language. Language retention, which is a key issue in ensuring empowerment and cultural vitality of Indigenous people, is 
also critical for maintaining the breadth of LIK (see Chapter 3, Cultures and Identities). The loss of LIK system, its replacement by the imported knowledge while may provide some new opportunities for local residents, also results in the deterioration of traditional economies and social institutions, brings about negative consequences that can negate benefits of modernization.

Arctic societies have a unique combination of human, social and civic capital that provides opportunities for enhancing material well-being while simultaneously ensuring cultural vitality and sustaining traditional livelihoods. Recent studies revealed that human capital accumulation and creativity of the Arctic labor force is not always associated with the western educational paradigm (e.g., Petrov, 2008). This is especially true for cultural capital (see Textbox 4.6 in Chapter 4, Economic Systems), a form of human capital based on economic engagement of cultural activities and practices. Cultural capital in northern communities is not correlated with levels of post-secondary education, but rather is strongly associated with the vibrancy of Indigenous culture and LIK (Petrov and Cavin, 2013).

Another strong economic endowment, associated with LIK and social and civic capital, is social economy. Indigenous societies have a longlived tradition of cooperation, sharing and community-building to sustain their prosperity. This knowledge finds its use in the growing social economy of the Arctic. For example, northern cooperatives in Canada are a major economic actor and an example of successful integration of western knowledge of business and technology and LIK (SERNNoCa, 2012). The Arctic Co-operatives Limited in Canada is a federation of 31 co-ops in NWT and Nunavut owned and controlled by 22,500 members. In 2011 co-ops reported CAD 197 million in total revenues and employed 800 workers (ACL, 2012). 


\section{Textbox 9.4}

\section{Local and Indigenous knowledge, culture and post-secondary education}

Across Norway, Sweden and Finland, numerous academic and vocational postsecondary programs are based in the Sámi culture. The universities in Tromsø (Norway), Umeå, (Sweden) and Oulu (Finland) are regarded as national hubs for Sámi education and research and all have specially organized Sámi study and research centers. Additionally in Finland both the University of Helsinki and University of Lapland offer Sámi language programs and Sámi-related programs as well as organized Sámi research. The Giellagas Institute for Sámi studies, located at the University of Oulu as an independent organization, has a nationwide responsibility to organize, introduce and provide Sami language and cultural studies and research at the academic level. One adult training center in Finland, the Sámi Institute in Inari, offers a range of programs for the Sámi area including Sámi language and reindeer herding programs and organizes university level courses together with universities.

The Sámi University College in Norway is a college specializing in Sámi higher education, running programs and administration in northern Sámi language. It runs several types of Sámi teacher training including virtual delivery of programs. The college offers up to master's degree in traditional Sámi craft (duodji), language and Sámi/Indigenous journalism. The college also offers programs including traditional knowledge, such as a reindeer husbandry bachelors program.

In Canada, a unique initiative for teaching and learning traditional knowledge is located in Clyde River, Nunavut. Part of Nunavut Arctic College, Piqqusilirivvik is a cultural learning center dedicated to enabling the transfer of traditional Inuit culture, knowledge, lifestyle, skills, and values, taught in the Inuit language and based on Inuit Qaujimajatuqangit guiding principles. Students 18 and over who can speak the Inuit language come from all over Nunavut to attend Piqqusilirivvik programs. The center houses students for four-month programs (two per year) and programming is based on the season cycle of the year and traditional Inuit activities. Programs involve a mix of land-based activities, classroom courses, and hands-on learning. Students also learn and use digital technology like cameras, GPS, and computer video editing programs to document their learning and share traditional knowledge. Piqqusilirivvik had its grand opening in May 2011. It has two satellite programs, in Baker Lake and Igloolik, Nunavut. 
The University of Alaska recently established a $\mathrm{PhD}$ program in Indigenous Studies with emphases in areas of research, education, Native languages, leadership, Indigenous knowledge systems and Indigenous sustainability. This new $\mathrm{PhD}$ is designed to integrate the tools and approaches of the natural and social sciences in a crosscultural and interdisciplinary framework for analysis to better understand the emerging dynamic between Indigenous knowledge systems, western science and higher education. Emphasis is placed on the interface between Indigenous knowledge and science on an international scale, with opportunities for collaboration among Indigenous peoples from throughout the Circumpolar region

\subsection{Summary and trends}

In the ten years since the first $A H D R$, significant changes have occurred in northern education. Post-secondary education attainment is increasing in many Arctic regions. New technologies are providing increased opportunities for distance education at all levels. There is growing recognition of the importance of local and Indigenous knowledge (LIK) at both the K-12 and post-secondary education levels. Indeed, the latest shifts in education systems described in this chapter indicate a movement towards integrating different knowledge systems (Barnhardt, 2005; Rasmussen et al., 2010) in order to maximize human development in the Arctic (Textbox 9.4). As western and LIK educational systems are increasingly intertwined, they serve individuals and communities in enhancing material and cultural well-being of northern populations. LIK not only enhances the opportunities for increased prosperity for Arctic communities, it gives these communities a competitive, yet untapped, edge in a globalized economy.

Education and human capital development is key to economic wellbeing, fate control and human development in general. And yet, numerous tensions obstruct the provision of both academic and career and technical education. For example, there is a tension between providing education that is local, place-based, and based on LIK, and schooling to prepare students to be globally competitive. Incorporation of local and Indigenous knowledge into educational and economic systems of the Arctic is a cornerstone of Arctic prosperity in the future. But the limited role of LIK in formal education and lack of LIK's utilization to enhance human capital capacity of Arctic regions curtails the competitiveness and well-being of northern communities and societies. Creating an edu- 
cation system based on LIK and one aimed at producing students prepared for the global economy are not inherently conflicting goals, and in some places (e.g., North Slope Borough School District in Alaska) these are seen as complementary goals. Still, many educators and policymakers see these as incompatible.

Another tension is around where schooling is provided. While mandating that students attend boarding schools is controversial, due in part to past abuses and the loss of culture resulting from these institutions, in some places residential schools are seen as preferred or only options. Providing access to a broad curriculum and resources is difficult in small and remote schools. Moreover, maintaining local schools in many remote northern communities is difficult due both to population outmigration and fiscal constraints.

Yet another tension exists around the use of Indigenous languages in education: There is both an increasing use of Indigenous languages in formal schooling and a continuing threat to Indigenous languages. There is a strong push in many northern school systems to offer education in the languages of the Indigenous people from those regions, particularly in Canada and across Norway, Finland and Sweden. At the same time, Indigenous languages continue to face threats across northern Russia and Siberia and in much of Alaska.

Gaps persist in education outcomes across northern regions, including between the Arctic and southern regions, urban/industrial arctic territories and the rest of the Arctic, and between Indigenous and nonIndigenous population in the Arctic. In addition, there is a growing gender gap in education and feminization of human capital: Women dominate the realm of education in most of the Arctic, especially in rural and remote communities. Across the Arctic girls outperform boys on standardized measures of achievement and graduate from high school at higher rates. Moreover, already in the 1990s women had become the majority group in relation to higher education in several countries, and by the late $1990 \mathrm{~s}$ virtually all regions in the Arctic had moved into this situation.

High dropout rates continue to be a major concern in the Arctic both from secondary schools and higher education institutions, especially among Indigenous students. Likewise, the high mobility of human capital in the Arctic is a considerable challenge; there is for much of the North a lot of population loss, and a prevalence of "brain drain", "brain turnover" (intensive in- and out-migration of human capital) and "brain waves" (surges and dips of human capital associated with the boom-and-bust economic cycles). 
Arctic communities will not progress without an education system that adequately prepares students to address all of the challenges raised in this report, from climate change to health threats to economic development needs. Our hope is that the trends toward increasing graduation rates, integrating LIK into education systems and growing opportunities for women are joined by better outcomes for male students, improved access to education for rural and remote students and a system that prepares students to be successful within local and global communities.

\subsection{References}

Aarsæther, N. (ed.) (2004). Innovations in the Nordic Periphery. Nordregio, Stockholm. AHDR (2004). Arctic Human Development Report. Einarsson, N., J. N. Larsen, A. Nilsson and O. R. Young (eds.). Stefansson Arctic Institute, Akureyri.

Alaska Department of Education and Early Development (AK EED) (2013). State Report Card 2012-2013. Juneau. http://education.alaska.gov/reportcard/20122013/reportcard2012-13.pdf (1st September 2014).

Alsop, J. (2010). History of Cape Dorset and the West Baffin Cooperative. Working Paper. University of Victoria, Victoria.

ASI (2014). Arctic Social Indicators II: Implementation. Larsen, J.N., P. Schweitzer, and A. Petrov (eds.). TemaNord. Nordic Council of Ministers, Copenhagen.

ASI (2010). Arctic Social Indicators - a follow-up to the Arctic Human Development Report. Larsen, J.N., P. Schweitzer, and G. Fondahl (eds.). TemaNord. Nordic Council of Ministers, Copenhagen.

Aylward, M. L. (2012). The Inuit Qaujimajatuqangit conversation: The language and culture of schooling in the Nunavut Territory of Canada. In: Bekerman, Z. and T. Geisen (eds.). International Handbook of Migration, Minorities and Education: Understanding Cultural and Social Differences in Processes of Learning, pp. 213-229. Springer, Netherlands. http://dx.doi.org/10.1007/978-94-007-1466-3_15

Aylward, M. L. (2010). The role of Inuit languages in Nunavut schooling: Nunavut teachers talk about bilingual education. Canadian Journal of Education, 33(2):295328.

Aylward, M. L. (2009). Journey to Inuuqatigiit: curriculum development for Nunavut education. Diaspora, Indigenous, and Minority Education, 3(3): pp. 137-158. http://dx.doi.org/10.1080/15595690902991022

Barnhardt, R and A.O. Kawagley (2004). Culture, chaos and complexity: catalysts for change in Indigenous education. Cultural Survival Quarterly, 27: 59-64.

Barnhardt, R., 2005. Creating a Place for Indigenous Knowledge in Education: The Alaska Native Knowledge Network. http://www.ankn.uaf.edu/Curriculum/Articles/ RayBarnhardt/PBE_ANKN_Chapter.html (1st September 2014).

Battiste, M. (2000). Maintaining Aboriginal identity, language, and culture in modern society. In: Battiste, M. (ed.). Reclaiming Indigenous Voice and Vision, pp. 192-208. UBC Press, Vancouver.

Bell, D. (1973). The Coming of Post-Industrial Society. Basic Books, New York.

Berger, P., and J.R. Epp (2007). There's no book and there's no guide: the expressed needs of Qallunaat educators in Nunavut. Brock Education, 16(2): pp. 44-56. 
Berger, P., J.R. Epp, and H. Møller (2006). The predictable influences of culture clash, current practice, and colonialism on punctuality, attendance, and achievement in Nunavut schools. Canadian Journal of Native Education, 29(2): 182.

Berger, T. R. (2006). The Nunavut project: Conciliator's final report: Nunavut land claims agreement: Implementation contract negotiations for the second planning period, 2003-2013. Bull, Housser \& Tupper, Vancouver.

Berkes, F. (1993). Traditional ecological knowledge in perspective. In: Inglis, J.T. (ed.). Traditional Ecological Knowledge: Concepts and Cases, pp. 1-9. Canadian Museum of Nature/International Development Research Centre, Ottawa.

Boolsen, M. W. (2009). The Education Reform 2009. http://www.wattboolsen.dk/ files/TheEducationalReform090620.pdf (1st September 2014).

CBC News (2012). Nunavut unveils new high school curriculum. 10th February 2012. http://www.cbc.ca/news/canada/north/nunavut-unveils-new-high-schoolcurriculum-1.1256723 (1st September 2014).

CETS 148 (1992). European Charter for Regional or Minority Languages. Council of Europe Treaty Series 148. http://conventions.coe.int/Treaty/en/Treaties/ Html/148.htm (1st September 2014).

Chevalier, J. F. (2012). Multilingual education in South Siberia: national schools in the republics of Altai and Tyva. Heritage Language Journal, 9(2): 1-15.

Christensen, S. (2012). Living lands: education and growth. Presentation at the 18th Inuit Studies Conference, Washington, D.C., 24th-28th October.

Copus, A., and D. Skuras (2006). Accessibility, innovative milleux and the innovative activity of businesses in the EU peripheral and lagging areas. In: Vaz, T. N., E.J. Morgan, and P. Nijkamp (eds). The New European Rurality: Strategies for Small Firms, pp. 29-40. Ashgate, Aldershot, England.

Cram, J. (1987). Northern Teachers for Northern Students: An Inuit Teacher Training Program, Education, Research, Information Systems and the North, Proceedings of the Association of Canadian Universities for Northern Studies (ACUNS) meetings, pp. 116-23. Yellowknife, 17th-19th April 1986.

Cruikshank, J. (2005). Do Glaciers Listen? Local Knowledge, Colonial Encounters and Social Imagination. UBC Press and University of Washington Press, Vancouver and Seattle.

Dahl, J., G. Fondahl, A. Petrov, and R. Sverre Fjellheim (2010). Fate Control. In: ASI, 2010. Arctic Social Indicators - a follow-up to the Arctic Human Development Report. Larsen, J.N., P. Schweitzer, and G. Fondahl (eds.), pp. 129-146. TemaNord. Nordic Council of Ministers, Copenhagen.

Darnell, F. and A. Hoëm (1996). Taken to Extremes. Scandinavian University Press. Oslo. de Krom, Keleutak, Quumaluk et Taylor (2011). Décoloniser un héritage linguicide en misant sur l'éducation bilingue au Nunavik: coordination de la politique, des programmes et de la recherche [Decolonizing the legacy of linguicide by focusing on bilingual education in Nunavik: Coordination of policies, programs and research], In: Petit, Bonnier, Viger, Aatami et Iserhoff (eds.), Les Inuit et les Cris du Nord du Québec, pp 273-288. Presses de l'Université du Québec, Quebec.

De la Fuente, À. and Ciccone, A. (2002). Human capital in a global and knowledge based economy. Final Report. Employment and Social Affairs, European Commission.

DeMarban, A. (2012). In Rural Alaska, villages fight extinction once schools close. Alaska Dispatch, 10th April 2012. http://www.adn.com/article/rural-alaskavillages-fight-extinction-once-schools-close (1st September 2014).

Dudeck, S. (2013). Challenging the state educational system in Western Siberia: Taiga school by the Tiuitiakha River. In: Kasten, E. and T. de Graaf (eds.). Sustaining Indigenous Knowledge: Learning Tools and Community Initiatives for Preserving En- 
dangered Languages and Local Cultural Heritage, pp. 129-157.

http://www.doria.fi/bitstream/handle/10024/88080/

SIK_dudeck_2013.pdf?sequence=1 (1st September 2014).

EU Commission (2013). Study to evaluate the performance of Higher (Tertiary) Education in Greenland. http://naalakkersuisut.gl/ /media/Nanoq/Files/ Attached\%20Files/Uddannelse/DK/Uddannelsesplaner/Study\%20to\%20evaluate\% 20Higher\%20Education\%20in\%20Greenland\%202013.pdf (1st September 2014).

Florida, R. (2002). The economic geography of talent. Annals of the Association of American Geographers, 94(2):743-755. http://dx.doi.org/10.1111/1467-8306.00314

Gaski, H. (1998). Sámi Culture in a New Era. In: Gaski, H. (ed.). Sámi Culture in a New Era, pp. 9-28. University of Washington Press, Seattle.

Gilmore, J. (2010). Trends in dropout rates and the labour market outcomes of young dropouts. Education Matters: Insights on Education, Learning and Training in Canada, 7(4).

Nunavut Government (2008). Nunavut Education Act, Chapter 15, Section 1(1).

Gouvernement du Québec (2011). Diplomation et qualification au secondaire [Secondary graduation and qualification] Édition 2011. Publication officielle du Ministère de l'Éducation, du loisir et du sport.

Greenland (2002) School Act, Chapter 2, Section 5.

Hadland, J. (2004). Alaska's brain drain: myth or reality. Monthly Lab. Review, 127: 9.

Hargreaves, L., R. Kvalsund and M. Galton (2009). Reviews of research on rural schools and their communities in British and Nordic countries: analytical perspectives and cultural meaning. International Journal of Educational Research, 48: pp. 80-88. http://dx.doi.org/10.1016/j.ijer.2009.02.001

Heleniak, T. 2010. Migration and population change in the Russian Far North during the 1990s. Migration in the Circumpolar North: Issues and Contexts, pp. 57-91.

Hill, A., D. Hirshberg and T. Argetsinger (2012). Formal Schooling of Indigenous Peoples in Remote and Rural Regions: Promising Models. Paper presented at the American Educational Research Association Annual Meeting, Vancouver, British Columbia.

Hill, A. and D. Hirshberg (2013). Alaska Teacher Turnover, Supply and Demand: 2013 Highlights. University of Alaska Anchorage Center for Alaska Education Policy Research, Alaska.

Hossain B. and L. Lamb (2012). The impact of human and social capital on aboriginal employment income in Canada. Economic Papers: A Journal of Applied Economics and Policy, 31 (4): pp 440-450.

Howe, E. (2011). Bridging the Aboriginal education gap in Saskatchewan. Gabriel Dumont Institute of Native Studies and Applied Research, 6.

Hull, J. (2005). Post-secondary Education and Labour Market Outcomes Canada, 2001. Prologica Research Inc., Winnipeg.

Jacobs, J., 1984. Cities and the Wealth of Nations: Principles of Economic Life. Vintage Books, London.

Johansson G., C. Paci and S. Hovdenak (2004). Education. In: AHDR, 2004. Arctic Human Development Report. Einarsson, N., J. N. Larsen, A. Nilsson and O. R. Young (eds.), pp. 169-184. Stefansson Arctic Institute, Akureyri.

Kativik School Board (KSB) (2013). Our organization. http://www.kativik.qc.ca/ en/our-organization (31st January 2013).

Keskitalo, J.H. (1998). The Saami experience: changing structures for learning. In: King, L. (ed.). New Perspectives on Adult Education for Indigenous Peoples, pp. 186200. UNESCO Institute for Education, Hamburg. 
Laidler, G. and A. Petrov (2013). The North: balancing tradition and change. In: R. Tremblay and H. Chicoine (eds.). Geographies of Canada. pp.393-452. P.I.E. Peter Lang, Brussels.

Lavrillier, A. (2013). Anthropology and applied anthropology in Siberia: Questions and solutions concerning a nomadic school among Evenk reindeer herders. In: Kasten, E. and T. de Graaf, (eds.). Sustaining Indigenous Knowledge: Learning Tools and Community Initiatives for Preserving Endangered Languages and Local Cultural Heritage, pp 105-127. http://www.doria.fi/bitstream/handle/10024/ 88080/SIK_dudeck_2013.pdf?sequence=1 (1st September 2014).

Martin, S. and A. Hill (2009). Webnote 5. The Changing Economic Status of Alaska Natives, 1970-2007. Institute of Social and Economic Research, Anchorage. http://www.iser.uaa.alaska.edu/Publications/webnote/WebNote5.pdf (1st September 2014).

McDiarmid, G. and A. Hill, A (2010). Alignment of Alaska's Educational Programs from Pre-School through Graduate Study: A First Look. ISER Working Paper 2010.1. Institute of Social and Economic Research, Anchorage.

McGregor, H. E. (2010). Inuit Education and Schools in the Eastern Arctic. UBC Press, Vancouver.

McGregor, H. E. (2012a). Curriculum change in Nunavut: towards Inuit Qaujimajatuqangit. McGill Journal of Education, 47(3):285-302. http://dx.doi.org/10.7202/1014860ar

McGregor, H. E. (2012b). Nunavut's Education Act: education, legislation and change in the Arctic. The Northern Review, 36(1): pp. 27-52.

Megatrends (2011). Rasmussen, R.O. (ed.). Nordic Council of Ministers, Copenhagen.

Morgan, K. (1997). The learning region: institutions, innovation and regional renewal. Regional Studies, 31:491-503. http://dx.doi.org/10.1080/ 00343409750132289

National Information Centre on Academic Recognition and Mobility (NIC ARM), n.d., Ministry of Education and Science of the Russian Federation.

NBS [Nunavut Bureau of Statistics] (2012). Nunavut Secondary School Graduation Rate by Region 1999 to 2011. Government of Nunavut. 26th April 2012. http://www.stats.gov.nu.ca/en/Social\%20education.aspx (2nd September 2014).

Nikolaev, D. and D. Chugunov (2012). The Education System in the Russian Federation. Education Brief. The World Bank, Washington, D.C.

Nordicity Group (2010). Economic Impact Study: Nunavut Arts and Crafts. Final Report. Iqaluit.

Nunavut Bureau of Statistics 26th April 2012. Nunavut Secondary School Graduates, 1999 to $2011 \mathrm{http} / /$ /www.stats.gov.nu.ca/Publications/Historical/Education/ Nunavut\%20Secondary\%20School\%20Graduates,\%201999\%20to\%202011 $\% 20(2 \% 20$ tables).xls (2nd September 2014).

Nunavut Department of Education (2007). Inuit Qaujimajatuqangit: Education Framework for Nunavut Curriculum. Iqaluit: Nunavut Department of Education, Curriculum and School Services Division.

NWTBS (Northwest Territories Bureau of Statistics) (2009). Education: Highest Level of Schooling. http://www.statsnwt.ca/education/highest-level/ (2nd September 2014).

Organization for Economic Co-operation and Development (OECD) (2011).Fejl! Hyperlinkreferencen er ugyldig. Education at a Glance 2011: OECD Indicators, OECD Publishing. http://dx.doi.org/10.1787/eag-2011-en (2nd September 2014).

Oxaal, Z. (1997). Education and Poverty: A Gender Analysis. Institute of Gender Development Studies, University of Sussex, Brighton. 
Petrov, A. (2013). From Sustaining Creativity to Creating Sustainability: Talent and Creative Capital for Sustainable Development in Arctic Urban Communities.

Presentation at the Arctic Urban Sustainability Conference, 30th-31st May 2013. George Washington University. 1-8

http://www.gwu.edu/ ieresgwu/assets/docs/

Petrov_conference_arctic_cities_short.pdf (2nd September 2014).

Petrov, A. (2011). Beyond spillovers: interrogating innovation and creativity in the Peripheries. In: Bathelt, H., M. Feldman, and D. F. Kogler (eds.). Beyond Territory: Dynamic Geographies of Innovation and Knowledge Creation, pp. 168-190. Routledge, New York.

Petrov, A. (2010). Post-staple bust: modeling economic effects of mine closures and post-mine demographic shifts in an Arctic economy (Yukon). Polar Geography, 33(1/2):39-61. http://dx.doi.org/10.1080/1088937X.2010.494850

Petrov, A. (2008). A talent in the cold? Creative class and the future of the Canadian North. ARCTIC - Journal of the Arctic Institute of North America, 61(2):162-176.

Petrov, A. (2007). A look beyond metropolis: exploring creative class in the Canadian periphery. Canadian Journal of Regional Science, 30(3):359-386.

Petrov, A. and P. Cavin (2013). Creative Alaska: creative capital and economic development opportunities in Alaska. Polar Record, 49 (4):348-361. http://dx.doi.org/10.1017/S0032247412000289

Petrov, A. and T. Vlasova (2010). Migration and socio-economic well-being in the Russian North: Interrelations, regional differentiation, recent trends and emerging issues In: Huskey, L. and C. Southcott, (eds.). Migration in the Circumpolar North: New Concepts and Patterns. Pp. 163-192. CCI Press, Edmonton.

Preston, J. P. (2008). The urgency of postsecondary education for Aboriginal peoples. Canadian Journal of Educational Administration and Policy, 86:1-22.

Psacharopoulos, G., and H.A. Patrinos (2004). Returns to investment in education: a further update. Education economics, 12(2):111-134. http://dx.doi.org/10.1080/0964529042000239140

Rasmussen, R, O., R. Barnhardt, and J.H. Keskitalo (2010). Education. In: ASI I, 2010. Arctic Social Indicators - a follow-up to the Arctic Human Development Report. Larsen, J.N., P. Schweitzer, and G. Fondahl (eds.), pp. 67-90. TemaNord. Nordic Council of Ministers, Copenhagen.

Rønning, W. and A. Wiborg (2008). Education for All in the Arctic? A Survey of Available Information and Research. The Nordland Research Institute, Bodø.

Rosstat (2012). Economic and Social Indicators in the Territories of the far North and Equated Areas. Moscow [in Russian].

Rosstat (2013). Results of the All-Russia Census of 2010. Moscow [in Russian] http://www.gks.ru/free_doc/new_site/perepis2010/croc/perepis_itogi1612.htm.

Russian Federation (2012). On Education in the Russian Federation. Federal Law No 273-FZ, 29th December 2012. http://cis-legislation.com/document.fwx?rgn=57340 (1st September 2014).

Schweitzer, P., S. Irlbacher Fox, Y. Csonka, and L. Kaplan (2010). Cultural Well-being and cultural vitality. In: ASI I, 2010. Arctic Social Indicators - a follow-up to the Arctic Human Development Report. Larsen, J.N., P. Schweitzer, and G. Fondahl (eds.), pp. 91-108. TemaNord. Nordic Council of Ministers, Copenhagen.

SERNNoCa (2012). Social Economy Research Network of Northern Canada http://yukonresearch.yukoncollege.yk.ca/sern/ (3rd November 3, 2014).

Sharpe, A., J. Arsenault, and S. Lapointe (2007). The Potential Contribution of Aboriginal Canadians to Labour Force, Employment, Productivity and Output Growth in 
Canada, 2001-2017: Centre for the Study of Living Standards, Ottawa.

http://www.csls.ca/reports/csls2007-04.PDF

Poppel, B., J. Kruse, G. Duhaime, L. Abryutina (2007). Survey of Living Conditions in the Arctic: SLiCA Results. Anchorage: Institute of Social and Economic Research, University of Alaska Anchorage.

Smith, G. (2003). Indigenous Struggle for the Transformation of Education and Schooling. Keynote address to the Alaska Federation of Natives, Anchorage, AK, 23rd October 2003.

State Polar Academy, nd. www.gpa-spb.ru (7th November 2014).

Statistics Iceland (2013). Education. http://www.statice.is/Statistics/Education (2nd September 2014).

UNESCO (United Nations Educational, Scientific, and Cultural Organization) (2013). Local and Indigenous Knowledge Systems. http://www.unesco.org/new/ en/natural-sciences/priority-areas/links/ (2nd September 2014).

UN DRIP (2007). United Nations Declaration on the Rights of Indigenous Peoples: resolution / adopted by the UN General Assembly, 2nd October 2007, A/RES/61/295.

Usher, A. (2006). Grants for Students: What They Do, Why They Work. Canadian Education Report Series. Online Submission.

Stonechild, B. (2006). The New Buffalo: The Struggle for Aboriginal Post-Secondary Education in Canada. University of Manitoba Press, Winnipeg.

U.S. Census Bureau (2012). American Community Survey. http://www.census.gov/acs/www/

Utdanningsdirektoratet (2010). Årsaker til nedlagde skular i perioden 2007/20082009/10. [Causes of school closures for the period of 2007/08 - 2009/10]. Oslo.

Van Drasek, B. (2012). Creative Russia: Spatial Dimensions of Creative Capital in Russia and its Northern Frontiers. Unpublished MA Thesis. University of Northern Iowa, Cedar Falls, Iowa.

Voswinkel, S. (2012). Survey of Yukon's Knowledge Sector: Results and Recommedations. Ylynx Management Consulting, Inc. and Yukon Research Centre, Yukon College, Whitehorse, YT.

WH (White House) (2011). The White House Initiative on American Indian and Alaska Native Education. http://www.ed.gov/edblogs/whiaiane/

Wyatt, T. R. (2012). Atuarfitsialak: Greenland's cultural compatible reform. International Journal of Qualitative Studies in Education, 25(6):819-836.

http://dx.doi.org/10.1080/09518398.2011.558033

YBS (Yukon Bureau of Statistics) (2008). Education. Information Sheet \#C06-09.

http://www.eco.gov.yk.ca/stats/pdf/2006censuseducation.pdf (2nd September 2014). 



\section{Globalization}

\section{Lead authors}

E. Carina H. Keskitalo, Umeå University, Sweden and Chris Southcott, Lakehead University, Canada

\section{Contributing authors}

Rasmus Ole Rasmussen (Nordregio, Sweden), Aina Tollefsen (Umeå University, Sweden), Susan Crate (George Mason University, USA)

\subsection{Introduction}

Arctic or far-northern areas are increasingly connected into a globalizing international system (Aarsæther and Bærenholdt, 2001; Keskitalo, 2008; Keskitalo, 2004; and Heininen and Southcott, 2010; Southcott, 2005). Impacts of globalization vary across a range of factors: in some cases globalization represents an intensification of processes with which Arctic communities have a long history of involvement, while in others, globalization brings new benefits and new challenges (Vaughan, 2007; and McGhee, 2004).

This chapter discusses the impact of globalization on human development in the Arctic, and describes the changes in systems due to globalization. In comparison with other chapters in this volume that focus more on specific aspects of human development, this chapter considers how human development across these various arenas may be changing as a result of globalization. The chapter describes how the influence of globalization in the Arctic region may be similar to or different from globalization processes in other parts of the world and how impacts vary among different northern regions. Relationships between globalization and specific factors such as age, gender, and identity are also described. 


\subsection{Globalization as a concept and its potential impacts on the Arctic}

This chapter describes three main types of globalization - economic, political, and cultural/social, and their relevance to human development in the North.iv The term globalization is defined here as the process of increasing economic, political and socio-cultural connections. However, while there is general agreement that globalization compresses time and space and increases global interaction, there is a lack of consensus about its essential components, and even more disagreement about the impacts on nations and communities (Ritzer, 2003).

Although networks and linkages have always connected the Arctic to the rest of the world, modern globalization means more connectivity through information and communication systems (including internet technologies), and expanded global trade networks. To a large extent, the Arctic is influenced by globalization in the same ways as other regions around the globe. However, given a relatively sparse population, some effects of globalization such as economic pressures towards increasingly large-scale organization may be felt particularly keenly in the region.

The impacts of globalization vary highly across the region, depending amongst other things on its large internal variation. The fact that we today talk about "the Arctic" as a region is itself a result of political globalization where eight separate states across a large proportion of the world's area have been able to be grouped together. Variations in impact of globalization are not surprising given that the region itself has differing patterns of historical development between the "Old World" of Europe, where processes originated much earlier in time and under less developed state power, and the "New World" of North America, where processes are of more recent origin and have resulted in large social change over a shorter period, with large impacts on local areas. Thus, while Indigenous communities in northern Canada, Alaska, Greenland and Russia have in some cases only been exposed to largescale external change for a few generations, the impacts of globalization have been more gradual and protracted in other areas such as the Fennoscandian North. Here, groups have interacted over historically longer times and comparatively smaller distances which are relatively well connected through physical infrastructure (roads and rails, for example) (Keskitalo, 2004).

The impacts of globalization also differ around the Arctic region due to the large variation internally in any given area. A group or individual may well oppose certain aspects of globalizing tendencies while embrac- 
ing and welcoming others. Some organizations or industries may be more equipped than others to "jump scale" from operating locally to globally (e.g., proficiency in international languages, large networks). Certain groups organizing as international non-governmental organizations may be able to drive their issues on the United Nations level, while others may continue to act locally. Some economic actors may have the capacity to target new markets in other areas of the world as conditions change, while others may not have financial capacity to reorganize (e.g., in forestry or fishing, in response to increased resource conflict or to changes that are concurrent with globalization, such as climate change or extreme weather impacts) (cf. Keskitalo, 2008).

A noodle shop in Reykjavik, Iceland, as an example of globalization in food culture

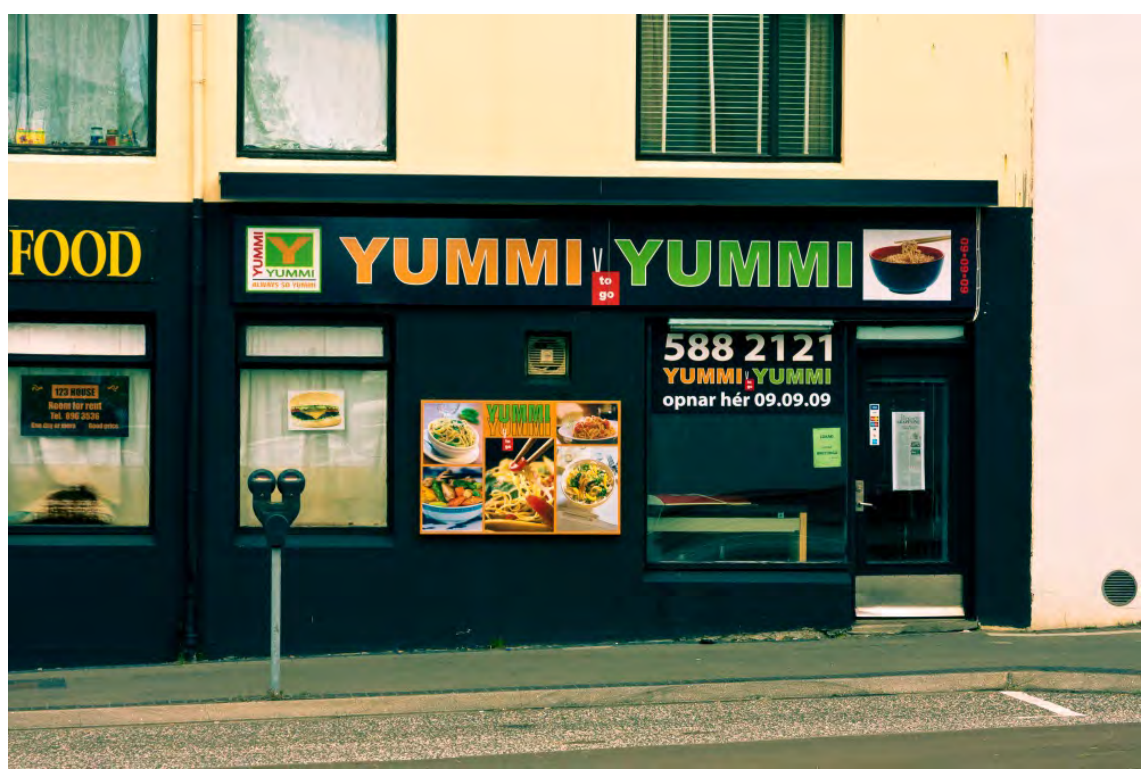

Credit: Amanda Graham.

\subsection{Economic globalization: local interests, external actors and unstable markets}

Economic globalization in the Arctic results in internationalizing the economic decision-making relevant to local and sub-regional economies. Mining, oil and gas, fishing, and forestry companies, for example, are increasingly multinational in nature: as a result, both their decision-making and primary concerns reflect interests beyond the local, making local situations relevant largely in terms of resource use rather 
than long-term employment (as would be the case more with a locally or even nationally based company). Economic globalization thus means that the role of the world market and competition for employment in resource extraction may involve distant locations that may be relevant to the same corporations. With the increase in large-scale international resource exploration, exemplified for instance by the current increase in resource development, mining, oil and gas regulation as well as local development benefits are increasingly subject to discussion, as competing land-use priorities, the extent of local employment, and long-term viability are considered (e.g. Knobblock and Pettersson, 2010) (see Textbox 10.1).

\section{Textbox 10.1}

\section{Globalization of economic decision-making relevant to local level}

Multiple examples can be identified of international companies that both originate in and come to influence Arctic states - but in most cases, moving decision-making farther from national, sub-regional and local contexts. The Canadian exploration company Dalradian Resources Inc. recently entered an agreement with private vendors for $5 \%$ of the aggregate land mass and mineral rights in Norway, and Statoil - a now global though originally Norwegian company, which acquired the North American Oil Sands corporation and oil sands leases in Alberta, Canada (New Europe, 2013; Statoil, 2013). In 2007, one of the world's largest aluminum producers, Alcoa Inc., which holds a large number of aluminum rights in Canada, the US, and Europe, opened a smelter in eastern Iceland, followed by another in Greenland, as part of an Arctic expansion (Alcoa 2013). Renewable resource companies follow the same pattern: forestry companies SCA and Stora Enso, of Swedish and Finnish origins, have operations in multiple countries (SCA, 2013; Stora Enso, 2013).

In some cases, companies based in far northern areas have gone global. One example is the Royal Greenland Trading Company, established in 1774 as a Danish trade company with a monopoly on trading in Greenland. Royal Greenland was set up as a separate company in 1985, and after Greenland obtained Home Rule in 1979 , the company became a cornerstone of the Greenland government's economic development strategy. In the first decades of Home Rule the strategy focused on a combination of small and large-scale activities, investing heavily in offshore trawlers and fishing industries in order to generate jobs in the larger towns. However, as village-based activities were declining, and prawn export became Greenland's dominant source of income, Royal Greenland became the world's largest exporter of coldwater shrimp, its operations concentrated in a few of the larger towns, including the capital, Nuuk. In the 1980s over 3,000 people were employed in the fish-processing industry in Greenland (Rasmussen 2000a; Rasmussen, 2008). After acquiring factories in Wilhelmshaven and Cuxhaven in Germany in 
the 1990s, and attempts at the division of Royal Greenland in Aalborg, Denmark, to increase competitiveness, the company expanded into new markets. Today, the company's Greenland operations, which include a total of 21 units, remain, providing jobs and income. A few of these units involve large-scale operations, but most involve small-scale production primarily for the home market. At present, Royal Greenland's global workforce numbers 1,888, of whom 793 are in Greenland and the remaining 1,095 at two plants in Denmark, two plants in Germany, one in Poland, and one in Canada (Royal Greenland, 2011). To local areas, the Royal Greenland story could be read as a success in terms of its continuation and development as a regional company and continual provision of local labor. On the other hand, it illustrates the decline in local low-skilled jobs that are replaced by technology - a common prerequisite for competition on a global market.

Historically, northern societies have often been seen as internal economic peripheries, or internal colonies, of nation states, which has an adverse impact on the long-term development of diversified economies in northern regions (Coates, 1985; Forsyth, 1994; Haycox, 2002). Some see globalization as the continuation and extension of these forces. The increased volatility of commodity markets, neo-liberal economic policies whereby regions are not compensated for their status as resource peripheries, and the limited competitive advantages due to long transport distances to markets, may have negative consequences for the Arctic. In general, the increased mobility of capital results in higher competition for capital and more difficulties for states to tax capital. This leads to a number of impacts with social consequences, which may include the decline of the welfare state, impacts on the power of labor unions, and impacts on the environment in the form of less stringent regulations. This may have particularly high impact on low populated areas with high resource values (Lyck, 2001; AHDR, 2004; Standlea, 2006).

On the other hand, economic globalization may also have a more positive impact on northern societies: it may contribute to increasing the speed of development, international labor mobility, and economic growth - for instance, in the tourism industry. Thus, some highlight that globalization might be viewed as an opportunity to break internal national colonialist or imperialist processes and to become better connected to, and better able to benefit from, international markets (McMicheal, 1996). Economic globalization may support easier access to global markets as the Internet and other tools increase interaction with consumers 
and buyers, and the potential to customize products and services to the market. Such developments could potentially also strengthen economic diversification and create opportunities to create and maintain employment at remote locations (see, e.g., Crate, 2006; Anderson, 2006). It has also been pointed out that at least some of the larger northern urban areas have managed to adapt and harness the potential of economic globalization to the benefit of the region (Cooke, 2001) - not the least drawing upon networks existing as part of advanced industrial states.

The potential to utilize economic globalization and an increasingly globalized market to support regional centers and diversification of the economy is to a large extent dependent on existing structure and legislation, e.g., on revenue and taxation from development, coupled with the ability to attract investments. For example, the case of diamond mining in the Arctic illustrates how benefits to local communities and governments differ widely based on the country in which mining is taking place. Within Russia, the physical act of mining diamonds has always been a state-controlled affair, with multi-nationals coming into the picture only to sell on the world market, whereas in other northern countries like Canada, multi-nationals have been the ones to stake the claims and operate the mining process (Crate, 2006; Crate and Yakovleva, 2008). Improved requirements upon regulation and contracting between these groups for mining operations, such as improved impact agreements, could support mutual long-term benefits such as environmental protection, compensation and profit sharing (Crate, 2006; Crate and Yakovleva, 2008; Kirsch, 2002) (see Textbox 10.2).

\section{Textbox 10.2}

\section{Globalization, Indigenous peoples and the oil and gas industry in Northern} Canada

Oil and gas developments have been important economic drivers in the Canadian North for almost 100 years. Large scale oil deposits were first discovered in the Northwest Territories in 1969 followed by a number of oil and gas discoveries in the years following. The problem with these deposits was that there was no way to transport the oil and gas profitably to market. In 1974 a group of international oil and gas companies was formed to propose the construction of a pipeline down the Mackenzie River Valley in order to get Arctic gas to southern markets. Initially the Indigenous population of the region was opposed to the pipeline. They were concerned with the impacts these developments would have on their traditional lifestyles. They were also against the idea that these developments would occur before the settlement of their respective land claims. Their opposition led to the establishment of a royal commission of inquiry headed by Justice Thomas Berger. This inquiry reported in 1978 that there be a moratorium on pipeline construction until Aboriginal land claims were settled. 
The settlement of land claims following this decision has served to significantly change the position of aboriginal peoples in the region, resulting in effective empowerment of Indigenous communities through these new comprehensive treaties (Dana et al., 2008).

Unlike the situation in Norway, for instance, where the state dominates oil and gas developments, the oil and gas industry in the North American Arctic is largely managed by global private sector companies that are increasingly aware that they need to negotiate benefits and environmental safeguards, or a social license, with local stakeholders. Land claim organizations such as the Inuvialuit Regional Corporation have been able to negotiate partnerships with major players in oil and gas development projects and are poised to become a major provider of subsidiary services (Nuttall, 2006). While concerns continue to exist in the region about the environmental and social consequences of oil and gas developments, and about the level of benefits that will go to local communities, positive attitudes to such developments may thus exist among many of the Indigenous peoples of these areas. However, this also means that treaty-based Indigenous organizations can appear to have vested interests in ensuring that projects such as the Mackenzie Gas Pipeline go ahead, sometimes resulting in difficult positions when global environmental NGOs work to counter new oil and gas initiatives.

Royal Arctic Line - a transport line owned by the Greenlandic Home Rule government

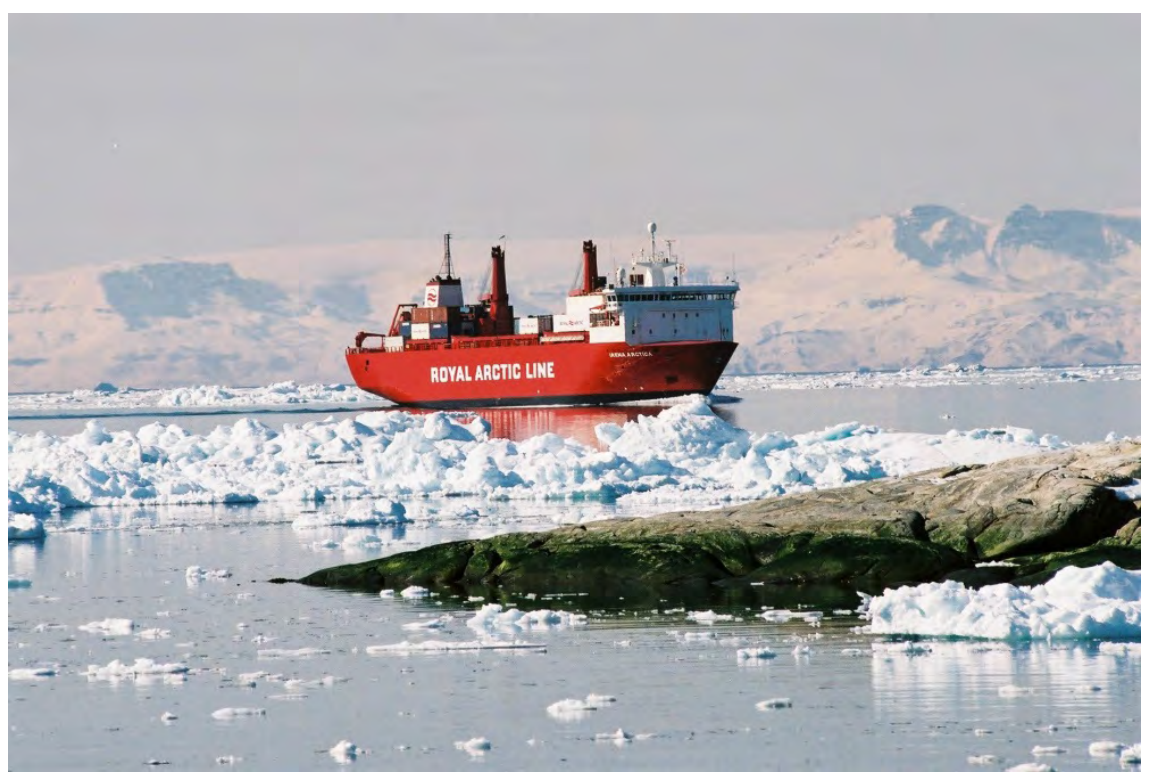

Credit: Harald Finkler. 
A variation in how areas are impacted may in particular also exist between urban and rural areas. More people globally live in cities today than ever before in history, at least partly driven by the increasing mobility of capital and economic globalization at large. At the same time, the increasing importance of international markets requires that business be transacted in the English language and that production be increasingly rationalized, meaning that fewer people are employed in traditional rural resource industries, even if their value to GNP remains high. This contributes to a transformation of northern areas similar to postindustrial developments and a focus on service economies occurring in many other areas. While the resource industry remains economically important, the more limited employment in resource industry over time complicates employment diversification and the potential to maintain services in rural areas in comparison with, for instance, urban regional centers. There is also an increasing difference between the internal migrations patterns of men and women, where women may be more likely to move out of the region for work (Hamilton and Seyfrit, 1993; Megatrends, 2011; see also Chapter 2, Arctic Population and Migration).

The ways in which these changes play out is strongly related to underlying structural impacts and the demographic situation (Huskey and Southcott, 2010), e.g., related to the aging (European) population vs. the younger (North American) population in northern areas. In northern Europe, many rural communities are characterized by a largely aging population as the general trend of an aging population is combined with outmigration for work. With regard to small-scale land ownership, for instance, family forestry in northern Europe is characterized by a trend of absentee owners: property owners may retain ownership of family land but no longer live at or depend on income from the property, as they work in regional urban centers. This also results in areas having to cope with highly varying service demands as large numbers of previous inhabitants return to what are now their second homes in summer (e.g., Stjernström et al., 2013). Such changes may impact both the composition of the local population as well as the level of control over land use at the local site (see Textbox 10.3). 


\section{Textbox 10.3}

\section{Gendered globalization: Greenland as an example}

Urbanization is a marked trend around the world, driven to a large extent by globalizing changes in employment patterns and values. Northern employment patterns and outdoor lifestyles have often been characterized as male oriented, with these historical patterns today possibly amplified by the increasing proportion of women in higher education that targets specialized employment in diversified employment markets. An example can be found in the case of Greenland, where a marked female deficit has characterized the population since the late 1950 s, largely as a result of changes in economic production in relation to work preference. This pattern has developed historically, following the process of modernization after WWII resulting in Greenlandic women marrying male workers from Denmark who were on temporary contracts in the building industry. When they left Greenland this resulted in the first wave of expanding gender imbalance. At this time the fishing industry, and particularly the rapidly developing shrimp fisheries, maintained the demand for local female workers, as these tasks were seen as traditionally female-oriented. However, when the peeling process, like many of the manual tasks, became automated, the need for labor in the fishing industry was sharply reduced, resulting in the second wave of gender imbalance. After Home Rule was established in Greenland in 1979, Greenlanders began to take over the government and administrative jobs previously held by Danes. In the first decade, however, the jobs were mainly taken by males, and emigration of women looking for education and jobs continued, creating the third wave of gender imbalance, which was only somewhat mitigated by the shift from an economy based on the production sector towards one based on the service sector, which supported traditionally female labor tasks. The imbalance in male and female emigration patterns, related amongst other to these changes in employment structure, has resulted in a current gender situation involving a 3\% deficit in women (Rasmussen, 2000b; Hamilton and Rasmussen, 2009).

In reaction to these structural changes, multiple solutions have been sought, most focused on increasing and diversifying employment. In rural Sweden, public expectations on second home-owners from Sweden, Finland, Germany, and the Netherlands are that these could to some extent offset employment-motivated emigration. There are also some cases of migration related to businesses that require large areas of land such as dog-farming or dogsledding businesses (e.g., Müller and Marjavaara, 2012). However, success in such ventures may to a large extent be dependent on the maintenance of social services such as healthcare and education at the local level despite the decrease in population. While 
tourism has often been looked to as a potential source for employment, studies indicate that while tourists are often interested in the "wilderness experience" in northern areas, they also require comparatively easy access to such sites, necessitating substantial infrastructure investment. Investments may, however, largely support large-scale tourism industries rather than target small-scale tourism specifically, as the former can gain access to media and organizational channels to reach international customers (Müller, 2011; Keskitalo and Lundmark, 2010). Globalization may thus, in many cases, support large-scale rather than smallscale developments, although synergy effects between these may exist.

“Say No to Oil": Protestors at Arctic Council Ministerial Meeting, Tromsø, Norway, 2009

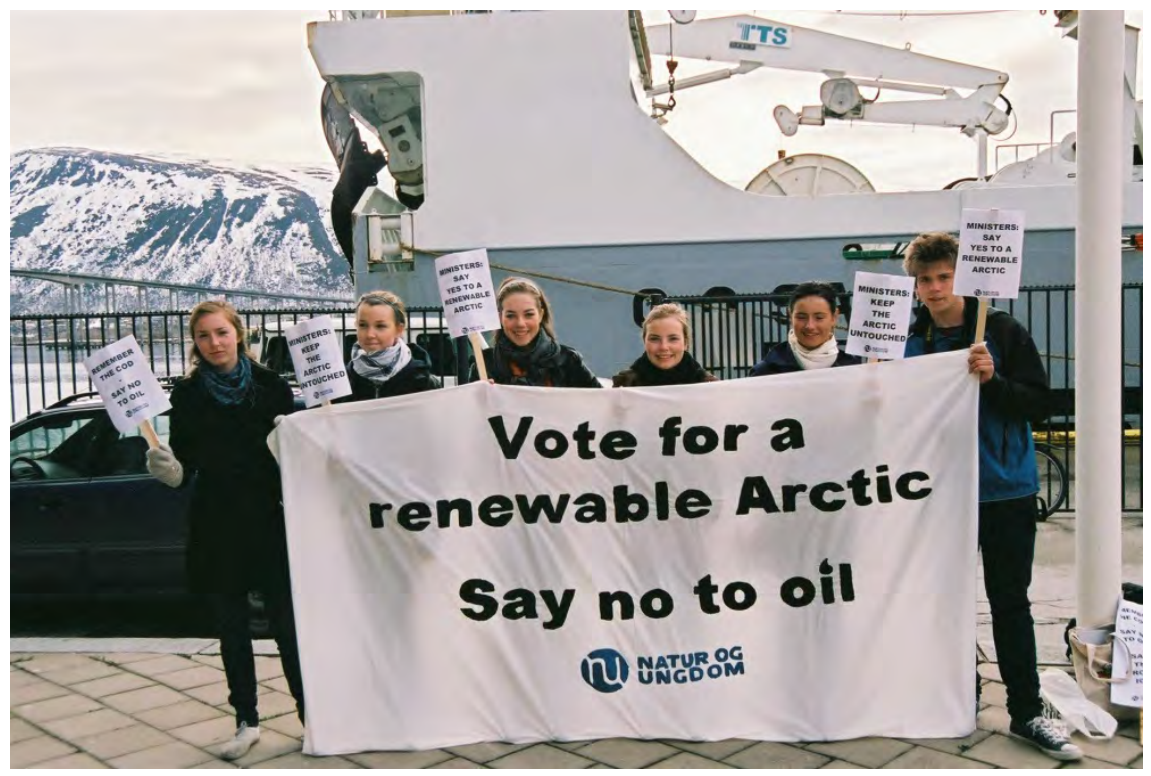

Credit: Harald Finkler.

Immigration and migratory workers are also changing the makeup of communities and of labor markets. Gender issues exist here with regard to different kinds of potential migratory workers, for example berry pickers may be both women and men while oil platform workers are predominantly male. In addition to gender disparities, many of these jobs may contribute less to local long-term employment and more to migratory or flexible work (see Textbox 10.4). 


\section{Textbox 10.4}

\section{The case of migrant labor in the wild-berry industry}

Research on migration and labor-market restructuring has highlighted trends towards globalizing labor markets with increased circularity, flexible employment, new forms of temporary contract laborers and influxes of undocumented migrant workers, especially in agriculture, construction and service sectors (Rye and Andrzejewska, 2010; Neergaard, 2009; Castles and Miller, 2009; Delgado Wise and Cypher, 2007; Schierup et al., 2006). The Scandinavian wild-berry industry is an example where both recruitment processes and regulatory frameworks are adjusting to new competitive global conditions (Woolfson et al., 2011; Eriksson and Tollefsen, 2013). Since its incorporation of a large foreign work force, the wild-berry industry in Sweden and Finland has been recognized as a potentially important economic sector (SIK, 2010; Jonsson and Uddstål, 2002; Richards and Saastamoinen, 2010), characterized by economic upgrading into a global commodity chain (Hedberg, 2013). The berry industry's buyerdriven value chain is controlled by lead companies operating in a global market, where Scandinavian berries are exported to China and Japan for processing into high-value products. The avoidance of responsibility and risk-sharing of lead companies and the pressures from intermediary berry companies to lower production costs have put low-wage workers in a highly vulnerable position (NAT, 2009; LO, 2013).

In Sweden, a large majority of the labor force consists of seasonal migrant workers from Asia and Eastern Europe, with Thai migrant berry pickers constituting the largest group of seasonal workers (Migrationsverket, 2012). Since the new Law on Labor Migration in Sweden in 2008, 51,806 temporary work permits have been issued, of which 18,610 were granted to Thai citizens (LO, 2013). EU enlargement in 2004 and 2007 made former berry pickers from Eastern Europe seek better-paid jobs in other EU countries, which propelled massive recruitment by berry companies of Asian workers to the Scandinavian berry industry. Labor rights in the industry have been weakly protected, with repeated reports of abuses, including human trafficking (NAT, 2009; Woolfson et al., 2011). Researchers have termed the last decade of Swedish labor migration policy "a new business-friendly 'guest-worker' system" (Schierup and Ålund, 2011:60) and the 2008 Act on Labor Migration has been strongly debated and partly reformed over time. Natural-resource based industries in the periphery produce disciplining practices of migrant workers that partly differ from those emerging within urban-based manufacturing, service and/or care work (Eriksson and Tollefsen, 2013). Nontimber forest products such as wild berries have historically and in a global perspective been overlooked and their governance usually neglected (Laird et al., 2010). The harvesting of non-timber forest products is 
generally conditional on "timber-centric forestry laws" (Laird et al., 2010) and other legislation related to access is currently under pressure from forestry, agriculture and private-land tenure laws (as documented in several studies, Laird et al., 2010).

In the Swedish case, these developments have led to new debates on the commercial exploitation of public access to forest land and berry-picking, which draw on longstanding historical disputes between proponents of private property rights and advocates of the public interest (Sténs and Sandström, 2012).

Globalizing market conditions may thus have a large impact on local employment and job creation. While many climate change studies focus on adaptation to climate change (e.g. Hovelsrud and Smit, 2010), adapting to economic globalization may be as, if not more, important to those in the targeted resource sector. For renewable resource uses, such as forestry, fishing, reindeer husbandry, and tourism in northern Europe, studies indicate that market conditions and income play a larger role in the concerns of practitioners than does adaptation to climate change. The ways in which these industries prioritize is largely related to maintaining employment during economic change, given the prices set for products on an international market. Climate change or environmental constraints are mainly considered to the extent to which they impact production, for instance, in limiting grazing opportunities in reindeer husbandry, impacting forest growth and harvesting conditions, or impacting access to fishing resources (Keskitalo, 2008).

At the least, these changes in the composition and societal structure of the population of far northern and rural areas contribute to different kinds of rural-urban dynamics, where we can see a trend toward relatively large changes in population structure and habitation patterns, as well as in occupations, involving increasing urbanization and changes for instance, from village to town, and from family farm to service occupations in relation to economic change and globalization (Westin, 2012). This begs the question of how we conceptualize such populations, and requires a further understanding of the Arctic not only in terms of the geographically large rural areas, but in terms of interaction with regional centers as well as larger global markets that often drive economic development. 


\subsection{Political globalization: are increasingly large scales of governance both empowering and limiting?}

In a manner similar to economic globalization, political globalization can be seen in a number of different ways and on a variety of levels, including international and supranational, national and local (see Textbox 10.5).

\section{Textbox 10.5}

\section{Globalization and political organization on the environment}

Increased global interest in the Arctic has partly focused on environmental concerns, and among these prominently on climate change. While globalization is not generally seen to be responsible for these environmental issues, both the increased exposure through global media, how these issues impact the Arctic and an increasingly global "cosmopolitan" concern about these issues (Giddens, 2002) mean that globalization and environmental concerns are linked (Leichenko and O'Brien, 2008).

Since the 1980s the world has witnessed an increasing international cooperation around environmental issues (De Sombre, 2005). The Arctic has benefitted from this in terms of attention. Increased cooperation among Arctic researchers and scientists have allowed the development of consensus positions concerning environmental challenges (e.g. ACIA, 2005). New international environmental organizations, both governmental and non-governmental, have taken hold of these positions and adopted them as key objectives, and environmental issues have also sometimes interrelated with the increasing voice given to minority and Indigenous rights. As the first Arctic Human Development Report pointed out, increased global concern about minority and Indigenous rights assisted amongst others Arctic Indigenous peoples in their struggle to voice their concerns, which included environmental challenges to the natural space that is important to local economies (Bankes, 2004).

The increasing global concern around environmental issues in the Arctic also means that new resource development projects in the region are increasingly under pressure to show that their operations will minimize impacts on the environment. In the past, resource extraction in the Arctic often paid limited attention to negative impacts on the local environment. The level of pollution around the Arctic Russian mining city of Norilsk and mercury pollution from the gold mines around the Canadian city of Yellowknife are vivid examples of highly impacting mining and resource exploitation. Currently, global public opinion is increasing pressure on companies through demands for corporate social responsibility and social licensing (O'Faircheallaigh and Ali, 2008). While the level of commitment to this idea varies across regions down to specific cases, with risks for "greenwashing", political organization on the environment plays a part in raising the focus on the environmental impacts of operations and on climate change in the Arctic. 
With regard to the Arctic, a well-established literature also exists on how during the 1990s political globalization helped establish the Arctic's regional actors as important players in international relations, and in effect develop the Arctic as an international region (Young, 2005; Heininen, 2005; Keskitalo, 2004). This process was largely related to state interests in security across East-West lines following the end of the Cold War, and resulted in numerous initiatives including the Arctic Council as well as the Barents Euro-Arctic Council (see Chapter 5, Political Systems and Geopolitics).

The development of the European Union (EU) and its relationship to continuously wider policy areas during this time can also be seen as one among several developments of world regions. On an international level related to northern cooperation, the EU has developed both EU-Russian northern cooperation (on a Finnish initiative) and EU Arctic policy (for instance in response to a meeting between the five coastal states bordering the Arctic Ocean, which thus excluded many EU interests) (see Chapter 6, Legal Systems). However, the strongest impact can be seen within EU member states: issues in which the EU has jurisdiction can fundamentally impact even local decision-making directly, for instance through directives that apply to all member states. As an example, the EU Water Framework Directive requires appointment of water authorities at the ecosystem level, rather than the established administrative regional level of the state. The directive further requires development of local, broadly participatory water councils at the local level to feed into the operations of the water authorities. This shifts power towards a broader and different selection of actors, resulting both in potentially substantial participation but also in a diffusion of power from the established administrative - and sometimes directly democratically elected units of the state (e.g., Keskitalo and Pettersson, 2012). The EU may also influence local government levels directly by providing an additional source of funding, which may to some extent support local prioritysetting, and may for instance contribute to Europeanization through the building of networks between local governments (e.g. Pierre, 2011).

Political globalization thus results in the transfer of power from state level to supranational level, but also to other levels. This often has been expressed as the "hollowing-out" of the state, and is frequently linked to economic globalization. "Hollowing out "is defined by a number of features whereby more limited resources for the state result in responsibility for specific measures being divested upwards or downwards (cf. Svallfors and Taylor-Gooby, 2012). General changes in state structure as a result of the hollowing-out of the state include the state not able to 
control companies moving abroad, and less tax coming in, which limits the state's possibilities to support economic development and social welfare. Limitations in funding for welfare areas such as health may impact opportunities, e.g., for youth or an aging population in rural areas and for attracting resources for diversification and employment. This means that the transformation of the welfare state in relation to globalization may have an increasingly significant impact on low-populated areas. The downscaling of the welfare state in Canada and the Nordic countries and the failing subsidy and support systems in Northern Russia have been seen to have negative consequences for the region (Beebe et al., 2004; Duhaime, 2004).

The economic features of globalization are therefore strongly linked to the political and other impacts of globalization, and are often seen as driving force behind changes in these areas. Political globalization is also linked to the advent of the communications revolution, which both enables and results in the development of interest groups and organization on larger levels, who now compete across global arenas for recognition and support (Castells, 2000).

As is the case with other processes of globalization, these processes result in differing responses. In general, globalization literature suggests that, as in the case of economic globalization, groups that may benefit from globalization include organized interests that are able to "jump scale" in order to gain international support. The first Arctic Human Development Report referred to this phenomenon when describing the increased global recognition of Indigenous peoples' rights and the increased transfer of legal authority to regional governments (Bankes, 2004). On the other hand, processes of decentralization of power beyond the state may lead to increasingly limited access and longer distances (physical and mental) to decision-makers. Processes of decentralization of power downwards, while contributing to increased local decision-making, may curtail the scope of such decision-making in cases where authority, but not resources, is decentralized (in line, e.g., with a hollowing-out of the state). Such processes may also contribute to increased inequality between smaller and larger municipalities or local governments (e.g. Pierre, 2011; Svallfors and Taylor-Gooby, 2012). 


\subsection{Cultural and social globalization: potentially increased differentiation at the same locality?}

Cultural and social impacts of globalization in the Arctic are often seen as very complex, as they differentiate among communities and groups down to an individual level. Many observers have mentioned the negative cultural impact that western culture has had on Indigenous communities in the Arctic, particularly in Canada, Alaska, and Greenland, the areas of the most recent settlement from the outside and where the impact of such settlement has been the greatest. At the same time, research on these issues tends to portray these impacts as the result of colonialism and/or modernization rather than globalization (Bjerregaard and Young, 1998; Freeman, 2000) (see Textbox 10.6 for a discussion of these terms).

\section{Textbox 10.6}

\section{Globalization and other concepts of social change}

In discussing the impacts of globalization, it is also useful to attempt to clarify its relationship to other large-scale forces of change. Terms that are often related to globalization in general include "colonialism", "imperialism" and "modernization". Both "colonialism" and "imperialism" refer in different ways to the control of a region by an outside region or people, with "colonialism", according to some definitions, referring specifically to a situation in which an Indigenous majority falls under the control of a foreign-based minority (Osterhammel, 2005).

While the integration of populations into different groups or nations of greater or lesser internal variety is a feature of all societies over time, both concepts highlight the power structures and force inherent in such developments. Colonialism may thus include decisions made not in the interests of the particular conquered region and, driven by the mentality of colonialism, under the assumption that the selfperceived superiority of the colonial civilization entitles it to rule (Osterhammel, 2005). Such concepts of power can also be seen as inherent in the definitional right imposed in concepts such as "frontier," defined as the boundary between civilization and nature, thereby defining previous residents as part of nature rather than of civilization. Colonialism thus refers to overt actions often within the context of nation-state power. The results of such processes have had a clear impact: for instance, today in areas such as Arctic Canada colonialism as the imposition of an external world view and organization is seen as at least partially to explain many of the social, cultural, and economic problems that this region currently suffers. While the term "globalization" relates largely to an extension beyond the nation-state and nation-state power, mechanisms inherent in colonialism with regard to what is today less overt force cannot be precluded, but are rather highlighted in some literature, emphasizing the complex, power-laden, and varying results of globalization (e.g., Banerjee and Linstead, 2001). 
The focus on the role of external powers has also been highlighted in concepts such as imperialism, sometimes seen as the "general mode of development of capitalism as an international system" (Liodakis, 2005: 343). In this regard, some argue that globalization is not fundamentally different from imperialism, in that it is to a large extent driven by large actors and interests, although those who now exert power do so in many more ways than during earlier periods of external domination of foreign lands (e.g., Petras and Veltmeyer, 2000; Dale, 1999). Relative to this asymmetry of power over globalization and globalizing features, and to the types of identities and world views that are expressed through them, globalization could by some definitions be seen as serving some imperialist aims (such as control over certain production by some specific entities) without explicit imperialism in the meaning of direct control of a foreign territory. However, as is the case with colonialism, increasing globalization may both support existing power structures and the provide tools to challenging them.

In general, it has been observed that the focus for instance of the 1950s and 1960 s on modernization has been largely replaced with a focus on globalization as a term (Jiafeng, 2009). "Modernization" was used to denote the development of a country or region, and often referred to large-scale developments, such as large-scale electrification and long-distance transportation. In this context, developments were often discussed in terms of the benefits of modernity, such as new technology (e.g., Black, 1966). However, in an Arctic context, "modernization" has been used, particularly in the Canadian North, to refer to Indigenous peoples moving into settlements in the 1950s and 1960s: in this case, with its reference both to physical changes and to the desirability of such changes, largely related to a view on development, a broader modernization process may thus also have contributed to colonialism, as it may have been driven by a colonial mindset and external decisions.

Great variety exists among the cultures of the Arctic, and how these cultures may be impacted by change (see Chapter 3, Cultures and Identities). As a result of impacts from the national (state) culture as well as other cultures in complex patterns of migration and influence (see, e.g., Box 10.4 on Thai migratory workers), the Arctic, like other regions, is increasingly impacted by what is often seen as a global entertainment culture and its values. Studies indicate relatively high shares of Internet users as a percentage of population ( $>70 \%$ ), in northern Norway, Sweden, Finland, Iceland, Alaska, and parts of Canada (Nordregio, 2011). Other studies cor- 
roborate that northern communities are increasingly places of modernity in the meaning of being seen as modernized - both with regard to living conditions and technology and in the self-images of the people living in these communities (Aarsæther and Bærenholdt, 1998).v

Such new communications linkages and identifications may provide access to a larger (external) scope of identities and aspirations to choose from, for instance in terms of places to live. In northern Canada, researchers have noted the impact of globalization in terms of providing a greater variety of options (Salokangas and Parlee, 2009). While such consequences may limit rural development and commitment to local areas as well as result in increasingly differentiated developments at the same location, they may, together with complex trends of migration and societal change, contribute to movement and over time limit cultural stress related to externally driven developments. However, lifestyle choices may also contribute to people's choosing to stay in northern areas, for instance in desirable locations (as described for the Nordic countries in Westin, 2012).

The development of hybrid or complex identities, in which individuals see themselves as a mix of traditional and modern elements, have been emphasized in research. With regard to the impact on highly localized as well as on Indigenous culture, Csonka and Schweitzer discuss the impact of new communications technology, noting that Indigenous culture is not disappearing, "rather, it is modernity that becomes indigenized" (2004: 51). In a manner similar to Robertson's notions of "glocalization" as concurrent local-global change (Robertson, 1995), Csonka and Schweitzer (2004) thus note the culturally empowering influences of global changes. Within this context, a reassertion of Indigenous culture has taken place, but also a redefinition of local identities in the wake of deindustrialization and with increasing awareness of for instance environmental and cultural values (see Chapter 3, Cultures and Identities. and Chapter 11, Community Viability and Adaptation). For instance, researchers in northern Canada have described the way in which global demand for Inuit art has not only improved the economic situation of communities such as Cape Dorset in Nunavut but also enhanced the voice of Inuit culture in the world in other spheres (Doubleday et al., 2004).

Changes due to social and cultural globalization may thus increasingly result in some homogeneity but also in increased heterogeneity in the same location, dependent on resources, choices, and societal subgroups, including across gender and class. The increasing focus on standing out in a more globalized media stream may, however, also result in further 
"exotification" of the Arctic, which may enhance the identification of traditional components that can be sold, e.g., for tourism or broader media consumption (Viken, 2006).

\section{Entertainment at the ICC General Assembly, 2010}

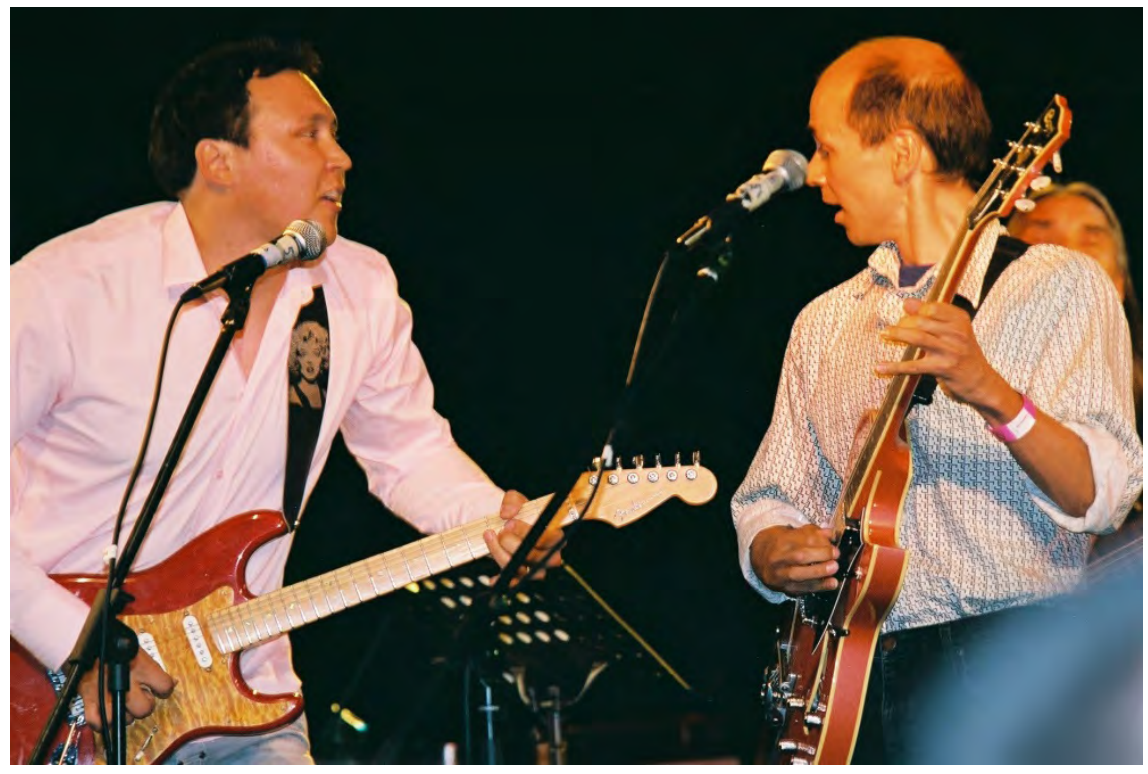

Credit: Harald Finkler.

\subsection{Summary of major trends and gaps in knowledge}

Globalization constitutes a trend with highly varying impacts, where the extent to which globalization plays a role may differ within the same community, down to the individual level. Differences within the Arctic include differential development tracks in terms of "Old World" and "New World", with trends differing between northern Europe and North America (Keskitalo, 2004). From this perspective, the "Arctic" can be seen as a region with highly differing characteristics and situations.

At the same time, many globalizing changes have similar impacts across most areas of the Arctic, largely related to its connotation of peripherality to major markets. Globalization represents an intensification of many of the effects brought about by modernity and by industrialization. Intensification of resource development in the North as a result of globalization may increase the conflicts between international economic forces and those of local use and local employment, and may largely 
decentralize economic decision-making from the regions it impacts. In many instances globalization means increased dependency of local interests on external powers and unstable markets. At the same time, the forces of globalization have also brought about many economic opportunities for northern areas, including increased development in resource use, creating some local employment. While politically globalization has partly been seen as leading to the weakening of the nation-state's ability to maintain the services relevant for all its population, it is also sometimes seen as resulting in increasing empowerment of new regional actors. Culturally, globalization brings with it increasing and diversified impacts, but also represents the ability to find new support for traditional and local cultures. Globalization may then also be linked to some degree of political and cultural empowerment, depending on group and accessibility to different structures of decision-making.

While a traditional focus in Arctic literature has so far resulted in an emphasis on areas that remain traditional rather than on the large changes taking place, and subsequently in a relatively limited focus on globalization in literature pertaining to the Arctic, globalization is an increasing impact in the areas. An increasing focus thus needs to be placed on how different state systems and regulations (for instance, revenue sharing systems) cause fundamentally different impacts and development trends in different countries and sub-regions. Local actors are here not necessarily passive objects of globalization, but may "resist" and/or co-opt the effects of globalization, and may adapt to globalization by using the same tools that globalization uses. They may, for instance, adopt external trends and images to make them fit local needs. At the same time, these relatively small-scale economies are also highly dependent on the existence of health care, education, and other social services, as well as on revenue from local resources, even if they are produced for global consumption, to be competitive in the future.

\subsection{Acknowledgements}

Carina Keskitalo gratefully acknowledges funding from the Mistra Arctic Futures program and the FORMAS PLURAL program for participation in the development of this report. Chris Southcott gratefully acknowledges funding from the Social Sciences and Humanities Research Council of Canada for the Resources and Sustainable Development in the Arctic (ReSDA) project. 


\subsection{References}

Aarsæther, N., L. Riabova, and J.O. Bærenholdt (2004). Community Viability, In: AHDR, 2004. Arctic Human Development Report. Einarsson, N., J. N. Larsen, A. Nilsson and O. R. Young (eds.), pp. 139-153. Stefansson Arctic Institute, Akureyri.

ACIA (2005). Arctic Climate Impact Assessment. Cambridge University Press, Cambridge. AHDR (2004). Arctic Human Development Report. Einarsson, N., J. N. Larsen, A. Nilsson and O. R. Young (eds.). Stefansson Arctic Institute, Akureyri.

Ali, S. and C. O'Faircheallaigh (2008). Earth Matters: Indigenous Peoples, The Extractive Industries and Corporate Social Responsibility. Sheffield: Greenleaf.

Alcoa (2013). Alcoa. http://www.alcoa.com/global/en/home.asp (31st October 2014).

Anderson, D. G. (2006). Is Siberian reindeer herding in crisis? Living with reindeer fifteen years after the end of state socialism. Nomadic Peoples, 10(2): pp. 87-97. http://dx.doi.org/10.3167/np.2006.100206

Bankes, N. (2004). Legal systems. In: AHDR, 2004. Arctic Human Development Report. Einarsson, N., J. N. Larsen, A. Nilsson and O. R. Young (eds.), pp. 101-117. Stefansson Arctic Institute, Akureyri.

Bannerjee, S. B. and S. Linstead (2001). Globalization, multiculturalism and other fictions: colonialism for the new millennium? Organization, 8(4): pp. 686-722. http://dx.doi.org/10.1177/135050840184006

Beebe, J., L. Zanasi, and N. Schacter (2004). Globalization and the North: Impacts of Trade Treaties On Canada's Northern Governments, Canadian Centre for Policy Alternatives, Ottawa.

Bjerregaard, P. and T.K. Young (1998). The Circumpolar Inuit: Health of a Population in Transition. Munksgaard, Copenhagen.

Black, C. (1966). The Dynamics of Modernization: A Study in Comparative History. Harper and Row, New York.

Castles, S. and J. Miller (2009). The Age of Migration: International Population Movements in the Modern World (4th ed.). Palgrave MacMillan, Basingstoke.

Castells, M. (2000). The Rise of the Network Society (2nd ed.). Blackwell, Oxford.

Coates, K. (1985). Canada's Colonies: A History of the Yukon and Northwest Territories. Lorimer, Toronto.

Cooke, P. (2001). Knowledge Economies: Clusters, Learning and Co-Operative Advantage. Routledge, New York. http://dx.doi.org/10.4324/9780203445402

Crate, S. A. (2006). Cows, Kin and Globalization: An Ethnography of Sustainability. Alta Mira Press, Walnut Creek.

Crate, S. A. and N. Yakovleva, N. (2008). Indigenous people, mining and the environment inRussia. In: O'Faircheallaigh, C. and A. Saleem (eds.). Earth Matters: Indigenous Peoples, the Extractive Industries and Corporate Social Responsibility, pp. 222244. Greenleaf, Sheffield.

Csonka, Y. and P. Schweitzer (2004). Societies and cultures: change and persistence. In: AHDR, 2004. Arctic Human Development Report. Einarsson, N., J. N. Larsen, A. Nilsson and O. R. Young (eds.), pp. 45-67. Stefansson Arctic Institute, Akureyri.

Dale, R. (1999). Specifying globalization effects on national policy: a focus on the mechanisms. Journal of Education Policy, 14(1): pp. 1-17. http://dx.doi.org/ 10.1080/026809399286468

Dana, L. A. Meis-Mason, and R. Anderson (2008). Oil and gas and the Inuvialuit people of the Western Arctic. Journal of Enterprising Communities: People and Places in 
the Global Economy, 2(2): pp. 151-167. http://dx.doi.org/10.1108/ 17506200810879970

Delgado Wise R. and J M. Cypher (2007). The strategic role of Mexican labor under NAFTA: critical perspectives on current economic integration. The Annals of the American Academy of Political and Social Science, 610: pp. 120-142.

DeSombre, E. R. (2005). Global Environment and World Politics. Continuum, London.

Doubleday, N. M., A. F., MacKenzie, and S. Dalby (2004). Reimagining sustainable cultures: constitutions, land and art. Canadian Geographe, 48(4): pp. 389-402. http://dx.doi.org/10.1111/j.0008-3658.2004.00067.x

Duhaime, G. (2004). Economic Systems. In: AHDR, 2004. Arctic Human Development Report. Einarsson, N., J. N. Larsen, A. Nilsson and O. R. Young (eds.), pp. 69-83. Stefansson Arctic Institute, Akureyri.

Eriksson, M. and A. Tollefsen (2013). Disciplining temporary workers: Thai seasonal labourers and the Swedish berry industry. In: Geiger, M. and A. Pecoud (eds.). Disciplining the Transnational Movements of People. Palgrave Macmillan, Basingstoke.

Forsyth, J. (1994). A history of the peoples of Siberia: Russia's North Asian colony 1581-1990. Cambridge University Press, Cambridge.

Freeman, M. M. R. (ed.) (2000). Endangered Peoples of the Arctic: Struggles to Survive and Thrive. Greenwood Press, Westport.

Giddens, A. (2002). Runaway World: How Globalization is Reshaping Our Lives. Routledge, New York.

Global Policy Forum, n.d., Defining Globalization. http://www.globalpolicy.org/ globaliz/define/index.htm (31st October 2014).

Hamilton, L. and R.O. Rasmussen (2009). Population, sex ratios and development in Greenland. Arctic, 63(1): pp. 43-52.

Hamilton, L., and C. Seyfrit (1993). Town-village contrasts in Alaskan youth aspirations. Arctic, 46(3): pp. 255-263. http://dx.doi.org/10.14430/arctic1351

Haycox, S. (2002). Alaska: An American Colony. University of Washington Press, Seattle.

Hedberg, C. (2013). "Grapes of Wrath"? Power spatialities and aspects of labor in the wild berry global commodity chain. Competition and Change 17(1): pp. 57-74. http://dx.doi.org/10.1179/1024529412Z.00000000025

Heininen, L. (2005). Impacts of Globalization, and the Circumpolar North in World Politics. Polar Geography, 29(2): pp. 91-102. http://dx.doi.org/10.1080/ 789610127

Held, D. and A. McGrew, n.d. Globalization, Entry for Oxford Companion to Politics. http://www.polity.co.uk/global/globalization-oxford.asp (31st October 2014). http://dx.doi.org/10.1080/789610127

Hovelsrud, G. and B. Smit, (eds.) (2010). Community Adaptation and Vulnerability in Arctic Regions. Springer, Dordrecht. http://dx.doi.org/10.1007/978-90-481-9174-1

Huskey, L. and C. Southcott (2010). Migration in the Circumpolar North: Issues and Contexts. CCI Press, Edmonton.

Jiafeng, W. (2009). Some reflections on modernization theory and globalization theory. Chinese Studies in History, 43(1): pp. 72-98. http://dx.doi.org/10.2753/ CSH0009-4633430107

Jonsson, L. and R. Uddstål (2002). En beskrivning av den svenska skogsbärbranschen [A description of the Swedish forest berry industry]. Umeå: Swedish University of Agricultural Sciences.

Keskitalo, E.C. H. (2008). Climate Change and Globalization in the Arctic: An Integrated Approach to Vulnerability Assessment. Earthscan, London. 
Keskitalo, E. C. H. (2004). Negotiating the Arctic: The Construction of an International Region. Routledge, New York.

Keskitalo, E. C. H. and L. Lundmark (2010). The controversy over protected areas and forest-sector employment in Norrbotten, Sweden: forest stakeholder perceptions and statistics. Society and Natural Resources, 23(2): pp. 146-164.

http://dx.doi.org/10.1080/08941920802688543

Keskitalo, E.C.H and M. Pettersson (2012). Implementing multi-level governance? The legal basis and implementation of the EU Water Framework Directive for forestry in Sweden. Environmental Policy and Governance, 22: pp. 90-103. http://dx.doi.org/10.1002/eet.1574

Kirsch, S. (2002). Anthropology and advocacy: a case study of the campaign against the Ok Tedi Mine. Critique of Anthropology, 22(2): pp. 175-200. http://dx.doi.org/10.1177/03075X02022002851

Knobblock, E. and Ö. Pettersson (2010). Restructuring and Risk-Reduction in Mining: Employment Implications for Northern Sweden, Fennia, 188(1): pp. 61-75.

Laird, S. A., R.J. McLain, and R.P. Wynberg, (eds) (2012). Wild Product Governance: Finding Policies that Work for Non-Timber Forest Products. Routledge, London \& Washington, D.C.

Leichenko, R., and K. O’Brien (2008). Environmental Change and Globalization: Double Exposures. Oxford University Press, Oxford. http://dx.doi.org/10.1093/acprof:oso/9780195177329.001.0001

Liodakis, G. (2005). The new stage of capitalist development and the prospects of globalization. Science \& Society, 69(3): pp. 341-366. http://dx.doi.org/10.1521/ siso.69.3.341.66525

LO (2013). Fusk och utnyttjande - om avregleringen av arbetskraftsinvandringen [Cheating and exploitation - concerning the de-regulation of labor immigration]. Stockholm.

Lyck L. (2001). Arctic economies and globalization. North Meets North. Proceedings of the First Northern Research Forum. University of Akureyri: Akureyri. pp. 68-71.

McGhee, R. (2004). The Last Imaginary Place: A Human History of the Arctic World. Key Porter, Toronto.

McMichael, P. (1996). Globalization: myths and realities. Rural Sociology 61(2): pp. 25-55.

Megatrends (2011). Rasmussen, R.O. (ed). Nordic Council of Ministers, Copenhagen. Migrationsverket (Swedish Migration Board) (2012). Beviljade uppehållstillstånd av arbetsmarknadsskäl efter medborgarskap 2000-2011 [Approved residency permits for employment purposes following citizenship 2000-2011] www.migrationsverket.se/download/18.78fcf371269cd4cda980004207/tabs5.pdf (31st October 2014).

Müller, D. K. (2011). Tourism development in Europe's "last wilderness": An assessment of nature-based tourism in Swedish Lapland. In: Grenier, A.A., and Müller, D.K., eds. Polar Tourism: A Tool for Regional Development. pp. 129-153. Presses de l'Université du Québec, Montreal.

Müller, D. K. and R. Marjavaara (2012). From second home to primary residence: Migration towards recreational properties in Sweden 1991-2005. Tijdschrift voor Economische en Sociale Geografie, 103(1): 53-68. http://dx.doi.org/10.1111/ j.1467-9663.2011.00674.x

NAT (Network against Trafficking and Exploitation of Migrant Workers) (2009). Labor Trafficking: The 2009 Blueberry Fiasco in Sweden, NAT Bulletin No. 1, 
http://www.scribd.com/doc/25137612/labour-trafficking-blueberry-fiasco-insweden (31st October 31, 2014).

Neergaard, A. (ed.) (2009). European Perspectives on Exclusion and Subordination: The Political Economy of Migration. Shaker Publishing, Maastricht.

Nordregio (2011). Internet in the Arctic. http://www.nordregio.se/en/Maps-Graphs/09-Other/Internet-in-the-Arctic/ (31st October 2014).

Nuttall, M. (2006). The Mackenzie gas project: Aboriginal interests, the environment and Northern Canada's energy frontier, Indigenous Affairs, 2-3: 20-29.

Osterhammel, J. (2005). Colonialism: A Theoretical Overview. Wiener, Princeton.

Petras, J. and H. Veltmeyer (2000). Globalization or imperialism? Cambridge Review of International Affairs, 14(1): pp. 32-48. http://dx.doi.org/10.1080/ 09557570008400327

Pierre, J. (2011). Why should we concern ourselves with urban governance? Revisiting the global-local nexus. In: Chu, C-P., A. Grasse, S-C. Park, M. Porsche-Ludwig (eds.), Local Governance in the Global Context: Theory and Practice, pp. 11-24. Lit Verlag, Berlin.

Rasmussen, R. O. (2008). Global North Atlantic fishing. Journal of Nordregio. 1(8).

Rasmussen, R. O. (2000a). Formal economy, renewable resources and structural changes in west Greenland. Etudes Inuit Studies, 24(1): pp. 48-78.

Rasmussen, R. O. (2000b). Topografisk Atlas Grønland [Topographic Atlas Greenland]. Det Kongelige Danske Geografiske Selskab Kort \& Matrikelstyrelsen., Copenhagen.

Richards, R. T. and O. Saastamoinen (2010). NTFP Policy, Access to Markets and Labor Issues in Finland: Impacts of Regionalization and Globalization on the Wild Berry Industry. In: Laird, S. A., R.J. McLain, and R.P. Wynberg (eds.). Wild Product Governance: Finding Policies that Work for Non-Timber Forest Products, pp. 287307. Routledge, London \& Washington, D.C.

Ritzer, G. (2003). Modern Sociological Theory (6th ed.). McGraw-Hill, Toronto.

Robertson, R., 1995. Glocalization: time-space and homogeneity-heterogeneity. In: Featherstone, M., S. Lash, and R. Robertson, (eds.). Global Modernities. Sage, London. Royal Greenland A/S (2011). Annual Report, 2010/2011.

Rye, J. F. and J. Andrzejewska (2010). The structural disempowerment of Eastern European migrant farm workers in Norwegian agriculture, Journal of Rural Studies, 26: pp. 41-51. http://dx.doi.org/10.1016/j.jrurstud.2009.06.003

Salokangas, R., and B. Parlee (2009). The influence of family history on learning opportunities of Inuvialuit youth. Études Inuit Studies, 33(1/2): pp. 191-207.

SCA (2013). Kortfakta om SCA [Short facts about SCA]. http://www.sca.com/sv/ Om_SCA/Kortfakta_om_SCA/ (31 October 2014).

Schierup C.U. and A. Ålund (2011). The end of Swedish exceptionalism? Citizenship, neoliberalism and the politics of exclusion. Race \& Class, 53(1): pp. 45-64. http://dx.doi.org/10.1177/0306396811406780

Schierup C.U., P. Hansen, and S. Castles (2006). Migration, Citizenship and the European Welfare State: A European Dilemma, Oxford University Press, Oxford. http://dx.doi.org/10.1093/0198280521.001.0001

SIK (2010). The Swedish Institute for Food and Biotechnology. http://engwww.sik.se (12th November 2014).

Standlea, D. (2006). Oil, Globalization, and the War for the Arctic Refuge. State University of New York Press, Albany.

Statoil (2013). Statoil Oil Sands. http://www.statoil.com/en/About/Worldwide/ NorthAmerica/canada/OilSands/Pages/StatoilInCanada.aspx (12th November 2014). 
Sténs, A., and C. Sandström (2012). Divergent interests and ideas around property rights: The case of berry harvesting in Sweden, Forest Policy and Economics, 33: pp. 56-62. http://dx.doi.org/10.1016/j.forpol.2012.05.004

Stjernström, O., S. Karsson, and Ö. Pettersson (2013). Skogen och den kommunala planeringen [Forest and municipal planning]. PLAN 1: pp. 42-45.

Stora Enso (2013). Stora Enso. http://www.storaenso.com/ (31st October 2014).

Svallfors, S., and P. Taylor-Gooby, (eds.) (2012). End of the Welfare State? Routledge, London and New York.

Vaughan, R. (2007). The Arctic: A History. Sutton Publishing, Stroud.

Viken, A. (2006). Tourism and Sámi Identity - An analysis of the tourism-identity nexus in a Sámi community. Scandinavian Journal of Hospitality and Tourism, 6(01): pp. 7-24. http://dx.doi.org/10.1080/15022250600560604

Westin, K. (2012). Hemma i Norrland: Om identitet och samhörighet med andra [At home in Norrland: Concerning identity and community with others]. In: Lidström, A. ed. Ett delat Norrland: på väg mot regioner [A shared Norrland: moving towards regions]. Umeå: Department of Political Science, Umeå University. Pp. 127-137.

Woolfson, C., P.Thörnquist, and P. Herzfeld Olsson (2011). Forced Labor in Sweden? The Case of Migrant Berry Pickers. Report to the Council of Baltic Sea States. http://www.diva-portal.org/smash/get/diva2:528568/FULLTEXT01.pdf (31st October 2014).

Young, O. (2005). Governing the Arctic: from cold war theater to mosaic of cooperation. Global Governance, 11(1): pp. 9-15.

Young, O. and N. Einarsson (2004). Introduction. In: AHDR, 2004. Arctic Human Development Report. Einarsson, N., J. N. Larsen, A. Nilsson and O. R. Young (eds.), pp. 15-25. Stefansson Arctic Institute, Akureyri.

\section{Endnotes for this chapter}

iv The well-known Global Policy Forum has four categories: economic, cultural, political, and legal (n.d.). David Held and Anthony McGrew refer to economic globalization and political globalization (Global Policy Forum n.d.). George Ritzer notes that globalization can best be analyzed culturally, economically, and politically/institutionally (Ritzer, 2003: 439).

$\mathrm{v}$ In the first $A H D R$, Aarsæther, Riabova and Bærenholdt discuss the potentially negative impact of cultural globalization but counter that, in reality these impacts may be producing positive effects (AHDR, 2004). 



\section{Community Viability and Adaptation}

\section{Lead authors}

Rasmus Ole Rasmussen, Nordregio, Sweden, Grete K. Hovelsrud, Nordland Research Institute, Norway, Shari Gearheard, National Snow and Ice Data Center, University of Colorado Boulder, USA

\section{Contributing authors}

Helene Amundsen (Centre for International Climate and Environmental Research - Norway), Matthew Berman (University of Alaska, Anchorage, USA), Brigt Dale (Nordland Research Institute, Norway), Klaus Georg Hansen (Ilisimatusarfik, Greenland), Lance Howe (University of Alaska Anchorage, USA), Henry Huntington (Huntington Consulting, USA), Noor Johnson (Smithsonian Institution, USA), Julia Olsen (Nordland Research Institute, Norway), Johanna Roto (Nordregio, Sweden), Lindis Sloan (Center for Knowledge and Gender Equality, Norway)

\subsection{Introduction}

This chapter emphasizes the linkages between sub-systems within communities, and how they interact and impact each other with different implications for future development of communities. The task here is to focus on evidence of linkages and feedbacks between social structures, socio-economic conditions, health and well-being and demography, as well as some of the overarching geopolitical, physical and biophysical sub-systems and globalization of communities and settlements. 
In this chapter, the terms "community" and "settlement" are distinguished from each other: community includes political, social and cultural linkages, while settlement refers to physical and territorial factors. The ways communities respond to challenges and opportunities differ within and between nations and regions, and within and between settlements in the same region. While it is not feasible to describe the full range of such differences in the Arctic, this chapter aims to illustrate different examples of "good practices" for responding to challenges.

The chapter emphasizes the complex linkages between community development and processes such as socio-economic impacts, globalization, and environmental change. While bio-physical systems may be characterized by delicate balances, thresholds, and tipping points, socio-economic systems may be characterized by the fluidity, flexibility and adaptation of communities.

The chapter is organized around the basis, dynamics, and perspectives of communities and how these are shaped. The second section introduces the types of settlement structures throughout the Arctic, emphasizing similarities and differences across the region. Next, we provide an overview of different types of factors contributing to both maintaining and changing communities. Different types of dynamics are identified and elaborated: first, the question of communities inside or between settlements; second, the consequences of more Arctic communities being developed as diasporas with important relations outside the Arctic; third, the urbanization process in the Arctic; fourth, the establishment of communities within settlements; fifth, the consequences of changes in the economic base; sixth, the impact of newcomers to the Arctic; and lastly, the impacts of on-going climate change. We then move on to discuss the perception of communities as seen by stakeholders as "insiders", i.e., how different groups have very different considerations and visions on community life, determined through such characteristics as gender, generation, and vocation. The fifth section assesses the trends and processes that influence adaptation and viability of settlements and communities in the Arctic.

The first AHDR identified three major challenges facing Arctic communities that are relevant to community viability: urbanization, commercialization, and privatization. In addition, globalization was noted as affecting the Arctic (see also ASI, 2010: 52), and will be partially included here (see also Chapter 10, Globalization). Also, the first AHDR was primarily based on selected case communities of approximately the same size and with similar socio-economic characteristics, which 
facilitated the comparison between them but also limited the representation of the variations of communities. This chapter uses cases to illustrate the variations between different types of communities and the complexity in generalizing between cases.

Since the first $A H D R$, the process of urbanization has been confirmed as one of the major factors in current human development and is discussed here in connection with settlement structures, demography, and social and economic consequences. The challenges of increased commercialization in the Arctic are less clear, and this chapter illustrates considerable variations throughout the Arctic. Currently commercialization in both national and local economies pertains more to large-scale industrial operations than to local renewable resources, but the importance for local economies has diminished with the expansion of large-scale resource extraction. The divide between the center and the periphery has increased since the first $A H D R$.

Privatization has become a major force in the present Arctic development context. Furthermore large-scale mining, energy exploitation and industries led by global companies have turned into major drivers of change. In the first $A H D R$ (2004), privatization was perceived as a public-private partnership between the community and the outside world, but such partnerships are not as common as were anticipated. Partnerships are indeed formed, but business decisions are often taken without full participation of communities. The partnerships are also often gender-biased with a focus on male employment skills and interests, while female qualifications and interests are not equally included (Sloan, 2006; Ingolfsdottir, 2011; Megatrends, 2011).

In the first $A H D R$ two developments that were predicted to become important for community development have not materialized: 1) development based on combined pre-industrial and welfare economies, and 2) jobs based on public initiatives. Instead, the current reality shows that community development is more dependent on innovation and new initiatives, combined with welfare systems and transfer payments.

Finally the important question of climate change impacts was almost totally absent in the chapter on Community Development in the first $A H D R$. It is clear that the current rate and magnitude of climate change and the consequences for the social and ecological systems have become a general characteristic of most discussions in relation to Arctic futures. Even if the direct consequences of climate change on communities currently are less critical than socio-economic challenges, the Arctic as a region is facing major questions on how to handle pre- 
sent and projected changes. The ten years that have gone by since the first AHDR show marked - and in some cases fundamental - changes in the Arctic communities as a result of the impacts of climate change.

\subsection{Community basis}

\subsubsection{Settlements and communities}

Settlements are defined here as the physical layout of multiple communities, containing different smaller entities. The structure, size and characteristics of settlements vary considerably across the Arctic. Therefore, the impact of current processes of globalization and connectivity also vary and may have greater effects on settlements that are more deeply grounded in history, reliant on natural resources and with close social relations and interdependencies within the settlement. The number, size and location of settlements, towns and cities are changing through history, due, in part, to natural catastrophes, decay or abandonment of settlements due to epidemics. Likewise the temporary characteristics of some settlements are connected to events such as booms and busts in fish stocks (Hamilton et al., 2005), or exploration of non-renewable resources such as oil, gas, and minerals (see Textbox 11.1).

A broad set of factors determines settlement patterns, the structural traits they have, and whether settlements of different types exist at any point in time. Important factors include demographic dynamics, natural reproduction, and migration and mobility characteristics of settlements.

\section{Textbox 11.1}

\section{Introduction to settlement development}

The resources of the Arctic have been of interest to Euro-American colonialism and capitalism for centuries, causing many similarities in the development of the settlement patterns throughout the Arctic.

Among the major similarities were the monopolistic conditions of colonialism, such as seen for example in the cases of the Royal Greenland Trading Company in Greenland, the Hudson's Bay Company in Canada, and the Russian Company in Alaska.

The result has been settlement patterns which can be described as a conglomerate of similar settlement policies, very different commercial and industrial policies, and at the same time closely related to the traditional use of renewable resources. 
Early resource usage required a dispersed and flexible settlement structure, but through the process of modernization and industrialization, it became necessary to connect places by creating communication infrastructures and labor markets, and to develop social institutions. But above all, changes in resource usage required much more centralized (as opposed to traditionally dispersed) structures (Rasmussen, 1998; Duhaime, 1987).

During the 1950s and 1960s, only limited resistance against centralization tendencies was articulated, or the resistance was suppressed by governing bodies. But with growing consciousness about traditions, especially connected to the effort towards self-determination during the 1970s, new perceptions of what could be an optimal settlement structure evolved. In the case of Greenland, the traditional settlement pattern was directly accentuated through Home Rule Policy, emphasizing that "the villages should be the backbone of the settlement policy." But even when the official policy was stressing the importance of a continuous existence of the smaller settlements, the concentration process continued through the process of urbanization, with growing populations in the larger settlements and a decline of the share of the population living in smaller settlements (Duhaime, 1991; Duhaime et al., 1996).

For most of the Arctic, the majority of the population is today living in large settlements - towns and villages. Looking at the settlements in the Arctic outside Russia, more than $75 \%$ of the settlements have a population below 1,000 inhabitants and encompass only just below $15 \%$ of the total population. In contrast, less than $25 \%$ of the inhabited places have more than 1,000 inhabitants, but encompass above $85 \%$ of the population. The number of larger settlements is increasing while the smaller places tend to decline both in number and in size.

This pattern - a few but large settlements combined with a large number of small places clustering around the larger places - is a general characteristic of the settlement structure. Most small settlements are placed in relation to a land-sea continuum and primarily based on the more highly productive parts of the sea, or inland along the large resources where resources for fisheries, hunting of sea and land mammals are abundant and where ample grazing resources are available. Another characteristic group in the Arctic settlement structure is the mediumlarge scale settlements dominated by administrative and educational activities. 
The map below provides an overview of all settlements in the Arctic with a focus on population change during the period 1990-2010. All settlements with less than 5,000 inhabitants are shown as dots while settlements with 5,000 or more inhabitants are shown as circles with an area equivalent to the population number. The colors indicate the direction of change, with yellow showing places where no changes have taken place, red indicating places with a declining population, and blue showing places with a population increase. The insert shows a map of the northern part of Scandinavia, Finland and the Kola Peninsula where the settlement density is highest. The map highlights a marked divide in the speed of change between the Arctic part of the eastern and the western hemisphere. Most of the smaller places in Fennoscandia and Russia have been declining markedly in size, while in Russia a substantial number of the larger places have also suffered a similar fate. Changes especially in terms of declining birth rates have been a major factor, while out-migration from smaller to larger places and continued migration out of the Arctic has been important in the Arctic as a general pattern. Urban areas have experienced major growth as educational opportunities are available and new jobs related to the knowledge and service economies have provided new opportunities for upcoming generations (Hansen and Rasmussen, 2013). See Textbox 3 where further detail on the present distribution and trend in settlement development is discussed. 


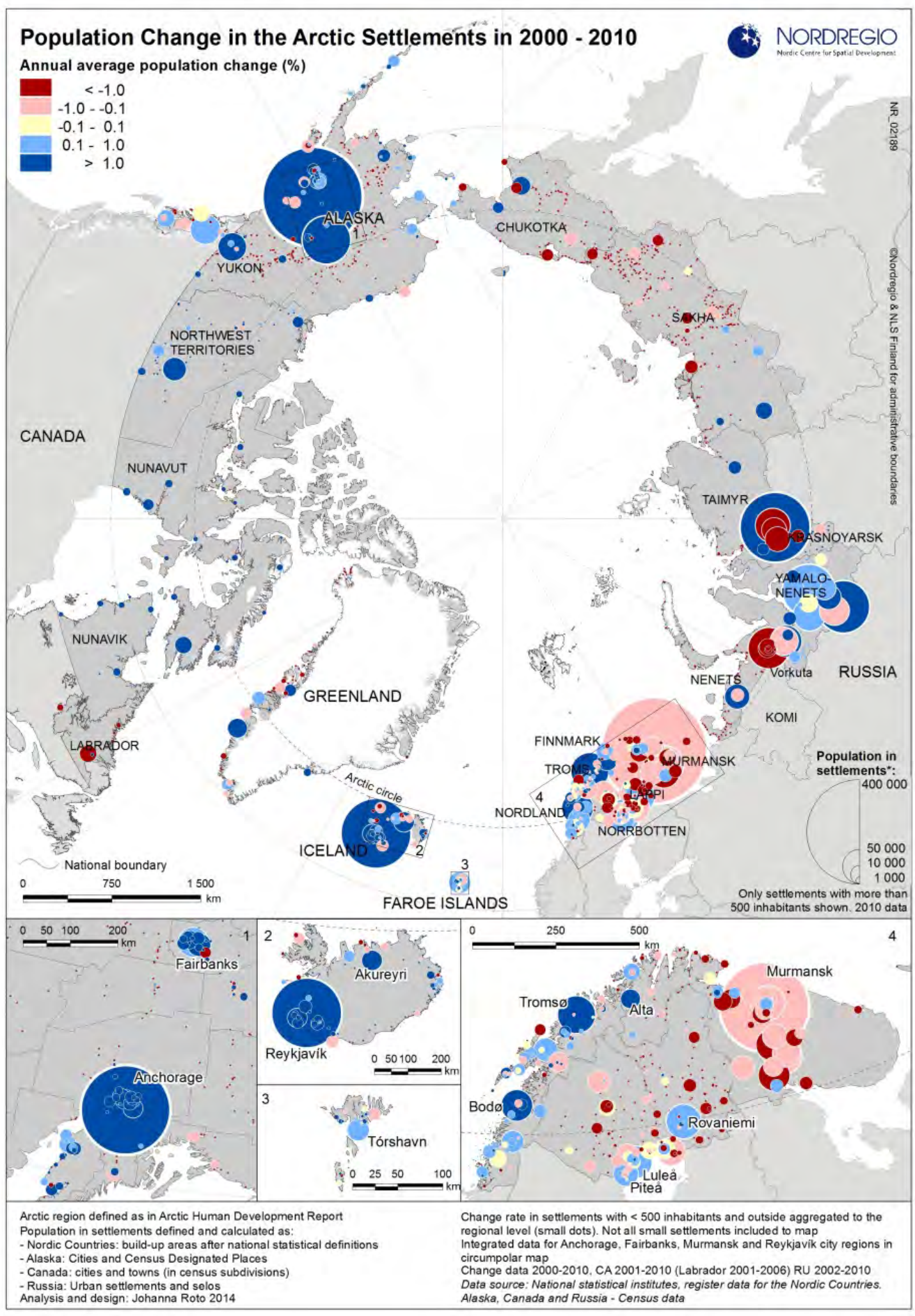

Part of textbox 11.1 
While settlements are considered as being individual physical settings, communities are social units within them. Communities can reach beyond individual settlements. Therefore, the viability of communities is connected both to physical space, the surrounding environment and a sense of common identity. As a consequence, communities are social constructs based on dynamic relationships between people, and can therefore serve as sites for negotiating community sustainability and viability (Davidson et al., 2003). In addition, people's ability to cope with change is influenced by power relations and the broader political context (see Chapter 5, Governance in the Arctic). Smaller communities in the Arctic are often characterized as being flexible and based on a diversified resource base.

\section{The community of Siorapaluk, Greenland, in 2007, one of world's northern most settlements}

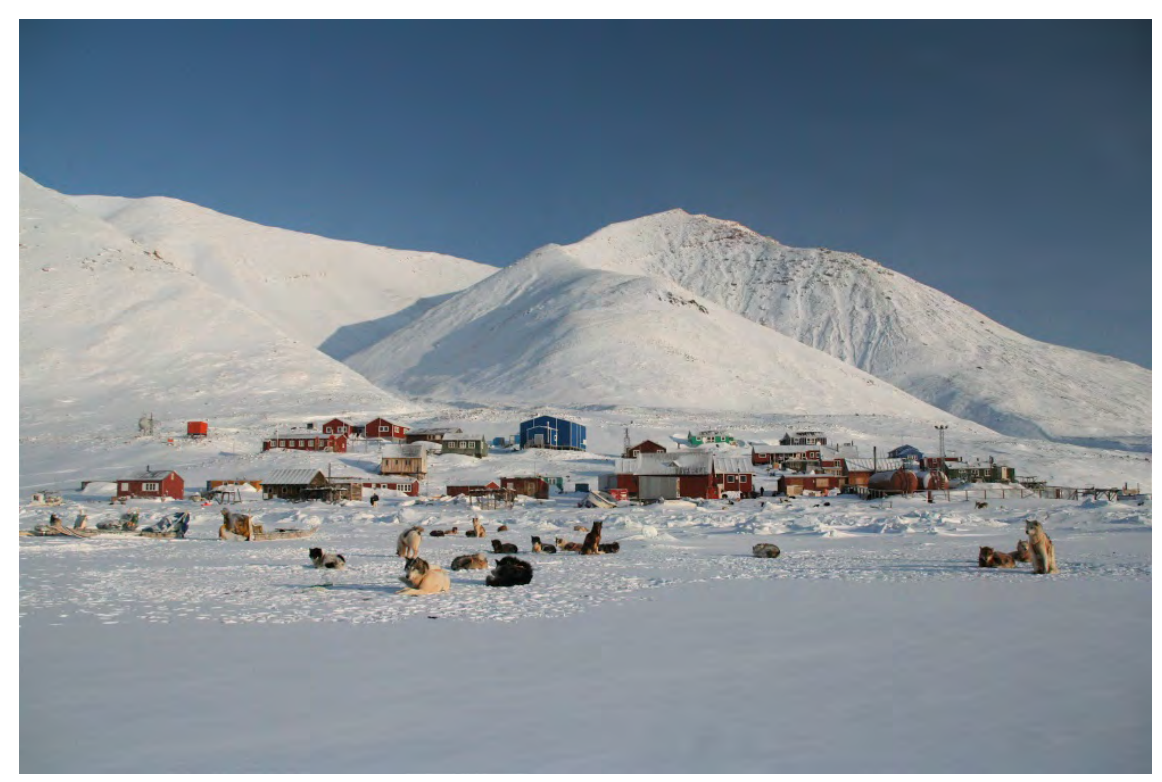

Credit: Shari Gearheard.

A focus on "community as a social unit" provides the context within which we may capture and understand the multiple factors creating viable and adaptive communities, or why a community is not viable or does not adapt to changes or risks (Harries and Penning-Rowsell, 2011). Interactions among different stakeholders across administrative and political scales can potentially enhance the adaptive capacity of communities, while a lack of tradition and methods for building institutional knowledge may affect communities negatively (Glaas et al., 2010). In addition, the 
viability of a settlement or a community is closely related to whether basic everyday security needs are met, ranging from concerns about the state of the environment, to the possibility of earning a living, to health care and education, to protection from imminent (physical) threats (Hoogensen et al., 2009; Dale, 2011; Hoogensen, 2012). In addition to socio-economic and ecological viability of communities, an understanding of how physical settlements are developed and maintained to ensure that communities can thrive is vital. This focus highlights the importance of population movements and subsequent processes of urbanization in some Arctic areas and de-population in others. It also reveals that there may be a competition between settlements (villages, towns, regions, and countries) when it comes to being attractive to new inhabitants.

Textbox 2 illustrates how an overarching management scheme aims for knowledge-based approaches which sets parameters for the way actors, both within and beyond local communities, may or may not utilize these resources.

\section{Textbox 11.2}

\section{Management of settlements and communities}

Most Arctic settlements did not appear randomly, but have evolved as part of development which requires conscious planning and management processes; in some cases involving the inhabitants of the settlements, and in other situations based on top-down processes (Duhaime, 1991).

While communities were formerly dependent on local resource extraction, the spectrum is now much wider and influenced by global development. Use of living resources has become polarized. On one hand with focus on effective and capital-intensive large-scale commercial fishing. These are usually based in larger settlements along the coast and generating products delivered to the world market. And on the other hand combinations of small-scale commercial fisheries, recreational and subsistence fishing which have evolved into distinct features of both coastal communities and inland fisheries along larger rivers and lakes. This raises significantly the issues of management of resources on the one hand - preserving the highly efficient distance fishing - but also the continued development of sustainable local fishing. These local fisheries also contribute to maintaining activities that are seen as an essential characteristic of coastal areas (Johnsen et al., 2005).

The increased focus on mineral and energy resources in the Arctic adds to the complexity, both in relation to activities on land and in the sea. Modernization and restructuring of public administration has been a central theme in the Nordic countries after World War II, with the first part of the process starting 
back in the 1960s, and another part of the process becoming a common theme for debate in all the Nordic countries during the past 10-15 years. The first part of the process may be characterized by two factors; a response to the significant changes in the industrial structure after World War II, and a response to the process of urbanization. In addition, the increased concentration of the population necessitated a shift from isolated small communities to more integrated functional units as part of the development of the Nordic welfare model. The pace of change has been largely related to the degree of dependence on local resources in combination with migration and increasing urbanization (Greve Harboe and Rasmussen, 2014).

These changes have resulted in demands for adjustments in the administrative hierarchies. In this connection the municipal structures and reforms have been important because this means both delegation and withdrawal of responsibilities from or to communities. It is a process which can be characterized as a double-edged sword: On one hand, the delegation of responsibilities takes place from state authorities to regions and municipalities which enables the municipalities to make decisions at a higher level. On the other hand, it increases the size of the municipalities whereby the distance between individuals and local decision-making powers are increased (Roto et al., 2013; Hansen and Rasmussen, 2013).

The processes of strategic, environmental, and social impact assessments are becoming an important part of planning processes where public hearings sometimes enable individuals and communities to become decisive in the planning process. There are national differences in the precise outlining of the processes, but also attempts to promote similarities and comparability.

Arctic settlement structures range from complex heterogeneous urban centers to smaller settlements where livelihoods are based on utilization of local resources. Settlements are typically located near the sea, or inland in connection with river and/or lake systems, where marine or fresh water resources are abundant. The increasing importance of urbanized centers and cities has evolved into a situation where they have become points of attraction of population - especially young persons and women - from the more peripheral settlements.

Globalization processes such as the industrialized utilization of natural resources for the global market are increasingly a major force of change in many Arctic communities. For example, in fishing communities where market mechanisms have strained the economy of small- 
scale producers, larger vessels increase their catch but reduce their number of employees. This is affecting communities where viability is linked to employment in fisheries. Additionally, onshore processing facilities have become more efficient and can handle increasing amounts of biomass, but are employing fewer people (Johnsen et al., 2005; Jentoft and Chuenpagdee, 2009). Similar situations characterize other uses of renewable resources and such changes emphasize three important dimensions of such uses: resource exploitation as a basis for (re)construction of identity, as a foundation for the development of ways of life, and in the context of economic and societal development.

\section{Hammerfest an oil and gas town, Norway}

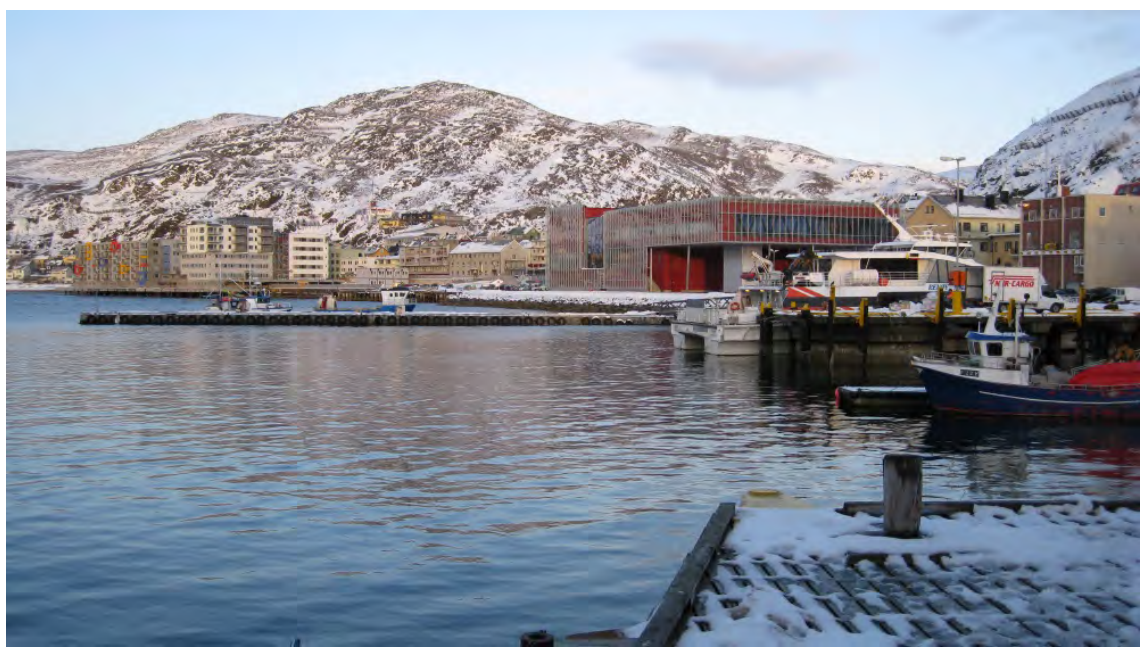

Credit Grete: K. Hovelsrud.

While the majority of the Arctic population lives in urban areas and towns, small settlements still outnumber large centers. Most small settlements, in differing ways, still utilize nearby natural renewable resources. An increase in medium-scale settlements dominated by administrative and educational activities is another typical feature of the Arctic. These small-scale urbanized hubs, with relatively stable economies, represent an important "backbone" in the Arctic settlement structure.

Finally, there are a few very large settlements or cities which continue to grow both in importance and in size due to their attractiveness as administrative, economic, cultural and educational hubs, providing a broad diversity of services, and thereby becoming attractive to younger people and especially younger women pursuing higher education opportunities and jobs requiring these skills. 


\subsubsection{Demographic challenges}

The different patterns of demographic change in the Arctic are a useful starting point for explaining why settlements and communities are exposed to different demographic challenges; not least by an increasingly diversified and mobile population. People move for a number of reasons, such as jobs, education, lifestyle preferences, and partaking in a globalized world. The move from a dependency on natural renewable resources (fisheries, forestry, hunting and gathering, herding) to a more diversified economy requires a specialized workforce, access to schools and universities, and lifestyle changes.

Chapter two, Arctic Populations and Migration, in this report provides details on different demographic components and an overview of the regional differences in demographic structures and transitions (see also ASI, 2010 on population indicators). A synthesis of such demographic characteristics highlights the role of population dynamics in community development and viability. If the birth rate does not outpace the death rate, or if out-migration exceeds that of in-migration, the population may be at risk of declining and the future of the settlement may be vulnerable. Gender imbalance may result if there are more women than men leaving the settlement. Yet another demographic vulnerability may be caused by a deficit of certain age groups, such as young people ages 15-24, leaving the settlements and looking for educational opportunities elsewhere.

\subsubsection{Cultural base of the communities}

Culture encompasses virtually all aspects of human life including language, knowledge, worldviews, beliefs, norms, values, social relationships, perceptions of risk, power relations, and understanding of and responses to the world we live in (Crate, 2011; see also ASI, 2010: 92, 101 , for further discussion of cultural aspects). Culture is dynamic and permeates rural and urban communities alike. It is important for maintaining identity and a sense of belonging. In a multi-cultural society, culture also carries potential for creating unequal power relations and conflict when some cultural features are promoted more than others. Cultural elements shape community viability, resilience, and both adaptive and maladaptive responses to socio-economic and environmental changes (see Chapter 3, Cultures and Identities; ASI, 2010 on indicators of cultural vitality).

Arctic communities often comprise numerous cultural and ethnic groups, and are highly dispersed with approximately one-third living in settlements of less than 5,000 people. The Indigenous peoples of the 
Arctic belong to the following major language families; Eskimo-Aleut, Na'Dene, Uralic-Yukagiran, Finno-Ugric and Altaic (AHDR, 2004: 47 (map)). The distributional relationship between Indigenous and nonIndigenous languages and cultures is highly variable within and between settlements, regions, and nations.

For many communities in the Arctic, culture and identity is closely related to farming, fishing, reindeer husbandry, hunting, sewing, handicrafts, and berry picking, whether full-time or as recreational activities. Such activities are also highly valued in more industrially-based communities, which are rapidly increasing in number. The continued preference for locally produced food suggests that an industrialized society does not necessarily exclude hunting and gathering activities.

\subsubsection{Food security, subsistence and informal economies}

Locally produced food plays an important role in the Arctic, including for health and cultural well-being (see Chapter 8, Human Health and WellBeing). Such foods are also sold and exchanged in both formal and informal markets and shared, bartered and exchanged within complex social and economic relationships. With increased reliance upon imported food stuffs in some communities, less locally produced foods are consumed, with implications for health and convenience. Imported food products are not replacing local products entirely because harvesting activities for household consumption are maintained in many instances. But more traditional sharing systems among many Indigenous peoples have changed, often resulting in a reduced supply of local foods (Duhaime and Bernard, 2008). This trend is reversing in other regions, such as Norway, where interest in local foods is increasing.

The role of local food products in the informal economy is difficult to assess in monetary terms, but it is clear that both the subsistence activities themselves and the consumption of the food-stuffs are considered vital to many household's well-being and cultural identity (see Textbox 11.3; also ASI, 2010, where "living close to nature" is identified as one of the components of well-being in the Arctic). Recent records of informal food distribution and transactions in Greenland - including home consumption, distribution to friends and families, products informally sold across municipalities, at the municipal Kalaaliminerniarfik (open air markets as described in Textbox 11.3), and distributed to homes for elderly people - show a contribution to the national economy of around $1.5 \%$ of GNP (Rasmussen 2005, Rasmussen, 2010). It has long been established that harvesting activities cannot take place without cash in- 
come, illustrating the close connections between the formal and informal economies and subsistence and commercial production (e.g. Dahl, 1989; Hovelsrud-Broda, 1999).

\section{Textbox 11.3}

\section{The role of local food and the informal economy}

Marquardt and Caulfield (1995), in their analysis of local market development in Greenland, demonstrate not only how subsistence activities in Greenland coexist with the formal economy, but also that the discourse in Greenland has included subsistence and informal marketing as important elements in economic development. Through their analysis, they show how the informal local markets - the Kalaaliminerniarfik - "brættet", arose in the 18th century to meet the needs of the employees of the church and the KGH (Royal Greenland Trade Company) They show how the local markets were used as a kind of "leveller" which removed differences in access to imported goods between Greenlandic hunters and those working for the colonial authorities.

The markets worked as redistribution channels through which hunters were able to obtain the necessary funds to buy European goods, while Greenlandic and Danish salaried employees were able to access valued country foods and locally produced items of clothing and other goods. Without the local markets a more clear separation might have developed between subsistence and commercial activities. The local markets enabled an integration of the two systems and ensured that the population of most of the major towns had access to nearly all types of country foods. It has further enabled the transition of these highly valued food items into shops and supermarkets as commercial food items throughout Greenland. The informal sector which provides traditional foods to almost all settlements in Greenland contributes approximately 1.5\% of the GDP while at the same time the commercialization of the products is adding substantially more to the economy (Rasmussen, 2005).

The situation in Greenland is by no means unique to the Arctic. Similar developments have taken place elsewhere, and the accompanying photos show how traditional food items are available through small informal market places as well as in the larger stores in a variety of places in the Nordic Countries and northwest Russia. 
Supermarket in Illulissat, Greenland

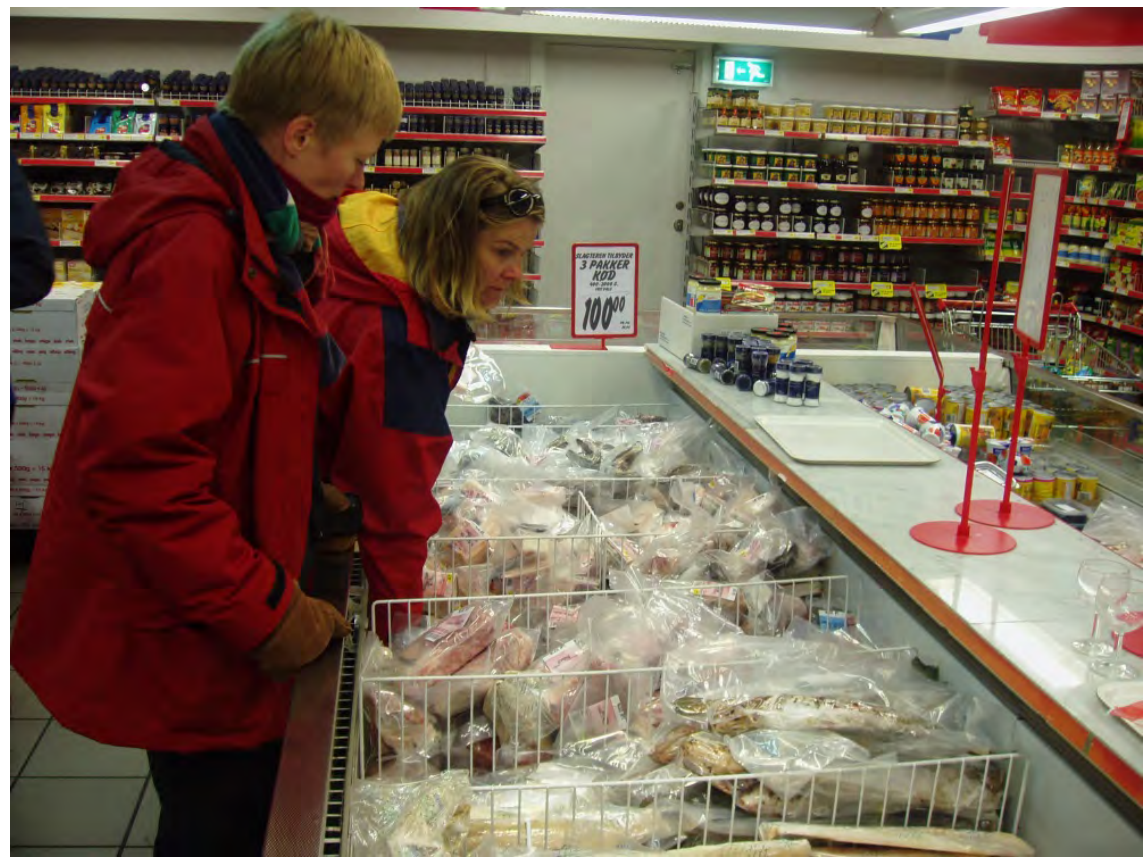

Credit: Rasmus Ole Rasmussen.

Sale of whale meat. Oslo, Norway

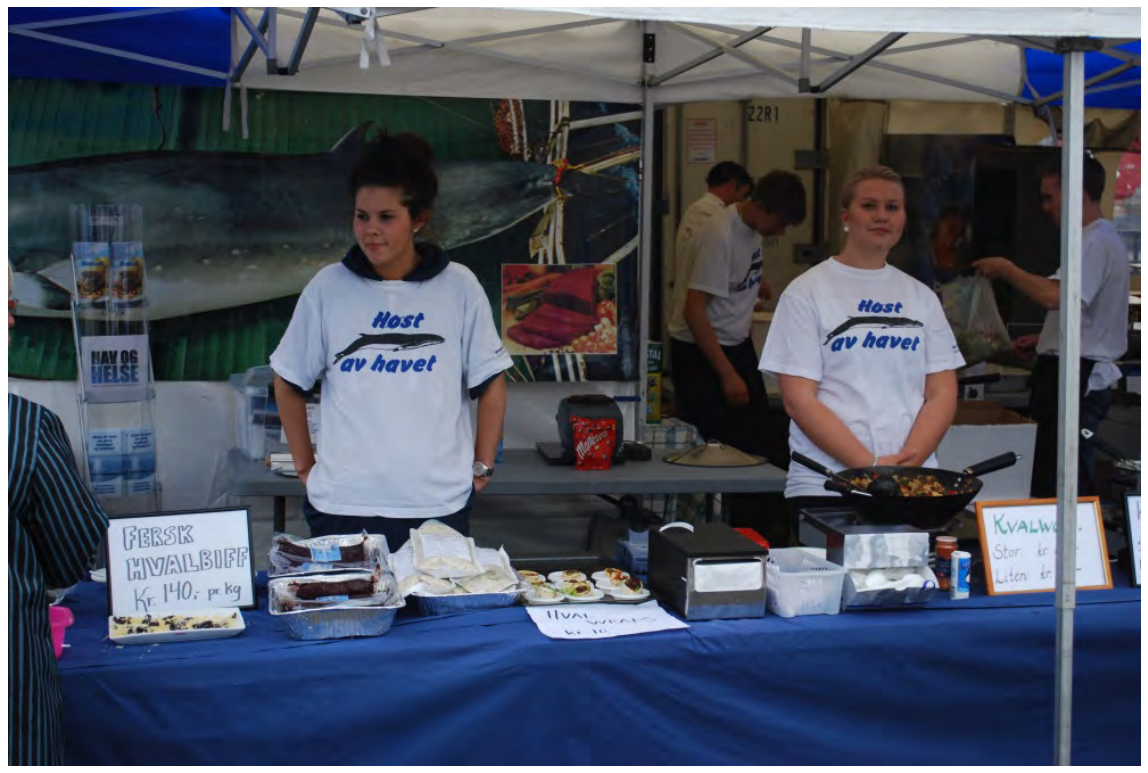

Credit: Rasmus Ole Rasmussen. 
There is a continuum of options for access to local versus imported food. The commercialization of local foods and distribution through supermarkets and specialty shops enables the population in most parts of the Nordic countries, Faroe Islands, and Greenland, to access many traditional food items. The right of common access (allemannsretten) in Fennoscandia provides an opportunity for all to pick berries, mushrooms and other sought after food stuffs, resulting in a situation in which many people are harvesting local foods. Historical use has become a popular legal right, and in some instances has caused conflict over the resources (i.e., cloudberry "wars" in northern Sweden and northern Norway). In Canada, where the sale of country foods is regulated by food safety laws, residents are trying to ensure access to local country foods through means such as elder/youth hunting/gathering programs, like one in Nain called "Aullak, Sangilivallianginnatuk: Going on the land, Growing strong" where volunteer hunters are teaching youth hunting skills and harvesting. The program is helping ensure access to local foods for households in need (thus supporting food security in the present) and supporting the future food security of the community (through skills development).

Research on food insecurity in Canada has highlighted the intersecting nature of environmental and social stresses, including on-going poverty, the high cost of store-bought food, and increasing difficulties in obtaining country foods (Chan et al., 2006). Food security in the Arctic, a key element in community viability, is in large parts of the region closely tied to the so-called, "Arctic public health dilemma" (Bonefeld-Jørgensen, 2009). One concern is that the accumulation of environmental toxins in the food chain ends up in traditional food sources that consist of nutrients and fatty acids essential for healthy living. In addition to threats posed by pollution from the south, large-scale industrial resource development mainly in the North American and Russian contexts also threaten food security, health, and well-being of many communities, mainly due to environmental pollution or the limitations it creates in terms of access to hunting, fishing and gathering areas (AMAP, 2004; Loring, 2010; Ford and Maeaumier, 2011). Local food gathering activities are important to health and well-being in Fennoscandia, but without having a strong subsistence component. Such activities are culturally and historically entrenched in the rural population at large, across settlements and communities. Fennoscandia has a long history of co-existence between Indigenous, non-Indigenous and different ethnic groups, creating a pattern of multiple and localized identities with strong ties to food. 
Local food-stuffs sold on the open market in Greenland, Kalaaliminerniarfik

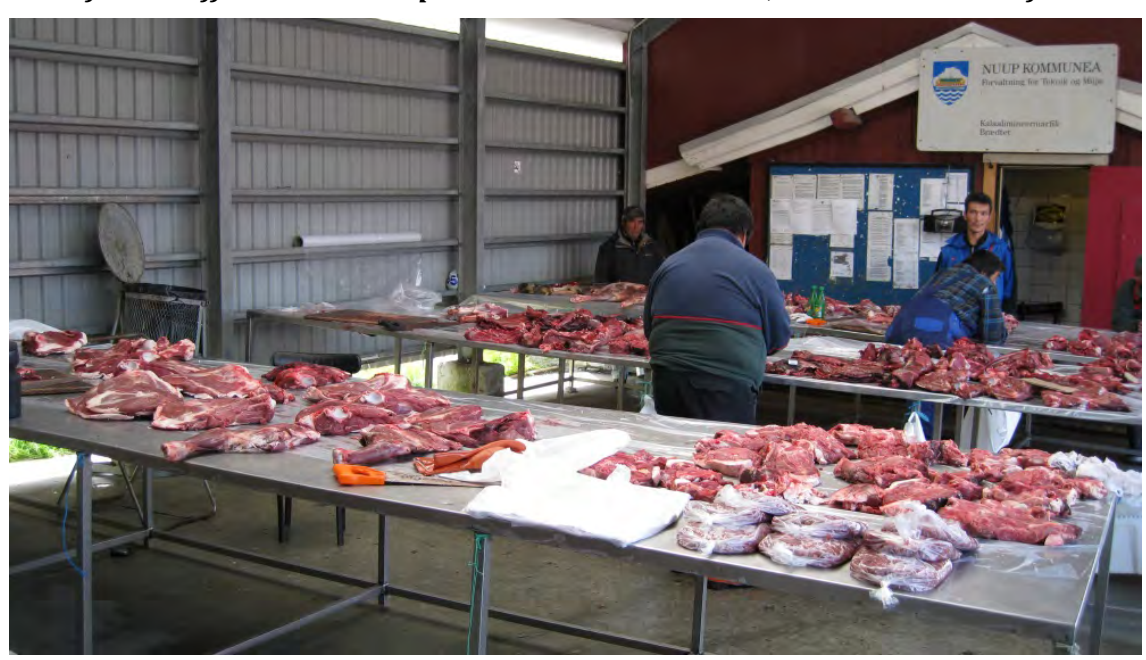

Credit Grete: K. Hovelsrud.

\subsection{Community dynamics}

The current rates and magnitude of demographic, social, economic, and environmental change in the Arctic have significant impacts on community viability and adaptation. The processes taking place are highly complex and interlinked. The purpose of this section is to highlight some of the processes and dynamics that have had an impact on communities in the past decade and may be expected to continue into the future. This section is closely linked to Chapter 10, Globalization.

\subsubsection{Urbanization}

Urbanization both in and outside of the region has a strong influence on Arctic communities (see also ASI, 2010: 52). The processes of urbanization are often met with scepticism in communities but are also seen as a necessary way of adapting to a changing world (Christiansen, 2013). As described in a recent report on megatrends influencing Arctic communities (Megatrends, 2011), urbanization was for a long time tied to industrial development, bulk trade from primary industry (mostly from the fisheries, hunting and herding) and public administration. With the emergence of a "knowledge society", educational possibilities also shape the future of Arctic settlements, as centers providing primary, secondary, and higher education become attractive as permanent places of residence (Dahlström and Hedin, 2010). Examples of this trend are the ways the establishment 
of universities in Tromsø, Norway (1972) and Nuuk, Greenland (1987) have spurred development and growth (Hedin, 2009).

In the Russian context, the strengthening of educational and research centers in Murmansk and Arkhangelsk in Russia has been important, although specific Russian circumstances have led to a present decrease in population in both cities. Additionally, the Arctic has been an important geopolitical arena for Russia. Development of urbanised settlements has also been characterized by military presence. From the mid1930s onwards, Soviet state policy in the Russian Arctic began to regulate the process of developing the Arctic regions and urbanizing the Soviet Arctic. The urge to use Arctic natural resources for the development of the national economy provided the driving force for these processes and political presence superseded the external threat factor. In 19331935, the issues of studying, developing and colonising the archipelagos of the Barents Sea came under the supervision of the Main Directorate of the Northern Sea Route, marking the transition to the centralised administration of these processes (Zahlkind, 2013). The fall of the Soviet Union in 1991 has resulted in a massive out-migration from the Northern periphery. Price liberalization, the fiscal decentralization and a shift in Russia's approach to the development of its Arctic and sub-Arctic regions have been the major causes for out-migration (Heleniak, 1999).

Another example of an urbanizing centre is Iqaluit, the capital of $\mathrm{Nu}-$ navut, Canada, where many Nunavut residents (a majority of them Inuit) move to seek education and government and administrative jobs. The challenge here is the possibility of extending rural and small community identity into an urban space (Abelsen, 2013). Indigenous identity, however, may not necessarily be either urban or rural. Many have skills in several cultures, and have multiple identities (see Chapter 3, Cultures and Identities). For example while living in cities, Indigenous mothers may make political demands for kindergartens and formal education systems to increase their children's possibilities to learn native language and culture during the city life. 


\section{Textbox 11.4}

\section{Urbanization}

"Urbanization is a global trend which will significantly contribute to the shaping of human life in the future. The Arctic region is no exception. Since the 1960s, most of the population growth in the Arctic has occurred in urban centers tied to industrial activities, social services and public administration" (Megatrends, 2011: 22).

The process of urbanization is partly the result of people seeking new opportunities. An increased population density enables better services such as childcare, education, and leisure. At the same time urbanization also presents a clear risk of generating disparities, economically as well as socially. In many ways it is a natural way to adapt to an ever-changing world, but at the same time it may present a threat to ways of living and the life people have become accustomed to.

The map shows the differences between the distributions of the population living in different settlement types. Small, predominantly rural villages typically with less than 1,000 inhabitants are marked by yellow; the larger villages and smaller towns with less than 10,000 inhabitants are shown by light blue colors. Large towns with between 10,000 and 50,000 inhabitants are shown with middle-blue colors, while the cities with more than 50,000 inhabitants is shown by dark blue.

Urban life has remained one of the distinctive characteristics of Russia's settlement pattern in its Far North during most of the last century, and today nearly $80 \%$ of the country's northern population are living in urban environments (Nord, 2005). Other countries exhibit similar trends, often with sharp disparities by age and gender (e.g., Hamilton and Seyfrit, 1994).

People move for many reasons, often attracted by the promise of work, higher salaries and a better social life, just as urban areas usually offer better opportunities, a diversity of economic activities and more options for education and social networks. While cities can be considered as economic development hubs of their regions, they may also foster social inequality, a dynamic that has also become a critical issue in the Arctic. 


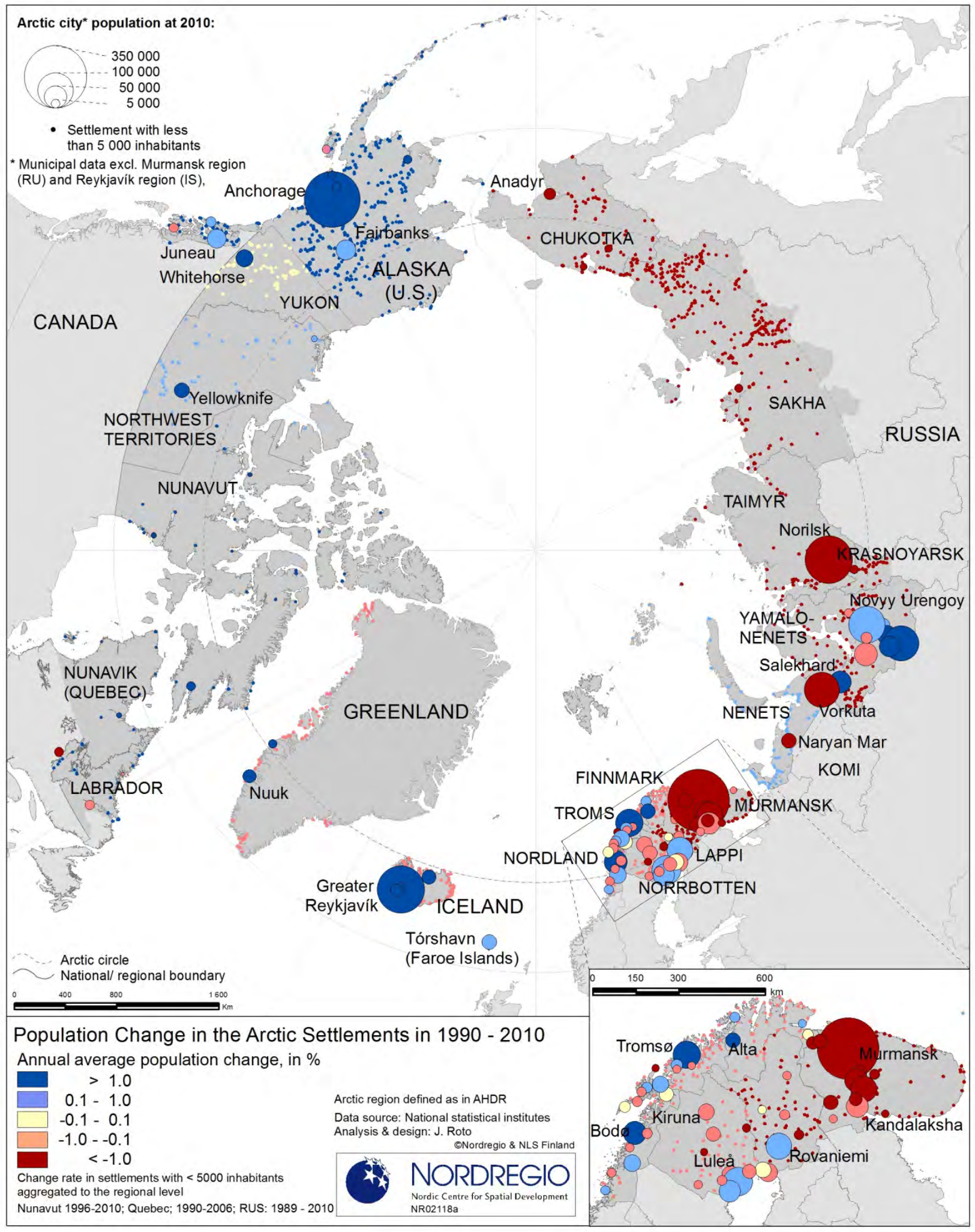

Part of textbox 11.4 
The large Sámi urban populations in the three Nordic countries of Finland, Norway and Sweden consist of a relatively young migrant population with cultural roots elsewhere. But there is an emerging Sámi presence and also promotion of Sámi culture in cities such as Troms $\emptyset$ and Umeå. In addition, cultural events, civic organizations, and educational institutions, such as Sámi University College in Kautokeino, contribute to an urban Sámi culture. Despite the fact that one third of the registered Sámi population is living in towns, only a few studies document the situation of this segment of the Sámi nation. The plurality of cultural arenas in urban settlements constitutes important meeting places for people and cultures from within and beyond the Arctic. In this context, a new generation of Sámi has emerged, regenerating and rejuvenating Sámi identity (Pedersen and Nyseth, 2013). Even though the urban Sámi communities have cultural roots elsewhere, a 30-year build-up of institutions such as kindergartens, primary schools, university studies and research institutions has made the urban Sámi more visible. Some have described the urban Sámi culture as being characterized by freedom, reflection and innovation (Dankertsen, 2006), with less stringent social control with respect to religious piety and traditional usage of symbols and artifacts than those characterizing many rural Sámi communities.

All residents of the Arctic countries are more or less affected by urbanity, the world market, and globalization with positive influences on and from Arctic culture, behavioral norms, language, food, music and fashion (Dybbroe, 2007: 2). Still, the focus is generally on the distinction between tradition and modernity as a way to approach social problems and social changes (Dybbroe, 2007). While urban development in the Arctic countries is not comparable with the city life of New York, London or Tokyo, the same anti-urban discourses are found in Arctic countries. For example earlier urban research on Greenlanders focused on how Indigenous populations have adapted to city living controlled by a colonial power. Despite the fact that the first signs of urbanity can be traced all the way back to the nineteenth century, Greenlandic literature is marked by many depictions of the city as a negative place to be for Greenlanders. Urbanization in Greenland has undoubtedly had a number of negative effects on many families, but it has also had a positive impact on life in Greenland; it has provided better housing, health care, education possibilities and wage labor. Recent research on Greenlandic urbanity has shown that towns and cities are increasingly seen as positive places to live (Sørensen, 2008).

For urban Sámi communities, there is a whole range of particular characteristics compared to more traditional Sámi communities in the 
rural peripheries (Pedersen and Nyseth, 2013). Their composition is more heterogeneous reflecting the differences in material and social contexts of the city, compared to the more rural Sámi communities, which tend to have more settler type characteristics.

Data from Statistics Canada show that Inuit made up a steady $60 \%$ of the Iqaluit population between 1996 and 2006, including many who moved there from across the territory, meaning that multiple dialects are spoken in the capital of Nunavut, and that Inuit residents are themselves a heterogeneous group (Shields and Weber, 2013). Furthermore, the data show that Iqaluit residents have become a more multicultural group, with a fifth coming from other provinces or territories in Canada, and a growing number being new Canadians such as Sudanese and Somalis.

Settlements in the Arctic may seem relatively isolated, but they are increasingly interrelated across boundaries through administrative, production and educational functions. As described above, throughout the Arctic, urban centers are growing in both numbers and size relative to rural settlements, with some small communities showing a clear decline, again both in size and numbers (Hamilton and Rasmussen, 2010). Urbanization of the Circumpolar Arctic in some ways reflects the global trends. The processes driving Arctic urbanization, however, differ in many respects from the global processes. Firstly, and in contrast to much of the rest of the world, the Arctic has never developed a significant agricultural base to drive the take-off of urbanization. The lack of agriculture also meant that the Arctic did not develop the internal transportation infrastructure to move agricultural products to markets. Instead, settlement of Arctic Indigenous peoples occurred largely as a consequence of colonial policies of the states that governed these regions from distant capitals. Arctic towns and cities built around exploitation of nearby resources such as gold mines tended to be highly seasonal and temporary. Exceptions were communities that developed as centers of public administration of the territories and those following direct government interventions for national defence or industrial policy such as the Russian cities of Murmansk and Norilsk, and Anchorage, Alaska (Armstrong et al., 1978).

Evidence suggests that the trend for rural Arctic areas is depopulation in line with areas outside the region (Heleniak et al., 2011; see also Chapter 2, Arctic Populations and Migration). This is dependent upon public policies, and is connected with questions of mobility, which is the topic of sections below. 


\subsubsection{The new Arctic diaspora}

The Arctic has been exposed to both substantial in- and out-migration of people during the last century. As discussed in the first ASI report, both in- and out-migration reflects the sum of various pull and push factors (ASI, 2010: 149). Historically, in-migrants reflect policies aimed at ensuring national presence in the region. This presence has often been connected to development strategies where new economic activities have required newcomers with specialized skills and knowledge. Typically, the people with these assets come from the southern areas of the respective Arctic nations. Many of these newcomers only live in the Arctic communities for a few years; others have become permanently settled. For many of the permanent settlers, ties back to their place of origin - their "homeland" - have been maintained; for instance, through cultural characteristics such as food habits and regular visits back to their communities of origin. The reverse process (i.e., people with origins in the Arctic moving south and settling there for longer periods of time, or permanently) has taken off since WWII and intensified so much during the last decades that it has a potential to become a major characteristic of Arctic Indigenous peoples. In spite of the geographical distance to their traditional homelands they have, in many cases, chosen to maintain personal, cultural, and economic ties to their families and communities of origin. This pattern of in-migrants and out-migrants has established what is generally referred to as a diaspora, or the movement of people away from their homeland (Rasmussen, 2013).

In its original meaning, "diaspora" describes the scattering of a population because of force or traumatic historical event (Cohen, 1997). A broadening of the concept recognizes that scattering processes connects to other causes and effects of mobility (Skeldon, 2001), such as labor. Furthermore, increasing mobility combined with the processes of scattering is no longer seen as one-directional (Brubaker, 2005). Evidence of return has become fairly abundant, captured by the term "counter-diasporic migration", referring to the process whereby the second generation relocates to the ancestral homeland. The question of counter-diasporic migration has become a critical issue in the former Danish colonies of Greenland and the Faroe Islands (and partly also Iceland). The main issue in this connection is options of "welleducated returners" as a means to cope with what is considered to be a stability-threatening high out-migration rate (see Chapter 9, Education and Human Capital). New media and other means of communication help in providing news and information links between homeland and host country, and serve as vehicles for community continuity. At the 
same time, in some areas such as Yakutia, Russia, media is drawing youth away from their communities to pursue a "better life" in the regional centers (Crate, 2006).

\section{Textbox 11.5}

\section{New Arctic diasporas}

"What distinguishes the diasporic condition from contemporary international migration and transnational communities are the historical continuity across at least two generations, a sense of possible permanence of exile and the broad spread and stability of the distribution of populations within the diaspora. In other words, 'time has to pass' before a migration becomes a diaspora” (Cohen, 1997: 185).

Mobility between Greenland and Denmark has been large since the 1960s when the modernization process led to a large influx of Danish labor to Greenland, and subsequently a large outflux of Greenlanders, not the least Greenlandic women who entered marriage with Danish craftsmen. Since the 1970s and especially in the last decades, mobility has been very closely related to education, and as a consequence a second outmigration-wave driven by the pursuit of education and the fact that many relationships between young Greenlanders and Danes have been established in connection with training in Denmark. This has led to the development of a diaspora with about one-fifth of persons of Greenlandic descent living permanently in Denmark, and the number is increasing.
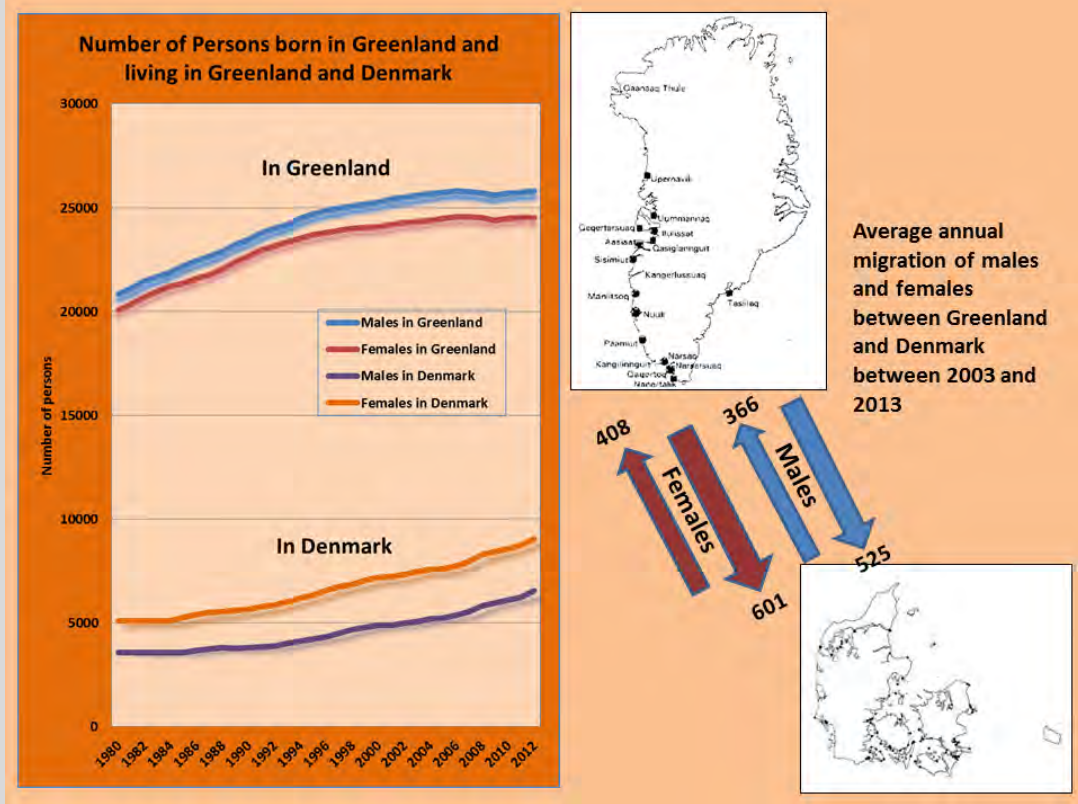
The illustration shows the population of Greenlanders born in Greenland in the period 1980 until 2012 subdivided between males and females. The top graph shows the group living in Greenland. It shows a marked stagnation in both males and females since the 1990s, while the graph below shows the population of Greenlanders born in Greenland but permanently living in Denmark. The population of Greenlanders living in Denmark has been increasing, and with the number of females increasing significantly. To the right, the graph shows the average annual migration of males and females between Greenland and Denmark, and the resulting net-outmigration.

In the Jewish diaspora the pronouncing of the wishful prayer "Next year in Jerusalem" is a manifestation of the unity within the diasporic community. A similar situation exists within the Greenlandic diaspora. Since Home Rule was established in Greenland 1979, 30th July to August 1st has become the world's largest Greenlandic cultural event outside Greenland. The event takes place in the amusement park Tivoli in the center of Copenhagen, and besides meeting with friends and relatives the arrangement includes cultural events such as kayak-turning, ritual mask dance and a lot of music events with both traditional and contemporary artists. Greenlandic country-food is available, just as a number of products from Greenland sold in booths.

In more recent years the event has been visited by around 100,000 participants yearly encompassing a mixed group of Greenlanders from Greenland, Greenlanders from Denmark, but also Danes who are married to Greenlanders, and Danes who have been employees and continue to feel an attachment to Greenland. 
So similar to the Jewish hopes for the future, Greenlanders have promoted their own wishful thinking:

VI SES NASTE ÅR I TIVOLI (See you next year in Tivoli)

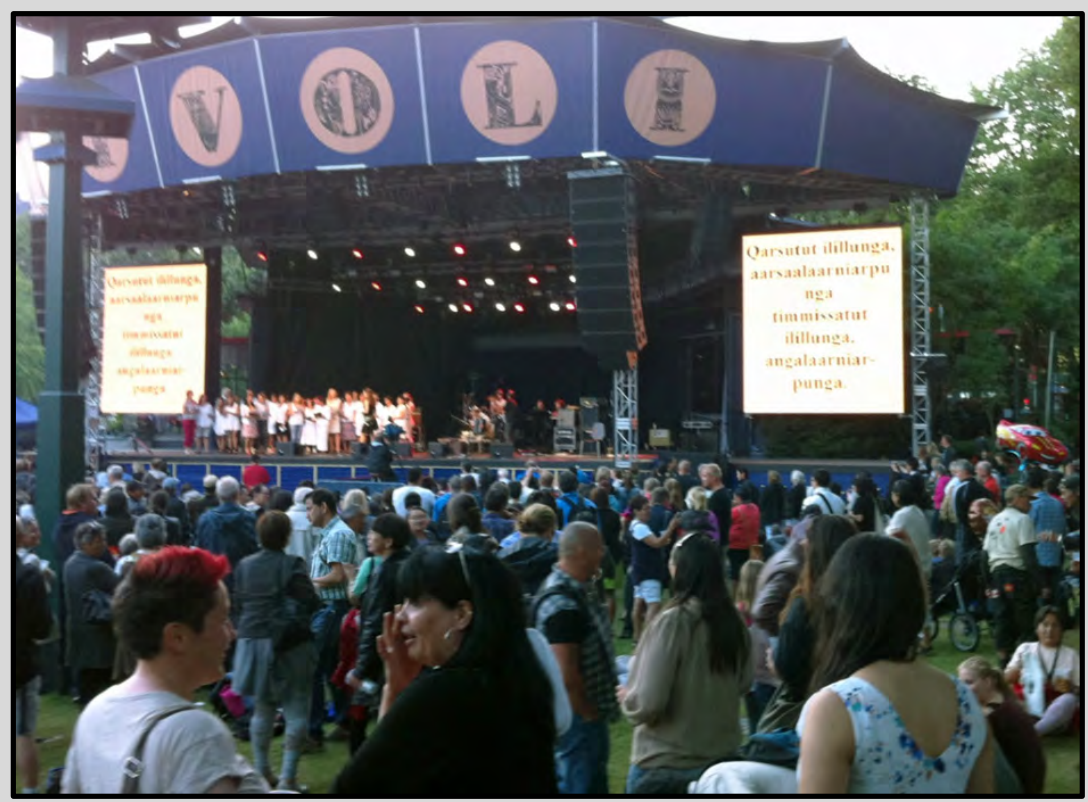

Photo: Karen Nathansen.

\subsubsection{New economic mechanisms}

There are differences in contemporary Arctic mobility characteristics between age groups, levels of education and gender that are connected to complex individual choices and preferences. In turn, these differences are shaped by family relations, community attachment, status and position within the community, and connected to the world outside the community and relations to the labor market. Mobility has increased in the past decades, both within and between communities in and outside the Arctic. Job opportunities, education, changes in social relations, and in some cases, impacts of climate change such as destruction of infrastructure due to coastal erosion or thawing permafrost, are reasons for moving. The on-going processes of globalization are clearly important contributors pertaining to job opportunities, educational options, and 
changes in social relations both inside and outside the communities (see also, Chapter 10, Globalization).

In this context, the "knowledge economy" becomes one of the major driving forces with respect to upcoming generations' decisions on whether to stay or leave their communities. The role of both economic and social innovations varies across different regions of the Arctic and the establishment of educational institutions in Arctic cities has in many areas played a role. An example is the Troms $\emptyset$ region in northern Norway, where the establishment of the northernmost university in the world was an important impetus for a rapid growth in population, as well as a diversification of business and community life. Over the past 40 years, Troms $\varnothing$ has grown from being a regional center with a population of approximately 35,000 , still struggling with the outflow of youngsters to the south seeking education and career opportunities, to becoming a net influx area with more than 70,000 inhabitants.

Expectations in relation to the welfare society for the older generations, and increased mobility where young people settle outside the Arctic and establish families and new networks are important aspects of migration. The influx of newcomers to the Arctic from, for instance, Southeast Asia, readily involves the service sector and newcomers tend to settle more permanently, adding to the diversity in the population structure. The future role and impact of large economic activities in relation to mining, resource processing and oil and gas extraction is currently a key debate as well (see Chapter 7, Resource Governance, for more indepth discussion on resource development activities and impacts in different Arctic regions).

\subsubsection{The "new" newcomers}

Newcomers to the Arctic, coming mainly for temporary and to some extent, permanent employment, have had an increasing influence on communities. In addition to seeking work, many people, predominantly women, come from other parts of the world to marry and start a new life in the North. The involvement and influence of people, either directly through working in industries, or indirectly through jobs created in adjacent activities, constitutes one of the most important links between industry and the local communities. The labor market is a crucial element in the interactions between the community and industry, such as mining and energy production, where the concept of labor market multipliers can be applied in order to provide a measure of the total involvement of the local labor market (Storey and Hamilton, 2004). The 
need for skilled labor generates a number of positive effects on communities. Advanced education opportunities for the local labor force to develop their qualifications also lead to a general improvement of local skills. This demand often results in a general improvement of available services, which creates new jobs and thereby a more open and dynamic economy. In addition, the pressure on more advanced qualifications leads to a more innovative milieu, which in turn has a significant role in the development of new economic activities. Such activities, however, may also have negative consequences for communities. New activities usually require qualified workers, and if looked for locally this may cause a drain on local human resources mainly because of higher salaries. If the qualifications are not accessible locally, the influx of new employees may put pressure on the existing physical and social infrastructure. The generally higher level of salaries in the new activities may create wage distortion - the Dutch Disease phenomenon - which in addition to draining qualified workers, may increase salaries and living costs, threatening existing businesses (Winther, 2000).

\subsubsection{Communities and climate change: impacts and adaptation}

In the Arctic, the many and myriad direct and indirect impacts of climate change have an effect on people (e.g. Hovelsrud et al., 2011; Kapsch et al., 2010). At the same time, peoples in the Arctic have throughout history and colonial encounters adapted to significant societal and environmental changes. The observed and projected impacts of climate change present both challenges and opportunities, albeit with regional and local differences in how people and institutions experience, understand, and respond to those impacts. Climate change is interlinked with the political, economic, cultural, and social context of a place. The impacts of climate change interact with other human-environment systems, can exacerbate existing challenges, and may create unexpected cascading effects that require adaptation (e.g. see Chapter 7, Resource Governance, for discussion of climate change and interactions with issues around resource development; also Hovelsrud et al., 2011). 
Textbox 11.6

\section{Potential impacts of climate change}

\begin{tabular}{|c|c|c|}
\hline $\begin{array}{l}\text { Cryospheric change } \\
\text { (exposure sensitivity) }\end{array}$ & Implications and challenges & $\begin{array}{l}\text { Adaptation strategies and governance } \\
\text { implications }\end{array}$ \\
\hline $\begin{array}{l}\text { Sea Ice } \\
\text { Shorter duration of } \\
\text { land-fast sea ice; } \\
\text { Poorer ice quality; } \\
\text { Reduced sea ice; } \\
\text { Longer and larger } \\
\text { open-water season at } \\
\text { the coast }\end{array}$ & $\begin{array}{l}\text { Land-fast ice travel and hunting } \\
\text { hampered; Safety of sea ice-based } \\
\text { hunting compromised; Changes in } \\
\text { fish stocks and fisheries, increased } \\
\text { access for fisheries, increased } \\
\text { traffic at sea, increased industrial } \\
\text { activity, oil and gas exploration; } \\
\text { Increased fetch (and storminess) } \\
\text { erodes coastline }\end{array}$ & $\begin{array}{l}\text { Changes in transport mode, shift to open- } \\
\text { sea hunting and fishing, new equipment } \\
\text { such as GPS and satellite phone required, } \\
\text { Fishery regulations must change, } \\
\text { International Maritime Organization (IMO) } \\
\text { standards for shipping in the Arctic, higher } \\
\text { costs of security provision, stricter } \\
\text { environmental regulations; Shoreline } \\
\text { stabilization and engineering options, } \\
\text { eventually relocation; authority to relocate } \\
\text { communities }\end{array}$ \\
\hline $\begin{array}{l}\text { Thawing permafrost } \\
\text { Increased coastal } \\
\text { erosion, increased } \\
\text { runoff, drainage of } \\
\text { forested areas }\end{array}$ & $\begin{array}{l}\text { Threatens coastal settlements; } \\
\text { Damage to poorly engineered and } \\
\text { constructed infrastructure; Release } \\
\text { of legacy pollutants that affect the } \\
\text { food chain and have negative health } \\
\text { effects; Tree death caused by } \\
\text { drought; increased forest fire } \\
\text { occurrence }\end{array}$ & $\begin{array}{l}\text { Shoreline stabilization and engineering } \\
\text { options, eventually relocation, authority to } \\
\text { relocate communities, design and } \\
\text { engineering to counteract permafrost } \\
\text { changes; Increased surveillance of } \\
\text { contaminants in traditional/local foods; } \\
\text { Investigation of health and ecosystem } \\
\text { effects on biota; Increased surveillance and } \\
\text { response capabilities to fires; altered } \\
\text { management regimes to enhance harvesting } \\
\text { of 'at risk' forests }\end{array}$ \\
\hline $\begin{array}{l}\text { Glacier and Ice caps } \\
\text { Retreating, Increased } \\
\text { calving }\end{array}$ & $\begin{array}{l}\text { Threat to planned infrastructure } \\
\text { development, marine transport, } \\
\text { fisheries, and oil and gas } \\
\text { exploration; Increased hydropower } \\
\text { production, reduced attractiveness } \\
\text { to tourists }\end{array}$ & $\begin{array}{l}\text { Improved mapping, surveillance, and } \\
\text { monitoring; Increased water storage } \\
\text { capacity }\end{array}$ \\
\hline $\begin{array}{l}\text { Snow } \\
\text { Increased frost and } \\
\text { thaw and ice cover on } \\
\text { tundra; Increased } \\
\text { amount of snow, } \\
\text { snowfall and winter } \\
\text { temperatures; } \\
\text { Increased growing } \\
\text { season; Increased } \\
\text { winter thaw, earlier } \\
\text { snowmelt, shorter and } \\
\text { milder winters }\end{array}$ & $\begin{array}{l}\text { Reindeer access to fodder altered, } \\
\text { increased mortality; Increased } \\
\text { hydropower production, damage to } \\
\text { forests, increase in avalanches; } \\
\text { Increased productivity in forestry } \\
\text { and agriculture; Increased tree } \\
\text { death for certain conifers and } \\
\text { damage to forests from insects and } \\
\text { pests, drought in dryer areas in } \\
\text { spring and summer, less frost } \\
\text { damage on pastures; Reduced } \\
\text { opportunities for winter tourism }\end{array}$ & $\begin{array}{l}\text { TEK - traditional ecological knowledge use } \\
\text { of castrated male reindeer to break the ice } \\
\text { crust; Flexibility in choice of pastures; } \\
\text { Increased water storage capacity; } \\
\text { Implications for fire regimes (see above); } \\
\text { Increased forecasting and surveillance of } \\
\text { hazards }\end{array}$ \\
\hline $\begin{array}{l}\text { Rivers and Lakes } \\
\text { Reduction in ice } \\
\text { duration and ice } \\
\text { thickness }\end{array}$ & $\begin{array}{l}\text { Reduced operation of ice roads, } \\
\text { increased barge transport on rivers } \\
\text { and lakes }\end{array}$ & $\begin{array}{l}\text { Concentrate on the coldest period of winter, } \\
\text { eventually construction of land-based road } \\
\text { or rail networks, development and } \\
\text { enhancement of infrastructure to support } \\
\text { such (e.g., dredging) }\end{array}$ \\
\hline
\end{tabular}

Source: AMAP, 2011. 
While there are regional and local differences in climate change impacts, there are some common drivers and processes across the Circumpolar region that shape community vulnerability and adaptation processes. These include but are not limited to access to resources and knowledge, attitudes and perceptions of change, economic and livelihood flexibility, enabling institutions, transfer of local and traditional knowledge, threats to cultural identity and well-being, and economic and employment opportunities (Hovelsrud et al., 2010). The capacity of communities to adapt is further determined by governance, path-dependencies, infrastructure, and connectivity (Keskitalo and Kulyasova, 2009) and shaped by community values and place identity. Research focussed on climate change as the primary source of community vulnerability misses other forces that are shaping vulnerability such as colonialism, globalization, social and economic change, or political and institutional factors including such as resource quotas (Jentoft et al., 2009).

Institutions and governance play significant roles in facilitating community responses to climate change and flexible institutions are needed to avoid the imposition of rules, frameworks, and discourses that may limit the ability of local actors to engage proactively with change on their own terms (Armitage, 2005). Particular attention has been paid to co-management infrastructures; researchers have concluded that in spite of discursively emphasizing a role for traditional knowledge, these institutions often privilege Western science in management decisions. Less attention has been paid to the role of community-based and community-led institutions in facilitating adaptation responses that are meaningful and relevant to communities. Examples exist around the Arctic where municipalities, communities and individuals are adapting, despite the lack of national policies for adaptation.

In Canada, there has been an increasing trend to engage Arctic residents in climate change research, including work on observing and documenting the impacts of climate change on environmental systems including sea ice and snow, wind and weather, and permafrost (e.g. Laidler et al., 2009; Gearheard et al., 2010; Tremblay et al., 2008). The resulting data often have greater relevance to locally identified priorities and may play an important role for communities as the region is made more accessible to extractive industries (e.g. oil, gas, mining). Climate change has an impact on sea ice and travel, hunting, subsistence activity and food security, viability of land skills and traditional knowledge, and health. Regional and community adaptation planning address hazards and risks in relation to infrastructure, including roads and buildings. 
Inuit hunters and Elders have become critical partners and leaders in climate change research in the Canadian Arctic, contributing their expert knowledge and skills to many projects and programs

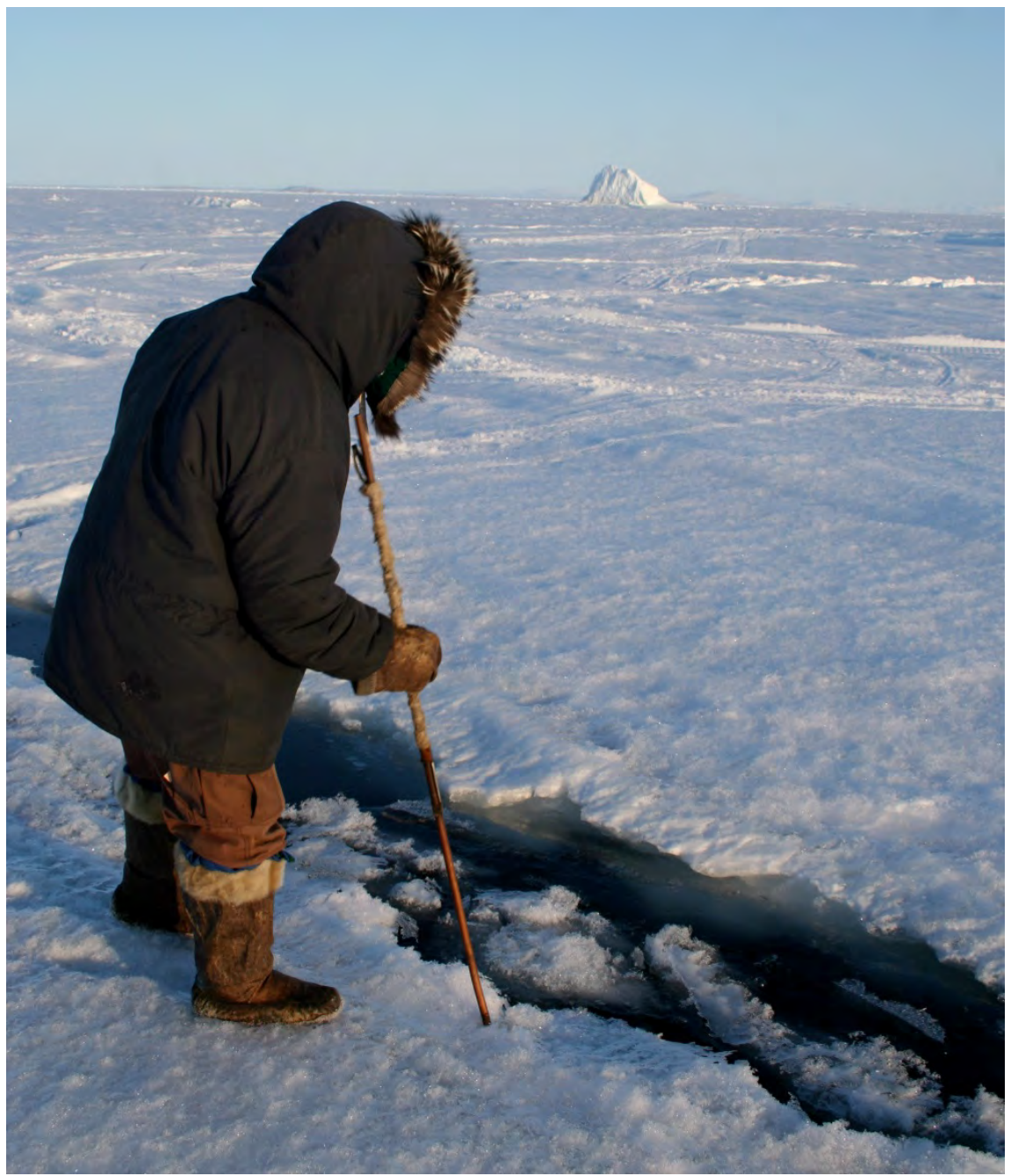

Credit: Shari Gearheard.

Nordic countries have a generally high adaptive capacity with respect to economy, technology, information and skills, infrastructure, institutions, and equity (Juhola et al., 2012). At the local/regional level, adaptive capacity is largely determined by socioeconomic conditions or social vulnerability, as well as being politically determined within governance networks (Keskitalo and Kulyasova, 2009). A lack of national legislation can hinder action at the local level or the flow of resources, but studies from Norway and Finland find voluntary adaptation initiatives at the local and regional levels, despite the absence of national policies, or the 
lack of knowledge transfer from national to local levels (Dannevig et al., 2012; Nilsson et al., 2010). This corresponds with studies that find that the way adaptation is handled as an issue at the national level coheres with national rationalities, while local and regional levels show diversities in the development of bottom-up adaptation technologies (Keskitalo, 2012).

In Greenland, significant decadal variations in ocean temperature have driven socio-economic shifts throughout its history, with three major shifts identified during the 20th century (Rasmussen, 2007). Communities in Greenland have adapted to combined social-ecological impacts on: 1) marine mammal stocks (increased ocean temperatures and decreased markets); 2), the cod stock (increasing cod and increasing markets); and 3) diversification with cooler ocean temperatures, decreasing cod stocks, better technology to fish for shrimp and deep water Greenland halibut and an increasing market. Marine mammal hunting was traditionally the main livelihood activity in Greenland and also the main focus of the colonial economic policy for Greenland well into the 20th century. The combination of increased human populations and declining European and North American markets for seal pelts and blubber created a need for diversifying commercial and subsistence activities. For the past ten years, communities located in areas where sea ice has been used as a platform for travel, hunting and fishing report a significant reduction in sea ice (Gearheard et al., 2013). This has resulted in significant reduction in sea ice dependent species such as ringed seals and narwhal, with consequences for hunting activities. Additionally, Greenland halibut fisheries are no longer taking place from the sea ice, but from smaller vessels on open water. Reduction in sea ice, decreasing hunting activities, increasing fisheries and a changing international market all have adaptation implications for Greenlandic communities and for resource management. 
A day at work in North West Greenland - hunters and fishers must navigate dangerous ice-infested water

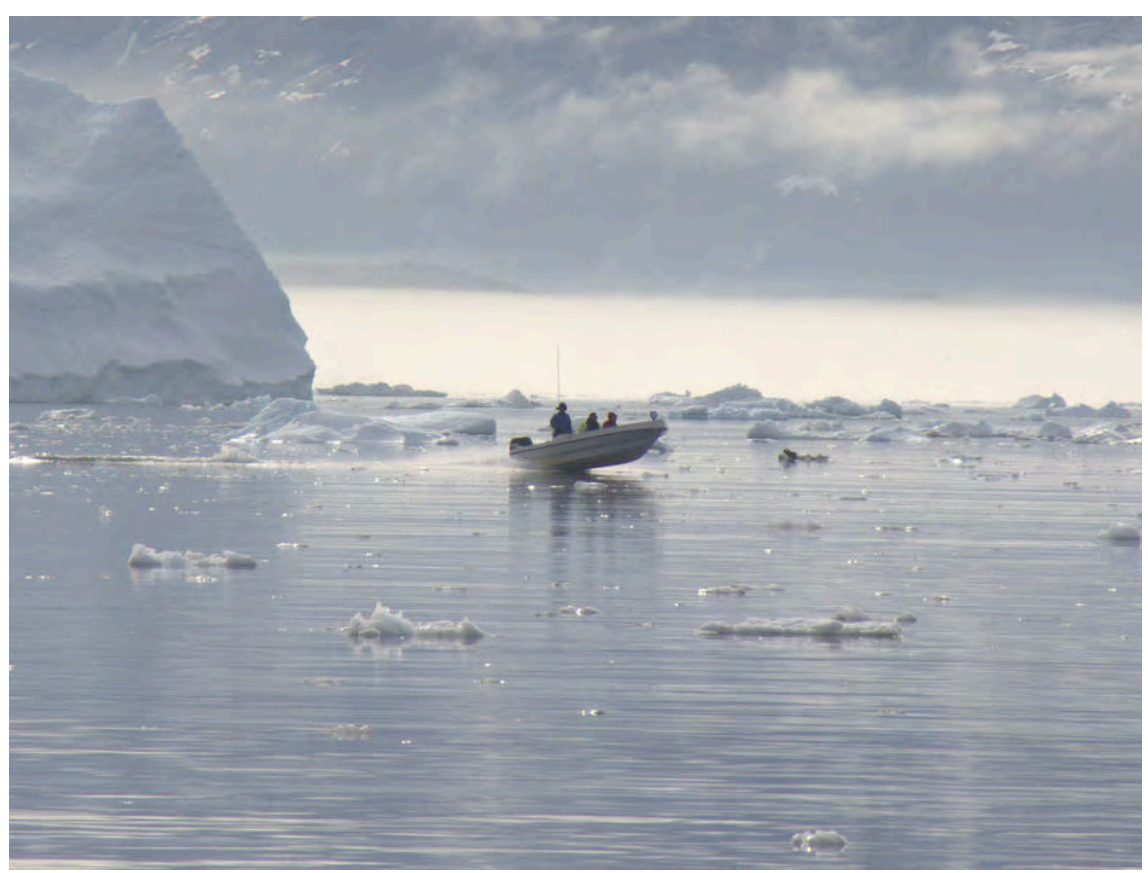

Credit: Grete K. Hovelsrud.

In Russia, the major political, economic and social upheavals of the last four centuries, together with the impacts of climate change, are influencing Russian Arctic communities (Forbes and Stammler, 2009). The importance of the Arctic regions for Russia is expected to increase because of increased mining and petroleum extraction activities. This increases the pressure on land use, and reduces the territory of the Indigenous peoples with consequences for communities (nomadic reindeer herders) migrating across the tundra. Thus, communities need to adapt to changing climatic, economic and political conditions (Vinokurova, 2011). The Russian government is increasingly aware that long-term socioeconomic development in the Russian North must be seen in the context of climate change impacts and through a regional approach to implementing climate adaptation strategies. For Indigenous peoples, these changes have major effects on cultural identity, health and well-being of individuals and communities. Other health risks for communities are caused by the influx of diseases from the south, from thawing permafrost that causes breakdowns in sanitary functions, the increasing presence of environmental toxins in food, air and soil, and changes in access to local foodstuffs and commercial food causing a switch from the former to the latter (Revich, 2009). 
In Alaskan communities, climate change poses a number of challenges, including physical threats such as erosion, service threats such as those affecting water supply or transportation, and cultural threats such as disruption of hunting and fishing. Together, the threats have been assessed in terms of human rights and justice, especially with respect to Indigenous communities (Bronen, 2011; Bronen and Chapin, 2013). Coastal or riverbank erosion as a major threat is not itself a product of climate change, but the rate of erosion may be increased through climate-induced changes in river flow, thawing permafrost, or loss of protective sea ice during stormy times of the year. Changes in settlement patterns and increased infrastructure build-up have exacerbated the problem (e.g., Brunner et al., 2004). Contamination of water supply and damaged roads and runways are caused by climate change. The use of frozen rivers and sea ice for travel is also becoming more hazardous as dangerous conditions become more common as a result of warmer winters and more snowfall, which combine to reduce ice thickness and stability.

Climate change poses a number of challenges for Arctic communities, and rural areas are more exposed due to fewer livelihood options and financial resources. Those communities are adapting in various ways, on their own and with outside assistance. Nonetheless, in many cases the rate and extent of change are greater than the ability to adjust. Particularly challenging is the fact that many impacts are interlinked, exacerbating the effects of physical or biological change, and requiring that effective responses overcome institutional and other barriers (see also, AMAP, 2011). Perhaps more importantly, the effects of climate change are occurring amid a number of other economic, societal, and cultural shifts, each of which has numerous and far-reaching impacts on community structure, function, and identity. Climate change thus acts not in isolation, but as an additional contributor to changes already underway. Community adaptation, therefore, must address many causes with many potential outcomes, which is why larger themes such as governance, justice, and food security are central to the long-term viability of Arctic communities. 


\subsection{Community perspectives}

\subsubsection{Communities and peoples}

Increasing globalization processes have the potential to transform Arctic communities by changing the connections that keep them together (see also Chapter 10, Globalization). This is a complex topic that requires attention to the fact that communities are heterogeneous entities, with different and sometimes conflicting interests and opinions about what is best for the community and the individual. Community resilience and ability to change are linked to cultural and community values, place identity, resource networks, institutions and services, and learning (Amundsen, 2012), all being driving forces in communities in parallel with globalization. In the face of globalization, people in many Arctic communities still express strong attachments to the places where they live and a willingness to work hard to be able to continue living in their community, to maintain social networks, a slow pace of life and safe communities even when challenged by factors such as outmigration, cuts in public services, and decline in the availability and accessibility of natural resources. At the same time, others are looking for alternatives (Carson et al., 2011). The different Arctic nations have different regional policies with respect to rural areas and infrastructural support, but even in Norway where the official policy is to fully maintain communities, outmigration is a fact on islands and in fjords. There is a general trend that women and youth leave communities for education and better jobs and men stay behind carrying out more traditional jobs and livelihood activities (Megatrends, 2011). This causes unsustainable gender, age and employment imbalances in communities. When women leave, fewer children are born, reducing the number of children in schools and the tax base by which municipalities support community services, further reducing population numbers and potentially leading to community abandonment (Martin, 2009). A stable population is crucial for maintaining viable communities and municipal services, therefore, many communities are trying to curb outmigration by creating jobs and activities that strengthen the attractiveness of places.

\subsubsection{Generation and generation shifts}

The impacts of changing gender balance and increasing generational divides are closely interlinked with the foundations for community viability. In many Arctic communities, younger generations are faced with the chal- 
lenges of maintaining culture, language, and traditions, while at the same time engaging with new technologies, economic opportunities, cultural influences, and globalization (see also ASI, 2010). In many rural communities, knowledge about community relevant topics such as the environment, resources, and cultural relationships and values is transferred between generations. In many Arctic Indigenous communities, there is concern and action towards ensuring that traditional knowledge is passed on to youth. The role, importance, and status of local and traditional knowledge is now widely accepted, and its transfer between generations is taking place through a number of locally-driven and research, government, and Indigenous organization sponsored activities.

Traditions and traditional knowledge are transferred from one generation to the next in an active process, through peoples' practical engagement with the environment and with each other. Myriad circumstances can influence how knowledge, values, skills, and traditions are shared between generations. The impacts of climate change have more recently spurred discussions about the ties between generations and the transfer of knowledge. In addition the youth - and communities are confronted with the need for other types of skills and knowledge in order to respond to the requirements of globalization going beyond previous experiences available in the community. The younger generations deal with still more demanding requirements regarding social, socio-economic and management capabilities, and interactions made possible by new means of media and communication. The expectations and perceptions of what constitutes a good life also have a generational character. The older generation of coastal fishers in the North Atlantic region accept that their occupation required long periods of absence from their families, but younger fishers prefer to come home every day and expect to own their own boat early on in their career (West and Hovelsrud, 2010). Attitudes towards work have changed and cultural identity tied to an occupation weakened, which may influence the glue of communities.

On the other hand, the value of a vibrant community is shared across generations and younger generations bring new perspectives and innovations that maintain strong communities. The use of social media has become extremely popular in Arctic communities in recent years and in many cases the technology is being used to promote traditional culture and language among youth as well as strengthening connections across remote communities. Many Indigenous peoples of the younger generations, such as the Sámi, are expressing their indigeneity more strongly 
than before, through social media and public participation wearing their traditional clothes and speaking their native language.

\section{Gender perspectives}

In most Arctic societies, gender roles have been historically distinguished along male and female social roles and responsibilities, but gender roles are increasingly changing along with changing community dynamics and perspectives. Communities are faced with new challenges with respect to industrial expansion and climate change impacts, and opportunities resulting from better connectivity, infrastructure, and being more connected to the world economy. It is increasingly clear that men and women meet these challenges differently, which is partly related to traditional gender roles and perhaps more importantly, related to the level of flexibility of communities and policies. These are in turn driven by national policies for regional and rural development. Gender differences in what is perceived as "the good life" has turned out to be one of the most important contemporary drivers of change in community structure, not only in the Arctic, but worldwide. In this respect, we find interlinked and interacting gender differences with respect to education, out-migration, decision-making and power relations. The trend since the 1990s is that women are more educated than men (Megatrends, 2011) and that educated women travel out of the communities for work while men stay behind (Sloan et al., 2006). But there are examples showing a reverse situation. In some settlements in the Sakha Republic (Yakutia), men leave the communities to take employment, and the women stay behind (Vinokurova, 2011). This sex-ratio imbalance in the communities has major consequence for community life (Hamilton and Rasmussen, 2010; Sloan et al., 2006). Whether women stay, return, or leave, is closely related to power relations, decision-making, and access to resources including knowledge and information.

There are major variations in these components throughout the Arctic, but it is clear that there are gender differences with respect to vulnerability, risk exposure-sensitivity and coping capacity (Bee et al., 2013). Under-representation of women in decision-making, underutilization of women's knowledge and management skills, and a weak representation of women's views on the relationship between humans and nature (Sloan, 2006; Ingolfsdottir, 2011) are all aspects that may influence women's decisions to leave the communities. Gender studies mainly focus on women, but there is an increasing call for more knowledge about the particular societal conditions that shape the vul- 
nerability and coping capacity of men. This includes the gender differences in how men and women perceive and adapt to climate change.

\section{Textbox 11.7}

\section{Gender perspectives on local and global scale}

Among the major concerns for Arctic communities are demographic challenges such as the aging of the population and increased mobility. Increasing mobility can be a challenge where the exodus of young people and especially young women has become a major factor. It creates the risk of inadequate number of children, and the tendency of former large families becoming families with one or two children is also an important issue (see Chapter 2: Arctic Populations and Migration).

Regions dominated by older people often display a majority of women due to the fact that women live longer than men. Research, however, shows that there are pull and push effects for women to leave sparsely populated areas. The table below shows some of the major differences as reflected through a survey from Greenland in 2011 (Hansen and Rasmussen, 2013). The patterns are similar to what has been found in other Northern communities (see, for instance, Sloan, 2006).

\begin{tabular}{|c|c|c|c|c|}
\hline \multicolumn{5}{|c|}{$\begin{array}{l}\text { Most important issues in relation to moving } \\
\text { Gender distribution and differences of answers }\end{array}$} \\
\hline & \multicolumn{2}{|c|}{ Percent of responses } & \multirow{2}{*}{$\begin{array}{c}\begin{array}{c}\text { interences } \\
\text { in } \\
\text { responses }\end{array} \\
\end{array}$} & \multirow{2}{*}{$\begin{array}{c}\text { Gender } \\
\text { characteristic }\end{array}$} \\
\hline & Men & Women & & \\
\hline Carreer opportunities & 9,9 & 7.5 & 2,4 & \multirow{6}{*}{$\begin{array}{r}\text { Clearly male } \\
\text { dominance }\end{array}$} \\
\hline Partner's education & 4,9 & 2,8 & 2,0 & \\
\hline Withdrawing from labor market & 2,9 & 1,3 & 1,6 & \\
\hline The option of getting a job & 9,9 & 8.4 & 1,4 & \\
\hline $\begin{array}{l}\text { The option of getting better working } \\
\text { conditions }\end{array}$ & 7.4 & 6.4 & 1,1 & \\
\hline $\begin{array}{l}\text { The option of getting another (better) } \\
\text { iob }\end{array}$ & 12,3 & 11.2 & 1,1 & \\
\hline Improved public transport options & 2.9 & 2,1 & 0,9 & \multirow{6}{*}{ Equal importance } \\
\hline $\begin{array}{l}\text { Does not feel "at home" where I'm } \\
\text { living now }\end{array}$ & 3,4 & 2,6 & 0,8 & \\
\hline Public service options & 4,7 & 3,9 & 0,8 & \\
\hline Better salaries & 8,6 & 7.9 & 0,7 & \\
\hline (Better) housing options & 5,2 & 4,5 & 0,7 & \\
\hline Improved health conditions & 2,1 & 1,5 & 0,6 & \\
\hline Improved leisure time options & 7.0 & 8,1 & 1,1 & \multirow{4}{*}{$\begin{array}{l}\text { Clearly female } \\
\text { dominance }\end{array}$} \\
\hline Better education options for children & 6,0 & 9,0 & 3,0 & \\
\hline Family matters & 5,7 & 10,5 & 18 & \\
\hline Better own education options & 7,3 & 12,4 & 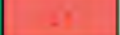 & \\
\hline Total & 1002.2 & 100,1 & & \\
\hline
\end{tabular}


As mentioned above, an important push is related to the limited education and job opportunities offered in smaller settlements with an economic base in the traditional exploitation of renewable as well as non-renewable resources. This is paralleled by a pull related to the opportunities of both jobs and educational opportunities available in larger towns and especially cities.

Furthermore, the situation is challenged by the fact that it is primarily women who respond to global challenges through education. An important consequence of this is changes in the labor market and declining workforces that are under pressure as a consequence of demographic change, changing production structures and the business environment. The interaction between these factors generates a need for a substantial modification of the local response to the impact of globalization of Arctic societies.

The calculation of the total number of women per 100 men is a good indicator of the result of differences in gender-based preferences in the Arctic. In order to adjust for the longevity among women compared to men in the Arctic the indicator has been adjusted according to the national averages. All blue and purple regions and settlements show a clear deficit of women. Places with surplus of women are first and foremost the larger cities where education and related job opportunities are available.

\subsubsection{Change and stability}

Balancing continuity and change is an important theme in all Arctic communities. Increasingly, researchers and community practitioners look to the concept of resilience to examine the question of why some individuals and communities are able to accommodate and adapt to change better than others (Folke, 2006). Components of community viability and resilience include social networks and support, safety nets, informal risk sharing mechanisms, learning, active involvement, collaboration across sectors and societal scales, environment and lifestyle, diverse and innovative economies, and leadership (Buikstra et al., 2010). In addressing the question of why some communities flourish and others do not, research findings point to innovation, individual and group agency, and local institutions as particularly important (Amundsen, 2012).

Indigenous concepts of resilience are expressed through specific stories and metaphors grounded in local culture and language (Kirmayer et al., 2011). Here, the sense of community is embedded in a social matrix that encompasses relations with animals and the environment (Stairs and Wenzel, 1992). Relational processes that support resilience include 
revising collective history, revitalizing language and culture, and engaging in political activism. Institutional responses and frameworks across scales are critical in supporting these processes, including devolving governance to local institutional authorities and supporting innovation and flexibility in local socio-economic organizations.

Some communities build on the local cultural history and make sure that adaptation processes ensure continuation of this history, especially with respect to maintaining the viability of the main and traditional livelihoods such as fishing, while others call for innovation and new sectors which will change the composition of the communities (Amundsen, 2012). For example, communities endowed with fisheries resources will continue to have a significant fisheries industry, but structural changes in the fisheries and society require new industries and sources of jobs. Change fosters stability and enables communities and settlements to utilize the potential that new markets and niches provide.

\subsection{Challenges influencing community viability in the Arctic}

While local level approaches are imperative it is also important to note that community responses are shaped by complex interactions in political and economic globalization, markets and national and international resource management (Keskitalo and Kulyasova, 2009). Aspects that may weaken the resilience of coupled social-ecological systems may also have a bearing on community viability, including breakdown of traditional institutions, rapid technological change and rapid changes in local socioeconomic conditions (Crona, 2006; Seixas and Berkes, 2003). It should also be noted that many changes initiated on a global scale may also have positive effects on, for example, community health, the possibilities for individual choice, and the possibility for a changed but still viable community future. The summary below assesses the trends and processes that influence adaptation and viability of settlements and communities in the Arctic. 


\subsubsection{Secure communities}

A community is viable when it is able to combine outside influences with local cultural and social competence and creativity. It is clear that local environmental variability interacts with socio-economic and sociocultural factors, and integrates individual entrepreneurship and collective action for change. The social and cultural variations that create settlements patterns and cultural expressions are broad in the Arctic, and hence the viability of these communities rests on highly variable skills and abilities. The forms and level of specific expertise that is needed vary greatly, depending on where a community is located, to what extent local traditions are dominant, or modernization and globalization processes have led to a dependency on more centralized and urbanized skills and knowledge.

\subsubsection{Centralization processes}

Arctic communities have shown an exceptionally high adaptability to their local environments. Both within and beyond the Arctic, the "knowledge society" demands formalized specialization, often based on the use and understanding of technology replacing manual labor. These processes coincide with the downscaling of labor intensive natural resource harvesting (e.g. fisheries, reindeer herding and farming), the steady rise in interest from extractive industries gaining access to resources in the Arctic, and in the spread of formalized educational institutions, around which centralization processes accelerate. Even though significant land use remains connected to traditional use of renewable resources (Rees et al., 2008), less labor is needed to extract and utilize these resources. Therefore, the need for a population living close to the resources diminishes, and with a decreasing number of communities engaging in hunting, gathering and herding for economic purposes, the financial basis is paid jobs, of which there are relatively few in many parts of the Arctic. Educational opportunities, sought after by young people in order to secure well paid jobs, are predominantly found in more urbanized centres. These centrifugal forces threaten to tap the Arctic of people, its most precious resource. But there are currents moving in the other direction, such as the new initiative called "smart specialization" within the new strategic research program of the European Union, Horizon 2020. In this scheme, a heightened focus is on the importance of viable regions in the EU. For the Arctic, this could mean that development, research, and production are merged geographically, ensuring hands-on approaches to innovation based on the needs of pro- 
duction. This would then enable businesses to develop research and development facilities close to the resource upon which this new knowhow is developed, ensuring a better utilization of the resource in the region where it is found.

Another trend is the so-called "lifestyle-based" move to the outskirts, to small-town life and to a life less involved in the high-speed economies of urban centers - a trend exemplified through "slow-city" - and "slowfood"-movements, but also characterized by sporty, outdoor activities (Knox and Mayer, 2013).

\subsubsection{Climate change and global risks}

Local communities face major combined and interlinked challenges such as climate change impacts and unwanted effects of economic globalization and ecosystem management, to which they can respond either as "powerless spectators" or as "adaptive managers", seeking to secure future viability by utilizing possibilities and evoking internal competences of resilience (Fabricius et al., 2007). These challenges are first and foremost emanating from the global scene, and are therefore not exclusive to the Arctic. Globally, climate change, industrialization processes and changing migration patterns due to population growth and pressures are major drivers for change. All of these drivers create possibilities, but can also have negative consequences for community viability. The impact of risky behavior reaches far beyond the risk taker as illustrated by anthropogenically driven global warming. The consequences of local, regional, national politics and priorities may very well decide the fates of others far away (Odland et al., 2002; Beck, 2009; Hovelsrud et al., 2011).

\subsubsection{Industrialization based on extractive industries}

As industrialization based on the extraction of natural resources such as petroleum, minerals and marine resources strengthens its position in the North, the risks and threats increase, both to traditional livelihoods and natural surroundings. But these processes also bring welcome changes. New jobs, improved infrastructure, schools and better primary health care are made accessible to larger parts of the population, and with these an increased possibility to choose lifestyle and professional careers. These "dual processes" are visible in many places in the Arctic, for example in the Nenets okrug in Russia, where the interactions between petroleum workers, the industry and the indigenous people are a 
complex mix of serving each other's needs. The Nenets provide meat for the workers whilst the petroleum industry enhances the options for communications to centres with health care, educational facilities and governmental institutions. At the same time, traditional reindeer herding seems to be vulnerable to the global trends these changes represent (Rees et al., 2008). In the Norwegian Arctic, the offensive tactics of the petroleum industry are met with skepticism by representatives of the traditional fisheries along the coast, who argue that the potential benefits do not outweigh the risks involved, either to the environment or to the way of life in the fishing villages. An alternative take on the modernization processes for these communities would be to combine the utilization of renewable resources with an influx of white-collar and specialized labor, combined with efforts aiming at strengthening the basis for (eco- and cultural) tourism and spin-off products from the traditional bulk-producing fishing industry (Dale, 2011).

\subsubsection{The mobility of goods, people, and ideas}

For centuries, people in the Arctic have travelled by sea, on sea ice and over land. The Arctic has been an important part of international trade systems for millennia, and has been subjected to legislative and taxation demands from nation states (Kurlandsky, 1997; Jaklin, 2006). More recently, natural resource extraction and, as a consequence, increasing physical and economic presence from industrial companies and governmental agencies, have led to new challenges and opportunities. This has in many communities spurred societal and cultural processes of change as well as re-evaluation and revitalization of the importance of cultural heritage, human-environment connections, and sense of place. Both the Arctic diasporas and the new newcomers to the Arctic partake in these processes, as they both blur the borders between "us" and "them" and utilize the potential for flow of goods, services and people that the modern world provides. Within and outside of the Arctic, communities adhering to an "Arctic" identity place less emphasis on physical settlements and historical roots as a clear denominator of identity and sense of belonging (Antonsen, 2011; Dale, 2011). At the same time, there is a revitalization of the importance of a common ancestry for some groups, be it based on being "a northerner" in a general sense, on ethnic affiliation, or on historically rooted family ties to a community or settlement. Without neglecting the fact that many Indigenous groups still experience stigmatization on the basis of their ethnic background, the notion of ethnic identity is balanced out by the positive connotations eth- 
nicity has for many peoples in the Arctic. Modern Sámi ethnicity may serve as an example of how these changes manifest themselves in the construction of individual identities. Being Sámi today is to adhere to the traditions of Sámi culture, as a provider of identity markers, but not necessarily to be involved directly in traditional Sámi trades such as fishing or reindeer herding. It is about language for many, but not for all; it is about wearing a traditional dress (gákti) but not for all. Still, for most Arctic residents, it is about being aware of the heritage, the common ancestry, and a sense of belonging to a community, which, in response to and being a part of shaping the globalized world, changes and adapts to new circumstances.

\subsection{Major trends}

We identify the following key trends:

- The overall demographic trend in the Arctic is outmigration from smaller communities to urban areas, with an increasing divide between centers and periphery.

- Mobility has been on the rise in the past decades within and between communities, and inside and outside the Arctic.

- Imported foods are on the rise in the Arctic, but in some areas, like Norway, interest in, and demand for, local food is rising.

- The rate and magnitude of climate change vary across the Arctic and between and within communities, and consequently the need for adaptive responses vary.

- Community connections, such as to the environment or among people within communities are also being transformed by increasing globalization. Once again, responses vary widely across the Arctic, but there is a trend toward outmigration as people seek opportunities and alternatives. In particular, there is a trend, though not the same everywhere, for women to leave and men to stay, creating a gender imbalance.

- Temperatures are increasing and will have consequences for communities and the environment. 


\subsection{Gaps in knowledge}

Gaps in knowledge pertain to the basis, dynamics, and perspectives of communities and how these are dynamically shaped. When attempting to understand the dynamic nature of how communities are shaped, maintained and functioning, we are up against a rather grand challenge. When we try to generalize across the Arctic and identify the gaps this challenge increases. There are significant gaps in knowledge about how we compare and analyze lessons learned across the communities in the region. While it is clear that one size does not fit all, policy makers and decision-makers could benefit from an increased understanding of the different drivers of change.

Communities are at different stages of development with different dynamics both internally and in how they relate to external processes. If we increase our knowledge about how we can create transferable lessons, planning for the future challenges would benefit. Another significant gap in knowledge is identified with respect to how communities throughout the Arctic will adapt to and handle the increased focus on the region both in terms of commercialization and climate change impacts.

We have insufficient knowledge about the best practices for communities to handle the increasing industrial developments such as large-scale mining, energy exploitation, and other industries led by global companies. Arctic communities face major challenges from such developments, and from climate change. We need more knowledge about creating best practices that are transferable to other communities and processes.

We need more and updated knowledge about how to assess the trends and processes that influence adaptation and viability of settlements and communities in the Arctic.

\subsection{Conclusion}

This chapter emphasizes linkages and feedbacks between social structures, socio-economic conditions, health, well-being, and demography within communities, as well as with some of the overarching geopolitical, physical and biophysical sub-systems and globalization of communities and settlements. It looks at a wide variety of inter-related challenges, opportunities, and stresses facing communities, highlighting the fact that communities respond differently within and between nations and regions, and within and between settlements in the same region. 
Communities and settlements in the Arctic are grappling with many different and interrelated issues. In the last ten years, urbanization has continued to be a major factor for human development with great variation from place to place. Urbanization has usually been connected to industrialization, but now educational opportunities are also driving growth and development of centers. The overall demographic trend in the Arctic is depopulation, with an increasing divide between centers and periphery.

Culture continues to play an important role in Arctic communities, touching all aspects of life including the dynamic relationships of people to place, to livelihoods, and to each other. Food and food security, an increasingly important and visible issue in the Arctic over the last decade, has key ties to culture, particularly in terms of subsistence activities and informal economies. Imported foods are on the rise in the Arctic, but in some areas, like Norway, local food is rising in interest and demand.

Mobility is on the rise in past decades within and between communities, and inside and outside the Arctic. A number of factors influence mobility, including education opportunities, jobs, changes in social relations, and in some cases impacts of climate change. Climate change, almost totally absent from the Community Development chapter in the first $A H D R$ (2004) a decade ago, features prominently in this chapter. Climate change impacts interact with coupled social-ecological systems and can exacerbate existing challenges, especially in rural areas where access to financial resources and options are fewer. Communities and individuals are adapting, based on time-tested strategies and with assistance and guidance from regional and national governments. The rate and magnitude of climate change vary across the Arctic and between and within communities, consequently the need for adaptive responses varies.

Community connections, such as to the environment or among people within communities are also being transformed by increasing globalization. Once again, responses vary widely across the Arctic, but there is a trend toward outmigration as people seek opportunities and alternatives. In particular, there is a trend, though not the same everywhere, for women to leave and men to stay, creating a gender imbalance. Since the 1990s, women tend to be more educated and tend to travel out of their communities. There are differences in gender in other ways, such as with respect to vulnerability, risk exposure, and coping ability, with men and women experiencing these differently. 


\subsection{References}

ASI (2010). Arctic Social Indicators - a follow-up to the Arctic Human Development Report. Larsen, J.N., P. Schweitzer, and G. Fondahl (eds.). TemaNord. Nordic Council of Ministers, Copenhagen.

Abelsen, M. (2013). Your region - your choice. In: Hansen, K.G., R.O. Rasmussen, R. Weber, (eds.), 2013. Proceedings from First International Conference on Urbanisation in the Arctic. Nordregio Working Paper 2013:6, Stockholm.

AMAP (2011). Snow, Water, Ice and Permafrost in the Arctic (SWIPA): Climate Change and the Cryosphere. Arctic Monitoring and Assessment Programme (AMAP), Oslo. http://amap.no/swipa/SWIPAContent.html\#Reports

AMAP (2004). Persistent Toxic Substances, Food Security and Indigenous Peoples of the Russian North. AMAP Secretariat, Oslo.

Amundsen, H. (2012). Illusions of resilience? An analysis of community responses to change in Northern Norway. Ecology and Society, 17(4): 46. http://dx.doi.org/ 10.5751/ES-05142-170446

Antonsen, K. M. (2011). Å velge bosted i Lofoten: en diskursanalyse av folkelige fortellinger om bostedsvalg og hverdagsliv [Choosing residency in Lofoten: a discourse analysis of folk tales about residency choices and everyday life].Geografisk institutt. Trondheim, NTNU. PhD.

Armitage, D. R. (2005). Community-based Narwhal management in Nunavut, Canada: change, uncertainty, and adaptation. Society \& Natural Resources, 18(8): PP. 715-731.

Armstrong, T., G. Rogers, and G. Rowley (1978). The Circumpolar North. A Political and Economic Geography of the Arctic and Sub-Arctic. Methuen, London.

Bee B., M. Biermann, and P. Tschakert (2013). Gender, development, and rightsbased approaches: lessons for climate change adaptation and adaptive social protection. In: Alston, M. and K. Whittenbury (eds.), Research, Action and Policy: Addressing the Gendered Impacts of Climate Change. Springer Dordrecht, Heidelberg. New York, London. http://dx.doi.org/10.1007/978-94-007-5518-5_7

Beck, U. (2009). World at Risk. Polity, Cambridge.

Bonefeld-Jørgensen, E. (2010). Biomonitoring in Greenland: human biomarkers of exposure and effects - a short review. Rural and Remote Health, 10: 1362.

Bonefeld-Jørgensen, E. (2009). Genetics and contaminants. In: Arctic Monitoring and Assessment Programme (AMAP), The AMAP Assessment Report 2009: Human Health in the Arctic, Oslo.

Bronen, R. (2011). Climate-induced community relocations: creating an adaptive governance framework based in human rights doctrine. New York University Review of Law and Social Change, 35(2): PP. 356-406.

Bronen, R. and F. S. Chapin III (2013). Adaptive governance and institutional strategies for climate-induced community relocations in Alaska. Proceedings of the $\mathrm{Na}$ tional Academy of Sciences, 110(23): PP. 9320-9325.

Brubaker, R. (2005). The "diaspora" diaspora, Ethnic and Racial Studies, 28(1): pp. 1-19. Brunner, R.D., A.H. Lynch, J.C. Pardikes, E.N. Cassano, L.R. Lestaa, and J.M. Vogel (2004). An Arctic disaster and its policy implications. Arctic, 57(4): PP. 336-346.

Buikstra, E., H. Ross, C. A. King, P. G. Baker, D. Hegney, K. McLachlan, and C. RogersClark (2010). The components of resilience - perceptions of an Australian rural community, Journal of Community Psychology 38(8): PP. 975-991.

Carson, D., R.O. Rasmussen, P. Ensign, L. Huskey and A. Taylor (eds.) (2011). Demography at the Edge. Remote Human Populations in Developed Nations. Ashgate, Surrey. 
Chan, H. M., K. Fediuk, S. Hamilton, L. Rostas, A. Caughey, H. Kuhnlein, G. Egeland, and E. Loring (2006). Food security in Nunavut, Canada: barriers and recommendations. International Journal of Circumpolar Health, 65(5): PP. 416-431.

Christiansen, P. (2013). Urbanisation - an obstacle or a precondition? In: Hansen, K.G., R.O. Rasmussen, and R. Weber (eds.). Proceedings from the First International Conference on Urbanisation in the Arctic, 28th-30th August 2012, Ilimmarfik, Nuuk, Greenland. Nordregio Working Paper 2013:6, Stockholm.

Cohen, R. (1997). Global Diasporas: An Introduction, University of Washington Press, Seattle. http://dx.doi.org/10.4324/9780203228920

Crate, S. (2011). We are going under water. Natural History, 119(5): pp. 20-29.

Crate, S. (2006). Elder knowledge and sustainable livelihoods in Post-Soviet Russia: finding dialogue across the generations. Arctic Anthropology, 43(1): pp. 40-51. http://dx.doi.org/10.1353/arc.2011.0030

Crona, B.I. (2006): Supporting and enhancing development of heterogeneous ecological knowledge among resource users in a Kenyan seascape. Ecology and Society, 11(1).

Dahl, J. (1989). The integrative and cultural role of hunting and subsistence in Greenland. Études/Inuit/Studies, 13(1): pp. 23-42.

Dahlström, M. and S. Hedin (eds.) (2010). Regional trajectories to the knowledge economy - Nordic-European comparisons (REKENE). Nordic Innovation Centre, Oslo.

Dale, B. (2011). Securing a Contingent Future: How Threats, Risks and Identity Matter in the Debate over Petroleum Development in Lofoten, Norway. Department of Sociology, Political Science and Community Planning. Tromsø, University of Tromsø. PhD.

Dankertsen, A. (2006). “Men du kan jo snakke frognerSámisk!”. Tradisjon og kulturell innovasjon blant samer i Oslo [But you can talk frognerSámi! Tradition and cultural innovation among the Sami people in Oslo]. Unpublished Master Thesis, Oslo University, Institute of Social Anthropology.

Dannevig, H., T. Rauken and G. Hovelsrud (2012). Implementing adaptation to climate change at the local level. Local Environment, 17(6-7): 1-15.

Davidson, D.J., T. Williamson, and J.R. Parkins (2003): Understanding climate change risk and vulnerability in northern forest-based communities. Canadian Journal of Forest Research, 33(11): pp. 2252-2261. http://dx.doi.org/10.1139/x03-138

Duhaime, G. (1991). La chasse inuit subventionnée: tradition et modernité [Sponsored Inuit hunting: tradition and modernity]. Recherches Sociographiques, 31(1): 45.

Duhaime, G. (1987). La régime économique de l'Arctique québécois [The economic system of Arctic Quebec]. In: Le Developpement des peuples du Nord. Actes du Premier colloque Quebec-Russie publié sous la direction de Gérard Duhaime. GETIC, Université Laval, Quebec.

Duhaime, G. and N. Bernard (2008). A Research Agenda to Address: the Global Challenge of Food Security. In: Duhaime, G., and N. Bernard (eds.). Arctic Food Security. Canadian Circumpolar Institute (CCI) Press, University of Alberta and Centre interuniversitaire d'etudes et de recherces autochtones (CIÉRA), Université Laval, Occational Publication No. 58.

Duhaime, G., P. Frechette, and V. Robichaud (1996). Changes and Stability in the Economic Structure of the Nunavik Region (Canada). Department of Agrifood Economics and Consumer Science, Edifice Paul-Comtois, Université Laval, Quebec.

Dybbroe, S. (2007). Is the Arctic really urbanising? Etudes/Inuit/Studies, 32(1): pp. 13-32.

EC, 2013. Smart Specialisation http://s3platform.jrc.ec.europa.eu/ home;jsessionid=vwNRR1RpXF1H7T1y5z4r7gLgClGCDKKQN8wQmxCx9VNZSsBQ 8pj2!-392399571!1367314942582 (28th October 2014). 
Fabricius, C., C. Folke, G. Cundill, and L. Schultz (2007). Powerless spectators, coping actors, and adaptive co-managers: a synthesis of the role of communities in ecosystem management. Ecology and Society, 12(1): 29.

http://www.ecologyandsociety.org/vol12/iss1/art29/ (28th October 2014).

Folke, C. (2006). Resilience: the emergence of a perspective for social-ecological systems analysis. Global Environmental Change, 16: pp. 253-267.

http://dx.doi.org/10.1016/j.gloenvcha.2006.04.002

Forbes, B.C. and F. Stammler (2009). Arctic climate change discourse: the contrasting politics of research agendas in the West and Russia. Polar Research, 28: pp. 28-42. http://dx.doi.org/10.1111/j.1751-8369.2009.00100.x

Ford, J. D. and M. Maeaumier (2011). Feeding the family during times of stress: experience and determinants of food insecurity in an Inuit community. The Geographical Journal, 177(1): pp. 44-61. http://dx.doi.org/10.1111/j.14754959.2010.00374.x

Gearheard, S., L. Kielsen Holm, H.P. Huntington, J.M. Leavitt, A. Mahoney, M. Opie, T. Oshima, and J. Sanguya (eds.) (2013). The Meaning of Ice: People and Sea Ice in Three Arctic Communities. International Polar Institute, Hanover.

Gearheard, S., M. Pocernich, R. Stewart, J. Sanguya, and H.P. Huntington (2010). Linking Inuit knowledge and meteorological station observations to understand changing wind patterns at Clyde River, Nunavut. Climatic Change, 100(2): pp. 267-294. http://dx.doi.org/10.1007/s10584-009-9587-1

Glaas, E., A. Jonsson, M. Hjerpe, Y. Andersson-Sköld (2010). Managing climate change vulnerabilities: formal institutions and knowledge use as determinants of adaptive capacity at the local level in Sweden. Local Environment, 15(6): pp. 525-39. http://dx.doi.org/10.1080/13549839.2010.487525

Greve Harbo, L. and R.O. Rasmussen (2014). State Interests in the Municipal planning - Case Greenland. Nordregio Working Paper. Stockholm.

Hamilton, L.C. and R.O. Rasmussen (2010). Population, sex ratios and development in Greenland. Arctic, 63(1): pp. 43-52. http://dx.doi.org/10.14430/arctic645

Hamilton, L.C., O. Otterstad and H. Ögmundardóttir (2005). Rise and fall of the herring towns: impacts of climate and human teleconnections. In: Hannesson, R. M. Barange and S.F. Herrick Jr. (eds.) Climate Change and the Economics of the World's Fisheries, pp. 100-125. Edward Elgar, Northhampton.

Hamilton, L. C., R.O. Rasmussen, N.E. Flanders, and C. Seyfrit (1996). Outmigration and gender balance in Greenland. Arctic Anthropology, 33(1).

Hansen, K.G. and R.O. Rasmussen (2013). Status of urbanisation processes in the Arctic. In: Hansen, K.G., S. Bitsch, and L. Zalkind, (eds). Urbanization and the Role of Housing in the Present Development Process in the Arctic. Nordregio Report 2013:3. Stockholm.

Hansen, K.G., R.O. Rasmussen, and J. Roto (2011). Demography in the Nordic Countries A Synthesis Report. Nordregio Working Paper 2011:9. Nordregio, Stockholm.

Harries, T. and E. Penning-Rowsell (2011): Victim pressure, institutional inertia and climate change adaptation: The case of flood risk. Global Environmental Change, 21(1): pp. 188-197. http://dx.doi.org/10.1016/j.gloenvcha.2010.09.002

Hedin, S., (ed.) (2009). Higher education institutions as drivers of regional development in the Nordic countries. Nordregio Working Paper, 2009:3. Nordregio, Stockholm.

Heleniak, T., T. Holzlehner, and E.V. Khlinovskaya Rockhill (2011). Der große Exodus: Demographische Trends an Russlands nördlicher Peripherie [The Great Exodus: Demographic Trends in Russia's Northern Periphery], Osteuropa, 2-3: pp. 371-386. 
Heleniak, T. (1999). Migration from the Russian North during the transition period. Social Protection Discussion Paper series; no. SP 9925. The World Bank, Washington, D.C. http://documents.worldbank.org/curated/en/1999/09/693405/ migration-russian-north-during-transition-period (28 October 2014).

Honigmann, J. J. and I. Honigmann (1970). Arctic Townsmen: Ethnic Background and Modernization. Canadian Research Centre, Saint Paul University, Ottawa.

Hoogensen G. (2012). Security by any other name: negative security, positive security, and a multi-actor security approach. Review of International Studies, 38(4): pp. 835-859. http://dx.doi.org/10.1017/S0260210511000751

Hoogensen, G., D. Bazely, J. Christensen, A. Tanentzap, and E. Bojko (2009). Human security in the Arctic - yes, it is relevant! Journal of Human Security, 5(2): pp. 1-10. http://dx.doi.org/10.3316/JHS0502001

Hovelsrud-Broda, G.K. (1999). The integrative role of seals in an East Greenlandic hunting village. Arctic Anthropology, 36(1-2): pp. 37-50.

Hovelsrud, G. K. and B. Smit (eds.) (2010). Community Adaptation and Vulnerability in Arctic Regions. Springer. Netherlands. http://dx.doi.org/10.1007/978-90-4819174-1 Hovelsrud, G. K., Dannevig, H., J. West, and H. Amundsen (2010). Adaptation in fisheries and municipalities: three communities in Northern Norway, In: Hovelsrud, G. K. and B. Smit (eds.), 2010. Community Adaptation and Vulnerability in Arctic Regions. Springer. Netherlands.

Hovelsrud, G. K., B. Poppel, B. van Oort, and J.D. Reist (2011). Arctic societies, cultures, and peoples in a changing cryosphere. AMBIO, 40(1): pp. 100-110. http://dx.doi.org/ 10.1007/s13280-011-0219-4

Ingòlfsdòttir, A. H. (2011). "Go north, young man" - gendered discourses on climate change and security in the Arctic. Nordia Geographical Publications. 40: pp. 89-98.

Jaklin, A. (2006). Historien om Nord-Norge [The Story of Northern Norway]. Gyldendal, Oslo.

Jentoft, S. and R. Chuenpagdee (2009). Fisheries and coastal governance as a wicked problem. Marine Policy, 33(4): pp. 553-560. http://dx.doi.org/10.1016/ j.marpol.2008.12.002

Johnsen, J. P., P. Holm, P.R. Sinclair, and S.A. Raanes (2005). The coming of the Cyborg fish. How resource management is made possible. Paper presented to the Mare Conference People and The Sea III, 7th-9th July. Amsterdam.

Juhola, S., L. Peltonen and P. Niemi (2012). The ability of Nordic countries to adapt to climate change: assessing adaptive capacity at the regional level. Local Environment. 17(6-7): pp. 717-34. http://dx.doi.org/10.1080/13549839.2012.665861

Kapsch, M.-L., H. Eicken, and M. Robards (2010). Sea ice distribution and ice use by indigenous walrus hunters on St. Lawrence Island, Alaska. In: Krupnik, I., C. Aporta, S. Gearheard, L. Kielsen Holm, and G. Laidler, (eds.), SIKU - Arctic Residents Document Sea Ice and Climate Change, pp. 115-144. Springer, Berlin.

Keskitalo, E.C.H. (2012). Climate change as governmentality: technologies of government for adaptation in three European countries. Journal of Environmental Planning and Management, 55(4): pp. 435-52. http://dx.doi.org/10.1080/ 09640568.2011.607994

Keskitalo, E. C. H. and A. Kulyasova (2009). The role of governance in community adaptation to climate change. Polar Research, 28(1): pp. 60-70. http://dx.doi.org/10.1111/j.1751-8369.2009.00097.x

Kirmayer, L. J., S. Dandeneau, E. Marshall, M.K. Phillips, and K.J. Williamson (2011). Rethinking resilience from Indigenous perspectives. Canadian Journal of Psychiatry, 56(2): pp. 84-91. 
Kirmayer, L. J., G.M. Brass, T. Holton, K. Paul, C. Simpson, and C. Tait (2007). Suicide Among Aboriginal People in Canada. Aboriginal Healing Foundation, Ottawa.

Knox, P. and H. Mayer (2013). Small Town Sustainability: Economic, Social and Environmental Innovation. Birkhaüser, Basel.

Kurlandsky, M. (1997). Cod. A Biography of the Fish That Changed the World, Penguin Books, New York.

Laidler, G. J., J. D. Ford, W. A. Gough, T. Ikummaq, A. S. Gagnon, S., Kowal, K. Qrunnut, and C. Irngaut (2009). Travelling and hunting in a changing Arctic: assessing Inuit vulnerability to sea ice change in Igloolik, Nunavut. Climatic Change, 94(3-4): pp. 363-397. http://dx.doi.org/10.1007/s10584-008-9512-z

Loring, P. A. (2010). Ways to help and ways to hinder. Climate, health and food security in Alaska. Unpublished PhD Dissertation, University of Alaska, Fairbanks.

Loring, P. A., and S. C. Gerlach (2009). Food, culture, and human health in Alaska: an integrative health approach to food security. Environmental Science \& Policy, 12: pp. 466-478. http://dx.doi.org/10.1016/j.envsci.2008.10.006

Martin, S. (2009). The effects of female out-migration on Alaska villages. Polar Geography, 32(1-2): pp. 61-67. http://dx.doi.org/10.1080/10889370903000455

Megatrends (2011). Rasmussen, R.O. (ed). Nordic Council of Ministers, Copenhagen.

Nilsson, C., R. Janssen, E. C. H. Keskitalo, T. Vlassova, M-L. Sutinen, J. Moen and F. S. Chapin, III (2010). Challenges to adaptation in northernmost Europe as a result of global climate change. AMBIO, 39(1): 81-84. http://dx.doi.org/10.1007/ s13280-009-0010-y

Odland, J. Ø., B. Deutch, J. C. Hansen, and I. C. Burkow (2002). The importance of diet on exposure and effects of persistent organic pollutants on human health in the Arctic. Acta Paediatr, 92(11): 1255-1266. http://dx.doi.org/10.1111/ j.1651-2227.2003.tb00494.x

Pedersen, P. and T. Nyseth (2013). Urban Sámi and the city as a frame for the development of a new Sámi cultural form in Scandinavia. In: Hansen, K.G., R.O. Rasmussen, R. Weber, (eds.), 2013. Proceedings from First International Conference on Urbanisation in the Arctic. Nordregio Working Paper 2013:6, Stockholm.

Rasmussen, R.O. (2013): Diasporas in the Arctic urbanization. In: Hansen, K.G., R.O. Rasmussen, R. Weber, (eds.), 2013. Proceedings from First International Conference on Urbanisation in the Arctic. Nordregio Working Paper 2013:6, Stockholm.

Rasmussen, R. O. (2010). Climate Change, the informal economy and generation and gender response to changes. In: Winther, G. (ed). The Political Economy of Northern Regional Development, Vol. I. TemaNord, 2010: 521. Nordic Council of Ministers, Copenhagen.

Rasmussen, R. O. (2007). Adjustment to reality - Social response to climate changes in Greenland. In: Borre Orbaek, J., R. Kallenborn, I. Tombre, E. N. Hegseth, S. FalkPetersen, A. H. Hoel, Arctic Alpine Ecosystems and People in a Changing Environment, pp.167-180. Springer, New York. http://dx.doi.org/10.1007/ 978-3-540-48514-8_10

Rasmussen, R. O., 2005. Analyse af fangererhvervet. Grønlands hjemmestyre [Analysis of the hunting sector. Greenlandic Home Rule, Nuuk.

Rasmussen, R. O. (1998). Settlement structure, resource management, and sustainable development - mega projects vs. local participation in Greenland. In: Duhaime, G., R.O. Rasmussen, and R. Comtois. Sustainable Development in the North: Local Initiatives vs Megaprojects, pp: 47-87. Proceedings of the Second Circumpolar Social Science Ph.D. Network Conference, held in Aguanish, Quebec, 1997. GETIC, Sainte Presses de l'Université Laval. 
Rees, W. G., F.M. Stammler, F.S. Danks, P. Vitebsky (2008). Vulnerability of European reindeer husbandry to global change. Climatic Change, 87(1-2): pp. 199-217. http://dx.doi.org/10.1007/s10584-007-9345-1

Revich, B. A. (2009). Climate change as a new risk factor for health in the Russian North. Human Ecology, 6: pp. 11-16.

Roto, J., J. Grunfelder and L. Rispling (eds.) (2014). State of the Nordic Region, 2013. Nordregio Report 2014:1. Stockholm.

Seixas, C.S. and F. Berkes (2003). Dynamics of social-ecological changes in a lagoon fishery in southern Brazil. In: Berkes, F., J. Colding, and C. Folke (eds.). Navigating social-ecological systems: building resilience for complexity and change, pp. 271298. Cambridge University Press, Cambridge.

Shields, R. and B. Weber (2013). Iqaluit - growth of an Inuit capital. In: Hansen, K.G., R.O. Rasmussen, R. Weber, (eds.), 2013. Proceedings from First International Conference on Urbanisation in the Arctic. Nordregio Working Paper 2013:6, Stockholm.

Skeldon, R. (2001). The dangers of diaspora: orientalism, the nation-state and the search for a new geopolitical order. In: Siddique, M. A. B. (ed.), International Migration into the 21st Century: Essays in Honour of Reginald Appleyard, pp. 109-125. Edward Elgar, Cheltenham.

Sloan L. (ed) (2006). Women and natural resource management in the rural north. Arctic Council Sustainable Development Working Group 2004-2006. Forlaget Nora, Norfold.

Sørensen, B. W. (2008). Perceiving Landscapes in Greenland. In: M. Jones and K. R. Olwig (eds.), Nordic Landscapes: Region and Belonging on the Northern Edge of Europe, pp. 106-38. University of Minnesota Press. Minneapolis.

State of Alaska (2009). Climate change in Alaska. http://www.climatechange.alaska.gov/ (21st February 2013).

Stairs, A., and G. Wenzel (1992). "I am I and the environment": Inuit hunting, community, and identity. Journal of Indigenous Studies, 3(1): pp. 1-12.

Storey, K. and L.C. Hamilton (2004). Planning for the impacts of megaprojects: two North American examples. In: R.O. Rasmussen and N.E. Koroleva (eds.) Social and Economic Impacts in the North. pp. 281-302. Kluwer Academic Publishers, Dordrecht.

Tremblay, M., C. Furgal, C. Larrivee, T. Annanack, P. Tookalook, M. Qiisik, E. Angiyou, N. Swappie, J-P. Savard, M. Barret (2008). Climate Change in Northern Quebec: Adaptation Strategies from Community-Based Research. Arctic, 61: pp. 27-34.

Vinokurova, L. I. (2011). Rural Yakutia: perception Indigenous population changes in environment. Arctic and North, 4: pp 154-61.

West, J. and G.K. Hovelsrud (2010). Cross-scale adaptation challenges in the coastal fisheries: findings from Lebesby, Northern Norway. Arctic, 63(3): pp. 338-354. http://dx.doi.org/10.14430/arctic1497

Winther, G. (2000). The Dutch disease phenomenon in a Greenland perspective. Northern Communities and the Global Economy: Circumpolar Arctic Social Sciences Ph.D. Network (CASS): Proceedings of the Fifth CASS Field Course, Alaska 2000, pp. 89-96. University of Alaska Fairbanks, Department of Alaska Native and Rural Development, Fairbanks. http://vbn.aau.dk/en/persons/gorm-winther(8d4ce1356ea0-4ead-b269-714066735f48).html

Zalkind, L. (2013). Review of Russian Arctic Regions: urbanization, economy, demography. In: Hansen, K.G., R.O. Rasmussen, and R. Weber (eds.), 2013. Proceedings from First International Conference on Urbanisation in the Arctic. Nordregio Working Paper 2013:6, Stockholm. 


\title{
12. Major Findings and Emerging Trends in Arctic Human Development
}

\begin{abstract}
Lead Authors
Joan Nymand Larsen, Stefansson Arctic Institute, Iceland and Gail Fondahl, University of Northern British Columbia, Canada

In this concluding chapter we highlight some of the main policy relevant conclusions from the Report's chapters, present key gaps in knowledge on Arctic human development, and offer suggestions for future research in this area of study. While the report is written for a broad audience of Arctic residents, students of the North, and stakeholders in the future of the Arctic, we intend this chapter to be useful for policymakers involved in decisions regarding various facets of Arctic human development.

The preceding chapters have shown that the Arctic is not a homogenous region. Impacts of globalization and environmental change differ within and among regions, between Indigenous and non-Indigenous northerners, between genders and along other axes. This volume has drawn attention to the diversity and important contrasts characterizing the Arctic. Nevertheless, in assessing the state of human development in the Arctic it is useful to identify a number of over-arching trends and issues that cut across various systems and thematic areas covered in this report.

While we have strived to avoid repetition, the inter-related nature of aspects of human development necessitates some reiteration when categorizing discussions under distinct headings. Under some headings, we have thus abridged our discussion to minimize repetition where key trends and gaps have already been detailed.
\end{abstract}

\subsection{Crosscutting trends}

Urbanization in the Arctic, and more broadly, "communities on the move," can be seen in the growing trend toward outmigration from local communities toward larger settlements and the increasing concentra- 
tion of population in urban centers. Associated with this mobility are "brain waves" and a "brain drain", linked in particular to changes in economy and resource development. Urbanization is distinctly gendered, with an increasing rate of female out-migration from smaller communities for educational opportunities and employment. We also note the emergence of "climigration" as a new dimension of Arctic mobility. Aging of the population, especially dramatic in some rural places, brings concomitant changes in depencency ratios.

Second, there is a dramatic burgeoning of interest in the Arctic from beyond the region, resulting largely from climate change and perceived resource development opportunities, and fuelled in part by media hype. Residents and outsiders both are redefining the "new Arctic", with northern identities becoming more of an asset and the Arctic more marketable. We cannot with full confidence forecast where this "new Arctic" is heading, as some trends contradict each other: for instance, increased interest in non-renewable resource development balances against global commodity price shifts that make such less likely; human and fiscal capacity challenges confront development projects; and numerous other disparities noted in the chapters make future trajectories hard to predict.

Third, disparities in formal educational achievement continue to be significant in the northern regions of the Arctic states, compared to national averages. With increasing global linkages come the intensifying need to build human capital and capacity. Although opportunities for formal education in the North have increased, both in urban centers and through distance education, we still observe (a heavily feminized) outmigration in pursuit of higher education. This situation perpetuates and exacerbates difficulties in meeting the ever-increasing demands for an educated workforce in the North.

Fourth, trends toward the increase in legitimate participation in Arctic decision-making and continued innovation in governance can be observed at all scales. We witness increased self-determination and autonomy in governance, including an increasing Indigenous representation in regional, national and international bodies. This entails ever-mounting demands on local and Indigenous representatives. It becomes increasingly important to find viable solutions to the growing human and fiscal resource challenges that accompany the increased participation opportunities. In terms of innovation, we note the trend both toward a broadening of informal resource govenance institutions and the elaboration of their relationships with more formal institutions.

And fifth, while we witness growing expectations for resource extractive industries, at the same time we also observe an increased interest in 
economic diversification, within and beyond extractive industries. The expansion of non-extractive industries is notable. While optimisim remains for large-scale industrial development, the high costs of doing business in the North remains a reality which climate change is not likely to change, and may indeed exacerbate in some places. Nevertheless, non-renewable resource development will remain a key economic base in the North in the near future, likely with ever-increasing efforts to locate production in order to minimize costs.

\section{Drying Fish, Alaska, USA}

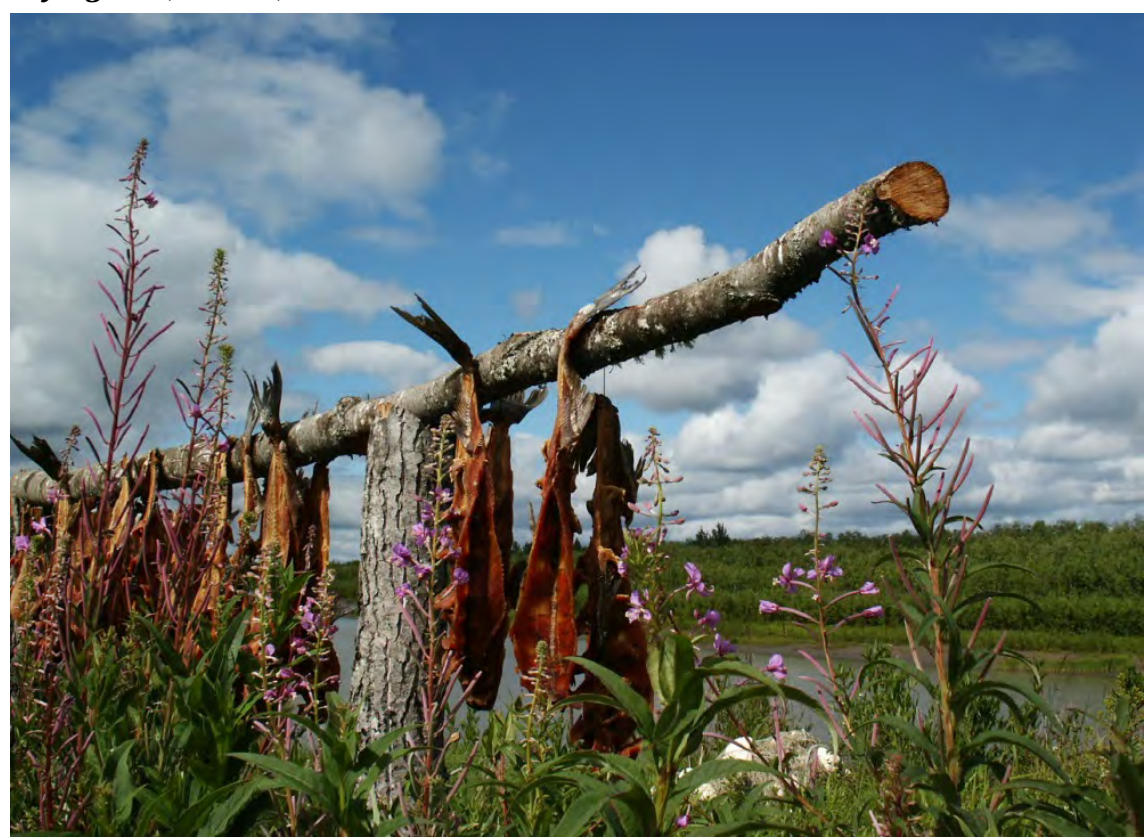

Credit: Diana Hirshberg.

\subsection{Policy-relevant conclusions}

\subsubsection{Arctic populations and migration}

- After decades of population growth, the size of the population of the Arctic appears to have stabilized at just over 4 million. At the same time, the booms and busts in the Arctic economy associated with the discovery and depletion of resources have always had, and will continue to have, a large influence on the size of regional populations. 
Demographic change and considerable demographic diversity characterizes the Arctic, with significant contrasts among its countries and regions. The Arctic population is experiencing robust growth in some regions, while substantial population declines typify most of the Russian Arctic. Overall, the population of the Arctic is projected to not increase by much in the near future. Most Arctic regions are at a late stage of demographic transtion, although exceptions to this are found in regions with large Indigenous populations.

The continued trend of outmigration from rural areas and increased concentration of the populations in the capital cities characterizes nearly all Arctic regions. Populations that are stable or declining, aging and losing females to outmigration, have implications for the allocation and distribution of human, financial and other resources.

So-called "climigration", mobility triggered by climate change, is an area that needs further study, in order to gain a fuller understanding of the impacts of climate change on migration and settlement patterns and choices. The gendered, age and "ethnic" dimensions of such mobility need to be investigated more thoroughly. Such mobility likely has marked repercussions for community viability in the Arctic, which we as yet do not fully comprehend and therefore for which we cannot adequately plan.

Given the speed of change, and the importance of demographic changes to Arctic human development, there is a need to further explore how demographic changes relate to social, cultural, economic, political, and enviromental changes. At the same time, many of the same trends and issues of a decade ago continue: the small size of the population, and outmigration remain important challenges.

\subsubsection{Cultures and identities}

- Interest in and awareness of the Arctic is growing, and Indigenous culture is increasingly seen as a resource in the North. Great cultural variation and complexity characterize the Arctic. For Arctic Indigenous peoples the challenge is not of choosing between "modernity" and "tradition", but to find a fulfilling combination of the two.

Several key trends noted a decade ago continue: increase connectedness between Arctic regions and non-Arctic national and international centers; decrease of local language use; and increased cultural revitalization efforts. A new trend is the marked increase in the global awareness of the Arctic region, which can be attributed in large part to the global climate 
change discourse and to expectations about future economic development opportunities in the North. Yet, as argued in Chapter 4 (Economic Systems), there is reason to be cautious about the current optimism surrounding resource development in the region, as costs are likely to remain high. Net costs and benefits to Arctic residents and cultures of the growth in interest in the Arctic remain uncertain, as regards distributional issues and environmental impacts of resource development.

While local cultures and identities continue to be vulnerable to modernization, globalization and urbanization, cultures in the Arctic, especially Indigenous cultures, have increasingly become a resource, in the sense of both a commodity and tool. A related trend that has strengthened greatly over the past decade is considering the North as marketable, which presents the Arctic region with a strategic advantage: being in and of the North presents new opportunities.

The rapid changes of a globalized world will continue to profoundly impact the younger generation. The future of Arctic cultures and identities will rest on the choices these young people make. It is critical to better understand the hopes and aspirations of Arctic youth, and how these are linked to the persistence and evolution of Arctic Indigenous cultures and identities.

\section{Barrow Teen Centre, Alaska, USA}

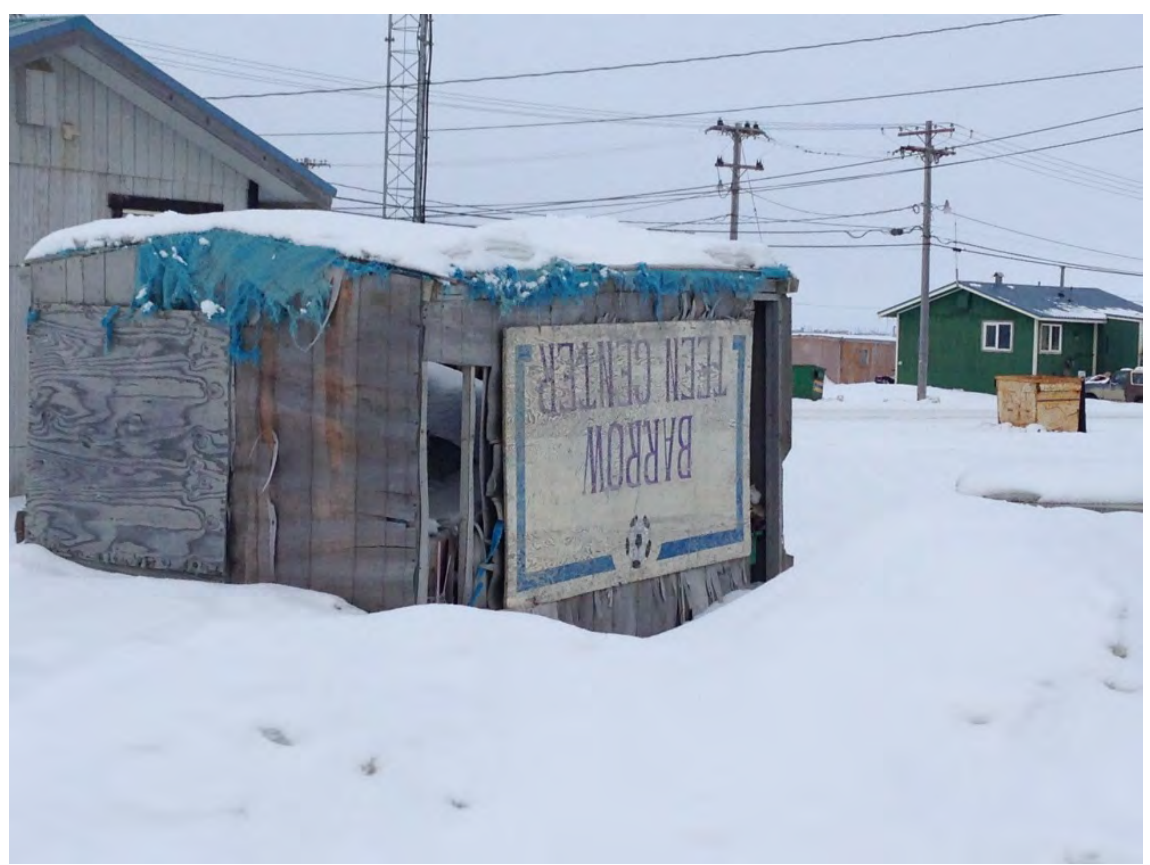

Credit: Diana Hirshberg. 


\subsubsection{Economic systems}

- The Arctic will remain a high cost region. The effects of environmental changes in the Arctic may in some cases benefit economic development, but in others will make resource development more costly. Natural resource production will continue as a driving force of the Arctic economy, although expectations of higher prices and lower costs for Arctic resources may be overly optimistic.

The Arctic economy continues to rest on three basic pillars: large-scale natural resource production for the international market, small-scale traditional production for local consumption, and transfers from higher levels of government. Over the past decade, the Arctic has experienced dramatic economic expansion and increased expectations for future investment in, and development of, oil, gas and other natural resources. But such expectations may prove overly optimistic, as the Arctic will remain a high cost region, not least because of persistent high costs of infrastructure and net costs of climate change. Uncertainty in commodity prices also hinders investment.

Diversity characterizes the Arctic economy. As well, local responses to world markets may diverge, reflecting differences in the concentration of resources, variation in the quality of resource concentrations (e.g. deposits), and the level of transport infrastructure.

While the first AHDR (2004) described the steep disparities between the value of what is produced in the Arctic and what actually remains there for consumption and investment opportunities, AHDR-II identifies a positive trend towards more economic benefits to the region. Recent institutional changes in the North have increased the local control and ownership of northern resources in some parts of the Arctic, enhanced the role of residents in decision-making about resource production, and improved the share of resource rents and income that stays in the region. While continued institutional change is likely to influence the future of the Arctic economy, questions remain on what types of institutions work best to improve the economic well-being of northern residents.

Traditional hunting, herding and fishing continue to be important to the economy of the Arctic, but questions remain about the role and significance of the traditional sector in the northern economy. The mix of formal and informal economic activity, and the changing relative shares of these, has significant implications for human development and the viability of Arctic coummunities. We need to understand better the in- 
teraction and interdependencies between these sectors, as well as their connections to cultural, mental and social well-being.

\section{Hurtig Ruten, Norway, 2009}

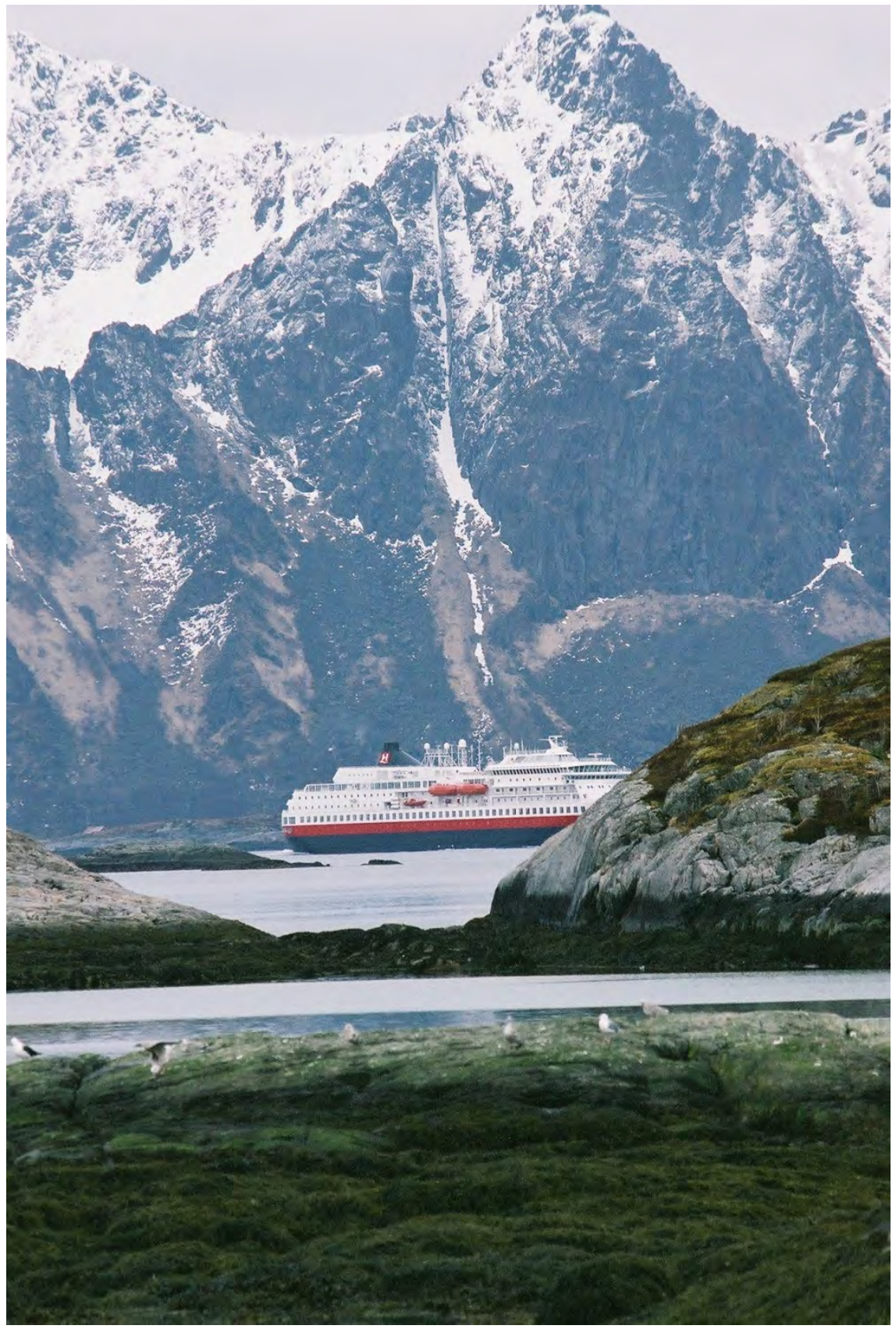

Credit: Harald Finkler. 


\subsubsection{Political systems}

- Devolutionary pressures continue to be a defining feature of political systems in the Arctic. Human and financial capacity challenges to devolution also persist. Whether and how these basic concerns of governance can be addressed will determine outcomes regarding social issues, economic opportunities, infrastructure, and land and environmental management.

Over the past decade, governance systems in the Arctic have continued to evolve. A key trend has been the continued process of devolution from central government to the regional and local levels. The efforts of Indigenous peoples to achieve self-determination and forms of selfgovernment - as seen, for instance, in the case of Nunavut and Greenland - have been fundamental to the emergence of new and innovative forms of governance and the growth of regional autonomy. While devolution must be seen as a success story it has not come without great challenges. The transfer of authority has brought significant benefits to the regional and local levels and their inhabitants, yet the attendant increased roles and responsibilities make great demands on human and financial capital. Finding creative solutions to the persistent gaps in human and fiscal capacity is crucial.

Likewise, while the increased representation of Indigenous organizations and regional governments in a growing number of international fora and committees, including the Arctic Council, is a success story, such representation puts further pressures on already limited resources. Growing demands on time and financial resources are certain to continue as non-Arctic parties become increasingly engaged in Arctic affairs. In the face of such increasingly vocal non-Arctic stakeholders, Arctic citizens and groups will want to ensure their voices continue to prevail.

\subsubsection{Legal systems}

- While there is a trend towards the increased adoption of Arcticspecific norms, global norms will continue to play a dominant role. Arctic states will continue to rely on international law and norms, Arctic-specific and global, to resolve territorial delimitation disputes in the Arctic.

Arctic-specific and global norms both play important roles in the legal systems that encompass the Arctic. While there is a trend towards the increased adoption of Arctic specific norms, global norms will continue 
to play a critical and central role. This has to do with the issue of scale, where challenges such as climate change and globalization cannot be solved locally or regionally, but rather necessitate regulations that extend beyond national borders. In terms of maritime boundary delimitation issues, despite media hype about lingering disagreements, Arctic states appear to be committed to resolving such disputes through cooperation and the application of global legal norms.

\subsubsection{Resource governance}

- Arctic resource governance continues to be innovative, while growing in complexity, and with increasing attention to the adoption of best practices.

Trends in resource governance first highlighted in AHDR (2004) continue: the growing importance of clearly delimited territorial rights; the incorporation of traditional and local ecological knowledge in decisionmaking; devolution of power to local decision makers; and the increased involvement of Arctic peoples in ownership and development of resources (see above). Emerging are trends of increasing complexity of resource governance, and of the adoption of best practices as ways to address uncertainty and unpredictability in a changing Arctic. Climate change, changes to Arctic economies, intensifying resource development, and changes in environmental and land use management, raise questions of how to best steward natural resources in the Arctic. Over the past decade, the extent of new challenges, including the increasing rate and magnitude of climate change, has elevated interest in adaptive resource governance, given its flexibility and its potential to better include different types of knowledge systems (traditional and scientific).

Both formal and informal institutions have an important role to play in promoting responsiveness to conditions of uncertainty and rapid change. Informal institutions at the regional and international levels can provide greater flexibility, but the enforcement of formal rights and specified laws can also be required. With consultation processes on the rise, ensuring meaningful consultation of local populations is paramount in decision-making processes regarding resources. 
Also on the rise is the adoption of best practices in Arctic resource governance. We need to further document these experiences, as well as the interplay of informal and formal institutions, and examples of effective consultation and of best practices in resource governance. Doing so will assist the transfer of their basic lessons both within and among different legal and management systems throughout the Arctic (and beyond).

\subsubsection{Human health and well-being}

- Among emerging threats for human health and well-being are the direct and indirect impacts of climate change, including worsening food and water security, changes in the pattern of infectious diseases, and impacts on health care infrastructure. Continuing threats to wellbeing also include mental health problems, high levels of suicide, accidents and domestic violence. The aging of the Arctic population demands attention.

The emerging threats related to accelerating climate change, the persistance of health outcome disparities experienced by the Indigenous populations, and the continuation of population aging all require the attention of policy makers and health service agencies. Complex interactions among genetic, economic, social, cultural, political and environmental factors affect human health and well-being in the Arcitc. Accelerating globalization and climate change pose new challenges for individuals and communities in the Arctic, and create new groups at risk of marginalization.

People in the Circumpolar North do not all enjoy the same health: substantial disparities exist across countries and regions, and within regions among population subgroups, particularly between Indigenous and non-Indigenous people. These disparities require the attention of policy makers and health service agencies.

Climate change is affecting many aspects of life, from stimulating migration to compromising food and water security, with implications for health and well-being. Globalization forces have contributed to increased rates of modern diseases such as obesity, diabetes and cardiovascular diseases. In many Indigenous communities in the Arctic we see a steady dietary transformation from "traditional" foods based on hunting and fishing to "western" foods. Water quality, an on-going issue in many Arctic communities, potentially faces further deterioration as the climate changes. Indicators of food and water security need to be incorporated into surveillance and monitoring programs across the Arctic. 
The observed aging trend in the Arctic (described in Chapter 2, Arctic Populations and Migration), a process expected to continue in the coming decades, has implications for health and well-being. The elderly may be able to make important contributions to Arctic development and community viability, if barriers to doing so are removed. We require a deeper understanding of the complexity of aging in Arctic populations, how Arctic societies will adapt to the aging population, and how the elderly can be better integrated into societal life.

Polaris Youth Centre, Salekhard, Russian Federation, 2006

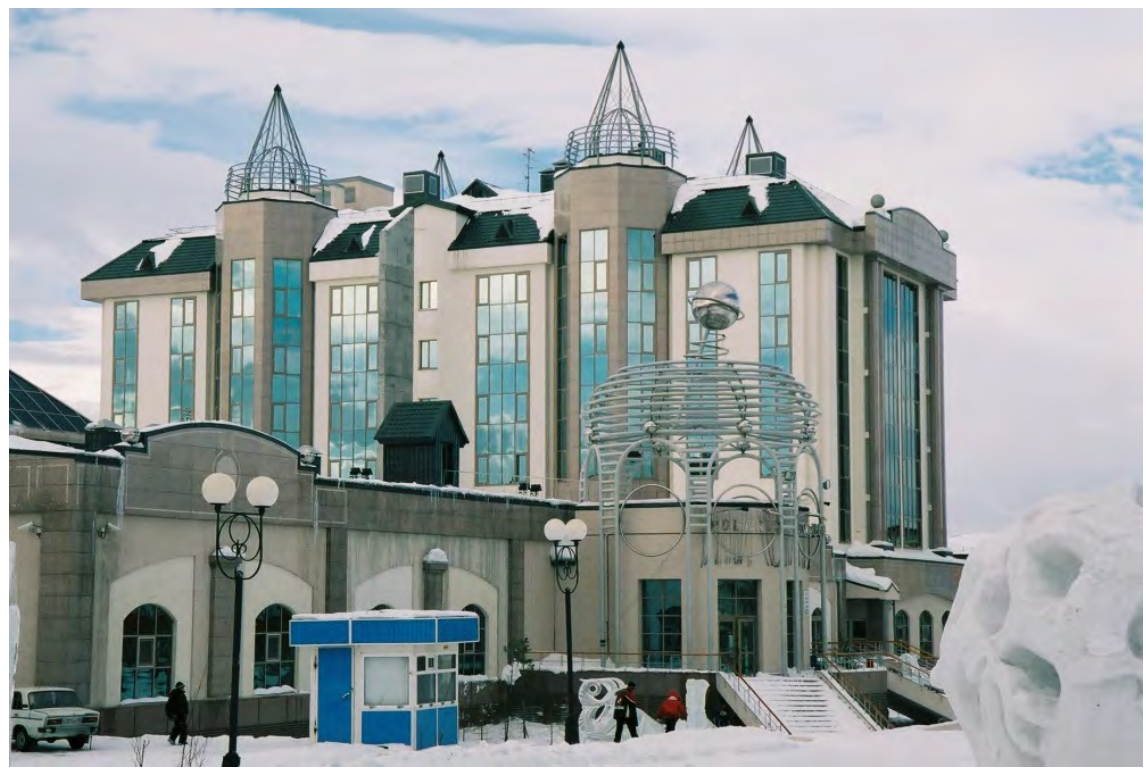

Credit: Harald Finkler

\subsubsection{Education and human capital}

- Disparities persist in education outcomes across the North. An increasing incorporation of Indigenous language instruction and the inclusion of traditional knowledge in formal schooling is counterpoised against a continuing erosion of Indigenous languages and traditional knowledge. The development of human and especially creative capital is crucial to the future of Arctic societies and economies.

The first AHDR (2004) emphasized as the most critical concerns in education the control of, relevance of, and access to, education in the Arctic. These concerns were contextualized in recognition of a legacy of western knowledge being prioritized over Indigenous knowledge. In the past 
decade, to address concerns over access, we see the expansion of both post-secondary institutions and distance education across the Arctic. In terms of the latter, we witness the case of the UArctic, where post secondary courses can today be accessed from just about any place in the Arctic, at least technologically. While institutions like the UArctic have made outstanding contributions to delivering post-secondary education in the Arctic, significant challenges persist. These include the delivery of primary and secondary education in rural and remote locations, including the recruitment and retainment of teachers.

Many other challenges continue to face education in the Arctic: providing education in Indigenous languages and with the integration of significant Indigenous knowledge content so that students perceive a relevance to their studies; addressing the low achievement and high dropout rates among Indigenous populations; and addressing the continued gender gap between males and females in formal educational attainment. We note improvements in the use of Indigenous languages in formal schooling and the growing recognition of the importance of local and Indigenous knowledge in (and beyond) the formal education system, although much work remains on this front. Maintaining the trajectories of these more positive trends and addressing challenges of better developing human capital across Indigenous and non-Indigenous, and male and female, Arctic residents will be fundamental to the future of human development in the region.

\subsubsection{Globalization}

- In many instances globalization means increased dependency of local interests on external powers and unstable markets. At the same time, the forces of globalization bring many economic opportunities to northern areas, including increased resource development and employment. Globalization brings greater cultural diversity, augmenting but also potentially attenuating local cultural traditions and institutions.

Ever-increasing connectivity to the rest of the world has intensified the effects of modernity and industrialization over the past decade. The Arctic's relatively small size explains why some effects of globalization are felt more strongly there than elsewhere. Increased economic globalization can result in heightened dependency of local interests on external powers and unstable markets. Developments flowing from economic globalization also provide benefits to the Arctic and its regions, in the 
form of investment and employment. Political globalization, while contributing to the weakening of the nation-state's role, also potentially increases the empowerment of new regional actors in the Arctic. Culturally, globalization not only erodes local cultural institutions, but paradoxically can offer new forces to support local cultural development. For instance, the marketability of the North, including northern cultural traditions, appears to be a growing Arctic asset (as noted above). Whether the Arctic will achieve net positive benefits from increased globalization - economic, political and cultural - hinges on the development of human capital, evolving political/governance institutions, and the application of legal instruments protecting the rights of indivduals and collectives, where necessary.

\subsubsection{Community viability and adaptation}

- Community connections are being transformed by increasing globalization, with responses varying widely across the Arctic. Differences exist in gender, age and ethnicity with respect to outmigration, vulnerability, risk exposure, and coping ability.

This report has documented the wide diversity across the Arctic in terms of the various human systems. The heterogeneity characterizing the region means that impacts and outcomes differ among Arctic communities. This diversity is well illustrated in the present discussions on communities in the Arctic and their ability to adapt in a time of rapid change. Communities and settlements in the Arctic are increasingly confronted with different and interrelated issues: increased mobility, including an increasing rate of out-migration; an increasing gender imbalance; an aging population. In some places immigration and the presence of shift-workers alter community cohesion. Such trends test the viability and adaptability of Arctic communities. The complexity of changes occurring in the Arctic today is amplified by the changing climate. 


\subsection{Gaps in knowledge}

This second assessment of the state of human development in the Arctic, and its focus on the major trends unfolding in these times of rapid change, has brought attention to a number of critical gaps in knowledge about human development and living conditions in the North. In this section we list the most critical cross-cutting gaps in our knowledge, those that extend across the various systems and thematic issues. In identifying such gaps, we address direct issues of climate change only incidentally, as other reports, including those being prepared for the Arctic Council (Arctic Resilience Report, Adaptation Actions for a Changing Arctic), as well as national reports (e.g. The Arctic in the Anthropocene [NRC 2014]) focus much of their attention to this aspect of change.

\subsubsection{Understanding the needs of Arctic youth and elderly}

The population is aging in many parts of the Arctic, triggering the need to better understand the social, cultural, economic and political role this sector of the population does and could play. At the same time, the future of the Arctic is connected with its youth. While demographic information has for the most part increased in availability and accessibility over the past decade (with exceptions due to changes in national censuses), there remains a need for more analysis of specific demographic cohorts, most notably youth and elderly.

\section{Youth}

The viability of Arctic cultures, languages, traditional activities, communities and settlements will rest on the choices young people make. Thus we need to better understand their aspirations, and the barriers to achieving these. Too many northern youth suffer from social alienation, which in its worst forms incites substance abuse and even suicide. We need to understand how formal education can be made more relevant, especially among Indigenous youth. What role can evolving technologies play in education and in local employment opportunities, including in the knowledge and creative economy? What is, and could be, the role of both education and the adoption of new technologies in shaping youth's attitudes toward traditional activities and northern settlements, as offering viable and attractive futures? What are the most strategic investments in human capital/capacity building that can be made to guarantee that youth - both male and female - wish to remain in or return to the North? 


\section{Elderly}

Aging in the Arctic is little studied - we need to understand its complexities. How will Arctic societies adapt to the aging population, and how can the elderly be better integrated into societal life? What role might elderly citizens contribute to economic, cultural and political development? How can increased old-age dependency rates be addressed? What role do the elderly play in contributing to community cohesion and viability, to connecting with Arctic "diasporas" abroad, and how does this differ across Arctic regions and settlement types? We also need to know more about the dimension of well-being of elderly, including changing roles in family structure, and prevalence of neglect or abuse.

\subsubsection{Gendered dimensions of Arctic change}

While the past decade has seen a substantial expansion of research on gender in the Arctic, we still note significant gaps in knowledge. Men and women are not affected equally by climate change and globalization, and their impacts on traditional and non-traditional economic activities in the Arctic, on political systems, on education and health care provision, etc. We lack knowledge about the gendered dimensions of contemporary cultural practices and expressions, including on the (re)negotiation of cultural and other identities. Gaps remain in our knowledge about the gendered aspects of domestic violence in the North. The gendered characteristics of decision-making regarding resources at different scales need further investigation, especially due to the dynamism that characterizes such gendered roles regarding control of and access to resources. Insufficient attention has been paid to the gendered nature of Arctic geopolitics, at all scales, but especially at the national and international scales: how does masculinism drive the very issues that are dealt with in national and international forums regarding the Arctic? Ways in which to effectively address the growing gender gap in education and human capital development also are critical to human development in the Arctic, given the impact gendered out-migration has on the social life and economy of Arctic communities.

\subsection{3 "Soft securities": food, water, and energy}

Food security is declining in the Arctic. Both environmental change (climate change and long-distance pollution) and globalization play a role here, as local foods become less available or attractive, and imported foodstuffs of lesser nutritional value and high cost gain popu- 
larity. Contradictory results of food insecurity in the North include both increased hunger and heightened prevalence of obesity and diabetes. We need to better understand the evolving role of traditional activities that produce "country foods" in northern societies, and their evolving interdependencies with the industrial sector. We also need to better understand the contributions of the traditional activities to cultural, social and mental, as well as physical, well-being: food is strongly tied to culture. What economic policies can enhance food security? How can local educational initiatives contribute?

Climate and other environmental changes affect water security as well, while global shifts in energy prices and related transportation costs compromise energy security in many Arctic settlements dependent in imported fuel. Research is needed here on innovative, economically effective ways of providing these key physical components to human wellbeing. A diverse set of solutions will likely be necessary, give the diversity of northern places. To track progress on food, water and energy security, we also identify the need for a specific set of indicators. Developing these with local community members, in order that they may understand and effectively take part in such monitoring, is critical.

\subsubsection{Arctic settlements and communities}

Questions remain on the economic role of large settlements in the economic growth of Northern regions. Urban settlements may help to generate new types of economic activity and change the cost of resource development. Better knowledge of the economies of these urban places in the North and their relationship to the economies of their surrounding regions will increase our understanding of the Northern economy.

The stakes - both costs and benefits - of larger scale resource development projects appear particularly high for smaller Arctic communities. Such projects potentially involve influxes of new residents (often temporary), the opportunity to negotiate employment and revenue sharing agreements, the threat of negative environmental impacts, and a potential loss of local "fate control". Where have Arctic communities most benefited from such developments, and what factors ensured positive outcomes? 
In terms of community dynamics in the Arctic, we poorly understand the role of Arctic diasporas in contributing to community identity, economic condition, and political strength. Similar information is needed about the "new newcomers". We also need to understand more about racisms and other exclusionary practices in the North: along what axes are they expressed and performed, how do they affect long-term and new-comer populations, and how can these be effectively addressed?

\section{Town of Nanortalik, South Greenland}

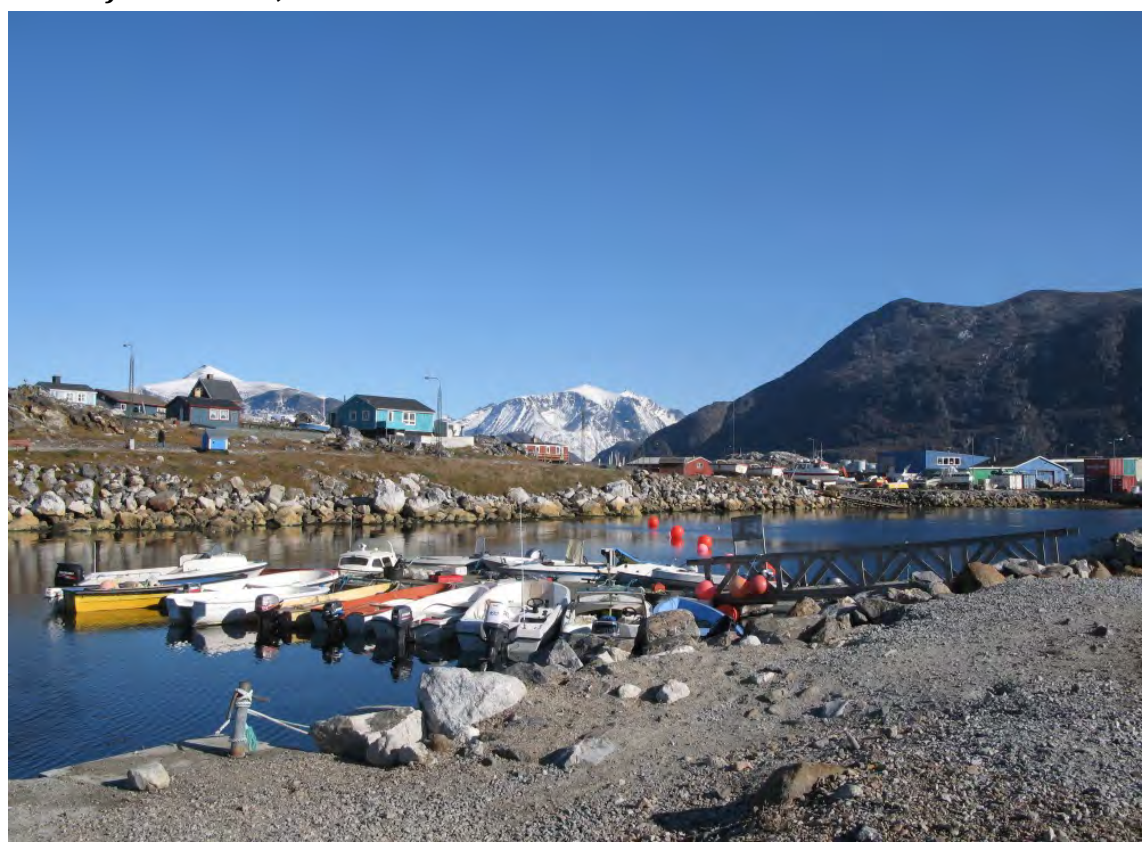

Credit: Joan Nymand Larsen

\subsubsection{Arctic institutions}

The introduction of a variety of new institutions represents one of the most significant changes in the Northern economy. Yet, we lack knowledge on what types of institutions work best to improve the economic well-being of northern residents; and what roles institutions, formal and informal, play in meeting future needs of human development in the North. New institutional arrangements provide residents of the North with more control over local resource projects. There is a need to know more about how informal institutions in the Arctic may complement formal institutions in achieve the best outcomes in resource management. 


\subsubsection{Global linkages and new Arctic actors}

Arctic studies have mostly focused internally on the region. Indeed, while in this report we have explicitly heeded global linkages, this has more been in terms of how global processes play out in the Arctic. Yet Arctic processes also impact the non-Arctic world at both the regional and global scale. We need to continue to work toward a more comprehensive understanding of the social, cultural, political, economic and environmental linkages between the Arctic and the rest of the world. How can we better evaluate the changing importance of the Arctic to the globe - economically, environmentally, and politically?

We need to better understand the ambitions of non-Arctic states in the Arctic, and the impacts these may have on Arctic future. How will increased interest by non-Arctic states in participation in forums, such as the Arctic Council, play out? How will their participation shape such forums and what will be the impact on the voices of Arctic participants (such as Permanent Participants in the Arctic Council)? How will these interests and activities vary across the non-Arctic states? And how will the role of other organizations, including global environmental, regulatory, and economic institutions, transform and adjust, as the political, economic and environmental landscape of the Arctic is altered?

\subsubsection{Promulgating and adopting best practices}

The Arctic has generated many innovations: in adaptive governance practices, in the inclusion of local and Indigenous voices in decisionmaking and also in research, in the development of power- and economic benefit sharing structures, and in initiatives related to distance education and telemedicine. Some of these innovations address the impacts of climate change and the myriad effects of globalization. While such innovations have strikingly contributed to Arctic human development, we still need greater insight into the conditions under which these innovations can be transferred to other northern places and what adaptations are required. How do we effectively transfer lessons learned in one part of the Arctic to other parts? We also need to address the challenges of capacity (human and fiscal), noted repeatedly in this report: doing so is essential to the co-production of the knowledge and strategies needed to address a changing Arctic. 
Best practices need to be identified and adopted for the preservation of Arctic languages and the incorporation of these languages and traditional knowledge into governance structures at all scales. We need to elaborate on best practices for establishing and maintaining genuine and trustworthy partnership between local communities and higher levels of government, local communities and researchers, and local communities and businesses. Acknowledging that local support for external resource development projects typically hinges on protection of local rights to the land and resources necessary to continue pursuing their traditional activities, we need to better understand the diversity of opportunities for ensuring such rights where resource development is also pursued.

We need to develop best practices for evaluating the impact of economic development initiatives in the Arctic, which fully calculate environmental, social and cultural costs and benefits. Better practices for remediation following resource development need to consider any benefits that infrastructure adjustments (e.g. adaptation rather than removal) might offer.

\subsubsection{Arctic social indicators and monitoring}

Efforts are needed to address the persistent gaps in data availability, the lack of common data protocols and the lack of primary data collection in order to enable the more complete measurement and tracking of Arctic specific social indicators. Also needed is work on the current set of ASI indicators and their technical definitions, in terms of fine-tuning these indicators to increase their strength and applicability, but also to reevaluate and adjust the set of indicators to better reflect the impact of global changes, including the complex interactions between bio-physical changes and changes in human systems. Additionally, we have highlighted the need to identify key indicators to enable us to better follow the changes in food, water and energy security in Arctic populations. Such indicators are recommended to be gender-sensitive, since such insecurities affect men and women differently.

Availability of, and access to, primary data presents significant challenges in devising and measuring indicators of human development in the Arctic. For instance, primary data collection on the size of the traditional economy is needed to help answer questions on the role and significance of the traditional sector in the Arctic economy across the Circumpolar North. Traditional harvesting plays an important economic and cultural role throughout the North, but comparing this activity to activities that take place in the resource and public sectors in many 
countries remains problematic. Developing measures of the extent and significance of this economic activity would allow a more complete picture of the Northern economy.

In general, data gaps and lack of primary data prevent us from making circumpolar wide comparisons. Especially difficult is the application of the indicators developed by the ASI project for the specific dimensions of Arctic human development identified by the first AHDR (2004) on "closeness to nature", "cultural well-being", and "fate control". While gathering, accesssing, and filling these data gaps remain formidable, challenges also extend beyond data to issues of indicator concepts, definitions and standardized measures, as well as questions of data storage and management. The implementation of an ASI monitoring system would contribute greatly to addressing these critical gaps and to tracking human development in a manner beneficial to policy-makers.

\section{The original 'Eskimo row', Disko Bay, Greenland}

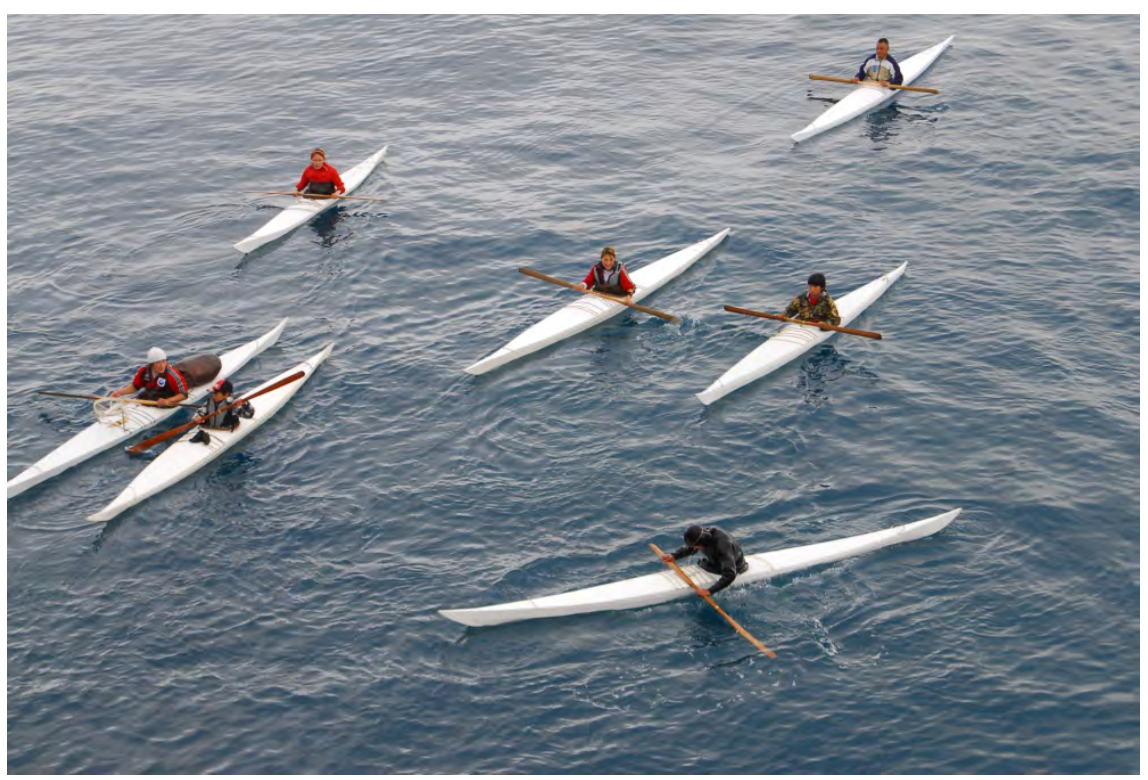

Credit: Peter Prokosch. 


\subsection{Conclusion}

The current assessment of human development in the Arctic has shown that the region continues to face significant challenges, e.g., outmigration from local communities, emerging health challenges, emerging threats related to climate change, persistent disparities between Indigenous and non-Indigenous standards of living, continued gaps in education between Indigenous and non-Indigenous people and between males and females, growing human and fiscal capacity challenges, violence and discrimination, and threats to traditional livelihoods. At the same time, Arctic success stories are many - and should be underscored. While the region is facing rapid change and complex challenges, we can distinguish adaptation, innovation and resilience as characterizing many northern communities. This assessment has noted the many contrasts between and within regions across the Arctic, between Indigenous and non-Indigenous peoples, between males and females. As emphasized in our focus on Arctic community viability and adaptation, many factors come into play in determining the success and ability of communities to adapt to change. Examples of Arctic success stories highlighted in this volume include:

- The increasing use of Indigenous knowledge: There is increasing use of Indigenous languages in formal schooling in the Arctic, and a growing recognition of the importance of local and Indigenous knowledge in many aspects of life in the Arctic. There is also awareness that the increased use and recognition of Indigenous knowledge represents a potentially important contribution to advancing the competitive edge of the region.

- The increase in Indigenous participation, control and ownership: Recent institutional changes in the North have increased the local control and ownership of northern resources in some parts of the Arctic, and have had an important effect on the relationship between international and local economies. The continued process of devolution from central government to the regional and local levels has placed more control in the hands of Indigenous and local communities.

- The continued growth of innovative governance arrangements: The efforts of Indigenous peoples to achieve self-determination and forms of self-government have been fundamental to the success of the emergence of new and innovative forms of governance, and the growth of regional autonomy. This has brought significant benefits to the regional and local level and its inhabitants. 
- The emergence of Arctic identities and a sense of Indigenous identity becoming an asset rather than a hinderance: culture, especially Indigenous culture, in the North has increasingly become a resource, both in the sense of a commodity and in the sense of a tool that makes external recognition easier. Also, there is a growing sense of the marketability of the North. This may provide the region with a strategic advantage, where being in the North presents new opportunities. We also see an emerging trend to the coexistence of tradition and modernity in individual and community lives.

We conclude this report by offering a number of suggestions for followup activities. These are based on discussions with lead authors and contributing authors during the AHDR-II process.

Dissemination: The production of a second assessment of Arctic human development represents a significant effort in terms of time and resources, and in it brings together many (and sometimes divergent) perspectives from a whole range of disciplines. We suggest wide dissemination, including through a series of well-targeted "townhall" meetings in Northern settlements - to address a range of issues discussed and brought to the forefront in this volume, as a highly effective dissemination and outreach activity. Such townhalls would serve the purpose of reaching a variety of stakeholders and provide an opportunity to discuss issues addressed in the AHDR that are of importance to local communities, just as it provides critical feedback to the AHDR-II group which can help identify gaps in knowledge and identify issues for the next volume. We also suggest translation of the report, or at least its introduction and conclusion, including into Russian, Inuktitut and Mandarin.

Monitoring: There remains a critical need to implement a system for collecting, storing, and tracking data on Arctic social indicators, to provide a tool for measuring changes in human development in the Arctic, as outlined in the section above.

Science Plan: Efforts should be made to support and undertake the drafting of a science plan based on the findings and conclusions of this report. The AHDR-II team is in a unique position to provide a science plan for addressing critical gaps in knowledge on Arctic human development and living conditions in the circumpolar region.

AHDR and the youth: The AHDR (Volumes I and II) has targeted a broad audience, and has been written in a style to increase its accesibility to a broad range of audiences - from local communities to Northern colleges and universities to policy makers and the Arctic Council. Yet, as emphasized in this volume, the future of the Arctic will be determined by 
the choices of the youth and their aspirations and priorities as concerns culture and identities, where to study and where to live, and what occupations and lifestyles to pursue. We suggest the production of a followup version of the $A H D R$ that targets specifically the youth - the next generation of Arctic residents, and a new audience for the AHDR - which would include issues and topics of interest and importance to this group. This would not replace the next decadal assessment of human development in the Arctic $(A H D R-I I I)$, but serve as a companion volume.

\subsection{Reference}

[NRC] Committee on Emerging Research Questions in the Arctic; Polar Research Board; Division on Earth and Life Studies; National Research Council (2014). The Arctic in the Anthropocene. Emerging Research Questions. The National Academies Press, Washington, D.C. 



\section{AHDR Sammenfatning}

Formålet med AHDR-II - Arctic Human Development Report: Regional Processes and Global Linkages - er at give en opdatering til den første AHDR (2004) om menneskelig udvikling i Arktis; samt at fremhæve de vigtigste tendenser og forandringer indenfor en række emner og tematiske områder omkring menneskelig udvikling i Arktis i det seneste årti; og på baggrund af denne opdatering og vurdering, at identificere og fremhæve nogle politisk relevante konklusioner samt mangler i vores viden om Arktis; og at sætte fokus på fremtrædende arktiske succeshistorier.

AHDR-II søger at belyse og vurdere de vigtigste ændringer og trends i menneskelig og samfunds udvikling i Arktis i det sidste årti indenfor følgende systemer og tematiske områder: demografi; migration; økonomi; uddannelse; sundhed og velvære; kultur og identitet; politiske systemer; ressource styring; retssystemer; globalisering; og bæredygtige samfund og tilpasning.

Rapporten indeholder en bred vifte af konklusioner der har politisk$\emptyset$ konomisk relevans. Baseret på disse resultater, peger vi på en række tværgående tendenser med relevans for beslutningstagere, herunder Arktisk Råd. Vi konkluderer blandt andet, at den kombination af hurtige og stressbetonede forandringer, som blev fremhævet i den første AHDR (2004), fortsætter i dag, men forstærket og med forøget hastighed. De samfunds- og miljømæssige ændringer konfronterer på forskellig vis arktiske beboere, lokalsamfund og socioøkonomiske sektorer, og udfordrer trivslen i Arktis.

Urbaniseringen i Arktis accelererer, og migrationen fra landdistrikterne mod større bosættelser og byer, samt en forøget koncentration af befolkningen, og fremkomsten af "climigration", er alle voksende tendenser. Interessen for Arktis er vokset markant, hvilket tildels kan tilskrives klimaændringer og stigende forventninger omkring udviklingsmuligheder indenfor mineral- og andre ressource sektorer. Der er høje forventninger for vækst i ressource udvinding, men samtidig er der tegn på vækst i andre sektorer, som f.eks. turisme. Arktiske identiteter ses i voksende grad som et aktiv, og samtidig er arktiske beboere selv ved at omdefinere, hvad det vil sige at være "nordbo". Der fore- 
kommer øget deltagelse i arktiske beslutningsprocesser og en fortsat innovation i regeringsførelse.

Rapporten identifierer en række arktiske succeshistorier. Den aktuelle vurdering af den menneskelige udvikling i Arktis har vist, at regionen fortsat står overfor store udfordringer, men samtidig med er der mange eksempler på arktiske succeshistorier, og de indkluderer blandt andet: En stigende brug af oprindelige folks viden og brug af de oprindelige sprog i folkeskolen i Arktis; forøget lokal deltagelse, kontrol og ejerskab, hvor man blandt andet ser, at de seneste institutionelle ændringer i Arktis har skabt mere lokal kontrol og ejerskab i ressource sektoren i nogle dele af Arktis, som så også har haft en vigtig indflydelse på forholdet mellem internationale og lokale økonomier. Ligeledes er der en fortsat vækst i innovative forvaltningsordninger. Indsatsen blandt oprindelige folk for at opnå selvbestemmelse og nye former for selvstyre har været grundlæggende for succes i nye og innovative former for styring, samt vækst i selvstyre. Og afslutningsvis, identiteten blandt folkefærd i Arktis ses i stigende grad som et aktiv, hvor kulturen, og især den indfødte kultur, fremstår som en form for ressource.

AHDR-II peger på manglende viden indenfor nogle vigtige felter, her $\mathrm{i}$ blandt en manglende viden om ungdommens og den ældre befolknings behov og ambitioner. Befolkningen bliver ældre i mange dele af Arktis, hvilket har øget behovet for mere og bedre viden omkring de sociale, kulturelle, økonomiske og politiske roller denne del af befolkningen kan spille. Ligeledes, fremtiden i Arktis afhænger i stor udstrækning af ungdommen, deres valg, ambitioner og håb om fremtiden, og det er et område hvor der er brug for mere viden. Der er også brug for mere arbejde omkring udarbejdelsen af arktiske sociale indikatorer og deres tekniske definitioner, som kan bidrage til at fremme deres styrke og anvendelighed. Der er også huller i vores viden om køn og kønsroller i Arktis, samt en specielt markant mangel på data om køn indenfor de forskellige AHDR systemer og tematiske områder. Der er ligeledes brug for mere viden om bysamfund og institutioner i Arktis, og deriblandt hvilken rolle forskellige institutioner - formelle og uformelle - vil eller kan spille for at imødekomme de fremtidige behov der vil opstå omkring ændringer i folkets velfærd og udvikling i Arktis. 


\section{norden}

\section{Nordic Council of Ministers}

Ved Stranden 18

DK-1061 Copenhagen K

www.norden.org

\section{Arctic Human Development Report}

The goals of the second volume of the AHDR - Arctic Human Development Report: Regional Processes and Global Linkages are to provide an update to the first AHDR (2004) in terms of an assessment of the state of Arctic human development; to highlight the major trends and changes unfolding related to the various issues and thematic areas of human development in the Arctic over the past decade; and, based on this assessment, to identify policy relevant conclusions and key gaps in knowledge, new and emerging Arctic success stories.

The production of AHDR-II on the tenth anniversary of the first AHDR makes it possible to move beyond the baseline assessment to make valuable comparisons and contrasts across a decade of persistent and rapid change in the North. It addresses critical issues and emerging challenges in Arctic living conditions, quality of life in the North, global change impacts and adaptation, and Indigenous livelihoods.

The assessment contributes to our understanding of the interplay and consequences of physical and social change processes affecting Arctic residents' quality of life, at both the regional and global scales. It shows that the Arctic is not a homogenous region. Impacts of globalization and environmental change differ within and between regions, between Indigenous and non-Indigenous northerners, between genders and along other axes. 